A Practical Guide to the ZigBee Mesh Networking Protocol

\title{
Building
}

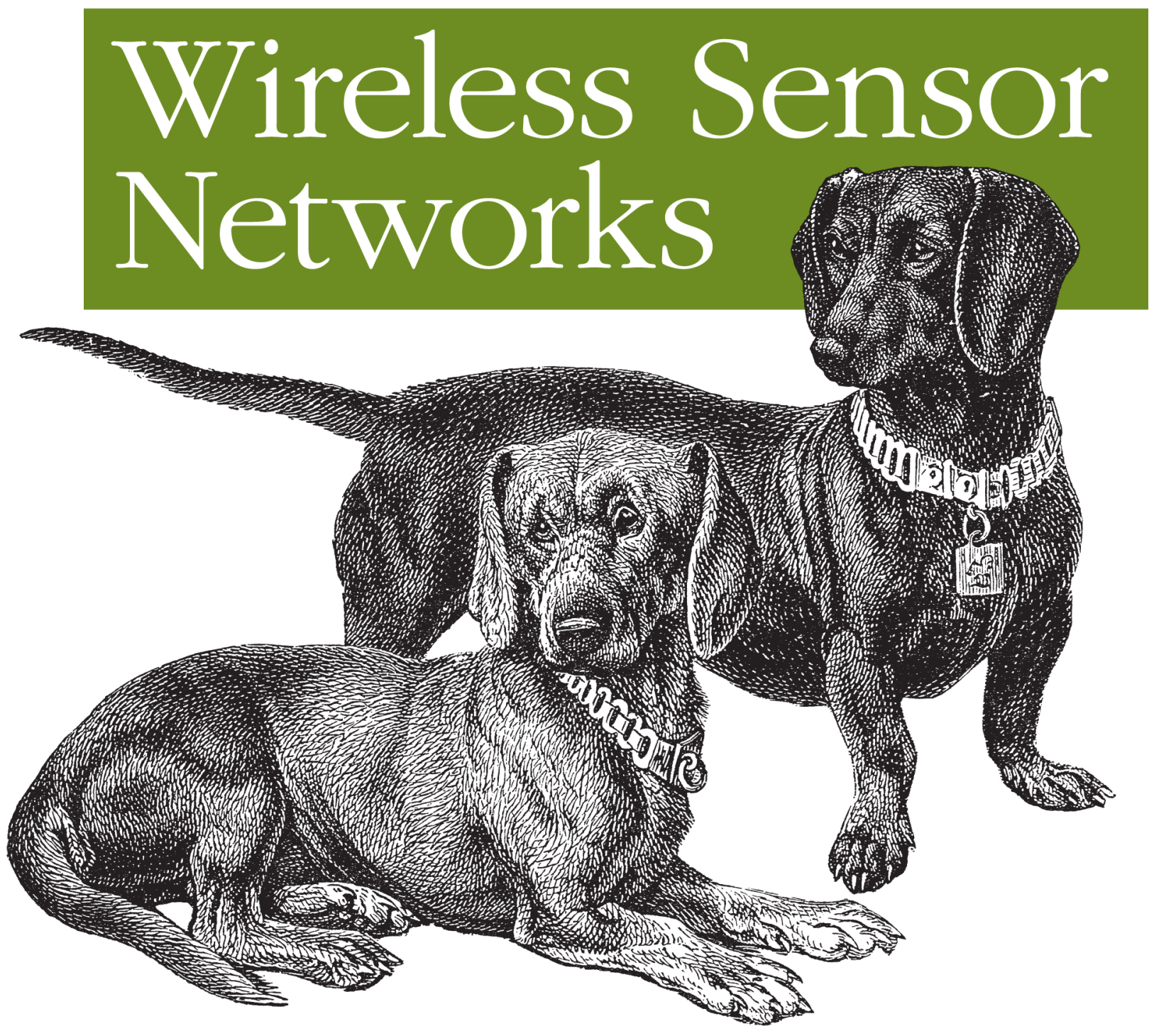




\section{Building Wireless Sensor Networks}

Get ready to create distributed sensor systems and intelligent interactive devices using the ZigBee wireless networking protocol and XBee radios. By the time you're halfway through this fastpaced, hands-on guide, you'll have built a series of useful projects, including a complete ZigBee wireless network that delivers remotely sensed data.

Radio networking is creating revolutions in volcano monitoring, performance art, clean energy, and consumer electronics. As you follow the examples in each chapter, you'll learn how to tackle inspiring projects of your own. This practical guide is ideal for inventors, hackers, crafters, students, hobbyists, and scientists.

Investigate an assortment of practical and intriguing project ideas

"It's about time! How terrific to finally bave a belpful guide for all the possibilities that these great little radios afford. If you want to make your electronics projects communicate without wires, this is the book you need." -Tom Igoe Associate Arts Professor, ITP/NYU

- Prep your ZigBee toolbox with an extensive shopping list of parts and programs

- Create a simple, working ZigBee network with XBee radios in less than two hours-for under $\$ 100$

- Use the Arduino open source electronics prototyping platform to build a series of increasingly complex projects

- Get familiar with XBee's API mode for creating sensor networks

- Build fully scalable sensing and actuation systems with inexpensive components

- Learn about power management, source routing, and other XBee technical nuances

- Make gateways that connect with neighboring networks, including the Internet

Robert Faludi, a professor at NYU and the School of Visual Arts, is an expert consultant on commercial projects, including large-scale home energy monitoring. His work has appeared in The New York Times, on CNet, on Good Morning America, and elsewhere. Robert is a CO-creator of the LilyPad XBee wearable radios and of Botanicalls, a system that allows thirsty plants to place phone calls for human help.

\section{O'REILLY $^{\circledR}$ oreilly.com}

Free online edition for 45 days with purchase of this book. Details on last page. 


\section{Building Wireless Sensor Networks}





\title{
Building Wireless Sensor Networks
}

\author{
Robert Faludi
}




\section{Building Wireless Sensor Networks}

by Robert Faludi

Copyright (C) 2011 Robert Faludi. All rights reserved.

Printed in the United States of America.

Published by O’Reilly Media, Inc., 1005 Gravenstein Highway North, Sebastopol, CA 95472.

O’Reilly books may be purchased for educational, business, or sales promotional use. Online editions are also available for most titles (http://my.safaribooksonline.com). For more information, contact our corporate/institutional sales department: 800-998-9938 or corporate@oreilly.com.

Editor: Brian Jepson

Production Editor: Adam Zaremba

Copyeditor: Sharon Terdeman

Technical Editors: Kate Hartman and Jordan Husney

Proofreader: Sada Preisch
Indexer: Angela Howard

Cover Designer: Karen Montgomery

Interior Designer: David Futato

Illustrator: Robert Romano

\section{Printing History:}

December 2010: First Edition.

Nutshell Handbook, the Nutshell Handbook logo, and the O'Reilly logo are registered trademarks of O'Reilly Media, Inc. Building Wireless Sensor Networks, the image of dachshunds, and related trade dress are trademarks of O'Reilly Media, Inc.

Many of the designations used by manufacturers and sellers to distinguish their products are claimed as trademarks. Where those designations appear in this book, and O'Reilly Media, Inc., was aware of a trademark claim, the designations have been printed in caps or initial caps.

While every precaution has been taken in the preparation of this book, the publisher and author assume no responsibility for errors or omissions, or for damages resulting from the use of the information contained herein.

RepKoverm

This book uses RepKover ${ }^{\mathrm{TM}}$, a durable and flexible lay-flat binding.

ISBN: 978-0-596-80773-3

$[\mathrm{M}]$ 


\section{Table of Contents}

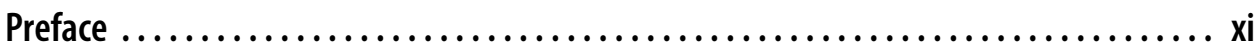

1. Getting Ready $\ldots \ldots \ldots \ldots \ldots \ldots \ldots \ldots \ldots \ldots \ldots \ldots \ldots \ldots \ldots \ldots \ldots \ldots \ldots \ldots$

Buying an XBee Radio $\quad 1$

Hardware 1

Antennas 4

Vendors $\quad 6$

Buying an Adapter 6

$\begin{array}{ll}\text { Digi Evaluation Board } & 7\end{array}$

USB Adapters $\quad 7$

Breakout Boards $\quad 10$

Arduino Board Adapter Hack $\quad 12$

What Are All Those Pins? $\quad 15$

$\begin{array}{ll}\text { Choosing a Terminal Program } & 15\end{array}$

Firmware Update Software $\quad 17$

Terminal Software for Configuring Settings 17

$\begin{array}{ll}\text { Summary } & 20\end{array}$

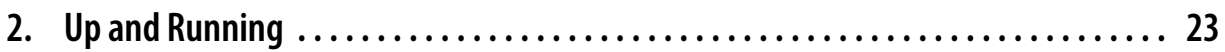

Radio Basics 23

Electromagnetic Spectrum $\quad 24$

Inverse Square Law 24

Introduction to ZigBee $\quad 25$

Network Topology 27

Addressing Basics 28

PAN Addresses $\quad 29$

Channels 29

All Together Now $\quad 29$

XBee Firmware Updates $\quad 32$

Reading Current Firmware and Configuration $\quad 35$

$\begin{array}{ll}\text { Configuring XBee } & 40\end{array}$ 
Settings $\quad 40$

Connecting from Windows 41

Connecting from Macintosh $\quad 43$

Command Mode and Transparent Mode $\quad 46$

AT Commands (Are Your Friend) $\quad 47$

Using AT Commands 49

Basic ZigBee Chat $\quad 50$

Parts $\quad 50$

Addresses $\quad 50$

Coordinator 51

Router 53

Two Computers 53

One Computer $\quad 54$

Chat 54

Troubleshooting $\quad 55$

Success! $\quad 56$

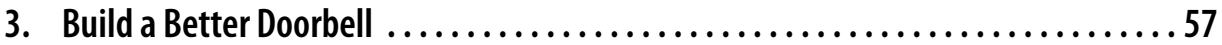

ZigBee and Arduino $\quad 57$

About Arduino $\quad 57$

Arduino Basics $\quad 59$

Connecting to Arduino 65

Doorbell Projects $\quad 67$

Parts $\quad 67$

Prepare Your Radios $\quad 68$

Connect Power from Arduino to Breadboard 68

XBee Breakout Board $\quad 68$

XBee Connections 69

Doorbell Introduction $\quad 72$

Switch Input...

....and Buzzer Output $\quad 73$

$\begin{array}{ll}\text { Configure Your XBees } & 75\end{array}$

Program the Arduino Doorbell $\quad 77$

Feedback Doorbell $\quad 80$

Feedback Light $\quad 81$

Program the Arduino Feedback Doorbell 81

Extra: Nap Doorbells and More $\quad 83$

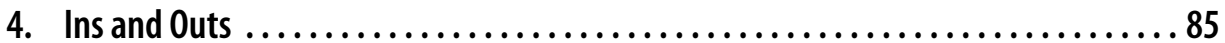

$\begin{array}{ll}\text { The Story of Data } & 85\end{array}$

Direct, Indirect, Subtext $\quad 85$

$\begin{array}{ll}\text { I/O Concepts } & 87\end{array}$

Why XBee Direct? 
XBee Direct Limitations $\quad 88$

XBee I/O Features $\quad 89$

AT Configuration I/O Commands $\quad 90$

Advanced I/O Commands 93

Romantic Lighting Sensor $\quad 93$

Basic Romantic Lighting Sensor 93

Prepare the Sensor Board $\quad 97$

Prepare the Base Station 99

Romantic Lighting Sensor with Feedback 104

API Ahead 109

5. API and a Sensor Network ................................. 111

What's an API? $\quad 111$

Protocols 113

Humans 113

Computers 113

XBee API Protocol 116

Start Delimiter 117

Length Bytes 118

Frame Data Bytes 118

Checksum 118

API Frame Types $\quad 119$

AT Commands $\quad 120$

AT Responses $\quad 122$

ZigBee Transmit Request $\quad 124$

ZigBee Transmit Status 127

ZigBee Receive Packet 129

I/O Data Sample Rx Indicator 131

Remote AT Command Request $\quad 135$

Remote Command Response $\quad 137$

Using What You Need 138

Libraries 141

Simple Sensor Network $\quad 142$

Parts 142

Prepare Your Coordinator Radio 143

Prepare Your Router Radios $\quad 144$

Prepare the Sensor Boards $\quad 144$

Prepare the Base Station 148

Program the Base Station $\quad 152$

Yay! 160

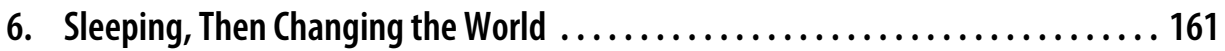

$\begin{array}{ll}\text { Sleep Mode } & 161\end{array}$ 
End Devices

Configuring Sleep 163

Easy Sleeping 167

$\begin{array}{ll}\text { Simple Sensor with Sleep Project } & 167\end{array}$

Parts $\quad 168$

Prepare Your End Device Radios $\quad 168$

Configure Your End Device XBees 168

Direct Actuation $\quad 171$

Direct Actuation Example $\quad 172$

Parts 172

Prepare Your Coordinator Radio $\quad 173$

Prepare Your Router Radios $\quad 174$

Prepare the Actuator Boards 174

Configure Your Router XBees 174

Prepare the Base Station 177

Simple Actuator Node Code in Processing 180

$\begin{array}{ll}\text { Summary } & 187\end{array}$

7. Over the Borders ............................................. 189

$\begin{array}{ll}\text { Gateways } & 189\end{array}$

XBee as Embedded Gateway $\quad 190$

Other Embedded Gateways 190

Internet Gateways 192

Internet Media 194

Computers Versus Dedicated Devices 194

ConnectPorts 195

Selecting a ConnectPort 196

Setting Up a ConnectPort 197

Configuring a ConnectPort 198

Remote Management 203

iDigi Connectivity Server 204

iDigi Features 204

Adding a ConnectPort 206

Viewing Configurations 209

Firmware Updates and Remote Reboot 210

Viewing an XBee Network $\quad 212$

XBee Internet Gateway (XIG) 214

Installing and Configuring XIG 216

Testing XIG 218

XIG Example $\quad 220$

Twitter Reader 221

Parts 222

Prepare Your ConnectPort with XBee Internet Gateway 223 
Prepare Your Router Radio

8. More to Love

235

Advanced ZigBee

ZigBee Stack Layers

Application Support Layer

Routing

Security

ZigBee Protocol References

243

Serial Flow Control

RTS and CTS

244

Sharing Data

245

Pachube

Simple Sensor Network with Pachube

API Key

Build the Simple Sensor Network in Chapter 5

Program the Base Station

Simple Sensor Network Pachube Code in Processing 250

The Future of ZigBee

Next Steps for You

Making Stuff

Sharing Your Work 



\section{Preface}

Building Wireless Sensor Networks is an essential guide for anyone interested in wireless communications for sensor networks, home networking, or device hacking. It is a first step in becoming proficient in making these systems. It is not a textbook on protocols or a complete guide to networking theory. No engineering or computer science background is expected or required. Those who have fooled around a bit with electronics or programming will certainly have a leg up, but in general, this book is aimed at hobbyists, students, makers, hardware hackers, designers, artists, and prototypers. In the chapters to come, you will scaffold your way up toward greater comfort and proficiency with hardware, software, radio, and communications. I'll explain everything necessary to get started, at least briefly. We'll create examples using accessible environments, such as Arduino for hardware and Processing for displays. And I'll provide a full range of resources, including helpful references to outside works for the electronics and networking novice. Whether you are a young inventor or an experienced engineer, this book focuses on getting your projects up and running as efficiently as possible.

All the projects you'll create in this book use radio signals that pass invisibly through the air. This "wirelessness" is essential whenever you want to place sensors where no cables can be installed, or where such tethering is undesirable. With radio, you can employ sensing and actuation in pristine natural settings, minimalist building interiors, or complex urban environments. Mobile devices like children's toys can benefit greatly by being communicative without being chained to the wall or to each other. Sensors can be attached to people or animals in a humane manner that doesn't hinder their movement. In short, lots of data can move freely from where it is gathered to where it can do the most good. That's why wireless is worth it.

The ZigBee protocol is a very popular way of creating radio sensor networks for a number of reasons. Wireless networks and connected devices in general tend to be used in situations where power is hard to come by and must be conserved. Many times the communications these networks send are small in nature, compared to systems that transfer huge files such as videos. Often, each device in the network transmits or receives unique information, so a robust system of individual addressing is extremely helpful. Security and design flexibility are frequently indispensable. That's why this book focuses on ZigBee, the protocol defined by various industry players who together 
form the ZigBee Alliance. In the past few years, ZigBee has found its way into commercial systems for home automation, smart energy systems, consumer electronics, industrial sensing, and health care. It features full addressing, many power-saving options, optimizations for efficiency in low-bandwidth applications, and a layered approach to communications design and security. Most importantly, ZigBee automatically forms entire networks that can heal themselves, routing around problem areas without manual intervention. Designers, hackers, inventors, artists, and engineers are currently making use of this popular wireless protocol to create the systems that inform, enable, and delight their various users.

We will make a new project in almost every chapter of this book to demonstrate how everyday people, not just electrical engineers and computer scientists, can develop these systems. A number of full sensor networks, an array of doorbells, a two-way lighting detector, a household control system, and several types of Internet-connected contraptions will be demonstrated step by step for you to build. After reading this book you'll have a solid understanding of what it takes to create scalable sensor and device networks because you'll have brought a variety of them into being with your own hands. This book's website makes even more resources available to you.

You may wonder what drives humans to create reactive sensor systems and connected devices. Since before written history, there have been people and cultures that believed every object in the world was imbued with spirits-that even rocks are alive. This worldview, termed animism by modern scholars, isn't something validated by science. And yet the tacit belief that objects are in some way alive seems to resonate as a fundamentally human way of thinking. "That mixer doesn't like it when the batter is too thick." "The DVD player doesn't want to eject that disk." "My computer hates me!" We seem to want our things to be alive and frequently consider them willful—-though, on an intellectual level we know they aren't. This book isn't about animism, of course; it's about making networks using ZigBee radios. However, one reason we do thisour motivation for making systems that are sensitive, active, reactive, and communicative-could just be some inherent desire to create the world we believe should exist: one where everything is imbued with a willful spirit and works together to help us live more richly. If so, this book is offered as a practical step in the right direction. I hope it will help you bring your own creations to life.

\section{How This Book Is Organized}

The chapters in this book are organized as follows:

Chapter 1, Getting Ready

This chapter offers a shopping guide and an introduction to all the major components we'll be using. We focus on just what you need to get up and running, including XBee radios, adapters, breakout boards, terminal programs, and software. 
Chapter 2, Up and Running

Right at the start of the book, you'll go from a bag of parts to a working ZigBee network in one chapter, taking the simplest path to early success. Radios, ZigBee, networks, and addressing are introduced, and then you'll configure your components to achieve a simple chat session.

Chapter 3, Build a Better Doorbell

This section focuses on creating something practical using the Arduino microcontroller system, which is briefly introduced. After getting up to speed on basic serial concepts and simple protocols, you'll execute a series of doorbell projects that increase in creative complexity as you gain skill.

Chapter 4, Ins and Outs

Here you'll take a closer look at the unique features of the XBee-brand ZigBee radios so we can start building fully scalable sensor networks. You'll begin with input/output concepts and commands, then immediately put these to use in a small set of progressively intricate projects.

Chapter 5, API and a Sensor Network

At this point you have everything you need to conquer the XBee's application programming interface. We start by laying a foundation of ideas and scaffold you up to a full understanding of the structured API communication frames. You are then ready to create a fully scalable sensor network of your own, using the complete example at the end of this chapter.

Chapter 6, Sleeping, Then Changing the World

We ease the development pace a bit here to address some nuances of ZigBee mesh networking, including sleep mode, end devices, and power management. Then it's time to change things in the physical world using direct actuation. This chapter features a powerful control project you can use to automate your home or turn just about anything on and off remotely.

Chapter 7, Over the Borders

In this chapter you learn to make gateways that connect ZigBee with neighboring networks, including a remarkably easy path to the Internet. You'll see full examples, showing how to allow anything to talk to everything everywhere-plus there's a special project for starry-eyed celebrity fans.

Chapter 8, More to Love

The final chapter is really a broader introduction. We explore advanced ZigBee techniques, demonstrate how to publish and share your data online, and then wrap things up with a peek at where ZigBee is headed.

Appendix, Resource Guide

To ensure that the book remains useful even after you have read it, we've included links to online resources and other texts for learning more about Arduino, Processing, Python, and ZigBee, along with a handy troubleshooting guide to get you unstuck from common mistakes. There are also tables to use as a fast daily reference to Digi radios, other brands of ZigBee modules, network analyzers, packet sniffers, 
connectors, shields, hexadecimals, binary numbers, ASCII codes, and finally a complete guide to XBee AT commands.

\section{About the Title}

You will notice that for a book called Building Wireless Sensor Networks, we spend quite a bit of time talking about actuation: outputs that make things happen in the physical world. The source of this is a deep-seated point of view that is backed up by some longstanding cognitive science.

"Thinking is for doing" is a phrase popularized by social psychologist Susan Fiske. Her point (and William James' when he commented similarly a century earlier) is that our brains exist first and last for creating physical actions. In fact, the brain is just the midpoint of the perception-action chain. The real action starts with our sensory systems. We see, smell, and feel, then we process those sensations for the purpose of choosing and executing our next move. Sensing never happens in a vacuum for its own sake. There's always a physical purpose. This is as true for wireless networks as it is for living organisms. The data we collect is always aimed at an action of some kind. Alarm systems trigger an immediate police response, while environmental sensing studies often have a much longer cycle that results in policies to guide real-world development. In both cases there's a purpose to the sensing that ends up, sooner or later, creating changes in the physical world. This book takes a comprehensive approach to cover both the input and output stages of the information-action cycle-sensing and actuation. In doing so, we hope to encourage projects to do more with data than simply collect it, hopefully enabling implementations that use their sensory input to create the rich physical experiences that humans crave.

\section{About the Examples}

All of the example circuits and code in this book are designed with clarity in mind. Astute electrical engineers will certainly notice that some corners have been cut. For example, we draw close to the rated output for the 3.3-volt pin on the Arduino board in some projects, and we rely on the microcontroller to throttle the current going to LEDs where we can. While that wouldn't be advisable in a commercial product, it does produce working circuits that are very simple for the beginner to build and understand. The same is true for the example code. Production-quality programming usually includes much more error correction and thriftier memory management than we offer here. Our purpose is to strip the code down to the basics as much as possible so that it can serve as a transparent tool for learning.

If you prefer to enhance the circuits and code to make them more robust, by all means do so! Feel free to share your suggestions or enhancements on the forums, and by sending them to us at bookquestions@oreilly.com. Feedback and community participation is always welcome! 
Additional code and circuit diagrams that are made available in the future will be linked from this book's website.

\section{Conventions Used in This Book}

The following typographical conventions are used in this book:

Italic

Indicates new terms, URLs, email addresses, filenames, and file extensions

Constant width

Used for program listings, as well as within paragraphs to refer to program elements such as variable or function names, databases, data types, environment variables, statements, and keywords

\section{Constant width bold}

Shows commands or other text that should be typed literally by the user

Constant width italic

Shows text that should be replaced with user-supplied values or by values determined by context
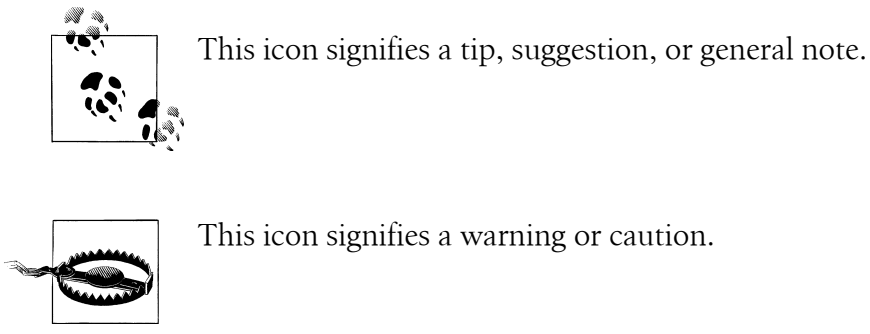

\section{Using Code Examples}

This book is here to help you get your job done. In general, you may use the code in this book in your programs and documentation, and the projects as a foundation for creations of your own. You do not need to contact us for permission unless you're reproducing a significant portion of the code or schematics. For example, writing a program that uses several chunks of code from this book does not require permission. Selling or distributing a CD-ROM of examples from O'Reilly books does require permission. Answering a question by citing this book and quoting example code does not require permission. Incorporating a significant amount of example code from this book into your product's documentation does require permission.

We appreciate, but do not require, attribution. An attribution usually includes the title, author, publisher, and ISBN. For example: "Building Wireless Sensor Networks by Robert Faludi. Copyright 2011 Robert Faludi, 978-0-596-80773-3.” 
If you feel your use of code examples falls outside fair use or the permission given here, feel free to contact us at permissions@oreilly.com.

\section{Safari ${ }^{\circledR}$ Books Online}

Safari`Safari Books Online is an on-demand digital library that lets you easily search over 7,500 technology and creative reference books and videos to find the answers you need quickly.

With a subscription, you can read any page and watch any video from our library online. Read books on your cell phone and mobile devices. Access new titles before they are available for print, and get exclusive access to manuscripts in development and post feedback for the authors. Copy and paste code samples, organize your favorites, download chapters, bookmark key sections, create notes, print out pages, and benefit from tons of other time-saving features.

O'Reilly Media has uploaded this book to the Safari Books Online service. To have full digital access to this book and others on similar topics from O'Reilly and other pub-

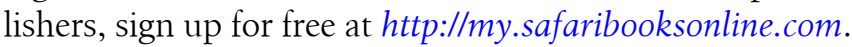

\section{How to Contact Us}

Please address comments and questions concerning this book to the publisher:

O'Reilly Media, Inc.

1005 Gravenstein Highway North

Sebastopol, CA 95472

800-998-9938 (in the United States or Canada)

707-829-0515 (international or local)

707-829-0104 (fax)

We have a web page for this book, where we list errata, examples, and any additional information. You can access this page at:

http://oreilly.com/catalog/9780596807733

To comment or ask technical questions about this book, send email to:

bookquestions@oreilly.com

For more information about our books, conferences, Resource Centers, and the O'Reilly Network, see our website at:

http://oreilly.com 


\section{Acknowledgments}

This book was strongly affected by my tag team of editors Brian Jepson and Tom Sgouros. Brian's fractured wit paired with his expert strategies constantly guided my hand, while Tom's attention to details and scientific discipline provided the rigor any technical book demands. Even when process and schedule left me breathless, I never lost appreciation for the wisdom and craft they supplied. I'm grateful for all their help.

My technical editors imparted a level of feedback that went well beyond their respective calls of duty. Kate Hartman, who encouraged this book from the get-go, spent many hours combing the text for confusing constructions and omitted explanations. Her project assessments and clarity of voice are felt throughout. Jordan Husney cheerfully reviewed many of these chapters from his perch at 35,000 feet. His deep knowledge of the ZigBee protocol is matched only by his competence as a wordsmith. Thanks to both for their efforts and uncommon friendships.

Building Wireless Sensor Networks is loosely structured around the Sociable Objects class I created at NYU's ITP graduate program in media and technology. There, Tom Igoe loaned me my first ZigBee radio, encouraged my projects, mentored my development as a teacher, and continues to be a seemingly bottomless well of excellent advice and terrible puns. This book almost certainly could not have happened without him. Clay Shirky, Nancy Hechinger, Marianne Petit, Dan Shiffman, Danny Rozen, and Dan O'Sullivan are but a few of the instructors who provided invaluable inspiration. George Agudow and the sensational staff at ITP have granted support to my work at every turn. My fellow resident researchers Jeff, John, Jenny, Kate, Gabe, and Demetrie influenced my ideas and enriched my experience during the year we were all lucky enough to work together. Almost all the concepts in this book were trialed by my Sociable Objects students and I am grateful for their feedback, which is incorporated throughout. Everyone in the ITP community owes a debt toward longtime Chair and perpetual guiding star Red Burns. Her steely stare, firm love, and rare brilliance continue to illuminate us all.

This book would have been immeasurably more difficult without Paul Cole's flexibility, generous spirit, and unflagging support. I am thankful for the grand company of my entire day job crew at GroundedPower, especially longtime collaborators Terence Arjo, Mike Bukhin, and Demetrie Tyler. They caught my bullets on countless occasions when I needed extra concentration for penning these pages. At SVA's MFA program in Interaction Design, Liz Danzico's words of wisdom and my graduate students' insightful creations brought depth to my thinking and clarity to my explanations.

My mother and father taught me to craft with words, wood, and wires—priceless skills that I am honored to share in some small measure here. I am lucky to come from two people with such talent, creative motivation, and quick-witted humor. My sister, Susan, and her partner, Russ, tirelessly guided me through the tricks of the writing trade. When enthusiasm flagged, Sue and Russ assured me that my writerly doldrums were distinctly underwhelming, cannily undermining my laments and restoring my cheer. I'm 
phenomenally lucky to have them in my life. Liz Arum bestowed suggestions, solace, affection, and perpetual patience as I alternately plodded and sprinted through the birthing of this book. Her family has pampered me with their hospitality, and her middle school students effortlessly completed several of the book's projects, just as she knew they could. I'm grateful to all of them, and to Liz especially.

One more thing: John Dobson's telescope-building class and indomitable spirit continue to be an inspiration in my work. If you ever get a chance to build your own sidewalk telescope, don't hesitate for a minute. Everyone deserves to meet the universe in person. 


\section{CHAPTER 1 \\ Getting Ready}

Let's get right down to business. This chapter offers a shopping guide and an introduction to all the major components you'll need to prep your networking toolbox with essential parts and programs. There are plenty of options, so we're going to focus on just what you need to get up and running. Check the Appendix for a comprehensive list of resources. For now, here are the essentials, distilled for your convenience.

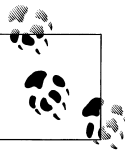

In this book we focus on XBee brand ZigBee radios because they have a host of features that make them especially easy for beginners to work with. Many other brands exist, however most are best suited to those with an electrical engineering background. If that's you, the resource guide at the end of this book lists other ZigBee options you can consider. Professional engineers often prefer XBees for prototyping or anytime a reduced development effort makes them the most cost-effective option.

\section{Buying an XBee Radio}

Digi International manufactures a bewildering array of XBee-branded radios. All told there are at least 30 different combinations of component hardware, firmware protocols, transmission powers, and antenna options. We'll first take a look at what's out there, and then narrow that down to the devices we will be using in this book.

\section{Hardware}

There are two basic varieties of XBee radio physical hardware:

\section{XBee Series 1 hardware}

These radios use a microchip made by Freescale to provide simple, standards-based point-to-point communications, as well as a proprietary implementation of mesh networking. We won't use the Series 1 hardware at all in this book. 


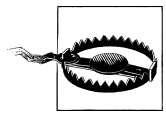

The sidebar "Series 1 Radios" on page 3 takes a quick look at the Series 1, but remember that the examples in this book won't work with Series 1 hardware.

\section{XBee Series 2 hardware}

The Series 2 uses a microchip from Ember Networks that enables several different flavors of standards-based ZigBee mesh networking. Mesh networking is the heart of creating robust sensor networks, the systems that can generate immensely rich data sets or support intricate human-scale interactions. Everything we do in this book from here on out will use the Series 2 hardware exclusively.

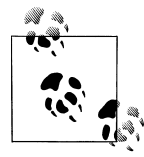

Digi has just released the newer Series 2B. Series 2B features include reduced power consumption, additional antenna options, and an optional programmable microprocessor. For the most part, Series 2 and $2 \mathrm{~B}$ are interchangeable.

Both the Series 1 and Series 2 radios are available in two different transmission powers, regular and PRO (see Figure 1-1). The regular version is called simply an XBee. The $\mathrm{XBee}-\mathrm{PRO}$ radio has more power and is larger and more expensive.

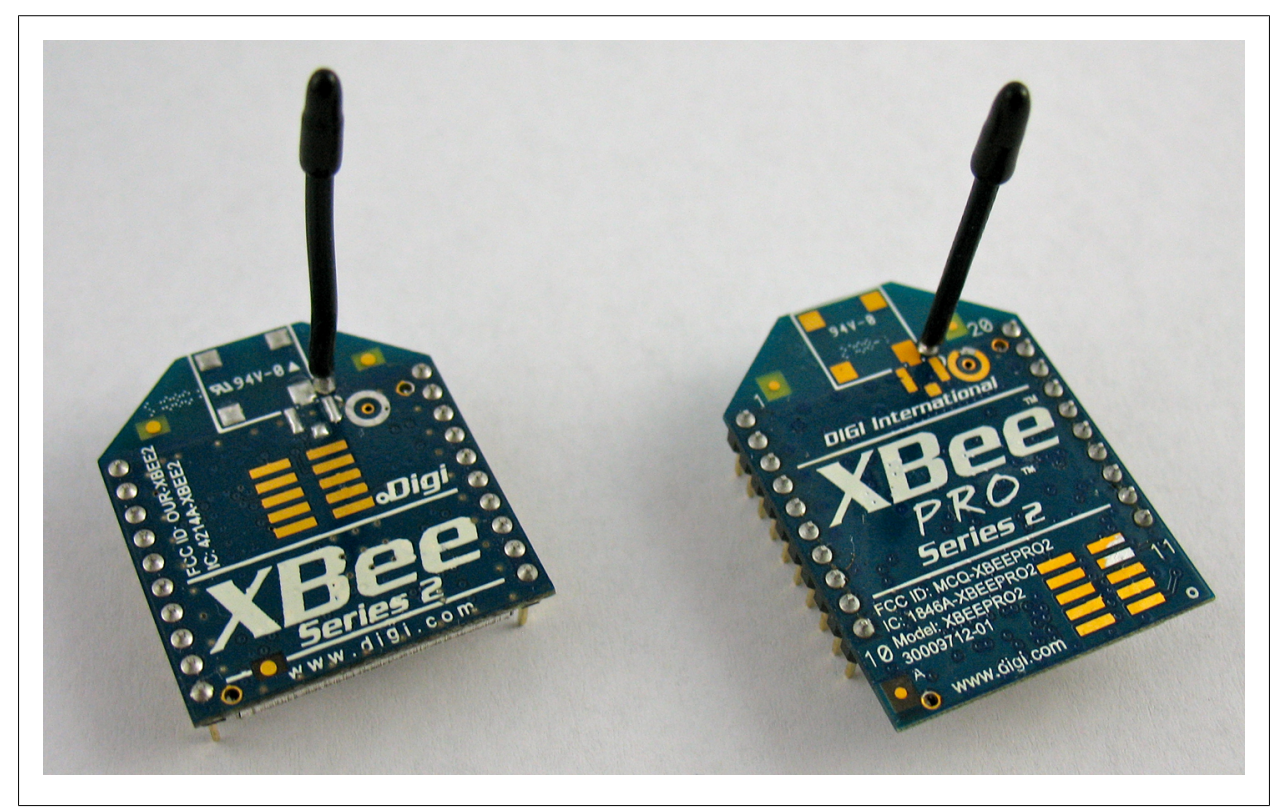

Figure 1-1. XBee radios in regular and $P R O$ flavors

The regular version is a slightly less expensive way to get started. For now, we won't worry about distinguishing between the regular and PRO radios because they are configured in the same way. 


\section{Series 1 Radios}

Series 1 XBee modules are quite popular with the do-it-yourself crowd, while Series 2 hardware supports the full ZigBee protocol. Series 1 is great for simple cable replacements and smaller-sized systems. Series 2 is designed with larger sensor networks in mind and is essential for the robust interactions with the ZigBee standards-based systems that are now being widely deployed in residential, academic, and commercial settings.

The Series 2 hardware has a little better range and uses slightly less power than the Series 1; yet these small improvements would not be a reason to choose one format over the other. Both use the same physical footprint and can be easily interchanged, often with only minor changes to the underlying software. However, the Series 2 will not talk to or interoperate with the Series 1 at all. Each network must use only one version. Table 1-1 shows a summary of the similarities and differences.

Table 1-1. Series 1 versus Series 2 for regular XBees

\begin{tabular}{lll} 
& Series 1 & Series 2 \\
\hline Typical (indoor/urban) range & 30 meters & 40 meters \\
Best (line of sight) range & 100 meters & 120 meters \\
Transmit/Receive current & $45 / 50 \mathrm{~mA}$ & $40 / 40 \mathrm{~mA}$ \\
Firmware (typical) & 802.15 .4 point-to-point & ZB ZigBee mesh \\
Digital input/output pins & 8 (plus 1 input-only) & 11 \\
Analog input pins & 7 & 4 \\
Analog (PWM) output pins & 2 & None \\
Low power, low bandwidth, low cost, addressable, standar- & Yes & Yes \\
dized, small, popular & & \\
Interoperable mesh routing, ad hoc network creation, self- & No & Yes \\
healing networks & & \\
Point-to-point, star topologies & Yes & Yes \\
Mesh, cluster tree topologies & No & Yes \\
Single firmware for all modes & Yes & No \\
Requires coordinator node & No & Yes \\
Point-to-point configuration & Simple & More involved \\
Standards-based networking & Yes & Yes \\
Standards-based applications & No & Yes \\
Underlying chipset & Freescale & Ember \\
Firmware available & 802.15 .4 (IEEE standard), & ZB(ZigBee2007),ZNet \\
Up-to-date and actively supported & DigiMesh (proprietary) & 2.5 (obsolete) \\
\hline & Yes & Yes \\
\hline
\end{tabular}


While this book uses the Series 2 hardware exclusively, what you learn here can help you with both series. Picking up the Series 1 commands should be a snap after reading this book because, for the most part, they are a subset of the Series 2 that we cover here. You will already know pretty much everything you need to work with them! Tom Igoe's excellent volume Making Things Talk (O'Reilly) has several appealing example projects for Series 1 XBees, and many more are available online.

\section{Antennas}

Radios need antennas to transmit and receive signals. There's more than one way to build an antenna, each with advantages and disadvantages. You probably won't be surprised to learn that Digi decided to offer plenty of choices. Here are the kinds of antenna options currently available (see Figure 1-2):

\section{Whip or wire antenna}

This is just what it sounds like - a single piece of wire sticking up from the body of the radio. In most cases, the wire antenna is just what you need. It's simple and offers omnidirectional radiation, meaning the maximum transmission distance is pretty much the same in all directions when its wire is straight and perpendicular to the module.

\section{Chip antenna}

Again, this is pretty much what it sounds like. The chip antenna is a flat ceramic chip that's flush with the body of the XBee. That makes it smaller and sturdier, but those advantages come at a price. Chip antennas have a cardioid (heart-shaped) radiation pattern, meaning that the signal is attenuated in many directions. However, if you're making a device where mechanical stress to the wire antenna might break it, or you need to put the radio in a very small space, then the chip antenna may be your best bet. Chip antennas are often the right choice for anything wearable.

\section{PCB antenna}

Introduced with the XBee-PRO S2B, the PCB antenna is printed directly on the circuit board of the XBee. It is composed of a series of conducting traces laid out in a fractal pattern. The PCB antenna offers many of the same advantages (and disadvantages) as the chip antenna with a much lower cost to manufacture.

\section{U.FL connector}

This is the smaller of the two types of external antenna connectors. More often than not, an external antenna is not needed: it is an additional expense if a simple wire antenna will do. However, when your radio is going to live on the inside of a metal box then the antenna will need to live on the outside. That way the signal is not attenuated by the enclosure. Also, it is sometimes advantageous to orient an external antenna differently than the XBee itself to or use a special-purpose antenna with a specific radiation pattern, such as a high-gain antenna that passes signals in a single direction over a broader distance. The U.FL connector is small, somewhat 
fragile, and almost always used with a short connecting cable that carries the signal from a remotely mounted antenna.

\section{RPSMA connector}

The RPSMA connector is just a different type of socket from the U.FL connector. It's bigger and bulkier, but you can use it with an external antenna, mounted directly to the XBee without a connecting cable. For most introductory projects, you're still best off with the simple wire antenna that is smaller, cheaper, and usually just as good.

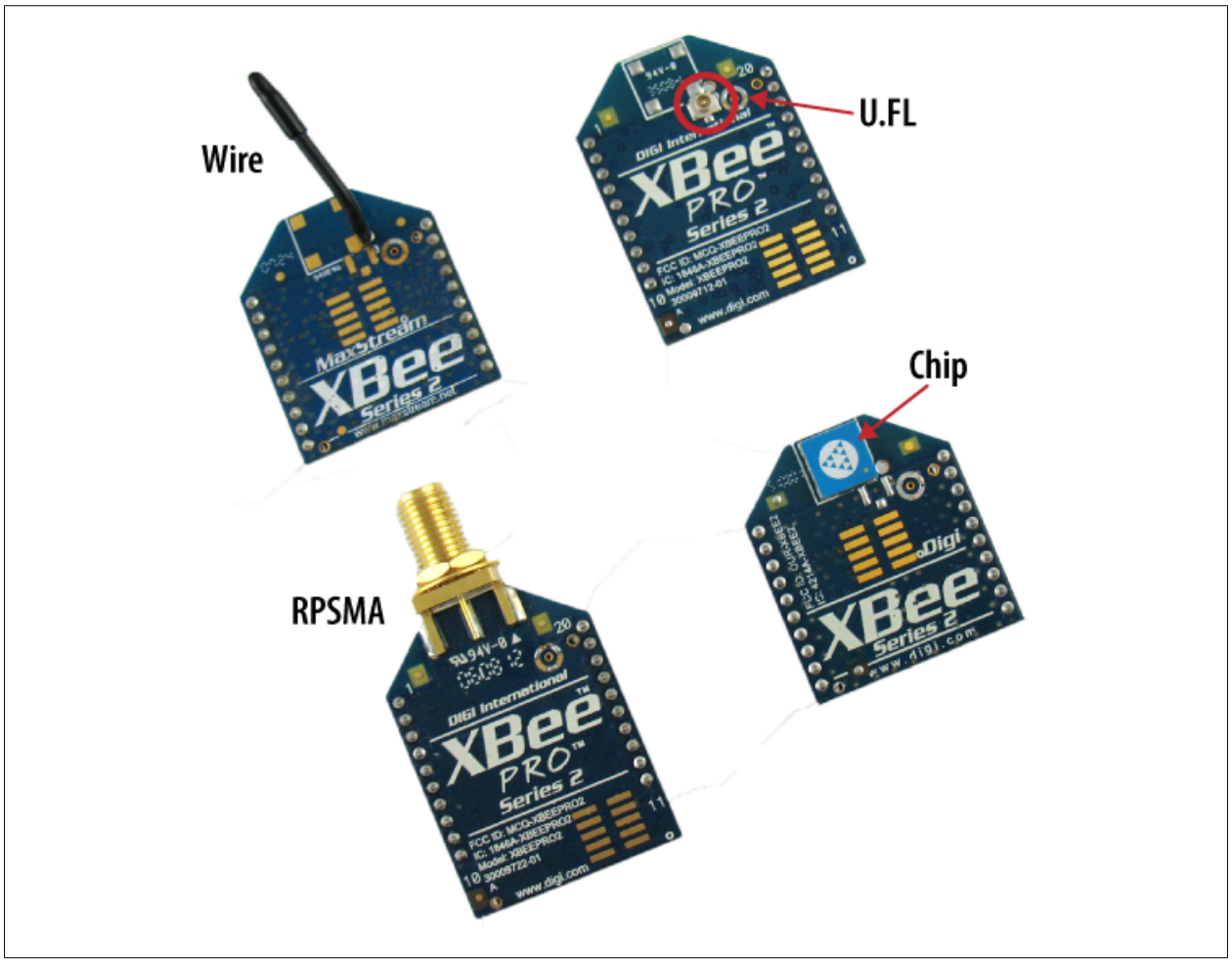

Figure 1-2. Antenna types

To keep it simple you can get started by purchasing two (or more) of the regular Series 2 XBees with wire antennas. Don't buy just one! You'll be as disappointed as a kid at Christmas who finds a single walkie-talkie under the tree. Here's the model number to get you started: XB24-Z7WIT-004. At the time of this writing, this module cost about $\$ 25$. 
If you need a chip antenna, the part number is XB24-Z7CIT-004. If you

require a PRO high-power radio, use part number XBP24BZ7WIT-004.

\section{Vendors}

Now that you know what you want to buy, you also need to decide where to buy it. $\mathrm{XBee}$ radios are available directly from Digi, and also from many online resellers. This is a list of vendors that typically stock XBees, as well as many other nifty electronic components you may need for your projects:

\section{Digi International}

Digi manufactures and sells all varieties of XBee radios and some interesting XBee starter kits, generally at the suggested retail price. They don't sell any other electronic components.

Maker SHED

MAKE: magazine (which is published by O'Reilly, the publisher of this book) offers a kit specifically designed for this book, via their in-house maker emporium. The kit includes many of the parts you'll need, including appropriate XBees.

SparkFun Electronics

SparkFun carries a rapidly growing array of prototyping supplies designed specifically for DIY electronics enthusiasts, including most of the XBee modules. You'll find documentation links for each part, as well as handy tutorials for using many of the components.

\section{DigiKey}

DigiKey offers a dizzying array of electronic components for the professional electrical-engineering market. It's normal to feel overwhelmed at first by the selection of about half a million different parts, but it's worth learning the ropes because you'll be able to buy almost anything you want and receive it the next day. The entire XBee line is usually represented at DigiKey (which has no relationship at all to Digi International).

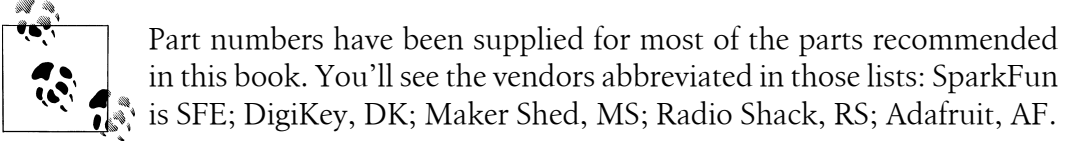

\section{Buying an Adapter}

You'll be using a computer to configure your XBee and to send and receive data directly from your desktop or laptop. The XBee is made to be soldered directly into a printed circuit board, so you'll need an adapter to connect it to your computer's USB port. If 
you need to connect to an older 9-pin or 25-pin serial port instead, check the Appendix for other options.

There are several different adapters available, along with a few handy hacks if you want to avoid buying one or if you get caught without one.

\section{Digi Evaluation Board}

If you buy a complete drop-in networking starter kit from Digi, such as their iDigi Professional Development Kit ZB (part no. XK-Z11-PD), it will include an evaluation board with a power supply, a USB connector, and some handy buttons and lights. The kits are a good value if you need everything they include. However, if you only want some radios and an adapter, other approaches are more cost-effective. Also, the Digi evaluation board is substantially larger than most third-party adapters, making it somewhat cumbersome to carry around. At the time of this writing, the development kit was available for $\$ 300$, though occasional promotions have brought it down to $\$ 150$. (See Figure 1-3.)

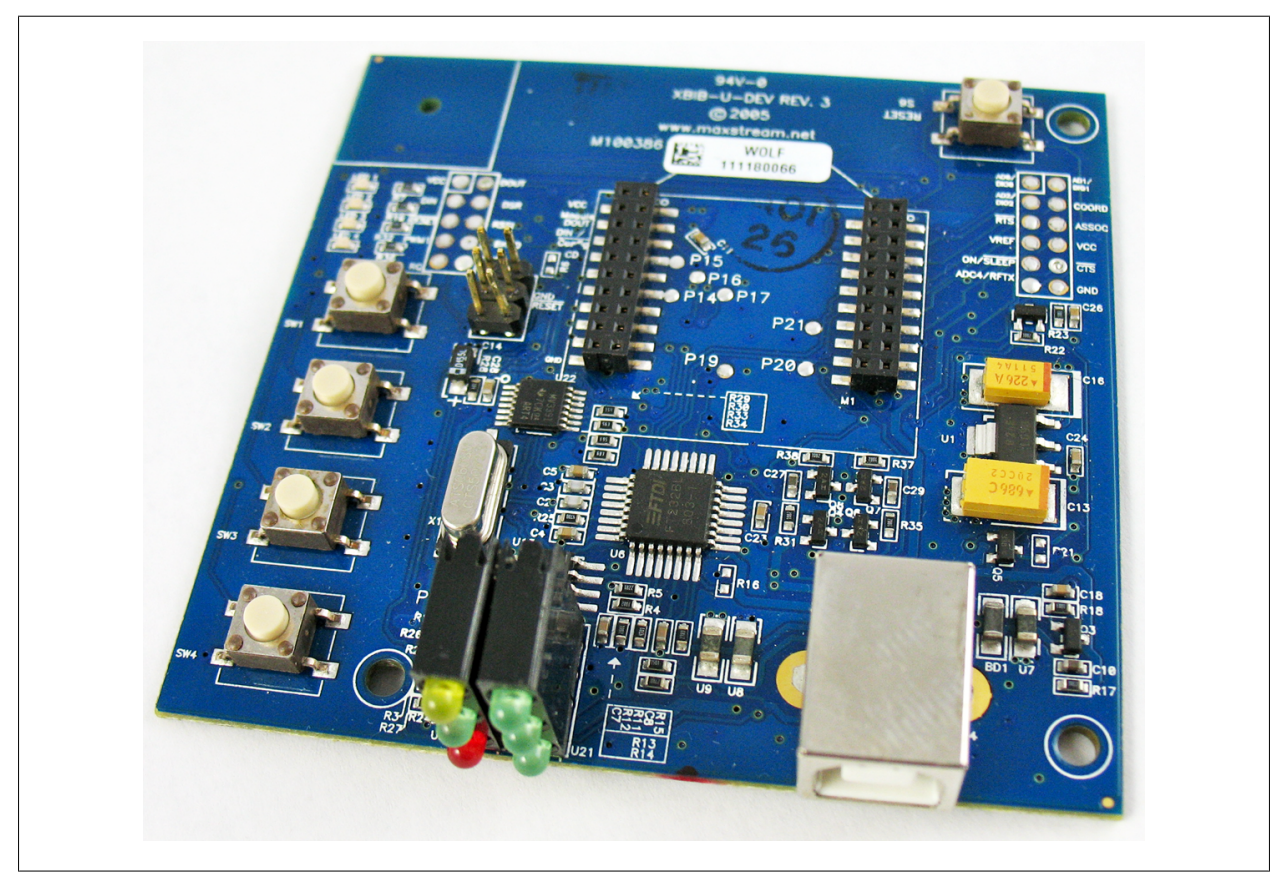

Figure 1-3. Digi evaluation board

\section{USB Adapters}

Several different XBee USB adapters are available from third-party manufacturers (see Figure 1-4): 


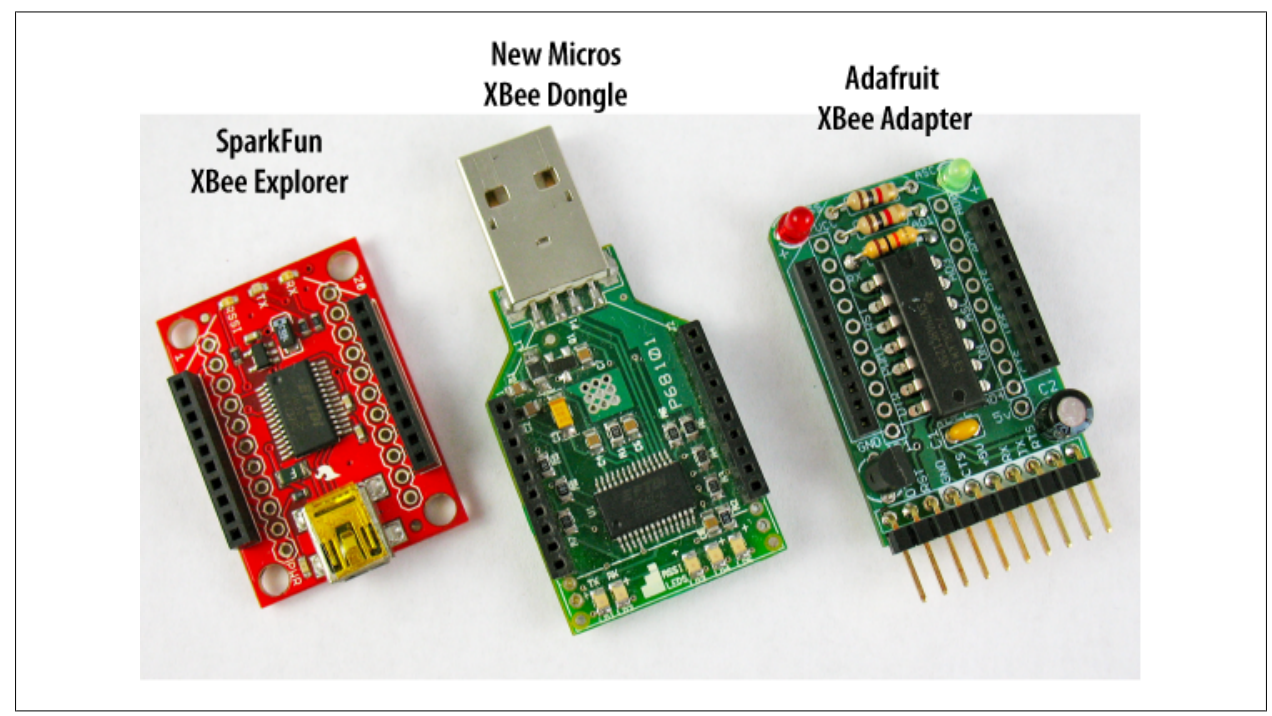

Figure 1-4. XBee adapters are available from many vendors in a variety of different formats

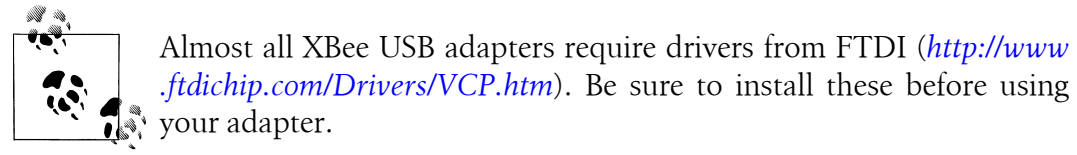

SparkFun XBee Explorer

The Explorer is a very popular adapter that uses a fairly standard USB A to miniB cable to connect with your computer. We'll be using it in most of the examples in this book. The cable is sold separately, but before you buy, check to see if you already have one. Many digital cameras come with this type of cable. Be aware that if you add male headers to use it in a breadboard, the pin order will not be the same as on the XBee. Check the data sheet carefully if you are using the Explorer with a breadboard setup. (About $\$ 25 ;$ ttp://www.sparkfun.com/commerce/product_info .php?products_id=8687.)

\section{Adafruit XBee Adapter Kit}

This is an inexpensive board that you'll need to solder together yourself. It also must be used with a special USB cable called the FTDI USB TTL-232, which can attach to its pin headers. The cable can be used with certain Arduino-type boards as well. Male headers can be added so that this adapter can be used in a breadboard. (About \$10; http://www.adafruit.com/index.php?main_page=product_infoEvcPath =29E.products_id=126. Cable about \$20; http://www.adafruit.com/index.php ?main_page $=$ product_infoEcPath $=29$ E products_id=70.) 
New Micros XBee Dongle

One of the smallest adapters, it needs no external cable. The Dongle does not provide any access to the radio beyond USB. Also, because it has no cable, its shape sometimes interferes with other cables or the computer casing. On the other hand, it's a very small all-in-one device that's easy to carry in a pocket. It's terrific for use on the go. (About $\$ 39$; http://www.newmicros.com/cgi-bin/store/order.cgi?form= prod_detaile $p$ art $=U S B-X B E E-D O N G L E-C A R R I E R$.

Gravitech XBee to USB Adapter

Like the Explorer, this is another simple adapter board that uses the USB A to miniB cable (not included). This one also has standard breadboard pinouts. (About \$28; http://store.gravitech.us/xbtousbad.html.)

\section{Breadboards}

Solderless breadboards (Figure 1-5) provide an easy test bed for hooking up electronic circuits without needing to make permanent connections. They consist of a plastic housing riddled with small holes. Metal clips that lurk beneath the holes in the breadboard provide a way to hold and connect components. Each metal clip is called a bus, and everything attached to the same bus is connected together electrically. Many breadboards have two power and ground buses running down each side, with shorter buses oriented to them at right angles (Figure 1-6). Components like LEDs, capacitors, radios, and microchips are placed in the shorter buses, called terminal strips. These are connected to the power buses and each other using jumper wires.

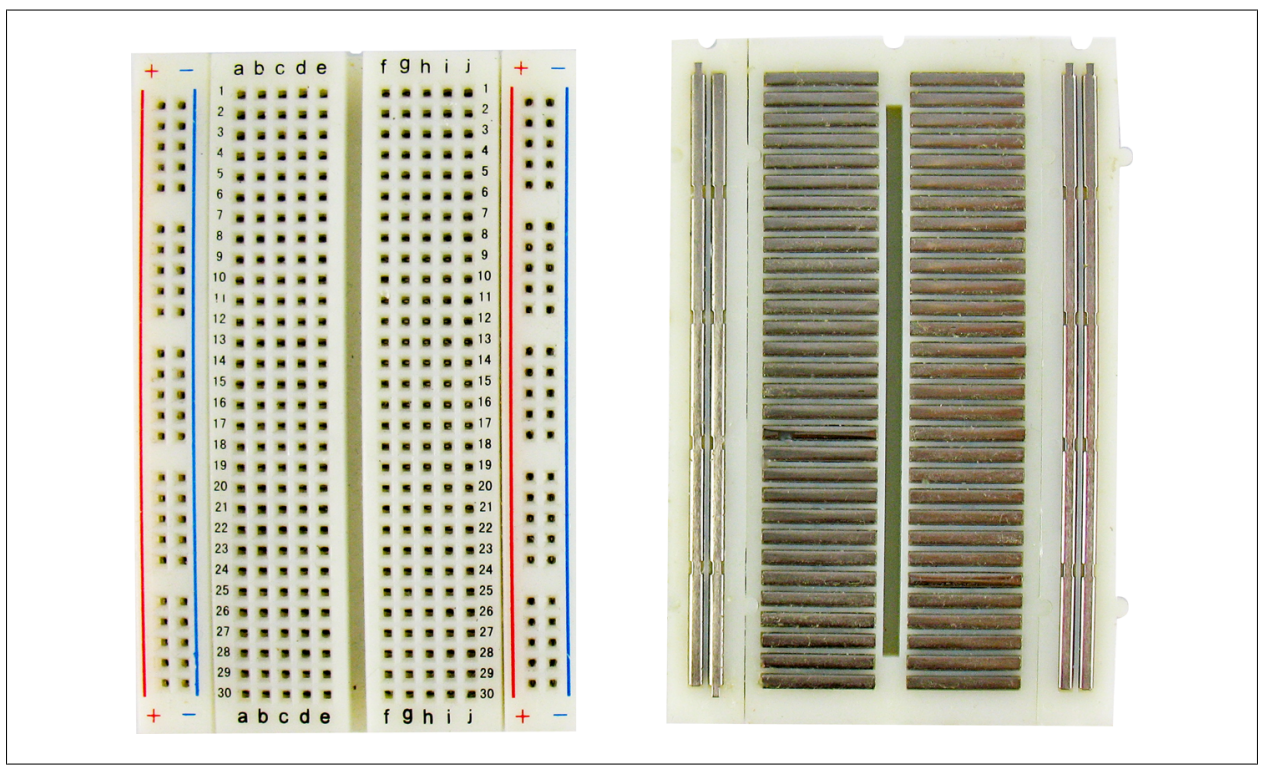

Figure 1-5. Breadboard front and back, showing metal clips 


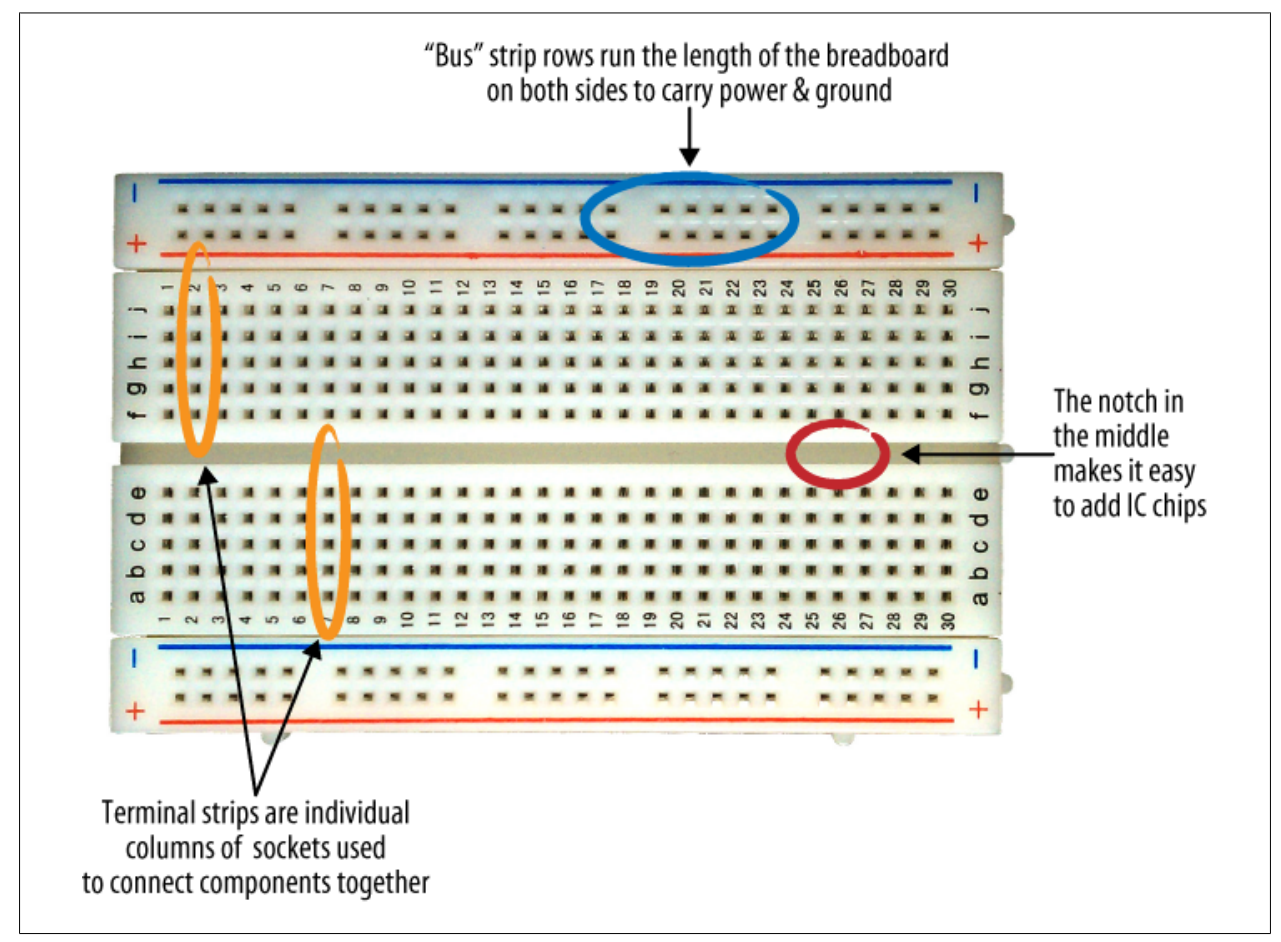

Figure 1-6. Breadboard with bus strips and terminal strips indicated

\section{Breakout Boards}

All XBee radios have 20 connection pins, each spaced $2 \mathrm{~mm}$ apart. The tight spacing of the pins helps to keep the radios very small, but doesn't allow them to fit into a solderless breadboard. Luckily, this is a very easy problem to solve. Simple XBee breakout boards that adapt to $0.1^{\prime \prime}$ breadboard spacing (see Figure 1-7) are available from:

- SparkFun

- Adafruit

- Cutedigi

- Gravitech

You will generally need to solder $2 \mathrm{~mm}$ female pin headers to one side of these breakout boards, and regular 0.1-inch male headers to the other side.

Note that the XBee Explorer (Figure 1-8), Adafruit XBee Adapter Kit, and the MCPros XBee Simple Board each have mounting holes for 0.1-inch male headers. Solder a set of male header pins into them to adapt these for breadboard use. 


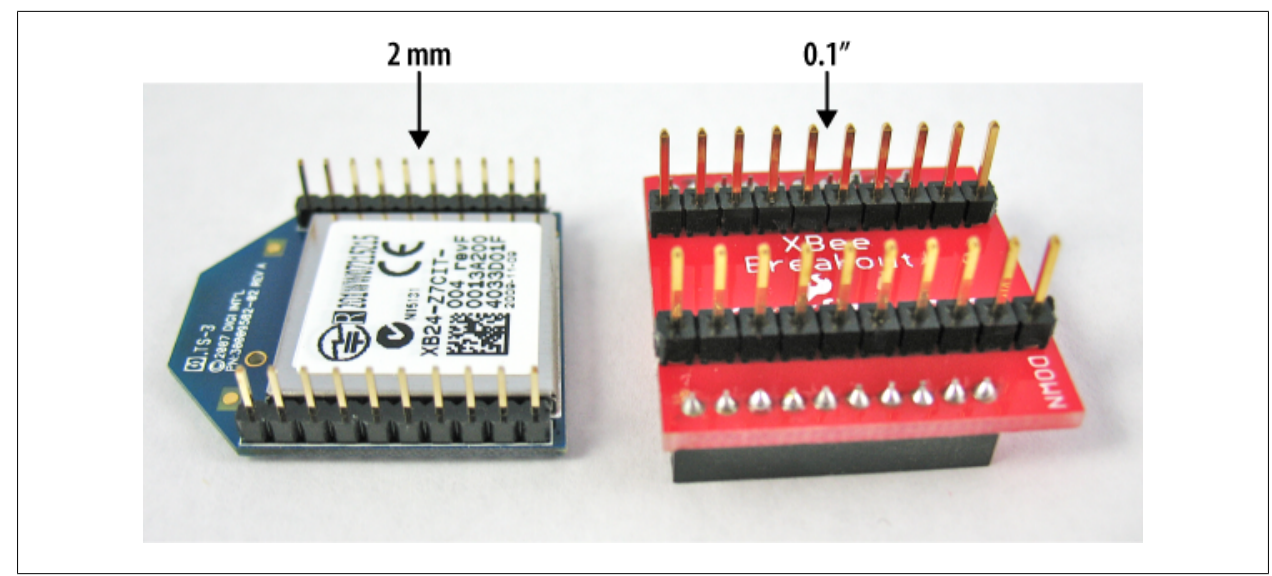

Figure 1-7. Breakout board showing pin spacing

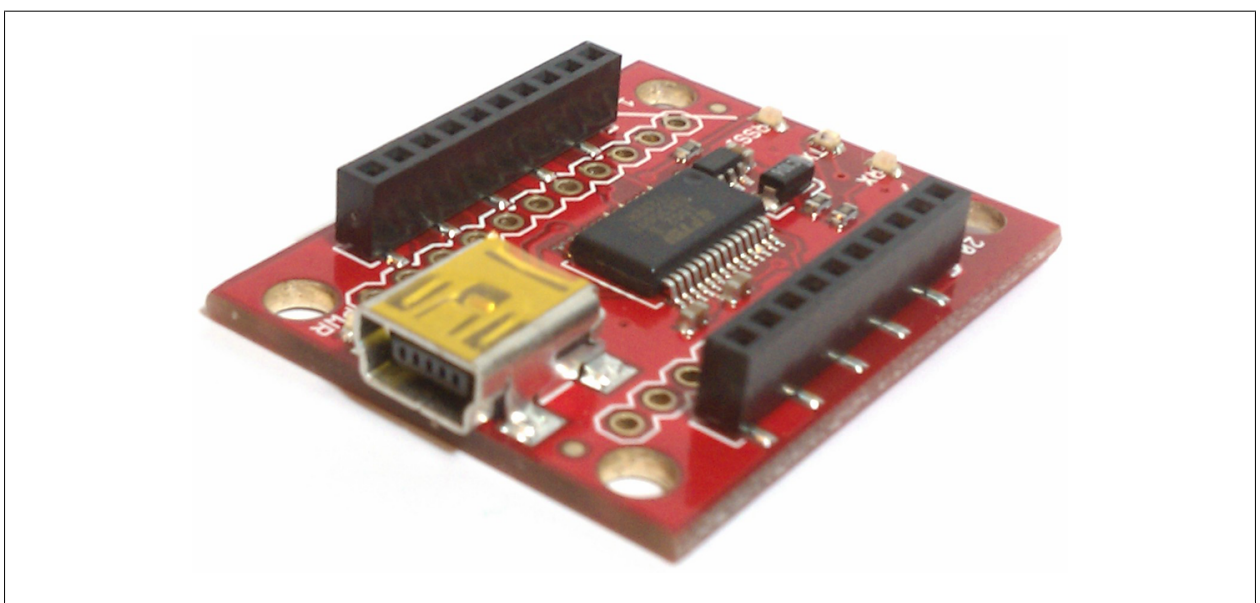

Figure 1-8. XBee Explorer board from SparkFun

\section{Adapters, Breakout Boards, and Shields}

In case you are still a bit mystified by the different ways that an XBee radio can be attached to another device, here's a quick review:

\section{Adapters}

Typically used to connect the XBee to a USB port on your computer. Some also provide breakout-board functionality.

Breakout boards

Used to plug your XBee into a standard breadboard and facilitate wired connections to other components, including the Arduino board. 
Shields

These attach an XBee directly to an Arduino microcontroller. Shields are printed circuit boards engineered to seat directly on top of an Arduino board. When you are not including other components, the shield eliminates the need for breadboards and wiring.

\section{Arduino Board Adapter Hack}

The Arduino microcontroller board we'll be using in Chapter 4 can be modified to function as an adapter for XBee radios. This is a useful hack if you don't want to buy an adapter-or anytime you find yourself caught without your regular XBee adapter setup. You'll still need a breakout board, however.

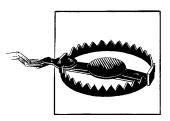

This hack allows you to connect to the XBee from a terminal program (described later in this chapter). It lets you use some features of X-CTU (also described later), but it does not support firmware upgrades. For that, you should use a proper XBee adapter.

Here's what you'll need:

- XBee radio (see "Buying an XBee Radio" on page 1)

- XBee breakout board (see "Breakout Boards" on page 10)

- Arduino microcontroller board with USB connection (Uno or similar) (SFE DEV-09950, AF 50)

- USB A-to-B-type cable (AF 62, DK 88732-9002, SFE CAB-00512)

- Solderless breadboard (AF 64, DK 438-1045-ND, SFE PRT-09567)

- Hookup wire (22 gauge or similar, different colors) or jumper wire kit (AF 153, DK 923351-ND, SFE PRT-00124)

- Wire strippers (AF 147, DK PAL70057-ND, SFE TOL-08696)

- IC extractor (DK K374-ND, RS 276-1581) or small flat-blade screwdriver (SFE TOL-09146)

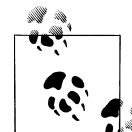

These part numbers are prefaced with abbreviations for the suppliers:

DK, DigiKey; SFE, SparkFun Electronics; AF, Adafruit; RS, Radio 2. Shack.

Insert the XBee into the breakout board, then mount the breakout board in the breadboard so that one set of legs is on each side of the breadboard's center channel. Cut four lengths of wire or select some precut jumper wires long enough to reach from the Arduino to the XBee. Use red, black, and two other colors of wire if you have them. 
Figure 1-9 shows the Arduino adapter hack breadboard layout, while Figure 1-10 shows the schematic:

1. Make sure that the Arduino is unplugged from the USB and disconnected from any other external power supply before setting up your wiring.

2. Carefully remove the ATMEGA chip from the Arduino, using an integrated circuit (IC) extractor or a small flat-blade screwdriver (when you replace it later, be sure the notch at one end of the chip is aligned with the notch in the socket). Or, if you don't want to pull the chip, program the Arduino with the following null code, which prevents the Arduino chip's bootloader from responding accidentally:

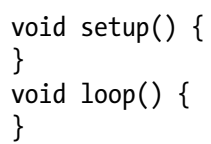

3. Connect a (red) wire from the $3.3 \mathrm{~V}$ socket on the Arduino so that it mates with the XBee's pin 1, the $3.3 \mathrm{~V}$ input pin in the upper-left corner of the XBee.

4. Next, connect a (black) wire from either GND socket on the Arduino so that it mates with pin 10 on the XBee in its lower-left corner.

5. Now wire up a connection from the TX pin (pin 1) on the Arduino to pin 2, the TX/DOUT pin on the XBee. See Table 1-2 and Figures 1-11 and 1-12 for a full list of the XBee's hardware pins and their functions.

6. Finally, wire a connection from the RX pin (pin 0) on the Arduino to pin 3, the RX/DIN pin on the XBee.

7. Check all your connections. It is very important that you supply only $3.3 \mathrm{~V}$ power to your radio.

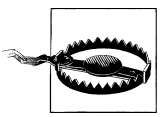

XBee radios will not work with voltages larger than 3.3. Giving them more than 7 volts will burn them out. When in doubt, remove the radio from your project and confirm the voltage with a multimeter (AF 71, DK BK117B-ND, SFE TOL-09141) before proceeding.

When you're done with the hack, set it aside for now. You won't need to power up this circuit until you get to "Configuring XBee" on page 40 in Chapter 2.

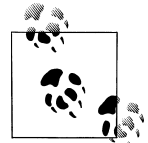

If you already have an Arduino Mini, you can use the same USB adapter you use for uploading code to the Arduino Mini as a connector for an

XBee on a breakout board. For this adapter, wire RX to RX on the XBee and TX to TX on the XBee (http://www.makershed.com/ProductDetails .asp?ProductCode=MKSP3). 


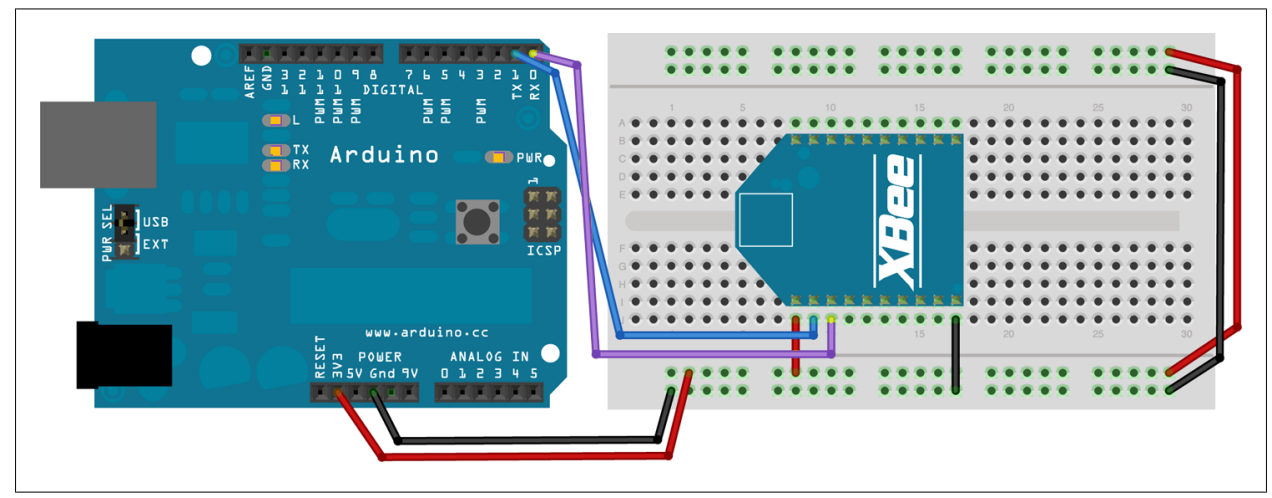

Figure 1-9. Arduino adapter hack breadboard layout

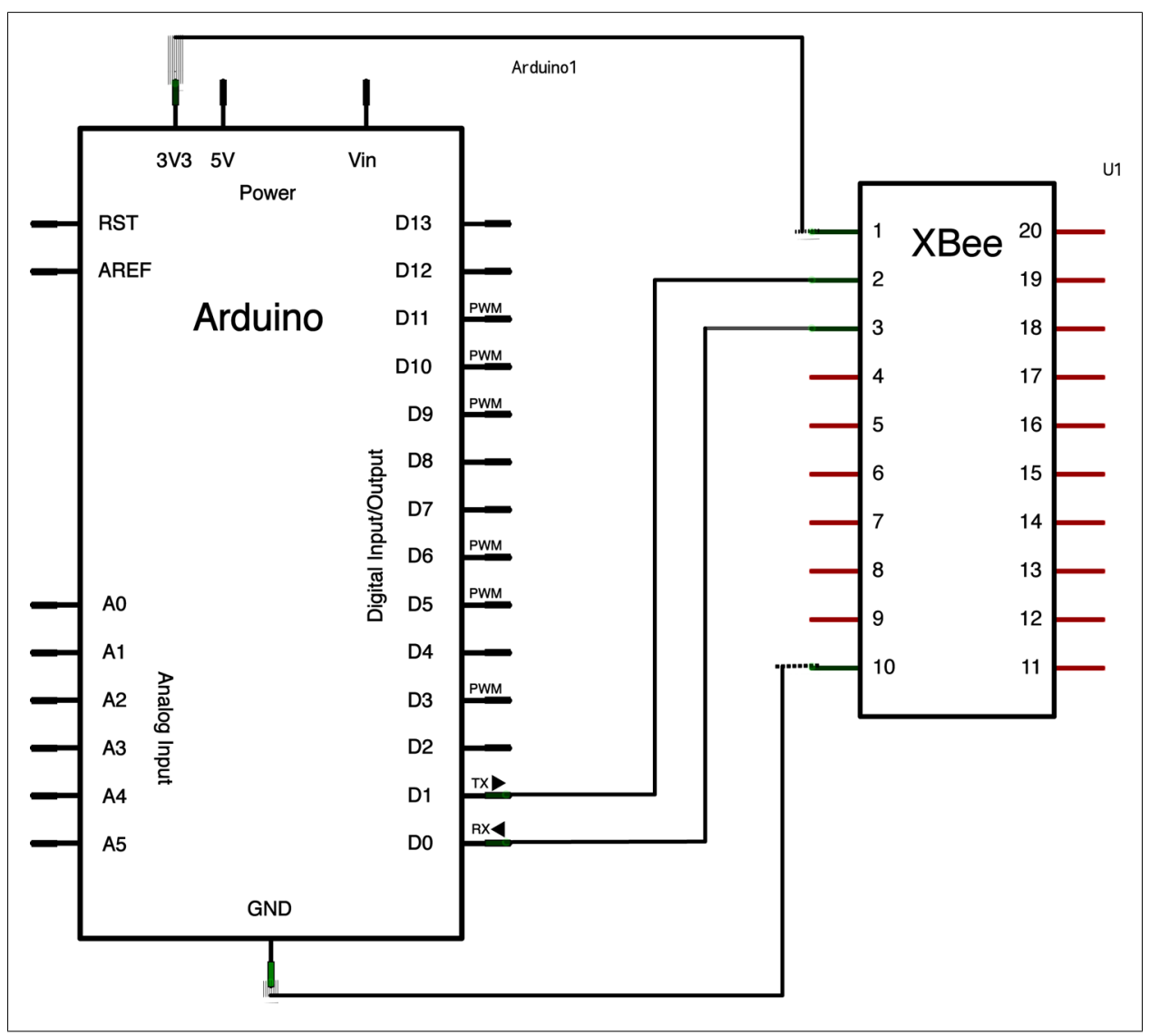

Figure 1-10. Arduino adapter hack schematic 


\section{What Are All Those Pins?}

Table 1-2. XBee pin descriptions

\begin{tabular}{|c|c|c|}
\hline Pin \# & Name(s) & Description \\
\hline 1 & VCC & 3.3 V power supply \\
\hline 2 & DOUT & Data Out (TX) \\
\hline 3 & DIN & Data $\ln (\mathrm{RX})$ \\
\hline 4 & DI012 & Digital I/0 12 \\
\hline 5 & RESET & Module reset (asserted low by bringing pin to ground) \\
\hline 6 & PWMO/RSSI/DI010 & Pulse-width modulation analog output 0, Received Signal Strength Indicator, Digital I/0 10 \\
\hline 7 & DI011 & Digital l/0 11 \\
\hline 8 & Reserved & Do not connect \\
\hline 9 & DTR/SLEEP_RQ/ DI08 & $\begin{array}{l}\text { Data Terminal Ready (hardware handshaking signal), Pin Sleep Control (asserted low), Digital } \\
\text { I/0 } 8\end{array}$ \\
\hline 10 & GND & Ground \\
\hline 11 & DI04 & Digital I/0 4 \\
\hline 12 & CTS/DI07 & Clear to Send (hardware handshaking), Digital I/0 7 \\
\hline 13 & ON/SLEEP & Sleep indicator (off when module is sleeping) \\
\hline 14 & VREF & Not used in Series 2 \\
\hline 15 & ASSOC/DI05 & $\begin{array}{l}\text { Association indicator: blinks if module is associated with a network, steady if not; Digital I/0 } \\
5\end{array}$ \\
\hline 16 & RTS/DI06 & Request to Send (hardware handshaking), Digital I/0 6 \\
\hline 17 & AD3/DI03 & Analog Input 3, Digital I/0 3 \\
\hline 18 & AD2/DI02 & Analog Input 2, Digital I/0 2 \\
\hline 19 & AD1/DI01 & Analog Input 1, Digital I/0 1 \\
\hline 20 & ADO/DIOO/COMMIS & Analog Input 0, Digital l/0 0, Commissioning Button \\
\hline
\end{tabular}

\section{Choosing a Terminal Program}

Each XBee radio has a tiny computer on board. This internal microcontroller runs a program, also known as firmware, that performs all its addressing, communication, security, and utility functions. You can configure this firmware with different settings that define things like its local address, which type of security is enforced, who it should send messages to, and how it should read sensors connected to its local input pins. 


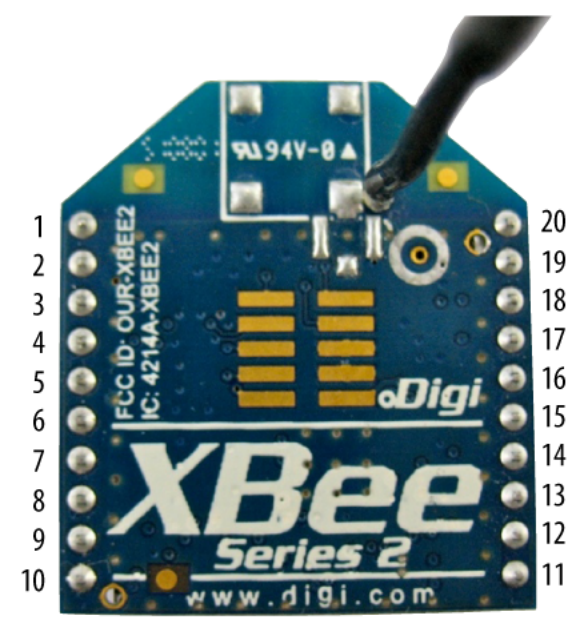

Figure 1-11. XBee physical pin numbering, front view

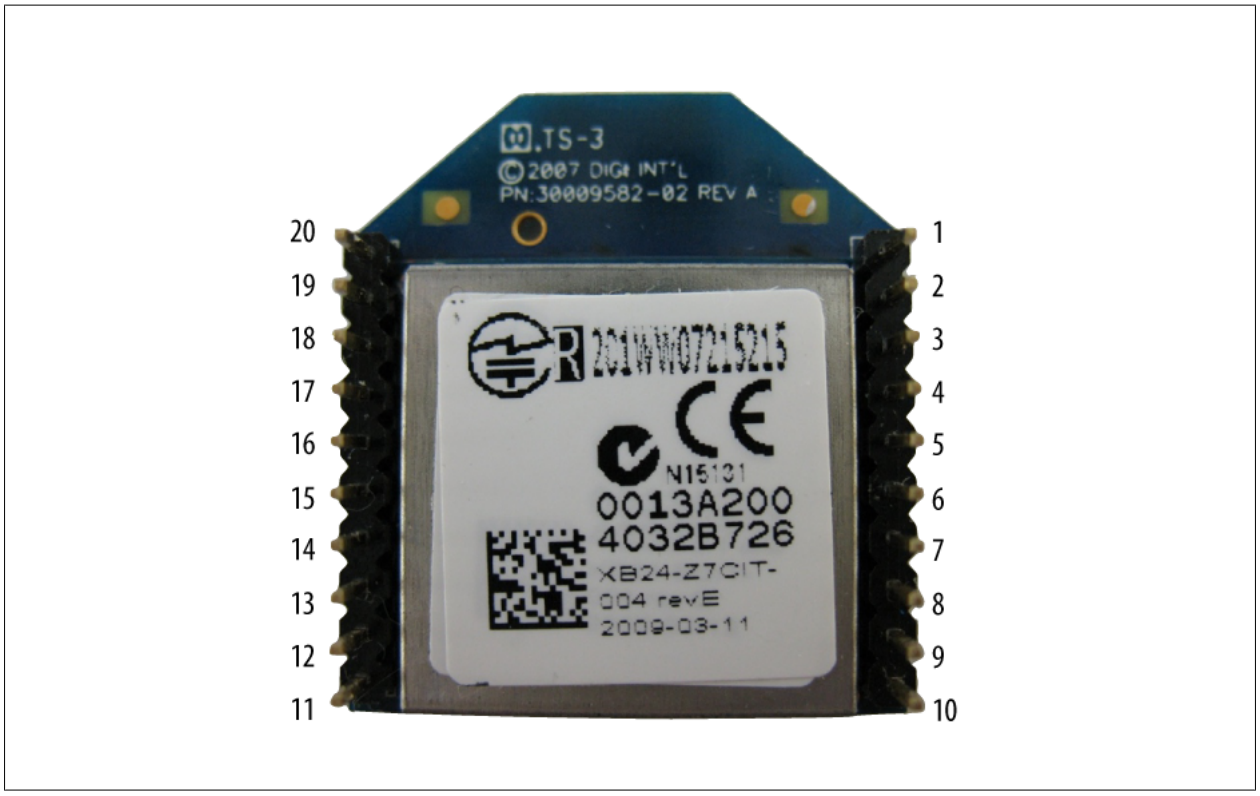

Figure 1-12. XBee physical pin numbering, back view 
To change or upgrade the firmware, we will use a program called X-CTU that you can download from the Digi website. On the upside, this program is totally free. On the downside, it runs only on Windows. Don't worry if you have limited access to Windows, though. Chances are you'll only need X-CTU initially, to load the proper firmware onto your XBee radio. Going forward, you can use serial terminal programs on Macintosh, Linux, or Windows to change many of the settings you'll be working with on a day-to-day basis. Let's take a look at some of these programs and how they operate.

\section{Firmware Update Software}

There is only one option for updating the low-level firmware on XBee radios: Digi's configuration tool, which is available for free.

\section{X-CTU}

The X-CTU program is the official configuration program for XBee radios. As noted, $\mathrm{X}-\mathrm{CTU}$ is available only for the Microsoft Windows operating system. If you have access to a native Windows computer, a Macintosh running Windows under Boot Camp or Parallels, or a Linux computer running the WINE Windows emulator (see "X-CTU in Linux" on page 33 in Chapter 2), you're all set. Luckily X-CTU is required only for updating firmware, which is a relatively infrequent task. It does have a number of other handy features, though, including fully commented setup commands, range tests, and easier access to the API features we'll be examining in Chapter 5 .

To use X-CTU, plug your XBee radio into a USB adapter and plug that adapter into one of your computer's USB ports. Next, launch the X-CTU program. It should show your USB connection as one of the available ports, similar to what you see in Figure 1-13. Select the appropriate port and then click on the Modem Configuration tab to get to a basic configuration screen (Figure 1-14). Clicking on the Read button will generally access the radio's setup, though this depends upon which firmware is currently loaded. Don't be concerned if you get an error message instead. We'll go over the details in the next chapter.

\section{Terminal Software for Configuring Settings}

Once you've loaded the firmware, you may want to use a different program to communicate with your XBee. It's very helpful to have some familiarity with one or more serial terminals because you may not always have access to X-CTU when you need it. At a friend's house, a hacking workshop, a public demo, or in the midst of a Maker Faire, you might need to check something or change a setting on a non-Windows computer. Or you may run into a Windows machine where you don't have the rights to download and install new software. Here's a host of different options that can save you in such cases. We'll talk about how to set them up and use them in the next chapter. 


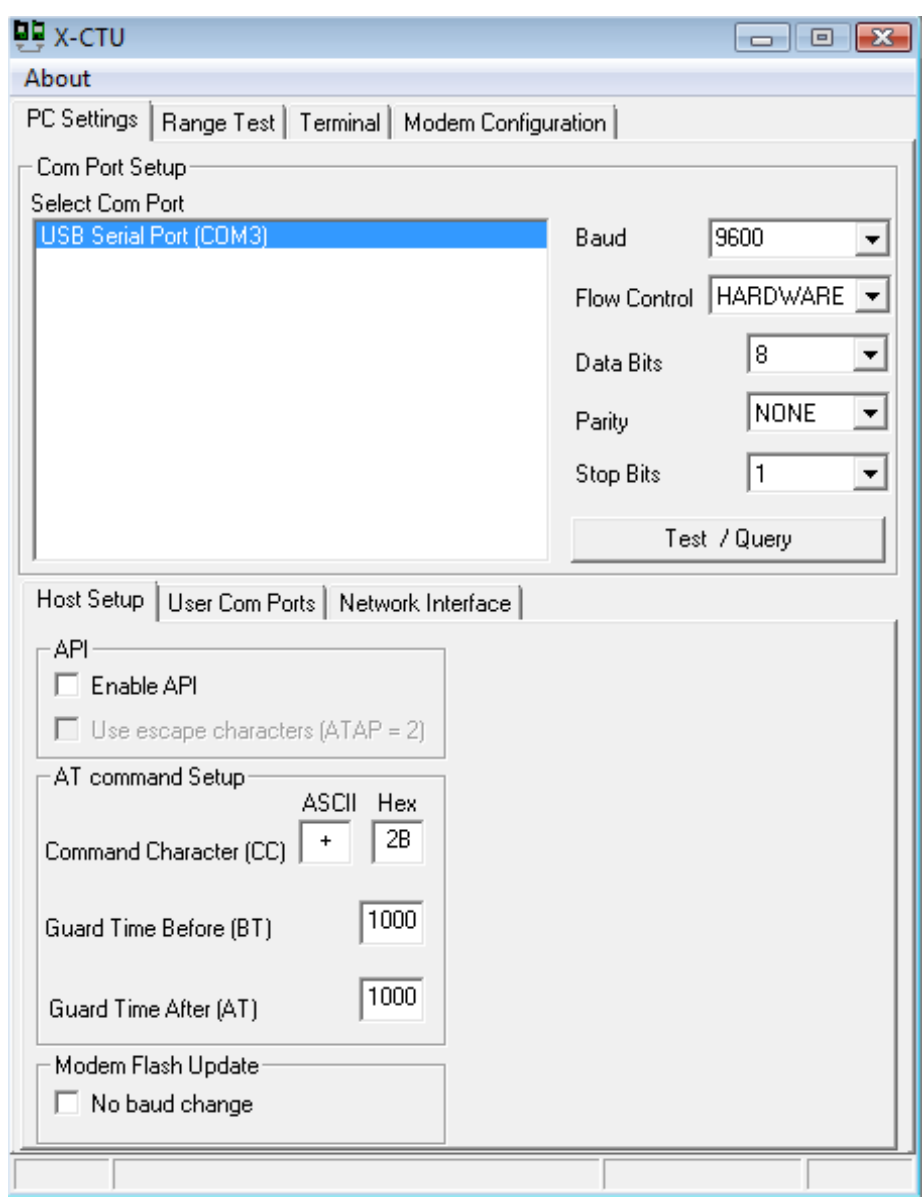

Figure 1-13. Using X-CTU

\section{CoolTerm}

CoolTerm is a terrific open source serial terminal program created by Roger Meier that runs well on both Windows and Macintosh. It's a relatively simple program that's perfect for most basic tasks you need to perform with XBee radios. CoolTerm is free. Consider making a small donation to show your appreciation and encourage continued support for the program (http://freeware.the-meiers.org).

\section{HyperTerminal}

Windows XP and older Windows versions come with a serial terminal program called HyperTerminal. If you are using Windows Vista or Windows 7, HyperTerminal may still be available as a free demo, or for purchase from http://www.hilgraeve.com/hyper terminal.html. 


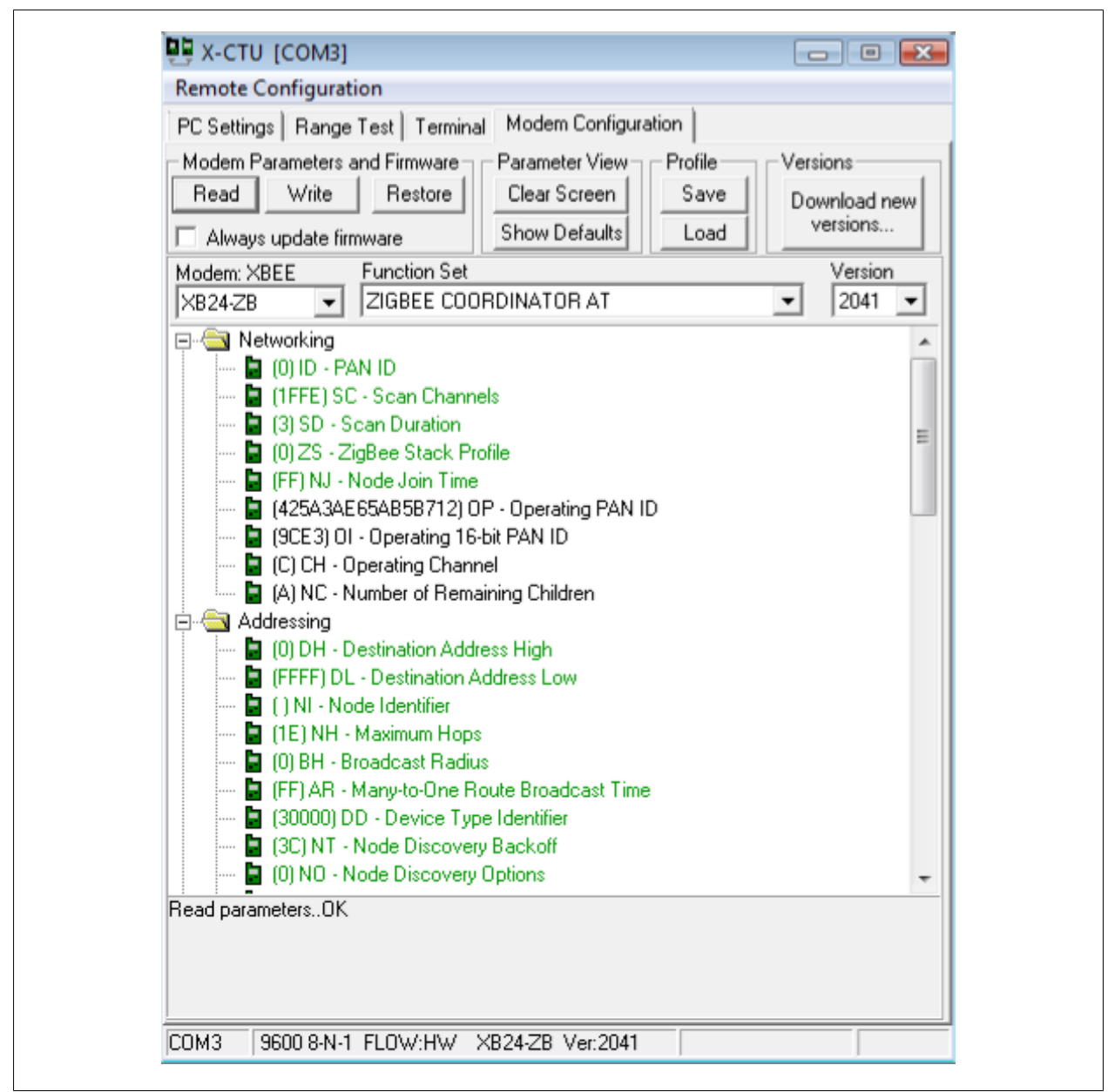

Figure 1-14. X-CTU Modem Configuration tab

\section{Tera Term}

Tera Term is a free, open source Windows program that performs a wide variety of terminal functions, including acting as a serial terminal. Those using Vista or Windows 7 will appreciate having a free option, since HyperTerminal is no longer bundled with Windows and must be purchased separately. This is the Windows software we'll use to demonstrate serial terminal use (http://ttssh2.sourceforge.jp/).

\section{ZTerm}

An old favorite terminal program on the Macintosh, ZTerm has been showing its age for quite some time. It was designed in 1992 and was last updated in 2002. Still, it is widely used and despite its anachronistic features and idiosyncratic design, it's been 
stable for almost 20 years. You'll find some brief setup documentation on my blog, and you can download ZTerm and pay its small shareware fee online (http://homepage.mac .com/dalverson/zterm/).

\section{screen}

For Linux users and for those comfortable in the Macintosh Terminal, there's a command-line program named screen that allows direct access to serial ports, including USB devices. On Mac OS X, the command ls dev/tty.* will list the available ports, returning a list like this:

/dev/tty.Bluetooth-Modem /dev/tty.Bluetooth-PDA-Sync/dev/tty.usb-A410032.

On Linux, try ls dev/ttyUSB*. Your serial port will probably be something like /dev/ ttyUSBO.

Once you know what your USB port is called, you can invoke the screen program, using the port and a data rate of 9600 baud. For example:

screen /dev/tty.usb-A410032 9600

To exit, type Ctrl-A followed by Ctrl- $\backslash$ and then $\mathbf{y}$ to quit.

The picocom program, described in the sidebar "A Serial Terminal Program for Linux" on page 40 in Chapter 2, is an alternative to screen and has certain features (such as local echo) that can be useful for working with XBees.

\section{Others}

Here are some other popular options for serial terminals. Some are free, and some aren't:

- RealTerm

- Termite

- PuTTY

- MacWise

\section{Summary}

Here is a basic shopping list that will work well for this book. Feel free to customize it according to your interests and the projects you have planned:

- Three XBee ZBs with wire antenna (Digi: XB24-Z7WIT-004, DK602-1098-ND)

- One or two XBee Explorers (SFE: WRL-08687)

- One or two USB A to Mini-B cables (SFE: CAB-00598)

- X-CTU for Windows (free)

- CoolTerm (free) 
Now that you know what to get, go do it! As soon as your components arrive, you will probably be itching to use them. The next chapter will help you transform your box of parts into a working ZigBee network. You'll be chatting wirelessly in no time. 

Here is the heart of the book. We go from a bag of parts to a working ZigBee network in one chapter, taking the simplest path to early success. Addresses, firmware, and configuration steps culminate in a simple chat session for a satisfying exchange of greetings. Hello world, you are up and running.

Let's get started.

\section{Radio Basics}

What exactly is this thing called radio? In any dictionary or encyclopedia, you'll find a definition that describes the transmission of information via modulation of waves in the electromagnetic spectrum. Whoa, that's pretty mysterious, especially when coupled with the mathematics and equations that describe the behavior of radio. These certainly help us work with the medium, yet they still may not answer the question of what it is. If you feel unsatisfied by the words or the math, that's OK. One helpful way to think of radio is as a well-described mystery. After all, we can't see radio. We can't touch radio or hear it or smell it or feel it. Billions of years of evolution haven't provided us with any direct sensory apparatus for perceiving the radio part of the electromagnetic spectrum at all. Our language around the phenomenon reveals this. The word radio comes from radius, the Latin for a ray or spoke in a wheel, something that propagates from a center outward. True, but pretty vague. Around the turn of the last century it was referred to as "ethereal communication" in a nod to the "ether" that was incorrectly thought to pervade outer space. That turned out to be just wrong. Today it's often referred to as wireless communication, but that's not what it is. That's what it isn't. Radio is also tomato-less, cheese-less, and bread-less, but that does no better to help us understand it. The element of mystery is fundamental to the human experience of radio, and a reassuring clue that your grasp on it will always be a little loose. Luckily, we do have a tremendous ability to describe radio's propagation, predict its behavior, and use it efficiently for a huge number of purposes. For example, you are about to use it in the creation of your own sensor mesh network, cleverly manipulating a phenomenon that is beyond your direct powers of perception. That's pretty neat. 


\section{Electromagnetic Spectrum}

Radio is only one slice of the broad array of energy we call the electromagnetic spectrum (see Figure 2-1). This spectrum includes high-power gamma rays that arrive from supernovas in outer space, the X-rays we use to sneak a peek at broken bones, microwaves that cook our food, infrared that we sense broadly as heat, and the one tiny slice of the spectrum that about a third of our brain is devoted to decoding: visible light. Radio waves are much longer than light waves and many can travel through opaque substances such as clothing, furniture, and brick walls. Radio energy requires no medium. It can propagate perfectly well in a vacuum and is therefore ideal for communications where metal wire connections are impractical, or where visual line of sight may be impeded by obstructions. When radio waves impinge on a conductor, like metal, they induce an electrical current that transforms their energy into another form. This means that radio will not travel well through metal walls, but it also means that we can employ metallic antennas to transduce radio energy into electrical signals that computers can detect and process. Engineers have a comprehensive body of theories, equations, and laws for predicting and manipulating the behavior of radio. Luckily, we can make do for the time being with just one of these tools, the inverse square law.

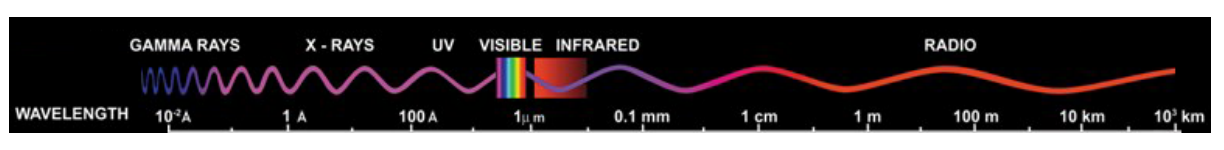

Figure 2-1. Chart of the electromagnetic spectrum

\section{Inverse Square Law}

Radio signals require a lot of power because, unlike messages running through a wire, they decay in an accelerated fashion. The reason for this is easy to understand. As radio signals radiate away from their source, they rapidly spread out like ripples in a pool. Sound works pretty much the same way, which is why it's easy to hear a whisper up close, but impossible to understand it even a few feet away. Both sound and radio decay according to the inverse square law. Each time you double the distance, you require four times the amount of power (as Figure 2-2 shows), so traversing long distances requires tremendous expenditures of energy compared to shorter ones.

ZigBee mesh networks are designed with the inverse square law in mind. Rather than using big batteries to generate the large amount of power needed to send a signal over a great distance, each radio needs only small amounts of power to go a short distance to its nearest neighbor in the network. By adding nodes to the network, great distances can be traversed without any node needing access to large amounts of energy. 


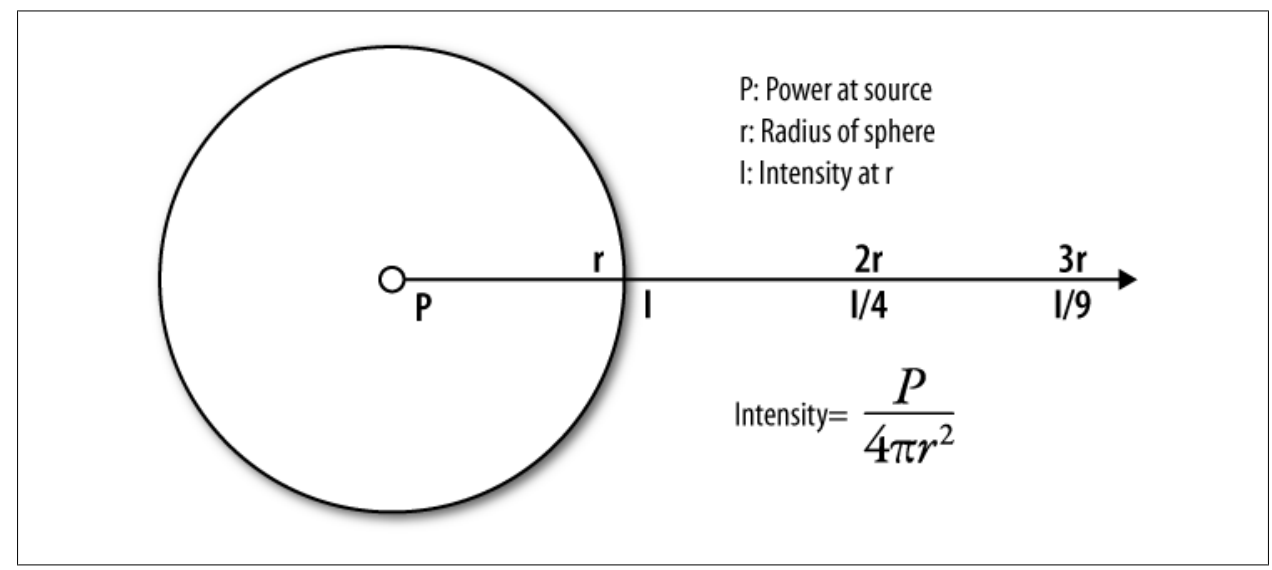

Figure 2-2. Inverse square law

\section{Introduction to ZigBee}

Many people think that ZigBee and XBee are the same thing. That's not true. ZigBee is a standard communications protocol for low-power, wireless mesh networking. XBee is a brand of radio that supports a variety of communication protocols, including ZigBee, 802.15.4, and WiFi, among others.

The ZigBee protocol is a standard the same way that Bluetooth is a standard. Any manufacturer's device that fully supports the ZigBee standard can communicate with any other company's ZigBee device. So just as your Motorola Bluetooth headset can communicate with your Apple iPhone, a CentralLite ZigBee light switch can communicate with a Black \& Decker door lock. How does this work? Well, just like a great cake, robust network protocols are all about layers.

Most modern network protocols employ a concept of layers to separate different components and functions into independent modules that can be assembled in different ways. We're not going to bother with a lot of network theory here, just enough for you to complete the tasks at hand.

Every network has a physical layer where signals are actually transmitted. For example, your computer may be connected via an Ethernet cable to the Internet. On the other hand, it may be going wireless with a WiFi connection, using radio signals to traverse the real world. That's all happening in the physical layer, and doesn't change a thing about, for example, what's going on at the application layer, which is where your web browser lives. Firefox doesn't care a whit if you switch from Ethernet to WiFi. It is protected by the interfaces between layers that allow each software and hardware module to change how it does its job, but still talk to the other layers in exactly the same way. 
Another way to conceptualize this is to consider your car. You can drive over concrete highways, asphalt driveways, metal bridges, and dirt parking lots without changing vehicles. Your tires provide an interface between the vehicle layer and the road layer. It would work just as well if you were driving a motorcycle or an ice cream truck. Either layer can be changed out independently without affecting the other.

The network layer below ZigBee that supports its advanced features is known as IEEE 802.15.4. This is a set of standards that define power management, addressing, error correction, message formats, and other point-to-point specifics necessary for proper communication to take place from one radio to another. XBee-brand radios can be purchased with or without ZigBee. For example, the XBee Series 1 hardware-which we don't work with in this book (but do mention in Chapter 1) —supports 802.15.4 directly in its native form. ZigBee is a set of layers built on top of 802.15.4. These layers add three important things:

\section{Routing}

Routing tables define how one radio can pass messages through a series of other radios along the way to their final destination.

Ad hoc network creation

This is an automated process that creates an entire network of radios on the fly, without any human intervention. Pretty cool.

\section{Self-healing mesh}

Self-healing is a related process that automatically figures out if one or more radios is missing from the network and reconfigures the network to repair any broken routes.

A ZigBee network is a little like a basketball team. Both are composed of various players, and each player specializes in certain types of actions. Without the different players, neither can function properly. Of course, ZigBee is not quite basketball. For one thing, the radios are not particularly tall. Also, there are really only three kinds of players, or device types. Every ZigBee network will have a single coordinator device. You can't call anything a network until you have at least two things connected. So every ZigBee network will also have at least one other player, either a router device or an end device. Many networks will have both, and most will be much larger than just two or three radios:

\section{Coordinator}

ZigBee networks always have a single coordinator device. This radio is responsible for forming the network, handing out addresses, and managing the other functions that define the network, secure it, and keep it healthy. Remember that each network must be formed by a coordinator and that you'll never have more than one coordinator in your network.

\section{Router}

A router is a full-featured ZigBee node. It can join existing networks, send information, receive information, and route information. Routing means acting as a 
messenger for communications between other devices that are too far apart to convey information on their own. Routers are typically plugged into an electrical outlet because they must be turned on all the time. A network may have multiple router radios.

\section{End device}

There are many situations where the hardware and full-time power of a router are excessive for what a particular radio node needs to do. End devices are essentially stripped-down versions of a router. They can join networks and send and receive information, but that's about it. They don't act as messengers between any other devices, so they can use less expensive hardware and can power themselves down intermittently, saving energy by going temporarily into a nonresponsive sleep mode. End devices always need a router or the coordinator to be their parent device. The parent helps end devices join the network, and stores messages for them when they are asleep. ZigBee networks may have any number of end devices. In fact, a network can be composed of one coordinator, multiple end devices, and no routers at all.

\section{Network Topology}

In basketball, once the players are selected, they still need to assemble as a team. ZigBee networks are the same way. They can connect together in several different layouts or topologies to give the network its structure. These topologies indicate how the radios are logically connected to each other. Their physical arrangement, of course, may be different. There are three major ZigBee topologies, illustrated in Figure 2-3:

Pair

The simplest network is one with just two radios, or nodes. One node must be a coordinator so that the network can be formed. The other can be configured as a router or an end device.

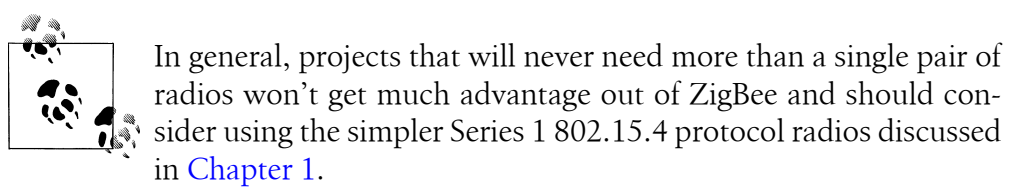

Star

This network arrangement is also fairly simple. A coordinator radio sits at the center of the star topology and connects to a circle of end devices. Every message in the system must pass through the coordinator radio, which routes them as needed between devices. The end devices do not communicate with each other directly. 


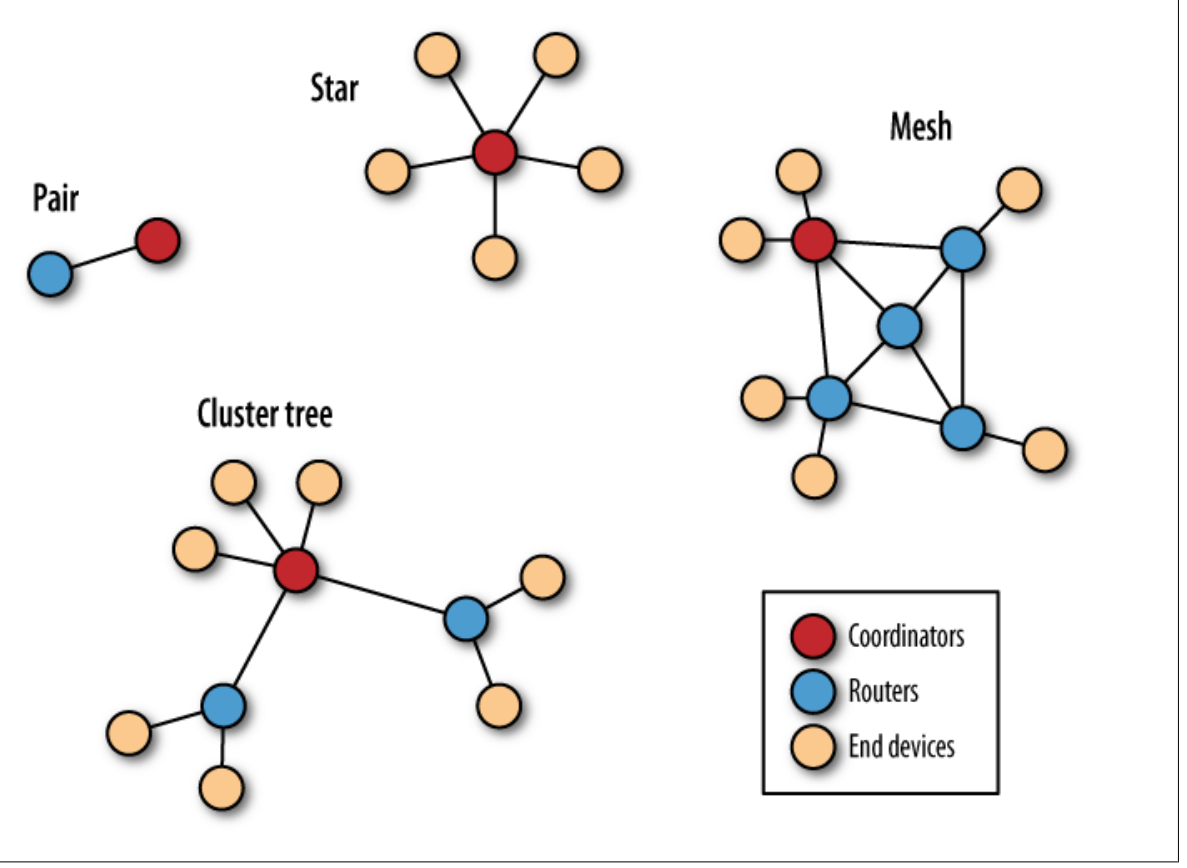

Figure 2-3. ZigBee pair, star, mesh, and cluster tree topologies

Mesh

The mesh configuration employs router nodes in addition to the coordinator radio. These radios can pass messages along to other routers and end devices as needed. The coordinator (really just a special form of router) acts to manage the network. It can also route messages. Various end devices may be attached to any router or to the coordinator. These can generate and receive information, but will need their parent's help to communicate with the other nodes.

Cluster tree

This is a network layout where routers form a backbone of sorts, with end devices clustered around each router. It's not very different from a mesh configuration.

\section{Addressing Basics}

Almost every person has an address where he can be reached, usually one that is unique to him. Many people have more than one. We have mailing addresses, email addresses, phone numbers, passport numbers, and the list goes on. Each kind of address or identifier serves a slightly different purpose. It's the same with radios (see Table 2-1). If you want to send a ZigBee message, you need to know the address of the destination radio. Just like with people, each radio is known by several different addresses, each of which serves a purpose. For starters, each radio has a unique and permanently assigned 64bit serial number. No other ZigBee radio on earth will have that same serial number. 
Then there's a shorter 16-bit address that is dynamically assigned to each radio by the coordinator when it sets up a network. This address is unique only within a given network, but since it's shorter, many more of them can be manipulated in the very limited memory available on a ZigBee chip. Finally, each XBee radio can be assigned a short string of text called the node identifier. This allows the radio to be addressed with a more human-friendly name. Four out of five humans prefer a friendly machine.

Table 2-1. Address types

\begin{tabular}{lll}
\hline Type & Example & Unique \\
\hline 64-bit & 0013A200403E0750 & Yes, always and everywhere \\
16-bit & 23F7 & Yes, but only within a network \\
Node identifier & FRED'S RADI0 & Uniqueness not guaranteed \\
\hline
\end{tabular}

\section{PAN Addresses}

In the United States, nearly every town has a Main Street. Thousands of different families live at, for example, 123 Main Street. We can tell them apart because while their street address is the same, their town or city is different. Each ZigBee network creates a virtual "city" in the same way, and labels that city not with a name but with a number, the Personal Area Network (PAN) address. This is another 16-bit address. There are 65,536 different PAN addresses available, each having the capability to generate another 65,536 16-bit radio addresses below it. In theory, therefore, this addressing scheme has room for more than 4 billion total radios, more than you'll ever need, no matter how ambitious a project you may have planned!

\section{Channels}

Even if all the addressing is perfect, your message still won't get through unless both radios are tuned to the same frequency. When the ZigBee coordinator picks a network PAN address, it also checks over all the available channels, typically 12 different ones, and picks a single one for that network's conversations. All the radios in that network must use the same channel. By default, XBee radios handle channel selection automatically so you usually don't need to worry about this, unless of course something goes wrong.

\section{All Together Now}

So for a message to get through from one radio to another, the radios need to be on the same channel and have the same PAN information, and the sending radio must know at least one of the receiving radio's addresses (see Figure 2-4). In addition, some networks have security protocols that require an exchange of keys; however, encryption and security protocols (discussed in Chapter 8) are not required for any of the projects 
we describe. For now, remember that you'll need a PAN and a radio address to get your messages through. You'll learn how these are set up in the next sections.
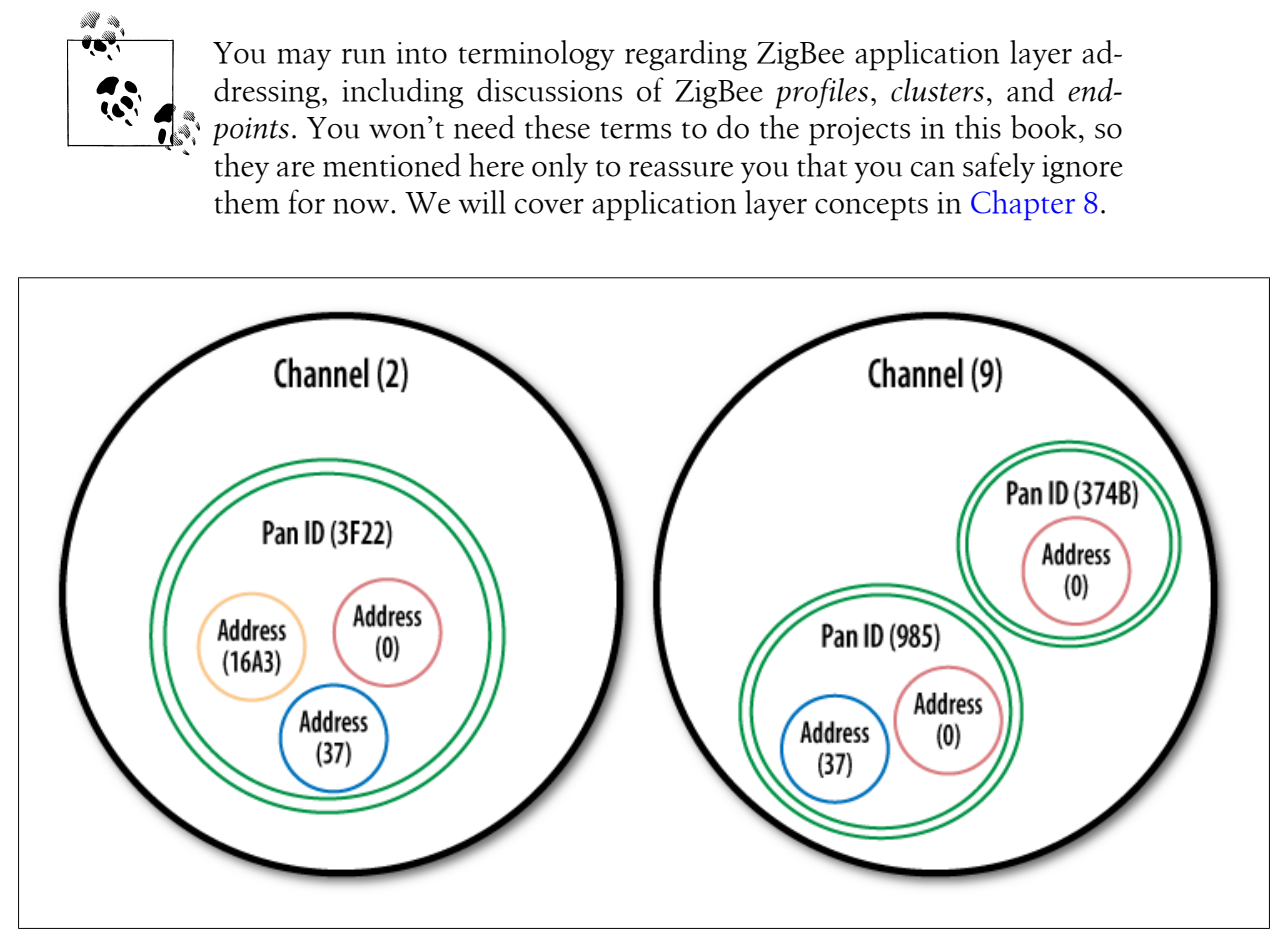

Figure 2-4. Venn diagram showing channel, PAN, and addressing

\section{Hexadecimals}

There's no question about it: if you want to use an XBee, you'll need to understand hexadecimal notation. Every time you set an XBee's address, configure one of its timers, or read the signal strength, the numbers you use are all formatted in base 16 .

Relax! It's pretty easy and we're going to show you everything you need to know about these special numbers. If you've worked with computers at all, you've almost certainly seen these numbers, called hexadecimals, hex, or base 16 (these all mean the same thing).

Normally, we express numbers in base 10, counting with numerals from 0 to 9 , then carrying to the next place to get 10 like this:

\section{$1 \ldots 2 \ldots 3 \ldots 4 \ldots 5 \ldots 6 \ldots 7 \ldots 8 \ldots 9 \ldots 10$}

In decimal, there's a ones place, a tens place, a hundreds place, and so on. Let's take the decimal number 7,453: 


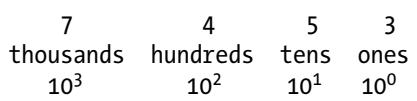

This probably looks pretty familiar. However, if you came from another planet and didn't know how to read decimals, you could multiply each number by its place to get the total value. $\left(7^{*} 1,000\right)+\left(4^{*} 100\right)+\left(5^{*} 10\right)+\left(3^{*} 1\right)=7,453$. Hold that thought; this method will come in handy below.

You might be interested to learn that decimal is only one of many ways to write down numbers. Imagine how compact your notation would be if you could count from 0 to 15 before you had to carry to the next place, for example:

\section{$1 \ldots 2 \ldots 3 \ldots 4 \ldots 5 \ldots 6 \ldots 7 \ldots 8 \ldots 9 \ldots \mathrm{UH}-\mathrm{OH}$ !}

We don't have a single numeral to express 10 ! We could make up a new squiggle and that would work fine, except it would be easier if we could use something already on the computer keyboard. So let's just use the letter A and say that stands for 10 . We can then use the letter B to stand for 11, and so forth:

$1 \ldots 2 \ldots 3 \ldots 4 \ldots 5 \ldots 6 \ldots 7 \ldots 8 \ldots 9 \ldots$ A...B...C..D ... ......10

It may look weird but we didn't make a mistake at the end. F stands for 15, and then to express 16 we carried so we had 1 in the sixteens place and 0 in the ones place:

$$
\begin{array}{cc}
1 & 0 \\
\text { sixteens } & \text { ones } \\
16^{1} & 16^{0}
\end{array}
$$

That's right, in hexadecimal the notation 10 means 16 . To avoid confusion, we usually mark hexadecimal numbers in a special way, with a leading zero and a letter $\mathrm{x}$, like this: $0 x 10$. The leading zero and $\mathrm{x}$ don't mean that we're multiplying by zero or anything. They're simply a prefix, like the dollar sign, to let us know what kind of notation will follow. So if you see the hexadecimal 0x7E2 here's how to break it down:

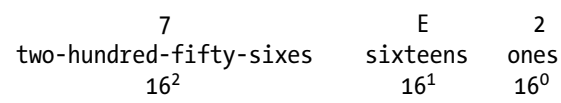

So what number is this anyway? Remember that multiplication exercise we did with decimals? Let's try it with this unfamiliar hexadecimal. $\left(7^{*} 256\right)+\left(\mathrm{E}^{*} 16\right)+(2 * 1)=$ ?? Oh bother, we need to translate that letter E into its decimal form. Let's do that right now: $(7 * 256)+(14 * 16)+(2 * 1)=2,018$.

Try translating these decimals into hex. The first few are filled in to get you started:

$10=0 x A$

$16=0 \times 10$

$17=0 \times 11$

$18=$

$160=$ (Hint: think of how many sixteens are in 160)

$256=$ 
Now try translating these hexadecimals into decimals:

$0 \mathrm{xFF}=$

$0 \times 3=$

$0 \times 4 \mathrm{~B}=$

$0 \times 4 C=$

$0 \times 186 \mathrm{~A} 0=$

That last one is hard, so it's only fair to tell you now that both Windows and Macintosh have hex calculators. On the regular Windows calculator, change the View menu from Standard to Scientific. On the Macintosh calculator (Figure 2-5), change the View menu to Programmer. Now, click the Dec and Hex buttons to switch from decimal to hexadecimal notation. You were promised easy, and what could be simpler than clicking a button? Enjoy.

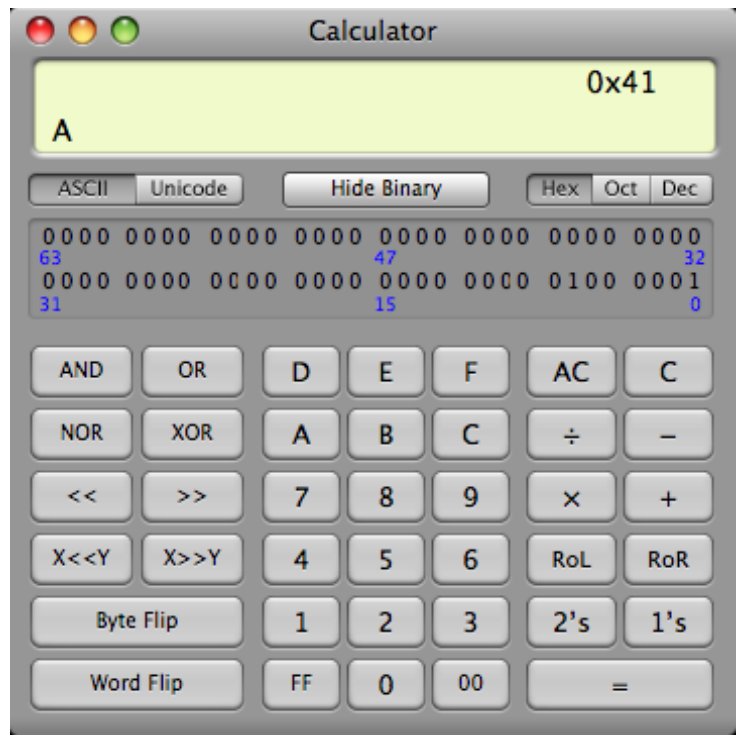

Figure 2-5. Mac calculator in programmer mode

\section{XBee Firmware Updates}

Your brain is brimming with facts, and your shiny new hardware sparkles with possibilities. The time has arrived to put your hands to work. Their first job will be to ensure that the right types and versions of the firmware are installed on your XBees. You'll be using the X-CTU program for this, so fire up the Windows operating system (or Linux; see "X-CTU in Linux" on page 33), then download and install X-CTU. 


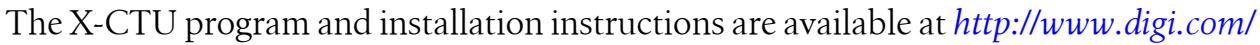
support/kbase/kbaseresultdetl.jsp?kb=125. During the installation process, if you are asked if you want to download new firmware versions, go ahead and get them. In addition to X-CTU, you'll need to install the appropriate drivers for your XBee adapter board. Most adapter boards, such as the SparkFun XBee Explorer and the New Micros XBee Dongle, use FTDI drivers. The drivers and installation instructions are located at http://www.ftdichip.com/FTDrivers.htm. Windows may be able to discover the drivers on its own if you have Windows Update enabled and are connected to a network.

\section{$X$-CTU in Linux}

To use X-CTU under Linux, you'll need to first install Wine, which lets you run Windows applications under the X Window System. On a Linux system, you can usually install Wine using your Linux package manager.

Next, using Wine, download the X-CTU installer and run this command:

wine 40002637_c.exe

(If the filename is not 40002637_c.exe, replace it with the name of the file you downloaded.)

Now create a symbolic link between the serial port that corresponds to your XBee and a Windows serial port, such as COM10:

In -s /dev/ttyUSBO /.wine/dosdevices/com10

The actual device filename (ttyUSBO in the example) will vary, so look at the output of the dmesg command shortly after you plug in the XBee adapter to see which device was added.

Next, launch X-CTU using a command something like:

wine .wine/drive_c/Program \Files/Digi/XCTU/X-CTU.exe

Click the User Com Ports tab and type in the name of the Com port you created (such as COM10), then click Add. Unfortunately, you will need to do this each time you launch X-CTU, as it does not remember the custom Com ports.

Plug one of your XBee radios into your XBee adapter and connect the adapter to one of your Windows computer's USB ports. Launch the X-CTU application. You should see your XBee's USB connection listed under Select Com Port. Click on the appropriate port to select it, as shown in Figure 2-6.

The default settings in X-CTU will usually work for brand-new XBee radios that were configured at the factory. The easiest way to confirm that everything is set up correctly is to click on the Test/Query button once you've selected a COM port. If all goes well, you'll see a message that communication with the modem is OK and that gives you the modem type and firmware version, as shown in Figure 2-7. 


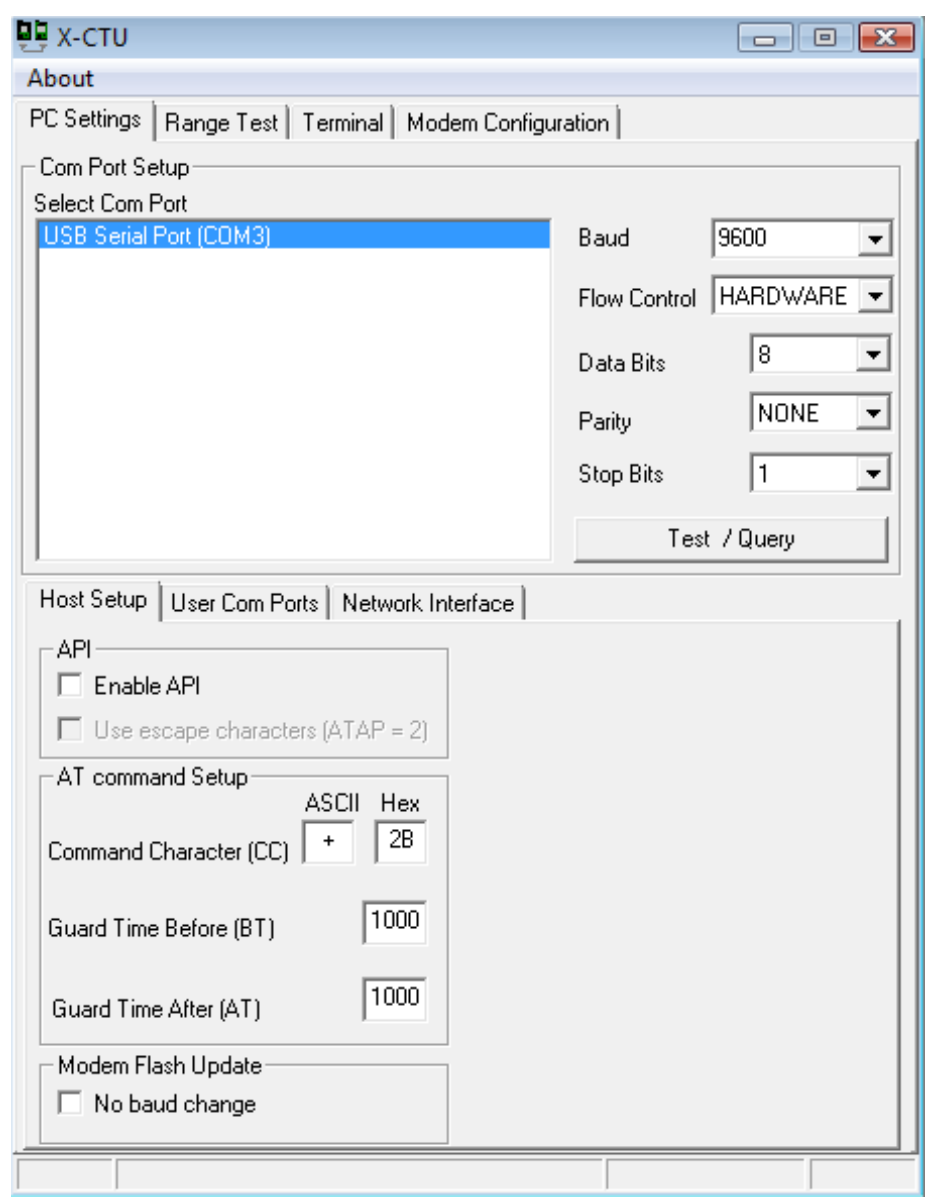

Figure 2-6. X-CTU starting screen

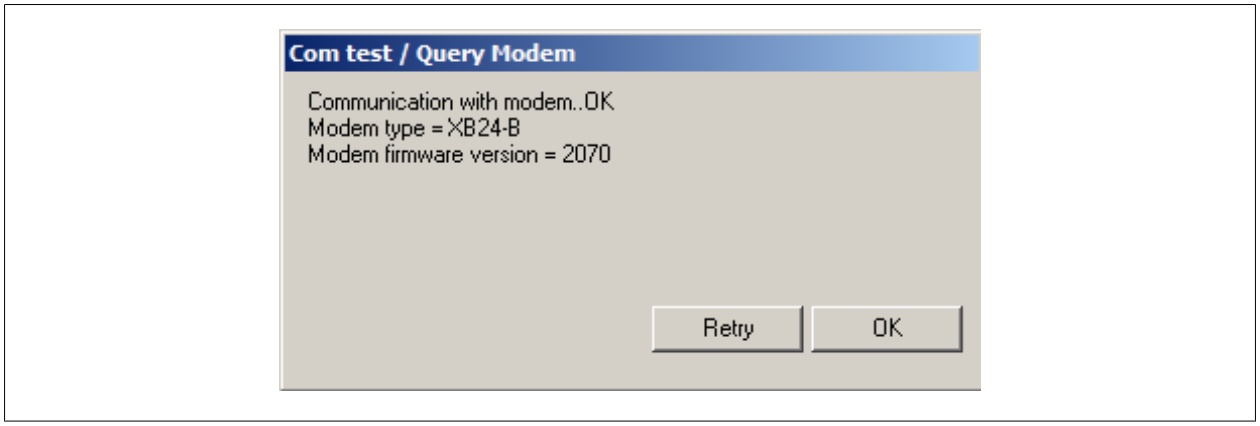

Figure 2-7. X-CTU test confirmation 
If you get the message "Unable to communicate with modem," make sure your XBee is seated properly in its adapter, that it isn't too far forward or back by a pin, and that it wasn't inserted backward (see Figures 2-8 and 2-9). Also, check to make sure you selected the correct COM port. (If you suspect that your XBee may be using the API firmware, try checking the Enable API Mode box. API mode is covered in Chapter 5.) It's also possible that your XBee has been configured to a baud rate different from the default of 9600 baud. Try switching to one of the other baud rates and trying again. If you still can't get an OK response to the test, don't despair. Most of the time, your hardware is just fine. There are plenty of other fixes you can attempt. Check the Appendix for additional troubleshooting steps, or contact Digi for technical support at $h t t p: / / w w w$.digi.com/support. Sometimes, just moving on to the next step helps with connection issues, so let's do that now.

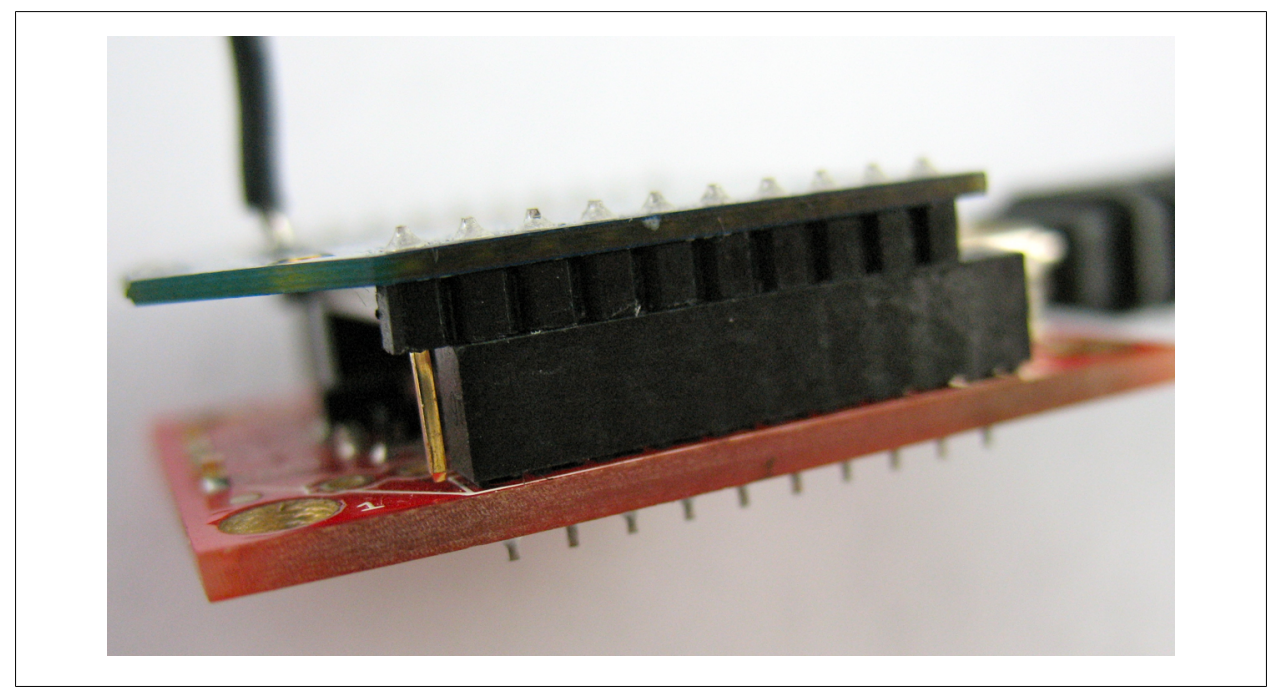

Figure 2-8. An XBee misaligned and seated incorrectly. Note that one of the metal pins is showing ahead of the socket. This radio will not work until it is properly reseated.

\section{Reading Current Firmware and Configuration}

Now that you've tested the XBee for basic communication, you'll want to take a look at what firmware it's sporting and how that firmware is currently configured. Switch to the Modem Configuration tab, then click on the Read button under Modem Parameters. If all goes well, this will populate the window below with all kinds of useful information, as shown in Figure 2-10. 


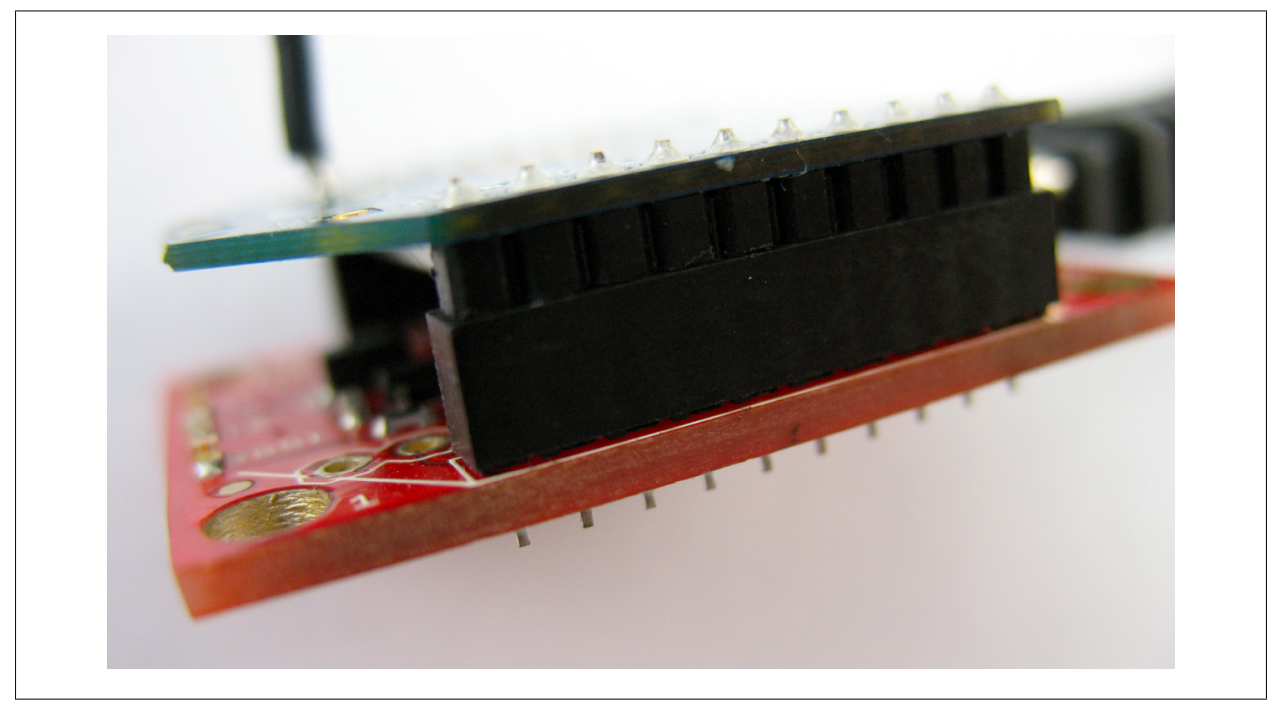

Figure 2-9. The XBee aligned and seated correctly in its adapter. All the metal pins are inserted into the sockets.

Note the "Download new versions" button. Use this button occasionally to have XCTU check the Digi website for new versions of firmware (click "Download new versions," then click Web).

\section{Linux Troubleshooting}

If you're running X-CTU under Wine on Linux, you may see a dialog box that tells you the modem configuration file could not be found. This dialog will offer to download the latest configuration files from the website. If it fails:

1. Visit the Digi FTP site.

2. Next, look in both the $x b e e \_s 2$ (series 2) and $x$ bee_zb (ZigBee firmware) subdirectories to find the firmware file that matches what you saw in Figure 2-7 (for example, XB24-ZB_2041.zip for a regular XBee, XBP24-ZB_2041.zip for an XBeePRO).

3. Then, find the highest numbered XB (for XBee) or XBP (for XBee-PRO) firmware in the $x b e e \_z b$ directory, sort by date, and download all of the most recent available ZIP files. For example, at the time of this writing, the most recent firmware files for the regular XBee were XB24-ZB_2070.zip, XB24-ZB_2170.zip, XB24ZB_2270.zip, XB24-ZB_2370.zip, XB24-ZB_2870.zip, and XB24-ZB_2970.zip.

4. In X-CTU, go to the Modem Configuration tab, click Download New Versions, and use the File option to install each of the files (one at a time, unfortunately) you just downloaded.

5. Click the Read button again, and X-CTU should recognize your XBee. 
The Modem type listed needs to be XB24-ZB or XBP24-ZB. Modem types XB24-B, XB24P-B, XB24-SE, and XB24P-SE can be updated to the $\mathrm{ZB}$ firmware. If you see another model listed when you Read from the radio, it may not be the correct hardware, in which case it will not work with this book. Chapter 1 has information on where to obtain the correct hardware.

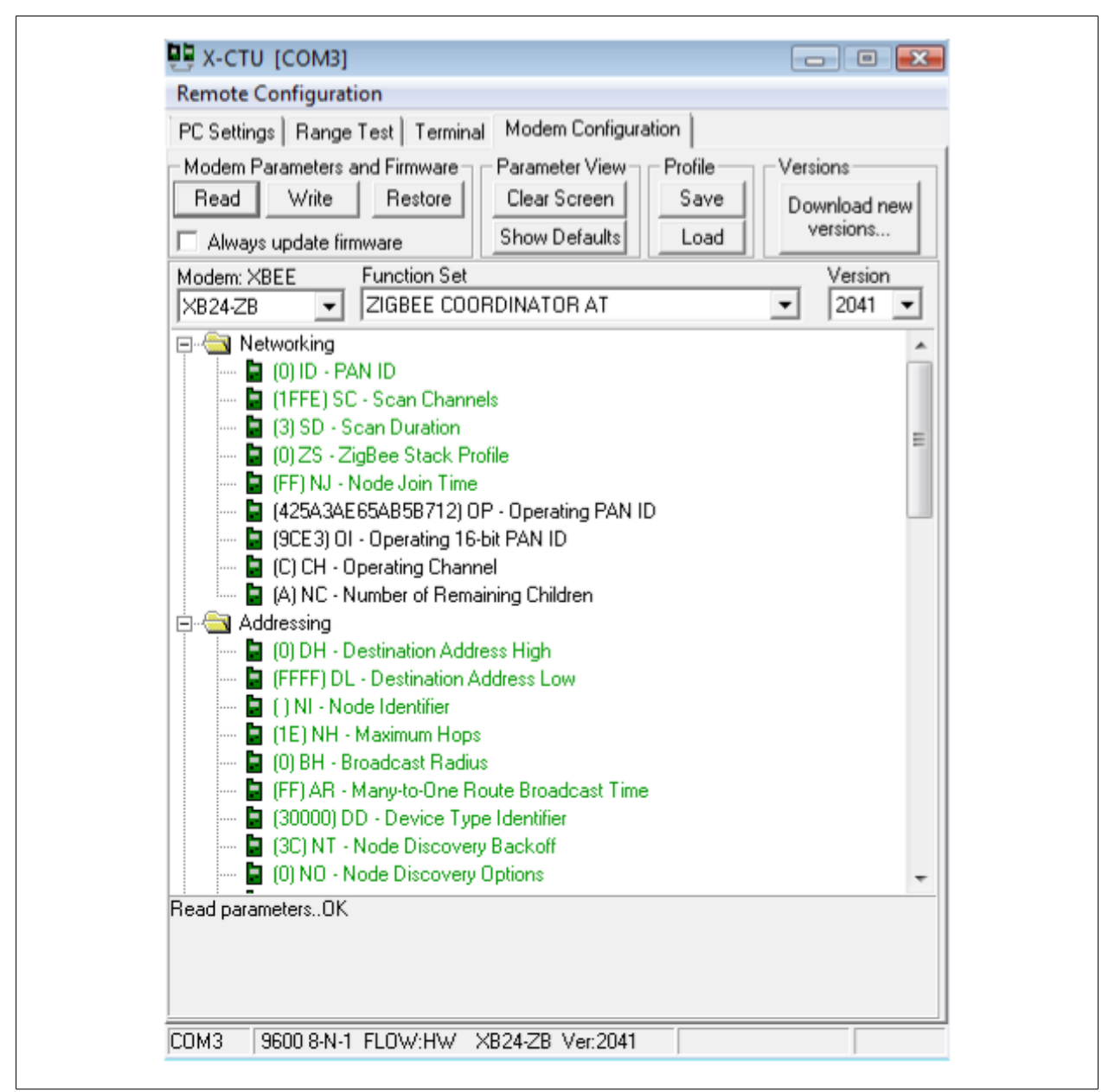

Figure 2-10. XBee coordinator AT configuration 
If you get a dialog box that says there's a problem (Figure 2-11), with a suggestion for pressing the XBee reset switch, try gently pulling the XBee out of its socket on the adapter and reseating it. Be sure to wait $10 \mathrm{sec}-$ onds for X-CTU to recognize the radio; after it does, it will close the dialog box on its own.

Doing this while the adapter is still plugged in effectively resets it. The Digi Evaluation Board has a reset button, so if that's your adapter, simply press the button.

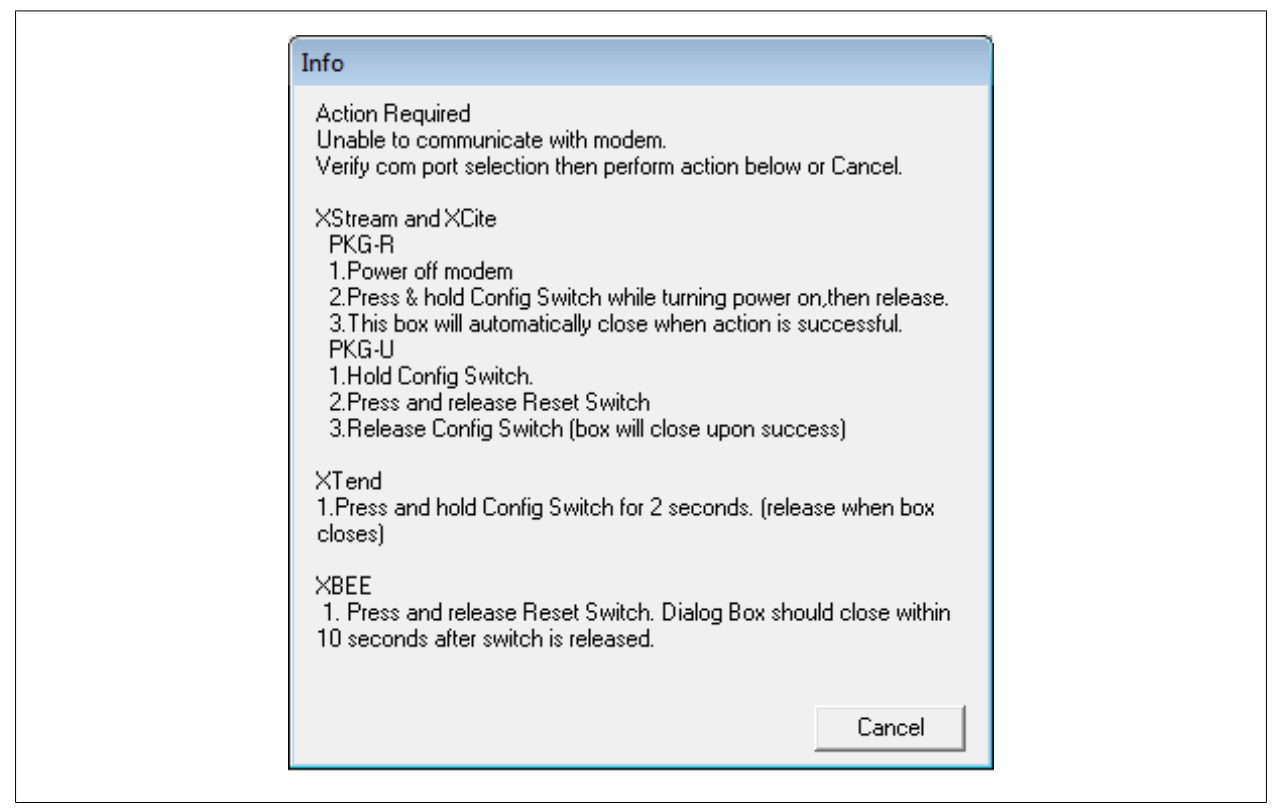

Figure 2-11. If you get this message, you can reset the XBee by gently pulling it out of its socket and reseating it

Let's configure the first XBee:

1. The class of radio modem is shown under Modem: XBEE (Figure 2-10). For everything we do in the main part of this book, it should be XB24-ZB (or XBP24-ZB if you're using the higher-power PRO version of the radios). If it's set to something else, select either of these options from the menu.

2. Under Function Set, you'll see a list of different firmware that can be loaded for this class of radio modem. To start, we'll be configuring one coordinator radio and one router, both in AT command mode. So for your first radio, if it's not already selected, choose ZIGBEE COORDINATOR AT for your function set. Any version 2070 or greater should be fine; in general you want the highest-number version (they're hexadecimals) listed for that particular function set. 
3. Click on the Write button to program your radio with the coordinator firmware. For later reference, use a piece of tape or a small sticker to identify this radio as the coordinator.

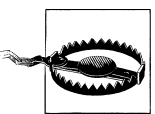

If you get a Windows error such as "Could not open output file. System error. Access denied.”, check that your account has administrator access.

Once you've installed your first radio with the coordinator AT command software, gently remove that radio from the adapter and carefully seat a second radio in the same adapter. Click on Read in the Modem Configuration screen to see what firmware is on that radio, then select XB24-ZB (or XBP24-ZB for PRO radios), ZIGBEE ROUTER AT, and the highest-number version available. Any version 2270 or greater should be fine. Again, click on Write to program your second radio with the router firmware. Mark the router radio as well to identify it.

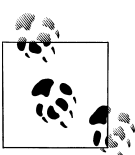

If your radio has API firmware and you had to check the Enable API box on the PC Settings tab, when you switch to AT command firmware, the last step of the update may fail with a message about an "Error Setting AT Parameters" (Figure 2-12). You can safely ignore this error, change back to the PC Settings tab, uncheck the Enable API box, and then select the Modem Configuration tab and Read in the Modem Parameters again. Phew!

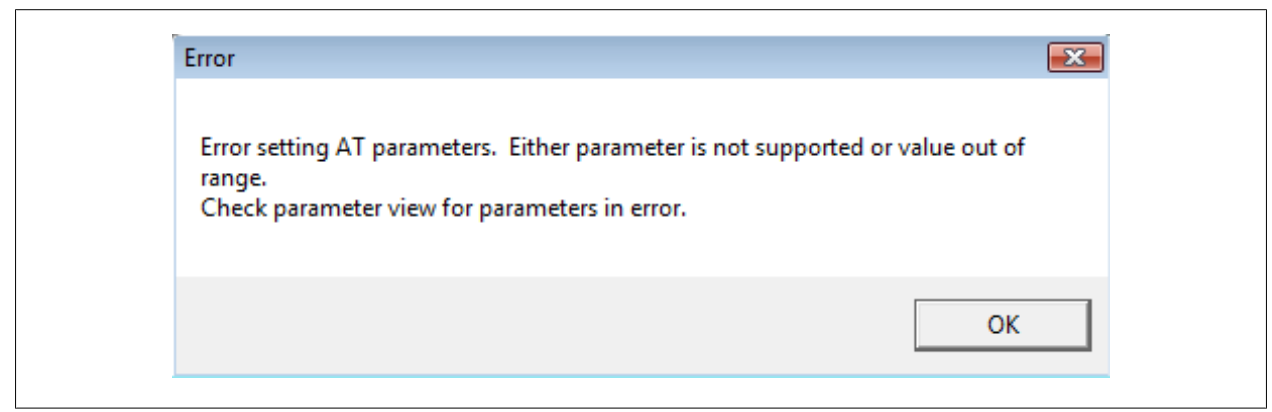

Figure 2-12. Error when switching from API to AT firmware

In addition to selecting firmware, you can use X-CTU to configure your radios' settings. Because you may not have full-time access to Windows and X-CTU, it's a good idea to learn how to change these settings with a regular serial terminal program. We'll start by setting up your XBees in this way. 


\section{Configuring XBee}

Any time you aren't able to use X-CTU, you can configure any XBee that's in AT command mode by using a serial terminal program. In the previous chapter we covered a variety of serial terminal software. Here we'll show two different programs, Tera Term for Windows and CoolTerm for Macintosh (CoolTerm also works on Windows).

\section{A Serial Terminal Program for Linux}

If you're on Linux, you'll probably find picocom to be a suitable terminal program. The newer versions of picocom support local echo, which lets you see what you're typing. To use picocom, you'll need to compile and install it, then run it at the command line. For example, to connect to the first USB-serial port (if you've only got one XBee plugged into your Linux system, it will probably be this port), use:

picocom --echo --imap crcrlf/dev/ttyUSBO

You can exit picocom by typing Ctrl-A followed by Ctrl-X.

\section{Settings}

No matter what program you use, you'll need to configure your software to use the communication settings shown in Table 2-2.

Table 2-2. Default XBee settings for serial terminal software

\begin{tabular}{ll} 
Baud & 9600 \\
Data & 8 bit \\
Parity & None \\
Stop bits & 1 \\
Flow control & None \\
Line feed & CR+LF or Auto Line Feed \\
Local echo & On \\
\hline
\end{tabular}

\section{Ports}

You always need to select the USB port your XBee adapter is attached to. On Windows, this will probably be listed as one of the COM ports; on Macintosh, as a port with the word usbserial in the title; and on Linux, as a port with ttyUSB in the title. Many people figure out which port is right via trial and error. Honestly, this isn't a bad way to do it. The other option is to remove the XBee adapter from the USB port and see which port name disappears from your port list. The port name that disappears is your XBee adapter. Windows users can also find a list of the active COM ports by selecting the Device Manager from the Windows Control Panel on the Start menu. Macintosh users 
can see a list of ports by opening the Terminal program, typing ls /dev/tty.* (Linux users should use ls /dev/ttyUSB*), and then pressing the Return key.

\section{Connecting from Windows}

To begin using your XBee via Tera Term on Windows, plug the XBee adapter into one of your USB ports and launch the Tera Term application. Tera Term can generally be selected right from the Windows Start menu. The opening screen (Figure 2-13) will prompt for a new connection.

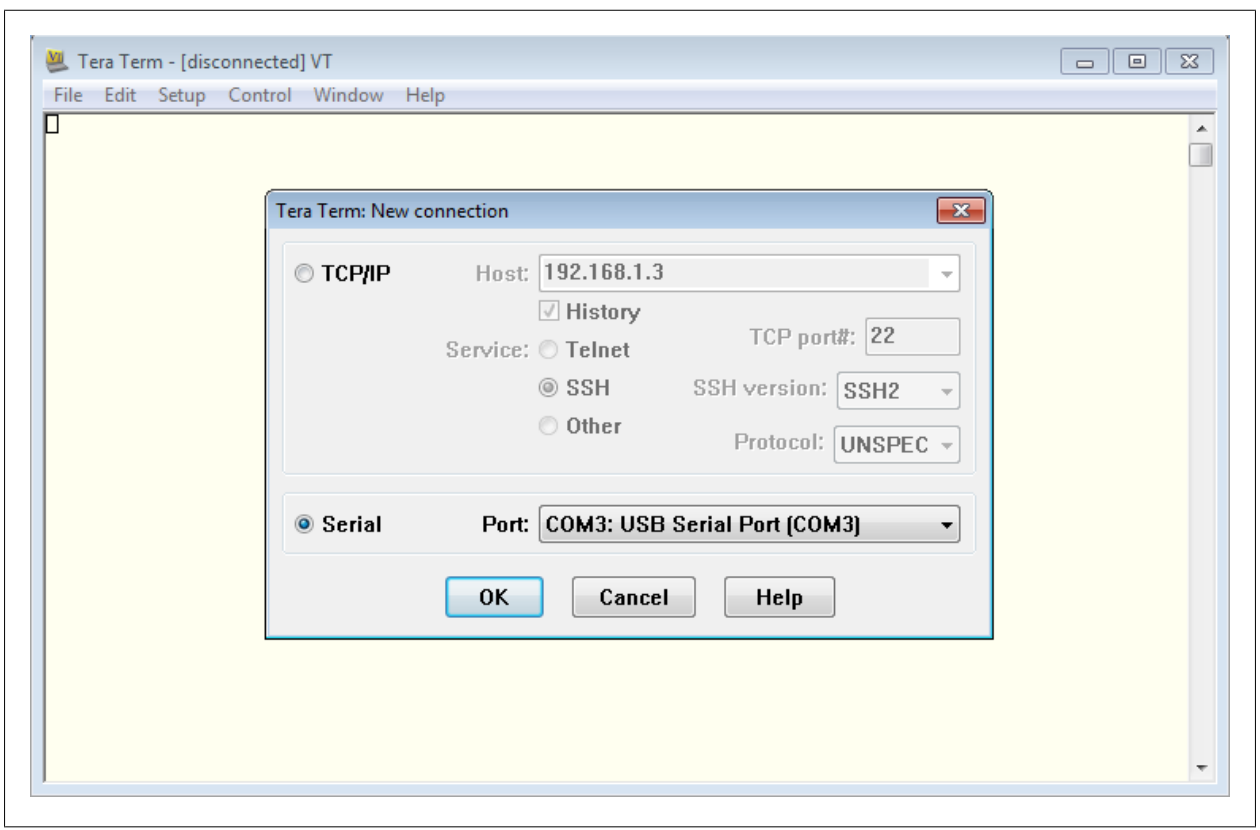

Figure 2-13. Tera Term opening screen

Select Serial on the "New connection" screen, then choose the port that is connected to your XBee adapter. Click on OK and you should see a blank Tera Term window. Choose Terminal from the Setup menu. In the dialog box that's presented (Figure 2-14), choose CR+LF for New-line Receive and check the "Local echo" box. Click OK to close this panel.

Next, select Serial from the Setup menu to confirm that the communication settings are correct. You've already selected your port, and the default of 9600 baud, 8 data bits, no parity, one stop bit, and no flow control will be perfect (Figure 2-15). Click OK to close the panel.

If you want to permanently save this setup, choose Save Setup... from the Setup menu and click the Save button. You're ready to get started with configuring your XBee! 
Terminal size

$90 \times 35$

$\nabla$ Term size $=$ win size

$\square$ Auto window resize

Terminal ID: VT100

Answerback:

Kanji [receive]

UTF-8 -

$\square 7$ bit katakana

locale: american
New-line

Receive: CR+LF -

Transmit: CR

V Local echo

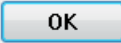

Cancel

Help

\section{$\square$ Auto switch [VT<->TEK]}

Kanji [transmit]

UTF-8 Kanji-in:

Kanji-out:

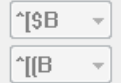

CodePage: 65001

Figure 2-14. Tera Term terminal setup

COM3:9600baud - Tera Term VT

File Edit Setup Control Window Resize Help

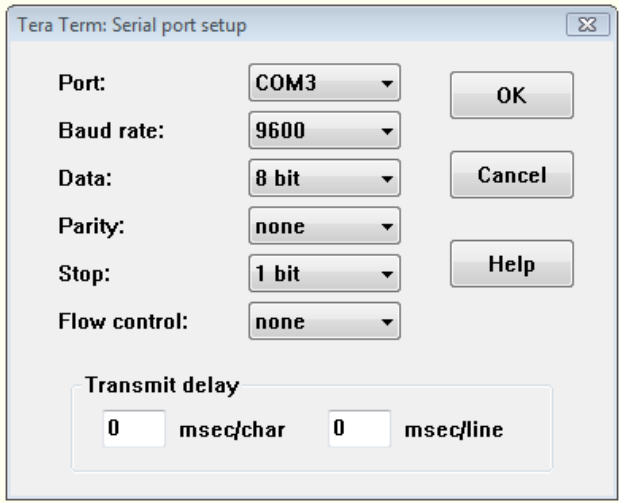

Figure 2-15. Tera Term serial port setup 
To confirm that your XBee is connected properly, you can try putting it into command mode. Type three plus signs in a row, but don't press Return, just wait a moment after entering them:

If you don't get a response, try typing the three plus signs again. Make sure you don't type anything before or after them. You should see an OK response (Figure 2-16). If you do, congratulations-you're successfully connected to your XBee! Skip to "Command Mode and Transparent Mode" on page 46 to continue.

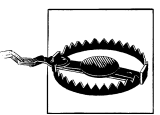

Your radio requires one second of guard time before and one second of guard time after you type the +++ or it won't go into command mode. Begin by typing nothing for at least one second, then type the three plus signs, and then don't type anything else for at least one second more. Remember, don't press the return key! That counts as typing something and will prevent you from going into command mode.

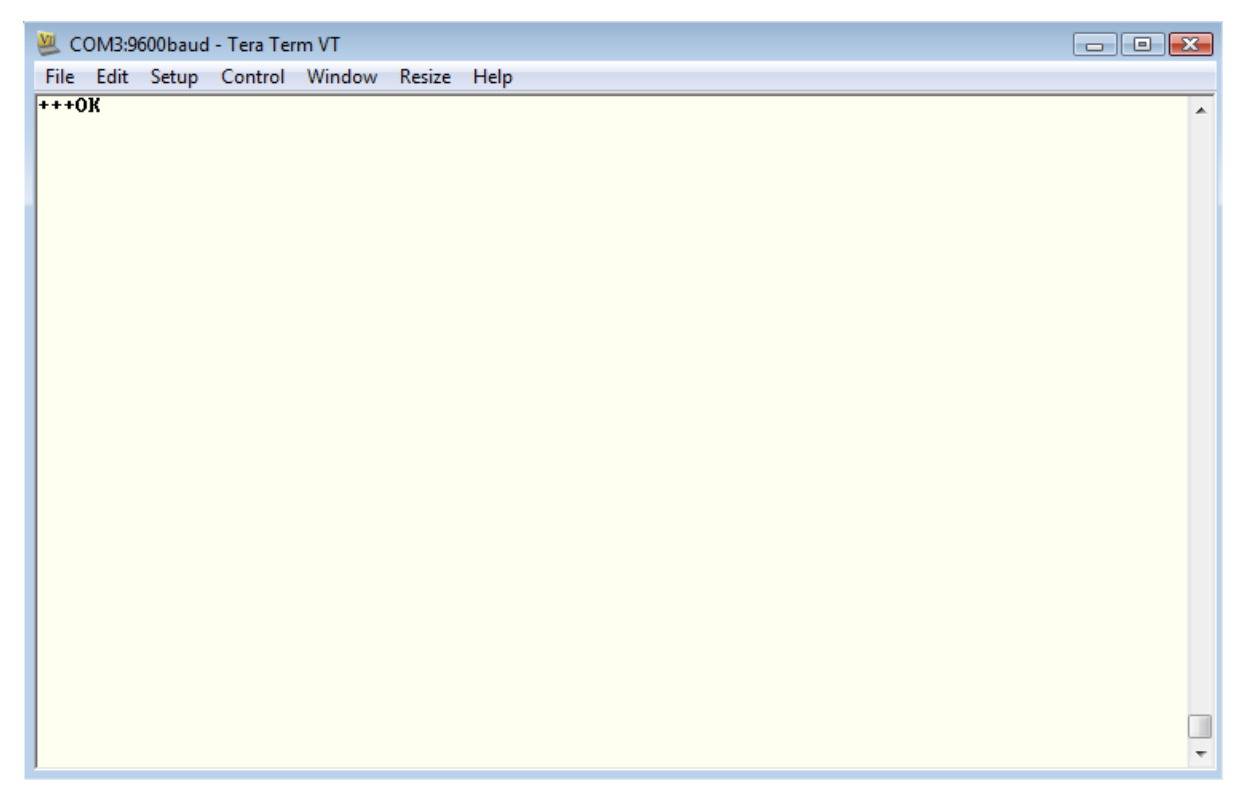

Figure 2-16. Tera Term with XBee in command mode

\section{Connecting from Macintosh}

To communicate with the XBee from the Macintosh OS, we will use an open source program called CoolTerm (Figure 2-17). CoolTerm is in ongoing development, so it will probably evolve rapidly and may behave somewhat differently or offer new features 
by the time you read this. Once you've downloaded and installed CoolTerm, doubleclick on the application to launch it. Click on the Options button to display the current settings (Figure 2-18). Choose the port your XBee adapter is connected to from the Device list at the top of the screen. The port will probably have usbserial as part of its title. The defaults of 9600 baud, 8-N-1 packets, and None for flow control will be perfect for initial connections to XBee radios. You'll want to check the Local Echo box so you can see the commands you're typing on-screen. Click the OK button to save your settings.

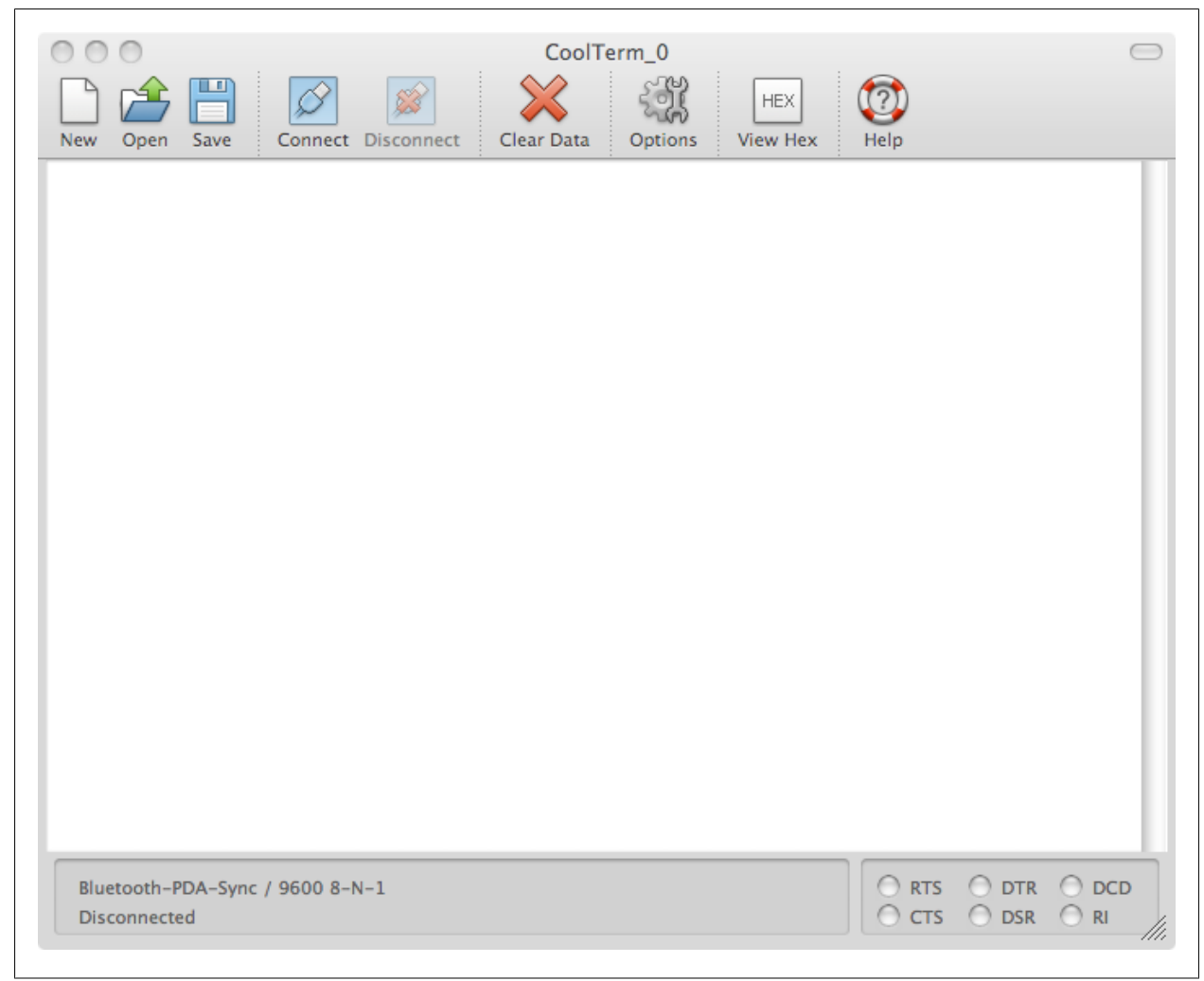

Figure 2-17. CoolTerm window

To open the serial connection, press the Connect button. Now you can put your XBee into command mode by typing three plus signs (+++) into the lower window. Don't press Return! The XBee should respond with OK about a second later. You'll see this response in the CoolTerm window (Figure 2-19). 


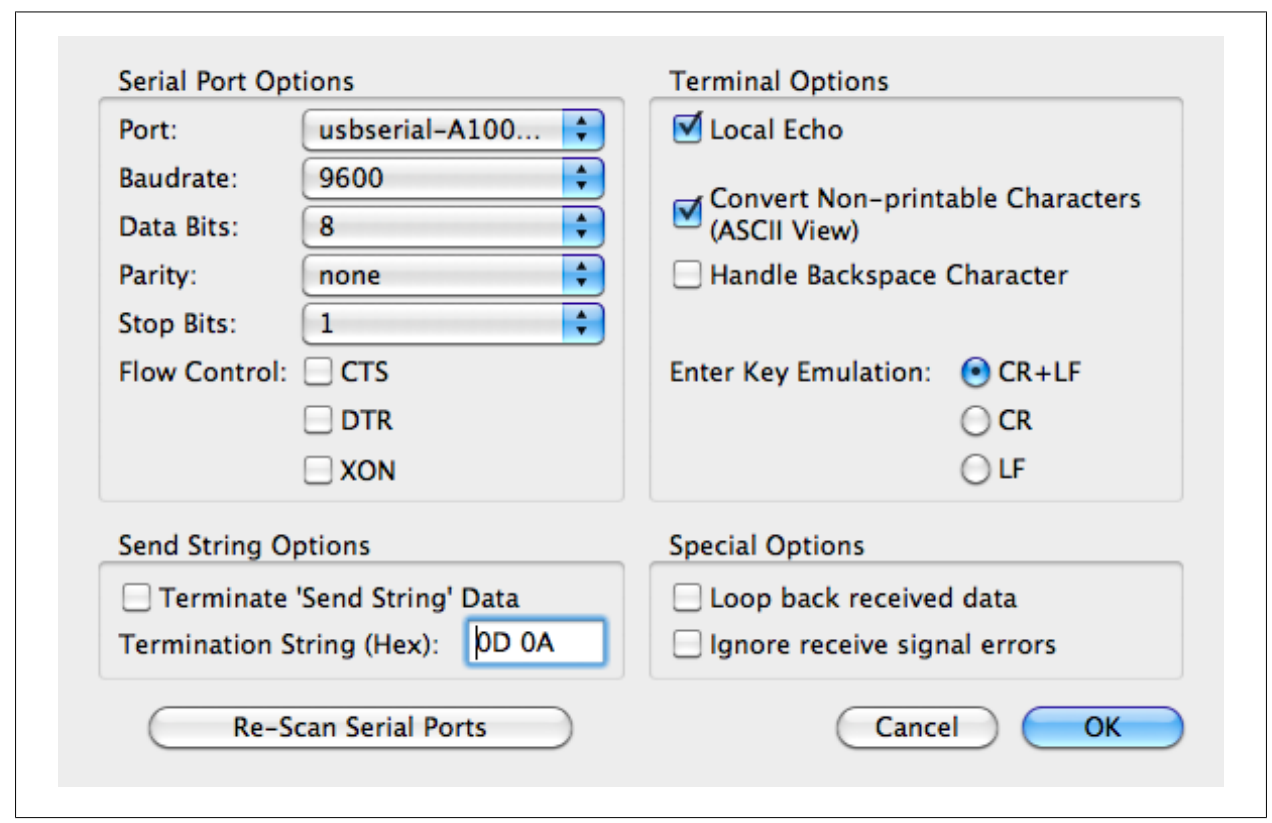

Figure 2-18. CoolTerm settings; select your port and check the Local Echo box

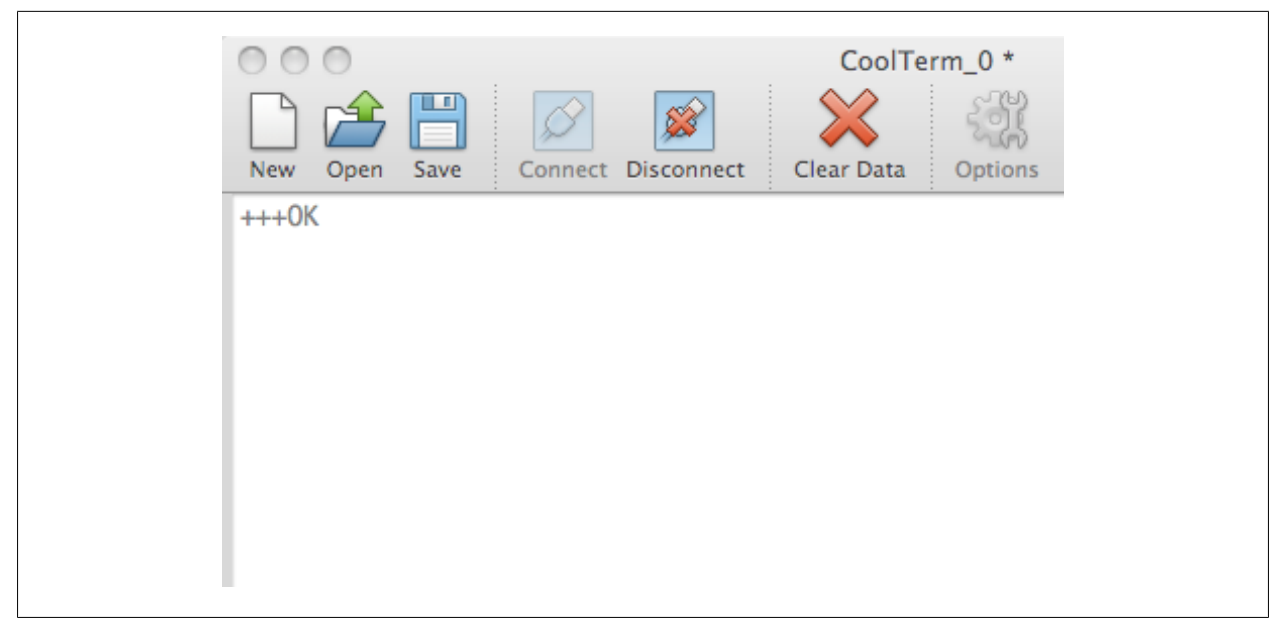

Figure 2-19. CoolTerm +++ with OK response for entering command mode

If you don't see the OK, check to make sure you've selected the correct port on the Options screen, and that you connected using the Connect button. Also make sure that you entered only +++. Don't press Return! The radio must get only the three plus signs or it won't go into command mode. 


\section{Troubleshooting}

Here are some things to check if you aren't getting an OK response from your radio when you try to put it into command mode:

- Is the radio connected properly to the XBee adapter?

- Is the XBee adapter plugged into the computer?

- Have you selected the correct port?

- Are you communicating at 9600 baud?

- Could your radio be set to some other baud rate?

- Could your radio be in API mode? See Chapter 5.

- Are you pressing the Return key after typing +++?

- Are you waiting a full second of "guard time" before typing +++?

- Are you waiting a full second of "guard time" after typing +++?

\section{Command Mode and Transparent Mode}

All XBees communicate over radio with each other in the same way. However, they can use their local serial connection in two very different ways. Radios configured for API mode utilize a data-enveloping format that's great for computers talking to each other but is not easily human-readable. We'll be covering this in a later chapter. XBees that are configured to use "AT" commands are designed for more direct human interaction. AT-configured radios switch back and forth between two modes:

\section{Transparent mode}

This is the default state for XBee radios using AT firmware. It's called transparent because the radio simply passes information along exactly as it receives it. Transparent mode is used to send data through the XBee to a remote destination radio. When data is received, it is sent out through the serial port exactly as it was received. What you send is what they get. Very simple.

Command mode

Sometimes we don't want to send any data at all. Instead, we want to talk directly to the local radio, perhaps to ask about its configuration or alter the way it behaves. In this case we want to talk to the radio rather than through it. Rather than passing along what we type, the radio should stop, listen, and react. This is called command mode.

Table 2-3 summarizes these modes.

Table 2-3. Transparent versus command mode for AT radios

Transparent mode

Talk through the XBee
Command mode

Talk to the XBee itself 
Transparent mode

Any data can be sent through

Default state
Command mode

Only responds to $\mathrm{AT}$ commands

+++ to enter mode

Times out after 10 seconds of no input

AT-configured XBees are normally in transparent mode. To get a radio to switch into command mode, we must issue a unique string of text in a special way. This is where those three plus signs come in (Table 2-4). When the radio sees a full second of silence in the data stream, followed by +++ and another full second of silence, it knows to stop sending data through and start accepting commands locally. (It's very unlikely that this particular combination would appear in the serial data by chance.) Once the radio is in command mode, it listens for user input for a while. If 10 seconds go by without any user input, the XBee automatically drops out of command mode and goes back into transparent mode.

Table 2-4. Entering command mode

\begin{tabular}{lll} 
Guard time silence & Command sequence & Guard time silence \\
One second before & +++ & One second after \\
\hline
\end{tabular}

Remember that you must not press Return or Enter after typing the +++ because it will interrupt the guard time silence and prevent you from going into command mode!

\section{AT Commands (Are Your Friend)}

The AT commands that XBee radios use for interactive setup are a descendant of the Hayes command set that was originally developed for configuring telephone modems. The Hayes command set was never a codified standard, but many other modem manufacturers styled their command set after Hayes and today a variety of communications devices use the same format to accept configuration messages from serial connections.

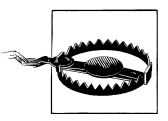

You always need to press Enter or Return after issuing an AT command. Now just to be clear, the deal is to never press Enter after +++ and always press Enter after your AT command. You'll probably make mistakes with this at first, but it will come naturally soon enough.

Every AT command starts with the letters "AT," which stands for "attention." The idea is to get the attention of the device, in this case our XBee radio. The AT is followed by two characters that indicate which command is being issued, then by some optional configuration values. Here's an example:

ATID $1966\langle C R>$ 
Don't type the $\langle$ CR $>$ literally. You just need to add a carriage return at the end, usually by pressing the Return key on the keyboard. It's a pretty simple structure that will be clear once you've issued a few commands. Here are some basic ones:

AT

When the AT command is issued by itself, the radio will simply return OK. This is like asking "Are you there?" and the radio replying "Yup!" If you type AT and press Return, and don't see an OK in response, you've probably timed out of command mode and will need to type the +++ to go back into it. This will happen a lot at first, but eventually you'll get used to the timing.

ATID

Typing ATID by itself will show you the Personal Area Network ID that is currently assigned to the radio. PAN addresses define the network that a radio will attach to, using a hexadecimal number in the range $0 \mathrm{x} 0-0 \mathrm{xFFFF}$. Adding an address after the ATID command will assign a new PAN address to the radio. This is demonstrated below.

ATSH/ATSL

Each XBee radio has a 64-bit serial number that serves as a permanent address that's unique to it in the world. The serial number address is split into two parts, a high part and a low part. This is because a single register is not big enough to hold the whole address. It can't be changed, so while typing ATSH or ATSL will show you the high and low parts of that serial number respectively, adding any address information after this command will cause an ERROR response.

ATDH/ATDL

These show or assign the destination address that the local radio will send information to. Typing ATDH will show you the current high part of the destination address, while putting address information after ATDH will set a new high part to the destination address.

ATCN

This command will drop you out of command mode immediately, returning the radio to transparent mode. You can also type nothing for 10 seconds and the radio will drop out of command mode automatically.

ATWR

This writes the complete current configuration to firmware, so that the next time the radio powers up it has the new configuration. ATWR is similar to a Save command on a computer that writes your document to the hard drive so it's stored even after the computer is turned off.

ATMY

This command shows you the current 16-bit address. The coordinator assigns this address dynamically so it can be displayed (but not set) for the Series 2 ZigBee radios. 


\section{Using AT Commands}

Issuing any of these AT commands is very easy on both Windows and Macintosh. Here are the steps:

1. Use +++ to ask the radio to go into command mode.

2. Wait for an OK response.

3. To read a register, type an AT command such as ATID, followed by a carriage return.

4. To set a register, type an AT command followed by the parameter you want to set, like this: ATID 1966, followed by a carriage return. The space before the parameter is optional so this also works: ATID1966.

Figure 2-20 shows how this looks in Windows.

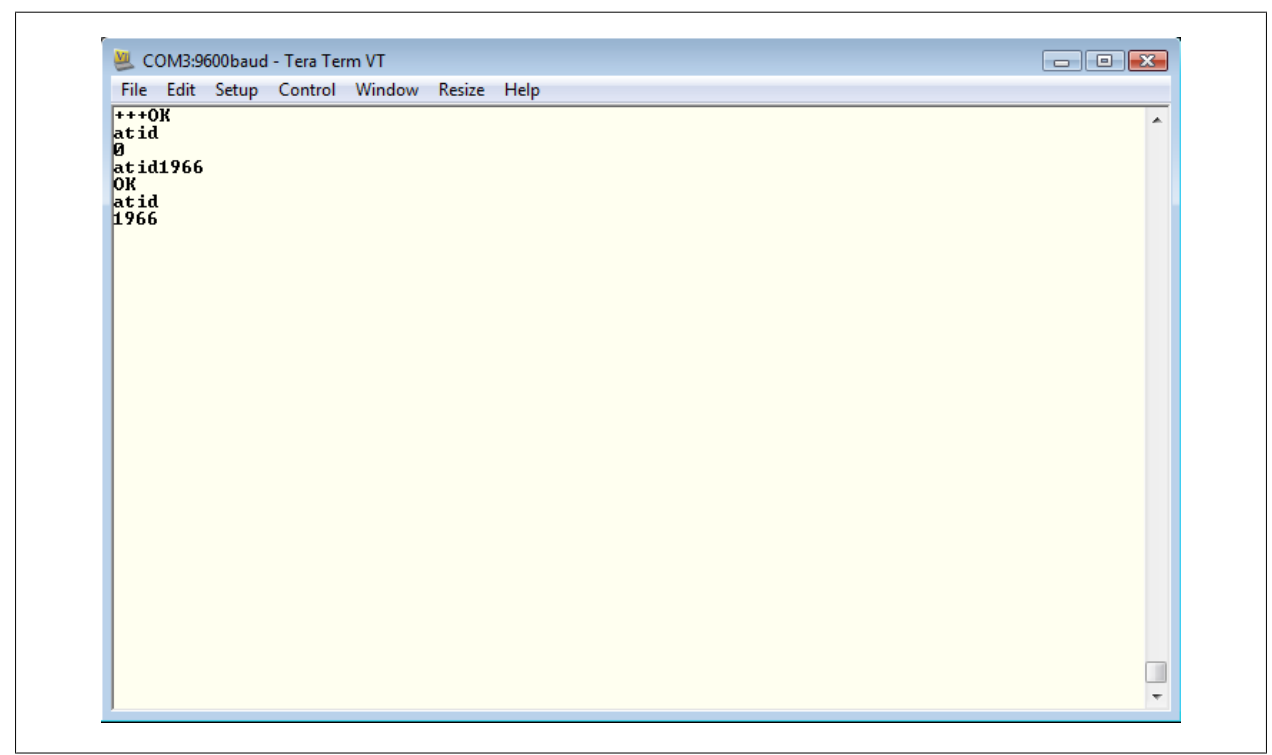

Figure 2-20. Reading, setting, and then rereading a register in Tera Term

In CoolTerm on a Macintosh, the procedure works pretty much the same. After putting the radio into command mode with +++, issue an AT command by typing it in the window, followed by a carriage return.

The results from querying with ATID, setting ATID 1966, and then querying with ATID again are shown in Figure 2-21.

Now that you know how to connect to your XBee, put it in command mode, and issue AT commands, you're ready to configure two radios to chat with each other. Let's start that scintillating conversation. 


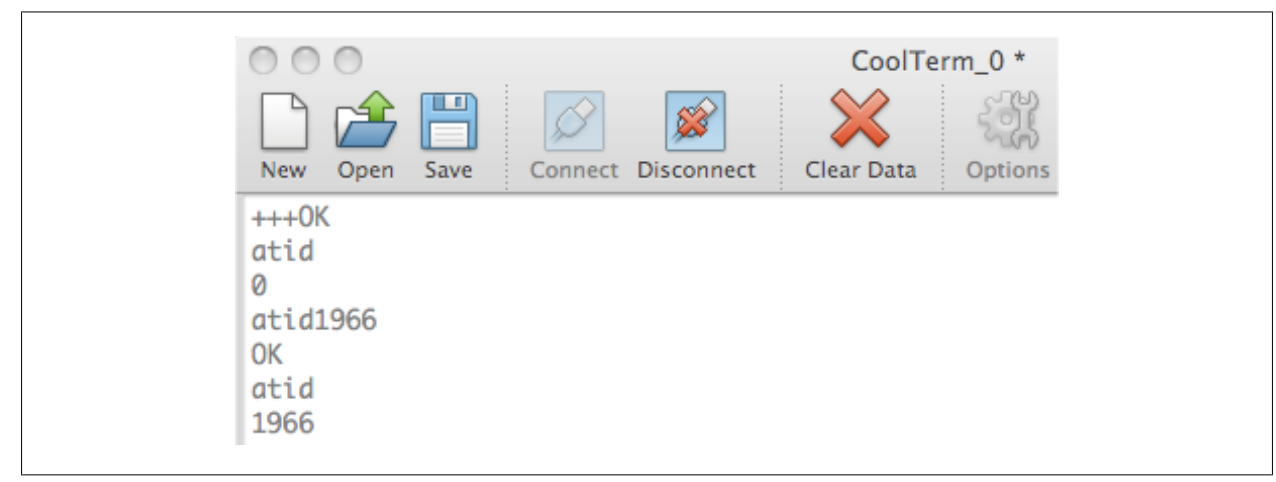

Figure 2-21. Reading and setting registers in CoolTerm

\section{Basic ZigBee Chat}

Networks are all about connections, so configuring a single radio doesn't qualify as making a network because it's not connected to anything. You need at least two radios to make a network, so here's what you'll need to create your first project-a simple ZigBee chat session.

\section{Parts}

- One XBee Series 2 radio, configured as a ZigBee Coordinator AT (Digi: XB24Z7WIT-004, DK 602-1098-ND).

- One XBee Series 2 radio, configured as a ZigBee Router AT (same as previous).

- Two XBee USB adapter boards (SFE: WRL-08687).

- Two computers, each running a serial terminal program, or one computer running two different serial terminal programs. Using two computers is less confusing, so find a buddy if you can.

\section{Addresses}

Every XBee radio has a 64-bit serial number address printed on the back (Figure 2-22). The beginning or "high" part of the address will be 0013A200, Digi's preassigned range of address space. The last or "low" part of the address will be different for every radio. It will look something like this: 4052DAE3.

Write down your coordinator and router addresses so you can refer to them later:

\begin{tabular}{ll}
\hline Coordinator address & Router address \\
$0013 \mathrm{~A} 200$ & $0013 \mathrm{~A} 200$ \\
\hline
\end{tabular}




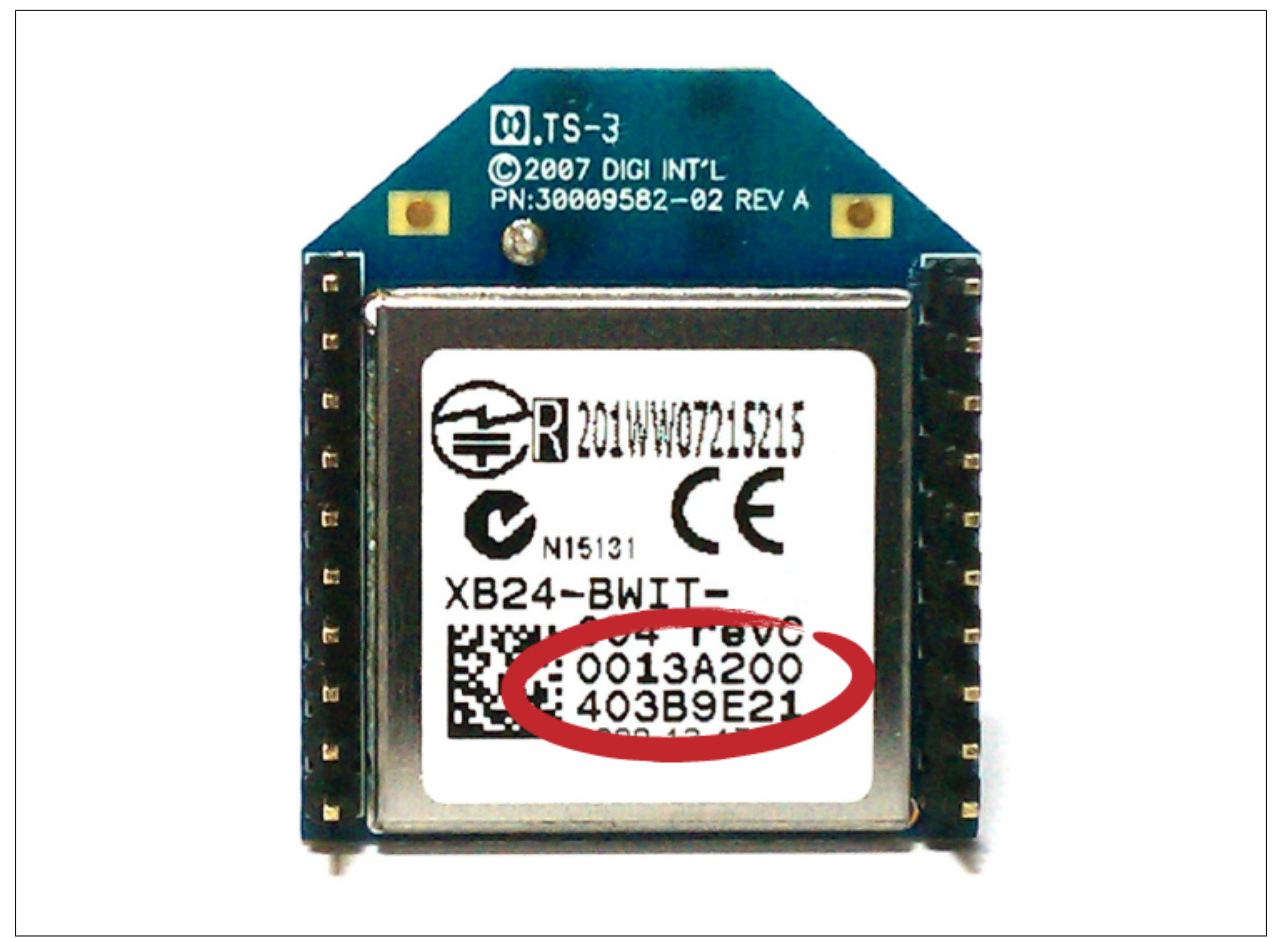

Figure 2-22. Back of XBee showing 64-bit address

\section{Coordinator}

Start with the XBee ZIGBEE COORDINATOR AT radio you configured earlier in this chapter. Remember that every ZigBee network must have one coordinator radio — and only one coordinator radio-so that the network can be properly defined and managed. Use your serial terminal program and AT commands (or X-CTU if you have access) to configure the coordinator radio with the settings in Table 2-5.

Table 2-5. Coordinator setup for paired chat

\begin{tabular}{lll} 
Function & Command & Parameter \\
PAN ID & ATID & 2001 (any address from 0 to FFFF will do) \\
Destination address high & ATDH & $\mathbf{0 0 1 3 A 2 0 0}$ \\
Destination address low & ATDL & 〈see your recorded Router Address \\
\hline
\end{tabular}

When you're finished, check your work by reissuing each AT command without any parameter so the radio can show you the addresses it's using (Figure 2-23).

As a final step, use the ATWR command to write the new configuration to your radio's firmware so it's saved for the next power-up. 


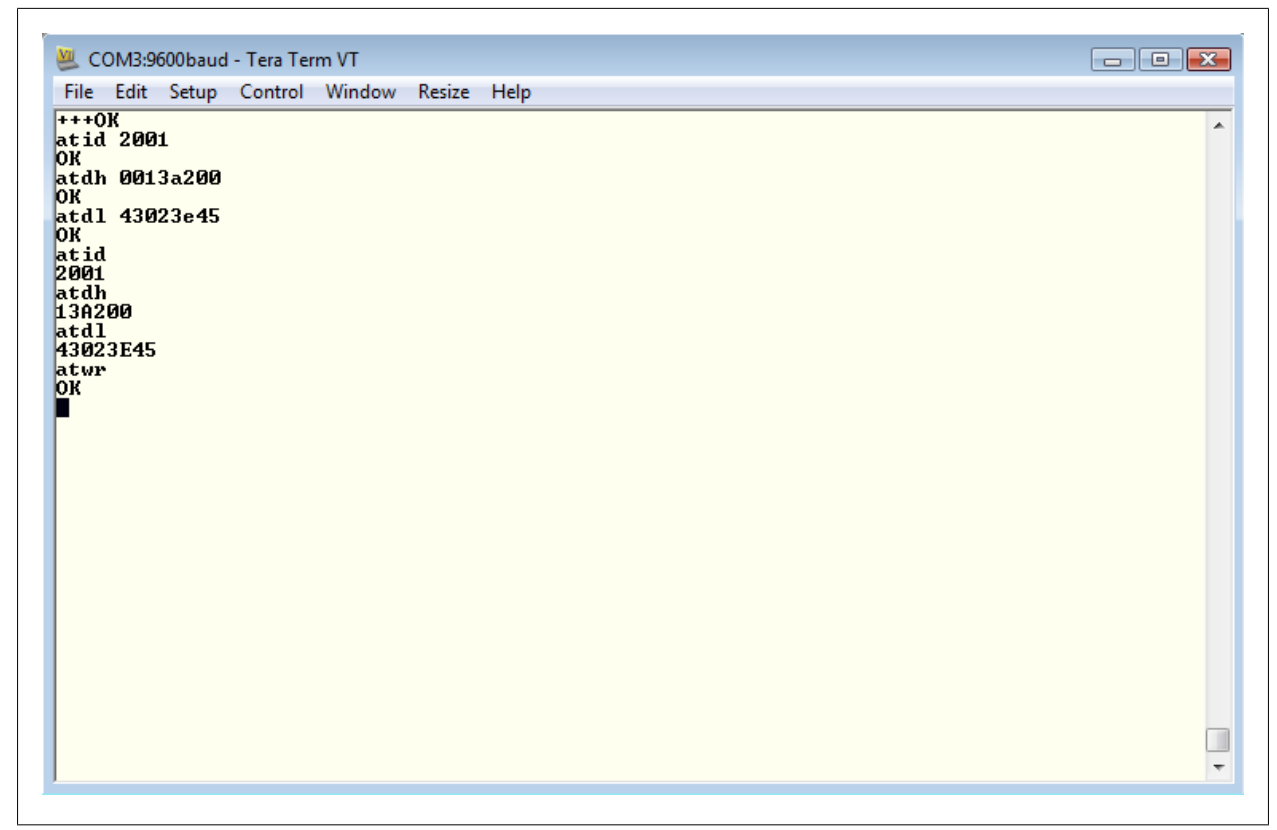

Figure 2-23. Setting and checking the coordinator radio

Here's what a session might look like:
+++
OK
ATID 2001
OK
OK
OK
ATID
2001
ATDH
$13 \mathrm{~A} 200$
ATDL
43023E45
ATWR
OK

ATDH $0013 A 300$

ATDL $43023 E 45$ 
You should get an OK response after issuing each command to set parameters, and another OK response when you write the changes to firmware. If you don't get an OK response, most likely you took more than 10 seconds to issue the command and you've dropped out of command mode. This can happen quite frequently when you're starting out, but you'll get better at it as you go along. The other common mistake is not issuing the ATWR command to save your changes, then losing your configuration when the radio is powered down.

The command mode timeout can be changed to a longer value with ATCT, but it's best to wait on doing this until you are more comfortable with the radios so you don't set the timeout to an impossibly short value by accident.

\section{Router}

Replace the coordinator radio with the XBee ZigBee Router AT radio you configured earlier in this chapter. Use your serial terminal program and AT commands (or X-CTU if you have access) to configure the router radio with the settings in Table 2-6.

Table 2-6. Router setup for paired chat

\begin{tabular}{lll} 
Function & Command & Parameter \\
PAN ID & ATID & 2001 (must be the same for all radios on your network) \\
Destination address high & ATDH & $\mathbf{0 0 1 3 A 2 0 0}$ \\
Destination address low & ATDL & $\langle$ see your recorded Coordinator Address \\
\hline
\end{tabular}

When you've finished configuring the radio, check your work by reissuing each AT command without any parameter so the radio can show you the addresses it's using (see Figure 2-24).

As a final step, use the ATWR command to write the new configuration to your radio's firmware so it's saved for the next power-up. Disconnect the XBee from the computer for now.

\section{Two Computers}

Get ready to chat. Connect your coordinator XBee via an adapter to one computer's USB port. Launch a serial terminal application, or use the Terminal tab in X-CTU. (Make sure you select the current port and configure the terminal application for the right baud rate and other settings.) Your router radio should be connected in the same way to the second computer, which should be running its own serial terminal application. 


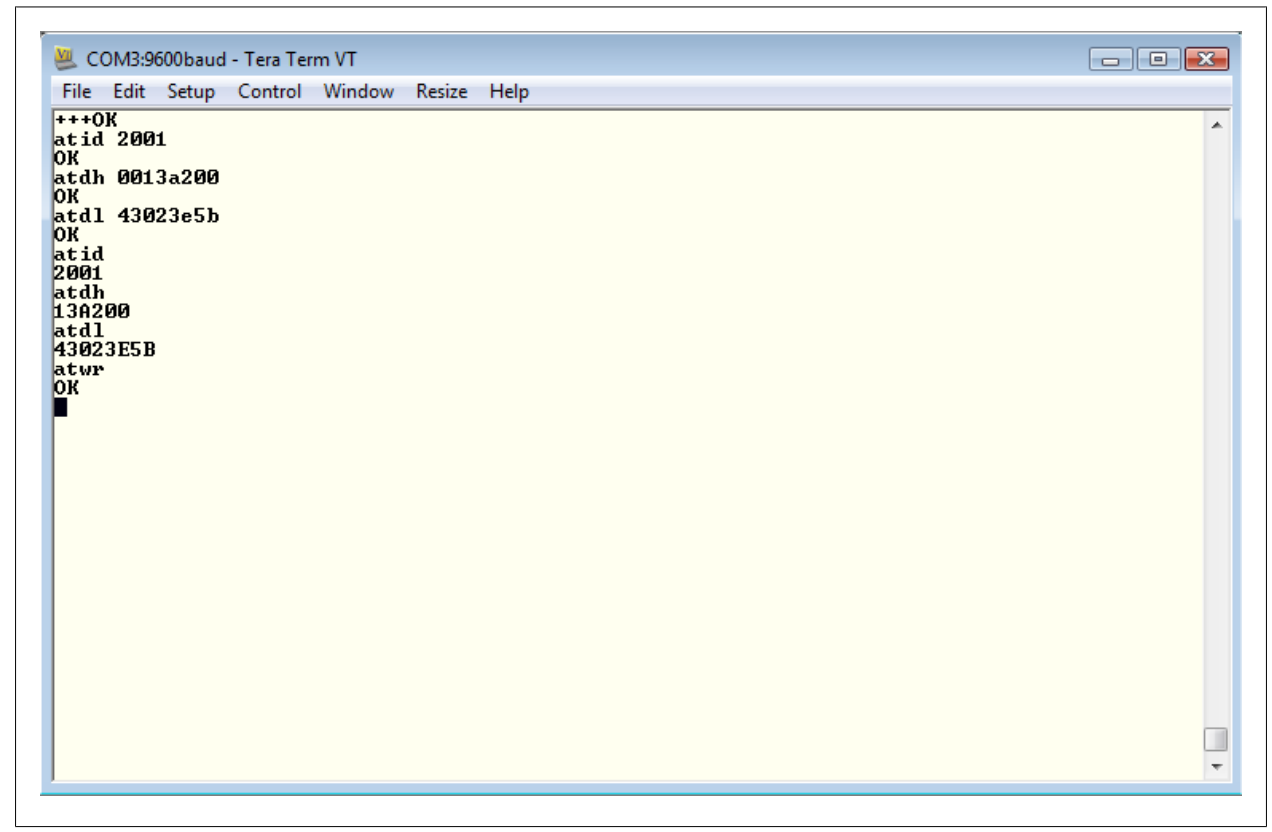

Figure 2-24. Setting and checking the router radio

\section{One Computer}

If you have only one computer, connect both radios to that computer's USB ports. Then choose two different terminal windows, like Tera Term and HyperTerminal on Windows, or CoolTerm and ZTerm on Macintosh. Pick one radio's port in one program and the other radio's port in the other program. Pretend that your first terminal program is one computer, and that your second terminal program is another one. Keeping all of this straight might make your head hurt a bit, but it's a valid test when you can't find a real second computer.

\section{Chat}

This is the moment you've been waiting for. If everything is set up properly, the text that you type in the serial terminal program on the first computer will be relayed to the second computer and appear on its serial terminal screen as well. Give it a try.

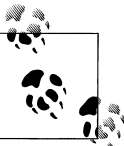

Remember that chatting will happen only when the radios are in transparent mode. If you are in command mode, type ATCN and press Return, or simply wait 10 seconds for command mode to time out. 


\section{Troubleshooting}

If everything works perfectly the first time around, that's GREAT! However, experience shows that it sometimes takes a few tries to get everything right. You've just set up a pretty complex system. Don't despair if your chat doesn't work right away. In almost every case, there's nothing wrong with any of your hardware or even with most of your setup. It takes only one wrong parameter to throw a wrench in the works. Learning how to find that wrench and fix it is an essential skill, so here are some tips on what to try if things don't work at first:

1. Start with the simple stuff. Make sure your radios are seated properly in the adapter boards and that all the USB cables are plugged in the way they should be.

2. Check that each radio is responding properly in the terminal window by trying to use +++ to put it into command mode. If you don't get an OK back, check your port selection, baud rate, and the other settings until you find the reason the radio is not communicating properly. (If you accidentally configured either radio with API firmware, it will not respond and you will need to change firmware to the AT version in X-CTU.)

3. If both radios are responding, use AT commands to check the settings. The most common problems are: not using the same PAN ID on both radios, not setting the destination address on each radio to the serial number of the other radio, and not saving the settings properly.

4. If the settings all seem to be correct, check to make sure that you have the coordinator firmware on one radio and the router firmware on the other radio. You can use the ATVR command to show which firmware is in use. The coordinator's version number will begin 20xx and the router's version number will begin 22xx. If you see other values, go back to X-CTU and load the proper firmware.

5. Sometimes the radios will be perfectly configured and connected, but your router will have joined a different network. This often happens in classroom situations, where many people are using the same PAN ID in the same room. Try using different PAN IDs for each pair.

6. A router will normally find the coordinator on the same PAN and join itself to the network. Very occasionally this won't happen properly. You can force each radio to rebuild its networking setup from scratch by issuing a network reset with ATNR. Do this on both radios, then recheck to see if they are now connecting properly.

7. Sometimes setting both radios back to factory defaults and reconfiguring them will flush out a bad setting that was left over from a previous setup, or an unrecognized typo. The ATRE command will wipe out your radio's custom configuration and leave the firmware set cleanly to factory defaults. Follow it with the ATWR command to write those defaults to the firmware, then go back to the configuration steps and try putting in your settings again. 
8. Don't forget that Digi's technical support is a great resource. While they needn't be your first step, if you're really stuck they can help you confirm that your radios are working properly at the hardware level. There are also a number of great online resources and forums you can read for ideas and where you can ask for more help. Check the resource guide in the Appendix.

\section{Success!}

When you do get the chat working, this is cause for a major celebration. Dance the hokey-pokey, sing Norway's national anthem, eat pudding, or do all three at the same time. Your very first ZigBee network is up and running! 
CHAPTER 3

Build a Better Doorbell

Now that the wheels are in motion, you're probably itching to create something practical. Let's get on with it. We'll briefly introduce the Arduino microcontroller system, with basic instructions for configuration and use. Since this isn't an Arduino book, we'll cover only what you need to know to get this project done. If you're new to Arduino and interested in learning more, ample references to other books and sites will be provided to help you learn whatever is beyond our fairly narrow scope. After getting up to speed on basic serial concepts and simple protocols, we'll execute a series of doorbell projects that build in creative complexity as you gain skill. Knock, knock. Who's there? Wireless interaction!

\section{ZigBee and Arduino}

Arduino and XBees can work extremely well together in wireless sensor systems. They are both great prototyping tools.

\section{About Arduino}

Arduino (Figure 3-1) is an open source microcontroller system that's very popular with prototypers, do-it-yourself enthusiasts, interaction designers, and educators. The system is designed to be easy to learn, easy to use, flexible, and fast to develop with. Microcontrollers are little computers that do specific jobs, such as taking input from switches and sensors and then deciding whether to turn on a light or ring a bell. They're widely used in portable devices, including the types you might want to use in a wireless sensor network. Here's how the Arduino project describes itself on its website:

Arduino can sense the environment by receiving input from a variety of sensors and can affect its surroundings by controlling lights, motors, and other actuators. The microcontroller on the board is programmed using the Arduino programming language (based on Wiring) and the Arduino development environment (based on Processing). Arduino projects can be stand-alone or they can communicate with software running on a computer (e.g., Flash, Processing, MaxMSP). 
The boards can be built by hand or purchased preassembled; the software can be downloaded for free.

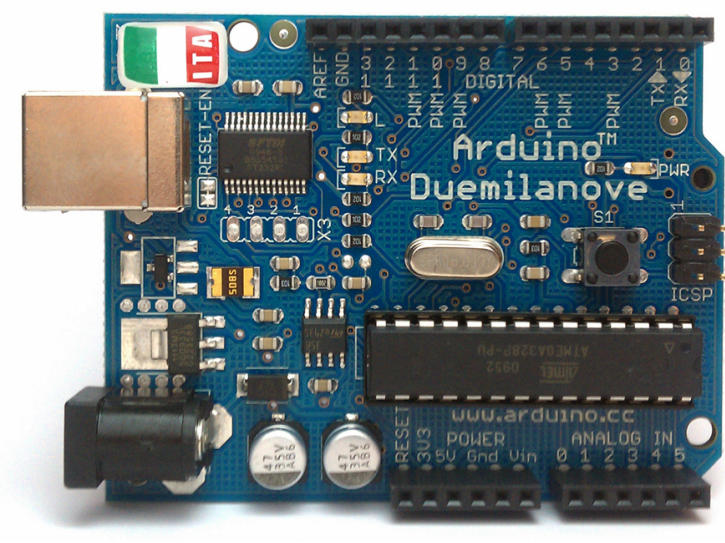

Figure 3-1. The basic Arduino USB board

In addition to Arduino, there are a slew of other microcontroller systems available, including the PIC chip, BASIC Stamp, Beagle Boards, and more. If you happen to prefer one of those platforms, simply use this book's examples and code as a guide.

We will see in upcoming chapters that the XBee is capable of doing some sensing and actuation without an external microcontroller, yet we're already starting with an additional piece of equipment. Actually, it's for a good reason. External microcontrollers bring several important advantages to a wireless project, including:

Local logic

While the basic XBee radios can be a source of sensor data or a trigger for local output, they can't be programmed to perform logical information processing. If your sensor or device needs local decision-making, you will almost certainly want to add a microcontroller to handle those processes.

\section{Additional input/output lines}

Series 2 XBee hardware comes with 10 digital input/output lines, 4 of which can be configured for analog (variable) input. While using an XBee, you can configure the basic Arduino to use up to 17 digital input/output lines, 6 of which can take analog input while 6 others offer hardware support for analog output. If you have extensive input or output needs, an external microcontroller may be just the thing. 


\section{Fast prototyping}

It is generally much easier to deploy and test a solution using a simple, high-level development system like Arduino than to mess with the XBee's application programming interface and data envelope frames. Even if you just want to do simple input/output on the XBee module, adding an external microcontroller will probably save you time as you try out your initial idea. If everything pans out, you can always slim your project down later.

\section{Lots of connection options}

With the help of an Arduino, your XBee can drive large motors, interact with GPS modules, drive LCD display screens, store data in local memory banks, and interact directly with the Internet via $\mathrm{WiFi}$ or your mobile phone. Working together, the possibilities are limitless.

\section{Arduino Basics}

Here's how to get ready to work with the Arduino microcontroller system.

\section{Buying an Arduino}

Arduino hardware comes in many flavors. The basic Arduino as of this writing is the Uno. This model supports 13 digital input/outputs along with 6 analog inputs. It can run off of USB power or via an external "wall wart" power supply. The onboard microcontroller supports up to $32 \mathrm{~K}$ of program code with $2 \mathrm{~K}$ of RAM. This may not seem like a lot, but in 8-bit microcontroller terms it's probably more than most prototypers need. The main Arduino website hosts an exhaustive list of sources at $h t t p: / / w w w . a r$ duino.cc/en/Main/Buy, or you can find it at Maker Shed, SparkFun, Adafruit, and many other online retailers.

There are plenty of other options if your project has special needs. For example, the Arduino Mega is good for very big jobs. It has 54 digital input/outputs and 16 analog inputs, along with 4 hardware serial connections. Should you want to go small, check out the Arduino Mini, which omits USB and female headers to allow a much smaller form factor, though at the expense of some prototyping ease. You'll find plenty of Arduino clones available too, all of which are configured a little differently to suit particular needs and tastes. If you feel bewildered by the options, the Uno is a fine choice for getting started. All the examples in this book are based on it.

\section{Don't forget the cable}

You'll want a USB cable for programming your Arduino board. For the Uno or Mega, you need the easily obtained A-to-B-style USB cable. Radio Shack carries these and you can also find them online at places like OutletPC.com, where they often are on sale for less than $\$ 1$. 


\section{Downloading the software}

The Arduino is programmed using an open source application that runs on your computer. This is known as the IDE (or integrated development environment) and you can download it for free directly from the Arduino website's software area. There are versions available for Macintosh, Windows, and Linux. Download the appropriate version for your computer. You'll find a basic guide to getting started at http://arduino.cclen/ Guide/HomePage.

\section{Using the Arduino IDE}

The Arduino IDE (Figure 3-2) is split into three areas. The blue area at the top of the window features a toolbar of buttons that control program behavior. The white area in the middle is where you enter and modify code. The black section at the bottom of the window is where status messages appear, and where you should look for error messages that can help you debug your code.

As described in the online Arduino guide, the toolbar buttons perform the following functions:

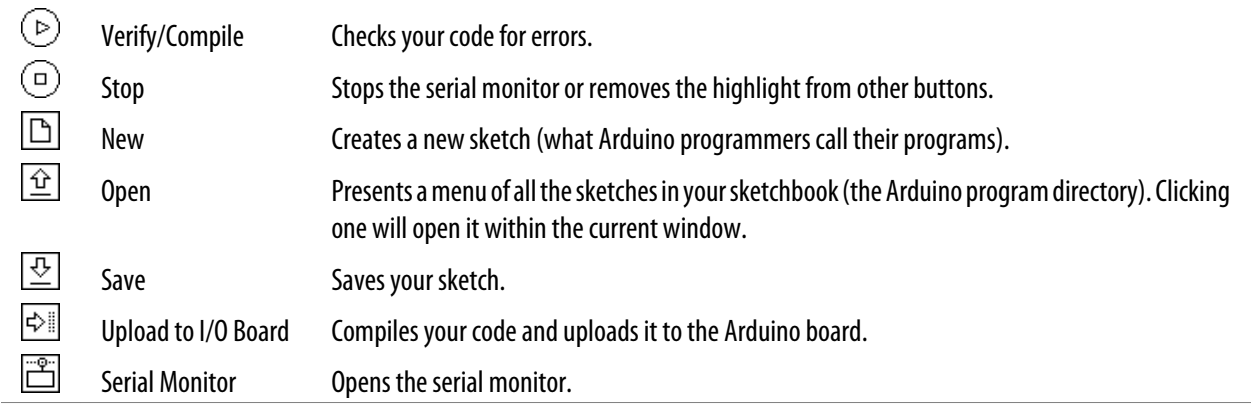

\section{Selecting the board and port}

To connect to your Arduino board, you must plug it into your computer using a USB A-to-B-style cable. Next, select the model of your Arduino board from the Board menu. Finally, select your serial port from the Serial menu. On Windows computers, the serial port will be one of the COM ports. On Macintosh, the serial port will have a name that includes usbserial, followed by some identifying letters and numbers. Once you've selected your board type and port, you're ready to do some programming!

\section{Code structure}

The Arduino language is based on $\mathrm{C} / \mathrm{C}++$ and as such it shares a specific set of structures that have been simplified for people new to programming. A simple program might look something like this:

// variable definitions always come first 


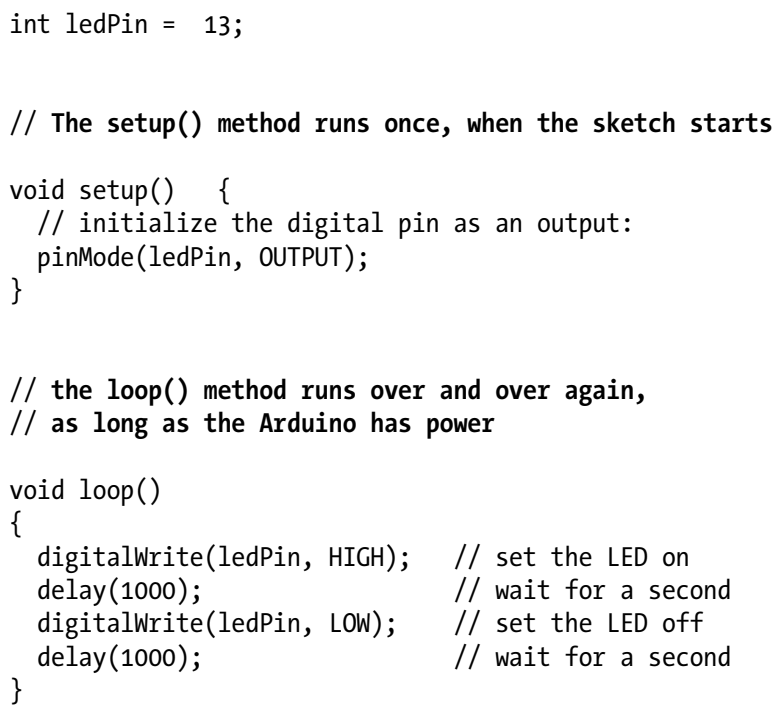

A basic program begins with statements that declare the names, types, and initial values for named containers that are used throughout the program, also known as global variables. Next comes a section that begins with void setup(). Everything between the curly braces for this section is code that runs only once, right after the Arduino is powered up or reset. Typically this section contains procedures that get the Arduino board ready to do its work, like initializing pins, setting up serial ports, and anything else that needs to happen only once, on startup. Finally, there's a section that starts with void loop(). The code contained in the loop section, between its curly braces, runs constantly. In the example above, this code will turn on an LED light, wait a moment, then turn it off and wait a moment. That's one blink of a blinking light. As soon as the first blink is complete, the loop code runs again, meaning the light will blink on and off indefinitely. Sometimes there will be other sections following the loop. These describe additional functions that are typically called from the main loop, but also could be called from setup or by each other. For full information on getting started with Arduino programming, take a look at the longer explanation at http://arduino.cc/en/ Tutorial/Foundations and the many examples that can be downloaded from http://ar duino.cclen/Tutorial/HomePage. You'll find the complete language reference at http:// arduino.cclen/Reference/HomePage. These resources are also available from the Help menu in the Arduino IDE. Figure 3-3 shows the Arduino board in detail.

\section{Buying electronics supplies}

Arduino projects almost always require additional components, such as switches, lights, sensors, knobs, wiring, or motors. Here are some resources where you can find the electronics goodies you need: 


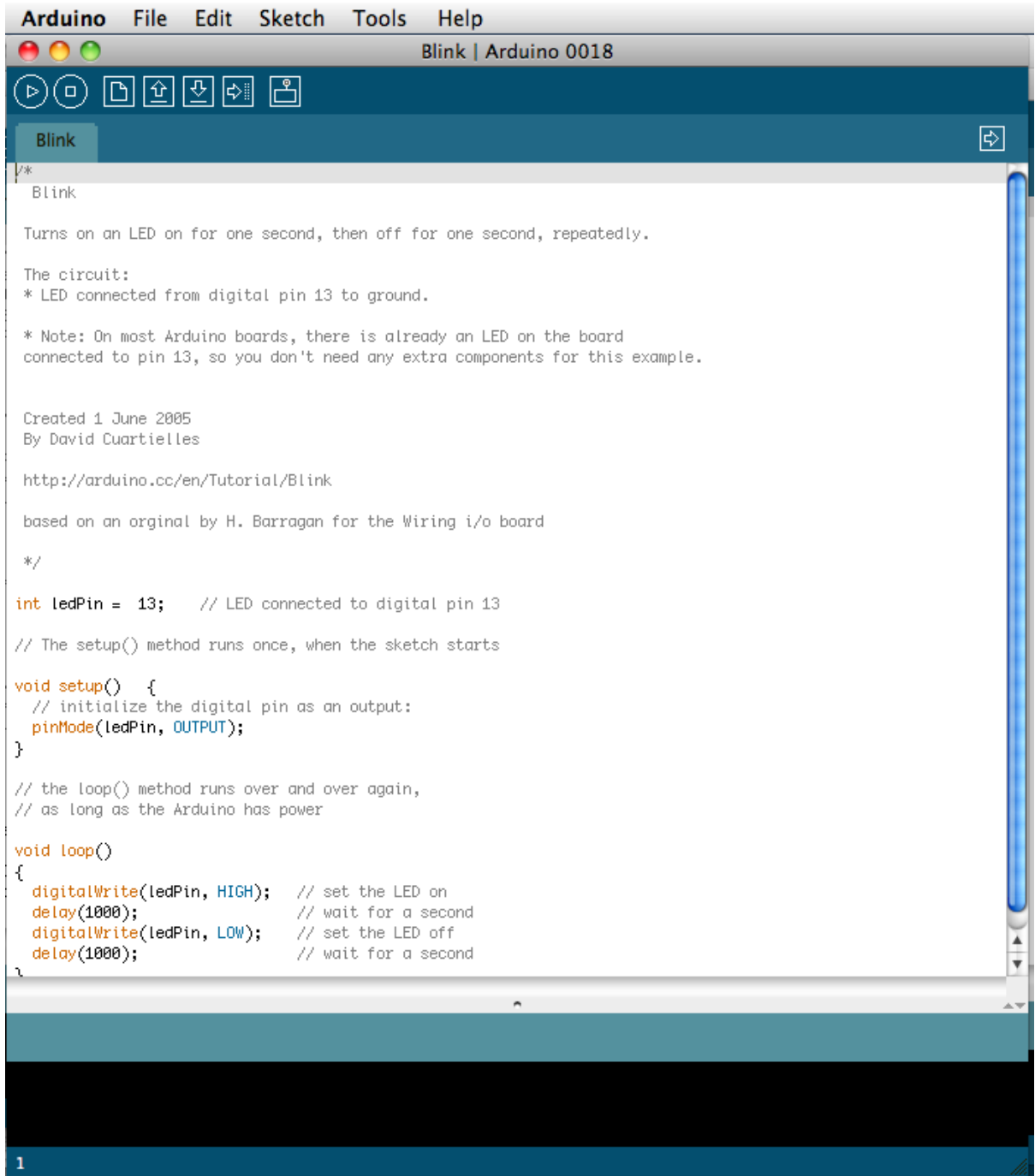

Figure 3-2. Arduino IDE programming software

\section{Maker Shed}

Has lots of projects and some good components, including a kit specifically designed for use with this book. 


\section{Adafruit}

Has a great collection of electronics kits with a small but useful selection of electronic components that are very appropriate for beginners.

SparkFun

Contains a wealth of terrific prototyping components, each well-explained for the amateur electronics enthusiast.

DigiKey

A complete resource for electrical engineering, DigiKey stocks half a million different components and can deliver any of them overnight. Take a deep breath before shopping because almost every part is available in a hundred different variations.

Jameco

Another electrical engineering resource, Jameco tends to have a more limited selection. This, along with its full-color catalog, can be a boon for those new to selecting components.

Mouser

Similar to DigiKey, Mouser carries a huge selection of parts. If you can't find it from one, check the other.

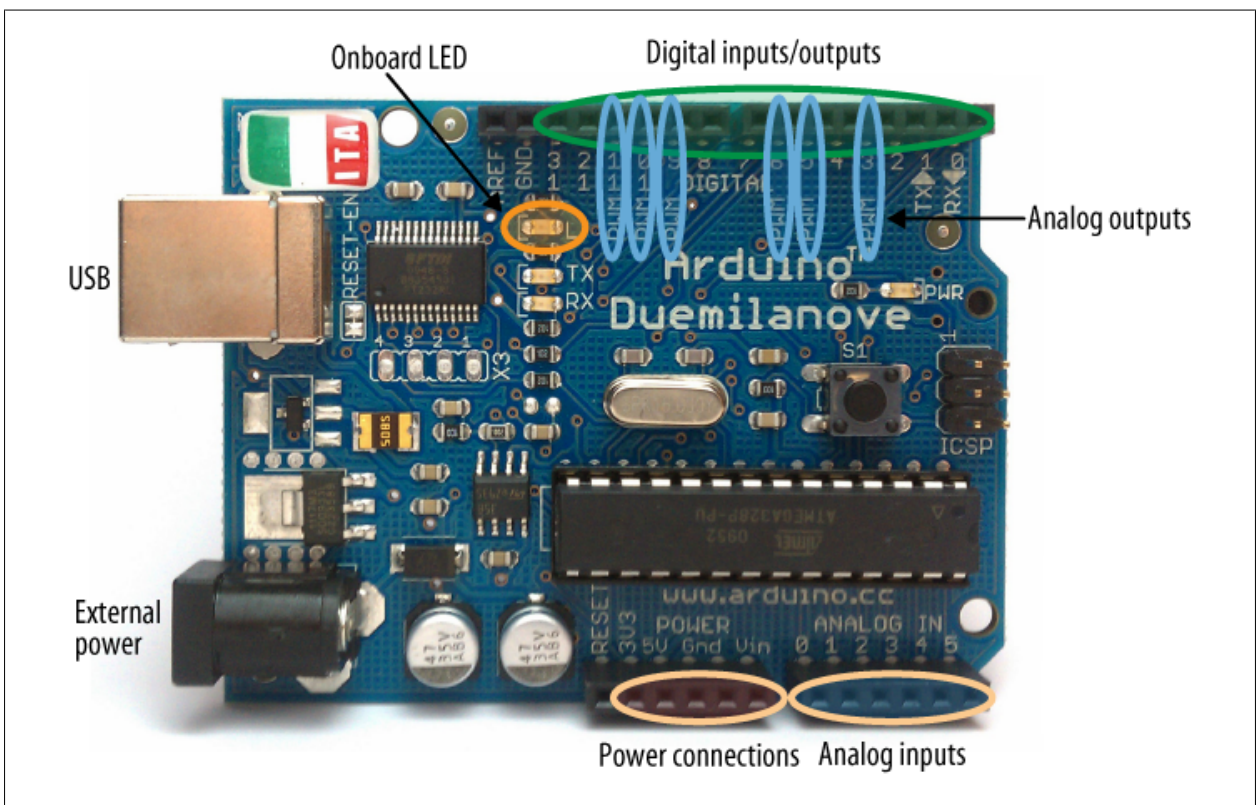

Figure 3-3. Arduino board features and connections. Notice that some pin connections can be configured for several different purposes. 


\section{Learning More About Arduino}

There's lots to know about the Arduino system, more than we could possibly cover in this chapter. Luckily, there are plenty of resources available to ease your journey from novice to expert.

On the Arduino site:

- The Getting Started guide (http://arduino.cc/en/Guide/HomePage).

- The Language Reference area lists all the commands and shows how to use them (http://arduino.cclen/Reference/HomePage).

- The Arduino Playground hosts a cornucopia of resources, including a wealth of completed projects and information on extending the basics with more advanced techniques (http://www.arduino.cc/playground/).

- The Hardware area lists most available boards as well as "shields" to extend them (http://arduino.cclen/Main/Hardware).

On the Internet:

- The LadyAda Arduino Tutorial (http://www.ladyada.net/learn/arduino/).

- The NYU ITP Physical Computing tutorials for getting started (http://itp.nyu.edu/ physcomp/Tutorials/) and the sensor wiki for understanding a variety of components (http://itp.nyu.edu/physcomp/sensors/).

- The SparkFun set of tutorials, from basic to iPhone (http://www.sparkfun.com/ commerce/tutorials.php).

- The Sheepdog Guides Arduino Course (http://sheepdogguides.com/arduino/ FA1main.htm).

- The Freeduino online index features links to guides and tutorials from all over (http://www.freeduino.org/).

In Arduino communities:

- The Arduino site forum is a good place to meet people, see if your question has already been answered, and if not, ask it (http://www.arduino.cc/cgi-bin/yabb2/ YaBB.pl).

- Both SparkFun and Adafruit have very active forums as well (http://forum.sparkfun .com/; http://forums.adafruit.com/).

At hacker spaces:

- The local one in New York is NYC Resistor (http://www.nycresistor.com/).

- Hundreds more from Maui to West Bengal are listed on Hackerspaces (http://hack erspaces.org/wiki/List_of_Hacker_Spaces).

In books:

- Getting Started with Arduino by Massimo Banzi (O’Reilly, 2008). 
- Practical Arduino: Cool Projects for Open Source Hardware by Jonathan Oxner and Hugh Blemings (Apress, 2009).

- Arduino Cookbook by Michael Margolis (O’Reilly, 2011).

- Physical Computing: Sensing and Controlling the Physical World with Computers by Dan O'Sullivan and Tom Igoe (Thomson, 2004) is a great reference to the process of building humanized interactive systems.

Finally, here's a brief history of the Arduino:

- Clive Thompson's great story about Arduino and open source hardware, "Build It. Share It. Profit. Can Open Source Hardware Work?” http://www.wired.com/ techbiz/startups/magazine/16-11/ff_openmanufacturing).

\section{Connecting to Arduino}

The solderless breadboard and XBee breakout boards described in Chapter 1 provide an easy way to link your Arduino board to an XBee. While the XBee has many pins, it takes only four of them to create a working connection so that your Arduino can communicate wirelessly, using its built-in serial communications protocol.

Remember that the XBee pins are spaced $2 \mathrm{~mm}$ apart, so the XBee can't be placed directly into a breadboard. A basic breakout board is the least expensive adapter for connecting to an Arduino. You can also use an XBee Explorer as a breakout board, but keep in mind that the pins are arranged differently. The four connecting wires will provide power, electrical ground, transmit, and receive. Table 3-1 shows the pin connections between Arduino and XBee, and Figure 3-4 shows them on an XBee breakout board.

Table 3-1. Pin connections between Arduino and XBee

\begin{tabular}{ll} 
XBee & Arduino \\
VCC or $3.3 \mathrm{~V}$ & $3 \mathrm{~V} 3$ \\
TX or DOUT & $\mathrm{RX}$ or 0 \\
RX or DIN & $\mathrm{TX}$ or 1 \\
GND & GND \\
\hline
\end{tabular}

Remember, if you are using the XBee Explorer you'll connect the same pins but their physical layout will be different, as shown in Figure 3-5.

After plugging your XBee into a small breadboard, you can use different colors of hookup wire to make the connections between your Arduino and XBee (see Figure 3-6). Once connected, the Arduino uses serial commands to send information out via the XBee, and to read in any information that's received. This is how our doorbells will operate. 


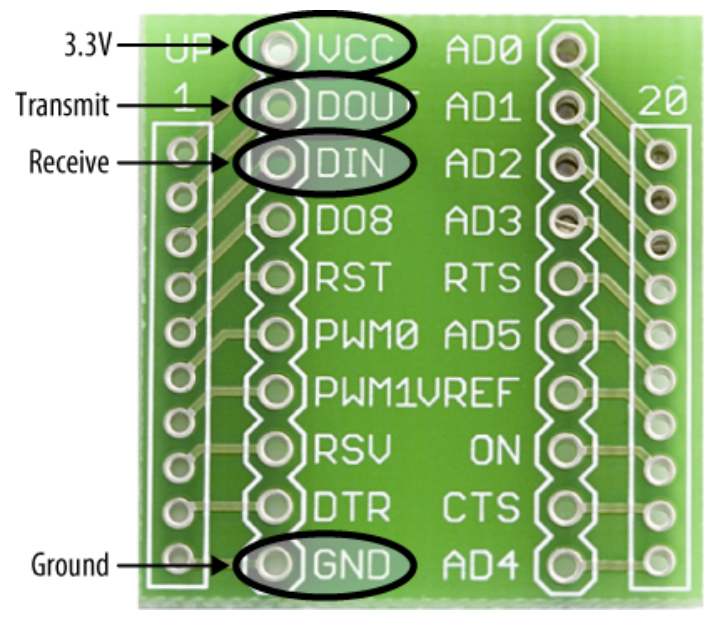

Figure 3-4. Breakout board pins for serial connection to Arduino

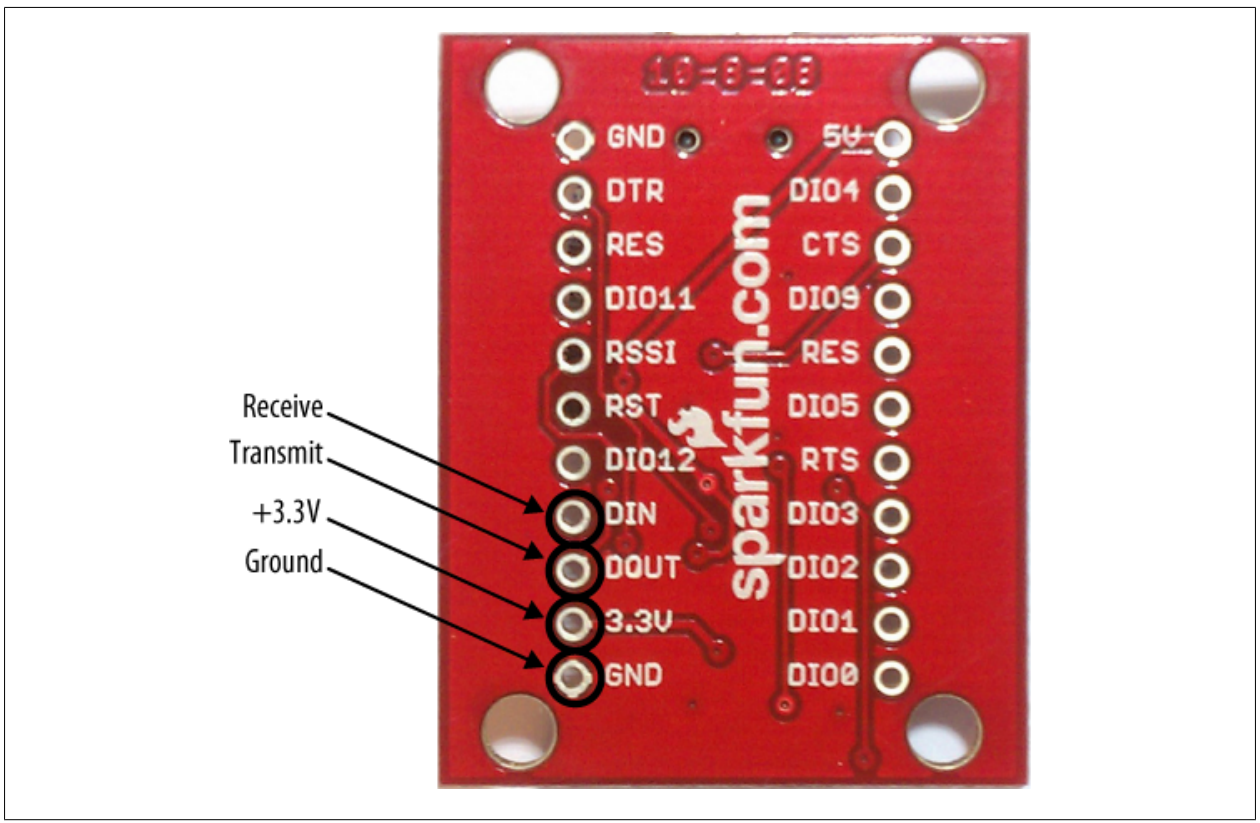

Figure 3-5. XBee Explorer pins for serial connection to Arduino 


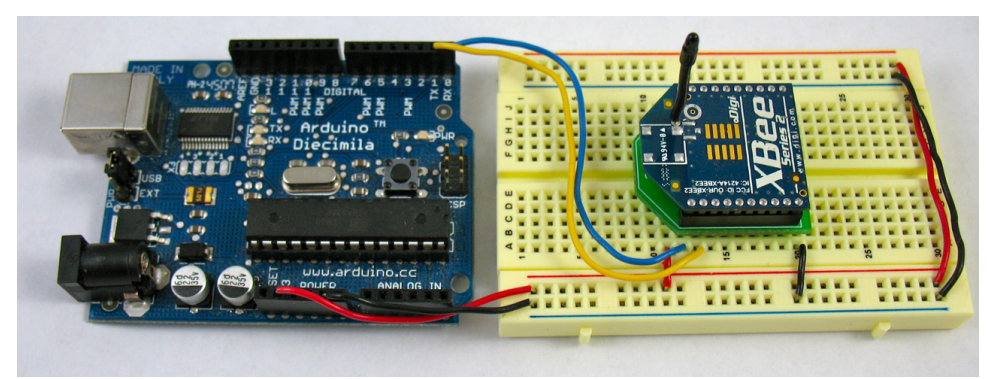

Figure 3-6. Arduino connected to an XBee radio, using a solderless breadboard and hookup wire

\section{Doorbell Projects}

You are now ready to create your first stand-alone wireless systems. The projects in this chapter use pairs of radios to help you learn networking basics. In a couple of chapters, you'll be creating much larger networks, using the skills you'll learn here.

\section{Parts}

- Two solderless breadboards (MS MKKN2, AF 64, DK 438-1045-ND, SFE PRT-09567)

- Hookup wire or jumper wire kit (MS MKSEEED3, AF 153, DK 923351-ND, SFE PRT-00124)

- Two Arduino boards (MS MKSP4, SFE DEV-09950, AF 50)

- USB A-to-B cable for Arduinos (AF 62, DK 88732-9002, SFE CAB-00512)

- An LED (try the $5 \mathrm{~mm}$ size, and make sure you don't buy any surface mount (SMT) parts) (DK 160-1707-ND, RS 276-041, SFE COM-09590)

- One 10K ohm resistor (DK P10KBACT-ND, SFE COM-08374)

- One momentary switch for input (DKEG2025-ND, RS 275-618, SFE COM-09179)

- One buzzer for output (DK 102-1621-ND, RS 273-060)

- One XBee radio (Series 2/ZB firmware) configured as a ZigBee Coordinator AT mode (Digi: XB24-Z7WIT-004, DK 602-1098-ND)

- One XBee radio (Series 2/ZB firmware) configured as a ZigBee Router AT mode (Digi: XB24-Z7WIT-004, DK 602-1098-ND)

- Two XBee breakout boards with male headers and $2 \mathrm{~mm}$ female headers installed (AF 126 [add SFE PRT-00116], SFE BOB-08669, PRT-08272, and PRT-00116)

- XBee USB Serial adapter (XBee Explorer, Digi Evaluation board, or similar) (AF 247, SFE WRL-08687)

- USB cable for XBee adapter (AF 260, SFE CAB-00598)

- Wire strippers (AF 147, DK PAL70057-ND, SFE TOL-08696) 


\section{Prepare Your Radios}

Every ZigBee network needs one and only one node configured as a coordinator. The other nodes can be configured as routers (or end devices):

1. Follow the instructions under "Reading Current Firmware and Configuration" on page 35 in Chapter 2 to configure one of your radios as a ZigBee Coordinator AT.

2. Using the same instructions, configure your other radio as a ZigBee Router AT.

3. Label the coordinator radio with a "C" so you know which one it is later on. Label the router radio with an "R."

\section{Connect Power from Arduino to Breadboard}

1. Hook up a red wire from the $3.3 \mathrm{~V}$ output of the Arduino to one of the power rails on the breadboard (see Figure 3-7).

2. Hook up a black wire from either ground (GND) connection on the Arduino to a ground rail on the breadboard.

3. Hook up power and ground across the breadboard so that the rails on both sides are live.

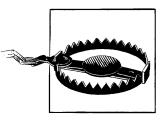

Make sure you are using $3.3 \mathrm{~V}$ power. The XBee will not run on 5 volts, and any more than 7 volts will permanently damage it.

\section{XBee Breakout Board}

Your XBee radio has pins spaced $2 \mathrm{~mm}$ apart. This helps keep the component small, but it means you can't use it directly on a standard 0.1 "-spaced solderless breadboard. To mate it with the breadboard, you need to use a breakout board. Basic breakout boards have no other electrical components. Another option is to use certain XBee USBserial adapters, such as the XBee Explorer, Adafruit XBee Adapter, or MCPros XBee Simple Board, all of which come with standard-spaced holes where you can solder on male headers. In this example, we'll just work with a basic breakout board:

1. Solder regular $0.1^{\prime \prime}$-spaced male headers onto the two center rows of holes on your basic XBee breakout board. The male headers come in long strips, and must be cut down to fit the breakout board before soldering. It's a good idea to place the male headers into the breakout board and insert them into the breadboard, as this helps with stability while soldering.

2. Next, flip the board over and solder two strips of female $2 \mathrm{~mm}$-spaced sockets onto the opposite side of the breakout board. 
3. Test-fit the XBee into the female sockets, being careful not to bend its pins (see Figure 3-8).

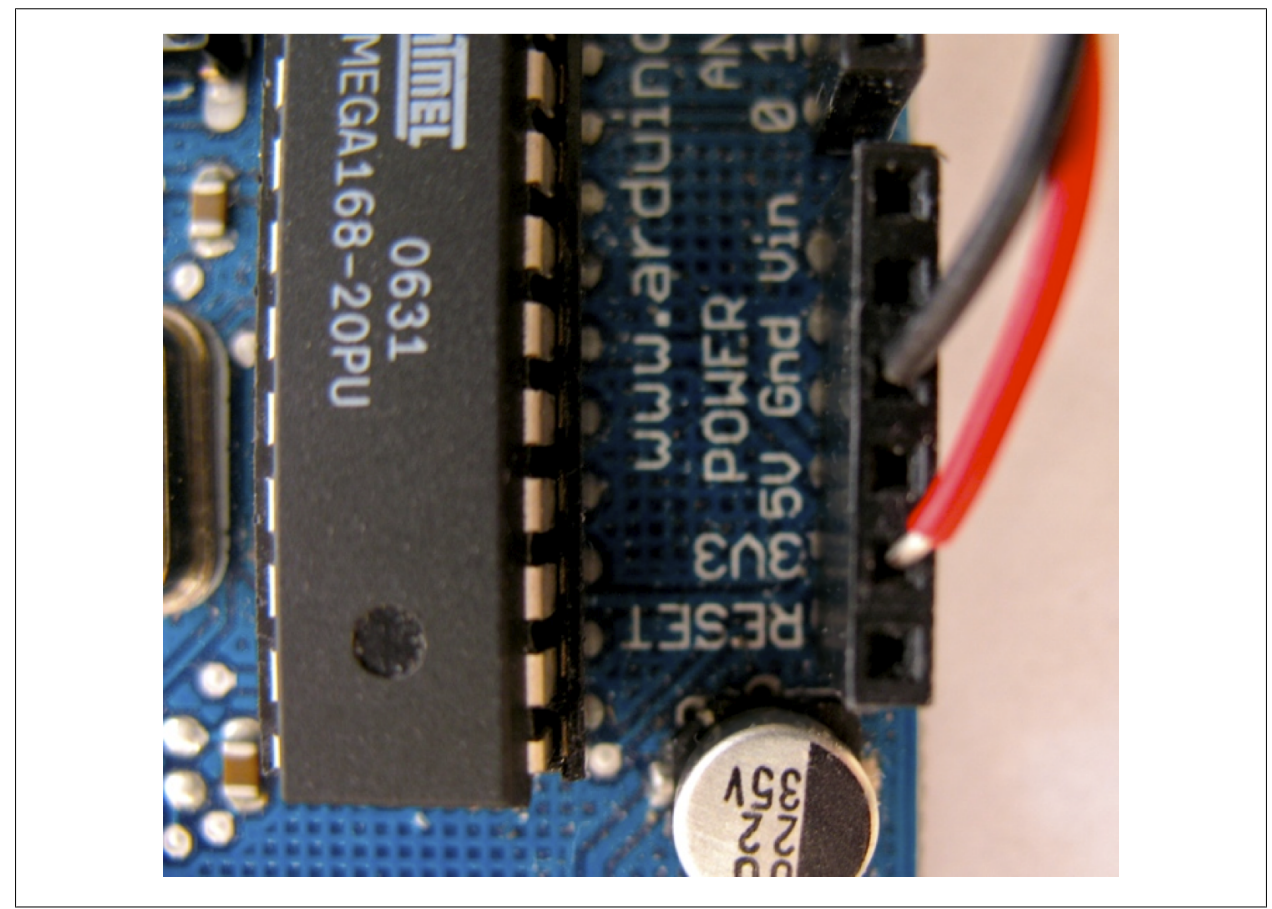

Figure 3-7. Power connections on Arduino
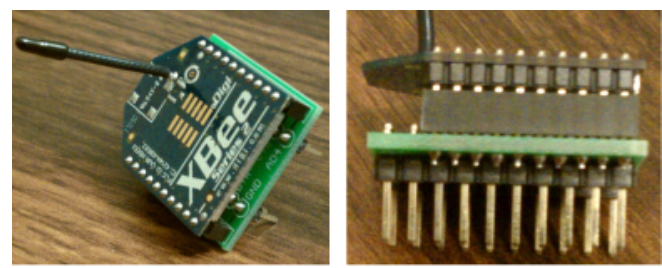

Figure 3-8. Finished breakout board with XBee mounted

\section{XBee Connections}

1. With the XBee mounted on its breakout board, position the breakout board in the center of your breadboard so that the two rows of male header pins are inserted on opposite sides of the center trough. 
2. Use red hookup wire to connect pin 1 (VCC) of the XBee to 3.3 volt power. See Figure 3-7.

3. Use black hookup wire to connect pin 10 (GND) of the XBee to ground.

4. Use yellow (or another color) hookup wire to connect pin 2 (TX/DOUT) of the XBee to digital pin 0 (RX) on your Arduino (see Figure 3-9).

5. Finally, use blue (or another color) hookup wire to connect pin 3 (RX/DIN) of your $\mathrm{XBee}$ to digital pin 1 (TX) on your Arduino. Figure 3-10 shows the connections to the XBee.

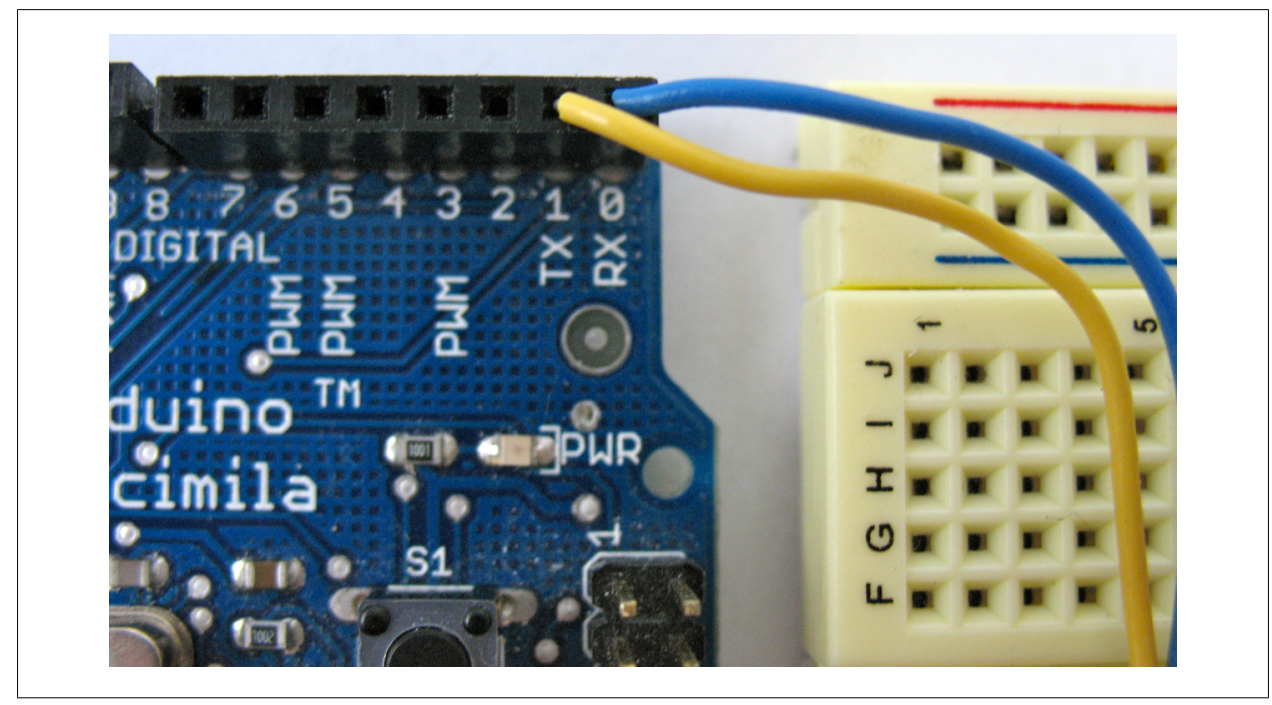

Figure 3-9. Transmit and receive connections on Arduino

Repeat these steps again with the other Arduino and XBee. Figure 3-11 shows the circuit diagram, and Figure 3-12 shows the schematic.

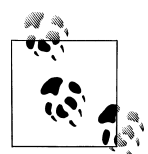

Sometimes it's a good idea to use a $1 \mu \mathrm{F}$ capacitor to decouple the power supply and filter out high-frequency noise that might interfere with your radio's ability to transmit or receive a clean signal. The Arduino typically provides clean enough power on its own. Decoupling is essential if you use a separate $3.3 \mathrm{~V}$ voltage regulator. In that case place the negative leg of the capacitor into ground and the positive leg into power, as near as you can to where your XBee is in the circuit. 


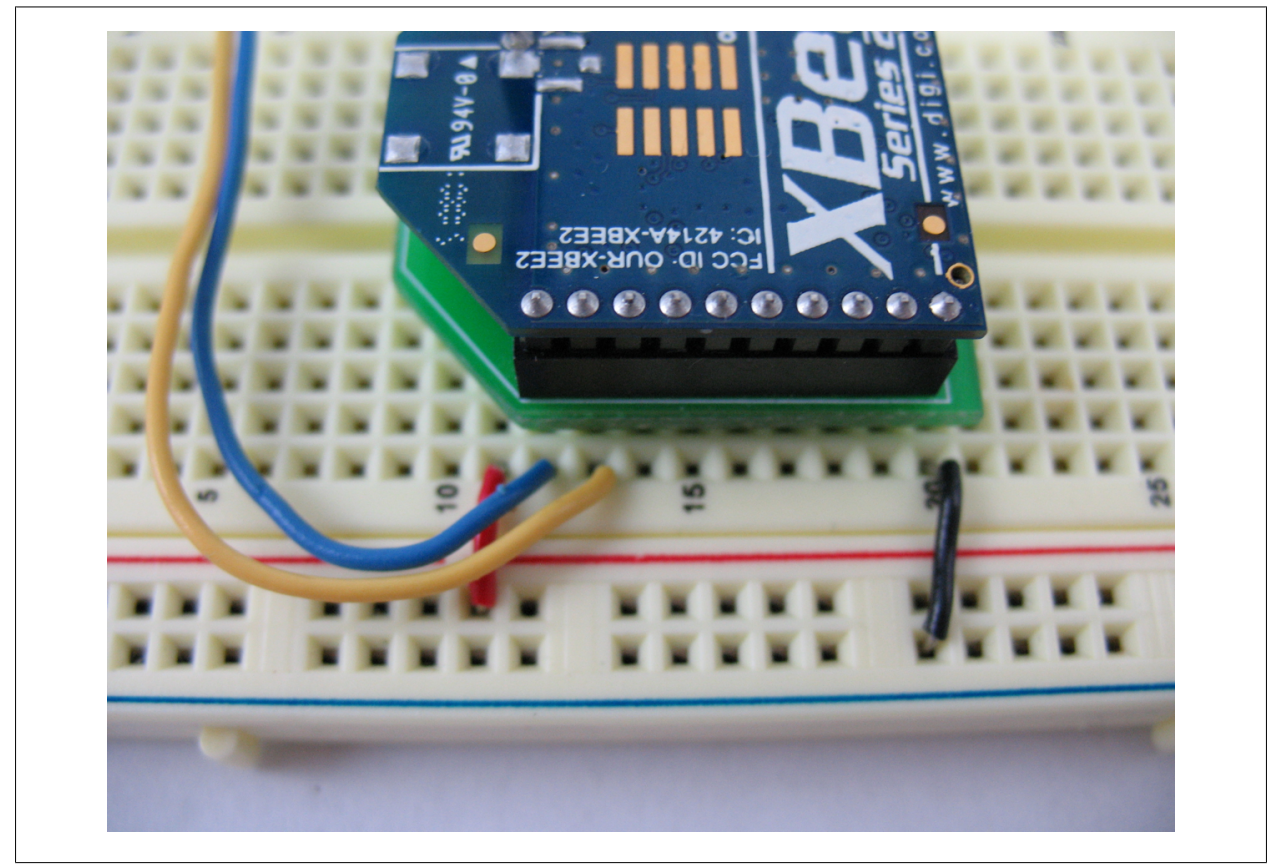

Figure 3-10. Power, ground, transmit, and receive connections on XBee. Note that Arduino TX goes to XBee RX and vice versa.

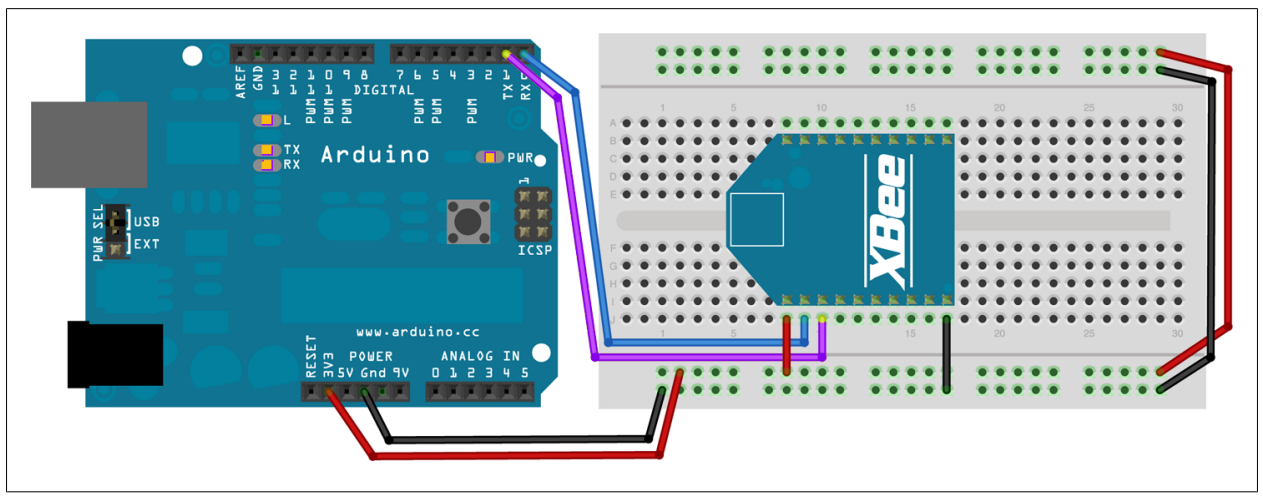

Figure 3-11. Arduino XBee TX/RX connection on breadboard 


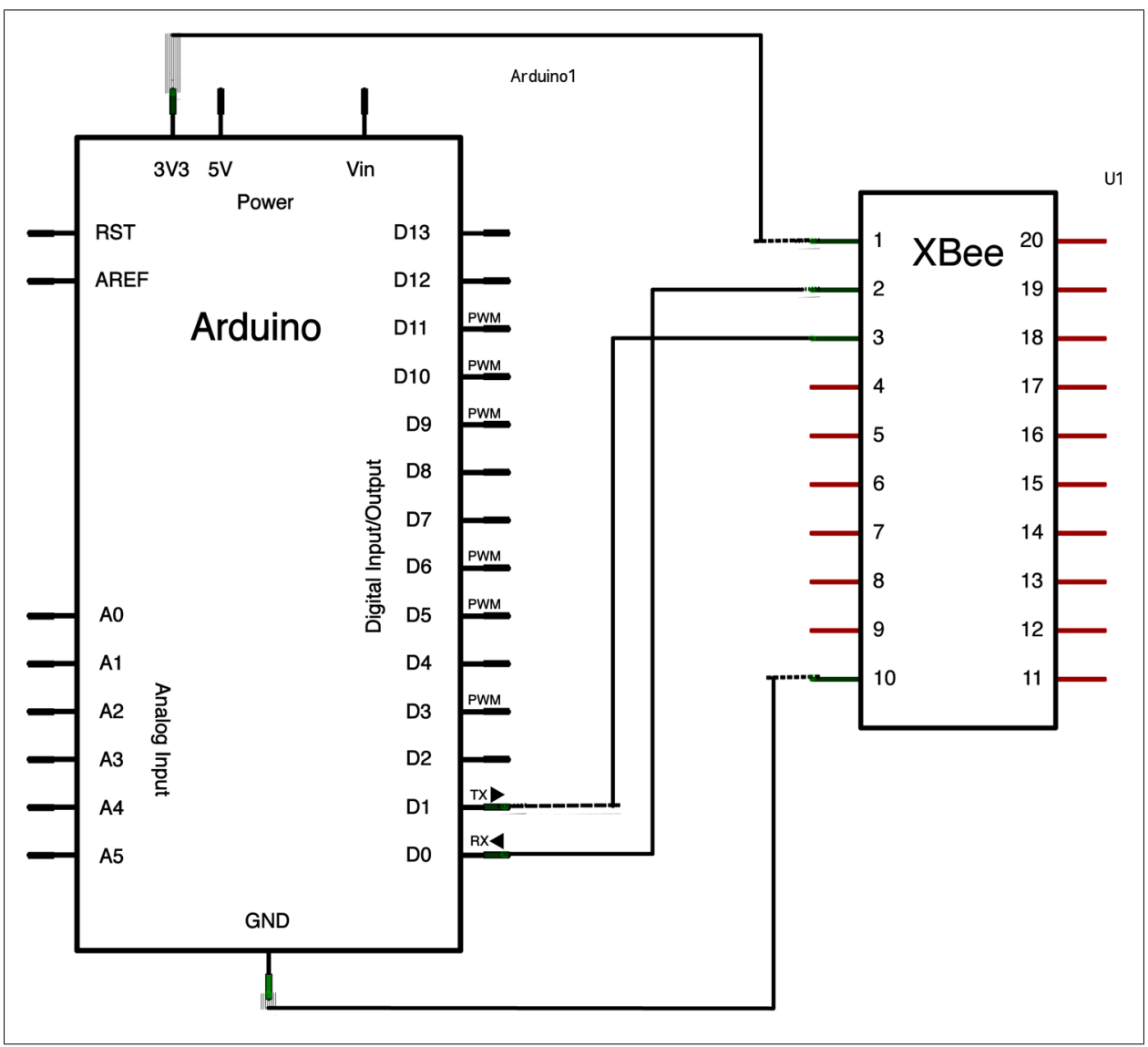

Figure 3-12. XBee Arduino TX/RX connections in schematic view

\section{Doorbell Introduction}

Radios aren't much fun on their own, so consider working with a friend on the next two projects, the first to make a simple doorbell and the second to make one with feedback. One of your boards will have the doorbell button input and the other will have a buzzer, speaker, or other noisemaker to act as the doorbell output. The two boards will run different Arduino code, so make sure you load the correct program on each.

\section{Switch Input...}

One of your boards will serve as the doorbell button: 
1. Pick the board with the coordinator to act as the doorbell button. (Either the board with the coordinator or the one with the router would work equally well, so the choice here is arbitrary.)

2. On that board, attach a momentary switch between power and Arduino digital input 2. Make sure you use a $10 \mathrm{~K}$ ohm pull-down resistor from digital input 2 to ground. This ensures the pin has a reference to zero voltage while the switch is open.

Figure 3-13 shows the circuit diagram, and Figure 3-14 shows the schematic.

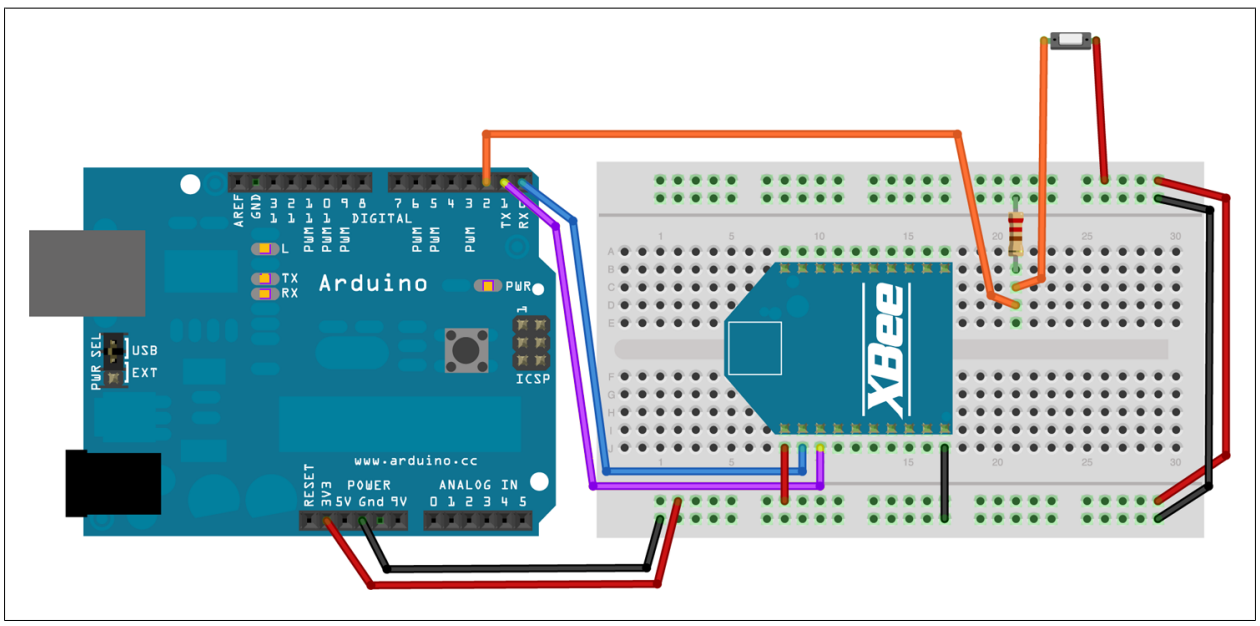

Figure 3-13. Basic doorbell: BUTTON system on breadboard. The button is represented here by the little white thingy, top right.

\section{...and Buzzer Output}

Your second board will act as the bell part of the doorbell. On the second board:

1. Attach the red power lead of your buzzer to digital pin 5 of your Arduino board.

2. Attach the black ground lead from your buzzer to ground.

3. If you are already familiar with analog output on the Arduino, you can also use a speaker or other sound output device, in which case employ what you already know to make the proper connections and adjust the Arduino code for that device. Remember that there are a lot of ways to make sound. If you decide to use a servo or to hack a toy, a relay might take the place of the buzzer. Imagination makes this project more fun, so go ahead and amuse your friends or confound your cat.

Figure 3-15 shows the circuit diagram, and Figure 3-16 shows the schematic. 


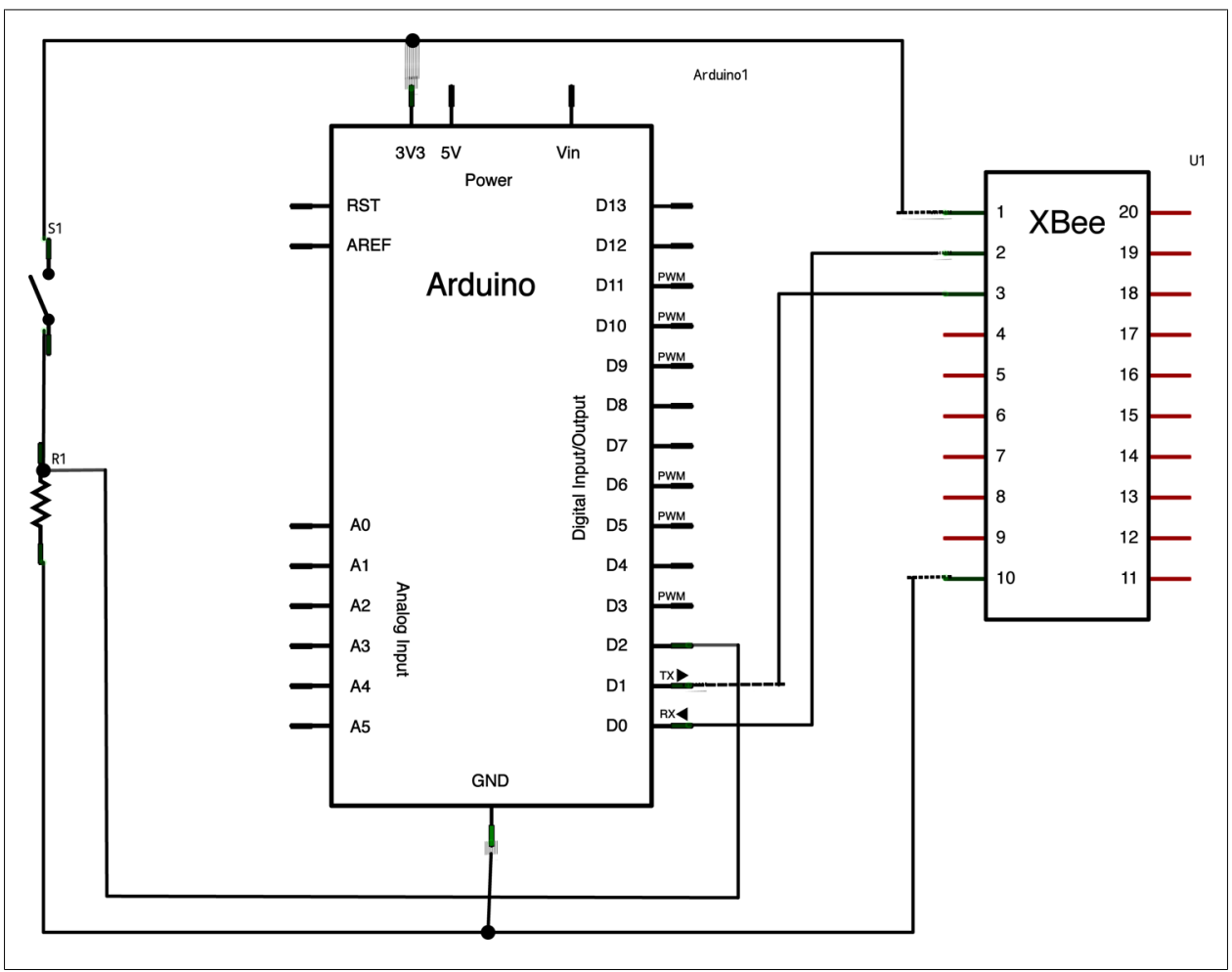

Figure 3-14. Basic doorbell: BUTTON system schematic

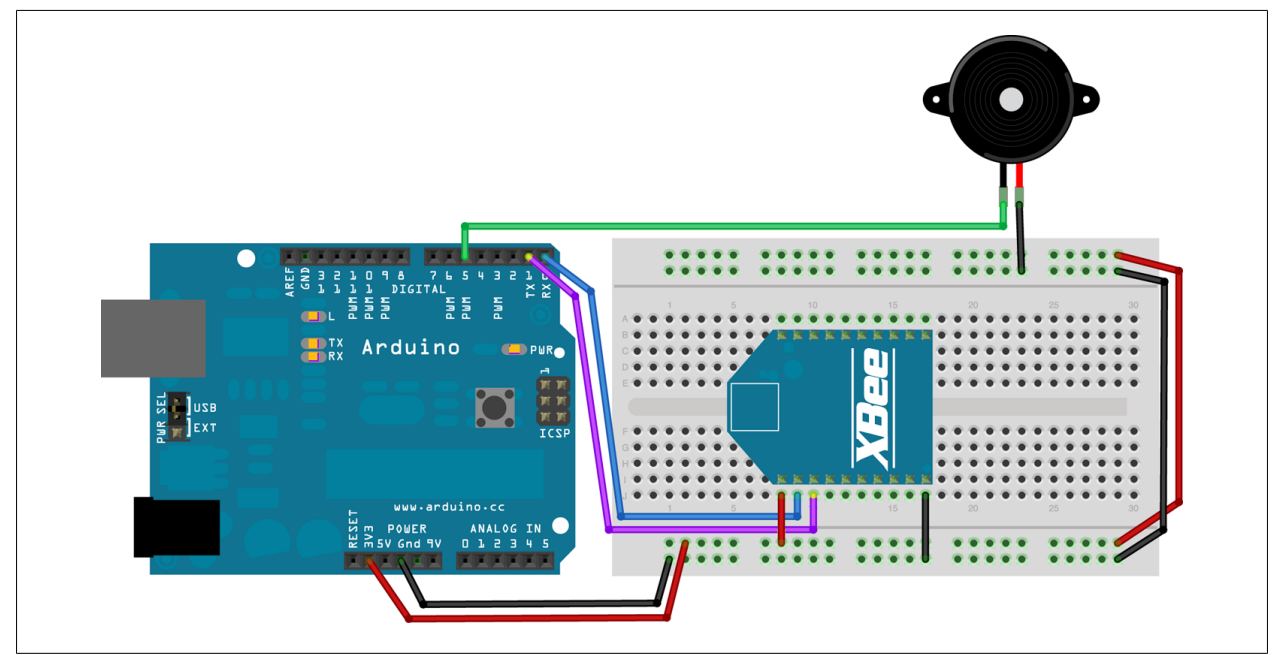

Figure 3-15. Basic doorbell: BELL system on breadboard 


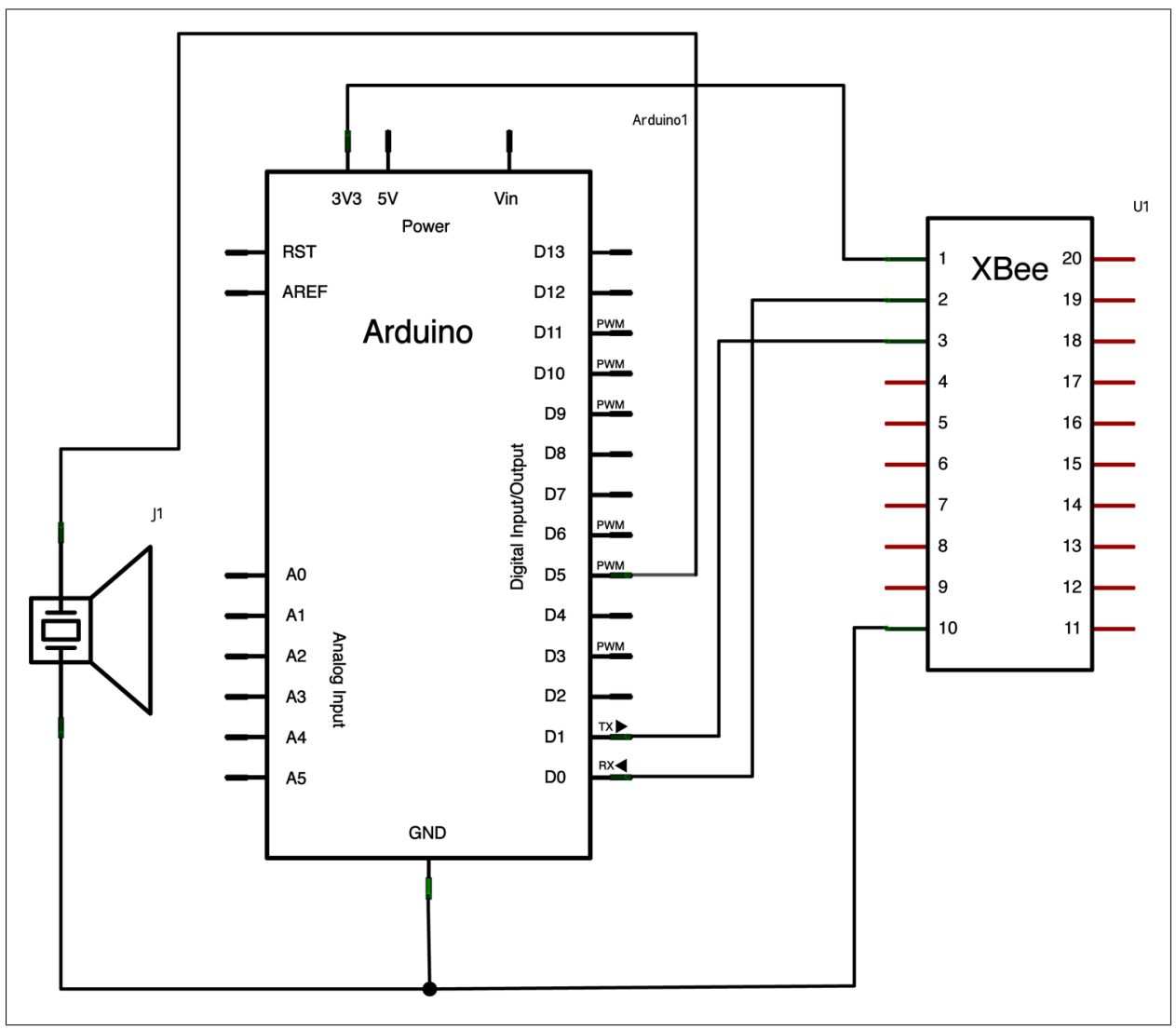

Figure 3-16. Basic doorbell: BELL system schematic

\section{Configure Your XBees}

Keep in mind that there are many ways to configure your XBee radios from your computer. We'll be using the CoolTerm (Mac, Windows) terminal program and an XBee Explorer USB adapter. (If you're on Linux, see the sidebar "A Serial Terminal Program for Linux" on page 40 in Chapter 2.) You could also use Digi's X-CTU program and the Digi evaluation board to accomplish the same task, or one of the many other combinations of serial terminal programs and USB adapter setups.

Remember that every XBee radio has a 64-bit serial number address printed on the underside. The beginning or "high" part of the address is 0013A200. The last or "low" part of the address will be different for every radio. It will look something like 4052DAE3.

Write down your coordinator and router radio's addresses so that you can refer to them during configuration: 
1. Select the coordinator XBee you labeled with a " $\mathrm{C}$ " and place it into the XBee Explorer. (Technically, either radio will work.)

2. Plug the XBee Explorer into your computer.

3. Run the CoolTerm program and press the Options button to configure it.

4. Select the appropriate serial port, which will probably have the words usbserial (Mac) or COM (Windows) in its name, and check the Local Echo box so you can see your commands as you type them.

5. Click on the Connect button to connect to the serial port.

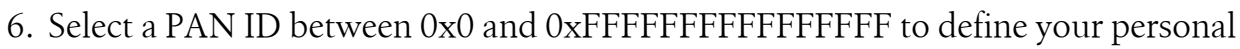
area network.

7. Put the radio into command mode by typing +++ (remember not to press Return). Next, enter ATID followed by the PAN ID you selected. For example, if you selected 0x2001 as your PAN ID, you'd enter ATID 2001 and press Enter on the keyboard. You should receive OK as a reply. If you don't receive an OK, you probably timed out of command mode and will need to start over with the +++ and try again.

8. Enter ATDH followed by the first "high" part of your radio's destination address. In this case we're making a pair of radios, so each one will have the other as its destination. All XBee-brand radios have the same high part-0013A200. Type ATDH 0013 A200 and press Enter on the keyboard.

9. Enter ATDL followed by the second "low" part of your radio's destination addressthe eight-character hexadecimal address of the router radio that follows 0013A200. Type ATDL followed by that second part of the destination address, then press Enter on the keyboard. (Don't forget to go into command mode first if you waited more than 10 seconds after your last command.)

10. To save your new settings as the radio's default, type ATWR and press Enter.

11. Remove the XBee from the serial adapter.

You set up your second radio in the same way:

1. Select the router XBee you labeled with an "R" and place it into the XBee Explorer.

2. CoolTerm should still be running; if not, repeat steps 2-6 above.

3. Select the same PAN ID you entered for your first radio above.

4. Type +++ to go into command mode. You should receive an OK reply from the radio.

5. Type ATID followed by the PAN ID you selected and press Enter on the keyboard. You should receive OK again as a reply. 
6. Enter ATDH followed by the high part of your radio's destination address-always the same for the XBees. Type ATDH 0013A200 and press Enter on the keyboard. You should receive an OK response.

7. Enter ATDL followed by the low part of your radio's destination address- the eightcharacter hexadecimal address of the coordinator radio that follows 0013A200. Type ATDL followed by that low part of the destination address, then press Enter. You should receive an OK response.

8. Again, save your new settings as the radio's default by typing ATWR and pressing Enter.

Your radios are now configured as a pair. Sometimes when people are first starting out with XBees, it takes a few tries to get everything typed in just right to pair the radios. If they don't work at first, don't panic; usually it's just because you missed a single step or made a typo. Try again. Remember that if you are in command mode and type an AT command without an argument, the radio will reply with the current setting. This is a good way to check that your configuration is correct.

\section{Program the Arduino Doorbell}

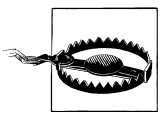

When uploading programs to the Arduino boards, disconnect the wiring from digital pin 0 (RX) first, then reconnect the wiring after loading. If you see an error message from "AVR dude," you probably forgot to do this.

There are two programs that run the doorbell system. One goes with the button and the other goes with the output buzzer or bell. Load this program onto your button board:

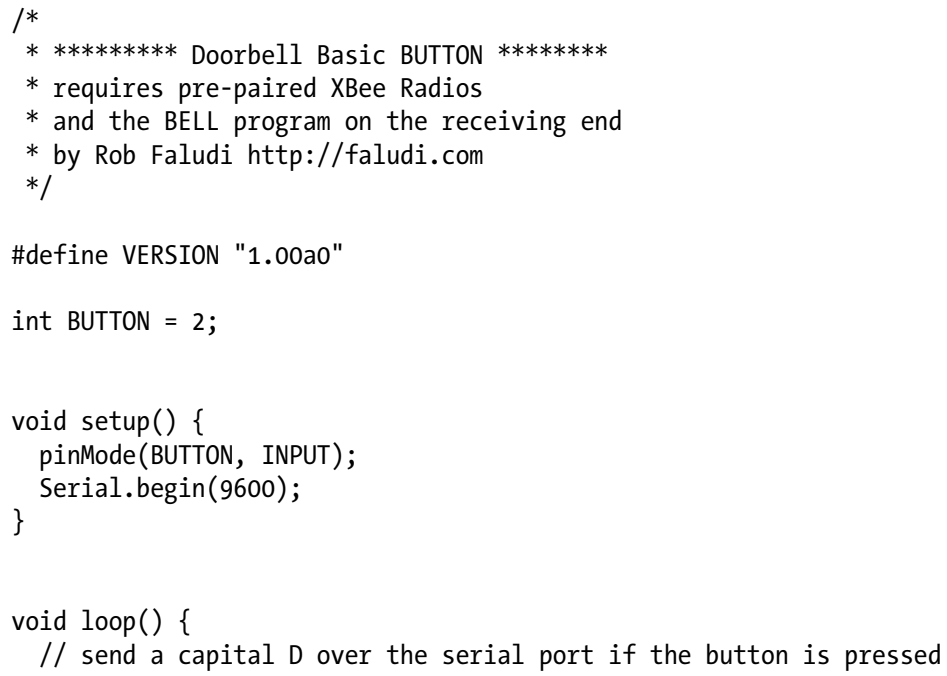




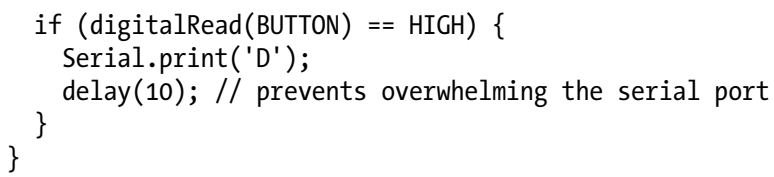

Program your second board with the bell code. This enables the board to receive a signal from the button board when its switch is activated and to ring the bell:

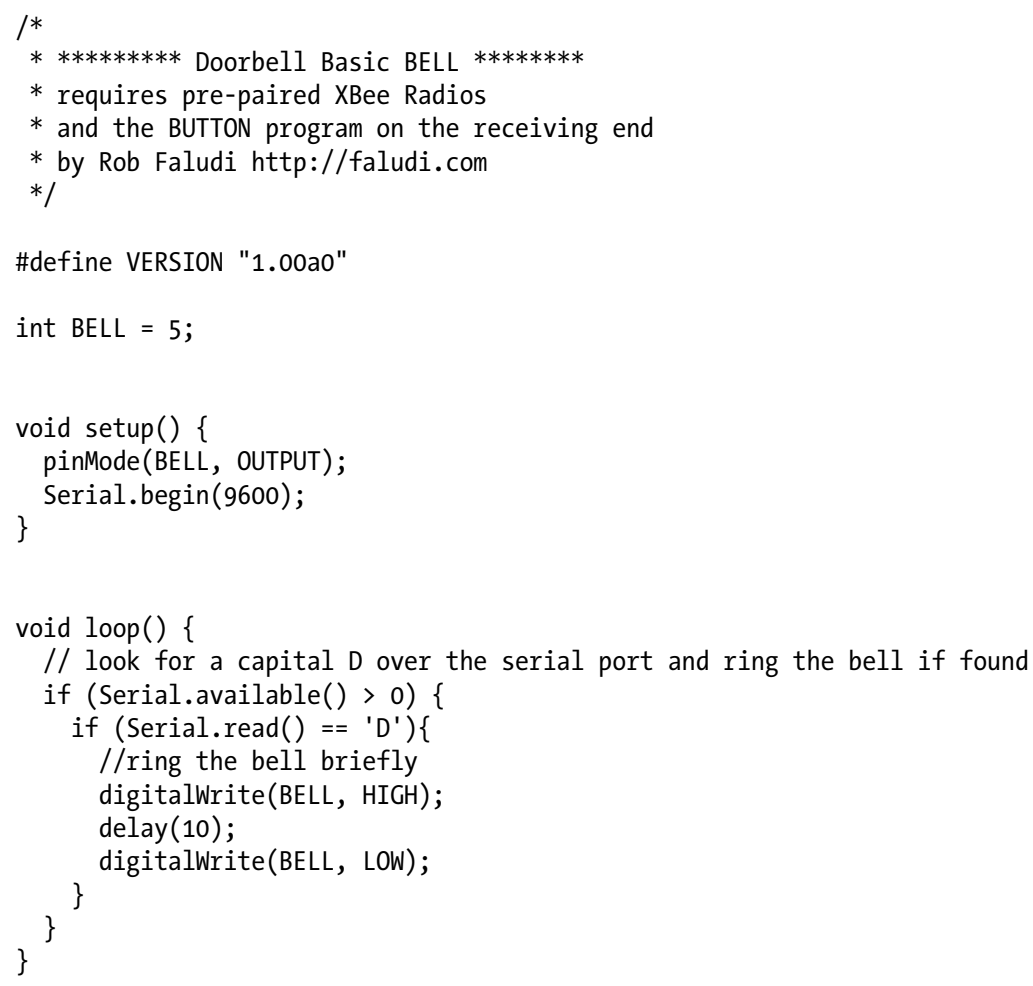

Don't forget to reconnect the wiring to digital pin $0(\mathrm{RX})$ after loading your code!

\section{Troubleshooting}

Sometimes it takes a few tries to get things right. This is a normal part of the learning process, so if your doorbell doesn't ring at first, keep up your good cheer and dig into figuring out the reason why:

1. Start with the simple stuff. Check to make sure your radios are seated correctly in the adapter boards, and that all the wiring is connected properly.

2. Use a serial adapter and a terminal program to check that the radios are paired to communicate with each other. You should be able to use them to chat between 
two computers, the way we demonstrated in the previous chapter. Check through the troubleshooting guide for the chat project if it seems like you're having radio issues.

3. If both radios are responding and paired properly, seat them back into the doorbell project. Make sure they're inserted into the sockets properly and facing the correct way.

4. Check your wiring again. The most common problems are simple electrical ones, where one wire is not making good contact or has accidentally been inserted into a socket one away from where it should be. Check for any wires where the bare ends might be touching each other and creating a short circuit.

5. Make sure that RX on the Arduino is connected to TX on the XBee and vice versa.

6. Use a multimeter to confirm that your XBee is getting 3.3 V power. Check that both Arduino boards have a power light illuminated.

7. Reload the Arduino code onto both boards. Remember that your button board takes one program, and that your buzzer board takes a different program. Make sure you're loading the right program onto the right Arduino board.

8. If you have any questions about Arduino, the best place to learn more is on the Arduino site itself. You'll find a complete reference guide there and extensive forums where you can search for answers or ask a question yourself.

\section{Revelations and Indications}

Electricity is invisible. Radio is invisible. How in the world are you going to confirm that your radio has power and is receiving information? There are three places on the XBee radio where you can attach a light to see what's going on:

1. Physical pin 13 is the On/Sleep indicator pin and can tell you if your radio is getting power and currently awake. As you are still a few chapters away from learning how to put the module to sleep, this indicator should always be on. Place an LED with the positive lead (the longer one) into a breadboard socket associated with XBee physical pin 13, and the negative lead (the shorter one) into the ground bus. If the LED lights up, your radio has power and is awake. If it doesn't, check to make sure you put the LED in the right way, with the shorter leg to ground, and that you attached it to the proper XBee pin. After that, check to see that the radio is seated properly in its breakout board, and that it's powered properly with 3.3 volts.

2. An LED placed between the Association indicator on physical pin 15 and ground will light steadily while the radio searches for a network, and then blink once it has associated itself with one. Coordinator radios always blink because they are always associated with the network they created themselves. Router radios (and end devices) will give a steady light when they are powered up and looking for a coordinator. When they find a network to join, their association light will start blinking. If you don't see a light at all, check for power problems. If the light is steady and not blinking, check the configuration of that radio to ensure that it has the same PAN ID as the coordinator and is within range of a radio that it can join. 
3. One of the most helpful indicators is on the other side of the XBee, on physical pin 6. Place an LED between this pin and ground. The Received Signal Strength Indicator (RSSI) will light up on this pin when the radio receives information that's addressed to it. By default, the RSSI light will remain on for 10 seconds after it receives information and then go out again. The RSSI LED will be slightly brighter when the signal is strong than when it is weak, but in practice this difference is extremely hard to see. If you think the radio should be receiving information but the RSSI light remains dark, check the configuration of the sender radio to make sure it is on the same PAN ID as the destination, is associated with the network, and has the destination address set correctly.

Figure 3-17 shows an XBee adorned with plenty of LEDs. The bottom right light is the On/Sleep indicator; the top right light is the Association indicator; and the left light shows Received Signal Strength for 10 seconds after data is received. Normally, the On/Sleep light should be on steadily, the Association light should be blinking, and the RSSI light should be on when data is received.

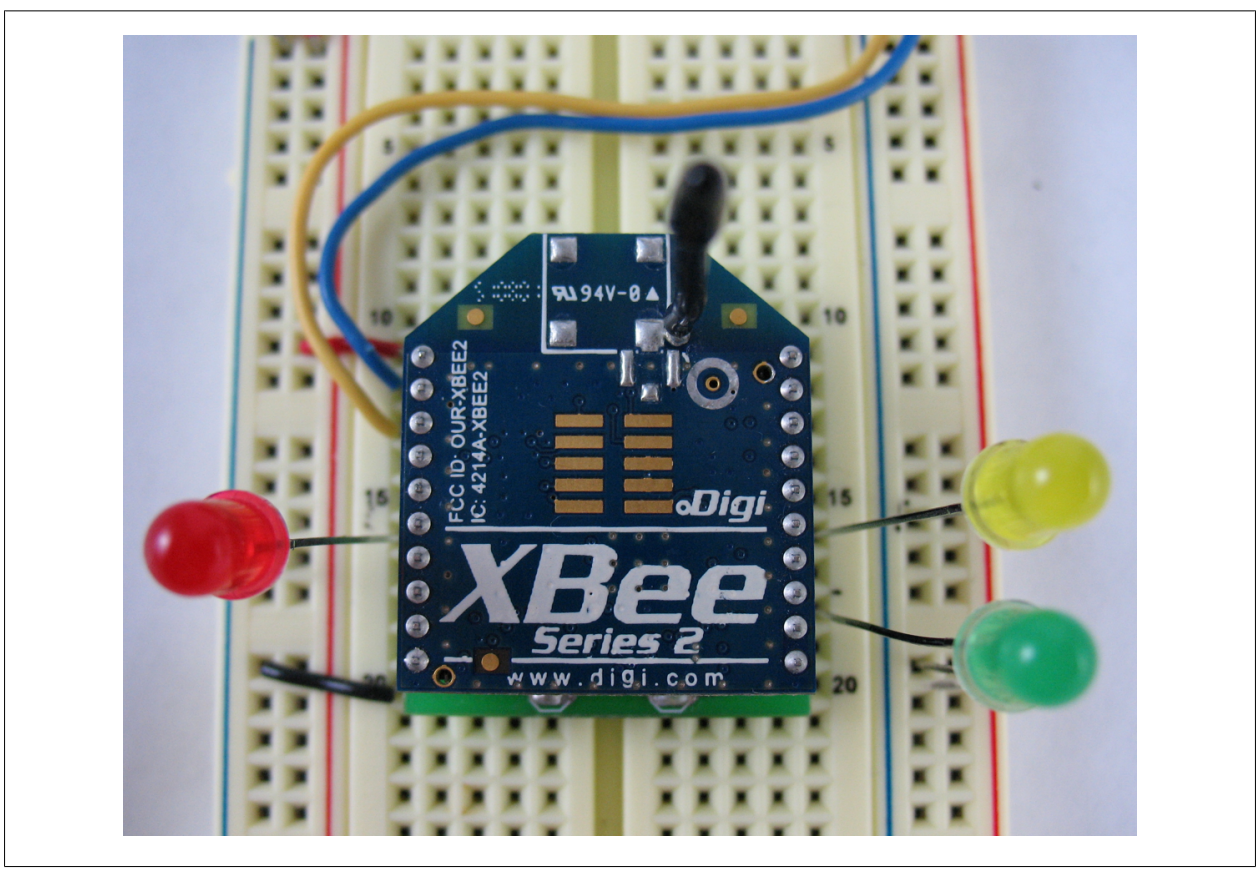

Figure 3-17. XBee with indicator LEDs attached.

\section{Feedback Doorbell}

The next project builds on the previous one-way signal to provide two-way feedback that the bell unit has received the doorbell button press and has rung. This is useful so the person at the door knows she actually rang the bell. 


\section{Feedback Light}

Add an LED as an output from Arduino digital pin 11 on the button board. Figure 3-18 shows the diagram, and Figure 3-19 shows the schematic.

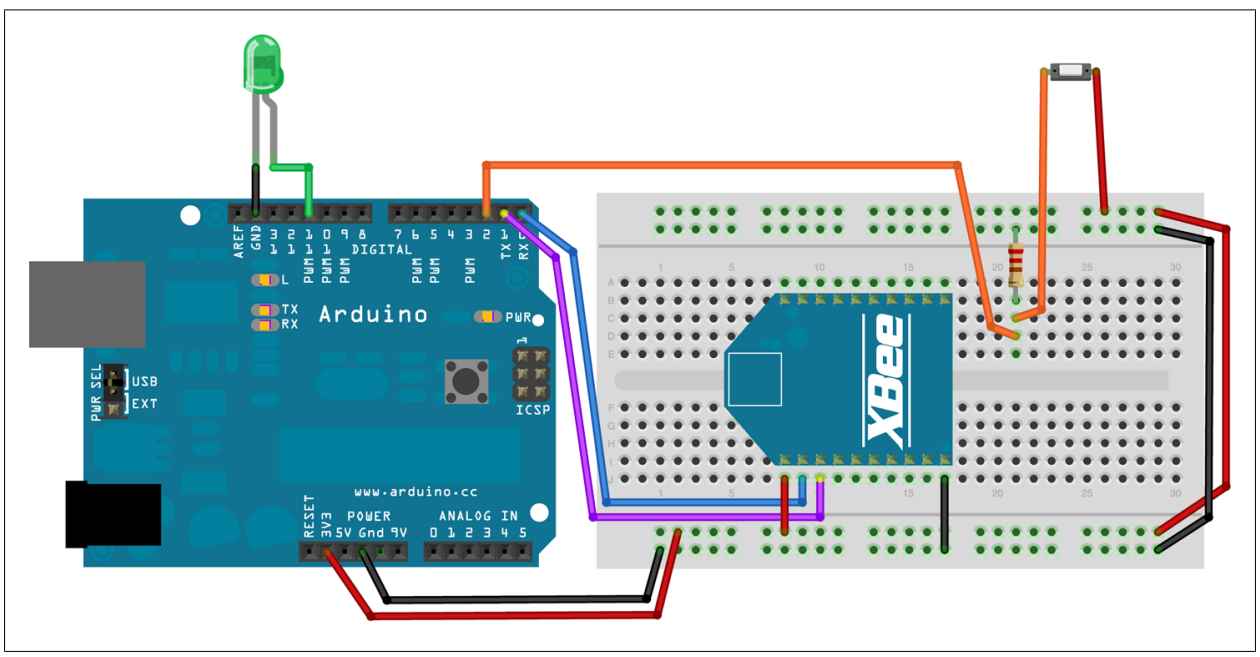

Figure 3-18. Feedback doorbell: BUTTON system on breadboard

\section{Program the Arduino Feedback Doorbell}

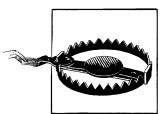

Remember to disconnect the wiring from digital pin 0 (RX) first, then reconnect the wiring after loading.

Use the following code for the button board with its new feedback light:

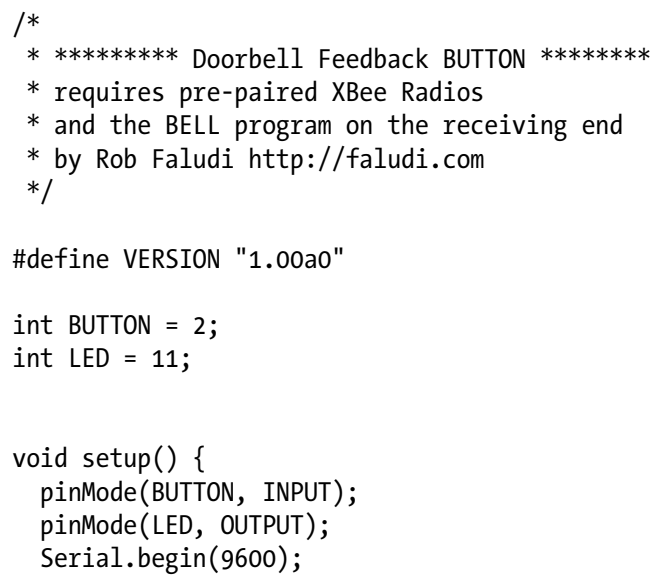




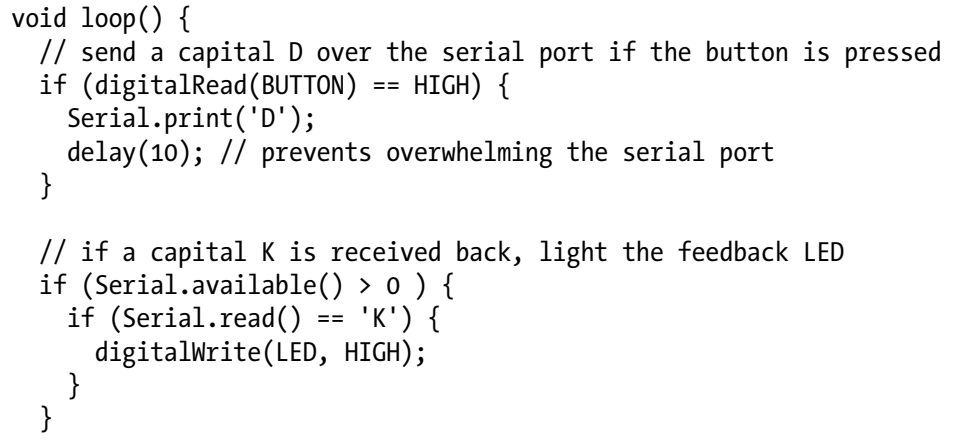

// when the button is released, turn off the LED

if (digitalRead(BUTTON) $==$ LOW \{

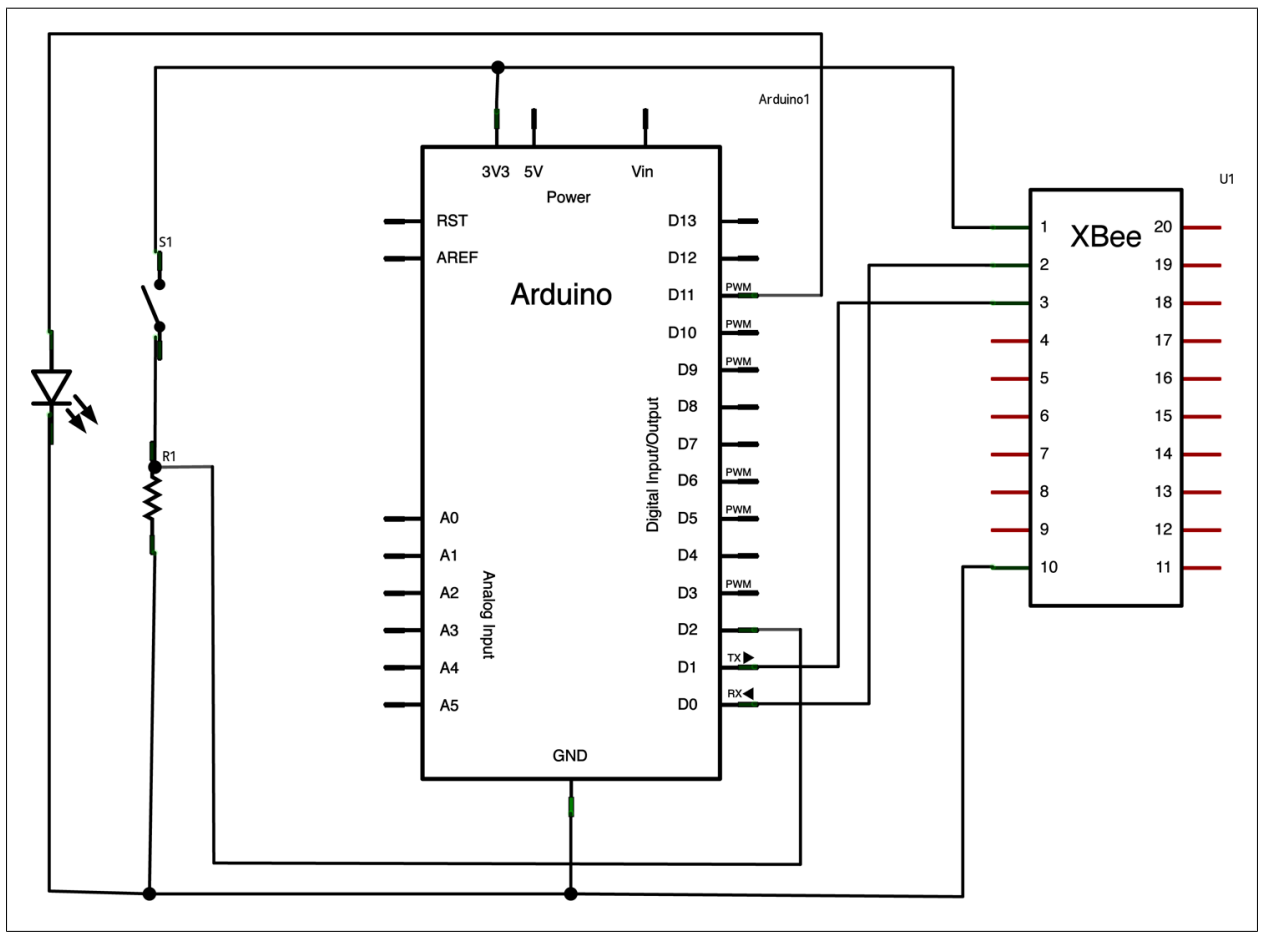

Figure 3-19. Feedback doorbell: BUTTON system schematic 
On the second bell board, use this code; it accepts the incoming ring request and responds that the bell has been rung:

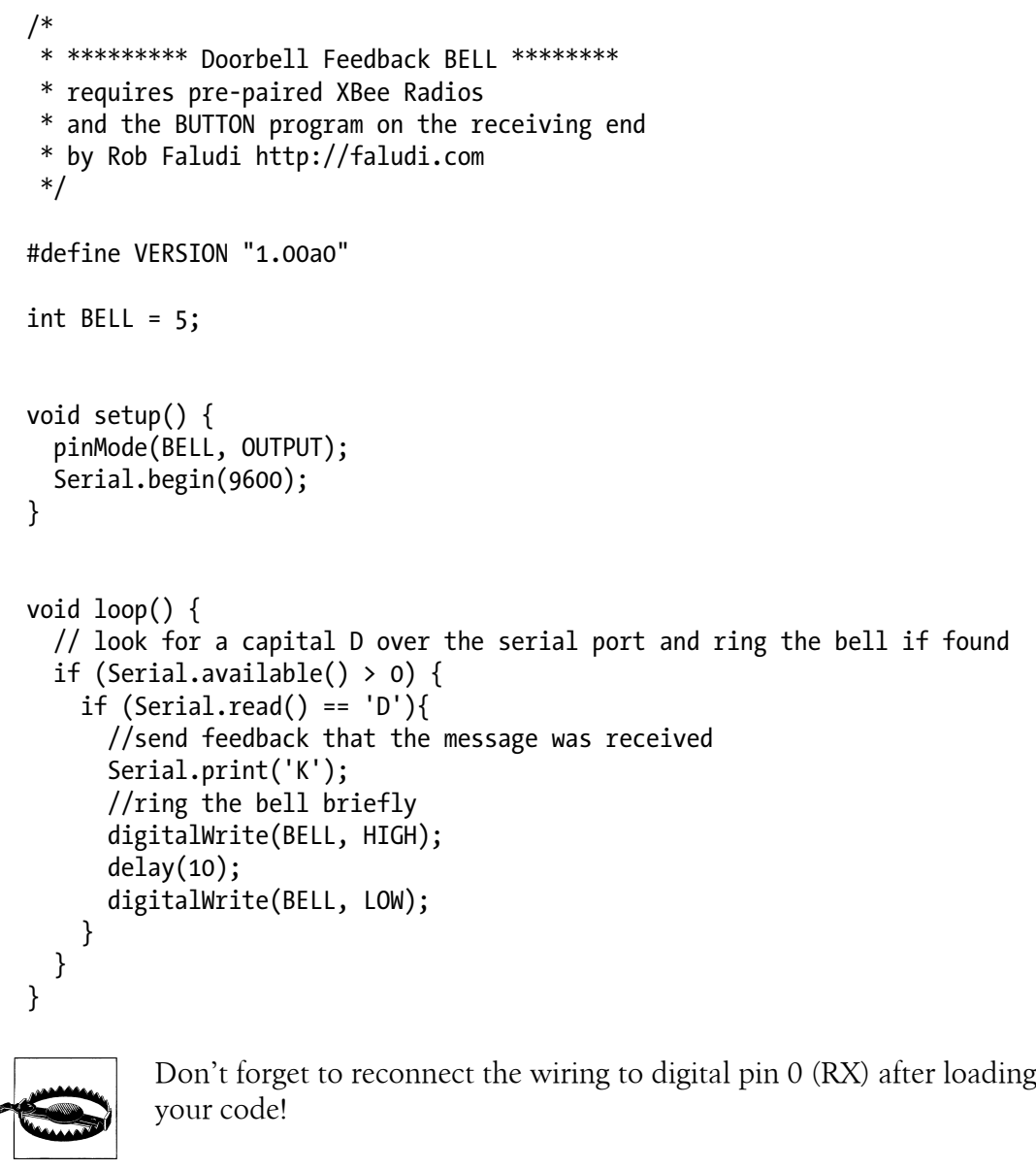

\section{Extra: Nap Doorbells and More}

There are many ways to take this project one step further. For example, let's imagine a situation where you wanted to take a nap and not be disturbed by the doorbell unless it was extremely urgent. In this case, initial presses of the doorbell button might only illuminate a signal light, rather than waking you with a bell. Eventually, after a large number of presses, the system would kick over into bell mode and wake you up. The caller would initially see a red light at the button to indicate that the bell hadn't been rung yet, then eventually after a large number of presses would see a green light to confirm that the bell had finally rung. Try creating this system or another of your choosing by extending the Feedback Doorbell system with new, useful features. For example, you could create a doorbell that rings only when the button is pressed in a 
special coded sequence, or a doorbell that can store and replay a history of its rings, or one with an SMS feature to send you a text message when somebody comes calling, or an LCD text display where the visitor can select messages to send with the ring. The possibilities are endless! 


\section{CHAPTER 4}

Ins and Outs

Congratulations-you now have configurations, communications, and some solid projects under your belt! It's time to take a closer look at the unique features of the XBee brand of ZigBee radio so we can start building fully scalable sensor networks. We begin with input/output concepts and commands, then immediately put these to use in a small set of progressive projects that whimsically inculcate the basics.

\section{The Story of Data}

Before getting into the technical aspects of sensing data, it's useful to take a step back and consider why it is we want to collect this type of information in the first place. After all, data has no value by itself. In its purest form, data is just a collection of numbers, and one set of numbers is as good as any other. Our real interest in data always comes from the story it might tell us. Gathering data is the first step in noticing new things in the world, proving a hunch, disproving a fallacy, or teaching a truth. It can also be a path to action. Patterns in data can trigger events, shape public policy, or just determine when it's time to feed the cat. We should always have a purpose in mind when collecting data because that purpose will guide us in how the data is collected. This doesn't mean we need to know what the data will tell us. Our purpose might be to simply gather results to examine for events or patterns that create new questions. This is known in science circles as exploratory data analysis - a well-accepted form of initial investigation. In other cases, our plan might be to seek out a highly specific event as a trigger for a fixed response. That sounds complicated, but really it describes most doorbells, including the ones you made in the last chapter. Data is collected from a button for the express purpose of triggering an audio alert. Simple enough, but what else could we learn from it?

\section{Direct, Indirect, Subtext}

A huge number of electronic sensors are available. Table 4-1 contains a partial list of those within reach of the average tinkerer. 
Table 4-1. Kinds of electronic sensors

\begin{tabular}{|c|c|c|}
\hline Sensor & Detects & $\begin{array}{l}\text { Example (SparkFun part num- } \\
\text { bers unless otherwise noted) }\end{array}$ \\
\hline Accelerometer & Accelerations (changes in speed) & SEN-00252 \\
\hline Capacitance & Electrical properties often associated with human touch & SEN-07918 \\
\hline Color & Wavelengths of light & SEN-08663 \\
\hline Flex & angular position and changes & SEN-08606 \\
\hline Force & Physical pressure in an analog scale & SEN-09673 \\
\hline \multirow[t]{2}{*}{ Gas } & Alcohol, methane, $\mathrm{CO}_{2}, \mathrm{CO}$, propane, and many others & SEN-08880 \\
\hline & & SEN-09404 \\
\hline GSR & Galvanic skin response, typically associated with emotional arousal & $\begin{array}{l}\text { http://www.extremenxt.com/ } \\
\text { gsr.htm }\end{array}$ \\
\hline Gyroscope & Rotation & SEN-09423 \\
\hline Hall effect & Magnetic fields & COM-09312 \\
\hline Microphone/acoustic & Sound & BOB-08669 \\
\hline Motion & Changes in relative distance & SEN-08630 \\
\hline Photocell & Light & SEN-09088 \\
\hline Potentiometer & Rotation or linear position on an analog scale & COM-09288 \\
\hline Pressure & Air or fluid pressure & SEN-09694 \\
\hline Pulse & Heartbeat rate & SEN-08660 \\
\hline Ranging & Distance between objects & SEN-00639 \\
\hline Rotary encoder & Rotation on a digital scale & COM-09117 \\
\hline Smoke & Airborne particles & COM-09689 \\
\hline Stretch & Physical deformation or strain & $\begin{array}{l}\text { http://www.imagesco.com/sen } \\
\text { sors/stretch-sensor.html }\end{array}$ \\
\hline Switch & Physical pressure on a digital scale & COM-09336 \\
\hline Thermistor & Temperature & SEN-00250 \\
\hline Tilt & Angular attitude & Adafruit 173 \\
\hline
\end{tabular}

Although the table describes detection of one phenomenon per sensor, each sensor is really capable of simultaneously detecting three distinct but intrinsically related categories of events:

Direct or proximal phenomena

These are the incidents that directly trigger the sensor apparatus. For example, in the case of a photocell, the proximal event would be photons striking the sensor. Sometimes the proximal phenomenon is not quite as obvious. For instance, a tilt sensor's proximal trigger would be the repositioning of a metal ball against two 
electrical contacts. A Hall-effect sensor reports changes in magnetic fields, though that's only rarely the phenomenon of interest.

\section{Indirect or distal phenomena}

Distal events are the remote causes of the local events actually triggering the sensor. The sun coming out from behind a cloud would be the distal phenomenon that results in a higher reading from a photocell. A window being opened might cause a Hall-effect magnetic sensor to move away from a magnet and open its contacts. These indirect events produce the proximal phenomena that our sensors can respond to, and they are frequently the ones we are most interested in.

Context and subtext

Sometimes neither the proximal or even distal events are what we're after. We aren't interested in magnetic fields at all. In fact, most window openings are not a cause for concern. What we really want to know is if a burglar is entering our house. Our sensor directly detects a change in a magnetic field. That change is an indirect result of a window changing position. But the context is human presence; in this case, definitely a presence that's undesired. Contextual leaps usually entail some degree of uncertainty. A window might swing open in a gust of wind. A houseguest might open up a window that we'd normally leave closed. This creates a need for determining more information to avoid false alerts or missed alarms. Sensing for multiple phenomena can reduce uncertainty. For example, security systems often include window sensors, motion detectors, and pressure mats. When all of these activate simultaneously, it is a more certain indication of criminal presence than hearing from any one on its own.

When choosing a sensor, always think about which category of events you're interested in detecting. Sometimes a surprising relationship can exist where a simple sensor can provide reliable indication of an intricate contextual event. A photocell can report when a bathroom cabinet is opened, by detecting that the interior is no longer totally dark. A microphone can detect the wind noise made when someone blows on a pinwheel, and therefore detect both pressure and presence. A switch on the handle of a toilet might indicate human absence if not triggered for two days, signaling an unsecured front door to lock itself.

Now that we've thought about sensing in theory, let's move on to the practical matter of getting the job done.

\section{I/0 Concepts}

Each XBee radio has the capability to directly gather sensor data and transmit it, without the use of an external microcontroller. This means that you don't always need something like the Arduino when building simple sensor nodes with XBee radios. In addition, the XBee offers some simple output functions so that basic actuations can also take place without an external microcontroller being present. For example, it's possible to send digital information directly to a standalone XBee radio to have it turn on a light 
or start up a motor. For clarity, we'll refer to these independent input/output functions as XBee direct, to distinguish them from the use of input and output that happens in conjunction with an external microcontroller.

\section{Why XBee Direct?}

There are lots of good reasons to use the XBee for direct input or output. By not having an external microcontroller, the overall size of your project is reduced. This is especially important when creating sensors that need to be inconspicuous or fit into tight spaces. By using the XBee alone you'll also save weight, which can be important if the system is to be lofted skyward in a kite or balloon, or worn on your body, or by your pet. When it comes to wearables, lighter is almost always better. Omitting an external microcontroller also reduces power consumption. This can be a critical advantage for projects that run on batteries, a necessary situation for any project that is truly wireless, and something we'll talk about more in Chapter 6 . Of course, eliminating the external microcontroller means saving money, and for sensor networks with hundreds of nodes, it can mean saving a lot of money. Finally, using the XBee alone is sometimes the leastcomplicated approach to a project. There's a lot going for the XBee direct model. However, there are also some important trade-offs to consider.

\section{XBee Direct Limitations}

Projects that use the XBee alone for its input/output features may face significant limitations compared to projects that incorporate an external microcontroller such as the Arduino. The XBee has limited input and output pins, with no simple way to extend them. Also, the Series 2 hardware that the ZigBee firmware requires doesn't currently support analog output at all, which means it can't be employed to dim a light or control the speed of a motor without additional electronic components. The single biggest limitation is that the basic standalone XBee radio doesn't allow access to any kind of logic. This means no decisions can be made on the local device and no standalone operations can be performed besides transmitting data or changing the state of digital pins as the result of remote commands.

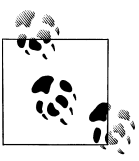

A new variation of the XBee radio was recently released that incorporates a second microcontroller to allow some forms of local logic. However, this comes at additional cost, will need to be accessed with special programming methods, and requires knowledge of C or Assembly, both lower-level approaches than using Arduino. 


\section{XBee I/O Features}

The XBee Series 2 hardware offers several flexible features for projects that need simple input and output. There are 10 pins that can be configured either as digital inputs for sensing switches and other things that operate like switches, or as digital outputs for controlling LEDs and small motors directly. Larger loads, including ones that run on alternating current, can be operated using these digital outputs via a relay. The first four of these pins can be configured as analog inputs for sensing a huge array of phenomena that scale over a range, like light, temperature, force, acceleration, humidity, gas levels, and so forth. On the Series 2 radios, there are currently no user-configurable analog or pulse-width modulated (PWM) outputs, so you cannot directly control the speed of a motor or the brightness of an LED light. However, the underlying chipset does support these types of outputs so perhaps they will be available in a future firmware upgrade.

XBees have all these different features available, but this doesn't mean you can use them all at once! There are only 10 pins total so you if you have all 10 digital inputs configured, you are out of pins and can't use any digital output or analog input. Happily, the pins can be used in a mix. For example, three analog inputs, four digital inputs, and three digital outputs would be fine. The only other thing to be aware of is that many of the 10 configurable pins are used for other optional duties. These other duties are important in many applications, but they've been carefully selected so that they are ones that don't tend to be needed in remote sensing and actuation projects. For example, some of the duties are serial hardware handshaking (CTS and RTS), an advanced feature that is generally not needed unless there is another microcontroller or logic-based device in the mix. Certain I/O pins do double duty as debugging light outputs for signal strength (RSSI) and association (ASSOC), which are handy for development but generally unimportant on a remote sensor that will not be viewed directly. There are also several pin-controlled sleep features (ON and SLEEP) that are not usually required for standalone sensing or actuation. Of course, on the off chance that one or more of those features is required, it would reduce the number of pins available only by one or two, so you'll generally have enough left over to cover the vast majority of application projects you can dream up. Table 4-2 shows the input/output pin names with physical numbers, corresponding AT commands, and other functions. Note that DIO8 and DIO9 are not supported in the current firmware so they can't be used for I/O at this time. Figure 4-1 shows the I/O pins on a breakout board.

Table 4-2. Input/output pin names with physical numbers, commands, and other functions

$\begin{array}{llll}\text { Pin name } & \text { Physical pin \# } & \text { AT command } & \text { Other functions } \\ \text { DI00, AD0 } & 20 & \text { D0 } & \text { Analog input, Commissioning Button } \\ \text { DI01, AD1 } & 19 & \text { D1 } & \text { Analog input } \\ \text { DI02, AD2 } & 18 & \text { D2 } & \text { Analog input } \\ \text { DI03, AD3 } & 17 & \text { D3 } & \text { Analog input }\end{array}$




\begin{tabular}{llll} 
Pin name & Physical pin \# & AT command & Other functions \\
D104 & 11 & D4 & \\
D105 & 15 & D5 & Association indicator \\
D106 & 16 & D6 & RTS \\
D107 & 12 & D7 & CTS \\
(D108) & 9 & None & Pin sleep control, DTR \\
(D109) & 13 & None & On/Sleep indicator \\
D1010 & 6 & P0 & Received Signal Strength Indicator (RSSI) \\
D1011 & 7 & P1 & \\
D1012 & 4 & P2 & \\
\hline
\end{tabular}

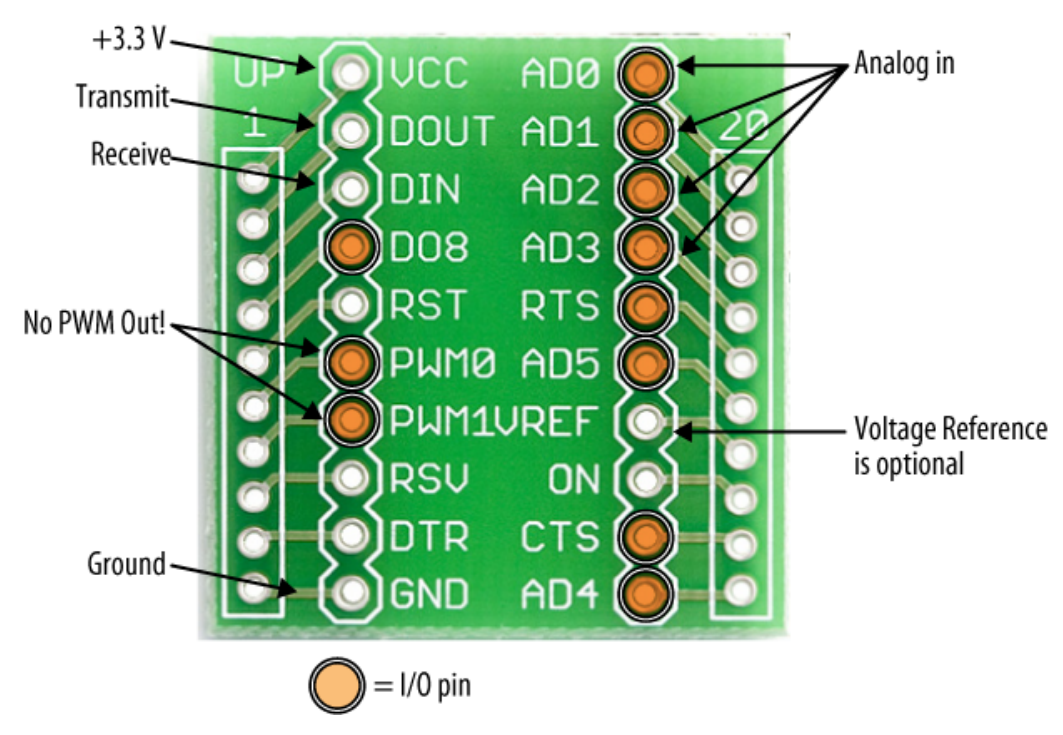

Figure 4-1. I/O pins as seen from below on a breakout board

\section{AT Configuration I/0 Commands}

To configure the XBee radio for direct input, output, or both, you'll use a set of AT commands that select each pin's mode and the sample rate for sending the data. There are several steps involved in getting this done, so read carefully through this section at least once before starting to configure your radio. 
Here's the basic I/O command set:

ATD0...ATD7

Configures pins 0 through 7 for $\mathrm{I} / \mathrm{O}$ mode (pins 8 and 9 are not supported in the current firmware version). The number after the $\mathrm{D}$ indicates which pin you'll be configuring. The command is followed by a numeric code that indicates whether the pin is tasked with digital input, output, analog input (pins 0 to 3 only), some other function, or nothing at all. For example, to configure I/O pin 2 as a digital input (code 3), the command would be ATD23. See the I/O settings codes in Table 4-3 for a complete list of the codes.

ATP0...ATP1

Configures pins 10 and 11 for I/O mode (there's a $\mathrm{P} 3$ for pin 12 , but it is not supported in the current firmware). Again, the number after the $\mathrm{P}$ indicates which pin you'll be configuring, and is followed by a numeric code to indicate what purpose the pin will serve- digital in, digital out, or nothing. For example, to configure I/O pin 11 as a high digital output (code 5) the command would be ATP15. Pins 10 and 11 do not support any analog functions.

ATIR

This sets the I/O sample rate- - how frequently to report the current pin state and transmit it to the destination address. The rate is set in milliseconds, using hexadecimal notation. So, for example, let's say you want to take a sample 10 times every second. There are 1,000 milliseconds in a second so we divide this by 10 to get 100 milliseconds. Now we just need to find the hexadecimal equivalent of 100 . This happens to be $0 \times 64$, so the command would be ATIR64. To disable periodic sampling, simply set ATIR to zero.

ATWR

Don't forget to write the configuration to firmware using ATWR so that the next time your radio powers up it retains the correct settings!

The settings codes for each I/O pin (Table 4-3) designate whether it will do nothing, perform a built-in function, take analog input, take digital input, or give digital output.

Table 4-3. I/O settings codes for use with ATDx and ATPx (where $x$ is the pin \#)

\begin{tabular}{ll} 
ATDx or ATP followed by: & Purpose: \\
0 & Disables I/0 on that pin \\
1 & Built-in function, if available on that pin \\
2 & Analog input, only on pins D0 through D3 \\
3 & Digital input \\
4 & Digital output, low (0 volts) \\
5 & Digital output, high (3.3 volts) \\
\hline
\end{tabular}


Analog input pins D0 through D3 read a range from 0 volts to 1.2 volts maximum. Voltages above 1.2 are ignored and result in the same maximum reading. Because most circuits using the XBee Series 2 run at 3.3 volts, if your input is a variable resistor, like a photoresistor, flex sensor, or force sensor, you'll need to create a voltage divider circuit that cuts maximum voltage by two-thirds to keep it within the range of the analog-digital converter (ADC).

The formula for voltage divider output between the two resistors is:

$$
V_{\text {out }}=\frac{R_{2}}{R_{1}+R_{2}} \times V_{\text {in }}
$$

A fast implementation for transforming a $3.3 \mathrm{~V}$ input into one that stays below $1.2 \mathrm{~V}$ max is to have the fixed resistor $R_{1}$ be twice the maximum resistance of the variable resistor $R_{2}$. So in the circuit shown in Figure 4-2, if $R_{2}$ is a flex sensor with a maximum resistance of $10 \mathrm{~K} \mathrm{ohm}$, then $R_{1}$ would be a $20 \mathrm{~K}$ ohm fixed resistor. Or, for a photocell rated at 300 ohms, a good choice of fixed resistor would be 600 ohms.

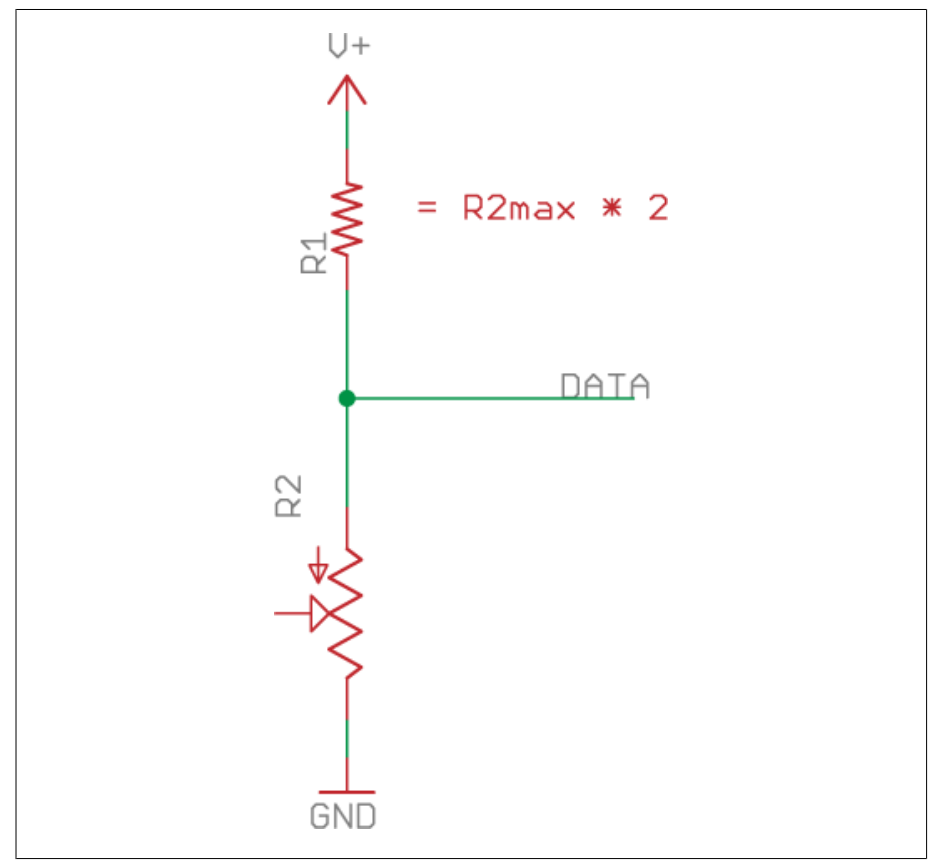

Figure 4-2. Voltage divider circuit to map $3.3 \mathrm{~V}$ range to $1.2 \mathrm{~V}$ range 


\section{Advanced I/0 Commands}

Several other AT commands may come in handy for projects with special I/O needs. These are worth knowing about even if you don't need to use them right away. The XBee manual has detailed specifications for each of these commands:

AT\%V

Returns the current supply voltage for the module. This is useful for keeping track of battery status.

ATPR

Configures the internal $30 \mathrm{~K}$ ohm pull-up resistors, using a binary value to set for each pin you've configured as an input. This is useful if your input component is a momentary digital switch that connects to ground, so you don't need to add the required external pull-up resistor. By default, the internal pull-ups are all enabled. ATIC

Configures the digital $\mathrm{I} / \mathrm{O}$ pins to monitor for changes in state, using a binary value to set for each pin. The pin(s) would also need to be configured as digital inputs. When change-detection is enabled, a sample is sent immediately any time a pin shifts from low to high or vice versa. This is useful if you are monitoring a switch, and care about triggering a transmission only when a button is pressed or released.

\section{Romantic Lighting Sensor}

Wireless networking is not nearly as tricky as navigating romance. Luckily, the former can help you with the latter, as this next project will demonstrate. Imagine for a moment that you are a brilliant engineer, hacker, interaction designer, or scientist - and perhaps you actually are. Let's say you've mastered math, manual skills, and usability, but nothing in your schooling has prepared you for the daunting task of setting a scene where love can blossom. What to do? The dining table is laid out perfectly; your date is moments away from ringing your wireless doorbell; now how to set the lights? We all know that glaringly bright lighting tends to hamper courtship. This is a date after all, not an interrogation. On the other hand, dimming the lights too far can seem creepy. What you need is a sensing system that lets you know you've lit things in the sweet spot for romance.

To get you started, here's a project that creates a remote wireless lighting sensor with a base station that lights a green LED when the mood is just right. It also happens to be a fine example for developing a variety of your own wireless I/O projects.

\section{Basic Romantic Lighting Sensor}

We'll start by creating a simple wireless lighting sensor that gives feedback at the base station. 


\section{Parts}

- Two solderless breadboards (MS MKKN2, AF 64, DK 438-1045-ND, SFE PRT-09567)

- Hookup wire or jumper wire kit (MS MKSEEED3, AF 153, DK 923351-ND, SFE PRT-00124)

- One Arduino board (MS MKSP4, SFE DEV-09950, AF 50)

- USB A-to-B cable for Arduino (AF 62, DK 88732-9002, SFE CAB-00512)

- Two AA battery holders with connection wires (RS 270-408, SFE PRT-09547)

- Two AA batteries (alkaline or NIMH rechargeable, fully charged) (RS 23-873, SFE PRT-09100 or PRT-00335)

- Two 5 mm LEDs (DK 160-1707-ND, RS 276-041, SFE COM-09590)

- One $20 \mathrm{~K}$ ohm resistor (or twice the max value of your photoresistor) (DK P20KBACT-ND, SFE COM-08374 * 2 in series)

- One 10K ohm photoresistor (also called an LDR or light-dependent resistor) (AF 161, DK PDV-P8001-ND, SFE SEN-09088)

- One XBee radio (Series 2/ZB firmware) configured as a ZigBee Coordinator API mode (Digi: XB24-Z7WIT-004, DK 602-1098-ND)

- One XBee radio (Series 2/ZB firmware) configured as a ZigBee Router AT mode (Digi: XB24-Z7WIT-004, DK 602-1098-ND)

- Two XBee breakout boards with male headers and $2 \mathrm{~mm}$ female headers installed (AF 126 [add SFE PRT-00116], SFE BOB-08276, PRT-08272, and PRT-00116)

- XBee USB Serial adapter (XBee Explorer, Digi Evaluation board, or similar) (AF 247, SFE WRL-08687)

- USB cable for XBee adapter (AF 260, SFE CAB-00598)

- Wire strippers (AF 147, DK PAL70057-ND, SFE TOL-08696)

\section{Prepare your coordinator radio}

Write down your coordinator and router radios' addresses (printed on the back) so that you can refer to them during configuration:

\begin{tabular}{ll} 
Coordinator address & Router address \\
$0013 \mathrm{~A} 200$ & $0013 \mathrm{~A} 200$ \\
\hline
\end{tabular}

1. Follow the instructions under "Reading Current Firmware and Configuration" on page 35 in Chapter 2 to configure one of your radios as a ZigBee Coordinator API. 
Your coordinator radio must use the API firmware for this project to work, because I/O data is delivered only in API mode. Be sure to select the API version for your coordinator!

When you change from AT to API mode using X-CTU, you may get an error message that the radio is no longer communicating. Go back to the PC Settings tab and check the Enable API box (Figure 4-3) to enable communications with your radio.

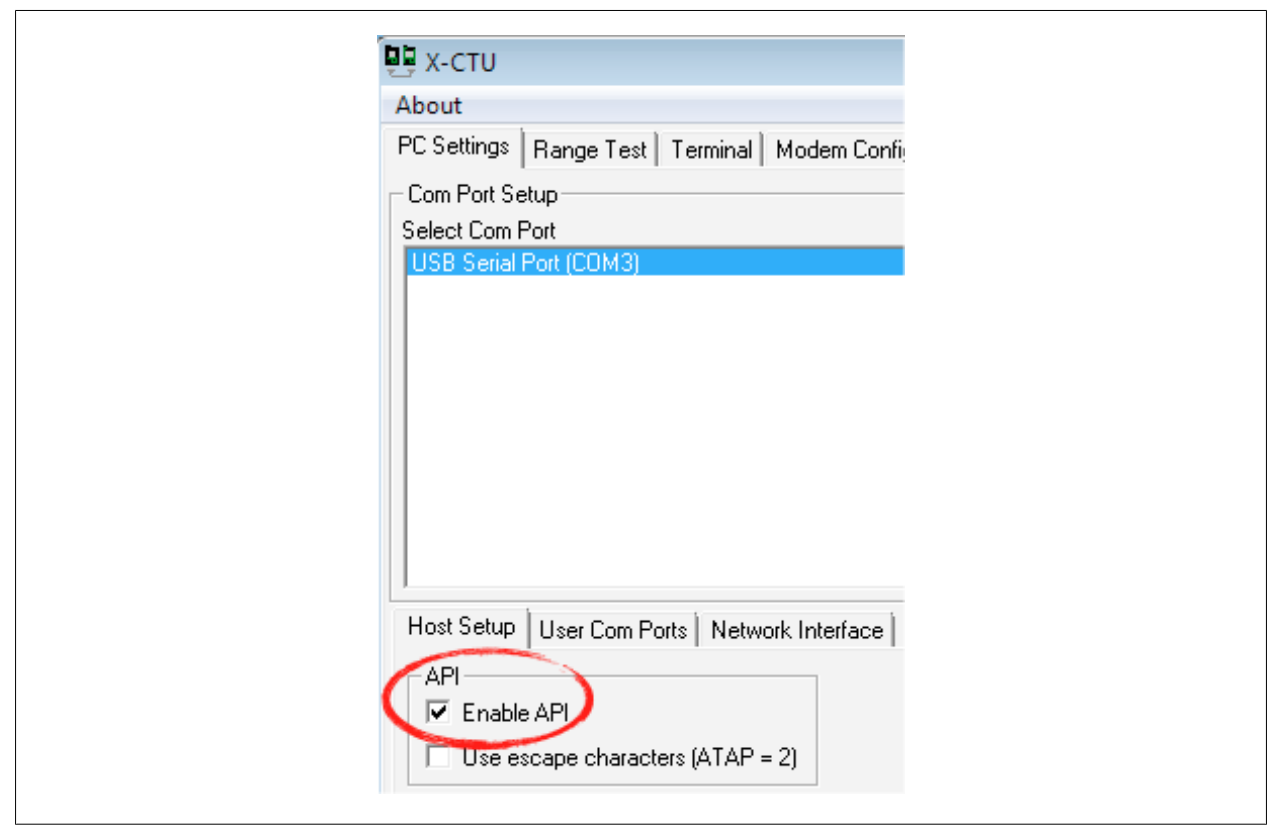

Figure 4-3. Enable API checkbox on PC Settings tab in X-CTU

2. Once a radio has been set to API mode, it can only be configured in X-CTU. You will not be able to make adjustments to this radio's configuration in CoolTerm or any other terminal program. Use X-CTU to configure the coordinator with a PAN ID (between $0 \mathrm{x} 0$ and $0 x F F F F F F F F F F F F F F F$ ) you've selected. Write down this PAN ID so you can program your router radio with the same one. Every radio in your network must use the same PAN ID so they can communicate with each other:

\section{Pan ID:}

3. Use X-CTU (Figure 4-4) to set ATDH to the high part of your router radio's address (always 0013A200 for XBees) and ATDL to the remainder of your router radio's address (the unique part of the number you noted above).

4. Click on the Write button to save your settings to the radio. 


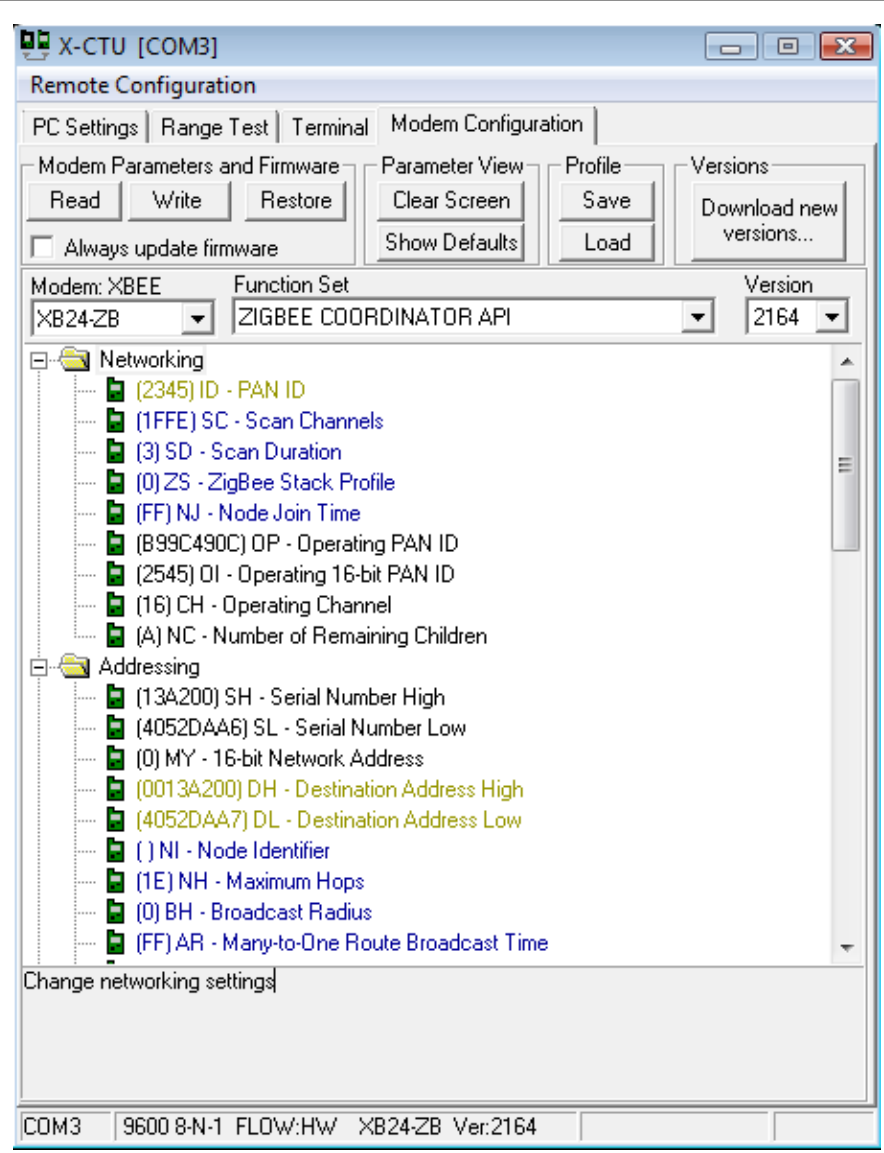

Figure 4-4. Setting ID, DH, and DL in X-CTU

\section{Prepare your router radio}

1. Follow the instructions under "Reading Current Firmware and Configuration" on page 35 in Chapter 2 to configure one of your radios as a ZigBee Router AT.

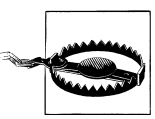

Your router radio will use the AT firmware, so you can easily configure it using a serial terminal. Be sure you select the AT version for your router!

When you change from an API radio to an AT radio, you may get an error message that the radio is no longer communicating. If so, go back to the PC Settings tab and uncheck the Enable API Mode box (Figure 4-5). 


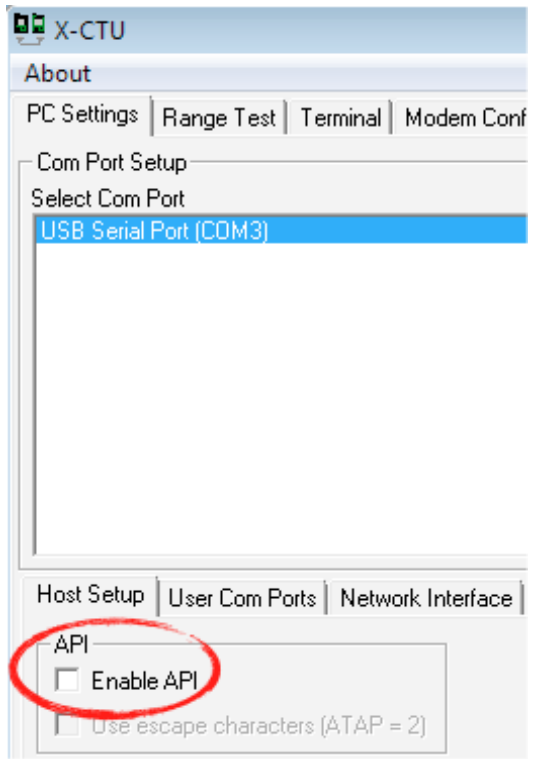

Figure 4-5. Disabled API checkbox on PC Settings tab in X-CTU

2. Label the coordinator radio with a "C" so you know which one it is later on. Label the router radio with an " $\mathrm{R}$."

\section{Prepare the Sensor Board}

We'll use the CoolTerm terminal program (Mac, Windows) and an XBee Explorer USB adapter again to set up your radios. (If you're on Linux, see the sidebar "A Serial Terminal Program for Linux" on page 40 in Chapter 2.)

\section{Configure your router XBee}

1. Select the router XBee you labeled with an "R" and place it into the XBee Explorer.

2. Plug the XBee Explorer into your computer.

3. Run the CoolTerm program and press the Options button to configure it.

4. Select the appropriate serial port, and check the Local Echo box so you can see your commands as you type them.

5. Click on the Connect button to connect to the serial port.

6. Type +++ to go into command mode. You should receive an OK reply from the radio.

7. Select the same PAN ID you entered for your first radio. PAN ID: 
8. Type ATID followed by the PAN ID you selected and press Enter on the keyboard. You should receive OK again as a reply.

9. Enter ATDH followed by the high part of your radio's destination address-always the same for the XBees. Type ATDH 0013A200 and press Enter on the keyboard. You should receive an OK response.

10. Enter ATDL followed by the low part of your radio's destination address-the eightcharacter hexadecimal address of the coordinator radio that follows 0013A200. Type ATDL followed by that low part of the destination address, then press Enter. You should receive an OK response. Remember that your destination will be the coordinator radio.

11. Enter ATJV1 to ensure that your router attempts to rejoin the coordinator on startup.

12. Enter ATDO2 to put pin 0 in analog mode.

13. Enter ATIR64 to set the sample rate to 100 milliseconds (hex 64).

14. Save your new settings as the radio's default by typing ATWR and pressing Enter.

It's not a bad idea to check your configurations after you enter them.

For example, to check that you entered the destination address cor-

rectly, from command mode type ATDL and press Return to see the current setting.

\section{Connect power from battery to breadboard}

Your remote sensor will use a breadboard connected to two AA batteries:

1. Hook up the positive (usually red) battery lead to one of the power rails on the breadboard.

2. Hook up the ground (usually black) battery lead to a ground rail on the breadboard.

3. Hook up power and ground across the breadboard so that the rails on both sides are live.

\section{Router XBee connection to battery}

1. With the router XBee mounted on its breakout board, position the breakout board in the center of your other breadboard so that the two rows of male header pins are inserted on opposite sides of the center trough.

2. Use red hookup wire to connect pin 1 (VCC) of the XBee to 3-volt battery power.

3. Use black hookup wire to connect pin 10 (GND) of the XBee to ground.

\section{Photoresistor input}

The battery-powered board with the router radio will be your remote sensor. On that board: 
1. Attach a photoresistor between ground and XBee digital input 0 (physical pin 20).

2. Make sure you use the $20 \mathrm{~K}$ ohm (or other value that's double your photoresistor's max value) pull-up resistor from digital input 0 to power. This ensures the sensor has a proper voltage divider circuit, which is required to get correct readings.

Figure 4-6 shows the layout of the board, and Figure 4-7 shows the schematic.

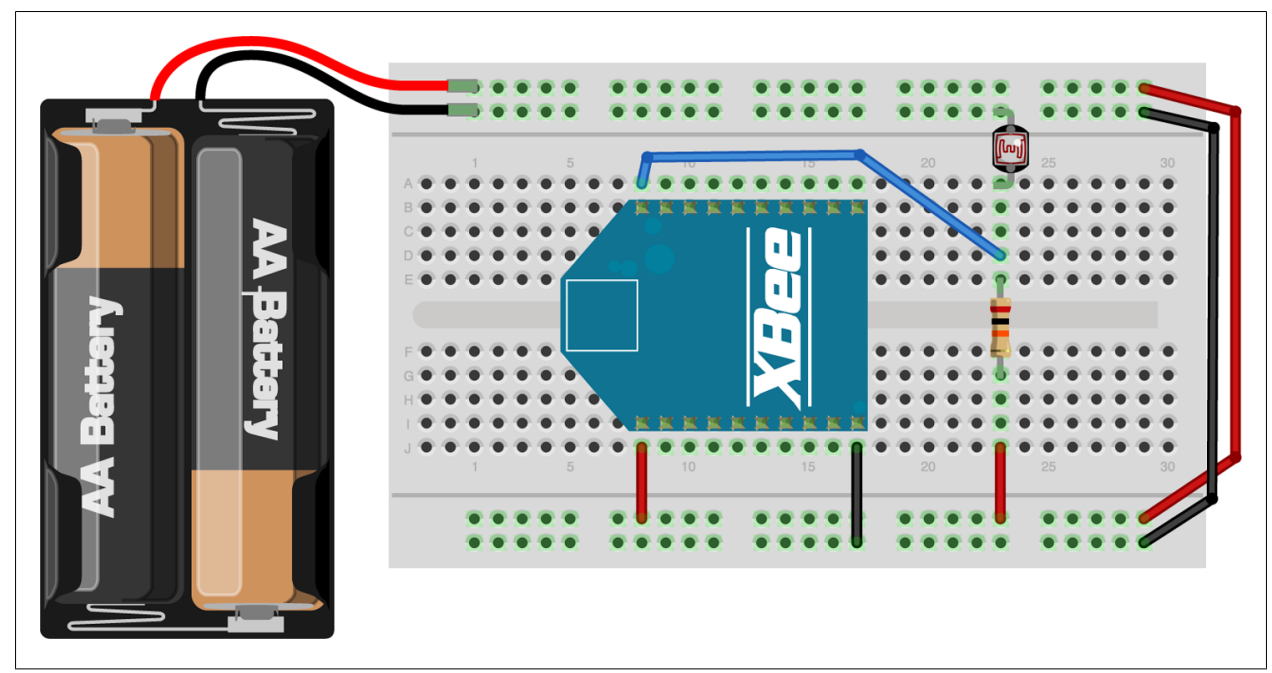

Figure 4-6. Romantic lighting sensor BASIC SENSOR breadboard layout

\section{Prepare the Base Station}

Your base station radio will use a breadboard connected to an Arduino board.

\section{Connect power from Arduino to breadboard}

1. Hook up a red wire from the $3.3 \mathrm{~V}$ output of the Arduino to one of the power rails on the breadboard.

2. Hook up a black wire from either ground (GND) connection on the Arduino to a ground rail on the breadboard.

3. Hook up power and ground across the breadboard so that the rails on both sides are live.

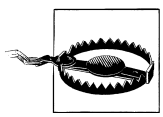

Make sure you are using $3.3 \mathrm{~V}$ power. 


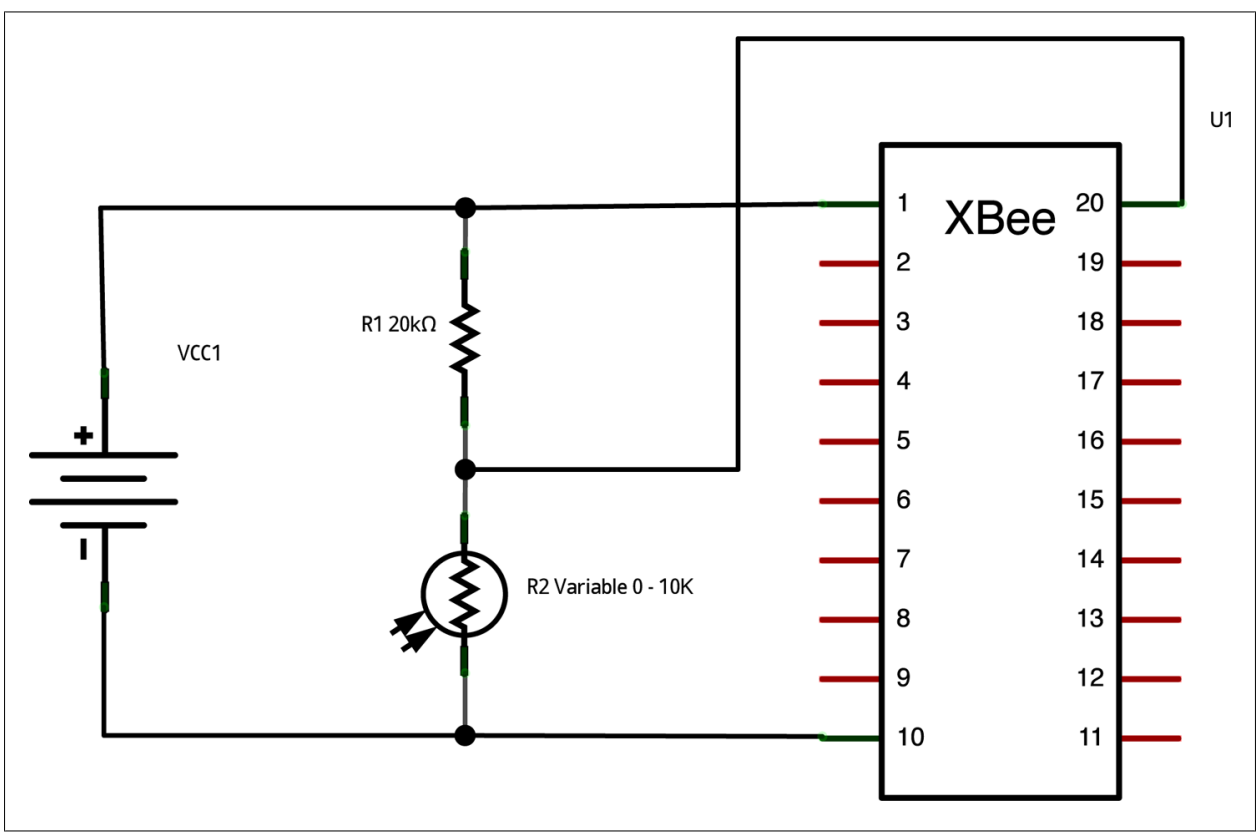

Figure 4-7. Romantic lighting sensor BASIC SENSOR schematic

\section{Coordinator XBee connection to Arduino}

1. With the coordinator XBee mounted on its breakout board, position the breakout board in the center of one of your breadboards so that the two rows of male header pins are inserted on opposite sides of the center trough.

2. Use red hookup wire to connect pin 1 (VCC) of the XBee to 3.3-volt power.

3. Use black hookup wire to connect pin 10 (GND) of the XBee to ground.

4. Use yellow (or another color) hookup wire to connect pin 2 (TX/DOUT) of the $\mathrm{XBee}$ to digital pin 0 (RX) on your Arduino.

5. Finally, use blue (or another color) hookup wire to connect pin 3 (RX/DIN) of your XBee to digital pin 1 (TX) on your Arduino.

\section{Light output}

1. Attach the positive (longer) lead of an LED to Arduino digital pin 11.

2. Attach the shorter ground lead from your LED to ground.

3. If you prefer to use another output, like an audio buzzer or pager motor, you can hook it up in the same way. Perhaps your romance chops are best demonstrated by a puff of scented air freshener. Then again, maybe a monkey playing the drums is more your style. The key to romance is being yourself, so don't hesitate to get creative! 
Figure 4-8 shows the layout of the board, and Figure 4-9 shows the schematic.

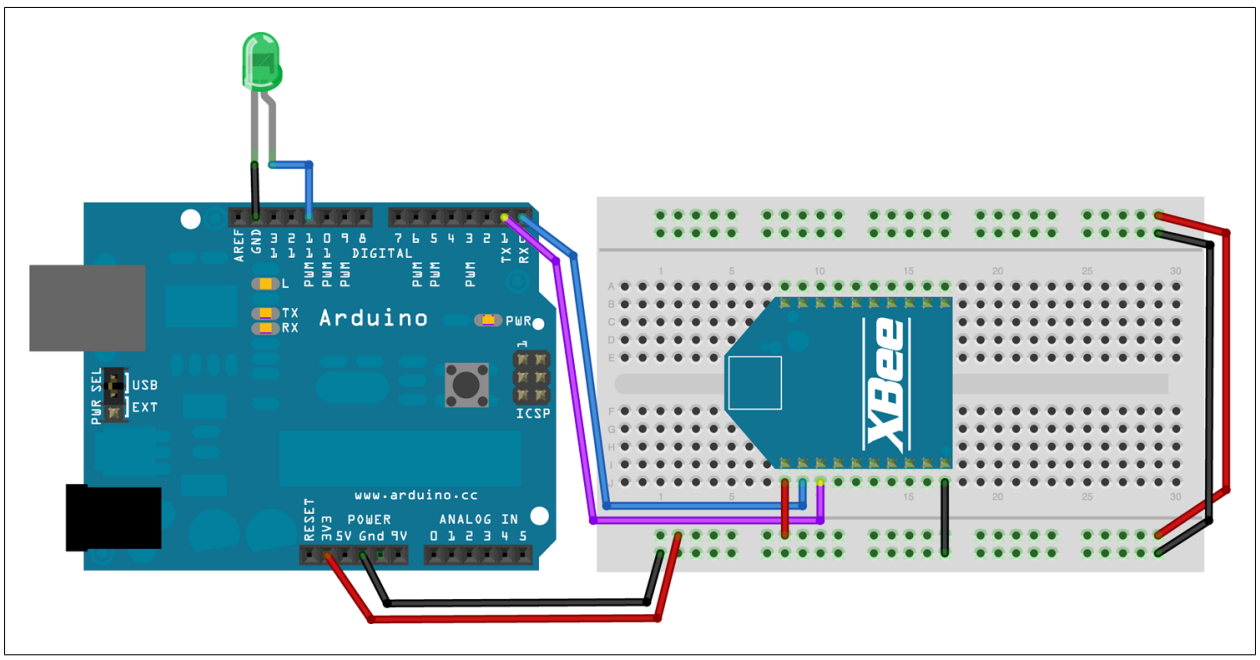

Figure 4-8. Romantic lighting sensor BASE breadboard configuration

\section{Program the romantic lighting sensor base station}

The romantic lighting sensor base station uses the following Arduino program. Upload it to your Arduino board and you're ready to test the mood:

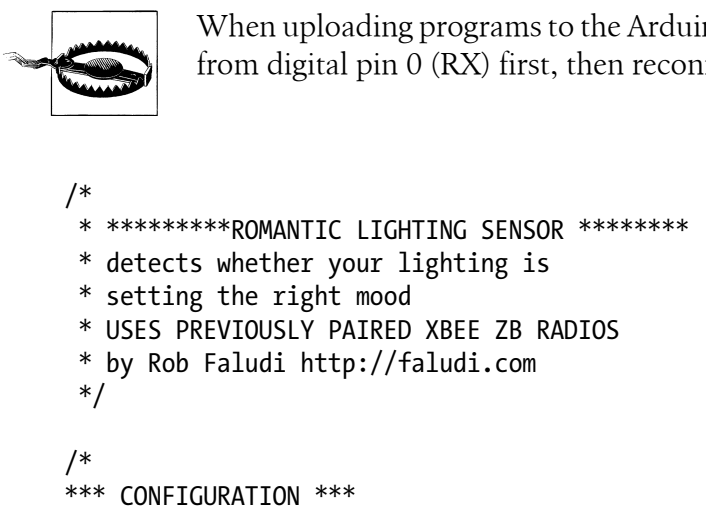

SENDER: (REMOTE SENSOR RADIO)

ATID3456 (PAN ID)

ATDH $\rightarrow$ set to SH of partner radio

ATDL $\rightarrow>$ set to SL of partner radio

ATJV1 -> rejoin with coordinator on startup

ATD02 pin 0 in analog in mode

ATIR64 sample rate 100 millisecs (hex 64) 
* THE LOCAL RADIO _MUST_BE IN API MODE *

RECEIVER: (LOCAL RADIO)

ATID3456 (PAN ID)

ATDH $->$ set to SH of partner radio

ATDL $\rightarrow>$ set to SL of partner radio

$* /$

\#define VERSION "1.02"

int LED = 11;

int debugLED = 13;

int analogValue $=0$;

void $\operatorname{setup}()\{$

pinMode(LED, OUTPUT);

pinMode(debugLED, OUTPUT);

\}

Serial.begin(9600);

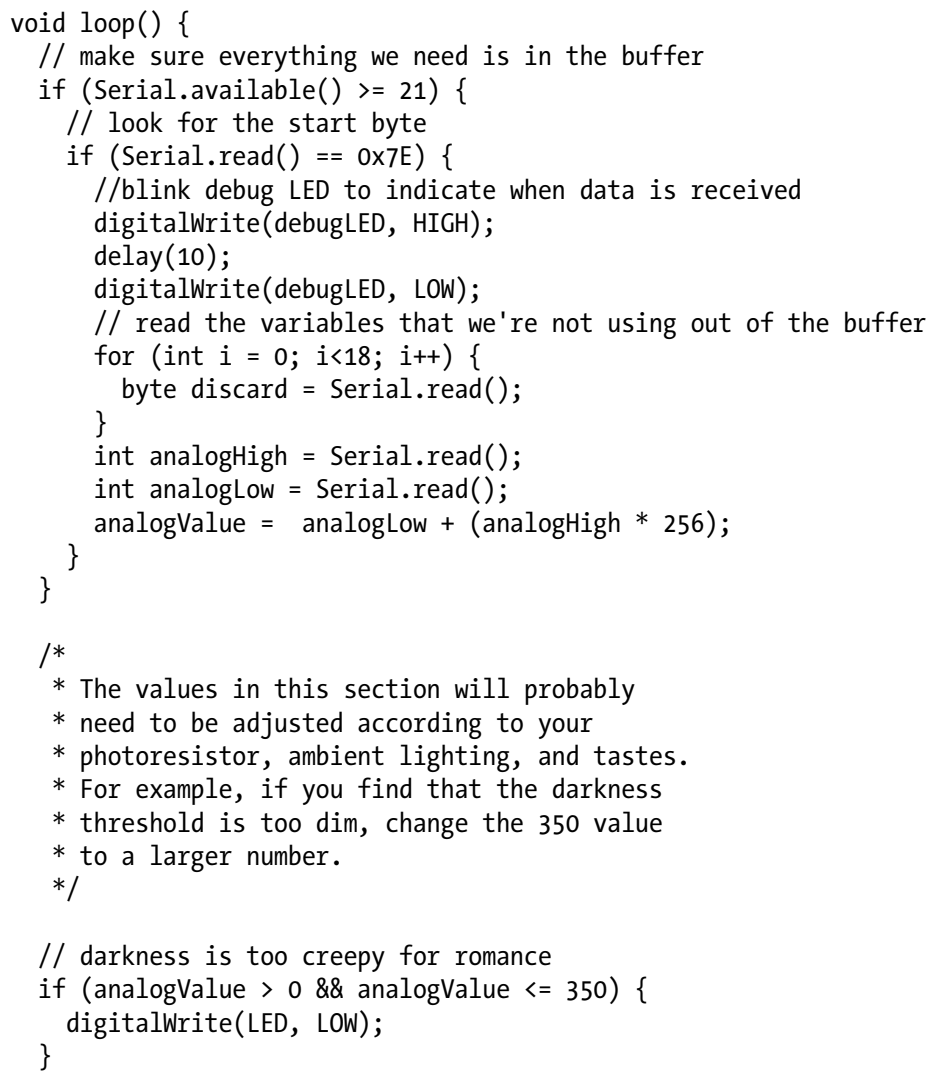



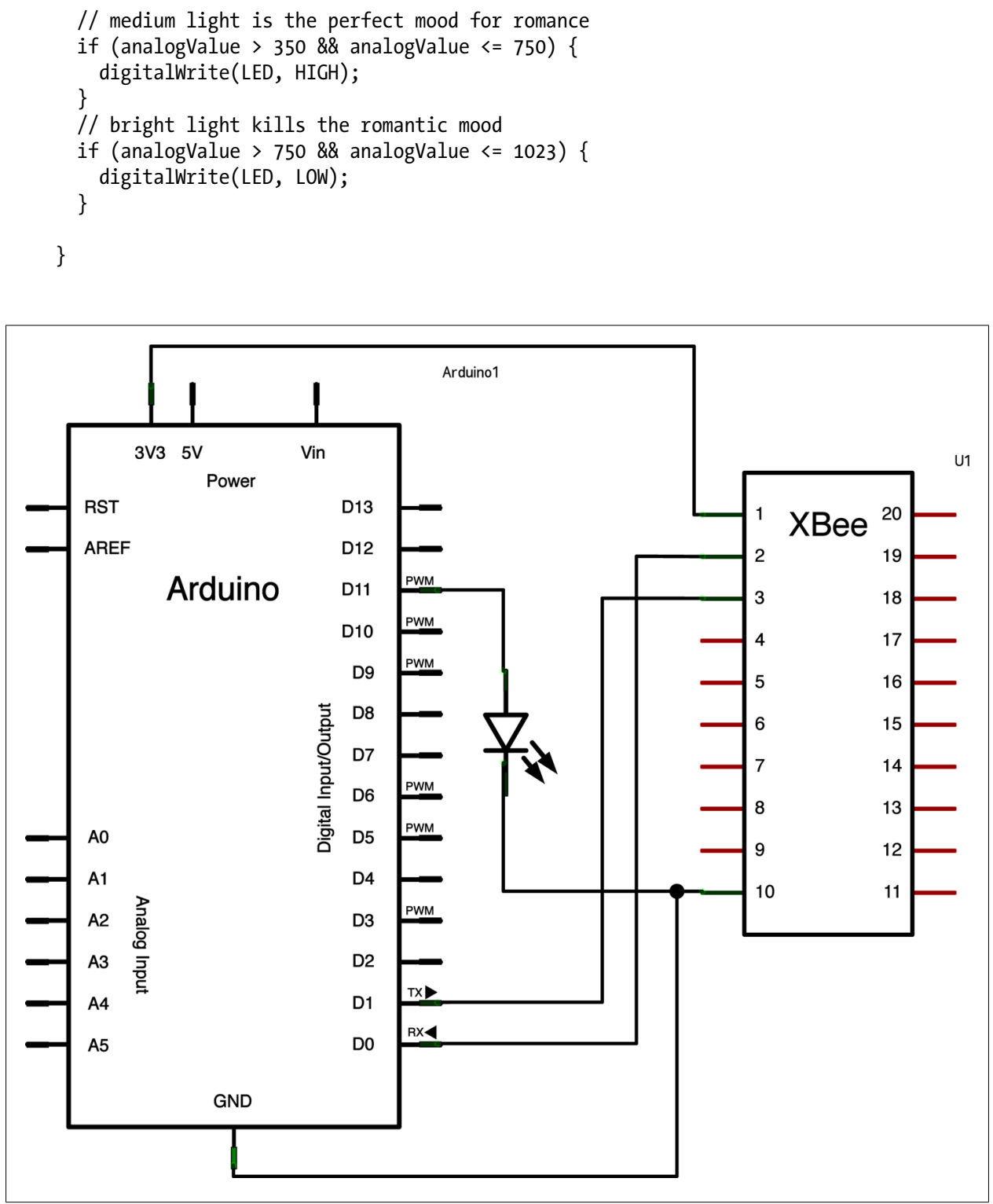

Figure 4-9. Romantic lighting sensor BASE schematic

\section{Troubleshooting}

If things don't work at first, here are some steps to take to try to figure out what's wrong:

1. Check all your electrical connections to make sure there are no loose wires and that all the components are connected properly. 
2. Check the coordinator configuration in X-CTU again, including that the correct modem type (XB24-ZB) and function set (ZigBee Coordinator API) have been selected. Also check that the PAN ID, destination high, and destination low are configured as you expect. Remember the destination is the other radio.

3. Check the router configuration in X-CTU to confirm that the correct modem type (XB24-ZB) and function set (ZigBee Coordinator AT) have been selected. Also check that the PAN ID, destination high, and destination low are configured as you expect, and that ATJV, ATD0, and ATIR have been configured as described above.

4. Make sure that the Arduino is programmed with the correct code for this project (the basic version above and the feedback version below have different code and must be matched to the correct board setup and radio settings).

5. The debug LED on the Arduino board (pin 13) will flash if you are receiving data. If this light is flashing but your output light doesn't change, try adjusting the sensor threshold values in the Arduino code.

6. An LED placed from the ASSOC pin of the XBee (physical pin 15) to ground should show a flashing light.

7. An LED placed from the RSSI pin of the XBee (physical pin 6) to ground should show a steady light when the radio is receiving information. If messages stop coming in, this light will time out and go dark after 10 seconds.

8. Use a multimeter to see if the voltage at the D0 pin of the XBee (physical pin 20) varies with changes in the lighting. It should be somewhere in the range between 0 and 1.2 volts and change as you shadow the light sensor with your hand.

9. We are not always able to see our own mistakes. Have a friend check everything for you. Sometimes only a second pair of eyes will catch the one (or more) issues standing in the way of success.

10. When all else fails: Try taking a break and coming back to the project after a good night's rest. Many of midnight's intractable puzzles are morning's simple fix.

\section{Romantic Lighting Sensor with Feedback}

The basic sensor works pretty well as long as you are at the base station. However, it's a pain to run back and forth between the sensor and the base to see if the mood is right. Let's improve on things by putting the feedback right where the sensor is. This is also a nice example to start with for any project where you want both sensing and actuation on a remote device.

\section{Add light output to the sensor}

On the sensor board:

1. Attach the positive (longer) lead of an LED to XBee digital input 1 (physical pin 19). 
2. Attach the shorter ground lead from your LED to ground.

Figure 4-10 shows the layout of the board, and Figure 4-11 shows the schematic.

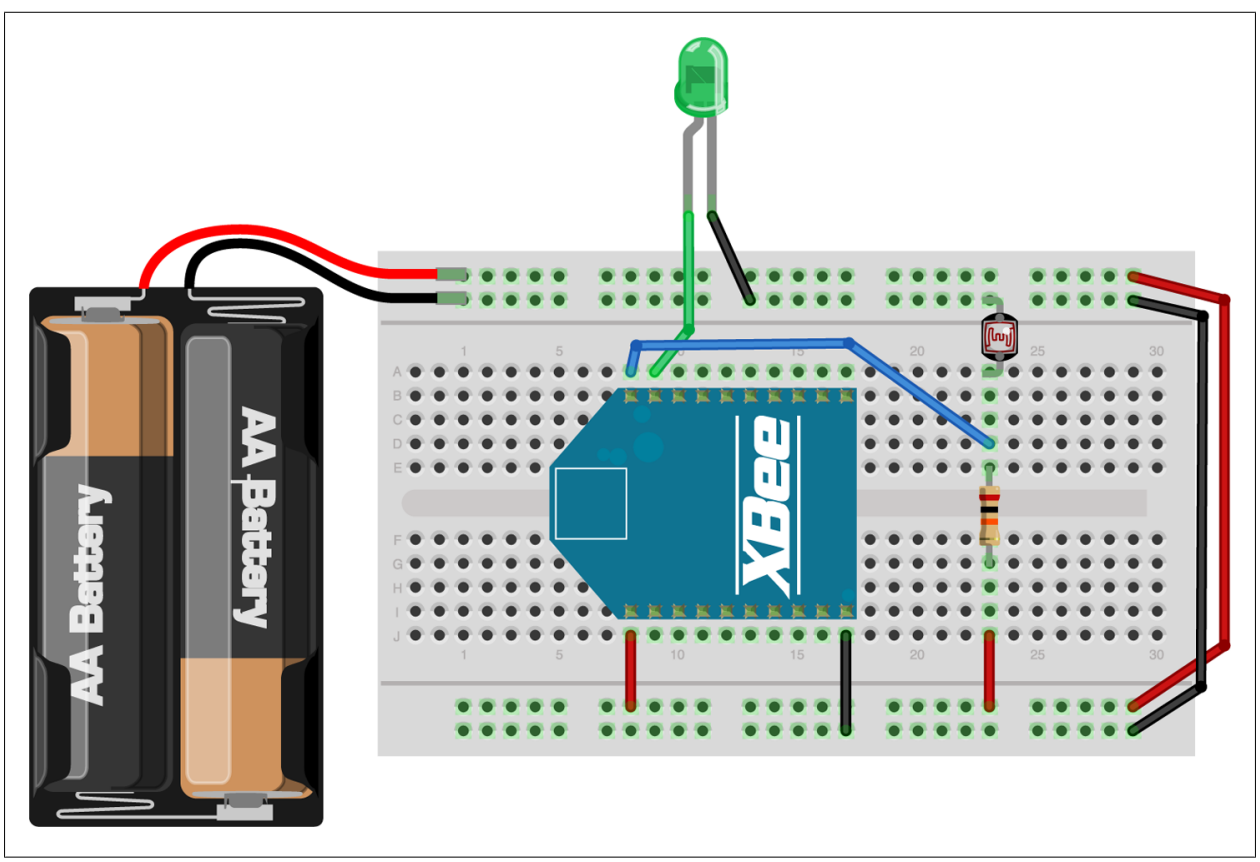

Figure 4-10. Romantic lighting sensor FEEDBACK SENSOR breadboard layout

\section{Configure your router XBee}

We'll use the CoolTerm terminal program and an XBee Explorer USB adapter again to set up the radios. The setup is the same as in the basic version above, with the addition of a digital output pin to control the sensor LED:

1. Select the router XBee you labeled with an "R" and place it into the XBee Explorer.

2. Plug the XBee Explorer into your computer.

3. Run the CoolTerm program and press the Options button to configure it.

4. Select the appropriate serial port, and check the Local Echo box so you can see your commands as you type them.

5. Click on the Connect button to connect to the serial port.

6. Type +++ to go into command mode. You should receive an OK reply from the radio.

7. Enter ATD14 to put pin 1 in low digital output mode.

8. Save your new settings as the radio's default by typing ATWR and pressing Enter. 


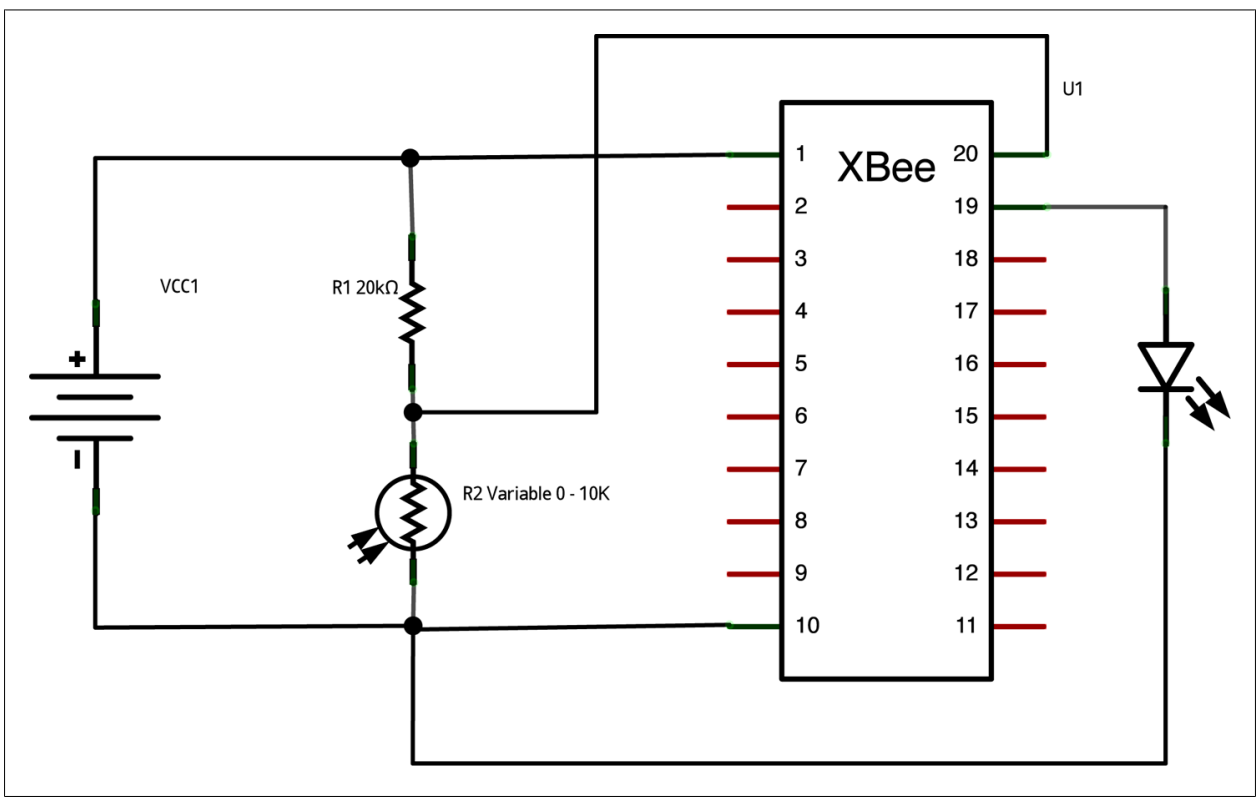

Figure 4-11. Romantic lighting sensor FEEDBACK SENSOR schematic

\section{Program the romantic lighting sensor with feedback base station}

The romantic lighting sensor with feedback base station uses the following Arduino program. Upload it to your Arduino board and you're ready to test the mood right from the sensor:

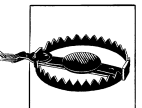

When uploading programs to the Arduino board, disconnect the wiring from digital pin 0 (RX) first, then reconnect the wiring after loading.

$/ *$

* **********ROMANTIC LIGHTING SENSOR WITH FEEDBACK*********

$*$ detects whether your lighting is

* setting the right mood and shows

* you the results on the sensor module

* USES PREVIOUSLY PAIRED XBEE ZB RADIOS

* by Rob Faludi http://faludi.com

*/

/*

*** CONFIGURATION $* * *$

SENDER: (REMOTE SENSOR RADIO)

ATID3456 (PAN ID)

ATDH $\rightarrow>$ set to SH of partner radio

ATDL $\rightarrow$ set to SL of partner radio

ATJV1 $\rightarrow$ rejoin with coordinator on startup

ATD02 pin 0 in analog in mode with a photo resistor 
(don't forget the voltage divider circuit--resistor to ground is good)

ATD14 pin 1 in digital output (default low) mode with an

LED from that pin to ground

ATIR64 sample rate 100 millisecs (hex 64)

* THE LOCAL RADIO_MUST_BE IN API MODE *

RECEIVER: (LOCAL RADIO)

ATID3456 (PAN ID)

ATDH $\rightarrow$ set to SH of partner radio

ATDL $\rightarrow$ set to SL of partner radio

$* /$

\#define VERSION "1.02"

int $L E D=11$;

int debugLED = 13;

int analogValue $=0$;

int remoteIndicator $=$ false; $\quad / /$ keeps track of the desired remote // on/off state

int lastRemoteIndicator $=$ false; $/ /$ record of prior remote state

unsigned long lastSent $=0 ; \quad / /$ records last time the remote was

// reset to keep it in sync

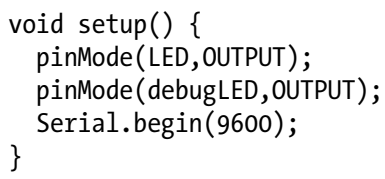

void $\operatorname{loop}()\{$

// make sure everything we need is in the buffer

if (Serial.available ()$>=23$ ) \{

// look for the start byte

if $($ Serial. $\operatorname{read}()==0 \times 7 \mathrm{E})\{$

//blink debug LED to indicate when data is received

digitalWrite(debugLED, HICH);

delay (10);

digitalWrite(debugLED, LOW);

// read the variables that we're not using out of the buffer

// (includes two more for the digital pin report)

for (int $i=0$; $i<20 ; i++)\{$ \}

byte discard $=$ Serial $\cdot \operatorname{read}()$;

int analogHigh $=$ Serial $\operatorname{read}()$;

int analogLow $=$ Serial $\operatorname{read}()$;

\}

analogValue $=$ analogLow $+($ analogHigh $* 256)$;

\} 


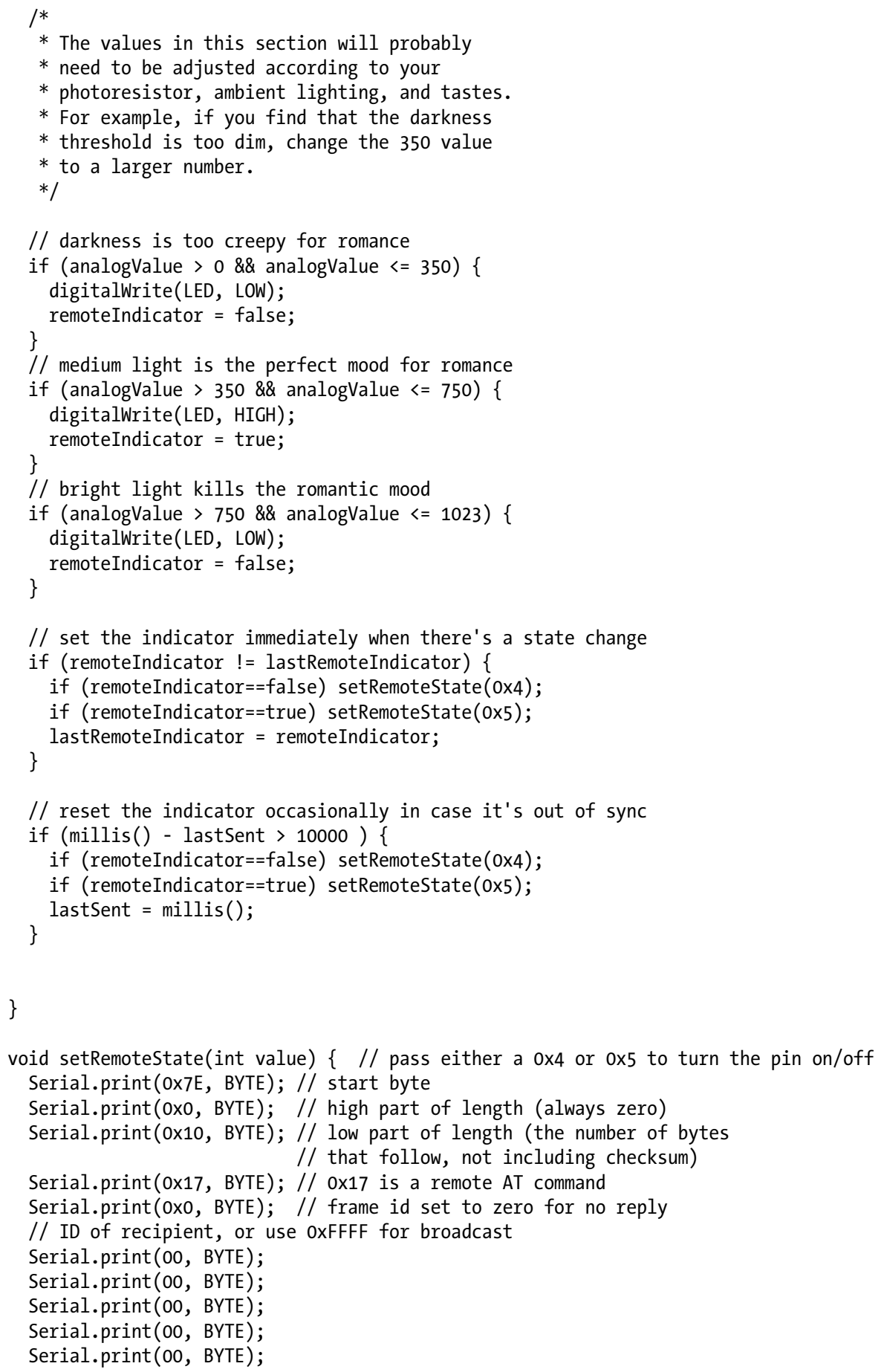




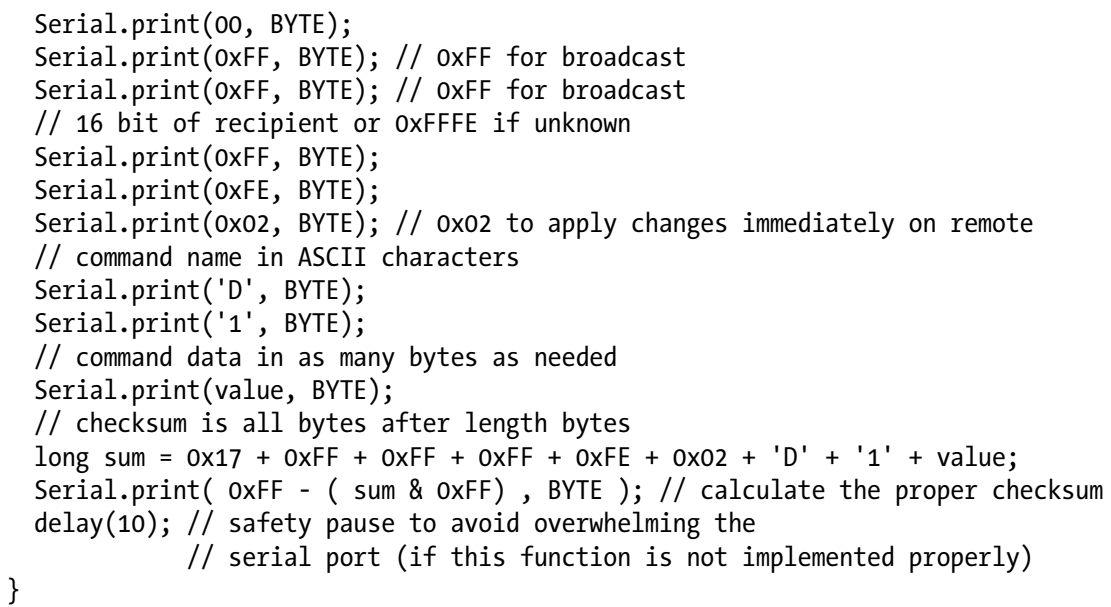

\section{API Ahead}

These last code examples contain something we haven't really looked at yet, API mode. The next chapter will explore the XBee Application Programming Interface, a highly structured way of communicating with your XBee radio. You've already used it, so let's find out how it works and why it is essential to certain projects. 



\section{API and a Sensor Network}

Here the plot heats up. You now have everything you need to conquer the XBee's application programming interface. This is something we need to do so we can use all the data our networks can provide. We will start with simple concepts and scaffold you up to a full understanding of the structured API communication frames. That will get you ready to create a fully scalable sensor network of your own, using the example at the end of the chapter.

\section{What's an API?}

An application programming interface (API) is simply a set of standard interfaces created to allow one software program to interact with another. APIs let one computer application request services from another application in a standard manner. For our purposes, the most important thing to note is that APIs are specifically engineered to enable computers to talk efficiently to other computers. They are not generally designed for direct human interaction.

So far, we've been using the XBee radios in transparent/command mode. For example, in the simple chat we set up in Chapter 2, we were able to type text at a keyboard to enter command mode, then issue AT commands by typing them right in. When we were done with configuration, we exited command mode and went right into transparent mode, where everything typed at the keyboard was transferred verbatim to the destination radio and read directly on the screen. This was a simple way to get started with wireless networking, and it's one of the great strengths of the XBee platform. It's very easy for humans to get started using direct interactions in the transparent/command modes. However, there is also a catch. When interactions are made easy for humans, they are not as robust, explicit, and efficient for computers. Computers care about things like algorithmic error correction, airtight mode identification, and efficient data transfer to get their job done quickly, predictably, and reliably. As the same time, they could care less about readability. Computers prefer to deal with numbers, and do best when the organization of these numbers provides an unambiguous and highly structured method for transfer. This is where API mode comes in to save the day. By 
providing an interface for programmatic communication with the XBee, API mode enables the radios to serve humans and computers equally well, each according to their needs.

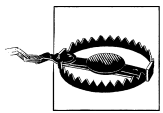

Transparent, command, and API interaction modes with an XBee are local to that particular radio. "Local" means that they apply to interactions with users, computers, or microcontrollers that take place via the XBee's serial connection (also known as its UART). Wireless communications between XBees are independent of the local interaction mode, as shown in Figure 5-1. So a radio in transparent mode can send to another in API mode just fine. It's only in local serial communications where transparent/command mode and API mode make a difference.

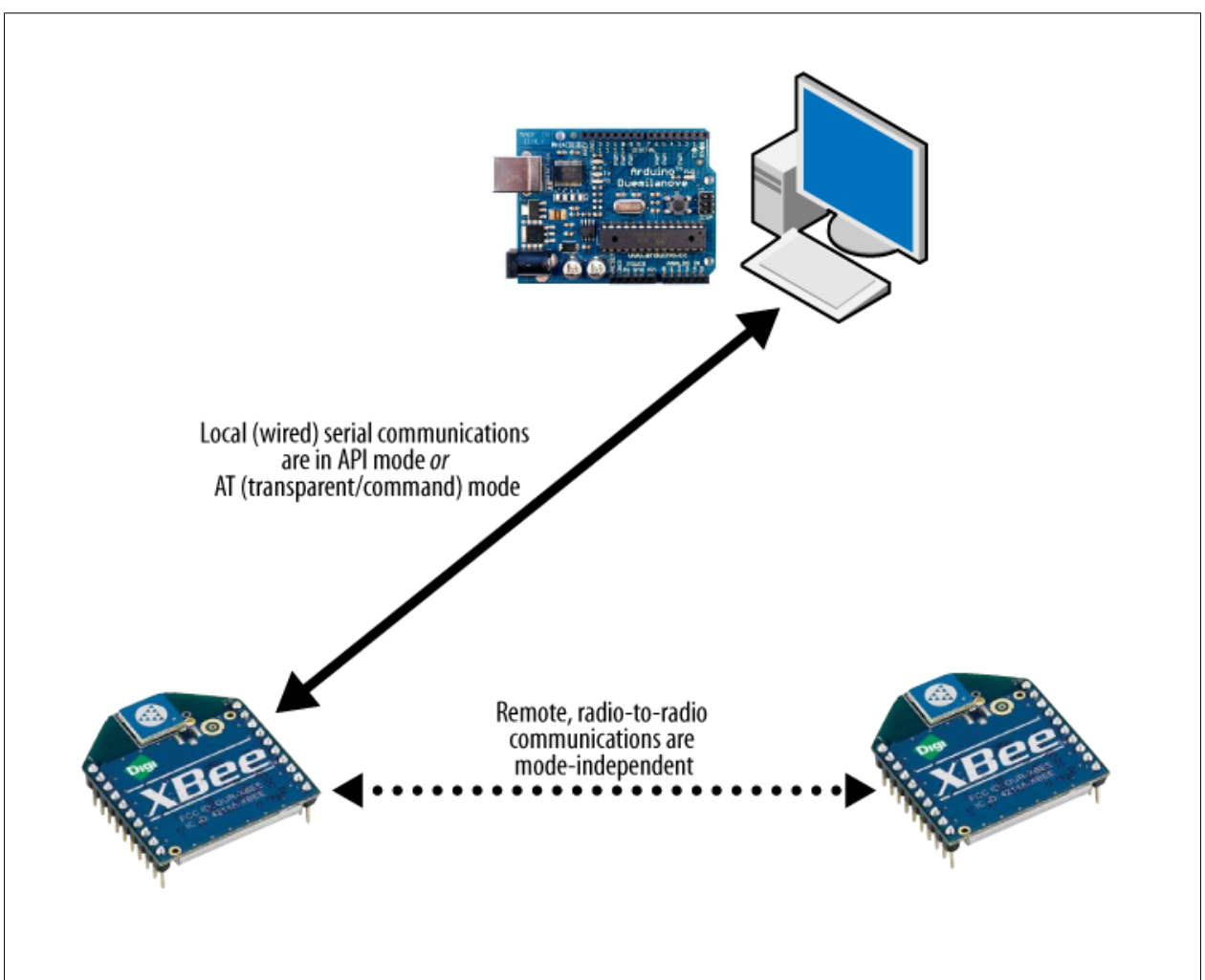

Figure 5-1. Local communications over serial wires happen in API mode or AT (transparent/ command) mode. Wireless communications are not affected by the choice of local modes.

Before we dive into the XBee API, let's review protocols in general and build a hypothetical one to examine just how they work. 


\section{Protocols}

Every transfer of information requires a protocol. Protocols are easy to understand; they're simply agreements on some rules for communication. There are established protocols for wireless computer communications, just as there are protocols for two human beings who want to have a casual conversation. Both people and computers face the same types of communication problems and we solve them in very similar ways.

\section{Humans}

Let's say that Jane and Michael have something to discuss. If Michael starts off in nonstop Chinese and Jane begins to shout over him in Icelandic, very little is going to be accomplished. It's best for them to agree on a common language first, and then follow some rules for speaking and listening so that an exchange of information can take place. If Jane begins speaking first, Michael will wait for her to finish, and then respond to her remarks. While Michael is talking, Jane will listen. If a fire truck goes by and Jane can't hear, she'll ask Michael to repeat whatever she missed. If what Michael says doesn't make sense to Jane, she'll either ignore his misspeak or request clarification depending upon the specifics of that particular statement. Much of the protocol of human conversation is so well ingrained that we usually don't think about the rules. We just follow them naturally. It's only when we discard protocol, by talking over each other, mumbling incoherently, or failing to voice our confusion that communications fall apart.

\section{Computers}

When computers talk to each other, they try to fulfill a given purpose in the simplest manner possible. In some contexts, that can be pretty simple indeed! For example, let's look at the least complicated communications protocol: basic streaming. One computer talks nonstop and the other listens. This is the perfect solution for conveying simple data from one point to another as long as some errors can be tolerated. More complicated protocols will define whether there's some kind of handshaking involved to set up the exchange, timing issues, what replies are sent in response to what messages, routing strategies, and so forth. But we don't need to worry about any of that for now because we're keeping it simple.

Let's say we want to send a number between 0 and 255 to represent in real time how bright it is outside. 
We use the range $0-255$ because 255 is the largest number that can be represented in a single byte of data. All common forms of serial communication break data up into bytes. A byte is a set of eight digital bits. A single bit can be either 0 or 1 , thereby representing two states. Add another bit and you now have four states: 00, 01, 10, and 11. A third bit allows for eight states (000, 001, 010, 011, 100, 101, 110, and 111 ), and so on and so forth until you get to eight bits that can represent 256 different states (including the zero state). That's a byte! In decimal, the numbers go from zero to 255 , and in hexadecimal notation they go from $0 \mathrm{x} 0$ to $0 \mathrm{xFF}$. For more on binary and bytes, see: $h t t p: / / e n$. wikipedia .org/wiki/Byte.

If we send our brightness data once every second, it would look something like this:

$136 \ldots 137 \ldots 137 \ldots 138 \ldots 138 \ldots 138 \ldots 139 \ldots 135 \ldots 128 \ldots 110 \ldots 125 \ldots 130 \ldots 136 \ldots$

Well, these numbers are just numbers so interpreting them requires a protocol to set an agreement about what they mean. Whatever is receiving them needs to already know that it's getting brightness data, and that the range from 0 to 255 represents from dark to dazzling. There is no way of telling if there's an error in the data or determining which sensor might be sending them to us. But if we're just making a single lamp that matches the current brightness from a single sensor on the roof, this may be all we need. If we get a wrong number every once in a while, the worst that may happen is the lamp might flicker for a moment. No big deal! So in this case we're all set. But what if our roof sensor is getting both light and temperature data? How can we tell the difference?

\section{Start bytes}

If we're sending two pieces of data, the first solution you might come up with is to always send them in order, first light and then temperature, like this:

$$
136 \ldots 14 \ldots 137 \ldots 14 \ldots 137 \ldots 14 \ldots 138 \ldots 14 \ldots 138 \ldots 15 \ldots 138 \ldots 15 \ldots 139 \ldots 15 \ldots
$$

For clarity in this example we've made the temperature numbers much smaller than the light numbers, but we certainly couldn't count on that always being the case in real data. If we plugged in our lamp at some arbitrary moment, we might see the following instead:

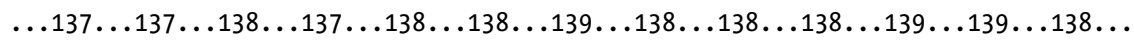

Which number represents light and which number represents temperature? There isn't any way to be sure, and that's no good. We have to come up with a better solution. Maybe we could add a special number at the beginning of the sequence, like 255, so that every time we see it we'd know the very next number would be a light value, followed by a temperature value. That would look like this:

$255 \ldots 136 \ldots 14 \ldots 255 \ldots 137 \ldots 14 \ldots 255 \ldots 137 \ldots 14 \ldots 255 \ldots 138 \ldots 14 \ldots 255 \ldots 138 \ldots 15 \ldots$ 
Great! Now our data is all organized in a sequence. The 255 in this case is known as a start byte. The start byte concept is so useful that you'll find it in many other protocols, including in the XBee's API. (By the way, we should make sure that our data values stop at 254 so that the start byte will always be unique.) For a computer to read this, we simply tell it to look for a 255, then read in the next byte as a light value and the third as a temperature value. It's a total solution, as long as the sequence and type of sensor values we're sending are fixed. But what if they aren't?

\section{Length byte}

What if sometimes our node sends light and temperature, but other times it sends light, temperature, and humidity? No problem. In this case we need to add a value to our protocol to indicate the length of data coming after the start byte:

$255 \ldots 2 \ldots 136 \ldots 14 \ldots 255 \ldots 3 \ldots 137 \ldots 14 \ldots 87 \ldots 255 \ldots 3 \ldots 137 \ldots 14 \ldots 89 \ldots$

In the sequence above, the numbers 2 and 3 indicate the length of the data. So this is the length byte. Now when a computer reads the sequence, it can know without a doubt that after the start byte, it gets a number that tells it how many more data values to read in.

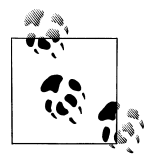

Protocol structures like these are often described as frames, packets, or envelopes. Each of those terms means pretty much the same thing-a repeating sequence containing useful data (sometimes called the payload) packaged with information about the data (sometimes called metadata). We'll use the term frame from here on out to describe our hypothetical protocol.

\section{Contents ID}

The length byte is handy, but it doesn't fully ensure that we know what the frame of data contains. For example, maybe sometimes we have light, temperature, and humidity, but other times we're sending pressure, rainfall, and wind speed. In both cases there are three pieces of data, so we also need to describe the contents of each frame. The simple thing is to add a contents byte, a number that acts as an ID for the type of data in a particular frame. We can decide arbitrarily that 1 will indicate a light/temperature/humidity frame and that 2 will indicate a pressure/rainfall/windspeed frame:

$$
255 \ldots 3 \ldots 1 \ldots 137 \ldots 14 \ldots 87 \ldots 255 \ldots 3 \ldots 2 \ldots 119 \ldots 28 \ldots 54 \ldots
$$

So our sequence here is start byte, length byte, ID byte, and then the data itself. This kind of predictable format is just what computers adore! It transmits everything we need to know, in as little space as possible. There's no limit to the type of useful metainformation we could add in this manner. For example, we might include an address byte to say which sensor node was sending the information, or a voltage byte to indicate the charge remaining in the sensor node's battery. As long as the sequence is 
predictable, it's an airtight method for communicating both the data and contextual information about that data.

There's one more item we should probably append to our message format. We've done a good job sequencing the numbers so that each one means something, but what if there's a transmission error? All methods of transmitting data are subject to corruption. Radio transmissions in particular are notoriously noisy. Static or interference of any kind could potentially introduce a stray bit into our data sequence. For example, in binary a single click of radio noise could easily turn a 21 into the number 149 . While there isn't any way to prevent corruption like this from happening, there are thankfully many ways of detecting it. Error-correction schemes can be rather complex, but the concepts that they use are quite simple to understand.

Jane and Michael are having a conversation across a noisy room. Michael wants Jane to bring him a glass of wine, a napkin, and a celery stick. He could yell to her, "Bring me a glass of wine, a napkin, and a celery stick! Three things!" That last part is for error correction. If Jane only heard the glass of wine and the celery stick, that's not going to match up with "three things." In this case, she'd probably yell back, "What?" to let Michael know he needed to repeat himself. Computers use the same strategies that people do to detect problems in their communications. This particular method would be described as a checksum, meaning a sum of items used solely to check for communication errors. Computer protocols often use more sophisticated arithmetic than simply counting the items, but the principle of sending some frame information followed by a number that can be used to check the frame is widely employed to detect errors in everything from spacecraft communications to credit card numbers.

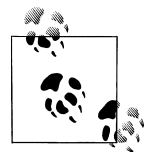

It's important to note that checksums don't provide a guarantee of errorfree communications. For example, if Jane brings Michael a beer, a fork, and a slice of cake, those are still three things, but definitely not the correct ones. More sophisticated checksums drastically decrease the probability of such "substitution" errors, but don't entirely eliminate them. For example, read about the cyclic redundancy check (CRC) at http://wikipedia.org/wiki/Cyclic_redundancy_check.

\section{XBee API Protocol}

Now that you know something about how protocols are designed, it should be fairly easy to understand the API format for XBee radios. The XBee API uses the same structures as our hypothetical protocol, and does so for the exact same reasons. The goal of API-mode communications is to transmit highly structured data quickly, predictably, and reliably. We will begin by taking a look at the structures shared by all API data frames and work our way into the specifics for each frame type. 
The tiny microcontroller inside the Series 2 XBee radio doesn't have enough room to hold all the instructions for both AT mode (transparent/command modes) and API mode. Therefore, different firmware must be loaded onto the radio with X-CTU depending upon which mode you'd like to use to communicate over the local serial port. All the ZigBee firmware versions end in either AT or API (see Figure 5-2) to indicate how they will talk to you on their serial ports.

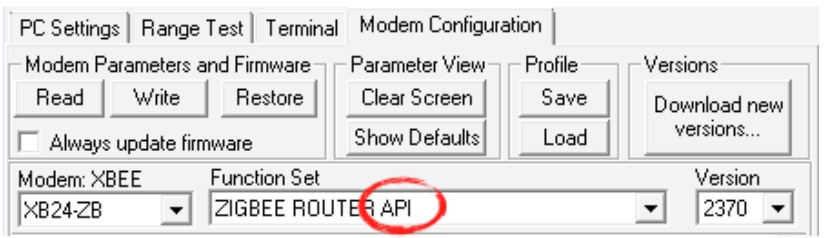

Figure 5-2. To use API mode, be sure to select a firmware function set that ends in "API"

The XBee API frame consists of a series of bytes, each new one building on the information already transmitted.

Let's dig in. You've already read about all the concepts that the API format uses, so hopefully each byte we discuss will now seem like an old friend. Table 5-1 shows the basic structure of the frame.

Table 5-1. Basic API frame structure

\begin{tabular}{lllllll} 
Start delimiter & Length & & \multicolumn{2}{l}{ Frame data } & & Checksum \\
Byte 1 & Byte 2 & Byte 3 & Byte 4 & $\ldots$ & Byten & Byten+1 \\
0x7E & MSB & LSB & APl-specific structure & Single byte \\
\hline
\end{tabular}

\section{Start Delimiter}

Every API frame begins with a start byte. This is a unique number that indicates we are at the beginning of the data frame. In our hypothetical example above, we used decimal 255 for this. The XBee API employs decimal 126 for the exact same purpose. Because everything in the API documentation is described in hexadecimal format, we'll do that here, too. Remember that hex is just notation: decimal 126 and 0x7E are exactly the same number, just written down in different styles. (If you need to brush up on hexadecimals, this is a good time to flip back to the sidebar "Hexadecimals" on page 30 in Chapter 2.)

If we start reading bytes that are arriving from the XBee's serial port in midstream, we won't know what they represent until we know their order. So the first thing to do is look for a start byte of 0x7E. Once we get that, we know where we are and everything else can fall into place. The start byte is like the front cover of a book. 


\section{Length Bytes}

The next two numbers we receive after the start byte indicate the overall length of the data frame. This lets us know how long to keep reading before we stop, in effect letting us know where the back cover of the book is. Right now the second byte, listed as MSB (most significant byte), is usually zero and the third one, listed as LSB (least significant byte), usually contains the entire length.

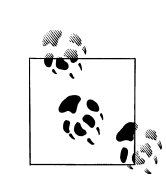

Because a very long data frame could exceed the number that can be described in a byte (remember, that's 0-255), we use a second byte to extend the value to a 16 -bit number $(0-65,535)$. In this case, the large part of the number will be covered in byte 2, the MSB, while the small part of the number would go into byte 3, the LSB. See the sidebar "Breaking Large Numbers into Bytes" on page 135 for more information.

\section{Frame Data Bytes}

The frame data is specific to each type of message we receive from the XBee radio. This is the guts of the information, and we'll expand on its internal structure below. Some frames will carry a great deal of internal data while the smallest frame contains only 2 bytes. For now, you can consider frame data to be like the inside pages of a book. Different kinds of books have different layouts, and the frame data functions in the same way. Remember that since we've read in the length byte, we already know exactly how long this frame data will be.

\section{Checksum}

The very last byte of the frame is always a checksum, so we can think of it as the back cover of our book. The checksum is calculated based on all the bytes that came before it. It's a simple sum of all the bytes that made up the frame, used at the receiving end to check and see if there was a transmission error. The calculation is regular arithmetic, designed to be extremely efficient for computers to process.

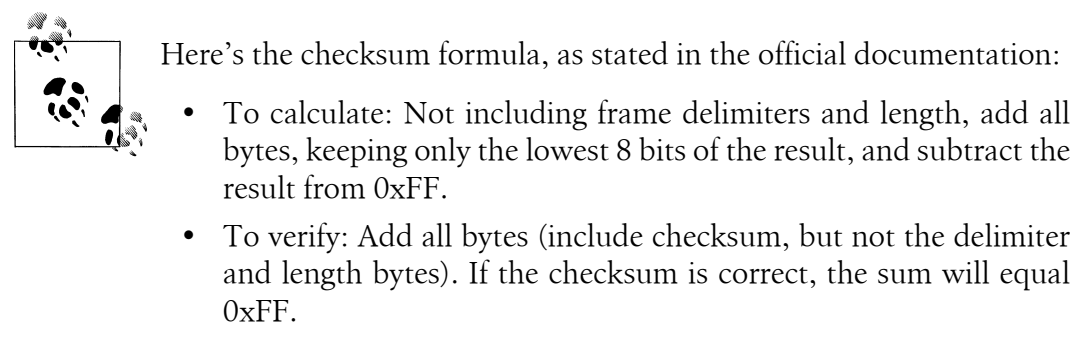


The checksum formula is mostly addition and subtraction, so it's very easy to program. (Keeping the lowest 8 bits of the result is accomplished in code with a bit mask operation that typically looks something like this: \& oxFF.) Usually you'd write a function in your program to do the whole checksum calculation for you. In most of our examples, even that isn't necessary because we use software libraries to do this work for us.

\section{API Frame Types}

Inside the general frame structure there are substructures that cover all the different kinds of data that you might want to send to and receive from your local XBee radio. Different types of frames contain different types of data structures in much the same way as different types of books contain different internal formats. When you pick up a cookbook, you expect to see a title page, an explanation of techniques, then a bunch of recipes (ingredients first), and finally a comprehensive index at the end. That's like one API frame type. A novel is totally different. After its title page, you expect to see a series of chapters, followed by an acknowledgments section that expresses gratitude to everyone ignored during the writing process, and finally a paragraph about the author's expensive schooling and trendy place of residence. The novel is like a second different API frame type. The cookbook and the novel both have front and back covers. Both have title pages. However, their internal structures follow different standardized patterns to help convey the different kinds of information the books contain.

There are more than a dozen different API frame types currently defined for the XBee ZB. We'll look at eight of them here.

The frame type byte tells us what type of API frame we are looking at. Knowing the frame type is crucial to knowing what information is coming next. For example, if the frame type is $0 \mathrm{x} 08$, that indicates it is an AT command frame. So by reading the first four bytes we will know:

- Where the frame begins (start byte)

- How long the frame is going to be (length bytes)

- What kind of frame we're looking at (frame type)

Every frame type is assigned a number. Table 5-2 lists the basic ones, including all the frame types we'll cover in this chapter.

Table 5-2. Some API mode frame types

$\begin{array}{ll}\text { Frame type } & \text { Description } \\ 0 \times 08 & \text { AT command (immediate) } \\ 0 \times 09 & \text { AT command (queued) } \\ 0 \times 17 & \text { Remote Command Request } \\ 0 \times 88 & \text { AT command response } \\ \text { 0x8A } & \text { Modem Status }\end{array}$




\begin{tabular}{ll} 
Frame type & Description \\
0x10 & TX request \\
0x8B & TX response \\
0x90 & RX received \\
0x92 & RX I/0 data received \\
$0 \times 95$ & Node Identification Indicator \\
0x97 & Remote Command Response \\
\hline
\end{tabular}

\section{AT Commands}

AT-type commands can be sent via API frames to configure your local radio. They can query the settings on the local radio or set parameters. These are all the same commands you typed in transparent/command mode.

Just like all the other frame types, AT command frames begin with our old buddy the start byte: $0 x 7 \mathrm{E}$ (see Table 5-3). This is followed by two bytes that indicate the frame length. There's also a checksum at the end. The data that makes the AT command frame unique goes in the Frame-specific section, starting right after the length byte.

Table 5-3. API format for AT commands

\begin{tabular}{|c|c|c|c|c|}
\hline Frame fields & & Offset & Example & Description \\
\hline Start delimiter & & 0 & $0 \times 7 \mathrm{E}$ & \\
\hline \multirow[t]{2}{*}{ Length } & & MSB 1 & $0 \times 00$ & \multirow{2}{*}{ Number of bytes between the length and the checksum. } \\
\hline & & LSB 2 & $0 \times 04$ & \\
\hline \multirow{6}{*}{$\begin{array}{l}\text { Frame-specific } \\
\text { data }\end{array}$} & Frame type & 3 & $0 \times 08$ & \\
\hline & Frame ID & 4 & $0 \times 52$ & $\begin{array}{l}\text { Identifies the UART data frame for the host to correlate with a } \\
\text { subsequent ACK (acknowledgment).Ifsetto0, no responseissent. }\end{array}$ \\
\hline & \multirow[t]{2}{*}{ AT command } & 5 & $0 \times 4 E(N)$ & \multirow{2}{*}{$\begin{array}{l}\text { The command name- - two ASCII characters that identify the AT } \\
\text { command. }\end{array}$} \\
\hline & & 6 & $0 \times 4 \mathrm{~A}(\mathrm{~J})$ & \\
\hline & $\begin{array}{l}\text { Parameter } \\
\text { value }\end{array}$ & & & $\begin{array}{l}\text { If present, indicates the requested parameter value to set the } \\
\text { given register. }\end{array}$ \\
\hline & (optional) & & & If no characters present, the register is queried. \\
\hline Checksum & & 7 & $0 \times 0 D$ & 0xFF - the 8-bit sum of bytes from offset 3 to this byte. \\
\hline
\end{tabular}

\section{Frame type}

The AT command frame is identified with $0 \mathrm{x} 08$. This lets the receiving radio know that the bytes that follow are going to be in the AT command frame order. 


\section{Frame ID}

Since we set a frame type of $0 \mathrm{x} 08$, the XBee receiving this data from us knows that the next byte contains a frame ID. The frame ID is simply a serial number that we attach to the command. Results will be tagged with the same ID. That way, if we've sent a number of commands, we can tell which ones came back OK and which ones might have been lost or gotten an error. By the way, if you set the frame ID to 0x0, you suppress any response from the XBee, but it will still carry out your command.

Generally, you'll set the frame ID to 0x1 for the first command you send, then 0x2 for the next one, and so forth, until you get to $0 \mathrm{xFF}$ (the largest number that a single byte can hold). At that point you can start over again with 0x1.

\section{AT command}

The two bytes that follow the frame ID contain the AT command itself. The letters AT are omitted. Since we already know this is an AT command frame, we just use the twoletter code of the command itself. For example, if we are sending the $\mathrm{NJ}$ command, the first byte will be the ASCII equivalent of a capital $\mathrm{N}$ in hexadecimal format: $0 \mathrm{x} 4 \mathrm{E}$. The second will be a capital $\mathrm{J}$, notated as $0 \mathrm{x} 4 \mathrm{~A}$.

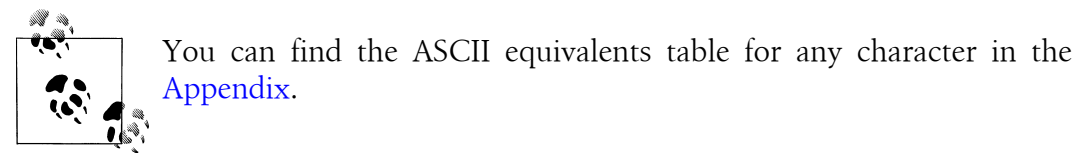

\section{Parameter value}

If the command you're sending requires a parameter, such as a specific register setting, those bytes will follow the AT command. If no parameter value is given, the command will be treated as a query, with the results sent back in a response frame as detailed below. Parameters that are larger than a single byte can be split across several bytes with the larger, "most significant" part of the split-up number coming first.

\section{Checksum}

The AT command frame, like all API frames, ends with a checksum as described above.

Here's what a whole AT command frame looks like. It's the same one as in Table 5-3:

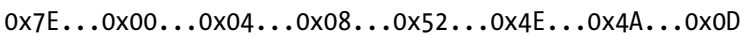


Mostly you'll use the API format coded into a program you modify that talks directly over the serial port to the local XBee. Alternatively, you might work with a software library that is programmed to talk in API format. However, it's entirely possible to figure out the bytes yourself and type them manually into any terminal program that provides a hexadecimal interface. Both X-CTU and CoolTerm have hex modes. In the $\mathrm{X}$-CTU Terminal tab, click on Assemble Packet to be able to type in hex, and click on Show Hex to be able to see the responses from your radio formatted in hex. In CoolTerm, select Send String from the Connection menu and click on the Hex button to type in hex. Click on the View Hex button to see hex-formatted responses from the XBee.

\section{AT Responses}

In API mode, every AT command sent to a local XBee radio can receive a response back from the XBee that contains the status of the command and optionally the registry value if one was requested in a query. This is a frame that the radio generates so you will read these but will never write one yourself. Table 5-4 shows the response format.

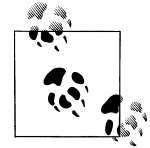

Whether your program cares about these responses will depend on your particular context. In some cases, the quick-and-dirty method of simply sending commands and ignoring the responses is a perfectly serviceable solution. For example, if you are prototyping an error-tolerant interactive sculpture project, then dealing with AT command responses may be far more trouble than it's worth. On the off chance that an error happens, your audience might not even notice. Naturally there are other applications where you wouldn't want to be so tolerant. If you're leaving a sensor network out in the desert by itself for a year, every detail must be addressed with strict response processing and error handling. The important thing is to pick the level of thoroughness that's appropriate to your project and not go overboard without a good reason.

Table 5-4. API format for AT command responses

\begin{tabular}{|c|c|c|c|c|}
\hline \multicolumn{2}{|l|}{ Frame fields } & Offset & Example & Description \\
\hline \multicolumn{2}{|l|}{$\begin{array}{l}\text { Start } \\
\text { delimiter }\end{array}$} & 0 & 0x7E & \\
\hline \multirow[t]{2}{*}{ Length } & & MSB 1 & $0 \times 00$ & \multirow{2}{*}{ Number of bytes between the length and the checksum. } \\
\hline & & LSB 2 & $0 \times 05$ & \\
\hline \multirow{2}{*}{$\begin{array}{l}\text { Frame- } \\
\text { specific data }\end{array}$} & Frame type & 3 & $0 \times 88$ & \\
\hline & Frame ID & 4 & $0 \times 01$ & $\begin{array}{l}\text { Identifies the UART data frame being reported. Note: If frame ID } \\
=0 \text { in AT command mode, no AT command response will be } \\
\text { given. }\end{array}$ \\
\hline
\end{tabular}




\begin{tabular}{|c|c|c|c|c|}
\hline Frame fields & & Offset & Example & Description \\
\hline & \multirow[t]{2}{*}{ AT command } & 5 & ${ }^{\prime} B^{\prime}=0 \times 42$ & \multirow{2}{*}{$\begin{array}{l}\text { The command name- }- \text { two ASCII characters that identify the AT } \\
\text { command. }\end{array}$} \\
\hline & & 6 & $D^{\prime}=0 \times 44$ & \\
\hline & \multirow{5}{*}{$\begin{array}{l}\text { Command } \\
\text { status }\end{array}$} & \multirow[t]{5}{*}{7} & \multirow[t]{5}{*}{$0 \times 00$} & $0=0 \mathrm{~K}$ \\
\hline & & & & $1=$ ERROR \\
\hline & & & & $2=$ Invalid Command \\
\hline & & & & $3=$ Invalid Parameter \\
\hline & & & & $4=$ Tx Failure \\
\hline & Command data & & & $\begin{array}{l}\text { Register data in binary format. If the register was set, this field } \\
\text { is not returned, as in this example. }\end{array}$ \\
\hline Checksum & & 8 & 0xFO & 0xFF - the 8-bit sum of bytes from offset 3 to this byte. \\
\hline
\end{tabular}

AT command response frames received back from the local XBee should look fairly familiar by now. There's a start byte, length bytes, a frame type, and a frame ID, followed by the type of AT command you sent. This is followed by the command status and data, which we'll look at in detail. As you might expect, the last byte is a checksum, calculated in the usual way.

\section{Frame type}

The AT command response frame type is always $0 \times 88$.

\section{Frame ID}

The frame ID you get back will be the same as the one you sent with the original AT command request. You can use the ID to match up your request with this response. Remember that if you set your request frame ID to 0x0, you won't get any response frame in the first place.

\section{AT command}

These two bytes will be the ASCII equivalents of the two command characters you sent.

\section{Command status}

This next byte indicates how your command fared. $0 \mathrm{x} 0$ indicates that everything went fine. It's like receiving an OK in transparent/command mode and should cause you and your program to do a happy dance. A value of $0 \times 1$ indicates that your command resulted in an ERROR. This means it was recognized but could not be carried out for some reason. Receiving 0x2 indicates that your command itself was invalid. Maybe you got one of the letters wrong? A value of $0 \times 3$ indicates that the command was recognized but the parameters you sent with it were out of range. Finally, $0 \times 4$ indicates a transmission failure. 


\section{Command data}

If you queried a register by sending a command with no parameters, these bytes will contain the response information. The response will be broken up into bytes and may represent a number or hex-encoded ASCII string.

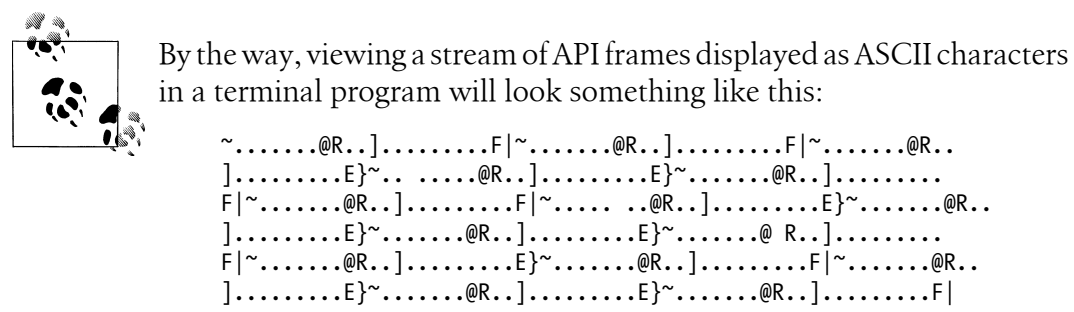

Note the repeating tilde $(\sim)$ character. This is the ASCII equivalent of 0x7E, the start byte, and is a clear indication that rather than seeing garbage, you are seeing good data being delivered in API mode. Switch into viewing hex to see the API frame contents properly.

\section{ZigBee Transmit Request}

Let's send some real data! This frame is how you tell your local radio to send information to some other remote radio. The ZigBee Transmit Request frame encapsulates your payload information (the data itself) with a batch of addressing and transmission options that describe how the payload should be delivered. This frame is a great example of how API mode facilitates something that can't easily be accomplished in transparent/ command mode: setting destination addresses on the fly. Now instead of issuing a +++ and a bunch of commands each time we want to change the destination address, we simply attach that destination to each a frame of data and send it on its way. This is a much more efficient process, especially if you have a network with hundreds of different nodes that you might need to use as destinations. Table 5-5 shows the ZigBee Transmit Request format.

Table 5-5. API format for ZigBee Transmit Request

\begin{tabular}{|c|c|c|c|c|}
\hline \multicolumn{2}{|c|}{ Frame fields } & Offset & Example & Description \\
\hline \multicolumn{2}{|l|}{$\begin{array}{l}\text { Start } \\
\text { delimiter }\end{array}$} & 0 & $0 \times 7 \mathrm{E}$ & \\
\hline \multirow[t]{2}{*}{ Length } & & MSB 1 & $0 \times 00$ & \multirow{2}{*}{ Number of bytes between the length and the checksum. } \\
\hline & & LSB 2 & $0 \times 16$ & \\
\hline \multirow{2}{*}{$\begin{array}{l}\text { Frame- } \\
\text { specific } \\
\text { data }\end{array}$} & Frame type & 3 & $0 \times 10$ & \\
\hline & Frame ID & 4 & $0 \times 01$ & $\begin{array}{l}\text { Identifies the UART data frame for the host to correlate with a } \\
\text { subsequent ACK (acknowledgment). If set to 0, no response is sent. }\end{array}$ \\
\hline
\end{tabular}




\begin{tabular}{|c|c|c|c|c|}
\hline Frame fields & & Offset & Example & Description \\
\hline & \multirow{8}{*}{$\begin{array}{l}\text { 64-bit } \\
\text { destination } \\
\text { address }\end{array}$} & MSB 5 & $0 \times 00$ & \multirow{8}{*}{$\begin{array}{l}\text { Set to the 64-bit address of the destination device. The following } \\
\text { addresses are also supported: } \\
\text { 0x0000000000000000 - Reserved 64-bit address for the } \\
\text { coordinator. } \\
\text { 0x0000000000000FFFF - Broadcast address. }\end{array}$} \\
\hline & & 6 & $0 \times 13$ & \\
\hline & & 7 & $0 \times A 2$ & \\
\hline & & 8 & $0 \times 00$ & \\
\hline & & 9 & $0 \times 40$ & \\
\hline & & 10 & $0 \times 0 A$ & \\
\hline & & 11 & $0 \times 01$ & \\
\hline & & LSB 12 & $0 \times 27$ & \\
\hline & \multirow{2}{*}{$\begin{array}{l}\text { 16-bit } \\
\text { destination } \\
\text { network } \\
\text { address }\end{array}$} & MSB 13 & 0xFF & \multirow[b]{2}{*}{$\begin{array}{l}\text { Set to the 16-bit address of the destination device, if known. Set } \\
\text { to 0xFFFE if the address is unknown, or if sending a broadcast. }\end{array}$} \\
\hline & & LSB 14 & OxFE & \\
\hline & $\begin{array}{l}\text { Broadcast } \\
\text { radius }\end{array}$ & 15 & $0 \times 00$ & $\begin{array}{l}\text { Sets maximum number of hops a broadcast transmission can take. } \\
\text { If set to } 0 \text {, the broadcast radius will be set to the maximum hops } \\
\text { value. }\end{array}$ \\
\hline & \multirow{7}{*}{ Options } & \multirow{7}{*}{16} & \multirow{7}{*}{$0 \times 00$} & $\begin{array}{l}\text { Bit field of supported transmission options. Supported values } \\
\text { include: }\end{array}$ \\
\hline & & & & 0x01 - Disable ACK \\
\hline & & & & $0 \times 20$ - Enable APS encryption (if EE $=1$ ) \\
\hline & & & & 0x40 - Use the extended transmission timeout for this destination \\
\hline & & & & $\begin{array}{l}\text { Enabling APS encryption decreases the maximum number of RF } \\
\text { payload bytes by } 4 \text { (below the value reported by NP). }\end{array}$ \\
\hline & & & & $\begin{array}{l}\text { Setting the extended timeout bit causes the stack to set the ex- } \\
\text { tended transmission timeout for the destination address. }\end{array}$ \\
\hline & & & & All unused and unsupported bits must be set to 0 . \\
\hline & \multirow{8}{*}{ RF data } & 17 & $0 \times 54$ & \multirow{8}{*}{ Data that is sent to the destination device. } \\
\hline & & 18 & $0 \times 78$ & \\
\hline & & 19 & $0 \times 44$ & \\
\hline & & 20 & $0 \times 61$ & \\
\hline & & 21 & $0 \times 74$ & \\
\hline & & 22 & $0 \times 61$ & \\
\hline & & 23 & $0 \times 30$ & \\
\hline & & 24 & $0 \times 41$ & \\
\hline Checksum & & 25 & $0 \times 13$ & 0xFF - the 8-bit sum of bytes from offset 3 to this byte. \\
\hline
\end{tabular}


Again, our frame begins with a start byte, length bytes, a frame type (in this case 0x10, indicating the ZigBee Transmit Request format), and a frame ID. This preamble is followed by addressing information that we'll look at in detail, and then by the data payload itself. The frame concludes as always with a single-byte checksum.

\section{4-bit destination address}

These eight bytes indicate the unique-in-the-world destination address for this transmission, for example 0x0013A200400A0127. There are two special addresses that you can also use. If you want to reach the network coordinator, you can set this address to 0x0000000000000000 (that's 16 zeros) and it will be routed automatically. To send a broadcast message that is delivered to all nodes on the network, set the 64-bit destination address to 0x000000000000FFFF. Check Chapter 7 for information about the ATND node discovery command that can be used to discover all the 64-bit addresses currently present on the network.

\section{6-bit destination network address}

These two bytes can be set to the 16-bit address of the destination radio, if you know what that is. Assigning this address manually is optional, but it will greatly speed up your transmission. This can be essential on larger networks. See "Limits of 64-bit Addressing" on page 126 for a description of the lookup process. If you don't know the 16-bit address that the coordinator has assigned for the destination, simply set these two bits to $0 \mathrm{xFF}$ and $0 \mathrm{xFE}$ respectively. This will cause an address lookup to occur so that the transmission can be properly delivered. 0xFFFE is also the proper 16-bit address setting for broadcast transmissions to be delivered to all the devices on the network.

\section{Limits of 64-bit Addressing}

Using 64-bit addressing to route messages requires broadcast transmissions to discover the 16-bit address. This is almost never a good idea when using the Series 2 on networks that are larger than around 10 nodes. Here's what happens during a transmission cycle when the 16-bit address is set to 0xFFFE for broadcast:

1. A broadcast is sent three times (a value controlled by the ZigBee stack profile) on the network asking to resolve the 64-bit address to the 16-bit network address. These broadcasts are very, very expensive in terms of routing and network overhead because they create three additional messages to every node on the network for every single message sent by any radio.

2. One or more nodes respond to the requester with a point-to-point frame containing the 16-bit address.

3. The transmission proceeds with the newly discovered 16-bit address being used.

If you have started with 64-bit addressing for your messages and your network grows, you will want to migrate your application toward either discovering and using the 
16-bit addresses in advance via the API or saving them offboard on your computer or device when it receives incoming data from the remote node. (If you do this, also consider tracking the TX status of any transmissions using the short address to see if the transmission fails so that you can invalidate the known 16-bit address to 0xFFFE and start the process again.) Remember that you don't need to worry about any of this if your network is relatively small, if messages are not sent too frequently, or if you are using a ConnectPort X gateway — as this is handled for you automatically. Phew!

Broadcast radius. Set this to $0 \mathrm{x} 0$. Each broadcast message can be constrained to a certain radius, usually defined by the default broadcast timeout value set in ATNH. This is an advanced setting for dealing with very specific application or network issues. You should almost always leave this at $0 \mathrm{x} 0$ and use the defaults.

Options. Set this to $0 \mathrm{x} 0$. As of this writing, there are no options defined for this frame type, though future versions of the firmware might implement additional features using this byte.

RF data. At long last we come to the payload. The payload is the data we wanted to send in the first place! It is the meat of our protocol sandwich (or the tasty eggplant, in case meat isn't your thing). Assemble your data into a string of bytes. On many small networks you can usually put up to 84 bytes in your payload transmission. Of course, if you keep your individual data transmissions small, you won't need to worry about this limit.

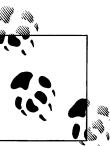

The exact number of allowed payload bytes in each frame is reduced when encryption or source routing are enabled (see Chapter 8 ). There's nothing in this book that requires you to use those features; however, if at some point in the future you decide to go with encryption or source routing, you can query the ATNP register to determine the current payload size limits for your network.

\section{ZigBee Transmit Status}

Another advantage to API mode is that transmissions don't just flow out into a virtual black hole. For each transmission where the frame ID is set to something other than 0x0, we receive back a full status report on any discovery, transmission, or delivery issues. Sometimes this doesn't matter one whit, especially if you're just doing a quick prototype or are running an application that's tolerant of an occasional failure. Transmissions to blink your holiday lighting don't require detailed status reports. Transmissions that monitor your home security probably do. Here's what that status message looks like. It contains all the now-familiar components (see Table 5-6). The frame type is set to 0x8B so you know it's a ZigBee Transmit Status. The frame ID will be the one you put in the original ZigBee Transmit Request that this Status frame is reporting on. There are also a few new components to indicate the transmit retry count, delivery status, and discovery status. 
Table 5-6. API format for ZigBee Transmit Status

\begin{tabular}{|c|c|c|c|c|}
\hline \multicolumn{2}{|l|}{ Frame fields } & Offset & Example & Description \\
\hline \multicolumn{2}{|l|}{$\begin{array}{l}\text { Start } \\
\text { delimiter }\end{array}$} & 0 & $0 \times 7 E$ & \\
\hline \multirow[t]{2}{*}{ Length } & & MSB 1 & $0 \times 00$ & \multirow{2}{*}{ Number of bytes between the length and the checksum. } \\
\hline & & LSB 2 & $0 \times 07$ & \\
\hline \multirow{23}{*}{$\begin{array}{l}\text { Frame- } \\
\text { specific data }\end{array}$} & Frame type & 3 & $0 \times 8 B$ & \\
\hline & Frame ID & 4 & $0 \times 01$ & $\begin{array}{l}\text { Identifies the UART data frame being reported. Note: If frame ID } \\
=0 \text { in AT command mode, no AT command response will be } \\
\text { given. }\end{array}$ \\
\hline & 16-bit address & 5 & $0 \times 7 D$ & If successful, this is the 16-bit network address the packet was \\
\hline & destination & 6 & $0 \times 84$ & $\begin{array}{l}\text { delivered to. If not successful, this address matches the destina- } \\
\text { tion network address that was provided in the Transmit Request } \\
\text { frame. }\end{array}$ \\
\hline & $\begin{array}{l}\text { Transmit retry } \\
\text { count }\end{array}$ & 7 & $0 \times 00$ & The number of application transmission retries that took place. \\
\hline & \multirow{17}{*}{ Delivery status } & \multirow{17}{*}{8} & \multirow{17}{*}{$0 \times 00$} & $0 \times 00=$ Success \\
\hline & & & & $0 \times 01=$ MAC ACK failure \\
\hline & & & & $0 \times 02=C C A$ failure \\
\hline & & & & 0x15 = Invalid destination endpoint \\
\hline & & & & $0 \times 21=$ Network ACK failure \\
\hline & & & & $0 \times 22=$ Not joined to network \\
\hline & & & & $0 \times 23=$ Self-addressed \\
\hline & & & & $0 \times 24=$ Address not found \\
\hline & & & & $0 \times 25=$ Route not found \\
\hline & & & & $\begin{array}{l}0 \times 26=\text { Broadcast source failed to hear a neighbor relay the } \\
\text { message }\end{array}$ \\
\hline & & & & $0 \times 2 B=$ Invalid binding table index \\
\hline & & & & $0 \times 2 C=$ Resource error, lack of free buffers, timers, etc. \\
\hline & & & & $0 \times 2 D=$ Attempted broadcast with APS transmission \\
\hline & & & & $0 \times 2 \mathrm{E}=$ Attempted unicast with APS transmission, but $\mathrm{EE}=0$ \\
\hline & & & & $0 \times 32=$ Resource error, lack of free buffers, timers, etc. \\
\hline & & & & 0x74 = Data payload too large \\
\hline & & & & $0 \times 75=$ Indirect message unrequested \\
\hline & Discovery status & 9 & $0 \times 01$ & $\begin{array}{l}0 \times 00=\text { No discovery overhead } \\
0 \times 01=\text { Address discovery }\end{array}$ \\
\hline
\end{tabular}




\begin{tabular}{llll} 
Frame fields & Offset & Example & Description \\
\hline & & 0x02 = Route discovery \\
& & $0 \times 03=$ Address and route \\
& & $0 \times 40=$ Extended timeout discovery \\
\hline Checksum & 10 & $0 \times 71$ & 0xFF - the 8-bit sum of bytes from offset 3 to this byte. \\
\hline
\end{tabular}

\section{Transmit retry count}

Every transmission will be attempted up to three times by the transmitting radio (other retries may happen invisibly along the mesh route). The count of these retries is listed in this byte. Retries are a normal part of wireless networking, so individually they are of no concern, though considered in aggregate they might indicate layout or interference issues. For now, there's no need to be particularly concerned about this count.

\section{Delivery status}

If this byte is $0 \mathrm{x} 0$, then hurray! Your transmission was successfully delivered to the destination address. Otherwise, the number you receive in this byte will indicate the kind of issue that prevented delivery, which is useful for debugging and possibly for deciding whether to send the information again. The error numbers are listed in Table 5-6. Many applications don't care why the error happened; they just need to know that it did. In this case, anything greater than $0 \mathrm{x} 0$ might tell your project to try the transmission again or to report an error to the user.

\section{Discovery status}

This byte gives a bit of information about how much overhead it took to discover the route for this transmission. In general, smaller numbers are better. For very large networks, you might want to keep an eye on this and consider using advanced source routing. For small networks like the ones we create in this book, the discovery status can be safely ignored.

\section{ZigBee Receive Packet}

Here's another API frame that gives us far more than we could get from simple transparent/command mode interactions. When a transmission is received in transparent mode, it comes with no indication of who the sender was. On a simple pair network that's fine because there's only one possible sender. But on a larger network, it's usually of considerable interest to know not only what was received but where it came from. So in addition to the usual preamble bytes, including the frame type of $0 \times 90$ to indicate a ZigBee Receive Packet and a frame ID that was sent by the transmitter, we get to see the 64-bit and 16-bit source addresses along with a receive options indicator, and of course the payload data itself, followed by a checksum. Table 5-7 shows this frame's format. 
Table 5-7. API format for ZigBee RX Packet

\begin{tabular}{|c|c|c|c|c|}
\hline \multicolumn{2}{|l|}{ Frame fields } & Offset & Example & Description \\
\hline \multicolumn{2}{|l|}{ Start delimiter } & 0 & $0 \times 7 \mathrm{E}$ & \\
\hline \multicolumn{2}{|l|}{ Length } & MSB 1 & $0 \times 00$ & $\begin{array}{l}\text { Number of bytes between the length and the } \\
\text { checksum. }\end{array}$ \\
\hline \multirow{21}{*}{$\begin{array}{l}\text { Frame-specific } \\
\text { data }\end{array}$} & Frametune & 3 & $0 \times 90$ & \\
\hline & \multirow{8}{*}{$\begin{array}{l}\text { 64-bit } \\
\text { source address }\end{array}$} & MSB 4 & $0 \times 00$ & \multirow{8}{*}{$\begin{array}{l}\text { 64-bit address of sender. Set to 0xFFFFFFFFFFFFFFFF } \\
\text { (unknown 64-bit address) if the sender's 64-bit } \\
\text { address is unknown. }\end{array}$} \\
\hline & & 5 & $0 \times 13$ & \\
\hline & & 6 & $0 \times A 2$ & \\
\hline & & 7 & $0 \times 00$ & \\
\hline & & 8 & $0 \times 40$ & \\
\hline & & 9 & $0 \times 52$ & \\
\hline & & 10 & $0 \times 2 B$ & \\
\hline & & LSB 11 & $0 \times A A$ & \\
\hline & \multirow{2}{*}{$\begin{array}{l}\text { 16-bit source } \\
\text { network address }\end{array}$} & MSB 12 & $0 \times 7 D$ & \multirow{2}{*}{ 16-bit address of sender. } \\
\hline & & LSB 13 & $0 \times 84$ & \\
\hline & \multirow{4}{*}{ Receive options } & \multirow{4}{*}{14} & \multirow{4}{*}{$0 \times 01$} & 0x01 - Packet acknowledged. \\
\hline & & & & 0x02 - Packet was a broadcast packet. \\
\hline & & & & 0x20 - Packet encrypted with APS encryption. \\
\hline & & & & 0x40-Packet was sent from an end device (ifknown). \\
\hline & \multirow{6}{*}{ Received data } & 15 & $0 \times 52$ & \multirow{6}{*}{ Received RF data. } \\
\hline & & 16 & $0 \times 78$ & \\
\hline & & 17 & $0 \times 44$ & \\
\hline & & 18 & $0 \times 61$ & \\
\hline & & 19 & $0 \times 74$ & \\
\hline & & 20 & $0 \times 61$ & \\
\hline Checksum & & 21 & $0 \times 0 D$ & 0xFF-the 8-bit sum of bytes from offset 3 to this byte. \\
\hline
\end{tabular}

\section{4-bit source address}

These eight bytes report the address that this transmission was sent from. It's how we can tell which radio is associated with the data we just received.

\section{6-bit source network address}

These two bytes tell us the short network address of the sender. Feel free to ignore this for now, but keep in mind that later it could be handy in case we want to speed up the 
reply process. If the 16-bit address is included in a future transmission frame, we can save time and some overhead by not forcing the network to look it up again.

\section{Receive options}

This byte provides just a little info. It indicates $0 x 1$ if receipt of transmission was acknowledged, or $0 \times 2$ if the received information was sent as a broadcast, in which case no acknowledgment will be sent. In most cases this byte can be safely ignored.

\section{Received data}

This is the data itself, organized as bytes in the exact same order it was in when the sender sent it. This data, by the way, could be anything from a doorbell push indicator to a poem. We refer to it as arbitrary data. It isn't arbitrary in the sense that it is random but rather because it doesn't need to follow a specific structure.

\section{I/0 Data Sample Rx Indicator}

We've covered all the basic API frame types used to issue local commands, transmit information, receive information, and check on the status of our commands and transmissions. Since you've made it this far, it should be pretty easy to understand the next API frame type. It contains the juiciest type of information — direct sensor data! This is how you will obtain real values from networks of remote sensors via the XBee's direct input/output functionality. Your room temperature, soil moisture, monkey-trap status, or whatever, will arrive encased in this frame type.

The ZigBee I/O Sample Rx Indicator is really just an extension of the ZigBee Receive Packet discussed above. The main difference is that instead of the payload having an arbitrary or unconstrained format, it is organized in a highly structured way that lets us decode a set of digital and/or analog samples that were taken directly by the transmitting XBee. It's important to note that I/O samples can't be received in transparent/ command mode at all. Using API mode is essential to receiving XBee direct I/O information. It's one of the most important reasons for us to cover the API in this book.

All I/O samples are received inside what otherwise would appear to be a simple ZigBee Receive Packet. The first clue that it's any different is its frame type of 0x92, which indicates that we'll be getting an I/O data sample in the payload. After that, everything is the same up until the first payload byte, which gives us the number of samples followed by the analog and digital channel masks that tell us how the sender's pins are configured. Then the digital and analog samples themselves are provided, all in a highly structured format that allows the data, when correctly interpreted, to be absolutely unambiguous. Table 5-8 shows the format for this frame. 
Table 5-8. API format for ZigBee I/O Data Sample Rx Indicator

\begin{tabular}{|c|c|c|c|c|}
\hline \multicolumn{2}{|l|}{ Frame fields } & Offset & Example & Description \\
\hline \multicolumn{2}{|l|}{ Start delimiter } & 0 & $0 \times 7 E$ & \\
\hline \multirow[t]{2}{*}{ Length } & & MSB 1 & $0 \times 00$ & \multirow{2}{*}{$\begin{array}{l}\text { Number of bytes between the length and the } \\
\text { checksum. }\end{array}$} \\
\hline & & LSB 2 & $0 \times 14$ & \\
\hline \multirow{22}{*}{$\begin{array}{l}\text { Frame-specific } \\
\text { data }\end{array}$} & Frame type & 3 & $0 \times 92$ & \\
\hline & \multirow{8}{*}{$\begin{array}{l}\text { 64-bit } \\
\text { source address }\end{array}$} & MSB 4 & $0 \times 00$ & \multirow{8}{*}{ 64-bit address of sender. } \\
\hline & & 5 & $0 \times 13$ & \\
\hline & & 6 & $0 \times A 2$ & \\
\hline & & 7 & $0 \times 00$ & \\
\hline & & 8 & $0 \times 40$ & \\
\hline & & 9 & $0 \times 52$ & \\
\hline & & 10 & $0 \times 2 B$ & \\
\hline & & LSB 11 & $0 \times A A$ & \\
\hline & \multirow{2}{*}{$\begin{array}{l}\text { 16-bit source } \\
\text { network address }\end{array}$} & $\begin{array}{l}\text { MSB } \\
12\end{array}$ & $0 \times 7 D$ & \multirow{2}{*}{ 16-bit address of sender. } \\
\hline & & LSB 13 & $0 \times 84$ & \\
\hline & \multirow[t]{2}{*}{ Receive options } & \multirow[t]{2}{*}{14} & \multirow[t]{2}{*}{$0 \times 01$} & 0x01 - Packet acknowledged. \\
\hline & & & & $0 \times 02$ - Packet was a broadcast packet. \\
\hline & Number of samples & 15 & $0 \times 01$ & $\begin{array}{l}\text { Number of sample sets included in the payload. } \\
\text { (Always set to 1.) }\end{array}$ \\
\hline & \multirow[t]{2}{*}{ Digital channel mask } & 16 & $0 \times 00$ & \multirow{2}{*}{$\begin{array}{l}\text { Bit mask field that indicates which digital } I / 0 \text { lines on } \\
\text { the remote have sampling enabled (if any). }\end{array}$} \\
\hline & & 17 & $0 \times 1 C$ & \\
\hline & Analog channel mask & 18 & $0 \times 02$ & $\begin{array}{l}\text { Bit mask field that indicates which analog } 1 / 0 \text { lines on } \\
\text { the remote have sampling enabled (if any). }\end{array}$ \\
\hline & \multirow{3}{*}{$\begin{array}{l}\text { Digital samples (if } \\
\text { included) }\end{array}$} & 19 & $0 \times 00$ & \multirow{2}{*}{$\begin{array}{l}\text { If the sample set includes any digital } I / 0 \text { lines (digital } \\
\text { channel mask }>0 \text { ), these two bytes contain samples } \\
\text { for all enabled digital } I / 0 \text { lines. }\end{array}$} \\
\hline & & 20 & $0 \times 14$ & \\
\hline & & & & $\begin{array}{l}\text { DI0 lines that do not have sampling enabled return } 0 . \\
\text { Bits in these two bytes map the same as they do in the } \\
\text { Digital Channels Mask field. }\end{array}$ \\
\hline & \multirow[t]{2}{*}{ Analog sample } & 21 & $0 \times 02$ & \multirow[b]{2}{*}{$\begin{array}{l}\text { If the sample set includes any analog input lines } \\
\text { (analog channel mask }>0 \text { ), each enabled analog input } \\
\text { returns a 2-byte value indicating the A/D measurement } \\
\text { of that input. Analog samples are ordered } \\
\text { sequentially from ADO/DIO0 to AD3/DI03, to the supply } \\
\text { voltage. }\end{array}$} \\
\hline & & 22 & $0 \times 25$ & \\
\hline \multicolumn{2}{|l|}{ Checksum } & 23 & $0 x F 5$ & 0xFF - the 8-bit sum of bytes from offset 3 to this byte. \\
\hline
\end{tabular}




\section{Number of samples}

This single byte indicates how many sampling collections are contained in this frame. Currently this is always set to $0 \mathrm{x} 1$ to indicate a single collection, because multiple collections are not yet supported on the Series 2 hardware. You can safely ignore this byte.

\section{Digital channel mask}

These two bytes indicate which of the sending XBee's pins are configured as digital inputs. Each hexadecimal can be translated to a binary number that will tell you which pins are configured as digital inputs. See the sidebar "Mapping Binary to Switches and Pins" on page 134 for information about how to do that. Once the numbers have been translated, you can read them using Tables 5-9 and 5-10.

Table 5-9. First byte of digital channel mask

$\begin{array}{llllllll}\text { n/a } & \text { n/a } & \text { n/a } & \text { D12 } & \text { D11 } & \text { D10 } & \text { n/a } & \text { n/a }\end{array}$

Table 5-10. Second byte of digital channel mask

$\begin{array}{llllllll}\text { D7 } & \text { D6 } & \text { D5 } & \text { D4 } & \text { D3 } & \text { D2 } & \text { D1 } & \text { D0 }\end{array}$

As an example, let's say you received $0 \mathrm{x} 0$ as the first digital channel mask byte and $0 \mathrm{x} 1 \mathrm{C}$ as the second one. The first byte in binary is 00000000 . (The space in the middle doesn't mean anything; it just makes the number easier to look at.) Using Table 5-11, we can see that none of pins D12, D11, and D10 are configured as digital inputs because they're all set to zero. 0 means that pin is not configured as a digital input; 1 means that it is. The second byte, $0 x 1 \mathrm{C}$, translates to 00011100 in binary. Placing this number into Table 5-12, we can see that pins D4, D3, and D2 are configured to be digital inputs because that's where the 1s show up.

Table 5-11. Example: first byte of digital channel mask showing that pins D10-D12 are NOT configured as digital inputs

\begin{tabular}{llllllll}
$\mathrm{n} / \mathrm{a}$ & $\mathrm{n} / \mathrm{a}$ & $\mathrm{n} / \mathrm{a}$ & $\mathrm{D} 12$ & $\mathrm{D} 11$ & $\mathrm{D} 10$ & $\mathrm{n} / \mathrm{a}$ & $\mathrm{n} / \mathrm{a}$ \\
0 & 0 & 0 & 0 & 0 & 0 & 0 & 0 \\
\hline
\end{tabular}

Table 5-12. Example: second byte of digital channel mask showing that ONLY pins D2-D4 are configured to be digital inputs

\begin{tabular}{llllllll} 
D7 & D6 & D5 & D4 & D3 & D2 & D1 & D0 \\
0 & 0 & 0 & 1 & 1 & 1 & 0 & 0 \\
\hline
\end{tabular}




\section{Mapping Binary to Switches and Pins}

The XBee API uses an elegant, if somewhat commonplace, trick to accomplish representing pin states as hexadecimals - using the arrangement of ones and zeros in binary notation to directly describe pins as being either on or off. We discussed binary briefly earlier in this chapter as a method for indicating a certain number of states. We can also map a number directly to a set of switches (or pins in this case) that can be either on or off. So if you had eight switches that were all off you could represent their on/off state with 0000 0000. (Remember, the space is just for readability.) If the first, third, and eighth switches were flipped on, the mapped number would look like 10000101. (Binary numbers increase from right to left, just like decimal numbers.) Translating that map from a binary number into decimal notation gives us 133, which in hexadecimal notation is $0 \times 85$. So by receiving the number $0 \times 85$ and looking at its binary equivalent, we can know which switches are on and off. Another example: if we received the hex number $0 x 1 C$, we could translate that to binary notation of 00011100 . So that means the third, fourth, and fifth switches are on. Remember that there are calculators available on most computers that will do the hex-to-binary translation for you.

\section{Analog channel mask}

There is only one byte for the analog channel mask. This is because we have only four analog inputs to consider. This mask uses the same system as the digital ones (see Table 5-13).

Table 5-13. Single byte for analog channel mask

$\begin{array}{llllllll}\text { (voltage) } & \text { n/a } & \text { n/a } & \text { n/a } & \text { A3 } & \text { A2 } & \text { A1 } & \text { A0 }\end{array}$

Using Table 5-13, we can decode the binary version of the byte into a pin configuration. For example, if we received $0 \times 2$ as the analog channel mask, that would translate into the binary number 00000010 . This would indicate that pin A1 is configured as an analog input, but none of the other pins are.

You may note that the highest bit in the analog channel mask indicates if system voltage readings have been enabled as part of the analog data set. By default they are not.

\section{Digital samples}

If you are receiving digital samples, these two bytes will appear and let you know whether the enabled pins are currently high or low, in the same arrangement as the mask. So if you receive 0x0 in the first digital sample byte and 0x14 in the second one, that indicates high voltage is being received only on pins D4 and D2. Any other pins configured as digital inputs are currently reading low. You can use the preceding tables to decode these samples, just like with the mask. 
These two bytes will be received only if at least one pin is enabled as a digital input. If your digital channel mask bytes are both 0x0, no pins have been enabled and these two bytes will be omitted.

\section{Analog samples}

The last component of the I/O Data Sample frame is a set of two bytes for each analog sample taken. We know how many to expect from the analog channel mask, which tells us what pins have been configured as analog inputs. So if data is being received from two analog pins, we can expect to receive four bytes of data in all. Each two-byte sample consists of a most significant and a least significant byte. This is because the sample itself is represented as a 10-bit number. 10 bits are enough to represent values from $0-1,023$, which gives us a pretty smooth resolution for our data.

\section{Breaking Large Numbers into Bytes}

Large numbers that are broken up into bytes for transmission can easily be reassembled once they are received. For example, the number 987 can't fit in a single byte. When the XBee radio needs to transmit this 10-bit number, it breaks it into two bytes. The lower part represents the part of the binary number that falls into the place values 0 255. The higher part represents the place values from 256 to 1,023 . We receive $0 \times 3$ as the first, most significant byte (MSB) and $0 \mathrm{xDB}$ as the second, least significant byte (LSB). Pasting these together gives us $0 \times 3 \mathrm{DB}$, and the decimal equivalent of that is 987. In code, we can accomplish this paste process arithmetically: multiplying the MSB 0xFF (same as decimal 255) and then adding the result to the LSB will give us the correct results:

$$
(0 \times 3 * 0 \times F F)+0 \times D B=0 \times 3 D B \ldots \text { same as } 987 \text { in decimal }
$$

\section{Remote AT Command Request}

Sending commands to configure the local radio is useful. Sending commands over the wireless network to configure remote radios is kind of exhilarating. It is also something you can accomplish only in API mode-yet another reason to master the API.

Any AT-type command that you can issue locally can also be sent wirelessly for execution on a remote radio. This is especially useful for remote actuation, where you might want to change the state of a digital output from low to high to trigger a realworld action. We'll use this command type to do just that in the next chapter. Table 5-14 shows the format of the Remote AT Command Request. 
Table 5-14. API format for Remote AT Command Request

\begin{tabular}{|c|c|c|c|c|}
\hline \multicolumn{2}{|c|}{ Frame fields } & Offset & Example & Description \\
\hline \multicolumn{2}{|l|}{$\begin{array}{l}\text { Start } \\
\text { delimiter }\end{array}$} & 0 & $0 \times 7 E$ & \\
\hline \multirow[t]{2}{*}{ Length } & & MSB 1 & $0 \times 00$ & \multirow{2}{*}{ Number of bytes between the length and the checksum. } \\
\hline & & LSB 2 & $0 \times 10$ & \\
\hline \multirow{21}{*}{$\begin{array}{l}\text { Frame- } \\
\text { specific } \\
\text { data }\end{array}$} & Frame type & 3 & $0 \times 17$ & \\
\hline & Frame ID & 4 & $0 \times 01$ & $\begin{array}{l}\text { Identifies the UART data frame for the host to correlate with a } \\
\text { subsequent ACK (acknowledgment).Ifsetto0, noresponseissent. }\end{array}$ \\
\hline & \multirow{8}{*}{$\begin{array}{l}\text { 64-bit } \\
\text { destination } \\
\text { address }\end{array}$} & MSB 5 & $0 \times 00$ & \multirow{8}{*}{$\begin{array}{l}\text { Set to the 64-bit address of the destination device. The following } \\
\text { addresses are also supported: } \\
\text { 0x0000000000000000 - Reserved 64-bit address for the } \\
\text { coordinator } \\
\text { 0x000000000000FFFF - Broadcast address }\end{array}$} \\
\hline & & 6 & $0 \times 13$ & \\
\hline & & 7 & $0 \times A 2$ & \\
\hline & & 8 & $0 \times 00$ & \\
\hline & & 9 & $0 \times 40$ & \\
\hline & & 10 & $0 \times 40$ & \\
\hline & & 11 & $0 \times 11$ & \\
\hline & & LSB 12 & $0 \times 22$ & \\
\hline & \multirow{2}{*}{$\begin{array}{l}\text { 16-bit } \\
\text { destination } \\
\text { network } \\
\text { address }\end{array}$} & MSB 13 & 0xFF & \multirow[b]{2}{*}{$\begin{array}{l}\text { Set to the 16-bit address of the destination device, if known. Set } \\
\text { to OxFFFE if the address is unknown, or if sending a broadcast. }\end{array}$} \\
\hline & & LSB 14 & $0 x \mathrm{FE}$ & \\
\hline & \multirow{6}{*}{$\begin{array}{l}\text { Remote } \\
\text { command } \\
\text { options }\end{array}$} & \multirow{6}{*}{15} & \multirow{6}{*}{$\begin{array}{l}0 \times 02 \\
\text { (apply } \\
\text { changes) }\end{array}$} & $\begin{array}{l}\text { Bit field to enable various remote command options. Supported } \\
\text { values include: }\end{array}$ \\
\hline & & & & 0x01 - Disable ACK. \\
\hline & & & & $\begin{array}{l}0 \times 02 \text { - Apply changes on remote. (If not set, } \mathrm{AC} \text { command must } \\
\text { be sent before changes will take effect.) }\end{array}$ \\
\hline & & & & $\begin{array}{l}\text { Ox40 - Use the extended transmission timeout for this } \\
\text { destination. }\end{array}$ \\
\hline & & & & $\begin{array}{l}\text { Setting the extended timeout bit causes the stack to set the ex- } \\
\text { tended transmission timeout for the destination address. }\end{array}$ \\
\hline & & & & All unused and unsupported bits must be set to 0 . \\
\hline & \multirow{2}{*}{ AT command } & 16 & $0 \times 42(B)$ & \multirow{2}{*}{ The name of the command. } \\
\hline & & 17 & $0 \times 48(\mathrm{H})$ & \\
\hline & $\begin{array}{l}\text { Command } \\
\text { parameter }\end{array}$ & 18 & $0 \times 01$ & $\begin{array}{l}\text { If present, indicates the requested parameter value to set the } \\
\text { given register. If no characters present, the register is queried. }\end{array}$ \\
\hline Checksum & & 19 & 0xF5 & 0xFF - the 8-bit sum of bytes from offset 3 to this byte. \\
\hline
\end{tabular}


The Remote AT Command Request is made up almost entirely of components we have already covered. The frame type is $0 \times 17$, followed by an ID, then the 64-bit and 16-bit addresses. The next byte is for remote command options, which we will look at below. That's followed by two bytes for the two characters of the command, one or more bytes to contain any parameter being sent, and finally the checksum. This is starting to get easy!

\section{Remote command options}

This byte can currently be set to one of two states. Normally you should set it to $0 \mathrm{x} 02$ to indicate that any changes requested by this remote AT command should be applied immediately. Occasionally you might not want to apply a command until a specific moment, for example if you wanted a bunch of output pins to all change at the same instant. To delay command execution, set this byte to be $0 \mathrm{x} 0$ and then issue an $\mathrm{AC}$ command later when you are ready for all your changes to take effect.

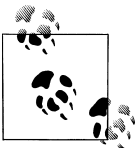

Applying a set of changes all at once is known in the computing world as atomicity. As with the original conception of an atom as an indivisible

f. bit of matter, atomicity is used when each change can't be separated out from the others.

\section{Remote Command Response}

Every Remote Command Request that is sent with a frame ID other than zero will receive a response frame to report on how the remote command fared. This frame type is $0 \times 97$, followed by the ID of the request, 64- and 16-bit address, the AT command you sent, a command status (just like the one for local AT commands), command data if you queried a register, and finally the checksum. There is nothing at all in this frame that is new to you. In fact, you may start to consider yourself something of an API expert! Table 5-15 shows the Remote Command Response format.

Table 5-15. API format for Remote Command Response

\begin{tabular}{lllll} 
Frame Fields & & Offset & Example & Description \\
\hline Start delimiter & 0 & 0x7E & \\
\hline Length & MSB 1 & $0 \times 00$ & $\begin{array}{l}\text { Number of bytes between the length and the } \\
\text { checksum. }\end{array}$ \\
\hline \multirow{2}{*}{$\begin{array}{llll}\text { Frame-specific } \\
\text { data }\end{array}$} & LSB 2 & $0 \times 13$ & \\
\cline { 2 - 5 } & Frame type & 3 & $0 \times 97$ & \\
\cline { 2 - 5 } & Frame ID & 4 & $0 \times 55$ & This is the same value passed in to the request. \\
\cline { 2 - 5 } & M4-bit source & MSB 5 & $0 \times 00$ & \\
(remote) address & 6 & $0 \times 13$ & The address of the remote radio returning this \\
& & 7 & $0 \times A 2$ & response. \\
& 8 & $0 \times 00$ &
\end{tabular}




\begin{tabular}{|c|c|c|c|c|}
\hline \multicolumn{2}{|l|}{ Frame Fields } & \multirow{2}{*}{$\begin{array}{l}\text { Offset } \\
9\end{array}$} & \multirow{2}{*}{$\begin{array}{l}\text { Example } \\
0 \times 40\end{array}$} & \multirow[t]{5}{*}{ Description } \\
\hline & & & & \\
\hline & & 10 & $0 \times 52$ & \\
\hline & & 11 & $0 \times 2 B$ & \\
\hline & & LSB 12 & OXAA & \\
\hline & \multirow{2}{*}{$\begin{array}{l}\text { 16-bit source } \\
\text { (remote) address }\end{array}$} & MSB 13 & $0 \times 7 D$ & Set to the 16 -bit network address of the remote. \\
\hline & & LSB 14 & $0 \times 84$ & Set to OxFFFE if unknown. \\
\hline & \multirow{2}{*}{ AT commands } & 15 & $0 \times 53$ & \multirow{2}{*}{ The name of the command. } \\
\hline & & 16 & $0 \times 4 C$ & \\
\hline & \multirow[t]{5}{*}{ Command status } & \multirow[t]{5}{*}{17} & \multirow[t]{5}{*}{$0 \times 00$} & $0=0 \mathrm{~K}$ \\
\hline & & & & $1=E R R O R$ \\
\hline & & & & $2=$ Invalid Command \\
\hline & & & & $3=$ Invalid Parameter \\
\hline & & & & $4=$ Remote Command Transmission Failed \\
\hline & \multirow[t]{4}{*}{ Command data } & 18 & $0 \times 40$ & \multirow{4}{*}{$\begin{array}{l}\text { Register data in binary format. If the register was } \\
\text { set, then this field is not returned. }\end{array}$} \\
\hline & & 19 & $0 \times 52$ & \\
\hline & & 20 & $0 \times 2 B$ & \\
\hline & & 21 & OXAA & \\
\hline Checksum & & 22 & 0xF0 & $\begin{array}{l}\text { 0xFF-the } 8 \text {-bit sum of bytes from offset } 3 \text { to this } \\
\text { byte. }\end{array}$ \\
\hline
\end{tabular}

\section{Using What You Need}

Now that you understand how API mode works and how the structures around it function, we can talk about using it. It's pretty simple to write your own microcontroller code to work with the API, especially if you use only what you need. While the API is capable of supplying your application with an airtight protocol that covers any possible radio configuration, in most cases you'll be using only a small subset of that radio's capabilities. For example, in many sensor network applications your I/O data samples will all be the same length. This means you can get away with never checking the length byte. It also means that your data will show up in the same place in every frame. There's no need to calculate the digital mask or look for digital samples if you already know that your network never uses digital inputs.

The romantic lighting sensor example in the previous chapter takes advantage of this minimalist strategy. Here's the code from the loop that reads the I/O Data Sample frame:

// make sure everything we need is in the buffer if (Serial.available ()$>=21$ ) \{

// look for the start byte 


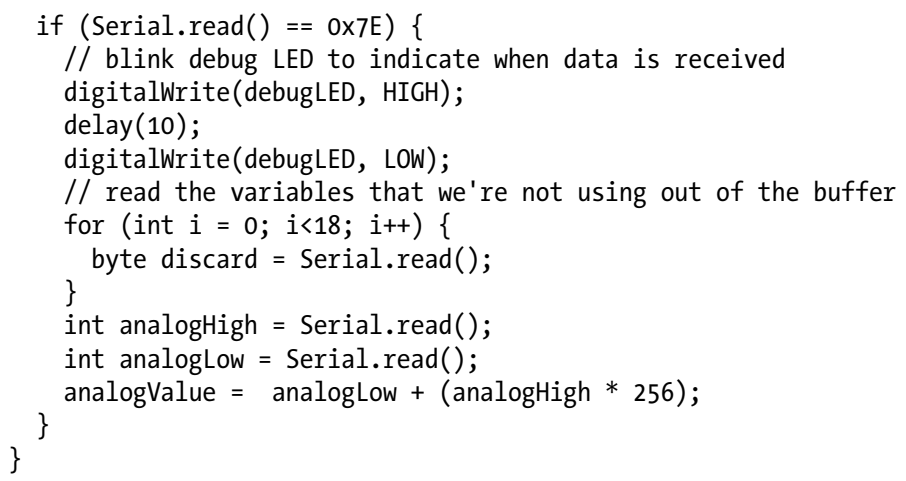

We start by seeing if there's potentially a full frame of information waiting in the buffer. Because we set the sensor radio's configuration ourselves, we already know how long a frame will be: 22 bytes, or in this case 21 because we ignore the checksum. So we begin by checking the Arduino's serial buffer to see if there's potentially a full frame of data waiting for us:

\section{// make sure everything we need is in the buffer if (Serial.available ()$>=21$ ) \{}

and then we read a byte in to see if it is a start byte of $0 x 7 \mathrm{E}$. If it isn't we'll skip to the next byte until we do find a $0 \times 7 \mathrm{E}$ and know we're most likely at the beginning of a data frame:

$$
\begin{aligned}
& / / \text { look for the start byte } \\
& \text { if (Serial.read() }==0 \times 7 E)\{
\end{aligned}
$$

When we know we're at the start of a data frame, we can skip over most of the frame contents. Length we already know. Same goes for frame type; we're only sending I/O sample frames so we can assume that whatever we receive will be the reply to one. The source address will always be the paired sensor, so that information can be ignored as well in this case. The receive options, number of samples, and all the channel mask information will never change in this project so we merrily read in all those bytes and throw them away:

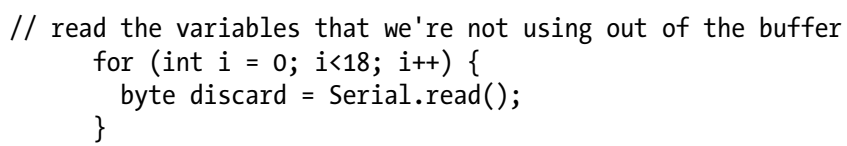

Finally we get to the bytes we do care about, the analog sensor readings from the remote photocell. The two bytes (MSB and LSB) get read into two variables:

$$
\begin{aligned}
& \text { int analogHigh }=\text { Serial } \cdot \operatorname{read}() \text {; } \\
& \text { int analogLow = Serial. } \operatorname{read}() \text {; }
\end{aligned}
$$

... and then pasted together arithmetically to reconstitute the original 10-bit sensor reading:

analogValue $=$ analogLow $+(\operatorname{analogHigh} * 256) ;$ 
This whole process is very fast to program and will run just fine. Granted, there are more careful ways to go about this. A commercial application might want to do some additional error checking. In this case, however, we simply write the code that suits our romantic lighting purposes and get on with making the evening's fondue.

You may also want to take a close look at the setRemoteState function in "Program the romantic lighting sensor with feedback base station" on page 106. That function issues a Remote AT Command Request to turn on an LED that is connected to the remote sensor XBee in that project. It has all the elements that you are now familiar with, so the code comments probably make a great deal of sense to you now. Here is that function again. Read it through and admire just how technically adept you have become:

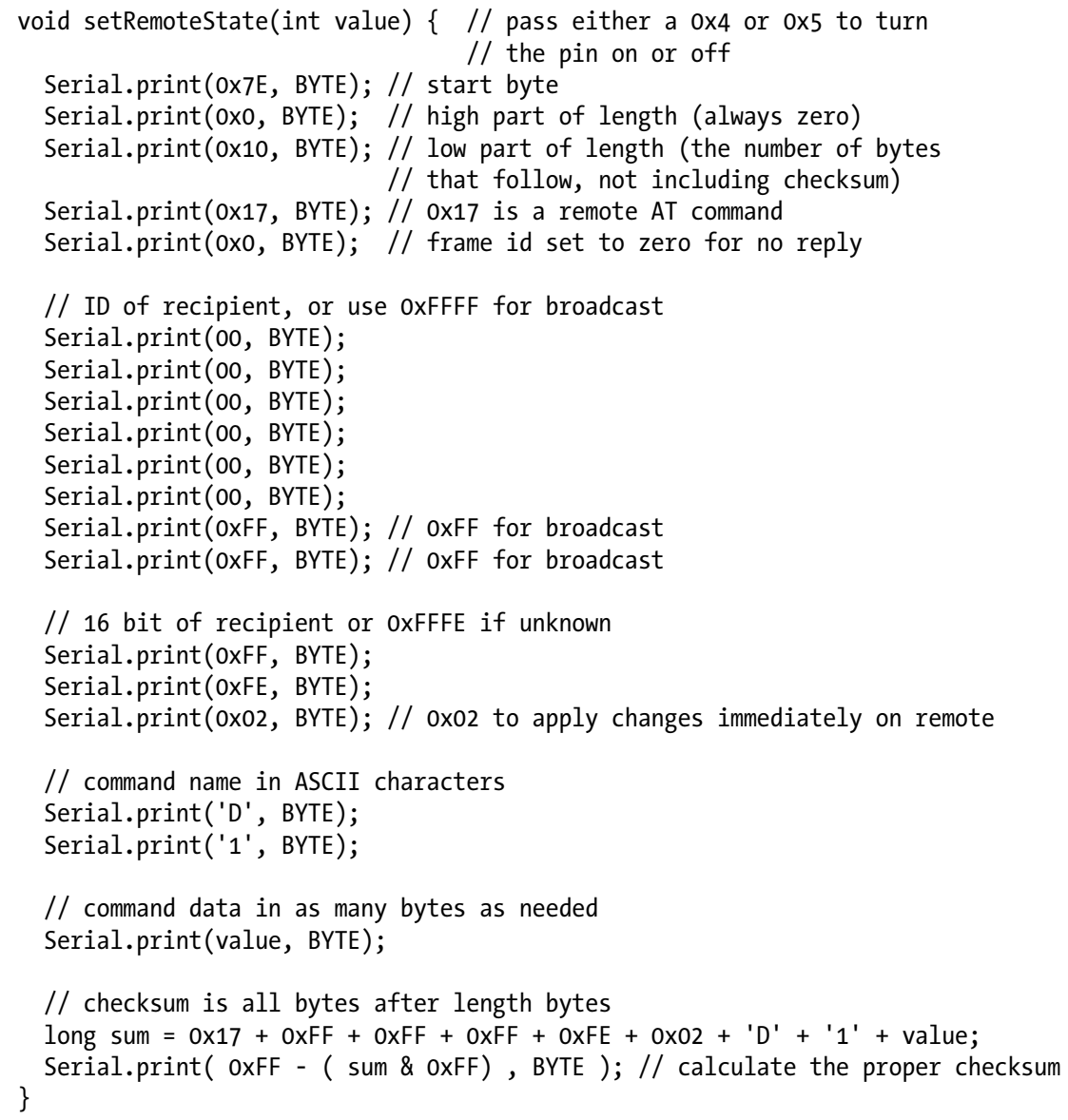




\section{Libraries}

Another way to take advantage of the API is to use a software library that parses the API frames for you and presents the internal information in a slightly more humanfriendly format. The upside is that everything is already written for you. The downside is that you may need to dig through considerable documentation to find the methods and attributes that apply to your situation. And you will definitely be glad that you know a thing or two about API frame format because most of these libraries use structures and terminology that exactly mirror it:

\section{Arduino $\mathrm{E} \mathrm{C} / \mathrm{C}++$}

This library by Andrew Rapp offers full support for both Series 1 and Series 2 XBee hardware in Arduino. It can be ported relatively easily into pure $\mathrm{C} / \mathrm{C}++$ environments as long as they support serial available/read/write/flush. (http://code.google .com/p/xbee-arduino/)

\section{Processing E Java}

Another good library by Andrew Rapp is written for use in Java and can be ported over to work well in Processing, as we have done for the example that concludes this chapter. (http://code.google.com/p/xbee-api/)

\section{Python}

Amit Snyderman created a library for Python environments that has been developed with significant contributions from several other developers. It requires the pySerial library. (http://code.google.com/p/python-xbee/)

Max/MSP

A community-developed patch is available for reading API info into the Max/MSP graphical programming environment for multimedia. (http://www.faludi.com/ xbee/max)

\section{PureData}

A similar patch has been ported over to the open source Pure Data multimedia graphical programming environment. (http://www.faludi.com/xbee/pd)

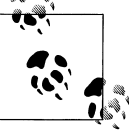

You should be aware that there are two slightly different API frame specifications used by these libraries. The first one is the default, preselected with ATAP 1 . The second can be selected by setting ATAP to 2, and it uses what are called "escaped" characters that avoid any possible confusion between data and control characters. The Java library we use in the example below does employ API operation with escaped characters. However, outside of being sure to select the proper ATAP setting when you configure your coordinator radio, you won't need to do anything differently in your code since the library will handle all the escaping for you. 


\section{Simple Sensor Network}

This project can serve as a model for almost any sensor network you'd like to build. You will create a set of inexpensive temperature sensors that are mesh-networked together to stream their data to a base station radio. This base station will be connected to a computer where the real-time temperature data will be visualized on the screen. In the next chapter we will discuss making the sensor nodes very power-efficient so that they can be run effectively from batteries. For now, these nodes can be powered from wall outlets so we can concentrate on the business of building our first complete wireless sensor network.

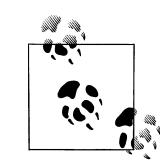

The example project in Figure 5-3 shows two sensor nodes and a base station. That's three radios in total. If you have only two radios, you can build it with a single sensor node and the base station. You can also create many more sensor nodes. If your network has more than 10 nodes, remember to extend the Processing code to change the display size and limits so that it can show all the data.

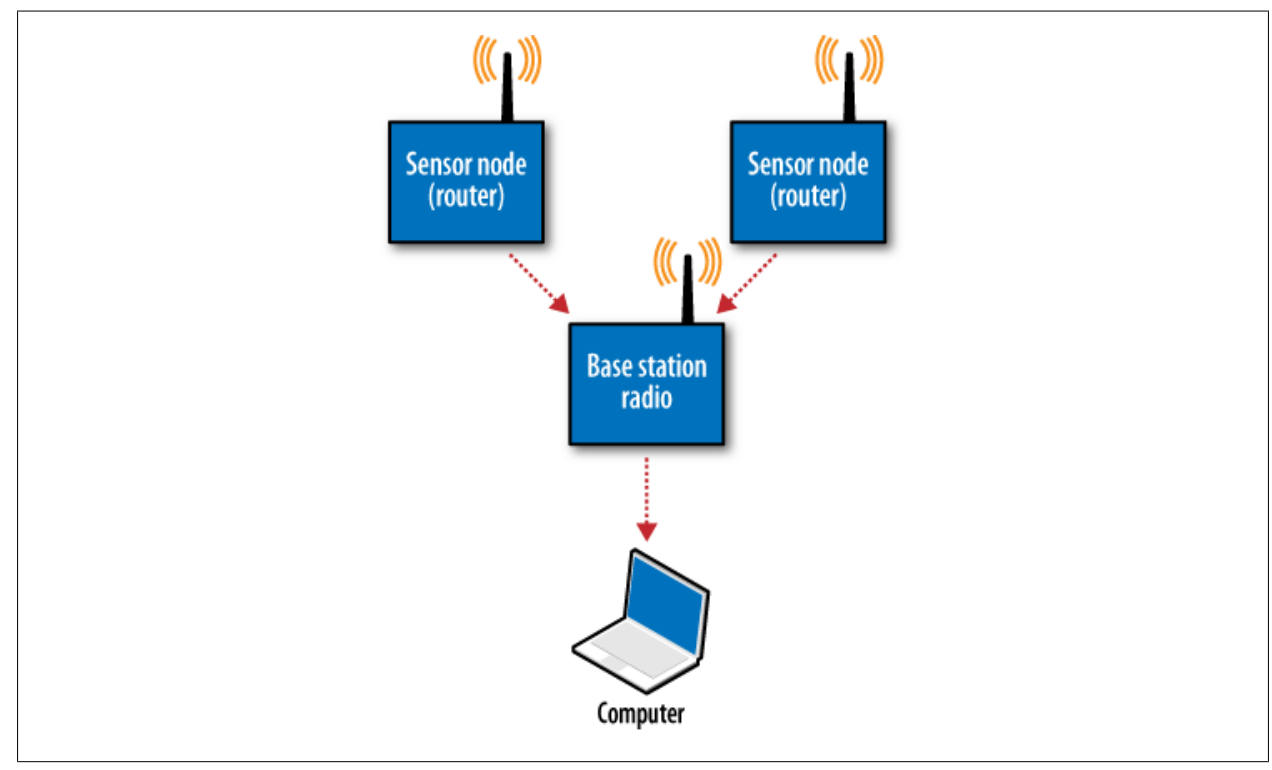

Figure 5-3. Simple sensor network

\section{Parts}

- Two solderless breadboards (AF 64, DK 438-1045-ND, SFE PRT-09567)

- Hookup wire or jumper wire kit (AF 153, DK 923351-ND, SFE PRT-00124) 
- Two 9-volt or 5-volt power supplies (9-volt batteries also work well for short-term use) (AF 63, or 80 with 9 V battery, RS 273-355, SFE TOL-08269 or TOL-00298)

- Two $3.3 \mathrm{~V}$ voltage regulators (TO-220 package) (DK 497-1491-5-ND, SFE COM-00526)

- Two DC power jacks (2.1 mm ID, 5.5 mm OD) (DK CP-024A-ND, RS 274-1577, SFE PRT-00119)

- Two 100K ohm resistors (DK P100KBACT-ND, RS 271-1347, SFE has an assorted resistor kit: COM-09258)

- Two 200K ohm resistors (DK P200KBACT-ND, or use two 100K resistors in series for each board)

- Two 300 ohm resistors (DK P300BACT-ND, RS 271-012)

- Two $10 \mu$ F electrolytic capacitors (DK P966-ND, RS 272-1025, SFE COM-00523)

- Two $1 \mu \mathrm{F}$ electrolytic capacitors (DK P993-ND, RS 272-1434)

- Two LM335 temperature sensors (in TO-92 packaging) (DK 497-2977-5-ND, SFE SEN-09438)

- One XBee radio (Series 2/ZB firmware) configured as a ZigBee Coordinator API mode (Digi: XB24-Z7WIT-004, DK 602-1098-ND)

- Two XBee radios (Series 2/ZB firmware) configured as a ZigBee Router AT mode (Digi: XB24-Z7WIT-004, DK 602-1098-ND)

- Two XBee breakout boards with male headers and $2 \mathrm{~mm}$ female headers installed (AF 126 (add SFE PRT-00116), SFE BOB-08276, PRT-08272, and PRT-00116)

- XBee USB serial adapter (XBee Explorer, Digi Evaluation board, or similar) (AF 247, SFE WRL-08687)

- USB cable for XBee adapter (AF 260, SFE CAB-00598)

- Wire strippers (AF 147, DK PAL70057-ND, SFE TOL-08696)

\section{Prepare Your Coordinator Radio}

1. Follow the instructions under "Reading Current Firmware and Configuration" on page 35 in Chapter 2 to configure one of your radios as a ZigBee Coordinator API. Note that your coordinator radio must use the API firmware for this project to work because I/O data is only delivered in API mode. Be sure to select the API version for your coordinator!

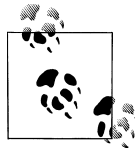

When you change from AT to API mode using X-CTU, you may get an error message that the radio is no longer communicating.

1. Go back to the PC Settings tab and check the Enable API box to enable communications with your radio. When you later change API mode to 2, go back to that tab and choose "Use escape characters $($ ATAP $=2)$." 
2. Once a radio has been set to API mode it can only be configured in X-CTU. You will not be able to make adjustments to this radio's configuration in CoolTerm. Use X-CTU to configure the coordinator with a PAN ID (between 0x0 and OxFFFFFFFFFFFFFFFF) that you've selected, then click Write. Write down this PAN ID so you can program your router radio with the same one. Every radio in your network must use the same PAN ID so that they can communicate with each other (there's no need to set the DH and DL in this case, because the coordinator will only be receiving data, not sending it):

Pan ID:

3. The software libraries that we are using in Processing require that the base station XBee be in API Mode 2 (API Operation with escaped characters). Use X-CTU to set AP (API Enable) to 2, and Write the configuration to your radio.

Be sure that you set the coordinator's API to mode 2, otherwise the project will not work!

\section{Prepare Your Router Radios}

1. Follow the instructions under "Reading Current Firmware and Configuration" on page 35 in Chapter 2 to configure each of your sensor node radios as a ZigBee Router AT. Your router radios will use the AT firmware so that you can easily configure them using a serial terminal. Be sure you select the AT version for your routers!

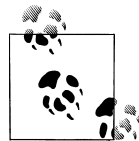

When you change from an API radio to an AT radio, you may get an error message that the radio is no longer communicating. If so,

. go back to the PC Settings tab and uncheck the Enable API Mode box.

2. Label the coordinator radio with a "C" so that you know which one it is later on. Each router radio can be labeled with an "R."

\section{Prepare the Sensor Boards}

We'll begin by configuring the router XBees. We'll use the CoolTerm terminal program and an XBee Explorer USB adapter again to set up your radios. For each of your sensor node radios:

1. Select a router XBee you've labeled with an "R" and place it into the XBee Explorer.

2. Plug the XBee Explorer into your computer. 
3. Run the CoolTerm program and press the Options button to configure it.

4. Select the appropriate serial port, and check the Local Echo box so you can see your commands as you type them.

5. Click on the Connect button to connect to the serial port.

6. Type +++ to go into command mode. You should receive an OK reply from the radio.

7. Select the same PAN ID you entered for your first radio above.

8. Type ATID followed by the PAN ID you selected and press Enter on the keyboard. You should receive OK again as a reply.

9. Every ZigBee coordinator always has 0 as its 16 -bit network address. In fact, that's the default destination address for any newly configured XBee radio. To use 16-bit addressing, the high part of your radio's destination address will be zero. Type ATDH $\mathbf{0}$ and press Enter on the keyboard. You should receive an OK response.

10. Enter ATDL followed by the low part of your radio's destination address, in this case also a zero because that's the fixed address for the coordinator. Type ATDL $\mathbf{0}$ and press Enter. You should receive an OK response.

11. Enter ATJV1 to ensure that your router attempts to rejoin the coordinator on startup.

12. Enter ATD02 to put pin 0 in analog mode.

13. Enter ATIR3E8 to set the sample rate to 1,000 milliseconds (hex 3E8).

14. Save your new settings as the radio's default by typing ATWR and pressing Enter.

It's not a bad idea to recheck your configurations after you enter them. For example, to recheck that you entered the destination address correctly, from command mode type ATDL and press Enter to see the current setting.

\section{Connect voltage regulator circuit and power jack to breadboard}

1. Wire up a breadboard with a 3.3-volt voltage regulator (LD1117V33) as shown in Figure 5-4. The regulator has three legs - typically, ground, output, and inputwhen viewed from the front (where the writing is). Sometimes these legs are in a different order, so find and check the data sheet if you're not sure! Input is where a high voltage, for example 5 or 9 volts, is applied to the regulator. Output is where you will get the regulated 3.3 volts. Ground is the common ground for your entire circuit, including input, output, and all the other components. Bring ground out to both blue ground rails that run along the sides of your breadboard. Bring 3.3volt output power to both of the red power rails. 


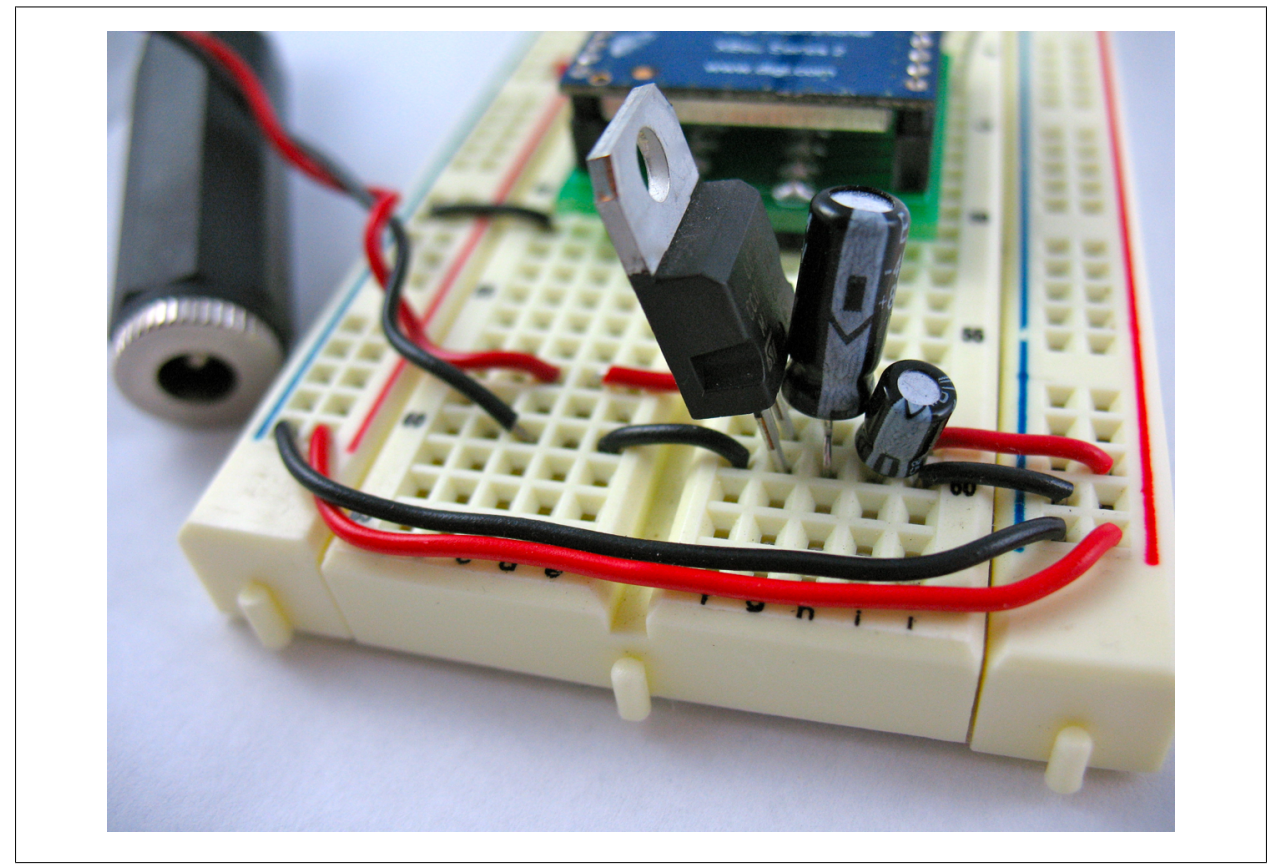

Figure 5-4. Voltage regulator circuit on breadboard

2. Solder a red wire (about $10 \mathrm{~cm}$ ) to the short center pin of your power jack, and solder a similar black wire to the longer outer pin, as shown in Figure 5-5. Don't allow the two connections to touch each other since that will create a short circuit when you power up!

3. Attach the red wire from the power jack, using the breadboard to connect it to the input pin of the voltage regulator. Attach the black ground wire to the ground pin of the voltage regulator in the same way.

4. Hook up the output pin of the voltage regulator to one of the power rails of the breadboard using a red wire. Hook up the ground pin to one of the ground rails on the breadboard.

5. Use the two capacitors to "decouple" the power supply in the following way: attach the short ground lead of the $10 \mu \mathrm{F}$ capacitor (also marked with a stripe on the capacitor's ground side) to ground near the voltage regulator. Attach the other positive lead of the $10 \mu \mathrm{F}$ capacitor to the voltage regulator's input pin. This will remove some lower-frequency noise coming from the wall power supply. Also attach the short ground lead of the $1 \mu \mathrm{F}$ capacitor to ground, and the other positive lead to the $3.3 \mathrm{~V}$ output pin. This will remove some higher-frequency noise coming out of the voltage regulator. Decoupling will prevent noisy power from reaching your radio and interfering with its signal. 


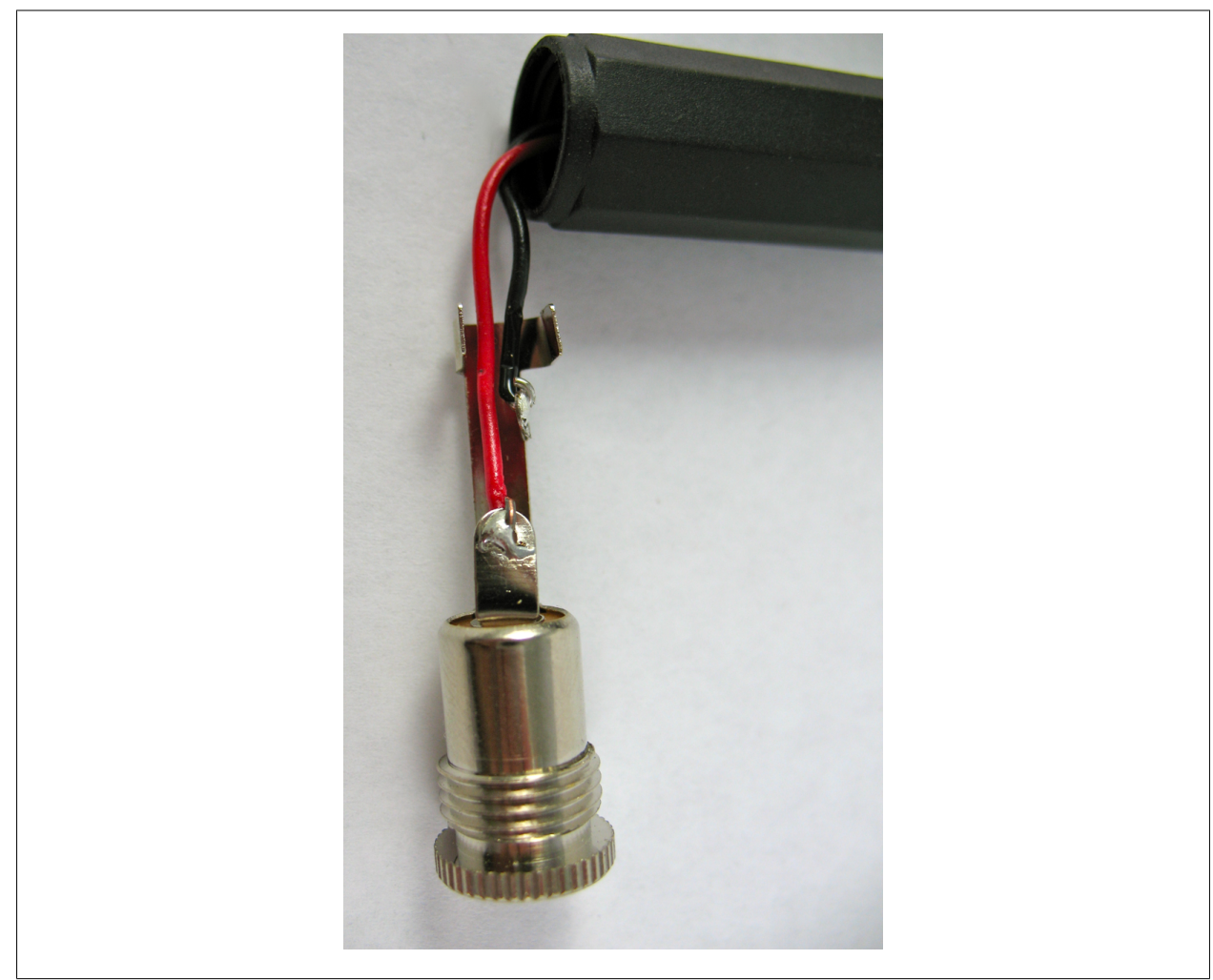

Figure 5-5. Power jack with wiring soldered in place

6. Hook up power and ground across the breadboard so that the rails on both sides are live.

It's a really good idea to check the voltage levels using a multimeter after you first wire up the breadboard for power. Make sure that your power rails have 3.3 volts on both sides where you expect it. You don't want to send 9 volts to your radio and cook it!

\section{Router XBee connection to power}

1. With a router XBee mounted on its breakout board, position the breakout board in the center of your other breadboard so that the two rows of male header pins are inserted on opposite sides of the center trough.

2. Use red hookup wire to connect pin 1 (VCC) of the XBee to 3.3-volt regulated power.

3. Use black hookup wire to connect pin 10 (GND) of the XBee to ground. 


\section{Temperature input}

This project uses the LM335 precision analog temperature sensor. This sensor has a linear output of $+10 \mathrm{mV}$ per degree Kelvin. It has an adjustment pin for calibration, but this can be safely ignored unless you mind one or two degrees of error at room temperature. You can get the entire data sheet for the LM335 at http://www.national .com/ds/LM/LM135.pdf:

1. The LM335 temperature sensor has three leads. When the sensor's flat side is facing you, the leads from left to right are adjustment, positive, and negative. Insert the LM335 so that each lead is in its own row on the breadboard.

2. Use a black wire to connect the rightmost, negative lead to one of the ground rails.

3. Insert the $300 \mathrm{ohm}$ resistor so that it's connected to power on one end and to the positive center pin of the LM335 on the other. You can use jumper wires to make the connection if that's more convenient in your breadboard layout.

4. Insert a $200 \mathrm{~K}$ ohm resistor so that it's connected to the positive center pin on one end and to an empty breadboard row on the other.

5. Use a jumper wire to connect between the unattached end of the $200 \mathrm{~K}$ ohm resistor to XBee digital input 0 (physical pin 20).

6. Insert a $100 \mathrm{~K}$ ohm resistor so that one end connects to XBee digital input 0 (and the $200 \mathrm{~K}$ resistor). The other end of the $100 \mathrm{~K}$ ohm resistor goes to ground. Again, use jumper wires as needed to complete these electrical connections.

\section{Second sensor board}

Create the second sensor board in the same way as the first. You can make as many sensor boards as you like. The system will work with as few as 1 or as many as 10 without any adjustment to the software. Figure 5-6 shows the breadboard layout for our simple sensor network, and Figure 5-7 shows the schematic.

\section{Prepare the Base Station}

\section{Connect to computer}

Your base station radio is simply an XBee serial adapter connected to your computer:

1. Select the coordinator XBee you've labeled with a " $\mathrm{C}$ " and place it into the XBee Explorer.

2. Plug the XBee Explorer into your computer. 


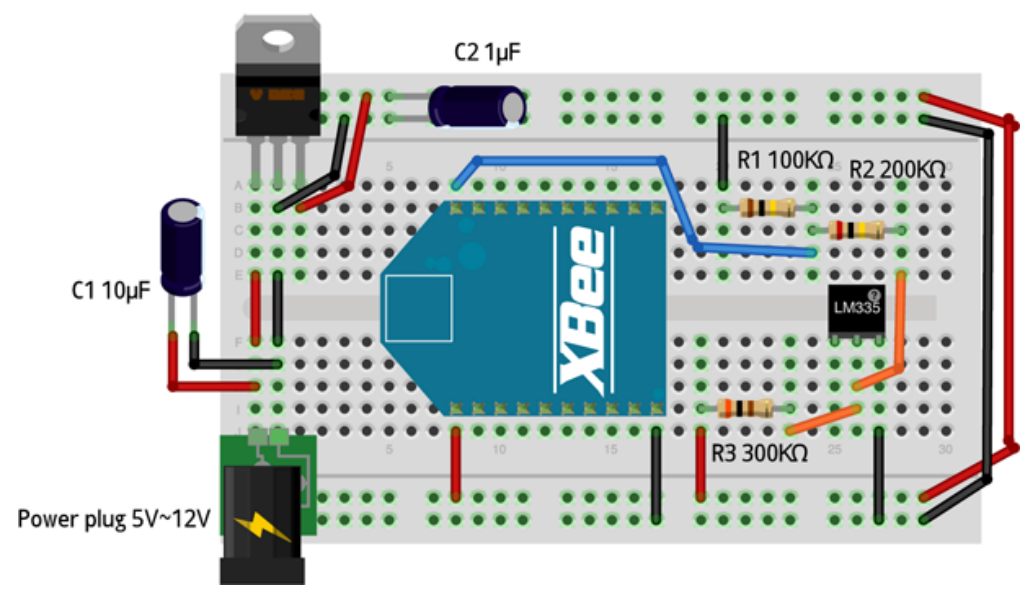

Figure 5-6. Simple sensor network LM335 breadboard layout

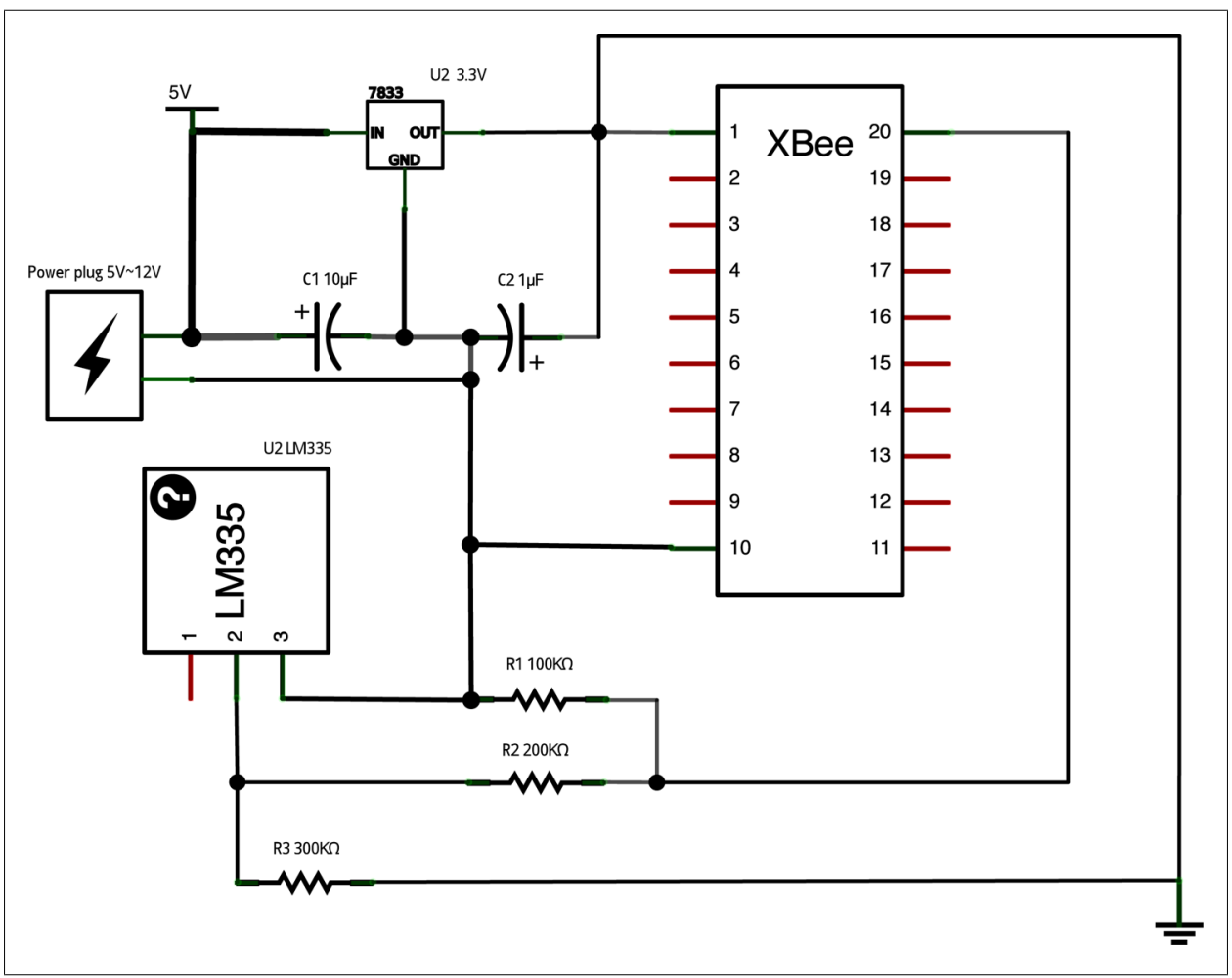

Figure 5-7. Simple sensor network LM335 schematic 


\section{All About Processing}

Processing is an open source software development environment designed for novice programmers and geared toward visual displays. It was originally created as a way of teaching beginning programmers and has evolved into a highly popular environment for artists, interaction designers, software hackers, students, and professionals to create visually oriented applications. Processing is available for free, under the GNU General Pubic License. It operates on Macintosh, Windows, and Linux and can export both fully functional web applets and standalone applications for all three platforms.

You can download Processing from http://processing.org/download (see Figure 5-8). On the Mac, you'll get a disk image $(. d m g)$ file that contains the Processing application. Simply drag it to your local Applications folder to install it and then double-click on the Processing icon to start the program. Windows downloads come in the form of a .zip file. Double-click the .zip file to open it, then drag the Processing folder into the Program Files directory (or any other location on your hard drive) and double-click processing.exe to begin. Linux users will download a tar.gz file. Processing can then be expanded in the terminal with tar $x v f z$ processing-xxxx.tgz (replacing $x x x x$ with the rest of the downloaded file's name, which is the version number). This will create a folder named something like processing-1.0. To start the program, change to that directory with cd processing-xxxx and run it with ./processing. If you run into any problems, check the troubleshooting page at http://wiki.processing.org/w/Troubleshoot ing for help.

The Processing Interactive Development Environment (IDE) is very easy to use, and has similar controls to the Arduino IDE. In fact, the Arduino development environment was based on Processing's and uses many of the same concepts, with one very important difference. Arduino is fundamentally a $\mathrm{C} / \mathrm{C}++$ environment, while Processing's underlying language is Java. So while the code syntax you use will look similar at first, be aware that there are distinct differences in the commands and structure. One good way to get to know Processing is by learning about it in the online Getting Started guide. The language itself is fully documented at http://processing.org/referencel (see Figure 5-9). There's also a comprehensive list of books at http://processing.org/learning/ books/, including Getting Started with Processing by Casey Reas and Ben Fry (O'Reilly) and Learning Processing by Daniel Shiffman (Kaufmann).

The Processing window has the same basic structure as Arduino's (see Figure 5-10). Programs are referred to as sketches. Buttons at the top allow the user to Run these sketches and Stop them; create New sketches; Open existing ones; Save them; and Export sketches as web applets. The center area is where code is edited, and the bottom of the window has a small gray space for messages, and for console output from the sketch. Sketches display visual output in a separate window. 


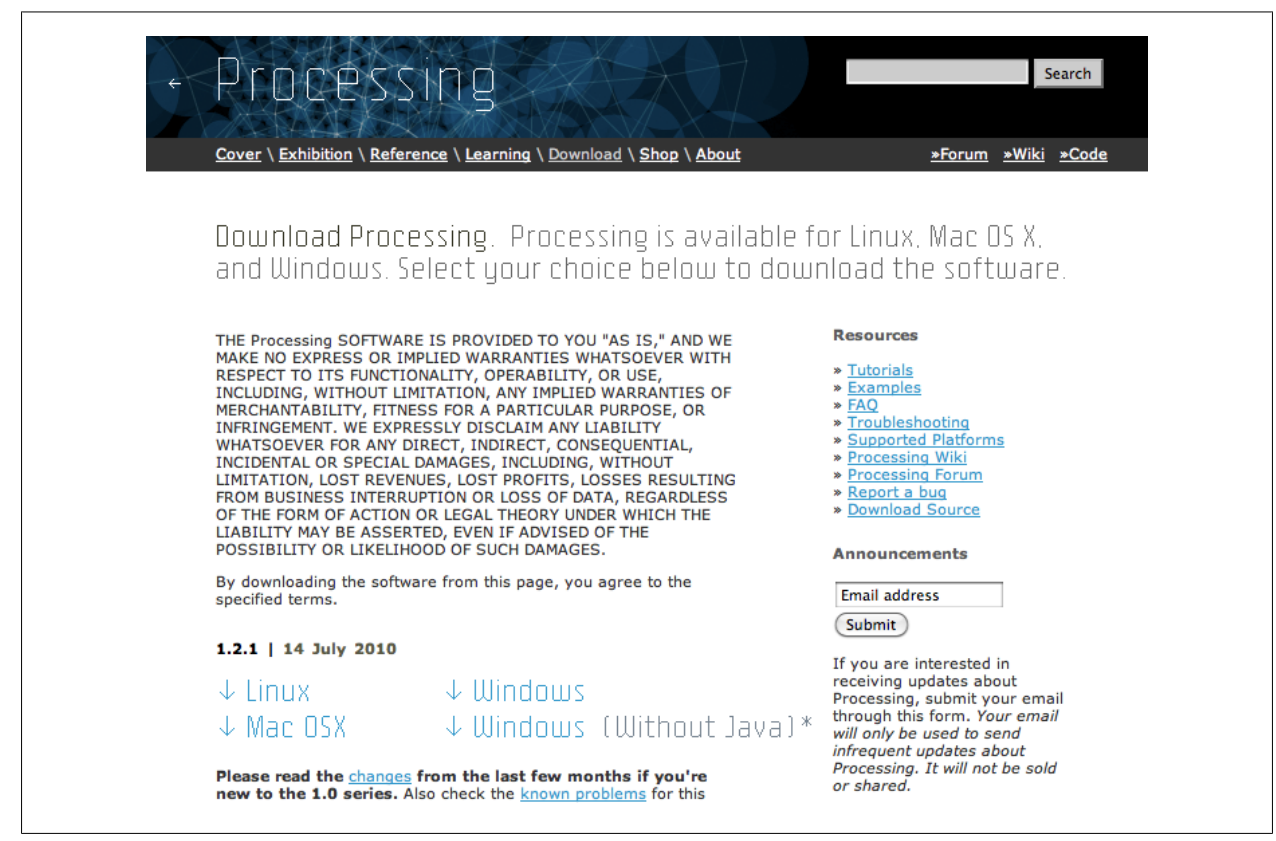

Figure 5-8. The Download page on the Processing.org website

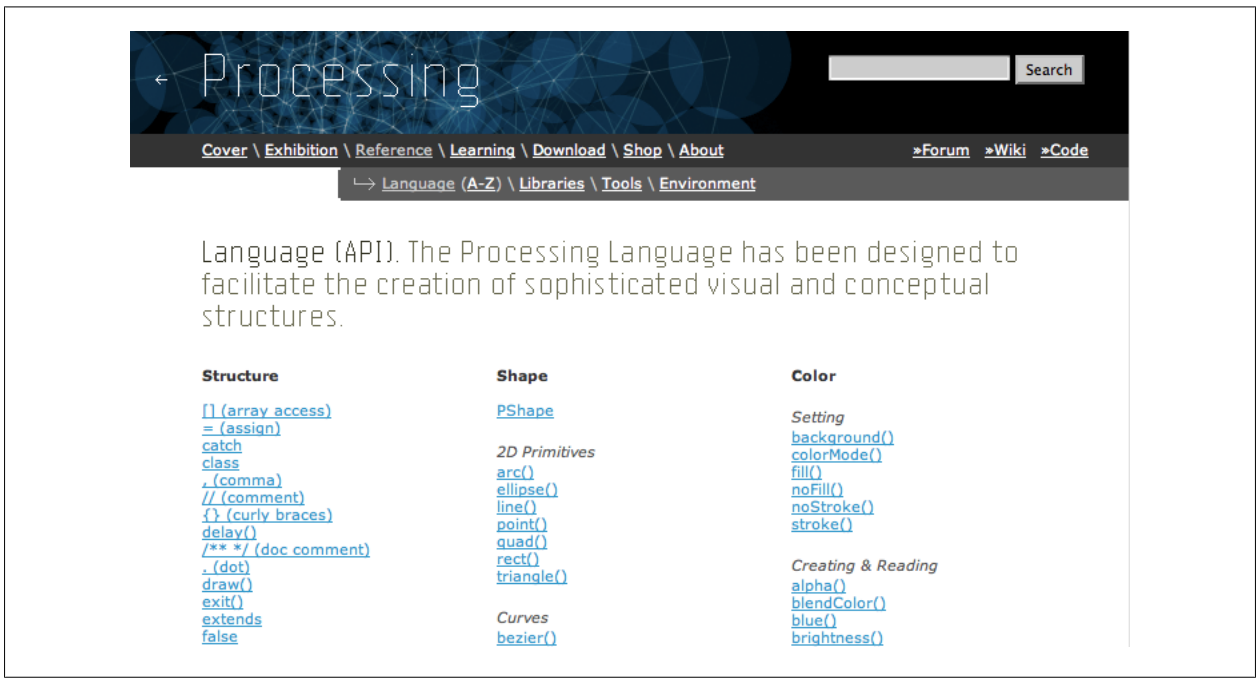

Figure 5-9. Processing's syntax and commands are fully documented on the website 


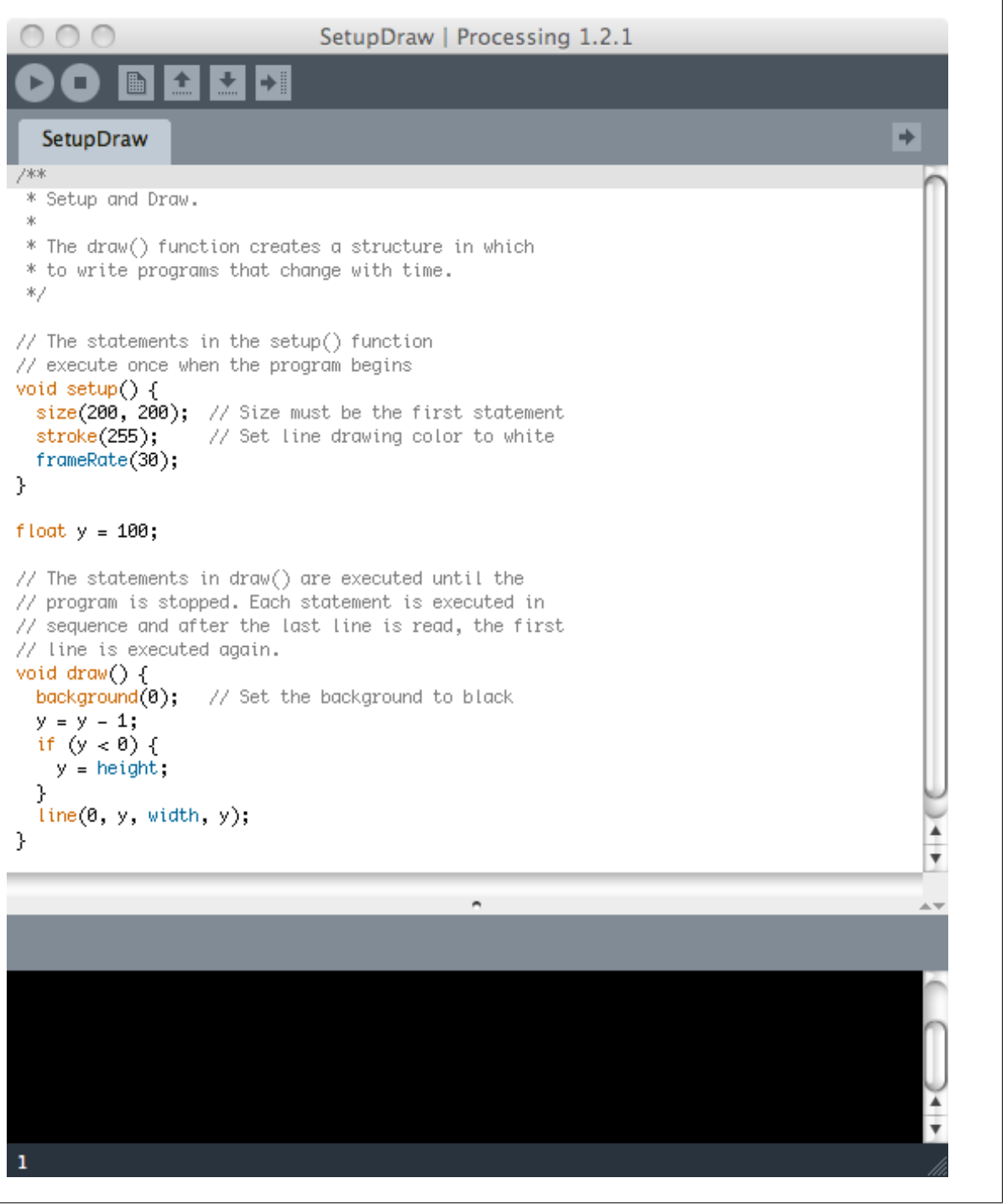

Figure 5-10. The Processing IDE with control buttons at the top, text area in the middle, and space for messages and console output at the bottom

\section{Program the Base Station}

The simple sensor network base station uses the following Processing program. Download the ZIP file of all the libraries and resources from this book's website. Inside the Processing sketch folder for the Simple Sensor Network program are two subdirectories called code and data (see Figure 5-11). The code folder contains the log4j.jar and xbee-api-0.5.5.jar library files. These contain all the code for communication with the 
XBee in API mode. The data folder holds the log4j.properties file, required by $\log 4$ j.jar. It also has a font file for a sans serif 10-point font used for screen display.

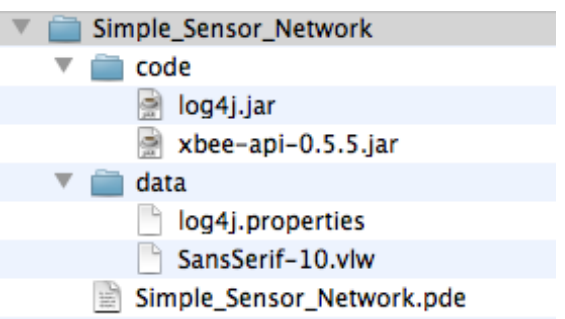

Figure 5-11. Directory structure for the Processing sketch program Simple Sensor Network, including all required libraries, config files, a font file, and the Processing ".pde” sketch itself

You must replace the COM port listed in this code with your actual COM port. Look for it in the code around line 20. Port names are listed in the console in Processing, as your program starts up.

Once you have loaded the files and directories onto your computer and opened the Simple_Sensor_Network.pde file in Processing, press the Run button (labeled with a triangle) to launch the display code. It will open in a new window and show a thermometer for each sensor node detected, as shown in Figure 5-12.

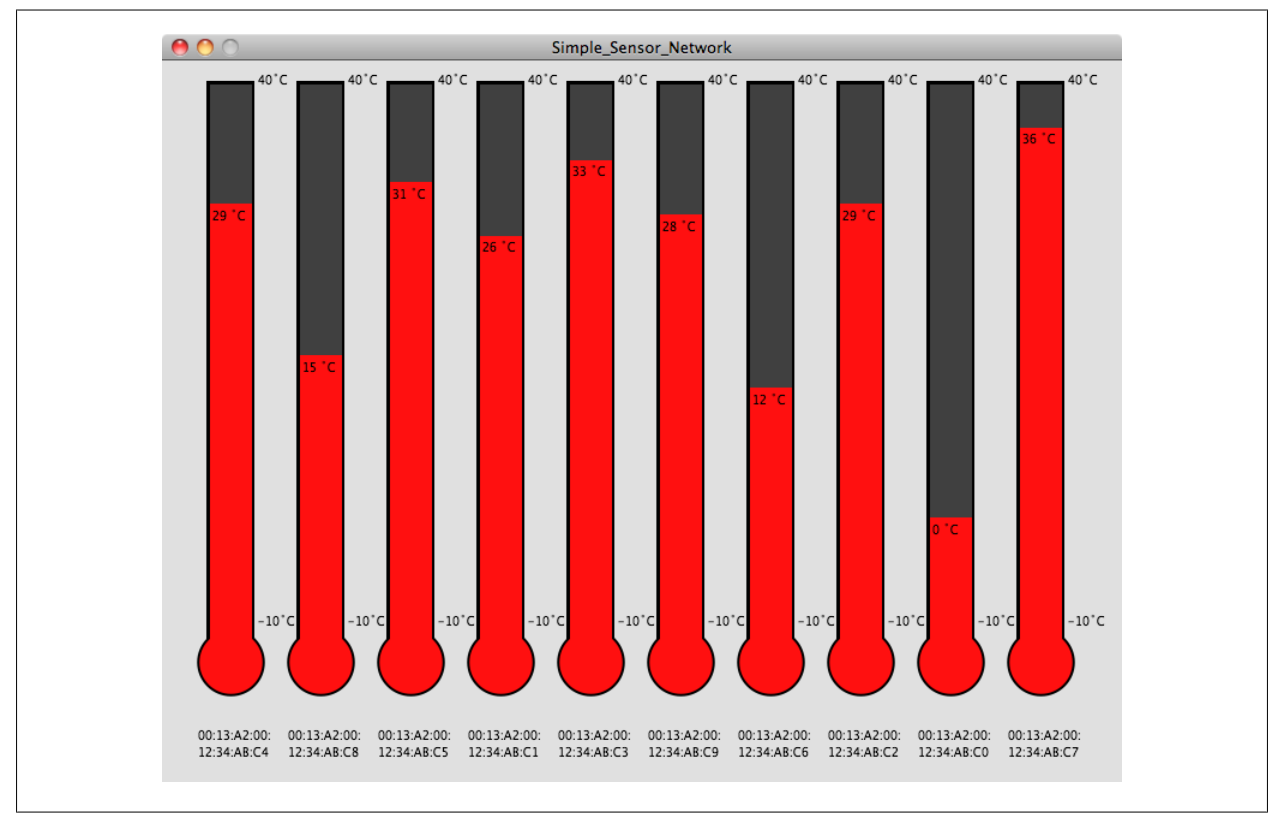

Figure 5-12. Simple Sensor Network temperature display screen in Processing 


\section{Simple Sensor Network display code in Processing}

Here's the source code for the Processing sketch. The comment shown in bold about the serial port highlights an essential change. Other commented instructions are only important if you didn't download the source from the website listed in the Preface:

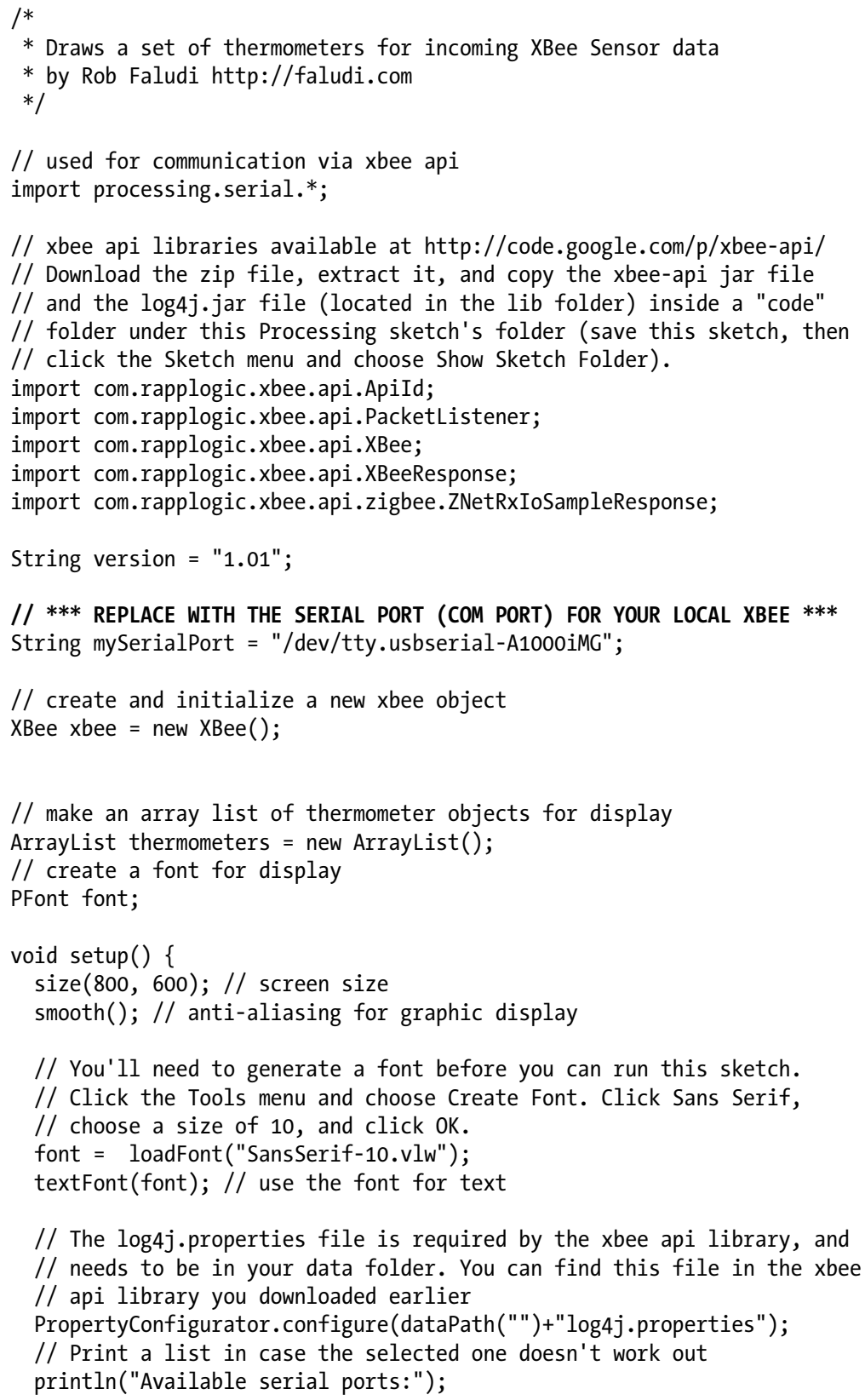




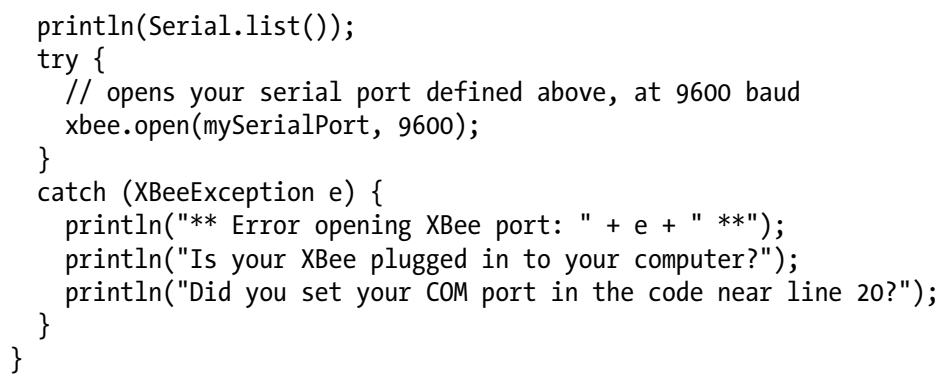




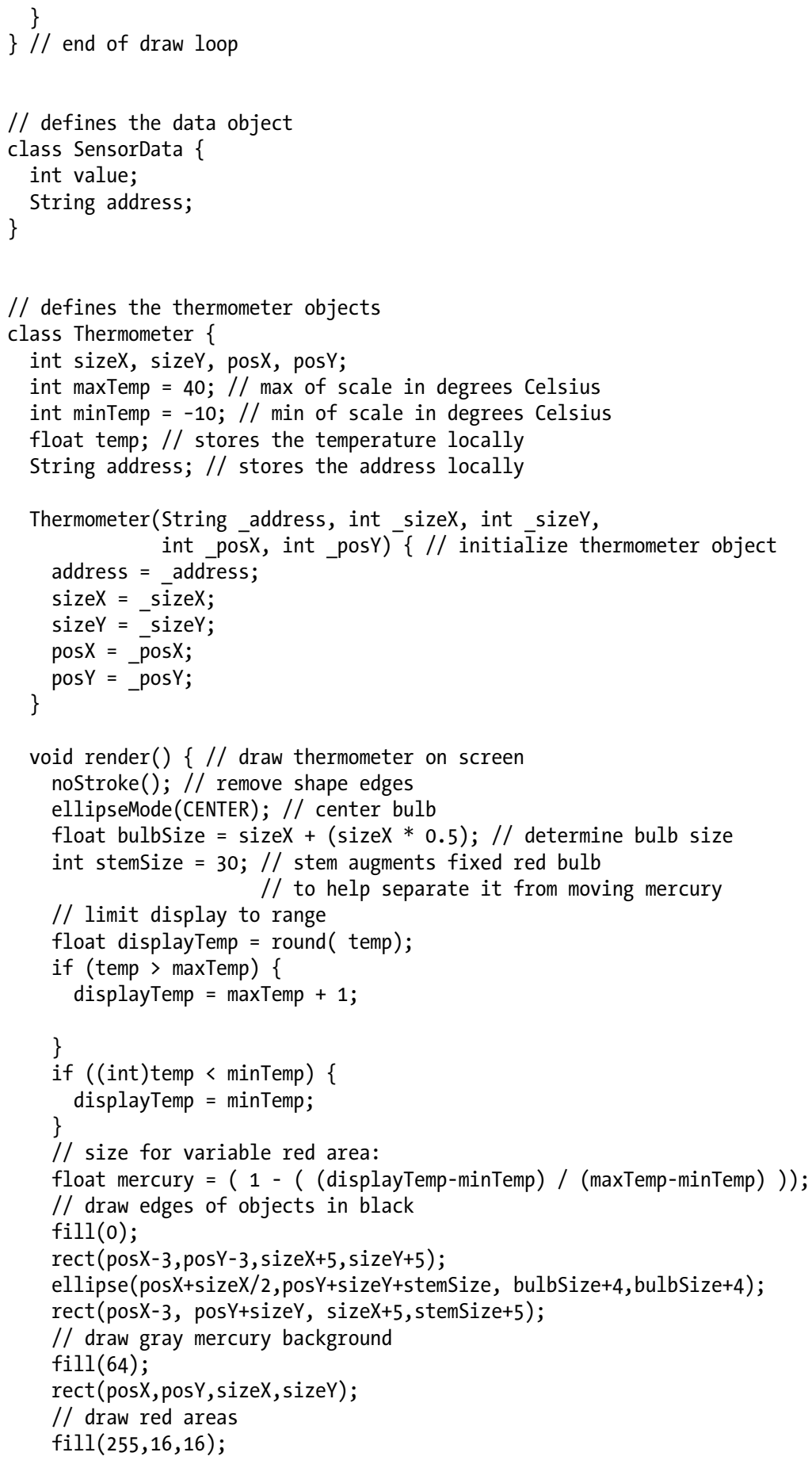




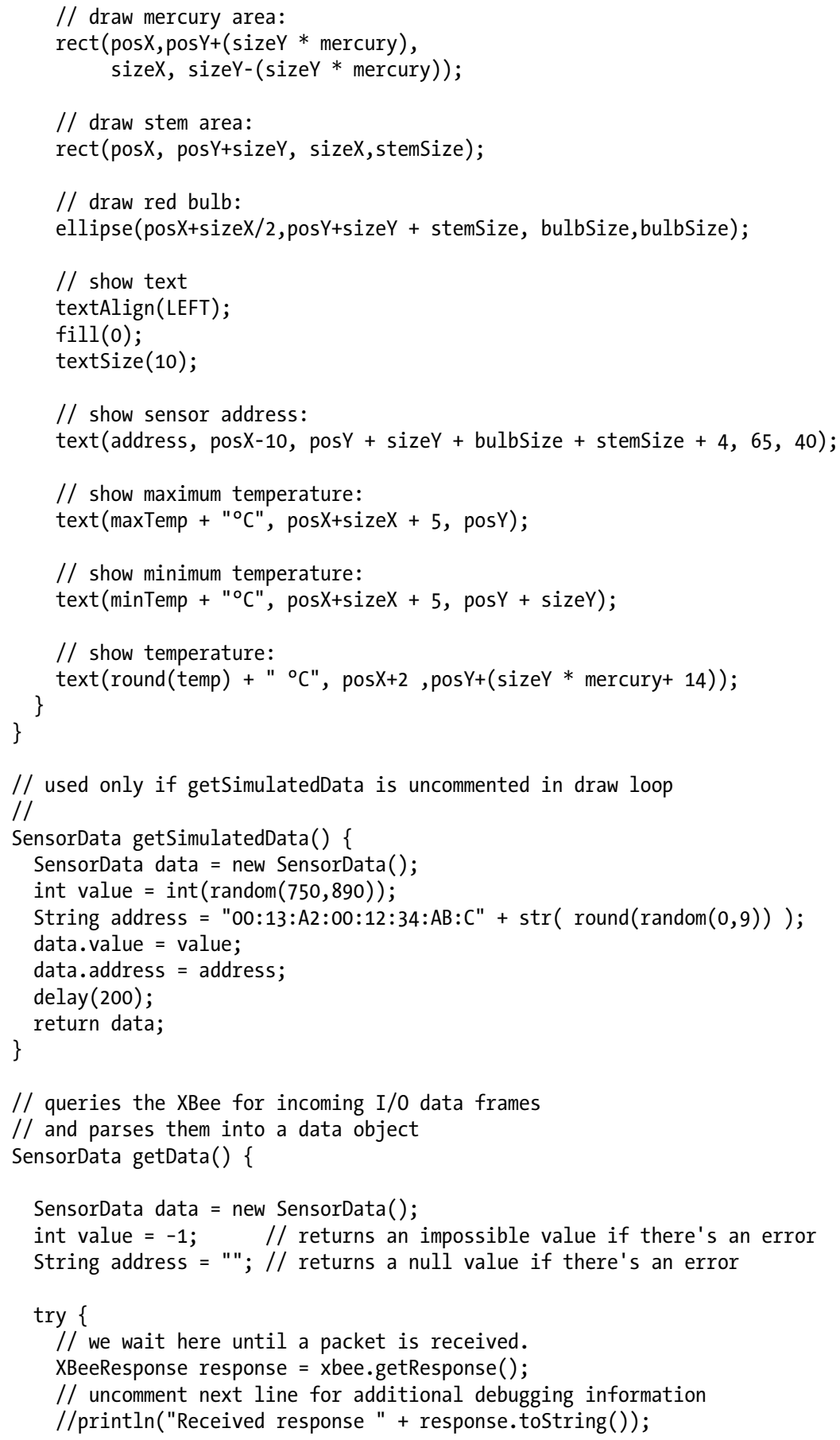




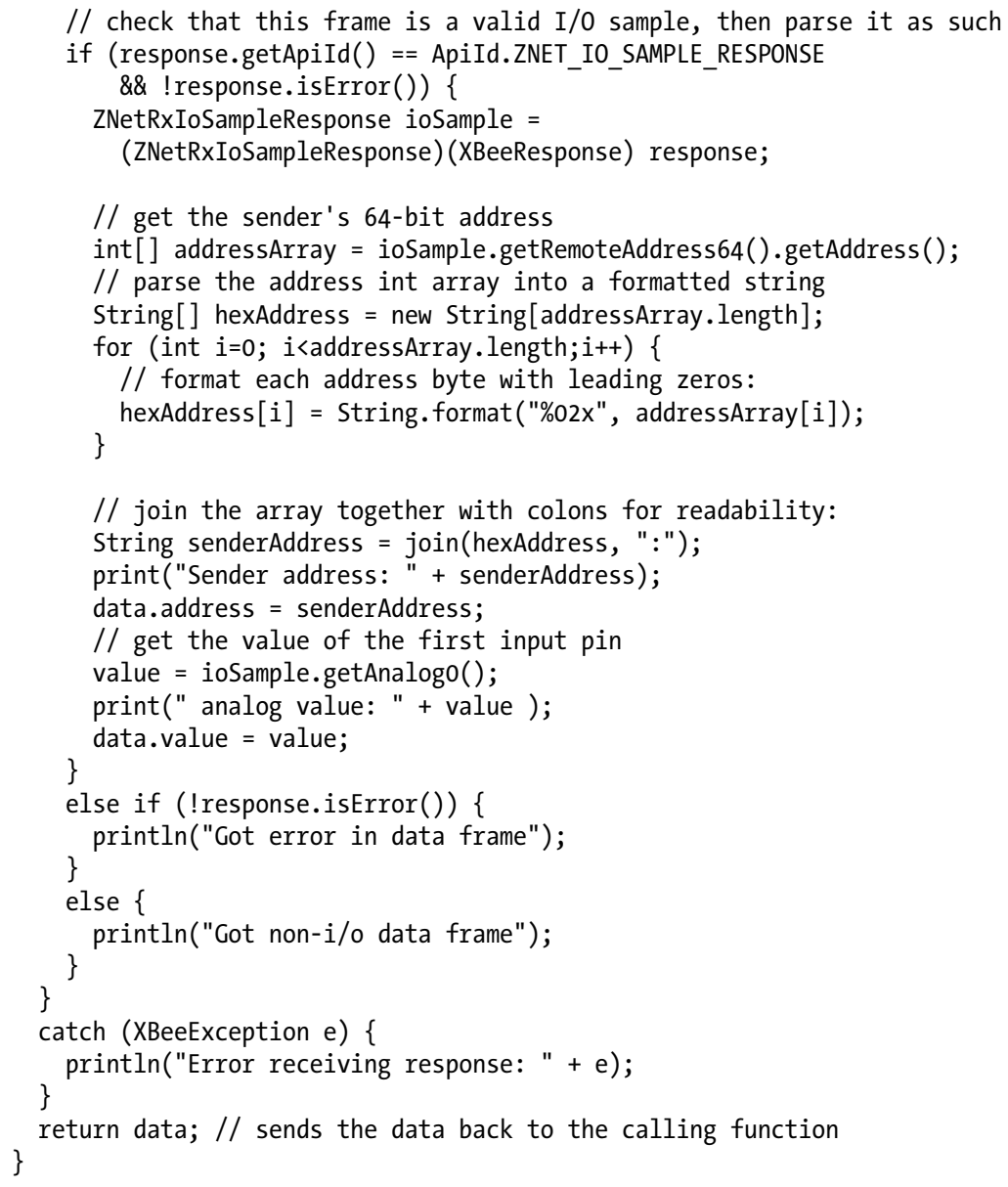

\section{Troubleshooting}

If things don't work at first, here are some steps to try:

1. Check all your electrical connections to make sure there are no loose wires and that all the components are connected properly.

2. Check the coordinator configuration in X-CTU again, including that the correct modem type (XB24-ZB) and function set (ZigBee Coordinator API) have been selected. Make sure that ATAP has been set to 2 for this project! Also check that the PAN ID is configured as you expect.

3. Check the router configuration in X-CTU to confirm that the correct modem type (XB24-ZB) and function set (ZigBee Router AT) have been selected. Also check that the PAN ID, destination high, and destination low are configured as you expect, and that ATJV, ATDO, and ATIR have been configured as described above. 
4. An LED placed from the ASSOC pin of each sensor XBee (physical pin 15) to ground should show a flashing light.

5. If your serial adapter has an RSSI light, it should illuminate when the radio is receiving information. If messages stop coming in, this light will time out and go dark after 10 seconds.

6. Use a multimeter to see if the voltage at the D0 pin of each sensor XBee (physical pin 20) varies with changes in the temperature. It should be somewhere in the range between 0 and 1.2 volts and change as you warm or cool the LM335.

7. If your temperature readings are somewhat off, you can calibrate the sensor by connecting a potentiometer across the LM335 with the output connected to the adjustment pin as in Figure 5-13. Also, check to see if it is next to another component on your circuit board that's generating a bit of heat, like the voltage regulator.

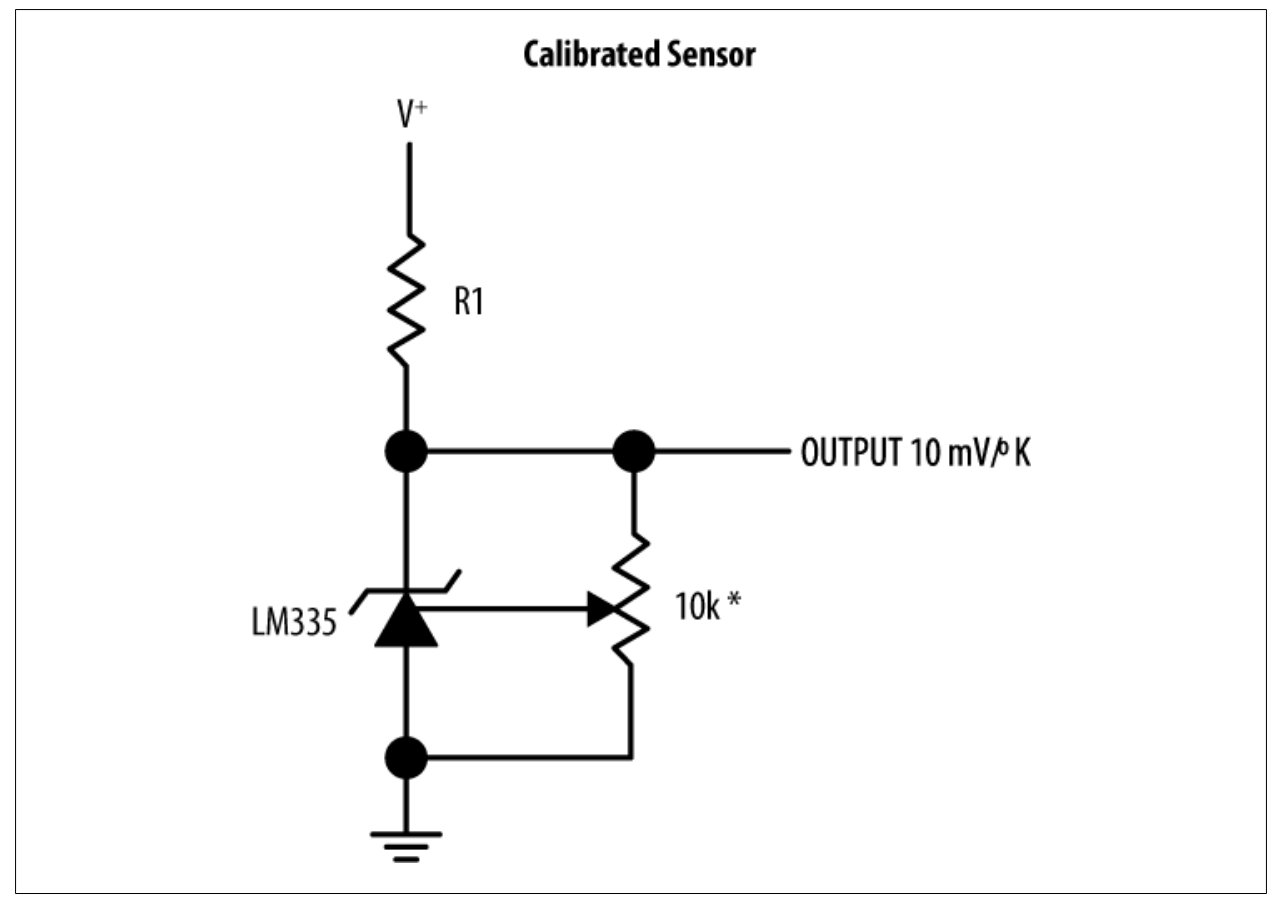

Figure 5-13. LM335 calibration schematic showing a potentiometer attached to power, ground, and the adjustment pin

8. We are not always able to see our own mistakes. Have a friend check everything for you. Sometimes only a second pair of eyes will catch the one or more issues that are standing in the way of success. 
9. When all else fails, try taking a break and coming back to the project after a good night's rest.

\section{Yay!}

You have learned a lot and built your very first complete wireless sensor network. Congratulations! While you won't always need to worry about the API mode, having some understanding of it will help you both in your work with the XBees and in general as new wireless communications protocols are developed and dispensed to the networking community. In the next chapter we will dial back the intensity a little to show you some useful power management tricks, and build a second network that allows you to control household appliances. Before that, take a moment to revel in securing your official Sensor Networking Merit Badge. You've earned it! 
CHAPTER 6

\section{Sleeping, Then Changing the World}

Whew! At this point, the book has covered a lot of ground and you have come a long way in a very short time. Now we'll ease the pace just a bit and address some nuances of ZigBee mesh networking, including sleep mode, end devices, and power management. Then it's time to change things in the world with direct actuation. This chapter features a powerful control project you could use to automate your home or to play Pong using the window lights in a skyscraper.

\section{Sleep Mode}

Going wireless often means cutting the electric cord. Projects that are mobile or remotely located frequently use batteries or another constrained power source that demands economizing on energy. XBee radios, like many other communications and microcontroller devices, can put themselves into a temporary sleep state where nearly no current is consumed. The trade-off is that during this state no activities can take place. During sleep, the device is almost completely turned off and is incapable of receiving or sending messages until it wakes back up. ZigBee mesh networking is specifically designed to smoothly handle communications on a network where at any moment many radios might be in this type of low-power state. In fact, by getting very stingy and using the right kind of power cell, we can envision ZigBee networks where batteries last many years at a time, while the networks still perform sufficient sensing and dependable actuation.

\section{End Devices}

We introduced end devices in Chapter 2. You may recall that end devices are essentially stripped-down versions of router radios. They can join networks and participate in communications, but because they power down intermittently, they explicitly do not act as messengers between any other devices. End devices always require a router or the coordinator to be their parent device. The end devices' parent helps them join the network to begin with and then stores messages for them when they are asleep. ZigBee 
networks may have any number of end devices. It's perfectly legitimate to create a network with a single coordinator acting as the sole parent for many end devices.

\section{Storing and forwarding}

When an end device is asleep, it is pretty much dead to the world. Any attempt to contact it by radio will fail because during hibernation the end device turns off its transmitter and receiver to conserve energy. That's why it needs a parent. One of the parent's jobs is to act as its mailbox, storing messages while the end device is asleep and forwarding them when the end device wakes back up. The portion of the protocol enabling network communications with sleeping end devices is automatically managed right inside the mesh network radios. ZigBee networks get this feature without any additional components or code required to manage the process.

\section{Constraints}

Alas, the power-saving lunch does not come entirely for free. The longer a node sleeps, the longer any message to it must be stored. XBee routers that act as the parent to end devices generally store only a single message, so communications must be carefully designed with those limitations in mind. Network chatter must be kept to a minimum to avoid overwhelming the parent device's storage resources and discarding important information. Also, radios configured as end devices are by definition incapable of acting as mesh routers themselves. After all, while the radio is asleep it is useless for retransmitting data and therefore presents a virtual cul de sac to the mesh network. On the upside, considerable power is conserved by forgoing these responsiveness and range extension features. You will want to carefully consider these trade-offs when designing your networks.

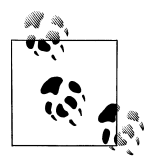

An XBee router must reserve memory to store mailbox data for each of its end device children. Usually the number of child nodes per router is

limited to about 8 or 10. If you like, you can check on how many remaining children a given router or coordinator will support with the ATNC command.

\section{Advantages}

Using sleep mode, an end device can stretch battery life from hours into days, weeks, and sometimes even years. For example, if full-time use of a (hypothetical) battery would drain it in four hours, putting the radio into a cycle where it slept for one second, then woke for one second before sleeping again could (roughly) double that battery's life to eight hours. Cyclically sleeping for 59 seconds and then waking for a single second might keep the same battery going for something on the order of 10 days. Taking this further, sleeping for the same 59.98 seconds and then only waking up for 20 milliseconds would extend the available power to over a year. Sleep mode can have tremendous 
benefits. Keep in mind, though, that real-world battery life predictions aren't quite so simple.

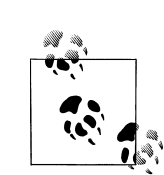

Batteries are physical, chemically active devices. Every type of battery chemistry has its own complex characteristics that bedevil any attempt to make simplistic calculations on power use. Factors like temperature, charge state, instantaneous draw levels, aging timetables, self-draining curves, and materials purity all weigh into the complex nonlinear functions required to properly estimate battery life. Nevertheless, sometimes simplistic estimations are all that's needed to select an appropriate sleep state that works within the context of your project. Build an ample safety factor into your back-of-the-napkin battery arithmetic and you'll find you can get a lot of reasonable estimations accomplished without getting mired in the awesome complexities of chemical engineering.

\section{Configuring Sleep}

There are six AT commands associated with sleeping (see Table 6-1). These commands work together to configure the specific behaviors that are most appropriate for your particular project. In many cases, you'll only really need to set three of them: Sleep Mode, Sleep Period, and Time Before Sleep. The other three commands are for more unusual configurations so they are less commonly needed.

\section{Sleep Mode}

The XBee ZigBee End Device radios have four basic sleep behaviors. These are set with the ATSM command:

\section{ATSM 0: Disables sleep mode}

The radio will always be awake and using power, but because it is running the end device firmware it will not route for other radios and it still requires a parent device.

ATSM 1: Pin wake mode

In this mode the XBee module will sleep when its sleep control pin-physical pin 9-is asserted or pulled high by connecting it to 3.3 volts (Figure 6-1). The radio will finish transmitting or receiving before it goes to sleep. When the sleep control pin is brought low by connecting it to ground, the radio will wake from sleep and be able to transmit and receive again. When asleep in this mode, the radio uses less than 10 microamps, a minuscule amount of power. Waking it up takes about 13 milliseconds. You use pin wake mode when there's another device-most commonly some kind of microcontroller - available to assert and de-assert the signal going to pin 9 .

\section{ATSM 2 and 3}

These modes are currently undefined. 


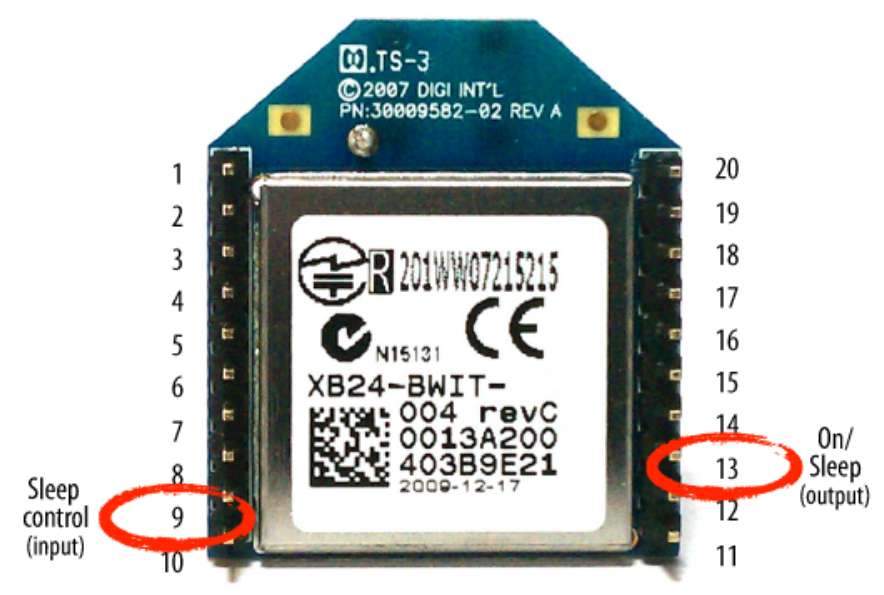

Figure 6-1. XBee sleep control pin 9 accepts external input for putting the module to sleep. The On/ Sleep pin 13 goes low when the radio sleeps and is brought high while the XBee is awake.

\section{ATSM 4: Cyclic sleep mode}

When an XBee radio is running independently and there is no other device to switch it in and out of sleep mode, it still has the internal capacity to sleep and wake on a fixed schedule. This is the most common way of conserving power when a radio is being used as a simple sensor node. You set how often the device sleeps and for how long using ATSP and ATST as described below. Upon wake-up, the end device will poll its parent to see if there are any incoming messages waiting for it. When asleep in cyclic mode, the radio uses less than 50 microamps, a very modest amount of power. Waking it up takes only 2 milliseconds.

ATSM 5: Cyclic sleep with pin wake

This is basically the same as regular cyclic sleep mode but with the option of also waking the module using physical pin 9.

\section{Sleep Period}

The length of time an XBee radio remains asleep is set with the ATSP command. The sleep period setting gets multiplied by 10 , with the result being the number of milliseconds of sleep time. SP will accept any setting in the hexadecimal range for $0 \times 20$ to 0xAF0. When multiplied by 10 , this means that the basic sleep period can be as short as 320 milliseconds and as long as 28 seconds. The sleep period can effectively be extended by setting the number of consecutive sleeps with ATSN and ATSO (as described below). 
The coordinator and router radios in an interactive network should have their SP register set to the same or greater value as the end devices. On parent nodes, SP defines how long to store messages in the forwarding buffer before discarding them. This way parents won't throw away messages before the child end-nodes have a chance to wake up and retrieve them.

\section{Time Before Sleep}

With the ATST command, you can set the minimum timeout for the radio to remain awake before returning into cyclic sleep. The XBee module will never go into its low power mode while a message is being transmitted or received, so think of this timeout as a period of silence that is required before the radio can fall asleep again. Time before sleep can be set to as little as $0 \mathrm{x} 1$ for one millisecond of timeout, or as high as $0 \mathrm{xFFFF}$ for about 65 seconds of delay before returning to low-power cyclic sleep.

\section{Advanced commands}

Several more sleep commands go beyond the basics. These are often used when an XBee radio is working with an external device, and controlling it with the XBee's internal timers. Imagine a weather station that might need to warm up for a short time before it is ready to supply a data sample. Alternately, imagine an application that uses the radio to remotely wake and activate a traffic information sign. If there's no incoming information, it's not necessary to wake the sign and consume valuable battery power for no reason. The following commands are intended to help in these special situations, and can also be employed in cases where a particularly lengthy sleep time is desired.

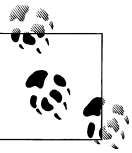

The On/Sleep pin, physical pin 13 on the XBee, goes high when the module is awake and low when it is sleeping. Attaching an LED to this pin gives a visual indication of the radio's current sleep state. This pin can also be used to control an external device-for example, so that it is powered up only when the radio is awake. (See Figure 6-1.)

\section{ATSN: Number of Sleep Periods}

This command specifies how many sleep periods to skip asserting the On/Sleep pin if no data is received on wake-up. Setting it to the default of $0 \mathrm{x} 01$ causes the On/Sleep pin to be asserted on every wake-up. Setting it higher-for example, to 0x09-would allow eight wake-ups with no incoming data to pass before asserting the On/Sleep pin. The maximum value for this register is $0 x F F F F$ or 65,535 wakeup checks before the On/Sleep pin is forcibly asserted. Remember that if incoming data is received during any wake-up, the On/Sleep pin will be brought high, no matter how ATSN is set. A secondary use of this register is as a multiplier for ATSP, when you need the radio to sleep for very long times. See ATSO below. 
ATWH: Wake Host

In some applications, a sensor or device might need time to be turned on, boot up, and stabilize before the XBee either sends received messages out of its local serial port or samples its local pins for I/O data. In these cases it's helpful to have a specific delay after the On/Sleep pin is asserted to turn on the attached device. The wake host delay can be as little as the default of $0 \mathrm{x} 0$ for no delay, or as long as $0 \mathrm{xFFFF}$ for about 65 seconds of waiting time before communication or I/O sampling.

ATSO: Sleep Options

There are currently three states for this register. $0 \mathrm{x} 0$ is the default setting of no options enabled. 0x02 tells the radio to always wake up for the entire ST time, even when no data is waiting to be sent or received. This is useful only in specialized external device control situations. The 0x04 option setting forces the radio to sleep continuously for the entire period specified by SN * SP, to a maximum total time of $1,834,980,000$ milliseconds or just over three weeks between wake-ups. This last option has the potential to allow the right battery to last for many years!

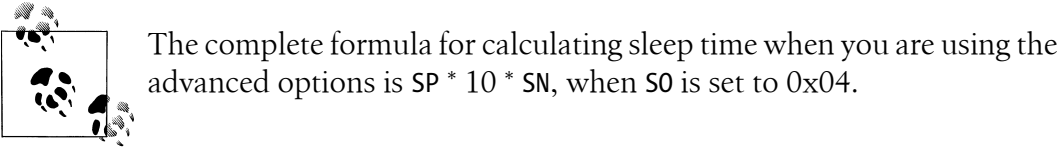

Table 6-1. Summary of AT commands for sleeping the XBee radios

\begin{tabular}{|c|c|c|c|c|}
\hline $\begin{array}{l}\text { AT } \\
\text { command }\end{array}$ & Name and description & $\begin{array}{l}\text { Node } \\
\text { type }\end{array}$ & Parameter range & Default \\
\hline \multirow[t]{4}{*}{ SM } & Sleep Mode. Sets the sleep mode on the RF module. & $\mathrm{E}$ & 0 - Sleep disabled & 0 \\
\hline & & & $\begin{array}{l}1 \text { - Pin sleep } \\
\text { enabled }\end{array}$ & \\
\hline & & & $\begin{array}{l}4 \text { - Cyclic sleep } \\
\text { enabled }\end{array}$ & \\
\hline & & & $\begin{array}{l}5 \text { - Cyclic sleep, pin } \\
\text { wake }\end{array}$ & \\
\hline SN & $\begin{array}{l}\text { Number of Sleep Periods. Sets the number of sleep periods } \\
\text { tonotassertthe On/Sleep pin on wake-upifnoRF data iswaiting } \\
\text { for the end device. This command allows a host application to } \\
\text { sleep for an extended time if no RF data is present. }\end{array}$ & CRE & 1-0xFFFF & 1 \\
\hline SP & $\begin{array}{l}\text { Sleep Period. Determines how long the end device will sleep } \\
\text { at a time, up to } 28 \text { seconds. (The sleep time can selectively be } \\
\text { extended past } 28 \text { seconds using the SN command.) On the } \\
\text { parent, this value determines how long the parent will buffer } \\
\text { messages for the sleeping end device. It should be set at least } \\
\text { equal to the longest SP time of any child end device. }\end{array}$ & CRE & $\begin{array}{l}0 \times 20-0 \times A F 0(\times 10 \\
\text { ms) (Quarter- } \\
\text { second resolution) }\end{array}$ & $0 \times 20$ \\
\hline ST & $\begin{array}{l}\text { Time Before Sleep. Sets the time-before-sleep timer on an } \\
\text { end device. The timer is reset each time serial or RF data is }\end{array}$ & $\mathrm{E}$ & $1-0 x F F F E(\times 1 \mathrm{~ms})$ & $\begin{array}{l}0 \times 1388(5 \\
\text { seconds) }\end{array}$ \\
\hline
\end{tabular}




\begin{tabular}{|c|c|c|c|c|}
\hline $\begin{array}{l}\text { AT } \\
\text { command }\end{array}$ & Name and description & $\begin{array}{l}\text { Node } \\
\text { type }\end{array}$ & Parameter range & Default \\
\hline & $\begin{array}{l}\text { received. Once the timer expires, an end device may enter low- } \\
\text { power operation. Applicable for cyclic sleep end devices only. }\end{array}$ & & & \\
\hline \multirow[t]{4}{*}{ so } & $\begin{array}{l}\text { Sleep Options. Configures options for sleep. Unused option } \\
\text { bits should be set to } 0 \text {. Sleep options include: }\end{array}$ & $\mathrm{E}$ & $0-0 x F F$ & 0 \\
\hline & 0X02 - Always wake for ST time & & & \\
\hline & 0x04 - Sleep entire SN * SP time & & & \\
\hline & Sleep options should not be used for most applications. & & & \\
\hline WH & $\begin{array}{l}\text { Wake Host. Sets or reads the wake host timer value. If the } \\
\text { wake host timer is set to a nonzero value, this timer specifies a } \\
\text { time (in millisecond units) that the device should allow after } \\
\text { waking from sleep before sending data out the UART or trans- } \\
\text { mitting an I/O sample. If serial characters are received, the } \\
\text { WH timer is stopped immediately. }\end{array}$ & $\mathrm{E}$ & $0-0 x F F F F(\times 1 \mathrm{~ms})$ & \\
\hline
\end{tabular}

\section{Easy Sleeping}

There are a lot of sleep options but the good news is that you only have to set them once for your application and, in most cases, you only need one or two settings. For instance, if we just want the XBee module to wake up briefly every five seconds, selecting ATSM 4, and ATSP 1F4 turns on cyclic sleep mode and sets the period of time the radio is asleep to 5000 milliseconds. Remember that all the commands use hexadecimals and that the SP register is always multiplied by 10 . So the hexadecimal $0 x 1 F 4$ translates to 500 in decimal, and when multiplied by 10 results in 5,000 milliseconds, or 5 seconds.

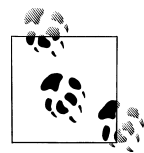

Waking up from sleep always triggers an I/O sample, as long as ATIR is set to a nonzero number and at least one pin is configured as a digital or analog input. Samples will continue at the IR rate until the ST timer has expired, and then the radio will sleep again.

\section{Simple Sensor with Sleep Project}

The simple sensor network from the previous chapter is a prime candidate for some power-saving assistance from sleep mode. You'll use the same base station configuration, but you can either add new end nodes to the network or replace your existing hardwired nodes with battery-powered ones. The following instructions are for a single sleeping node. Create as many of these as you like; just remember the base station still needs to be a coordinator radio, so its configuration should remain the same. 
The parts listed below supply enough battery voltage to continue using the voltage regulator circuits you already built. Alternatively, you could remove the voltage regulation and use two AA batteries to power the end nodes.

\section{Parts}

- 9-volt batteries (RS 23-866)

- 9-volt to barrel jack adapters (SFE PRT-09518)

...or

- AA batteries (RS 23-942)

- 4xAA to barrel jack connectors (SFE PRT-09835)

...and

- XBee radios (Series 2/ZB firmware) configured as a ZigBee End Device AT mode (Digi: XB24-Z7WIT-004, DK 602-1098-ND)

\section{Prepare Your End Device Radios}

Follow the instructions under "Reading Current Firmware and Configuration" on page 35 in Chapter 2 to configure each of your actuator node radios as a ZigBee End Device AT.

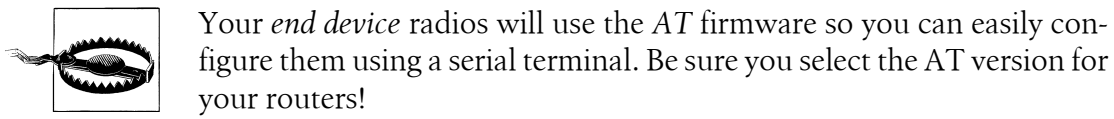

Each end device radio can be labeled with an "E."

\section{Configure Your End Device XBees}

We'll continue to use CoolTerm and an XBee Explorer USB adapter to set up the radios. For each of your sleeping end device sensor node radios:

1. Select an end device XBee you've labeled with an "E" and place it into the XBee Explorer.

2. Plug the XBee Explorer into your computer.

3. Run the CoolTerm program and press the Options button to configure it.

4. Select the appropriate serial port and check the Local Echo box so you can see your commands as you type them.

5. Click on the Connect button to connect to the serial port. 
6. Type +++ to go into command mode. You should receive an OK reply from the radio.

7. Select the same PAN ID that you entered for your original simple sensor network.

8. Type ATID followed by the PAN ID you selected and press Enter on the keyboard. You should receive OK again as a reply.

9. Every ZigBee coordinator always has 0 as its 16-bit network address. Type ATDH $\mathbf{0}$ and press Enter on the keyboard. You should receive an OK response.

10. Enter ATDL followed by the low part of your radio's destination address ( 0 , the address of the coordinator). Type ATDL $\mathbf{0}$ and press Enter. You should receive an OK response.

11. Enter ATD02 to put pin 0 in analog mode.

12. Enter ATIR3E8 to set the sample rate to 1,000 milliseconds.

13. Enter ATSM4 to put the radio into cyclic sleep mode.

14. Enter ATSP64 to sleep the radio for one second $(100 * 10 \mathrm{~ms})$.

15. Enter ATST14 to time out and sleep 20 milliseconds after each sample transmission.

16. Save your new settings as the radio's default by typing ATWR and pressing Enter.

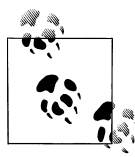

Once you put your radio into sleep mode it may appear to become unresponsive. That's because it is sleeping! Don't panic if you can't

. wake it up. See the sidebar "Wake-Up Issues and Reset Strategies" on page 170 for more information on waking a sleeping radio.

\section{Add sensor nodes...}

1. If you've decided to add new temperature sensor nodes, make them exactly like those in the previous chapter. The radios you just configured can be plugged right into these new sensor boards.

2. Power your new sensor boards with a battery pack to make them totally untethered.

\section{...or replace sensor nodes}

1. If you don't want to add any new sensor nodes, you can also replace the radios in your existing sensor boards with the ones you just programmed for sleep mode.

2. Power your newly sleeping sensor boards with a battery pack for full mobility.

3. Run the Simple Sensor Network program in Processing to test your new and/or replaced sensor nodes.

Figure 6-2 illustrates the simple sensor network with end devices. 


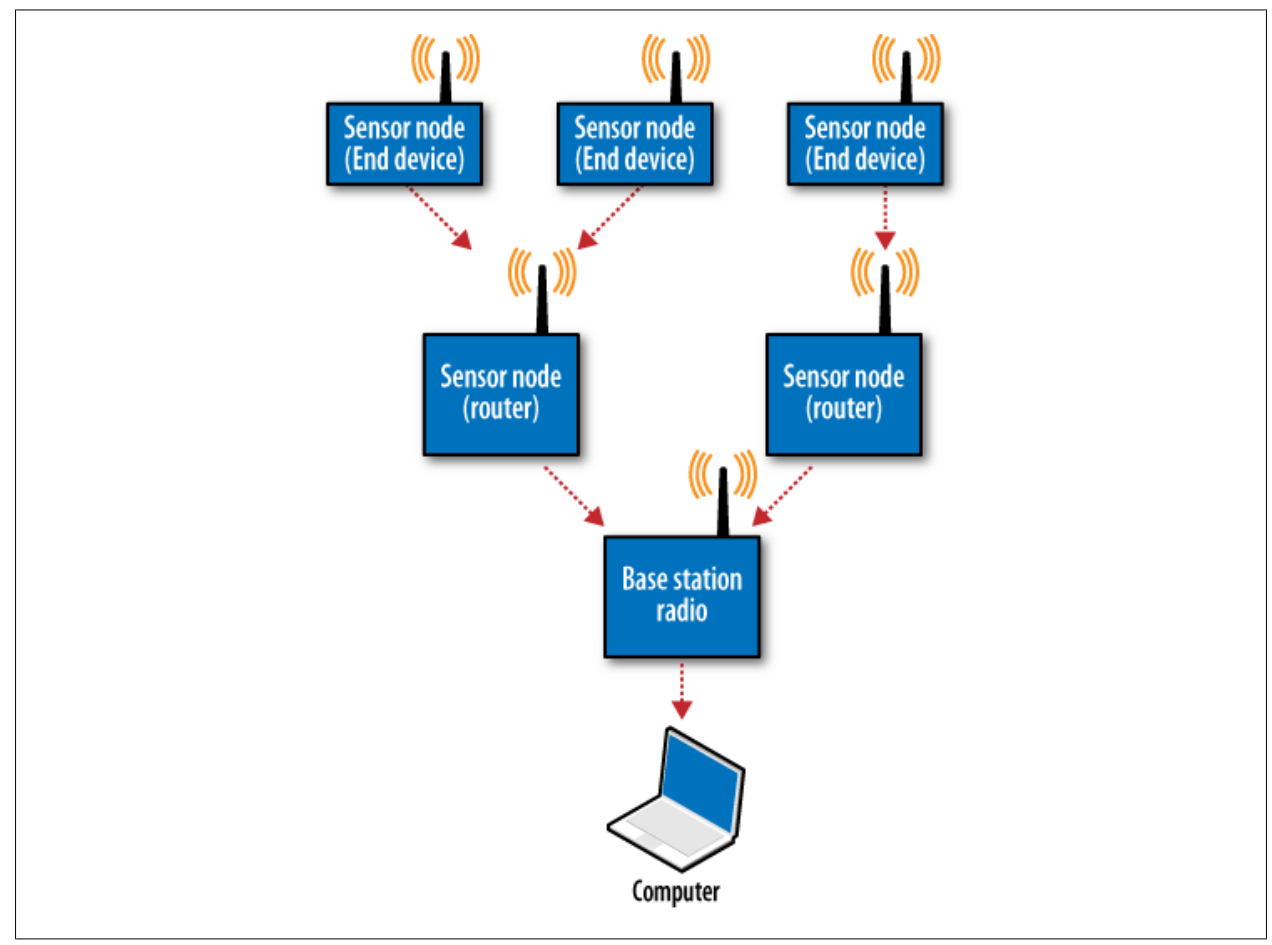

Figure 6-2. Simple sensor network with sleeping end devices

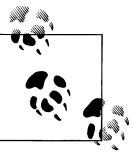

In the Simple Sensor with Sleep project, it is not necessary to add the SP setting to the parent routers or coordinator. This is because they

won't be storing any outgoing messages so the buffer timeout won't ever be used. However, it doesn't hurt and it is a good habit to have. Set ATSP to 1F4 on every device in the network, but only if you want to.

\section{Wake-Up Issues and Reset Strategies}

Once you set ATSM (sleep mode) to something other than zero, there will be times when the radio refuses to respond at all. This is because it is sleeping! Don't worry; you can still wake the radio up to talk to it. Here are some strategies to rouse your snoozing radio for reconfiguration:

- If the radio is in sleep mode 1 (pin sleep) or sleep mode 5 (cyclic sleep with pin wake-up), bringing physical pin 9 high by connecting it to 3.3 volts will wake the radio.

- For radios in sleep mode 4, there are a few options. Assuming the radio is using AT command firmware, you could keep issuing the +++ sequence with a onesecond or longer pause in between each attempt. Sooner or later the radio will respond with an OK and you can take it out of sleep mode (ATSMO) or issue other 
commands. If you've set the sleep period (ATSP) to be very long or the time before sleep (ATST) to be very short, it may take quite some time to get lucky enough to issue the command just as the radio wakes up. Be patient and don't forget to pause at least one second between +++ attempts.

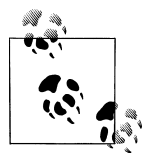

If you are using a full-featured serial adapter, you may be able to see the CTS indicator in your terminal program light up when the radio is awake. Both CoolTerm and X-CTU have this indicator. Time your +++ attempts to happen when CTS is active for fastest results.

- X-CTU is your wake-up buddy. If the radio is sleeping when you attempt to configure it with X-CTU, X-CTU will show a dialog box (refer back to Figure 2-11) that suggests resetting the radio. If your adapter board doesn't have a reset button, carefully lifting the radio out of its serial adapter sockets (yes, while the socket is still plugged into USB) and reseating it will effectively reset it and should wake it up.

- If all else fails, you can always force-download new firmware to the radio. This type of drastic step is usually not necessary. On the PC Settings tab, set Flow Control to Hardware. On the Modem Configuration tab, check the Always Update Firmware checkbox. In the Modem pop-up list, select XB24-ZB along with the function set you desire, such as ZigBee End Device AT. Press the Show Defaults button and then the Write button. You may be asked to reset the radio, in which case carefully unseat it and reseat it in its serial adapter sockets. It may take several tries to get the firmware to reload, but patience tends to pay off here.

- When all else fails, don't forget that Digi has excellent tech support staff that can probably help you resuscitate your radio (http://digi.com/support).

\section{Direct Actuation}

Creating sensor networks is a lot of fun and tremendously useful. There are plenty of reasons to collect data from multiple nodes and bring it to a central location. There are equally great reasons to take commands from a central location and create real events in multiple physical locations remotely. The XBee radio is capable of receiving commands that set its digital I/O output pins to trigger real-world events without the use of any external microcontroller. By itself, the XBee can power an LED, sound a small buzzer, or even operate a tiny motor. Many more devices can be operated directly from the XBee with the use of a relay. Relays are really just electrically operated switches. They allow low currents to turn on and off devices that require much higher currents to operate. Relay-type devices for our purposes include transistors that can transform low current outputs into medium ones, as well as the larger mechanical and electrical relays that typically use medium currents to switch high-power loads, such as large DC motors or even wall-plug A/C appliances. The example at the end of this chapter will 
have you turning on and off home electronics quite reliably with just an XBee and a set of relays doing all the actuation work.

Naturally, not every application that wants to have real-world effects can forgo an external microcontroller. Many devices will benefit from additional local decisionmaking. For example, a lamp that can be turned on remotely may behave more intelligently if it first checks a photocell to see whether the room is already flooded with daylight. Many applications also require more digital outputs than the 10 that XBee radios provide. A simple 12-segment bar graph is going to need a microcontroller to provide the required outputs to drive its display. Don't be afraid to use an external microcontroller if it makes your project easier to prototype, or of course if it is required to enable basic functionality.

\section{Direct Actuation Example}

Remote control impresses people quite a lot, even though it isn't terribly difficult. This project will allow you to control lamps and other small home appliances wirelessly from your computer. It can serve as the basis for any number of interesting control systems, from basic automated home lighting to that complex interactive robotic opera that's been kicking around in your head the past few years. Or you could create Pong on the side of a building (http://blinkenlights.net/blinkenlights). A wireless switch is a wonderful thing.

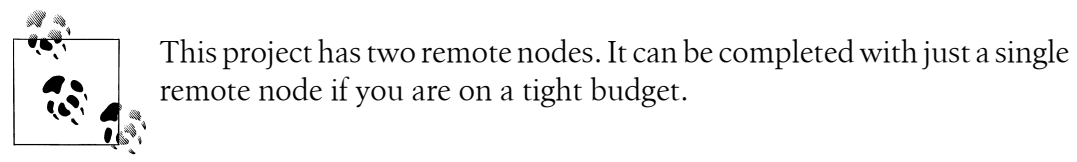

\section{Parts}

- Two solderless breadboards (AF 64, DK 438-1045-ND, SFE PRT-09567)

- Hookup wire or jumper wire kit (AF 153, DK 923351-ND, SFE PRT-00124)

- Two 9-volt or 5-volt power supplies (9-volt batteries also work well for short-term use) (AF 63 or 80 with 9 V battery, RS 273-355, SFE TOL-08269 or TOL-00298)

- Two $3.3 \mathrm{~V}$ voltage regulators (TO-220 package) (DK 497-1491-5-ND, SFE COM-00526)

- Two DC power jacks (2.1 mm ID, 5.5 mm OD) (DK CP-024A-ND, RS 274-1577, SFE PRT-00119)

- Assorted 5 mm LEDs (DK 160-1707-ND, RS 276-041, SFE COM-09590)

- Two 10K ohm resistors (DK P10KBACT-ND, SFE COM-08374)

- Two $10 \mu \mathrm{F}$ electrolytic capacitors (DK P966-ND, RS 272-1025, SFE COM-00523)

- Two $1 \mu$ F electrolytic capacitors (DK P993-ND, RS 272-1434) 
- Two 2N3904 transistors (DK 2N3904TFCT-ND, RS276-2016, SFE COM-00521)

- Two PowerSwitch Tails, 5-volt relay for A/C loads (http://powerswitchtail.com or SFE COM-09842)

- One XBee radio (Series 2/ZB firmware) configured as a ZigBee Coordinator API mode (Digi: XB24-Z7WIT-004, DK 602-1098-ND)

- Two XBee radios (Series 2/ZB firmware) configured as a ZigBee Router AT mode (Digi: XB24-Z7WIT-004, DK 602-1098-ND)

- Two XBee breakout boards with male headers and $2 \mathrm{~mm}$ female headers installed (AF 126 [add SFE PRT-00116], SFE BOB-08276, PRT-08272, and PRT-00116)

- XBee USB serial adapter (XBee Explorer, Digi Evaluation board, or similar) (AF 247, SFE WRL-08687)

- USB cable for XBee adapter (AF 260, SFE CAB-00598)

- Wire strippers (AF 147, DK PAL70057-ND, SFE TOL-08696)

- Small screwdriver (RS 64-069, SFE TOL-09146)

- A lamp or any other small A/C appliance that draws less than 10 resistive amps

\section{Prepare Your Coordinator Radio}

1. Follow the instructions under "Reading Current Firmware and Configuration" on page 35 in Chapter 2 to configure one of your radios as a ZigBee Coordinator API.

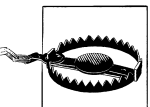

Your coordinator radio must use the API firmware for this project to work because $\mathrm{I} / \mathrm{O}$ data is only delivered in API mode. Be sure to select the API version for your coordinator!

2. Use X-CTU to configure the coordinator with a PAN ID (between $0 \mathrm{x} 0$ and OxFFFFFFFFFFFFFFFF) that you've selected. Write down this PAN ID so you can program your router radios with the same one. Every radio in your network must use the same PAN ID so that they can communicate with each other:

\section{Pan ID:}

3. The software libraries that we are using in Processing require that the base station XBee be in API Mode 2 (API Operation with escaped characters). Use X-CTU to set ATAP to 2, and Write the configuration to your radio.

4. Label the coordinator radio with a "C" so that later you'll know which one it is. 
The XBee Java API Library communicates using escaped character mode, as described in a note under "Libraries" on page 141. Be sure that you set the coordinator's API to mode 2; otherwise the project will not work!

\section{Prepare Your Router Radios}

1. Follow the instructions under "Reading Current Firmware and Configuration" on page 35 in Chapter 2 to configure each of your actuator node radios as a ZigBee Router AT.

2. Your router radios will use the AT firmware so you can easily configure them using a serial terminal. Be sure you select the AT version for your routers!

3. Each router radio can be labeled with an "R."

\section{Prepare the Actuator Boards}

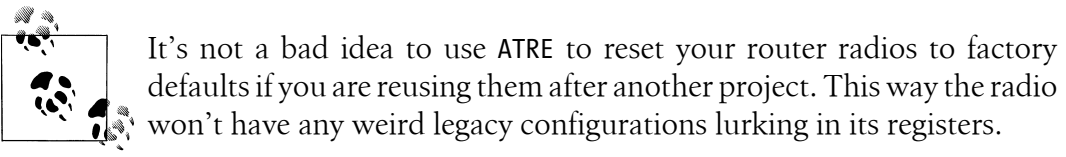

\section{Configure Your Router XBees}

We'll use the CoolTerm terminal program and an XBee Explorer USB adapter again to set up your radios. For each of your sensor node radios:

1. Select a router XBee you've labeled with an "R" and place it into the XBee Explorer.

2. Plug the XBee Explorer into your computer.

3. Run the CoolTerm program and press the Options button to configure it.

4. Select the appropriate serial port, and check the Local Echo box so you can see your commands as you type them.

5. Click on the Connect button to connect to the serial port.

6. Type +++ to go into command mode. You should receive an OK reply from the radio.

7. Select the same PAN ID you entered for your first radio above.

8. Type ATID followed by the PAN ID you selected and press Enter on the keyboard. You should receive OK again as a reply.

9. Every ZigBee coordinator always has 0 as its 16-bit network address. Type ATDH $\mathbf{0}$ and press Enter on the keyboard. You should receive an OK response. 
10. Enter ATDL followed by the low part of your radio's destination address, in this case also a zero because that's the fixed address for the coordinator. Type ATDL $\mathbf{0}$ and press Enter. You should receive an OK response.

11. Enter ATJV1 to ensure that your router attempts to rejoin the coordinator on startup.

12. Enter ATD04 to set pin 0 as low digital output to begin with.

13. Save your new settings as the radio's default by typing ATWR and pressing Enter.

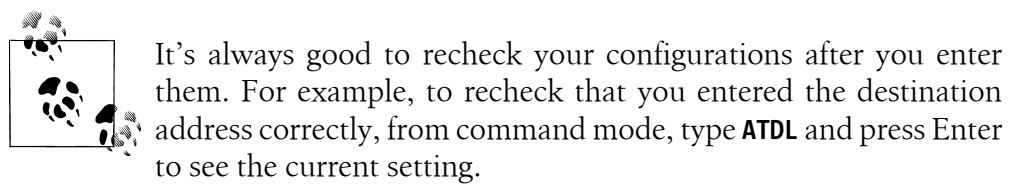

\section{Connect voltage regulator circuit and power jack to breadboard}

1. Wire up a breadboard with a 3.3-volt voltage regulator (LD1117V33) as shown. The regulator has three legs - typically, ground, output, and input - when viewed from the front (where the writing is). Sometimes these legs are in a different order, so find and check the data sheet if you're not sure! Input is where a high voltage, for example 5 or 9 volts, is applied to the regulator. Output is where you will get the regulated 3.3 volts. Ground is the common ground for your entire circuit, including input, output, and all the other components. Bring ground out to both blue ground rails that run along the sides of your breadboard. Bring 3.3-volt output power to both of the red power rails (refer back to Figure 5-4).

2. Solder a red wire (about $10 \mathrm{~cm}$ ) to the short center pin of your power jack, and solder a similar black wire to the longer outer pin (refer back to Figure 5-5). Don't allow the two connections to touch each other since that will create a short circuit when you power up!

3. Attach the red wire from the power jack, using the breadboard to connect it to the input pin of the voltage regulator. Attach the black ground wire to the ground pin of the voltage regulator in the same way.

4. Hook up the output pin of the voltage regulator to one of the power rails of the breadboard using a red wire. Hook up the ground pin to one of the ground rails on the breadboard.

5. Use the two capacitors to "decouple" the power supply in the following way: attach the short ground lead of the $10 \mu \mathrm{F}$ capacitor (also marked with a stripe on the capacitor's ground side) to ground near the voltage regulator. Attach the other positive lead of the $10 \mu \mathrm{F}$ capacitor to the voltage regulator's input pin. This will remove some lower-frequency noise coming from the wall power supply. Also attach the short ground lead of the $1 \mu \mathrm{F}$ capacitor to ground, and the other positive lead to the $3.3 \mathrm{~V}$ output pin. This will remove some higher-frequency noise coming out of the voltage regulator. Decoupling will prevent noisy power from reaching your radio and interfering with its signal. 
6. Hook up power and ground across the breadboard so that the rails on both sides are live.

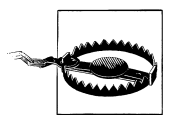

It's a really good idea to check the voltage levels using a multimeter after you first wire up the breadboard for power. Make sure that your power rails have 3.3 volts on both sides where you expect it. You don't want to send 9 volts to your radio and cook it!

\section{Router XBee connection to power}

1. With a router XBee mounted on its breakout board, position the breakout board in the center of your other breadboard so that the two rows of male header pins are inserted on opposite sides of the center trough.

2. Use red hookup wire to connect pin 1 (VCC) of the XBee to 3.3-volt regulated power.

3. Use black hookup wire to connect pin 10 (GND) of the XBee to ground.

\section{Transistor and relay output}

This project uses the PowerSwitch Tail A/C relay (see Figure 6-3). This relay is usually activated by at least $5 \mathrm{~V}$ of direct current. The XBee can't provide enough voltage or amperage by itself to drive that relay, so we use an NPN transistor as an electronic switch to send 5 or 9 volts directly to the relay. Think of it as a switch that throws another switch. You can get the data sheet for the 2N3904 transistor at http://www .fairchildsemi.com/ds/2N\%2F2N3904.pdf, and the schematic for the PowerSwitch Tail

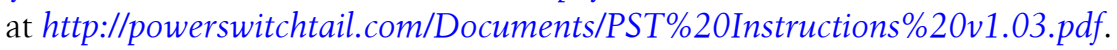

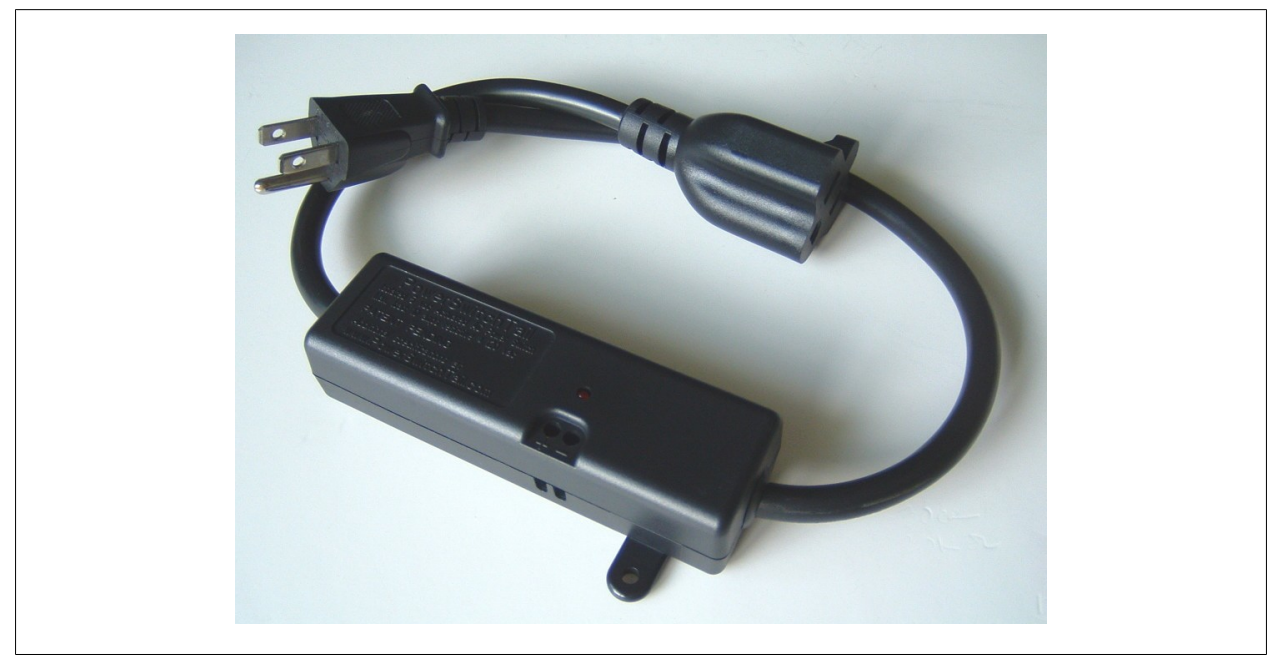

Figure 6-3. PowerSwitch Tail 5 V relay for A/C loads 
1. The 2 N3904 transistor has three leads. When the sensor's flat side is facing you, the leads from left to right are emitter, base, and collector. Insert the 2N3904 so that each lead is in its own row on the breadboard.

2. Use a black wire to connect the leftmost emitter lead to one of the ground rails.

3. Insert the $10 \mathrm{~K}$ ohm resistor so that it is connected to XBee digital output 0 (physical pin 20) on one end and to the center base pin of the 2N3904 on the other. You can use jumper wires to make the connection if that's more convenient in your breadboard layout.

4. Connect a black wire from the rightmost collector pin of the 2 N3904 to the negative screw terminal on the PowerSwitch Tail. It should be marked with a - (minus sign). Use a small screwdriver to tighten the screw terminal so that the black wire is securely attached.

5. Connect a red wire from the input voltage coming from your DC power jack (the same pin that feeds the input of your voltage regulator with 5 to 9 volts) to the positive screw terminal on the PowerSwitch Tail. That screw terminal should be marked with a + . Use a small screwdriver to tighten the screw so that the red wire is securely attached.

\section{Second actuator board}

Create the second actuator board in the same way as the first. You can make as many actuator systems as you like. The software will work with as few as one or as many as five without any adjustment to the software. Figure 6-4 shows the breadboard layout for the simple actuator node, and Figure 6-5 shows the schematic.

\section{PowerSwitch Tail A/C relay}

Plug one end of the PowerSwitch Tail into an A/C wall outlet. The other end can have a lamp or any similar appliance that draws less than 10 amps at $120 \mathrm{~V}$ plugged into it. See the data sheet for additional information (http://powerswitchtail.com/Documents/ PST\%20Instructions\%20v1.03.pdf). You'll be able to turn this device on and off wirelessly, right from your computer using the on-screen switches displayed by the Processing program.

\section{Prepare the Base Station}

Your base station radio is simply an XBee serial adapter connected to your computer.

\section{Connect to your computer}

1. Select the coordinator XBee you've labeled with a " $\mathrm{C}$ " and place it into the XBee Explorer.

2. Plug the XBee Explorer into your computer. 


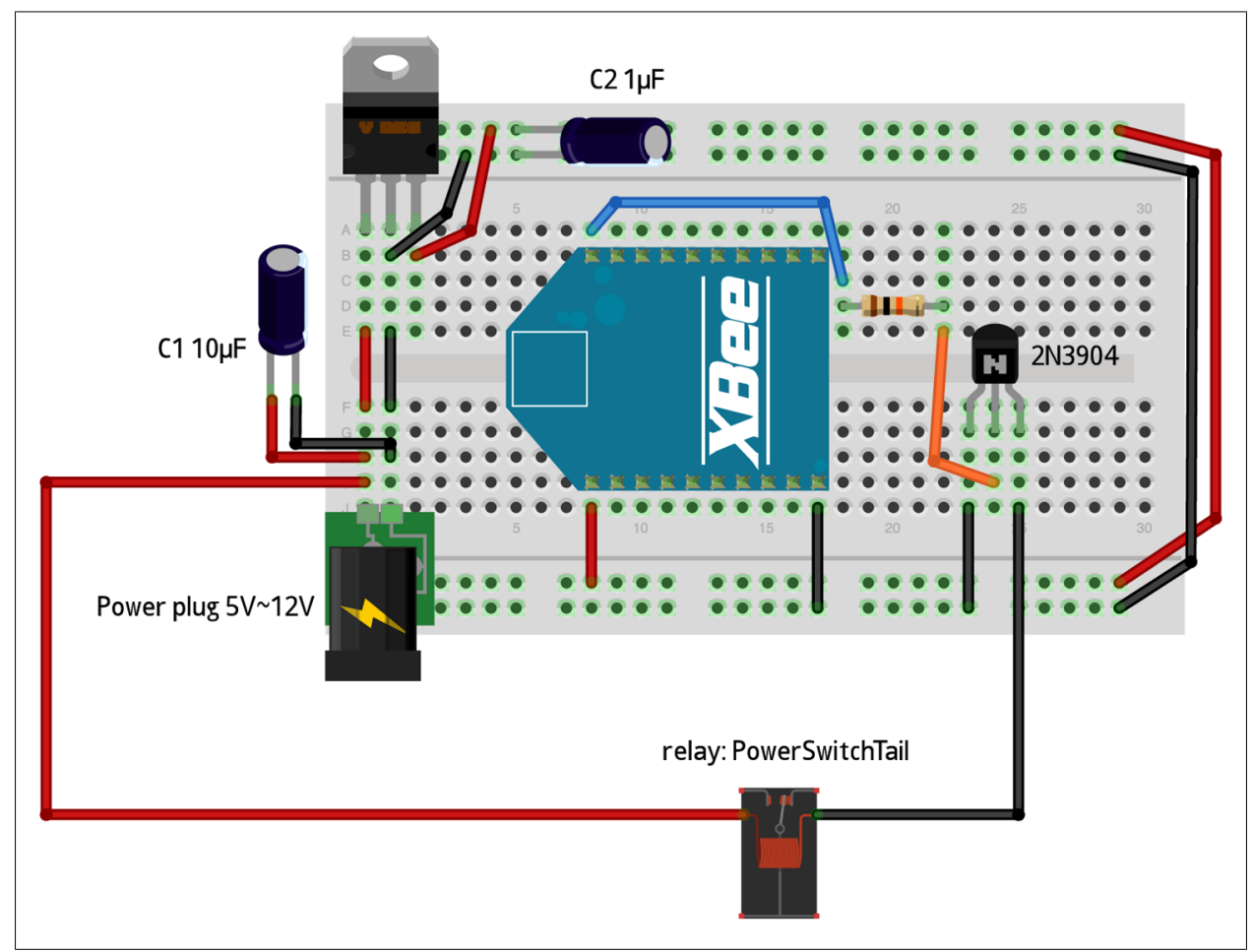

Figure 6-4. Simple actuator node breadboard layout

\section{Program the actuator network base station}

The simple actuator network base station uses the Processing program that follows. You can download a ZIP file of all the libraries and resources from this book's website. You can also find the XBee API library at http://code.google.com/p/xbee-api/ and Processing at http://processing.org/download for Linux, Macintosh, or Windows.

Inside the Processing sketch folder for the Simple Actuator Network program are two subdirectories called code and data (see Figure 6-6). The code folder contains the $\log 4$ j.jar and xbee-api-0.5.5.jar library files. These contain all the code for communication with the XBee in API mode. The data folder holds the log4j.properties file, required by $\log 4 j$.jar. It also has a font file for a sans serif 10-point font used for screen display and two .jpg images for the on and off switch positions.

If you download the file from this book's website, simply unzip it and launch the Simple_Actuator_Network.pde file using Processing.

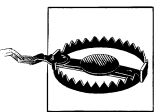

Be sure to replace the COM port listed in this code with your actual $\mathrm{COM}$ port. Port names are listed in the console in Processing, as your program starts up. 


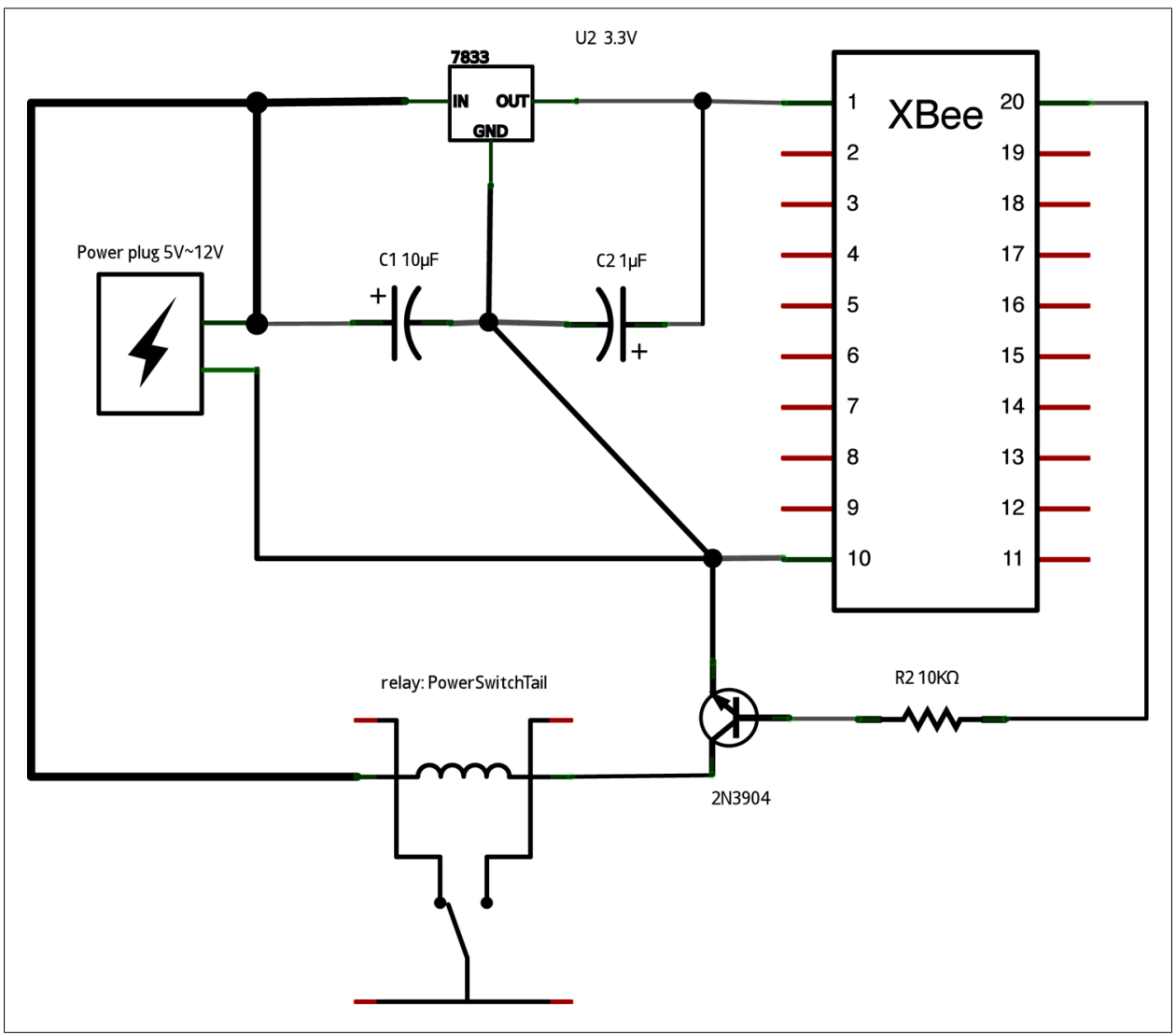

Figure 6-5. Simple actuator node schematic

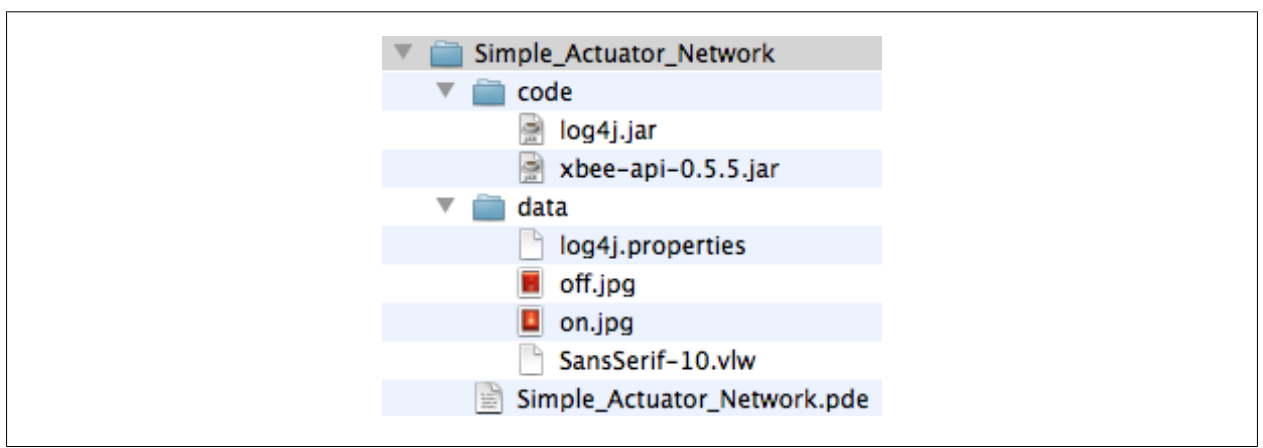

Figure 6-6. Directory structure for the Processing sketch program Simple Actuator Network, including all required libraries, config files, a font file, two image files, and the Processing “.pde” sketch itself 
Once you have loaded the files and directories onto your computer and opened the Simple_Actuator_Network.pde file in Processing, press the Run button (labeled with a triangle) to launch the display code. It will open in a new window and show a switch for each actuator node detected, as shown in Figure 6-7.

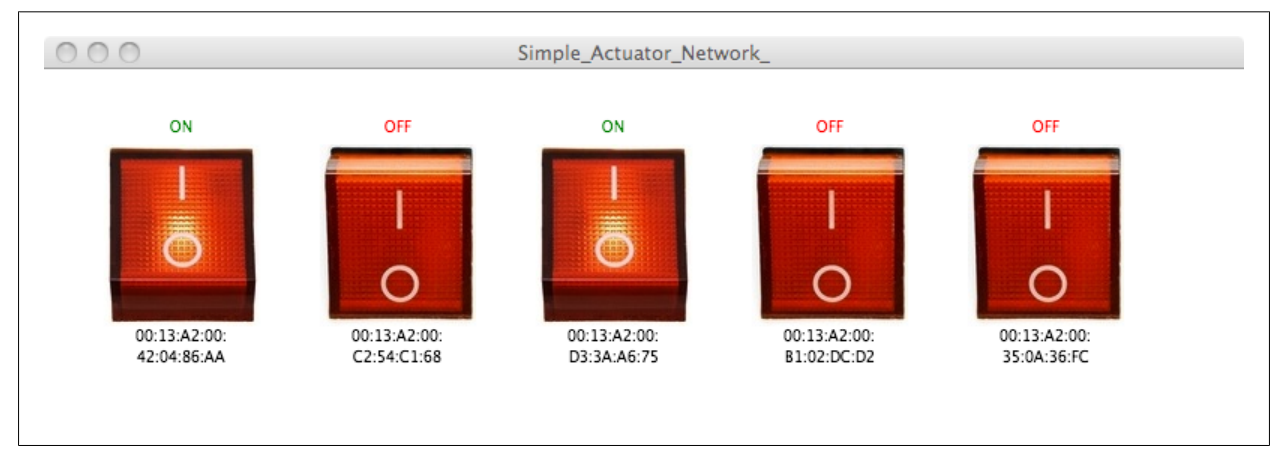

Figure 6-7. Simple Actuator Network switch display screen in Processing

\section{Simple Actuator Node Code in Processing}

Here's the source code for the Processing sketch. The comment shown in bold about the serial port highlights an essential change. Other commented instructions are only important if you didn't download the source from the website listed in the Preface (however, you'll still need to download this source code to obtain the on.jpg and off.jpg images used in this example):

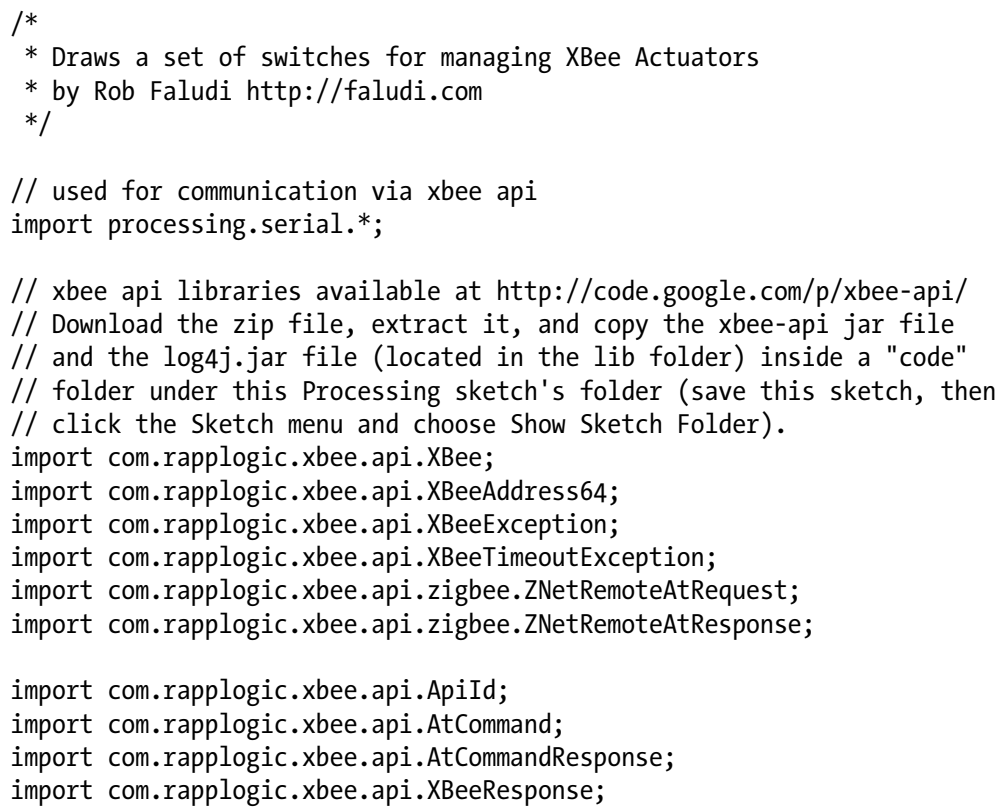


import com.rapplogic.xbee.api.zigbee.NodeDiscover;

String version $=" 1.02 "$;

// *** REPLACE WITH THE SERIAL PORT (COM PORT) FOR YOUR LOCAL XBEE *** String mySerialPort = "/dev/tty.usbserial-A1000iMG";

// create and initialize a new xbee object

$\mathrm{XBee}$ xbee $=$ new $\mathrm{XBee}()$;

int error $=0$;

// make an array list of thermometer objects for display

ArrayList switches = new ArrayList();

ArrayList nodes = new ArrayList () ;

// create a font for display

PFont font;

float lastNodeDiscovery;

void $\operatorname{setup}()\{$

size $(800,230)$; // screen size

smooth(); // anti-aliasing for graphic display

// You'll need to generate a font before you can run this sketch.

// Click the Tools menu and choose Create Font. Click Sans Serif,

// choose a size of 10 , and click OK.

font $=$ loadFont ("SansSerif-10.vlw");

textFont(font);

// The log4j.properties file is required by the xbee api library, and

// needs to be in your data folder. You can find this file in the xbee

// api library you downloaded earlier

PropertyConfigurator.configure(dataPath(" )+"log4j.properties");

// Print a list in case the selected serial port doesn't work out println("Available serial ports:");

println(Serial.list());

try \{

// opens your serial port defined above, at 9600 baud

\} xbee.open(mySerialPort, 9600);

catch (XBeeException e) \{

println("");

println(" ** Error opening XBee port: " + e + "**");

println("");

println("Is your XBee plugged in to your computer?");

println("Did you set your COM port in the code near line 30?");

\} error=1;

// run a node discovery to find all the radios currently on the network

// (this assumes that all the network radios are Actuator nodes) 


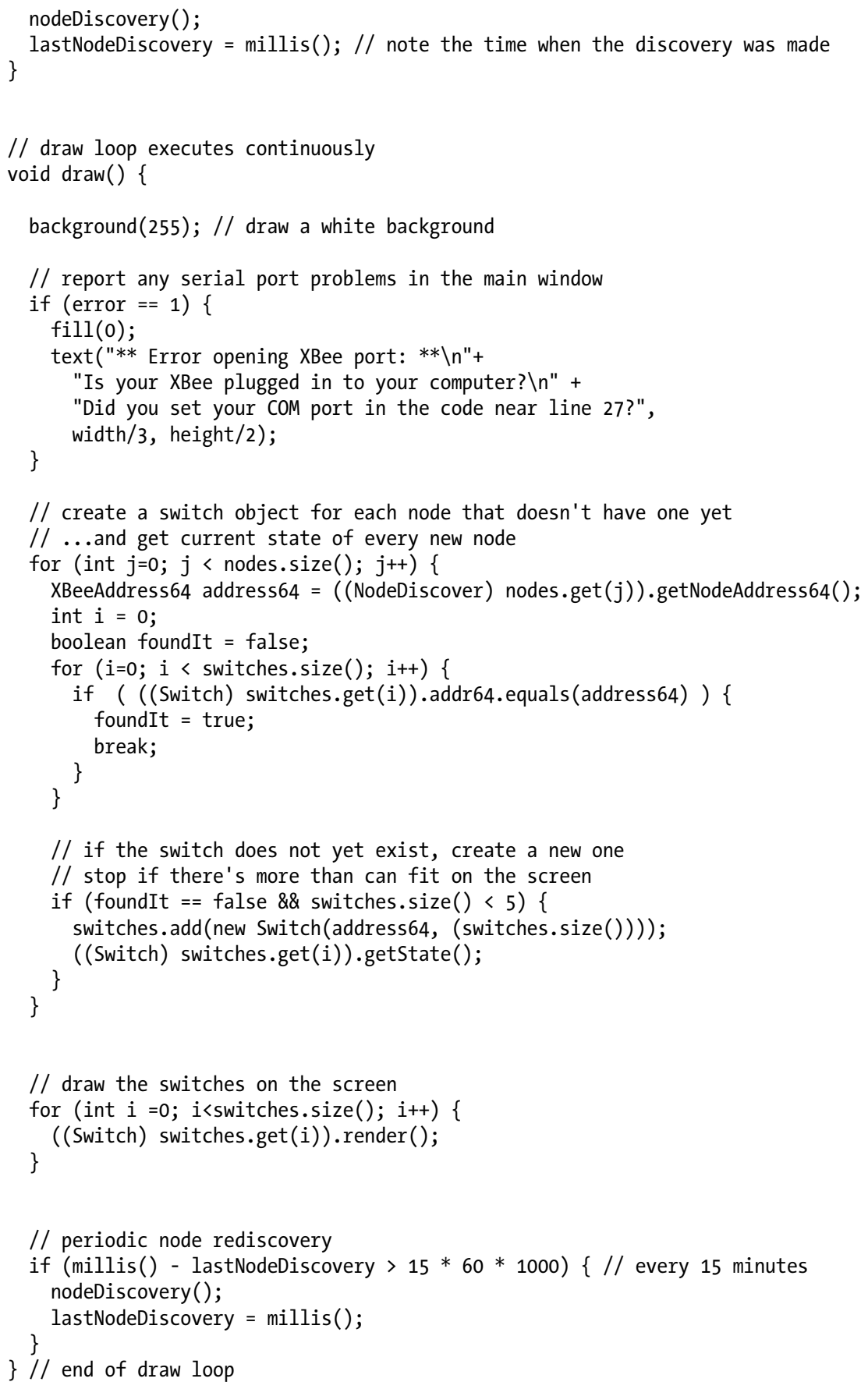


// function to look up all the nodes on the network

$/ /$ and add them to an ArrayList

void nodeDiscovery() \{

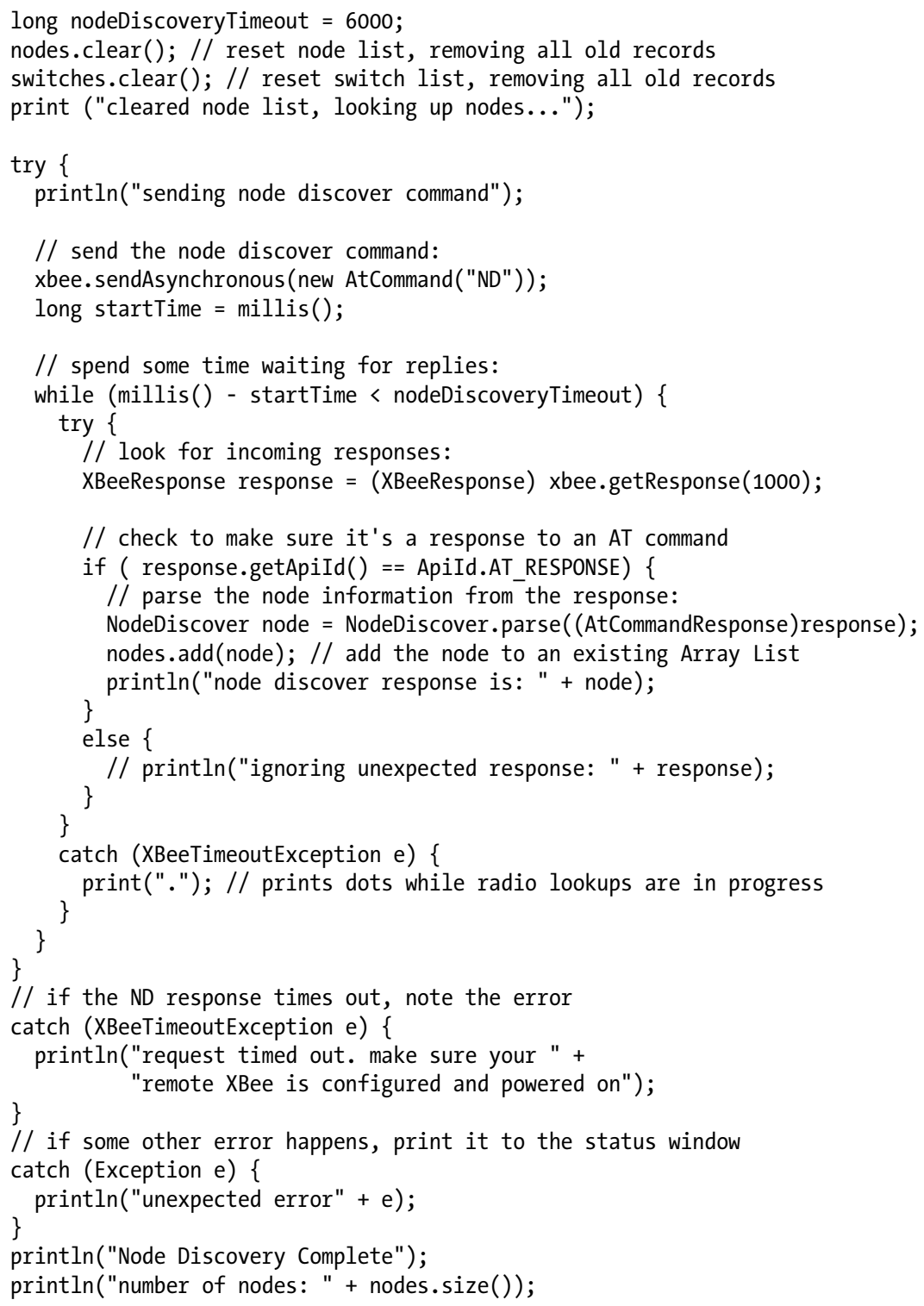


// this function runs once every time the mouse is pressed void mousePressed() \{

// check every switch object on the screen to see

// if the mouse press was within its borders

$/ /$ and toggle the state if it was (turn it on or off)

for (int $i=0 ; i<$ switches.size(); $i++)\{$ \}

((Switch) switches.get(i)).togglestate();

\}

// defines the switch objects and their behaviors

class Switch \{

int switchNumber, posX, posY;

boolean state $=$ false; // current switch state

XBeeAddress64 addr64; // stores the raw address locally

String address; // stores the formatted address locally

PImage on, off; // stores the pictures of the on and off switches

// initialize switch object:

Switch(XBeeAddress64_addr64, int _switchNumber) \{

on = loadImage ("on.jpg");

off = loadImage("off.jpg");

addr64 = _addr64;

switchNumber = _switchNumber;

pos $X=$ switchNumber $*$ (on.width +40$)+40$;

$\operatorname{pos} Y=50$;

// parse the address int array into a formatted string

String[ ] hexAddress = new String[addr64.getAddress().length];

for (int $i=0$; $i<$ addr64.getAddress (). length; $i++$ ) \{

// format each address byte with leading zeros:

\}

hexAddress[i] = String.format ("\%02x", addr64.getAddress()[i]);

// join the array together with colons for readability:

address = join(hexAddress, ":");

\}

println("Sender address: " + address);

void render() \{ // draw switch on screen

noStroke(); // remove shape edges

if(state) image(on, posX, posY); // if the switch is on, draw the on image

else image(off, posX, posY); // otherwise, if the switch is off,

// show text

// draw the off image

textAlign(CENTER);

fill $(0)$;

textSize(10);

// show actuator address

text(address, pos X+on.width/2, posY + on.height +10$)$;

// show on/off state 


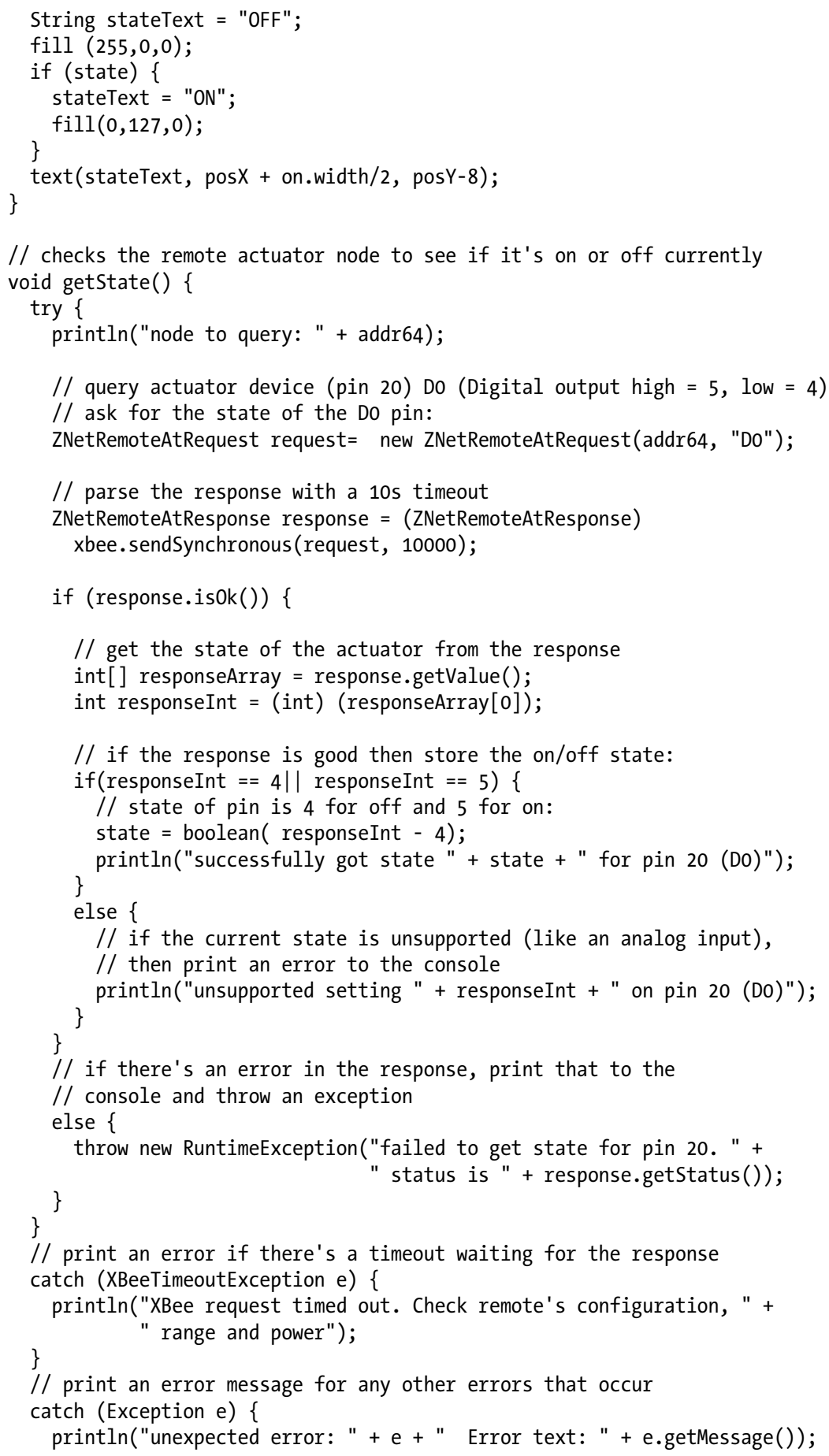


// this function is called to check for a mouse click

// on the switch object, and toggle the switch accordingly.

// it is called by the MousePressed() function so we already

// know that the user just clicked the mouse somewhere void togglestate() \{

// check to see if the user clicked the mouse on this particular switch if (mouseX $>=$ posX \&\& mouseY $>=$ posY \&\&

\{ mouseX $<=$ posX+on.width \&\& mouseY $<=$ posY+on.height)

println("clicked on " + address);

state = !state; // change the state of the switch if it was clicked

try \{

// turn the actuator on or off (pin 20)

$/ /$ Do (Digital output high $=5$, low $=4$ )

int [ ] command $=\{$

4

\}; // start with the off command

if (state) command[0]=5; // change to the on command

$/ /$ if the current state is on

else command[0]=4; // otherwise set the state to off

ZNetRemoteAtRequest request $=$

new ZNetRemoteAtRequest(addr64, "DO", command);

ZNetRemoteAtResponse response $=$

(ZNetRemoteAtResponse) xbee.sendSynchronous(request, 10000);

// if everything worked, print a message to the console

if (response.is0k()) \{ \}

println("toggled pin 20 (DO) on node " + address);

// if there was a problem, throw an exception

else \{

throw new RuntimeException(

"failed to toggle pin 20. status is " + response.getStatus ()$)$;

\} \}

// if the request timed out, print

// that error to the console and

// change the state back to what

// it was originally

catch (XBeeTimeoutException e) \{

println("XBee request timed out. Check remote's " +

"configuaration, range and power");

\}

state $=$ !state;

// if some other error occured, print that

// to the console and change the state back

// to what it was originally

catch (Exception e) \{

println("unexpected error: " + e + 


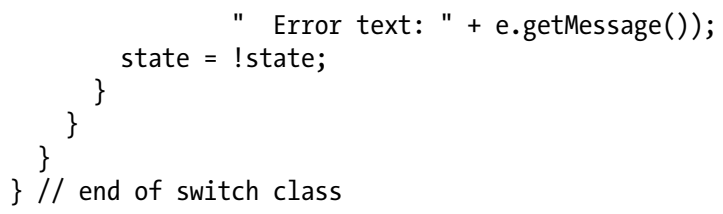

\section{Summary}

One minute you're collecting meaningful data from a sensor network, and the next you're remotely activating your home appliances. It is not a stretch to consider yourself a wireless networking expert at this point, easily traversing multiple nodes in a single bound! Now you may be wondering how to make the next big leap. How do you push your powers beyond the surly bonds of ZigBee to touch other networks, like the Internet? The next chapter will unfurl the glory of gateways, opening up a pathway for your wireless networks to talk to almost anything or anyone, in whatever protocol is spoken in that realm. Take a moment to pat yourself on the back first. You deserve it. 



\section{Over the Borders}

ZigBee is only one of the great flavors of networking. In this chapter we learn to make gateways that cross borders to connect with neighboring networks, including a remarkably easy path to the Internet. You'll see full examples, showing how to allow anything to talk to everything everywhere; plus there's something special for you starryeyed celebrity fans. Let this chapter be your passport.

\section{Gateways}

The great thing about standards is there are so many of them. Bluetooth, IPv6, UDP, ZigBee, SMS, VoIP, WiFi, Ethernet, 4G, SMTP, and TCP/IP all define different networking protocols and layers within those protocols. There's no such thing as a perfect network; that's why there are so many different ways to get networking done. Each protocol is designed to solve a specific type of problem. Most do a great job at their task. For example, Bluetooth performs solidly when connecting up eight local devices as a personal area network. At the same time, many of the engineering choices that solve one kind of problem create barriers when the protocol is pushed into unfamiliar territory. Bluetooth's simple pairing and addressing schemes don't readily scale to networks of hundreds or thousands of nodes, while other protocols may sacrifice such simplicity for flexibility and scalability. Luckily, there's no need to stick to a single protocol. Each can do what it's best at, and connections can be made so they all work well together. Using the best network means using many networks. Gateways are the glue that holds them all together.

A gateway is any device that provides connectivity between different networks. In some cases, the two networks use the same protocol and are separated by a gateway for traffic or security reasons. These won't be our focus here. We're mostly interested in gateways that expand our capabilities by opening up a world beyond the local mesh. These gateways will allow the information we've made available with ZigBee to traverse a whole web of interesting networks, accomplishing worthwhile and sometimes extraordinary tasks. 


\section{XBee as Embedded Gateway}

Any XBee radio that is using local serial communications is actually acting as a gateway between two very important protocols. As you are well aware of by now, the XBees use ZigBee to communicate wirelessly between radios. They simultaneously use TTL (sometimes called board-level or logic-level) serial to communicate over metal wiring to other local devices, such as microcontrollers and desktop computers. Everything that happens on the RX and TX pins of the XBee is using TTL serial. Everything that happens over our XBee's radio antenna is ZigBee. It's the XBee's internal circuitry and software that serves as a translator between these two protocols. This is how your computer or Arduino, which only speak to the XBee using serial over wires, gain the capability to talk using radio waves to remote devices. The XBee acts as their gateway and extends them onto ZigBee networks. That's pretty important, and it's only the tip of the iceberg when it comes to the power of gateways.

\section{Other Embedded Gateways}

Many other gateway modules are available to connect from the TTL serial communications commonly found on circuit boards to a myriad of other useful protocols. In many cases, simply wiring TX/RX on the XBee directly to RX/TX on the other embedded module effectively creates a bare-bones gateway between the two protocols, as long as the other device has been properly configured for transparent retransmissions. Here are just a few of the embedded possibilities you could explore:

\section{Bluetooth}

The Bluetooth protocol is commonly used for small, short-range mobile personal area networks. Roving Networks makes a variety of embedded modules, including the RN-41, available on a breakout board for prototyping from SparkFun (SFE WRL-00582). This module could be used to link your ZigBee network to Bluetooth Serial Port Profile to communicate directly with certain mobile phones. (See http: //www.sparkfun.com/.)

CAN (or CAN-bus)

Controller-Area Networking is a standard form of communication used widely in the automotive industry for moving information between the various devices and subsystems inside cars and trucks. Every time you take a drive, your brakes, engine, airbags, and transmission are probably communicating with CAN. The Microchip MCP2515 CAN controller and MCP2551 CAN transceiver are available on an Arduino shield that you could use to stream data to and from your car. (See http:// www.skpang.co.uk/content/view/32/42/.)

Ethernet

This is a big category. Ethernet is the primary wired interface to the Internet, and embedded modules are just one of many ways to bridge our communications worldwide. One useful embedded gateway is the Lantronix XPort, which can transparently connect TTL serial signals (RX/TX) to Ethernet and TCP/IP, 
thereby forging a circuit-board-level connection to the Internet (module: http:// www.lantronix.com/device-networking/embedded-device-servers/xport.html,

breakout board: SFE BOB-08845). Later in this chapter we will examine some external Internet gateways, including a powerful system that makes device communications almost as simple as using a web browser.

GPRS, 3G, 4G cellular modems

Getting data out into the world isn't all about wires. As long as your project is not too far away from the places frequented by humans, you are probably within range of the mobile wireless network. The Telit GE865, for example, is an embedded module that can bridge from your XBee's TX/RX pins to GPRS mobile data networks and all the way out to the Internet. It can also provide additional onboard logic using its built-in Python interpreter. Since the GE865 doesn't have pins that connect to a breadboard, you'll almost certainly want to start with an evaluation system (SFE CEL-09342) that makes the module ready for prototyping.

\section{HomePlug}

The HomePlug Alliance specifies a protocol for creating networks over residential electrical wiring. Though just one of many powerline networking protocols, it has gained traction recently as a popular profile for managing audio/visual systems. There is a HomePlug modem board available (SFE SEN-09080) to gateway TTL serial to HomePlug, and it even includes a pin-compatible XBee header so you can plug a radio right into it! Also see X-10 below for an older method of accomplishing communications over power lines.

\section{$R F$ without protocols}

Certain very inexpensive transmitter modules (SFE WRL-08946) can be paired with receiver modules (SFE WRL-08950) for a low-cost one-way link. In general the reliability and flexibility of these connections is poor enough that they can't be recommended. However, their limitations often create a fine demonstration of how much ZigBee's addressing, network infrastructure, and error handling are helping you out. Proceed with caution, but don't be afraid to try them.

RFID

Radio Frequency ID tags (RFID) are small microchips that typically use radio energy as a passive power source for transmitting a serial number. RFID readers (for example, SFE SEN-08419) can detect these transmissions and repeat them as a TTL serial TX that could be retransmitted through your XBee radio. RFID is a lot like a bar code and sometimes disappoints those who try to extend it beyond its limits.

USB

Computers commonly communicate with other devices using the Universal Serial Bus protocol. Several different USB-to-TTL serial gateways have already been described in Chapter 1 since they are required for serial configuration of the XBee. Many of these use a very popular microchip from FTDI so computer drivers are readily available (http://www.ftdichip.com/FTDrivers.htm). This FTDI chip is 
available on breakout boards (SFE BOB-00718), making it easy to create generic connections between board-level TTL serial and USB host devices like computers. WiFi

Another very popular on-ramp to the Internet is $\mathrm{WiFi}$, the wireless networking protocol generally used by laptop computers and many smartphones. There are so many modules to keep track of in this space that an entire book could be written about them. Two worth knowing about are the Lantronix MatchPort because its configuration matches the Ethernet XPort, and the WiFly module because it's available on a breakout board (SFE WRL-09333) for prototyping. Remember that WiFi connections need to be configured with a different system name and security key each time they attach to a new network, so unless your device sits in one place, or incorporates a screen and keyboard, WiFi might not work for your project. Think this through before getting started.

$X-10$

One of the oldest home automation protocols is X-10. Originally devised as a powerline networking protocol, it has since been extended to wireless as well. Newer protocols like HomePlug may someday render X-10 obsolete but today it remains in wide use so you might have cause to use a gateway module like the TW523 to control existing home automation systems.

\section{Z-Wave}

The Z-Wave Alliance defined this proprietary wireless communications protocol, which has gained some traction in the home automation market. Like ZigBee, it is designed for low-power, low-bandwidth data interactions. It operates in a different frequency spectrum (900 MHz versus ZigBee's $2.4 \mathrm{GHz}$ ) and, unlike ZigBee, the protocol itself is only available under a nondisclosure agreement. Development kits (DK 703-1056-ND) are probably the best way to start working with Z-Wave, though they are far more expensive than the modules themselves (DK 703-1023ND).

\section{Internet Gateways}

Of all the places to take your data, nothing is quite as compelling as passing it through a gateway to the Internet. That's because the Internet reaches almost everywhere and has the capability to touch almost anything. Some people think of the Internet as mostly web pages, but that's only one of a dazzling array of destinations for the data streaming from your device or sensor network. Really the Internet is a vast collection of pathways and services that has already grown so complex that it is sometimes described as beyond the grasp of human comprehension. Luckily you don't need to understand the whole thing to move information from place to place in a reasonably efficient manner. There are a lot of reasons to do it: 


\section{Data storage}

Any sensor network that is devoted to amassing data will need to store that data someplace. It may be fine to bring the data onto a local computer and work with it there, but in many cases that isn't going to be enough. If there's a lot of data it may be more than you can store locally. Also there may be other people who are interested in access to your data, such as your project partners, colleagues, clients, customers, or even the general public. The great thing about the Internet is that storage can really be anywhere. It's quite common to stash data in places whose physical location is totally unknown to the user. Amazon's S3 service, for example, synchronizes your data onto a suite of servers located around the world. Customers of the service do not generally know the actual locations and physical storage media that hold their data. They usually don't care because access is far more important than the mechanics involved. Whether your data resides on a private MySQL database with a hosting provider such as Dreamhost or HostMonster, or in a shared compendium of public data like Pachube's system, the advantages of outsourcing your data storage will be the same. Storage can be endlessly expanded and access can occur from any place at any time.

\section{Data presentation}

Everyone can see the Internet, so it's a good place to show your data. You may decide to roll your own display using something like Processing, or hook up with any of the online data visualization services like Google Charts or Microsoft's Pivot Viewer. Data sharing sites like Pachube (see Chapter 8) often embed other sites' visualization services, so you may be using these big companies' systems indirectly. Once your data is on the Internet, there's no end to the ways it can be displayed to tell its story.

\section{Remote actuation}

Objects are on the Internet, and some of them move. Robots, door locks, plant watering systems, lights, bells, whistles, and interactive kinetic sculptures all can take data from remote sensors to perform actions based upon real-world information. Remember that a sensor can be as simple as a switch. Perhaps you want to make a lamp in London react to the current amount of daylight in Delhi. Or maybe you want the tide in Tiburon to create motion in Melbourne. Unless you're going to lay the cable yourself, the Internet is the best way to join your widely separated inputs and outputs together to create something amazing.

\section{Everything}

There's no reason to do just one of these things. You will have the most fun doing them all at the same time, uploading your data to a system that creates remote actuations (perhaps generating sensor data of its own at the other end), and creating an interface to control the systems and present their resulting tale. The Internet is big and it is new, so it presents endless opportunities to do things that have never been done before. Make your mark. 


\section{Internet Media}

The why of Internet gateways should be clear and compelling. Let's take a look at the how. In most cases you'll be choosing among three options for your physical network connection: wired Ethernet, local WiFi connections, or mobile data connections that use the cell phone network. Of course there are other possibilities, like old-school telephone modems or amateur packet radio that might apply to special situations, but those won't be discussed here:

\section{Ethernet}

Ethernet uses physical wiring, which means your gateway will be tethered in place. Ethernet connections are fast and very reliable because their wires are not exposed to much noise disruption. Configuration is often not necessary at all. Ethernet is also very cheap to physically implement so generally this will be the lowest-cost option.

WiFi

WiFi provides a high-bandwidth wireless connection that's locally available in many homes, schools, businesses, and even some public parks. Configuration is almost always required to select the network you want to attach to and to issue the password or keys that secure each system. WiFi connections (wireless using IEEE $802.11 x$ standards) typically communicate using unlicensed parts of the radio spectrum, which means they need to be tolerant of noise, and their physical components are more expensive. Generally, using a WiFi gateway will cost more and be somewhat less reliable than using Ethernet; however, in certain locations it may be the only practical choice.

Mobile data

This type of connection goes by various names, including carrier wireless, cellular data, mobile Internet, GPRS, 2G/3G/4G, and several other vague or inscrutable monikers. Data plans are offered by large carriers such as Orange, AT\&T, Verizon, or NTT. Mobile data is available at most locations that have mobile phone coverage. The components are similar in cost to $\mathrm{WiFi}$, but the connection itself is generally far more expensive. An account with a carrier is required and needs to be provisioned in advance. Data charges can quickly accumulate into an exorbitant bill. Even so, mobile data reaches to places where Ethernet and WiFi are not a possibility. Because configuration is not location-specific, this is the best choice for any gateway that will be moving from place to place. If you want to wire up a freight truck with sensors, mobile data services will almost certainly be part of your solution.

\section{Computers Versus Dedicated Devices}

Most Internet gateways are either implemented on a personal computer or manufactured as dedicated devices. It is certainly possible to build your own dedicated gateway 
from scratch, though in most cases it will cost more and do less than one of the commercially available ones.

Computers are a good choice for gateways in quick prototype systems. Most likely you already have a computer, and that computer almost certainly comes with all the equipment needed to connect to both Ethernet and WiFi for access to the Internet. It's a safe bet that it also has a serial port (probably USB) for plugging in your XBee radio. In a certain sense using your computer is free, assuming you already own one. It also contains an extremely powerful processor that will have no problem performing complex manipulations to the information passing through it. So for a quick prototype, you can write some code in any number of languages (including Processing) to translate your data from ZigBee to TCP/IP.

Many people find that personal computers don't work as well for projects installed over the long term. For one thing, computers run a very complex operating system that needs regular upgrades to keep it stable and secure. In the course of running other programs, you may slow down or crash the system, disabling your gateway at the same time. Your computer also uses quite a bit of electricity, takes up a fair amount of physical space, and in the case of a personal laptop, periodically gets moved away from the location where the gateway operates. This is where a dedicated device can do a better job.

Dedicated gateways are simple pleasures. They typically come in the form of a nondescript rectangular box. For ZigBee gateways there's a radio to talk to your wireless sensor network, often a small processor chip, and another module to talk to whatever you're gatewaying to, typically either an Ethernet module, WiFi radio, or mobile data system. These types of gateway devices rarely have any kind of screen or keyboard. Configuration is usually done through a web browser or another type of remote connection. They also tend to be small, use far less power than a full-size computer, and run a slimmed-down operating system that has only a few essential features. Finally, they tend to cost less than a full computer and run stably without rebooting, potentially for years at a time. Some of them are even designed to use renewable energy sources, operate outdoors, or survive in harsh environments with extended temperature ranges. There are many flavors and brands of gateway. Since we're already working with XBee radios, we'll examine the ConnectPort line of devices made by Digi International. These are specifically designed to work seamlessly with all the features that the XBee has to offer. Other ZigBee gateways are available from Pervasa, RFM, Crestron, Exegin, AMX, Alektrona, and many others.

\section{ConnectPorts}

Digi's ConnectPort line of gateways provides many advantages: they use the XBee radios you're already familiar with, and they have an easy-to-use web interface and an internal Python programming language interpreter. Having Python inside means you can write and run programs to manipulate your incoming and outgoing data. You can provide your sensor readings in just the way a remote system wants them, interpret 
remote commands to turn them into meaningful actions, or do a little of both at the same time so that your sensors and actuators can work locally together, only contacting the outside world when it's essential to do so. The internal Python interpreter comes preloaded with XBee libraries that make it very easy to script commands, communications, and transformations right inside the gateway.

\section{Selecting a ConnectPort}

All of the ConnectPort models described below (and shown in Figure 7-1) support connections between ZigBee and another Internet medium. Many are optionally offered with 802.15.4 Series 1 radios (not compatible with ZigBee), so be sure you choose the ZigBee version when you purchase your ConnectPort! Some support serial/USB connections to control devices that are plugged into the gateway itself. Here we'll focus on the Internet connectivity because that's what's important for our networks:

\section{ConnectPort X8}

This gateway supports ZigBee, Ethernet, WiFi, two different mobile data networks, two USB ports, one standard serial port, and a local sensor port. This Cadillac of the ConnectPort line costs from $\$ 800$ to $\$ 1,000$ depending upon configuration.

\section{ConnectPort X5}

Designed for Vehicle Area Networks (winkingly abbreviated VAN), this gateway comes with ZigBee, satellite radio, mobile data, WiFi, and a GPS feed. It is a rugged unit designed with fleet trucks in mind. The X5 runs about $\$ 1,000$.

\section{ConnectPort X4}

The $\mathrm{X} 4$ router is available with Ethernet or WiFi and a slot for a mobile data radio along with one USB port and one plain serial port. A ConnectPort X4 runs from $\$ 450-700$ depending upon configuration.

\section{ConnectPort X3}

This is a brand-new and fairly inexpensive option for mobile data connections from ZigBee. The X3 offers a GSM/GPRS radio that with the addition of a data plan will let you connect from just about anywhere. The base cost is about $\$ 250$.

\section{ConnectPort X2}

Here's the simplest option. The base X2 comes with a ZigBee radio and one Ethernet port. (There's also a WiFi version that costs more). The X2 isn't brimming with memory but you can get a surprising amount done inside its $8 \mathrm{MB}$ of RAM. The $\mathrm{X} 2$ was originally manufactured with a metal enclosure and an external antenna that retailed for about $\$ 200$. Recently a slimmed-down design has been released at $\$ 99$, with a plastic case that allows for an internal antenna (X2-Z11-EC-A). That's the one to get started with. 


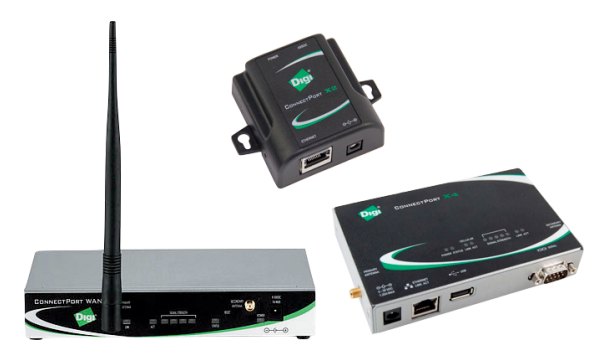

Figure 7-1. The ConnectPort line of gateways, including, from left to right: the X8 with a wide offering of interfaces, the inexpensive X2 for Ethernet, and the midrange X4, which can also come configured for WiFi or mobile data

\section{Setting Up a ConnectPort}

It is extremely easy to set up a ConnectPort. This section will demonstrate setup and configuration for the low-cost ConnectPort X2 ZB Ethernet (Digi X2-Z11-EC-A). Setting up the $\mathrm{X} 4$ or $\mathrm{X} 8$ is very similar:

1. Begin by plugging the ConnectPort into a wall outlet using its supplied power adapter.

2. Next, use a standard Ethernet cable to attach the ConnectPort to any available Ethernet jack on your Internet router. Your home network is probably already configured to assign IP addresses using DHCP, in which case simply powering it on and plugging it into the Ethernet port will allow it to configure its own network settings. If not, you'll have an opportunity to do manual configuration in the next step.

3. Connect a computer running Windows to the same network as the ConnectPort. In many cases, using the local WiFi connection will be fine, but if you aren't sure, plugging into the same Ethernet switch as your ConnectPort will ensure that you're using the same local network. Business and educational networks often have additional restrictions that your system administrator will need to help you with.

4. Some ConnectPorts come with a software CD that includes a Windows program called Device_Discovery.exe. Insert the CD into your Windows computer and launch Gateway Software $\rightarrow$ ConnectPort $\mathrm{X} \rightarrow$ Configuration $\rightarrow$ Device Discovery. This will open a window that will show you all the devices on your local network. (If you don't have the CD, the program can also be downloaded from Digi's website at $h t t p: / / w w w$.digi.com/support/getasset.jsp?fn $=40002265 \varepsilon t p=4$.)

\section{Troubleshooting}

If you don't see any devices listed in the Device Discovery program, check to make sure that the ConnectPort is showing a power light on the front, and that it is properly connected to Ethernet. A yellow link light on the Ethernet jack will illuminate as long as there is some kind of Ethernet connection active. Also make sure your computer is 
on the same network. Some home Internet setups are multiple boxes that operate on different TCP/IP networks from each other. If possible, try plugging your computer into the same set of Ethernet jacks as your ConnectPort, turn off WiFi, and see if clicking on "Refresh view" convinces the ConnectPort to pop up in your device list, as shown in Figure 7-2.

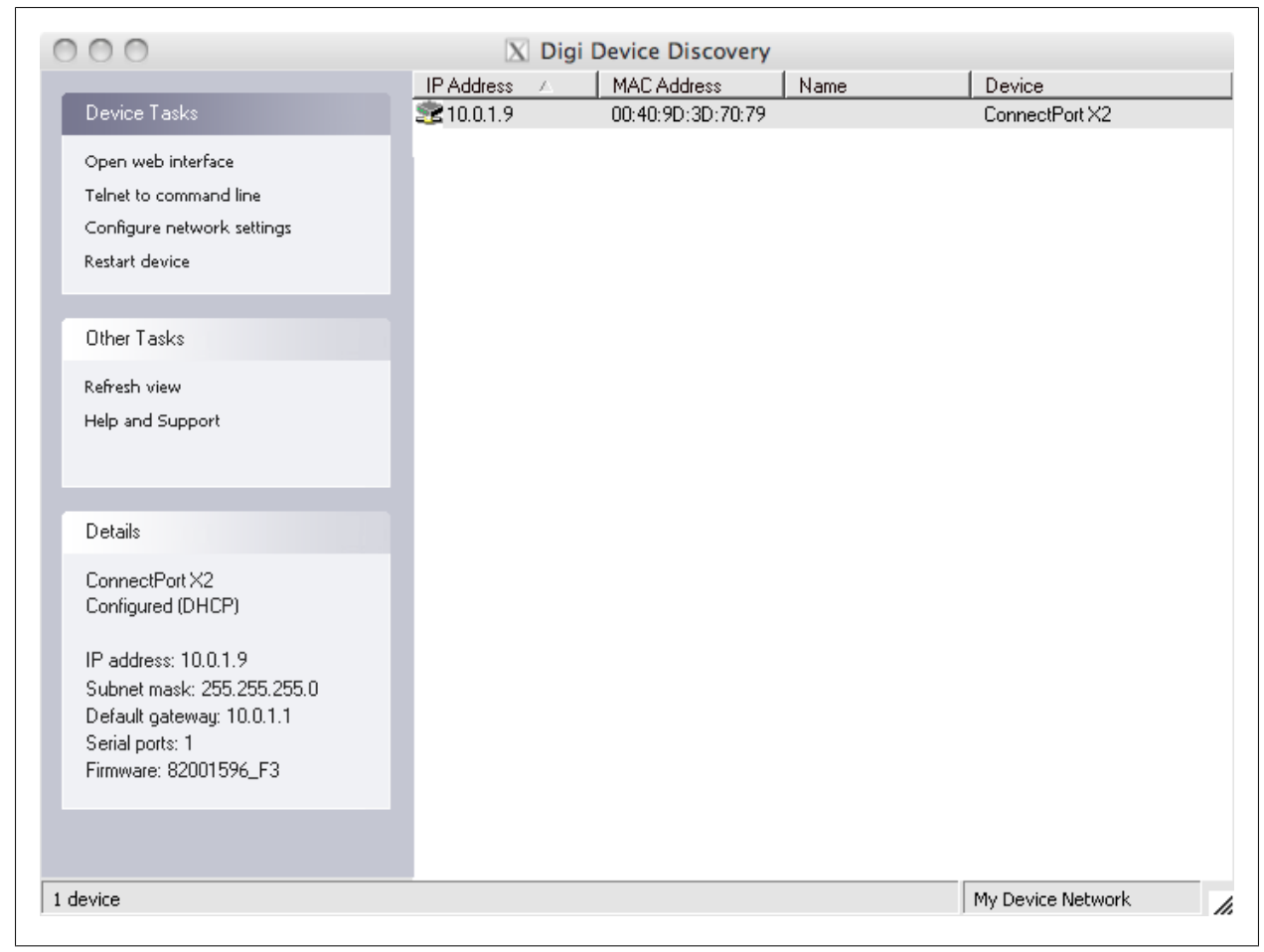

Figure 7-2. Using Device_Discovery.exe to locate the ConnectPort on your local TCP/IP network

If your network does not assign IP addresses automatically (via DHCP),

click on the listed device to select it, then click "Configure network set-
tings" under Device Tasks to enter the IP address information for your network.

\section{Configuring a ConnectPort}

Once you can see the ConnectPort listed in the Device Discovery window, make a note of its IP address. In most cases double-clicking on the device in the list will open a web browser; however, if it doesn't, you can also simply type its IP address into your browser's URL field. For example, if the IP address listed is 10.0.1.9, putting http:// 10.0.1.9 into your web browser should open up a configuration screen similar to the one shown in Figure 7-3. 


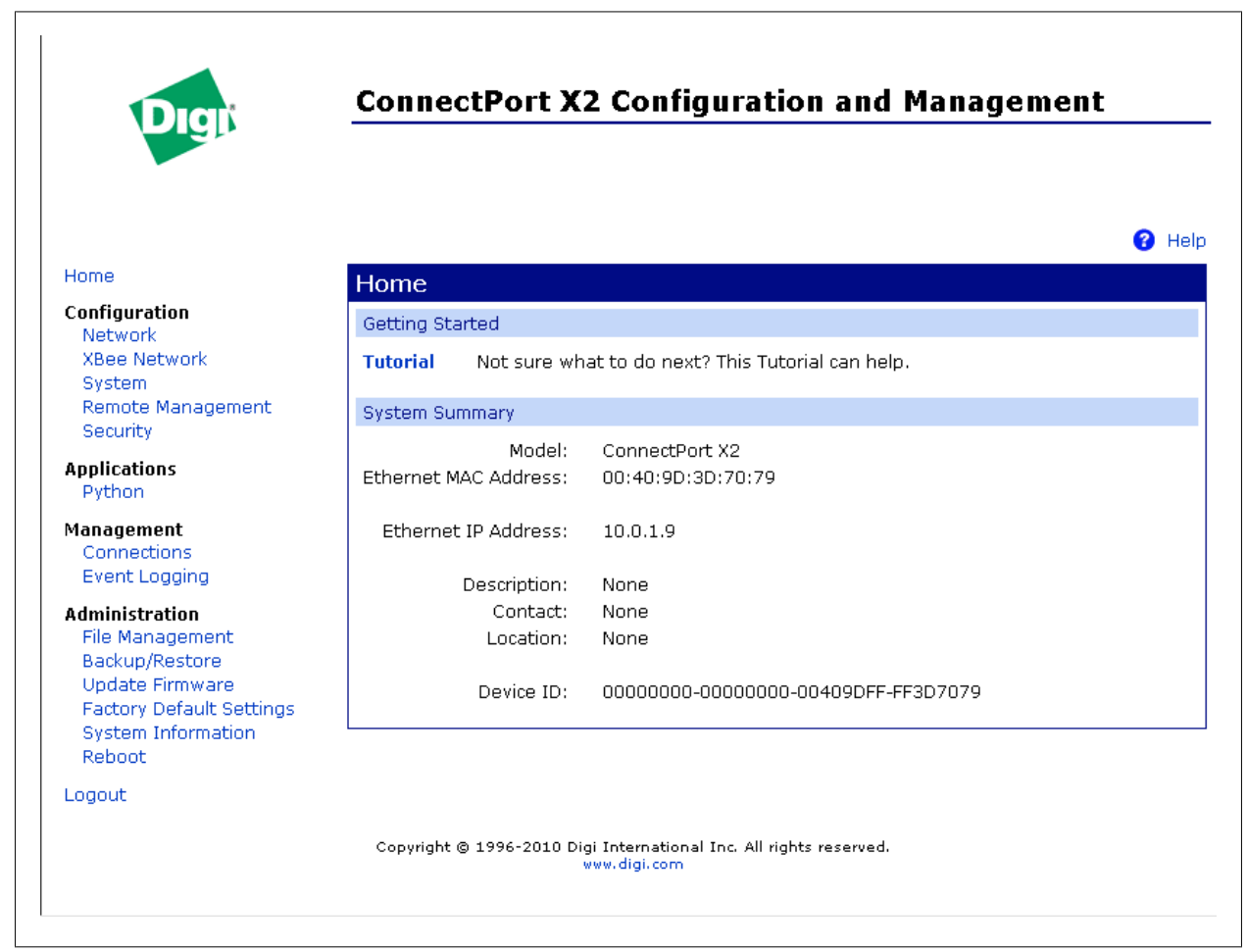

Figure 7-3. ConnectPort Home screen accessed using a web browser

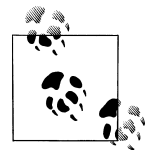

If you are familiar with the Telnet program, you can use it to contact the ConnectPort by connecting to its IP address on the default port 23 Q.3: options.

The ConnectPort comes preconfigured to obtain its IP address automatically via DHCP. If you'd prefer that it had a fixed address, or if you need to change any other TCP/IP network settings, they can be accessed by clicking on Network, displaying the screen shown in Figure 7-4.

Inside the ConnectPort is a ZigBee radio that is preconfigured to be a network coordinator. To view or change its settings, first click on XBee Network to see a list like the one shown in Figure 7-5. This list will include the internal radio, as well as any other devices that have joined the ZigBee network. 


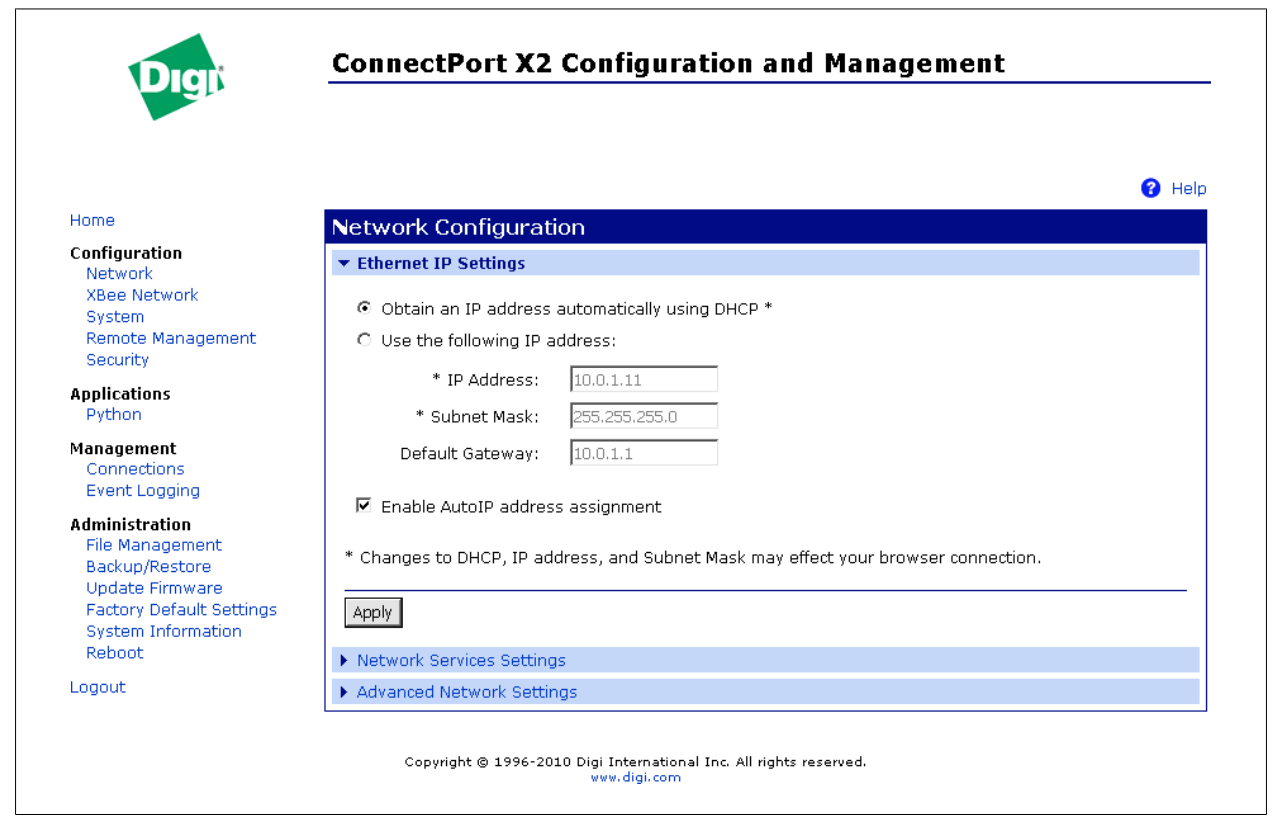

Figure 7-4. Network configuration for the ConnectPort's TCP/IP connection to the Internet

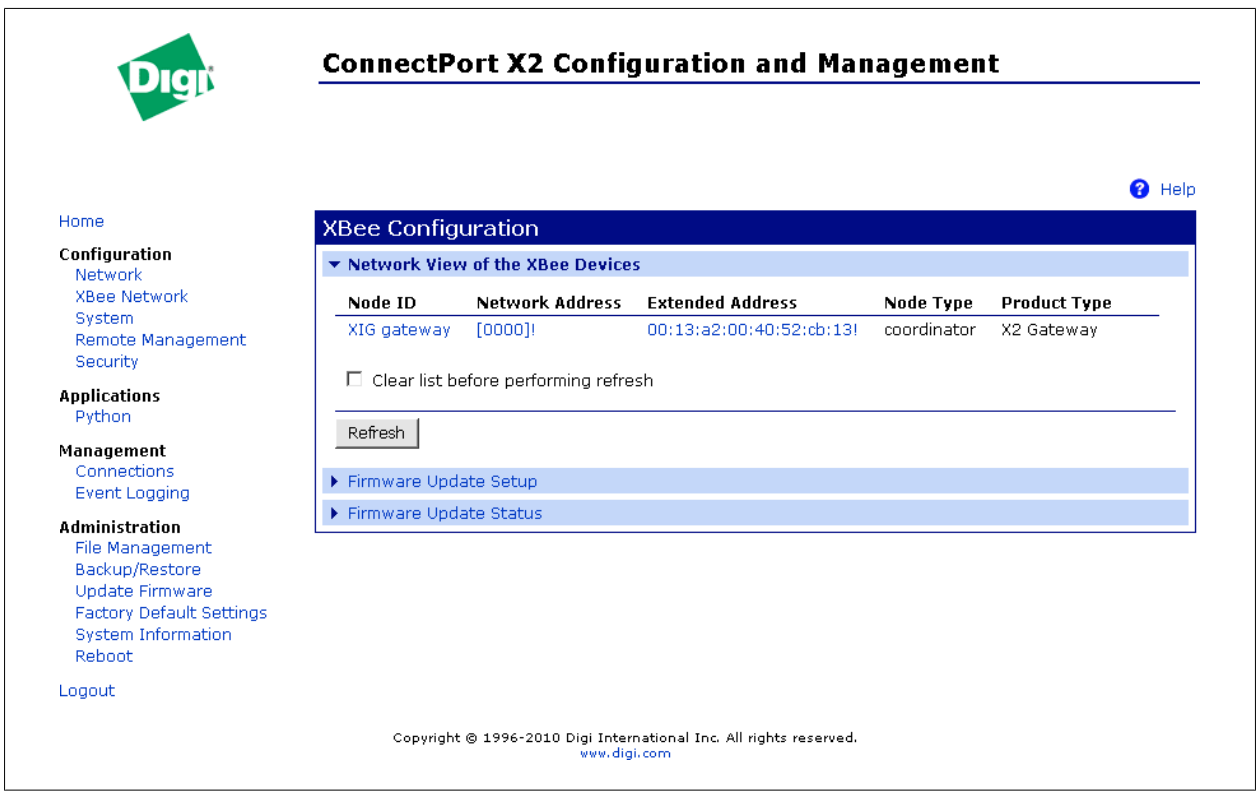

Figure 7-5. XBee Network lists the radio inside the ConnectPort, as well as any other radios that have joined the network 
Each radio listed in the XBee Network screen can be configured through your browser. This includes the local radio inside the ConnectPort. But wait, there's more. It also includes any other remote radio that has joined the ConnectPort's network. You can now change the configurations of all the radios on your network from the comfort of your laptop computer by clicking on that XBee in the list to see its detail screen, as shown in Figure 7-6. These can be changed right from your browser! You may notice that these figures show a node ID that labels the radio. See the sidebar "Naming Radios" on page 202 for information about how to set and discover these node identifiers.

Be careful changing the settings on remote radios. If you make a change that accidentally causes one to leave the network, you may then need to physically access that radio to fix the situation, which might be difficult if your remote radio is miles away, or on the ceiling, or strapped to an angry goat. Think before you click!

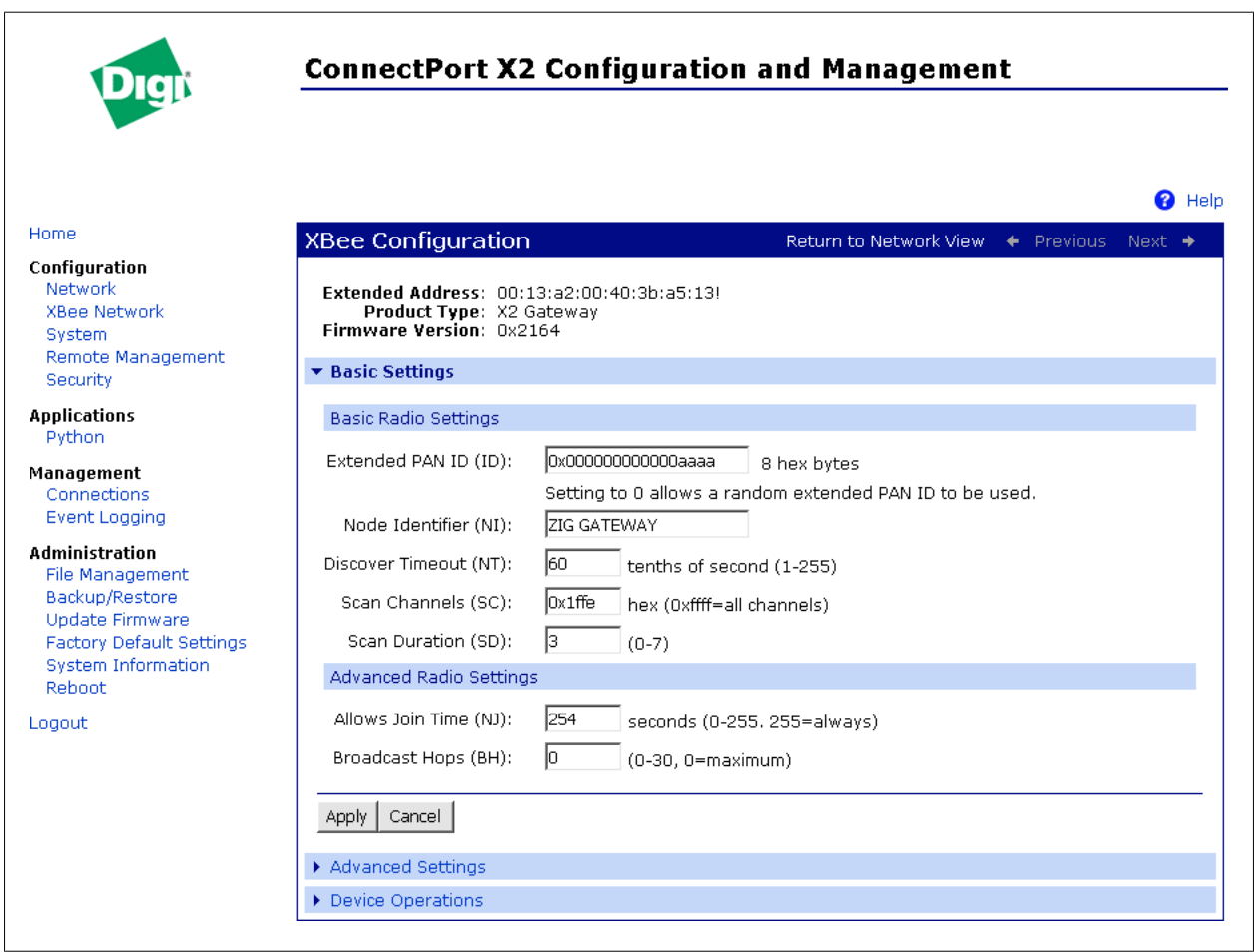

Figure 7-6. XBee Configuration details show both basic and advanced settings for every radio joined to your ConnectPort's network 
We will look at several other configuration screens in detail when we set up the XBee Internet Gateway later in this chapter. In the meantime, you might want to browse around and familiarize yourself with the various settings. The Help link at the top right of each screen will link you to a basic explanation of the functions. Of course, you should be somewhat cautious about changing any settings you don't understand, but remember that measured bravery is the prime gateway to technical expertise.

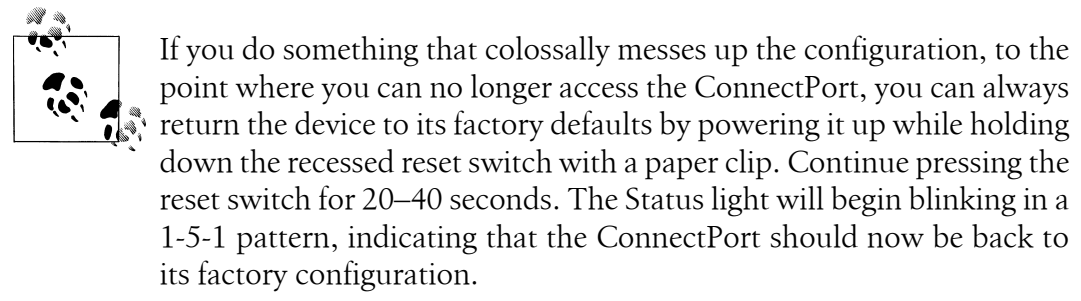

\section{Naming Radios}

Each ZigBee radio has at least two addresses: its permanent and unique 64-bit address and its coordinator-assigned 16-bit address. Human beings have a much easier time remembering names than numbers, especially long numbers. In a welcome nod to the human race, XBee radios offer another, more humane option. Each radio can be configured with a node identifier text string that names it over the network. The ATNI command will set this Node Identifier with any phrase up to 20 characters long. For example, to set the name of your radio to Mary's Temperature Sensor, the command in AT mode would be ATNI Mary's Temperature Sensor (the space after ATNI is optional but makes for easier reading). Typing ATNI by itself and hitting Enter will cause the local radio to reply with its current node identifier. In most cases, though, you will want to use this human-friendly name to find a remote radio that's out on your network.

The Node Discovery command ATND will start this process. In AT mode (transparent/ command mode), the Node Discovery command will be broadcast to the entire network and each radio that can hear it will respond with a block of information about itself, including its node identifier if that's been set. That format for each block you receive will be:

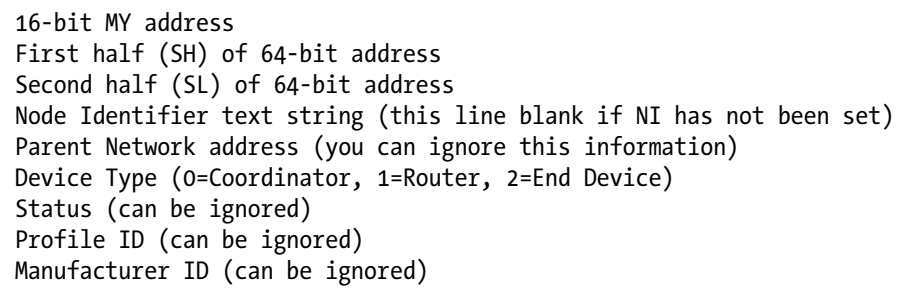

In API mode, issuing this command (inside an AT command frame, of course) will result in a separate response frame being returned for each radio. The command data will contain response blocks similar to the one above. 
It's also possible to configure any radio's destination address by using the ATDN command to tell it the destination's node identifier you'd like it to speak to. In AT command mode, ATDN Mary's Temperature Sensor will attempt to discover that node's numeric address over the air, and if it's found will automatically set the DL and DH registers to the appropriate 64-bit address. When the ATDN command is successful, it returns OK to let you know, then exits command mode immediately. If the ATDN command fails to find the radio you requested on the network, it will return ERROR and remain in command mode. If you are using API firmware, issuing this command inside an AT Command frame will result in a response frame that contains both the 16- and 64-bit addresses of the remote radio, along with a success or error indicator in the Command Status byte. The Destination Node command allows you to implement a whole system of radio naming and name lookups that bypass using any numeric addresses in your code. Consider this if your project involves creating many duplicate networks where each node is in a role that could be called by a specific name, no matter what its physical or assigned numeric address might happen to be.

\section{Remote Management}

You may still be glowing with excitement about your newfound ability to configure radios wirelessly from the ConnectPort's web interface. It is certainly pretty cool but it does require that you have direct network access to your ConnectPort, something that's not always possible once you leave the location where your ConnectPort lives. In most cases a firewall, network address translation, or other network obstacle of some kind means you'll need to be physically near the device and plugged into the same network to access the ConnectPort's web interface. But if you think that's going to limit your powers, well hang on to your hats and glasses because the ride is about to get about 10 times better. At the Remote Management screen you can configure your ConnectPort to open a special connection that links it to a central access server at Digi International called iDigi. Once that link is set up, logging on to the iDigi server's web interface from anywhere gives you full configuration access to all of your ConnectPorts and every radio that's joined to any of those ConnectPorts . This is a massively powerful feature. It's like suddenly discovering you are in command of a robot army, willing to do your worldwide bidding. Here's how to get started:

1. Click on Remote Management in the web interface to show the configuration screen. Check the box for "Enable Remote Management and Configuration using a client-initiated connection," then enter developer.idigi.com for the Server Address as shown in Figure 7-7. This is where you can link to a central server and command your robot army of sensor networks.

2. Check the box to "Automatically reconnect to the server after being disconnected." The default setting of 1 minute should be fine. This will ensure a persistent connection. 


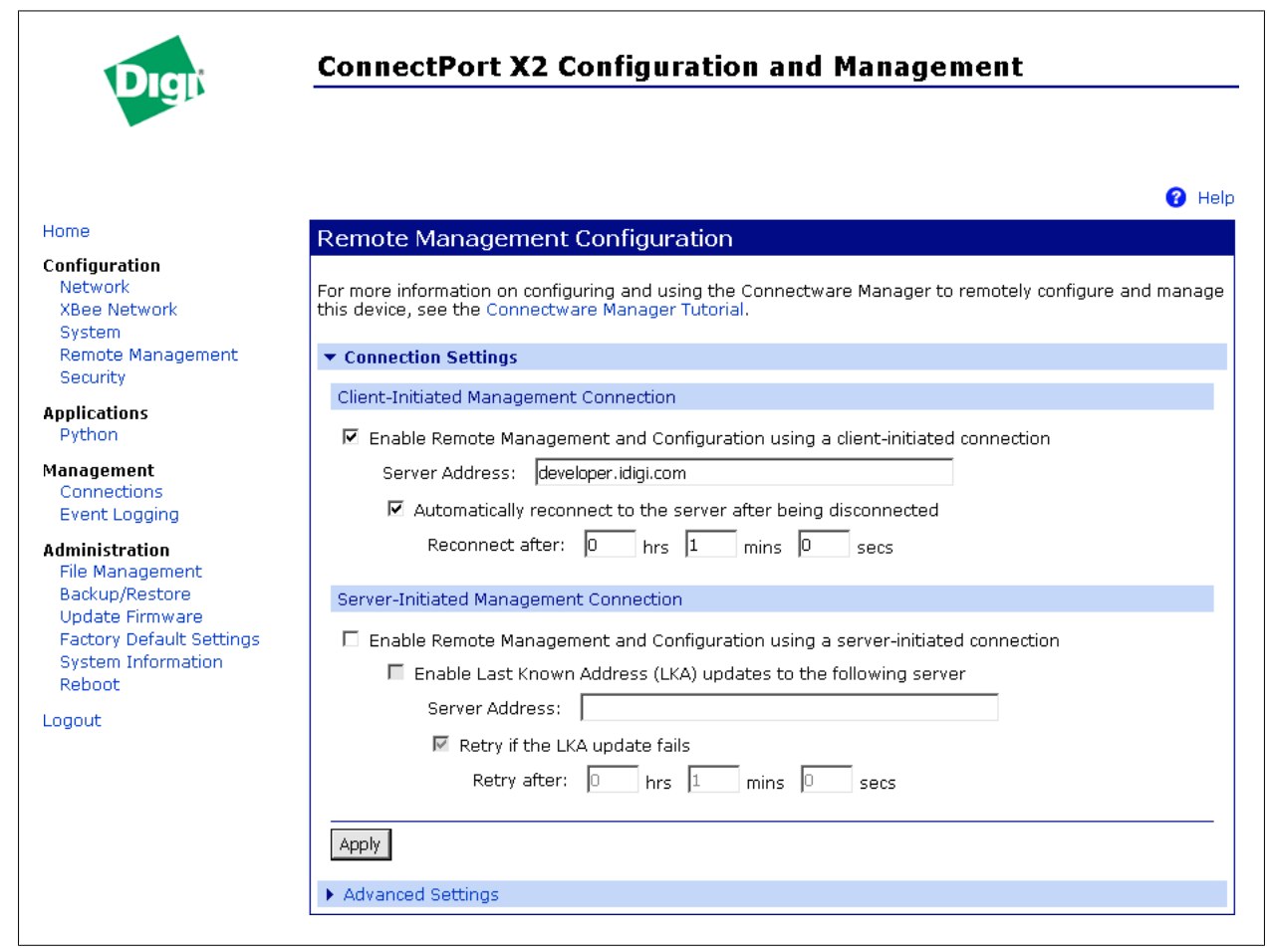

Figure 7-7. The Remote Management Configuration screen on the ConnectPort

3. Click the Apply button at the bottom of the screen to save your changes. The ConnectPort will now attempt to make an outgoing connection to the iDigi server.

\section{iDigi Connectivity Server}

iDigi is a cloud service that aggregates ConnectPorts and their networks so that they can be accessed and configured remotely from anywhere in the world. Currently anyone can set up a free account to control up to five ConnectPorts, along with an unlimited number of radios connected to each of those ConnectPorts. To begin, go to http:// developer.idigi.com (Figure 7-8). Before you can log in, you'll need an account. Select the New User link and fill out the registration forms (Figure 7-9). At the end you'll be taken back to the login screen where you can now access your account. Enter your newly selected username and password to begin your remote networking adventure.

\section{iDigi Features}

The iDigi service is intended as a platform for application-to-device messaging, data storage, and administration. We'll mostly be considering the administration features here, because you'll want to use them with your ConnectPort. 


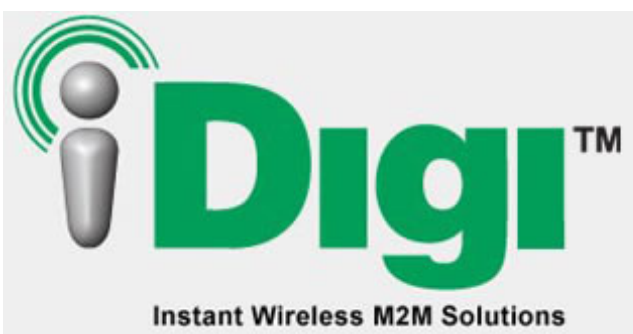

Instant Wireless M2M Solutions

\section{Log in to the iDigi Platform}

User Name:

Password:

Log on

Forgot your user name or password? Are you a new user?

\section{Privacy Policy I Contact us}

IDigi is a Digi International brand. Copyright (c) 1996-2010 Digi International Inc. All rights reserved.

Figure 7-8. iDigi login screen at http://developer.idigi.com

\section{iDigr}

\section{iDigi Registration}

Company:

First Name:

Last Name:

Email:

Job Title:

Phone:

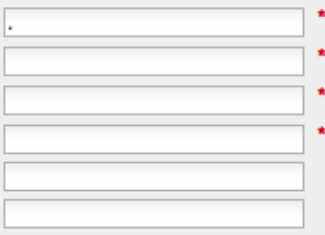

* Asterisk indicates required field.

Your privacy is important to us! We do not share your contact information with third parties. Privacy Policy

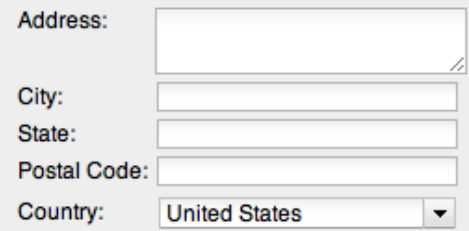

Country:

United States 
On every iDigi screen (see Figure 7-10), you have access to a menu that includes:

Home

This contains the Welcome link that describes the system and a Documentation area that connects to support forums and various downloadable documents.

\section{Management}

Here is where most of your iDigi work will get done. It's where you can administer your ConnectPort devices and their networks of XBees, along with iDigi's data storage and messaging features.

Devices

Every piece of equipment that connects directly to iDigi will be listed here. You'll want to add your ConnectPort to this list, and we'll show you how below.

XBee Networks

Once some ConnectPort devices have been added, their ZigBee networks can be discovered. They will then be listed here where they can all be remotely configured.

Storage

This is where uploaded data can be stored. Check the online documentation for more information about storage features.

Web Services Console

Web services are a standard for exchanging information that use HTTP browser protocols and URLs as an application programming interface. This is where the application-to-device messaging features of iDigi are implemented. Check the documentation area for an entire manual on these services if you're interested in learning more about them.

Subscriptions

The Summary and Details links under Subscriptions show you the features and limitations associated with your account. Contact iDigi if you need to raise the number of devices or allowable traffic limits for your project.

\section{Administration}

The My Account, Messages, and Operations links are where you can update your settings, read about system updates, and review a logfile of the operations that you've performed during your iDigi session.

\section{Adding a ConnectPort}

Your first order of business is to add your ConnectPort to the Devices list so you can begin managing it.

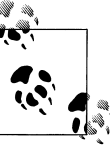

It is best to complete the local configuration steps described in "Remote Management" on page 203 before attempting to add your device to

iDigi. This will ensure that your ConnectPort is preconfigured to open a socket connection to iDigi even if it can't be discovered automatically. 


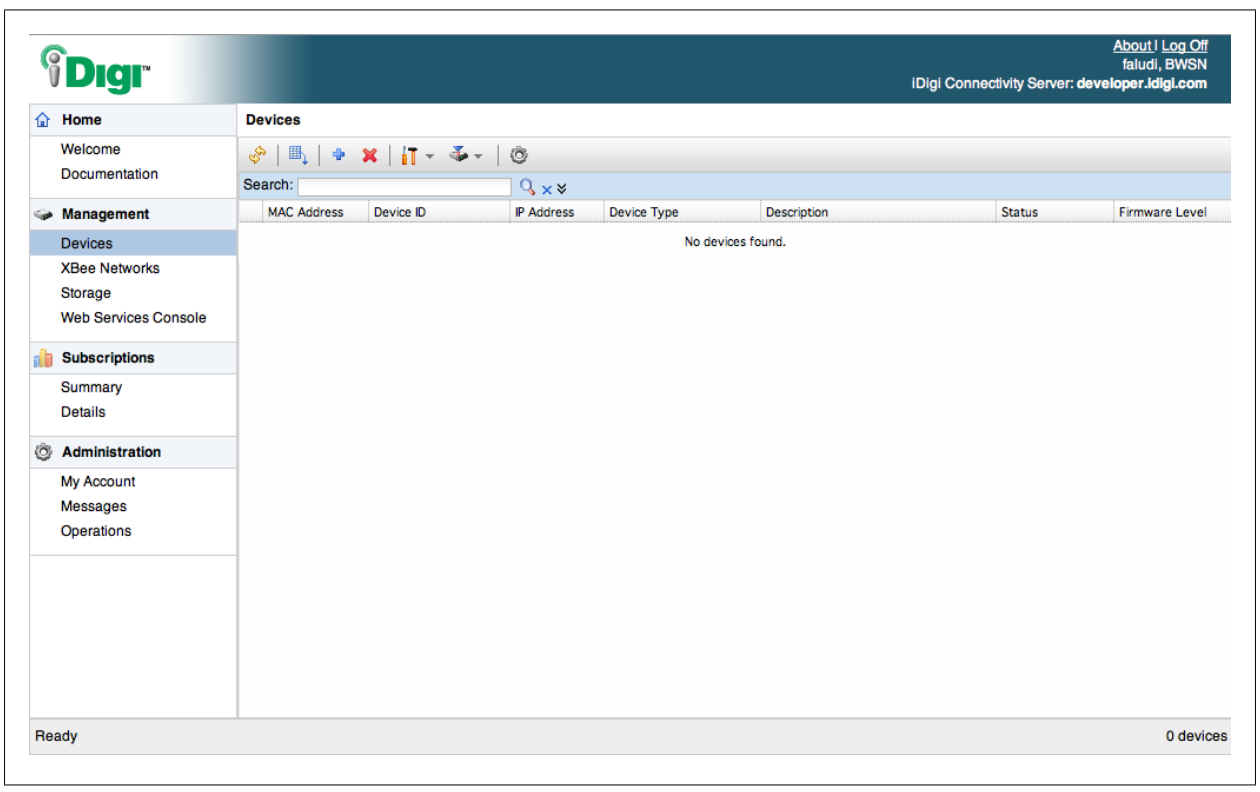

Figure 7-10. iDigi Devices screen, before any devices have been added

Select the Devices link and then click on the blue plus sign icon on the button bar to begin adding a new device. You'll see a dialog box called Add Devices, as shown in Figure 7-11.

If your ConnectPort is on the same local network as the computer where you are using iDigi's web interface, it may be listed here automatically. If it is, simply click on the device in the list and then click the OK button to add it to iDigi. Not all browsers or networks will allow this automatic discovery to happen properly, so if you don't see your device listed, try clicking the Add Manually button to see the display shown in Figure 7-12.

To add a ConnectPort manually, enter its MAC address (the unique hardware address assigned to every Ethernet device) in the MAC field and click on the Add button. You can find the MAC address printed on the back of each ConnectPort. It will begin with 00409D, which is the official prefix for all Ethernet addresses assigned to Digi devices. You can either use the XXXXXX:XXXXXX or XX:XX:XX:XX:XX:XX format. In Figure 7-12 the MAC address entered is 00:40:9D:33:B7:0C; yours, of course, will be different. Once you enter that address and click on the Add button, you can click the OK button at the bottom of the screen to complete the process of adding the new device to your iDigi account. A green bar at the top of the screen will briefly appear to indicate that your device was added successfully, as shown in Figure 7-13. 


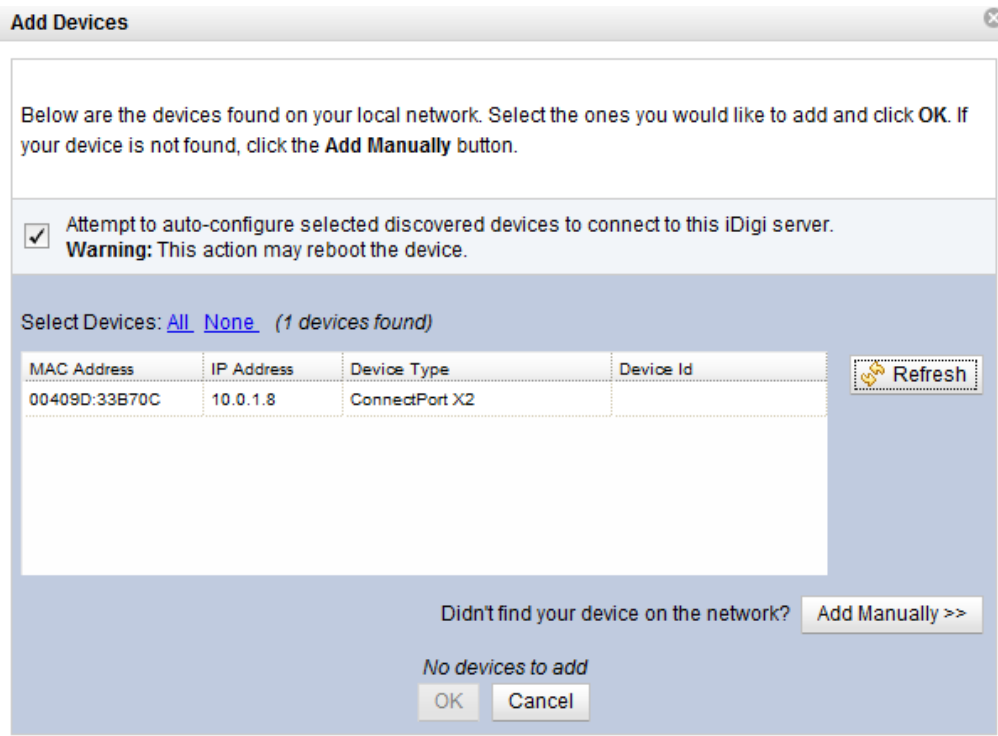

Figure 7-11. The iDigi Add Devices dialog box will automatically attempt to discover any devices that are on your local network

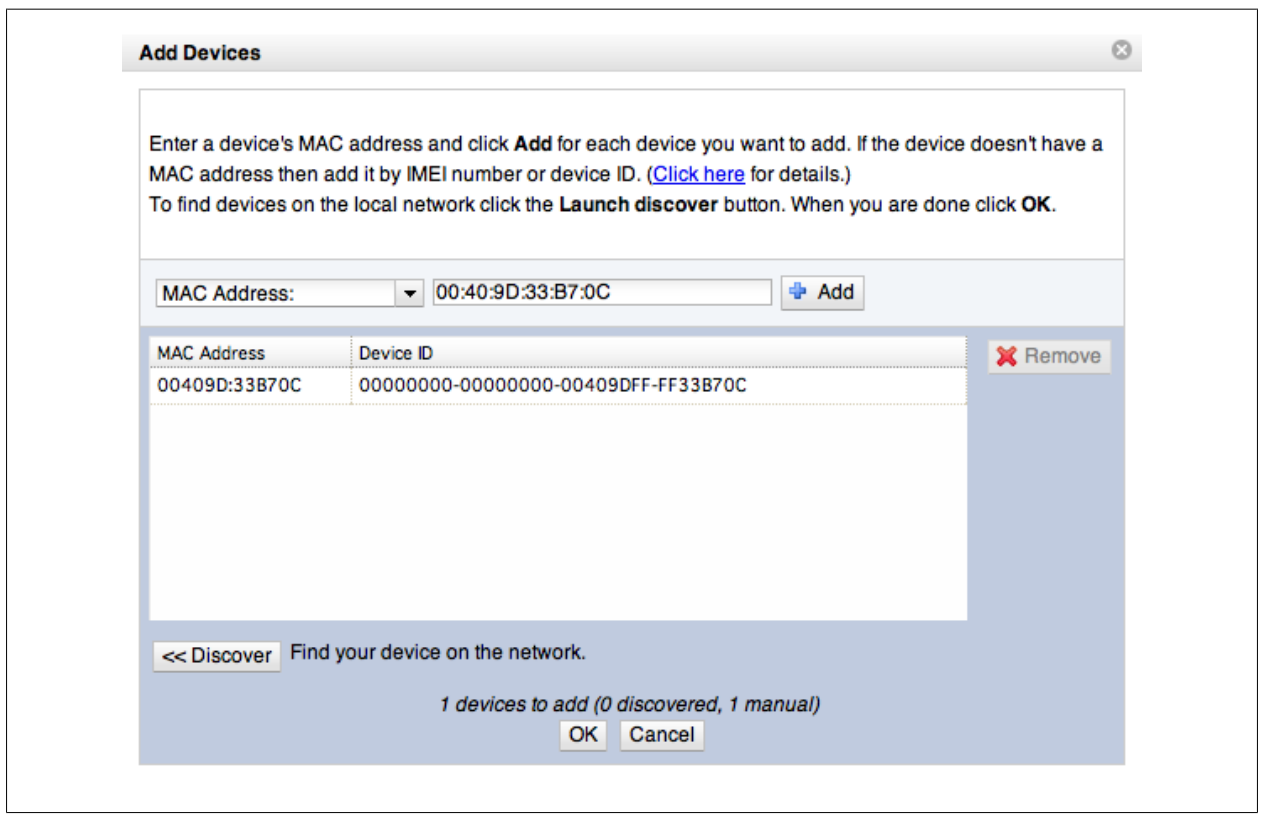

Figure 7-12. iDigi's Add Manually feature lets you search iDigi for any ConnectPort that is already configured for remote administration 


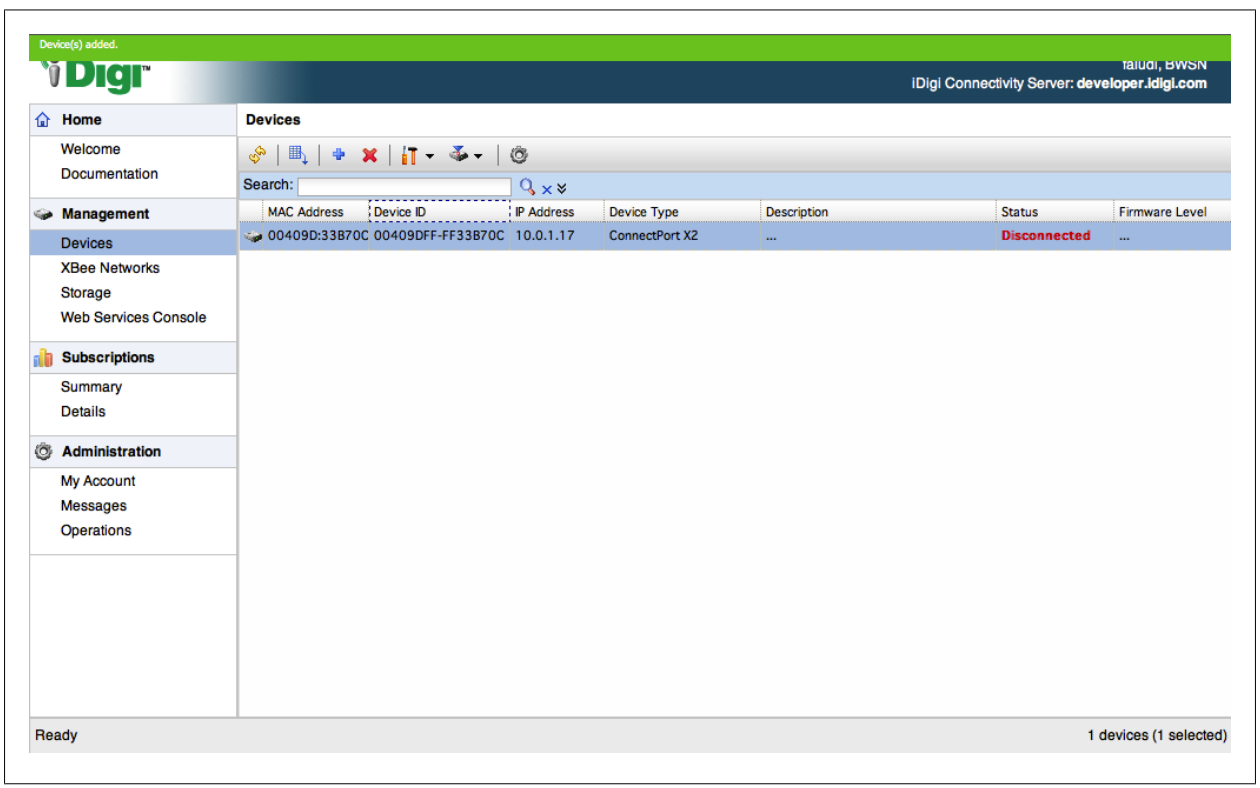

Figure 7-13. Devices newly added to iDigi will be confirmed by an announcement in green at the top of the screen

If your device's status is initially listed as Disconnected, try waiting a minute and then clicking on the circling yellow arrows in the button bar to refresh the list. Also, check your ConnectPort's connection to the Internet, and be sure that you ran through all the steps listed in "Remote Management" on page 203.

\section{Viewing Configurations}

Once you have added your ConnectPort to the Devices list and its Status is listed as Connected, simply double-click on the listed device to open up its properties page. You'll first see a Home screen as shown in Figure 7-14, along with a list of links to configure the various properties of the ConnectPort. Any system information that has been entered will be shown here as well. Now you can perform many types of configuration from anywhere in the world that you can find an Internet connection!

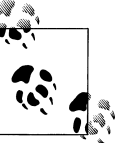

Some features differ between the direct web interface and the iDigi interface. For example, iDigi does not currently show you a list of active connections or read the event log. These features may be added in the future, as iDigi is under active development. 


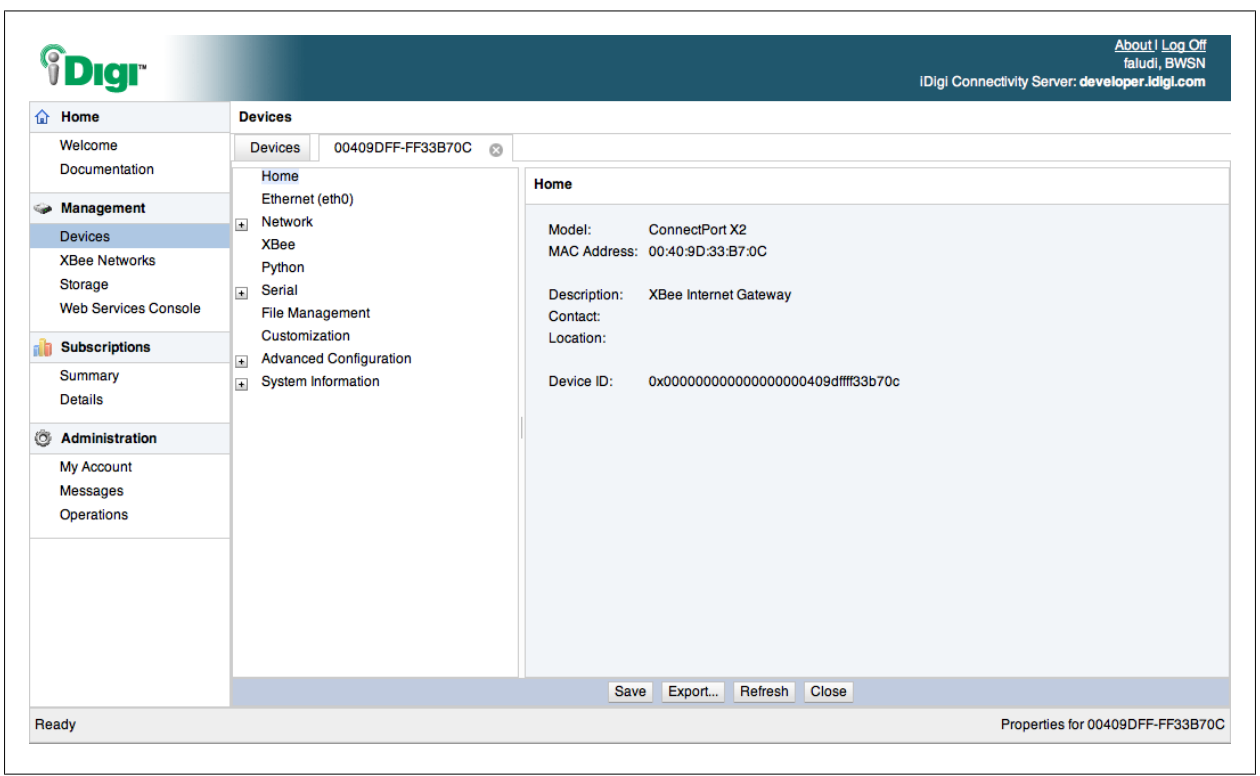

Figure 7-14. The ConnectPort's Devices Home screen on iDigi lists its MAC address and model

For example, the Python link shows a screen (Figure 7-15) that lists all the programs and libraries loaded onto this device. iDigi gives you full access to remotely upload and delete these files, as well as to indicate which ones should start up automatically when the device is powered on. We will talk more about Python files in "XBee Internet Gateway (XIG)" on page 214.

Another useful screen is the System screen (Figure 7-16) under the Advanced Configuration link visible in Figure 7-15. Here you can enter a text description for your ConnectPort that will show up in the device list. It's very helpful to set this information if you have a number of ConnectPorts, so that it's easy to see in the listing which one is which.

\section{Firmware Updates and Remote Reboot}

Several more essential features can be accessed via the Devices list. (Clicking on the Devices tab at the top of the screen will take you back there.) Each ConnectPort can remotely receive upgrades to its internal OS firmware, as well as upgrades to the firmware that drives its internal XBee radio. (We'll talk about configuring the other XBees in the network below.) The Firmware icon in the button bar (Figure 7-17) displays a menu that includes these options. 


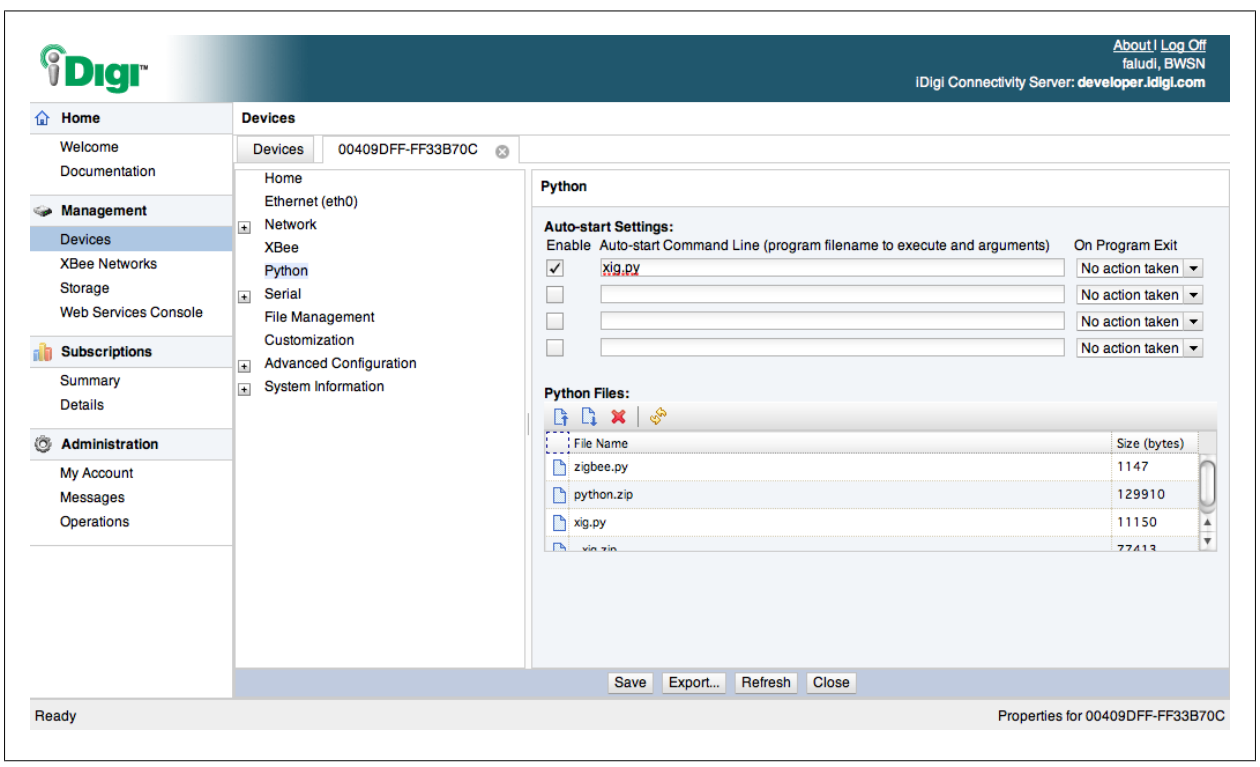

Figure 7-15. ConnectPort's Devices Python administration screen on iDigi; program files and startup can be managed here

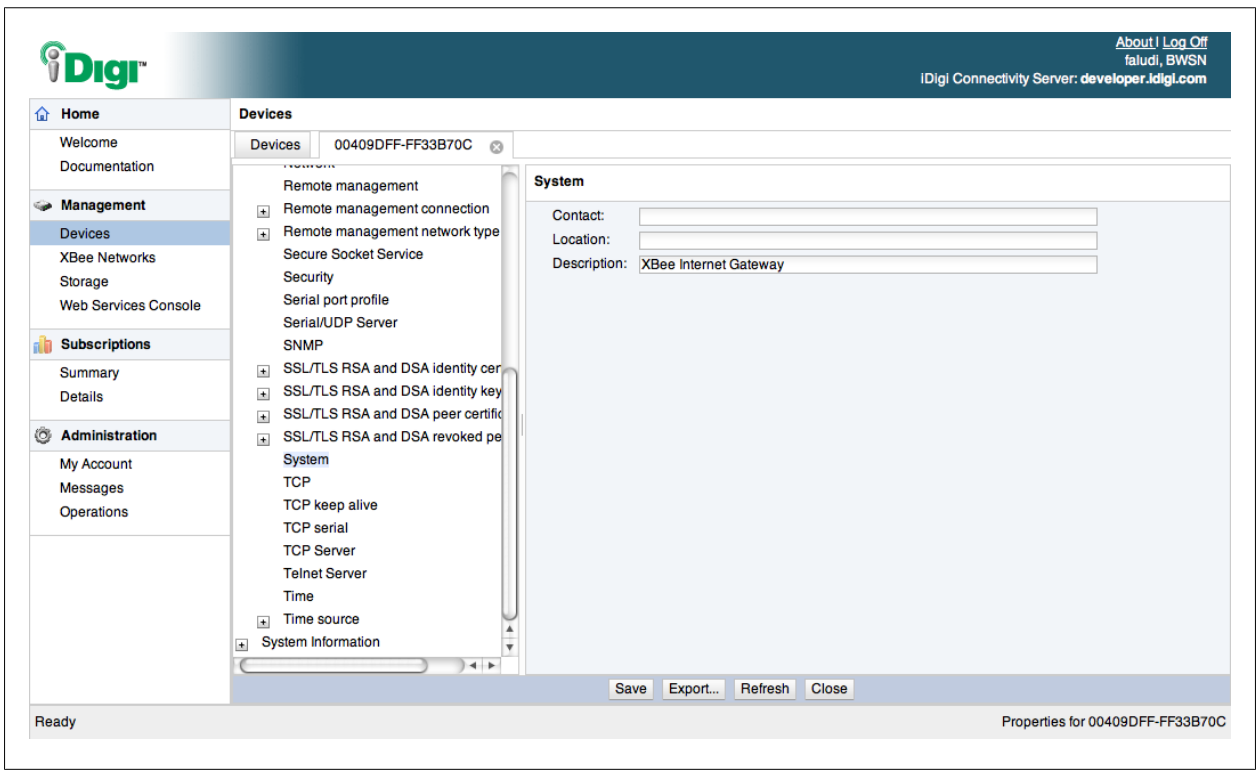

Figure 7-16. System information, including a description, can be entered under the Advanced Configuration link 


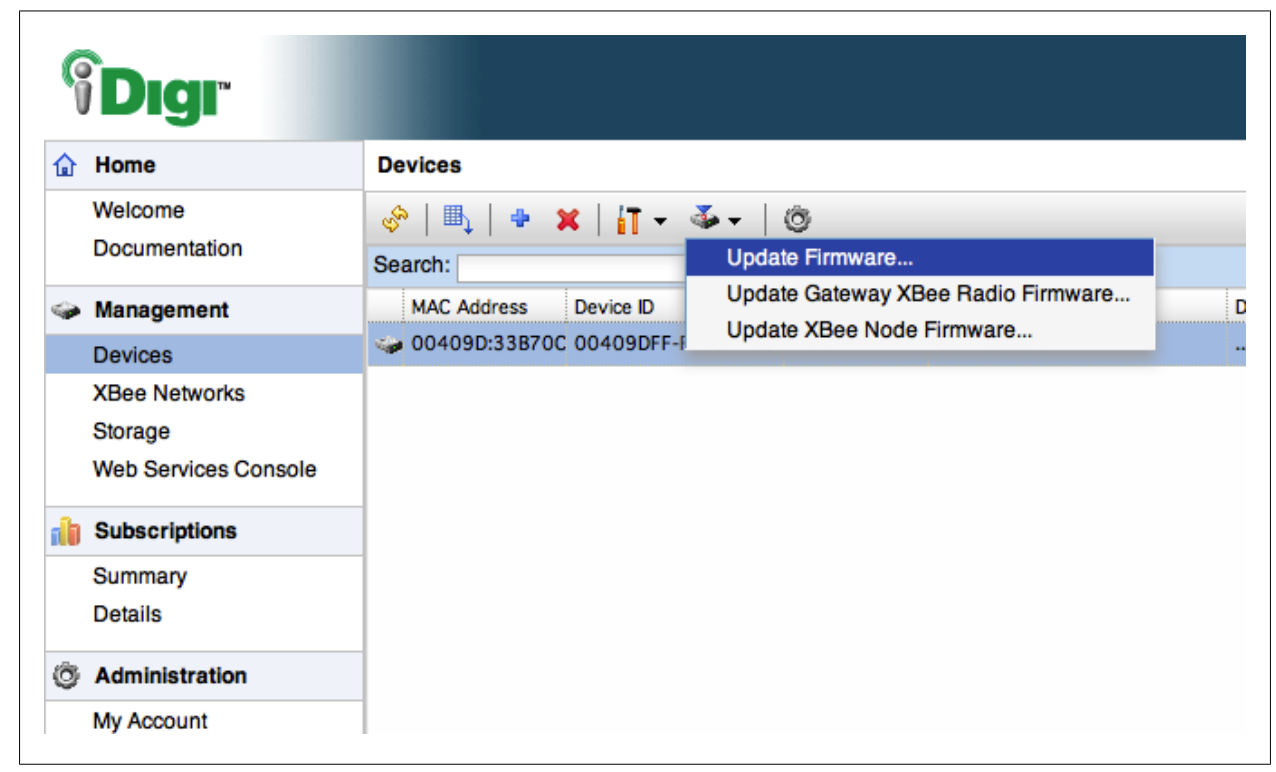

Figure 7-17. The firmware update menu on iDigi gives remote access for upgrading and changing the low-level device firmware

Firmware for the ConnectPort and the XBees can be downloaded to your local hard drive from the Support area on the regular Digi website. Back at iDigi, you can select the appropriate menu item to upload firmware with the .bin extension for the ConnectPort and with an .ebl extension for the Gateway XBee. Updating the firmware on remote XBee nodes takes two steps. First, select the Update XBee Node Firmware menu item to place the appropriate .ebl files onto the ConnectPort. For example, to update to the version of the ZigBee Router AT that's current as of this writing, you could upload a file called XB24-ZB_2270.ebl that's available in the Digi website's Support area. The second step is to make sure that the ConnectPort is configured for over-the-air firmware updates. Navigate to the device's properties and select the XBee link to show a screen like the one in Figure 7-18. Check all four boxes to ensure that your update is distributed automatically over the air to any radio that's out of date. You can also upload and delete XBee firmware files using the interface on this screen. Over-the-air firmware updates are an extremely powerful feature of iDigi. As long as your radios are joined to a ConnectPort, you are able to send them new firmware over the Internet using their own radio connection. Amazing!

\section{Viewing an XBee Network}

It's easy to examine your remote XBee networks with iDigi. Click on the XBee Networks link to see a list of all the radios that have currently been discovered (Figure 7-19). Initially you'll probably see only a single radio, the XBee that's inside the ConnectPort gateway. If other radios have joined your ConnectPort's network, you can discover 
them by clicking on one of the flashlight icons in the button bar- the first to do a regular discovery and the second to clear the cache and rediscover the network from scratch.

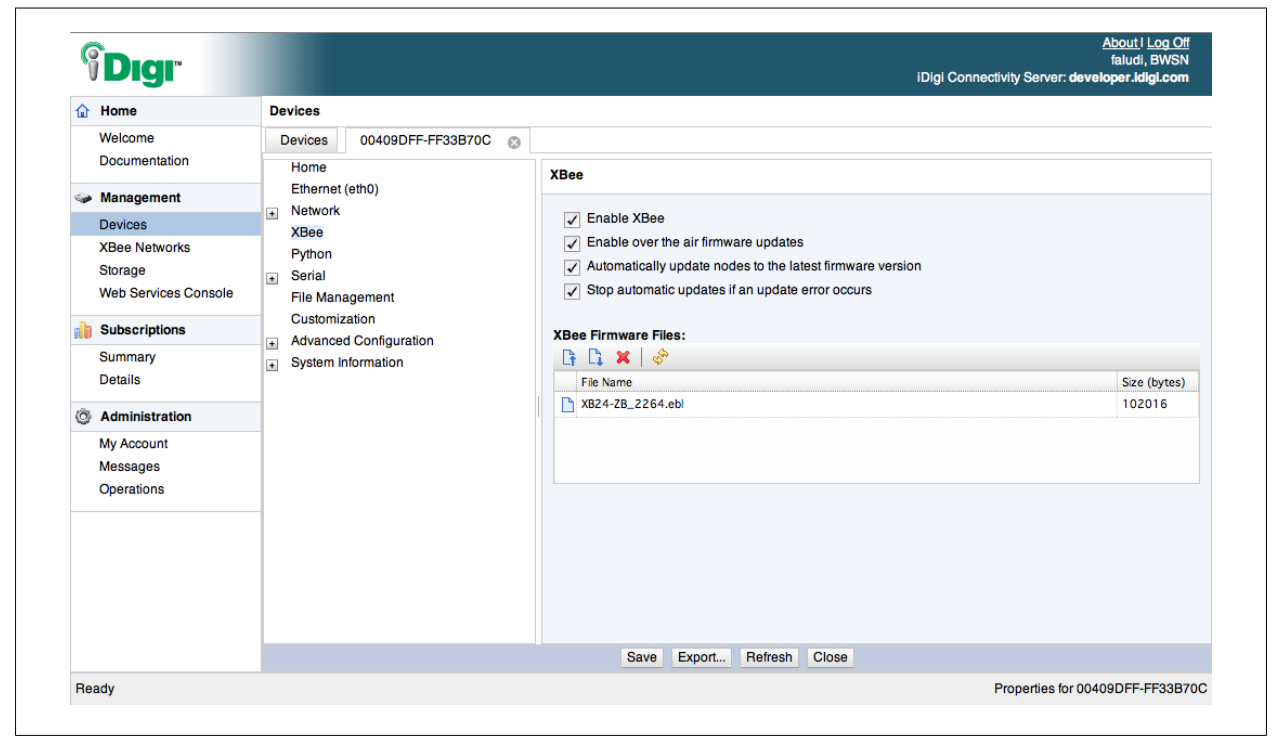

Figure 7-18. XBee firmware updates can be automatically distributed from the ConnectPort, using the configuration and files listed on the device's XBee screen

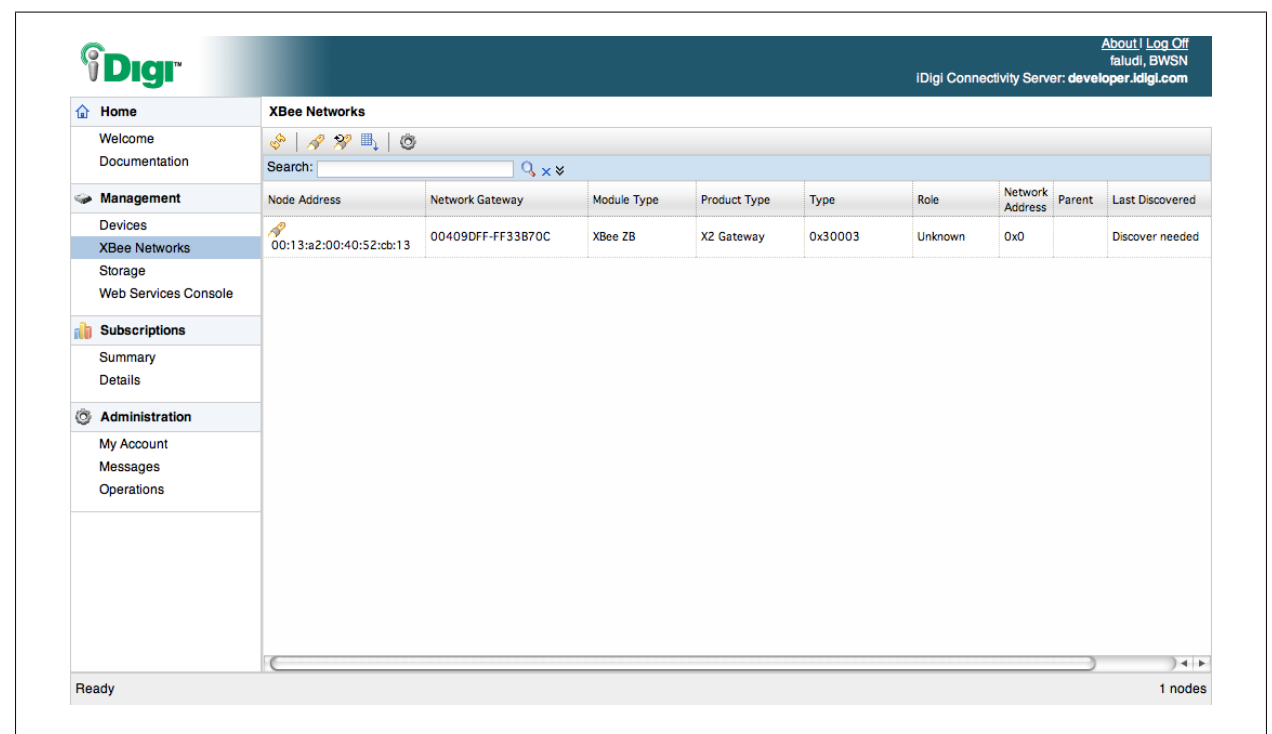

Figure 7-19. In iDigi, XBee Networks lists all the radios that have currently been discovered on all your networks, in this case just the internal gateway radio that displays initially 
To configure any of the remote XBees or the gateway XBee, simply double-click on its name in the list to open up a tab with its properties, as shown in Figure 7-20. Most of the interesting settings are on the Advanced screen (Figure 7-21). Some of these are old friends because they are the exact same settings you've been configuring with AT commands all along. Now you can change those settings from anywhere! For example, you can change the node identifier by entering a new one in the field called "Node identifier" on the Basic screen and then pressing the Save button at the bottom. (See the sidebar "Naming Radios" on page 202 for more information.) Use a reasonable amount of caution when you make changes because they will be executed immediately, and any changes that cause your remote radios to leave the network might require physical access to get them to rejoin.

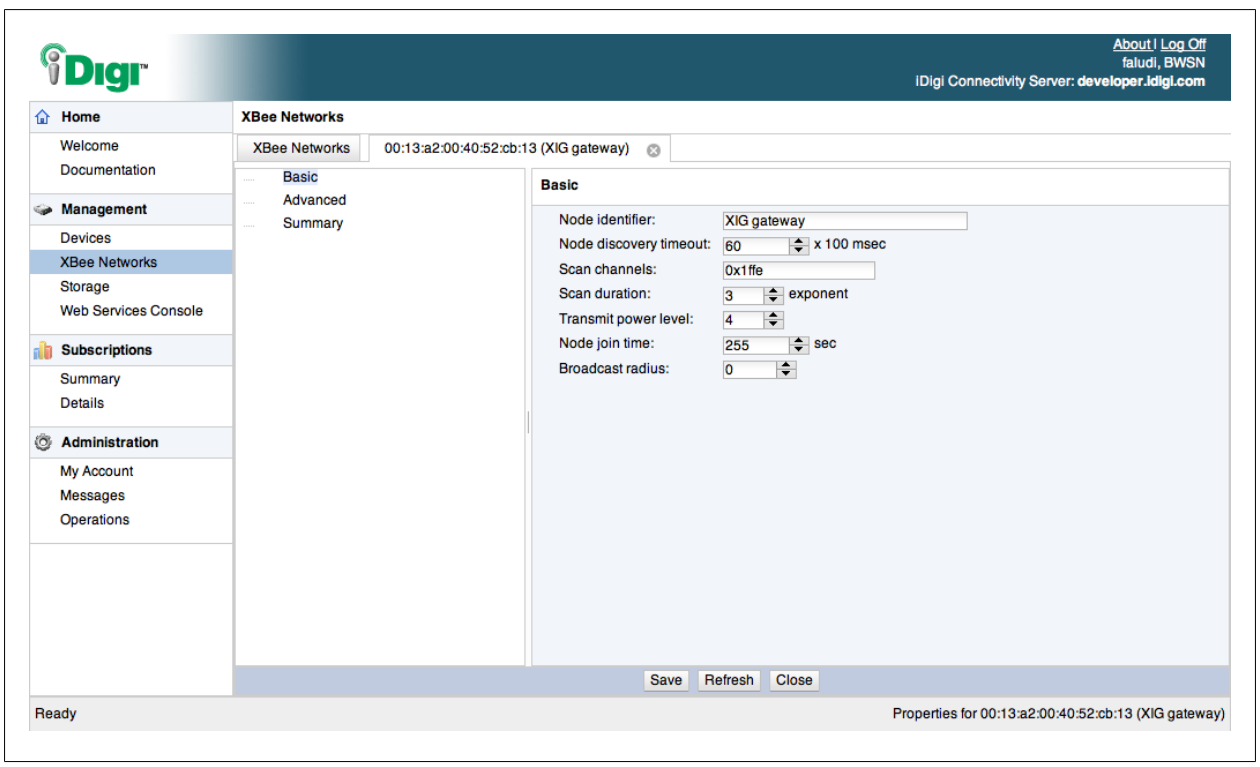

Figure 7-20. Basic view of an XBee's properties shows a few frequently used settings

Now that you've had a solid tour of the ConnectPort and iDigi's management services, you are probably eager to create a working system of your own. The next section will show you how to install and run the XBee Internet Gateway, getting you ready for the example Twitter Reader project at the end of this chapter.

\section{XBee Internet Gateway (XIG)}

The ConnectPorts are very flexible and powerful devices that can connect your ZigBee network to any Internet service in pretty much any way you like. The seemingly unlimited range of options can sometimes feel overwhelming to a beginner. Rather than learn about TCP/IP addressing, port numbering, DNS, application-layer protocols, and 
Python programming — terrific as all those things are to know-you probably would like to start with something simple that opens a path between your prototype and the teeming mass of terrific services that are available on the Web.

The XBee Internet Gateway is a Python program that can be loaded onto any ConnectPort right out of the box. It's an interface that mirrors the interactions humans have in web browsers. Once XIG is running, any radio that sends it a standard plain-text URL will receive back the regular results from that URL. Redirects to other pages, timeouts, security, retries, and so forth, are all handled behind the scenes just like they are in a web browser. Take a look at View Source in your browser; you can see the web page's underlying HTML. With the XIG, the radios in your project see the exact same thing. Each can send out a URL and receive back the source for that web pagewhatever it is. This simple service shifts all the hard stuff about interacting with the Web to the gateway. There's no need to handle security, domain lookups, or redirects locally. That's all taken care of for you by the XIG on the ConnectPort, giving your prototype a very simple yet completely flexible pathway to any web service you can imagine.

$\mathrm{XIG}$ is an open source team effort lead by your author, Jordan Husney, and Ted Hayes, with valuable support from a community of commercial and educational users. You

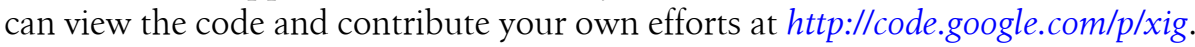

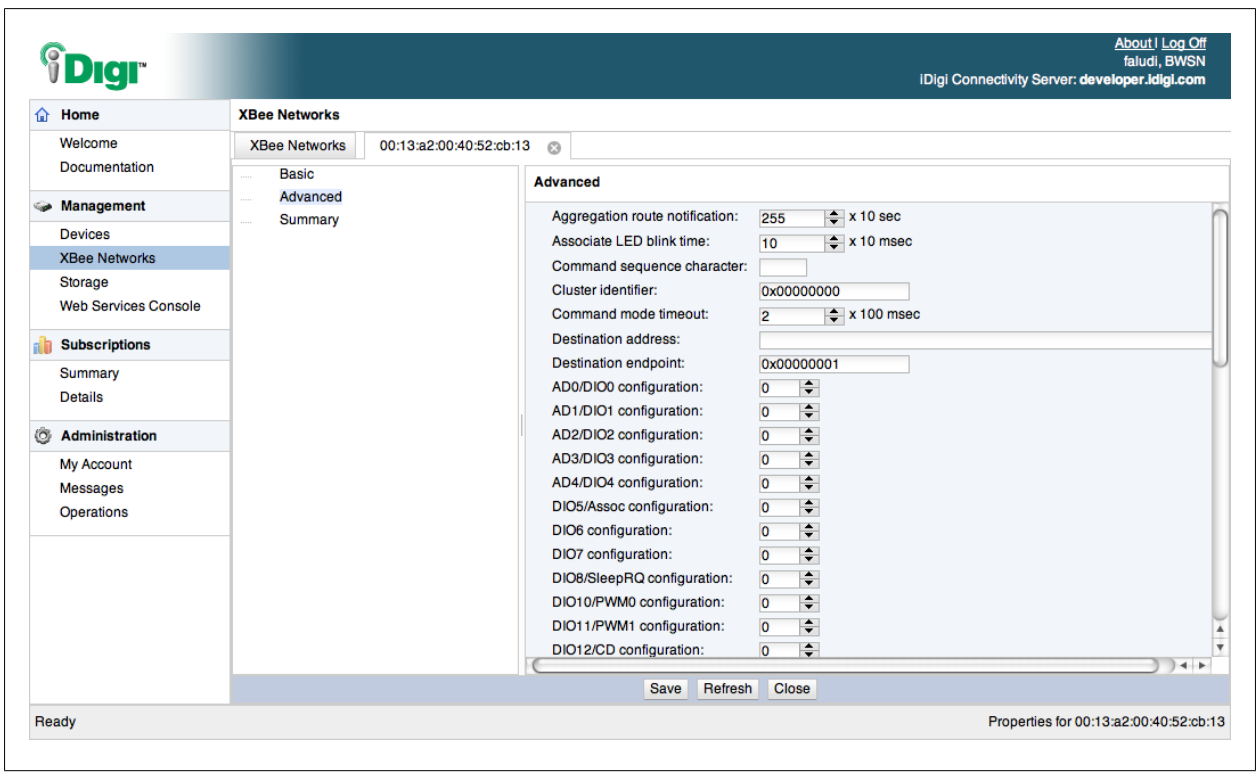

Figure 7-21. Advanced view of an XBee's properties shows many familiar AT command settings 


\section{Installing and Configuring XIG}

Setting up the XIG is easy, now that you're familiar with the general administration of a ConnectPort gateway. If you haven't already, follow the instructions in the previous sections "Setting Up a ConnectPort" on page 197 and "Configuring a ConnectPort" on page 198. Next, download the XIG code, which is linked from this book's

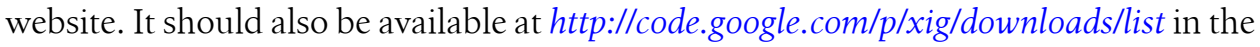
form of a ZIP file that contains xig.py and_xig.zip. Make sure the main file (xig-x.x.xbin.zip) gets unzipped, but leave_xig.zip and any other internal files in their compressed form.

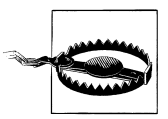

The XBee Internet Gateway is still undergoing active development and may have been upgraded and changed by the time you read this. Check this book's website (see the Preface) to make sure you have the latest files and instructions.

Once you are looking at the ConnectPort's administration interface, click on the Python link to begin uploading files. The ConnectPorts all come with the required python.zip libraries preloaded. There may be other resource files here as well, including zigbee.py, which is not needed for this project but can safely remain in the directory. Click the Browse button to navigate to and select each file you'd like to upload, starting with xig.py. Click the Upload button and wait for a File Uploaded message to appear at the top of the screen, as shown in Figure 7-22. Repeat this process for_xig.zip.

Next, click the Auto-start Settings link at the bottom of the screen. Check the first Enable box and then type xig.py into the first Auto-start command line field, as shown in Figure 7-23.

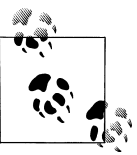

For XIG version 1.1.0, it's necessary to manually configure the Extended PAN ID for the ConnectPort's XBee radio. Click on the XBee Network link to view a list of the radios in the network (Figure 7-5). Select the gateway's radio-its node type will be listed as "coordinator"-to view its Basic Settings (Figure 7-6). Enter OxAAAA in the Extended PAN ID field and click the Apply button.

It's fine to pick any other PAN ID; just remember what it is and substitute it in the examples below. Also keep in mind that future versions of the XIG might configure the PAN ID automatically. Check the README file that comes with your download for the latest information. 


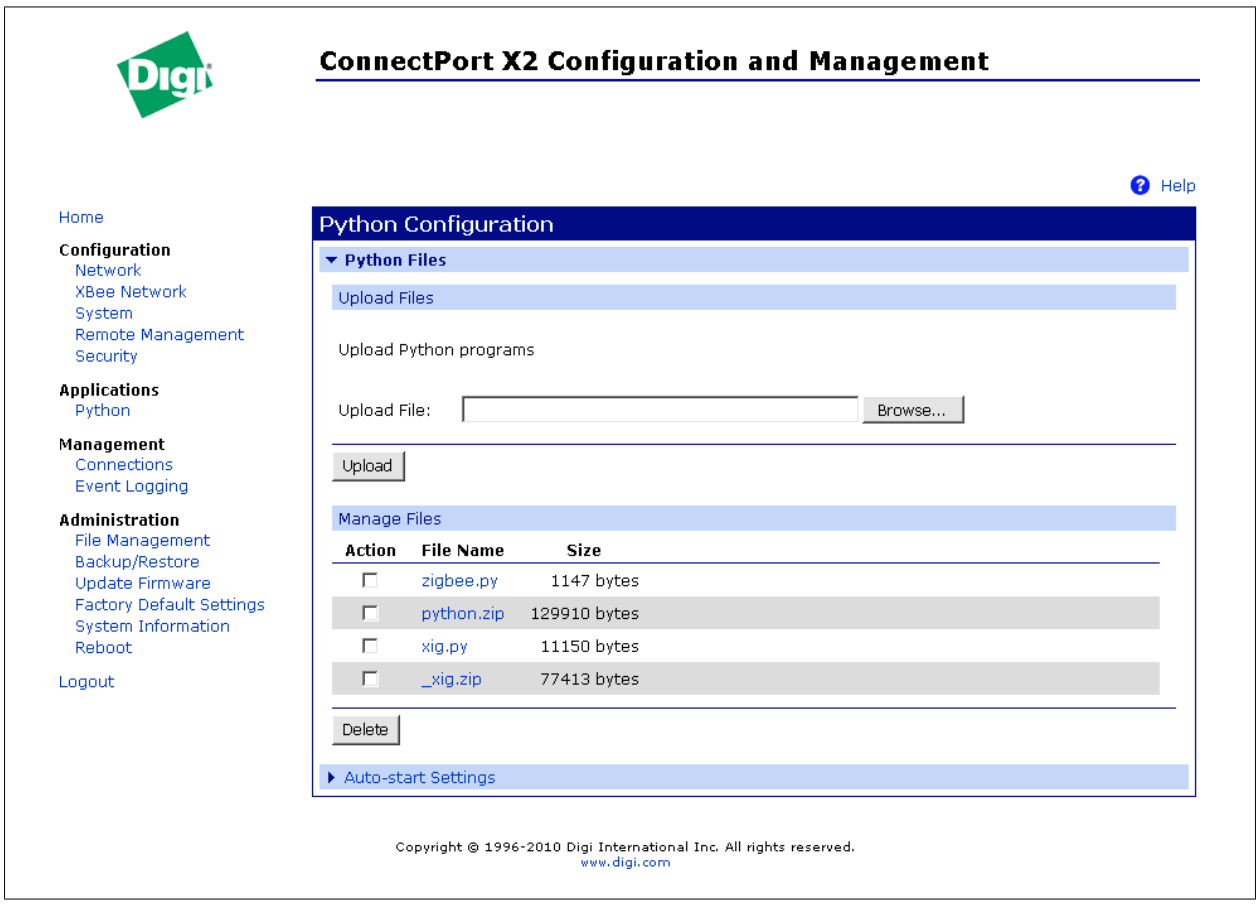

Figure 7-22. XIG files uploaded to ConnectPort

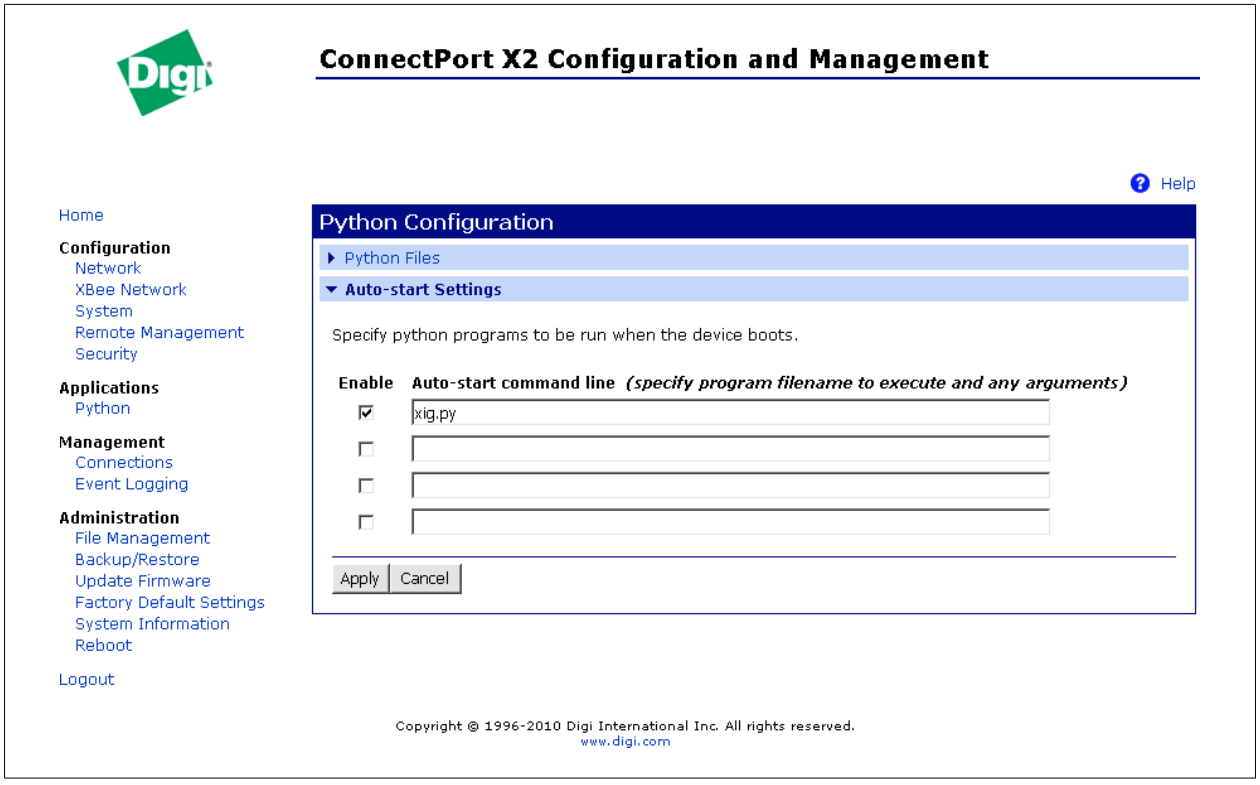

Figure 7-23. Auto-start configuration for XIG on ConnectPort 
It's a good idea to set some security on your XIG. It will be connected directly to the public Internet so protecting it with a password will prevent anyone else from trying to get in and mess with your configuration. Click the Security link to enter a password (Figure 7-24). You can also change the username if you like for an extra layer of security. After you click the Apply button to make this change, you'll immediately be prompted for your user ID and password.

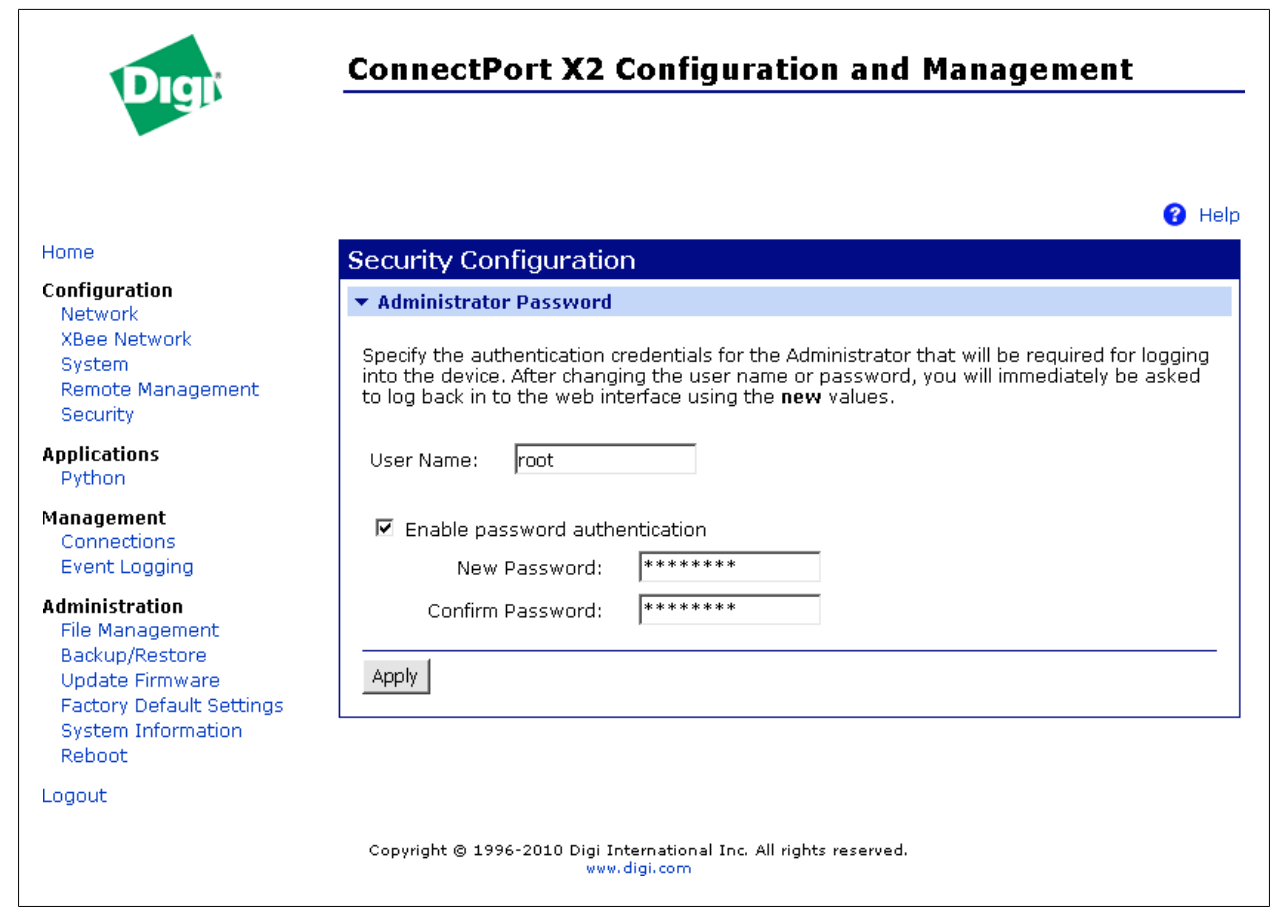

Figure 7-24. Entering security information on the ConnectPort

Finally, select the Reboot link and press the Reboot button. The XIG will now run automatically at startup.

\section{Testing XIG}

Before you start hooking up any projects, it's a good idea to confirm that your XIG is working properly. This can be done with the help of our old friend CoolTerm, or any other serial terminal program:

1. Use X-CTU to configure an XBee as a ZigBee router in AT mode.

2. Place that router into an XBee Explorer, plug it into your computer, and run the CoolTerm program.

3. Press the Options button in CoolTerm to configure the serial connection. 
4. Select the appropriate serial port and check the Local Echo box.

5. Click on the Connect button to connect to the serial port.

6. Type +++ to go into command mode.

7. Type ATID followed by the PAN ID. The recommended PAN for the XIG is AAAA, SO ATID AAAA will get you configured.

8. Every ZigBee coordinator always has 0 as its 16-bit network address, and this is the default destination address for any newly configured XBee radio. Enter ATDL $\mathbf{0}$ and ATDH $\mathbf{0}$ to be sure that you are in the default configuration for the destination address.

9. Enter ATJV 1 to ensure that your router attempts to rejoin the coordinator on startup.

The XIG often needs to send a lot of data, so it can be helpful to raise the baud rate. This is optional:

1. To raise the baud rate, type ATBD followed by the code for the baud rate you'd like to use-in this case 7 for 115,200 bps, which is the fastest speed-so type ATBD 7 and hit Enter.

2. Once the baud rate has been raised, you'll need to select the same baud rate in CoolTerm (and later on, the same baud rate in your own project). Click Disconnect to drop the serial connection, then Options to open the settings, and switch the Baudrate setting to 115200. Press OK and then Connect to reconnect to your XBee. It should now be responding at the higher baud rate.

To check the XIG, try typing the word help in the connected CoolTerm window, then pressing Return. You should get a text response with basic information on using the XIG. If that works, try entering in a URL like $h t t p: / / w w w$.faludi.com/test.html. The HTML source for that URL should be returned, something like this:

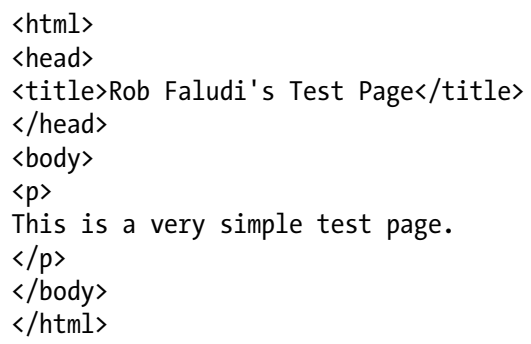

If you have problems, try double-checking the baud rate. You can make sure that the router XBee has joined a network by using ATAI and looking for a response of 0 . You may also want to confirm your router is connected to the right network by issuing the ATND command and seeing if the gateway radio's listing comes back. Once you have confirmed everything is working, you're ready to make some stuff that's connected to the Internet! 


\section{XIG Example}

PHP is a very common language for writing applications that run on web servers. A full explanation of PHP and Internet protocols is beyond the scope of this book (see http: //oreilly.com/pub/topic/php for some books and resources on PHP). We will supply a very simple example that you can upload to your server. Use this as a starting point for creating simple URLs that your project can connect to via the XIG. On the XIG website, there is more example code that can be used to download and upload information for sensing and control.

Here's a quick look at some PHP code. Place it in a file on your server called XIG_download_example.php. When you access it online, whatever value you put in the $\$$ value variable will be returned to your web browser. In this case, you'll simply see an 8.

\section{XIG download example in PHP}

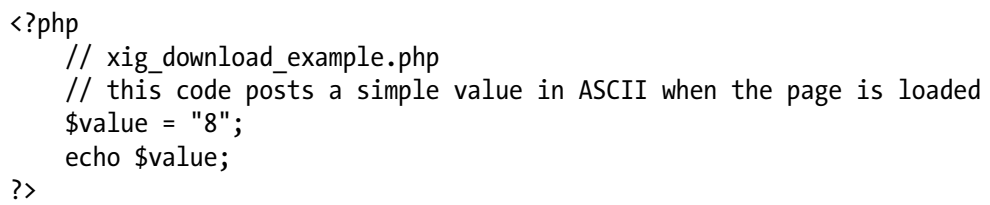

To read this in on an Arduino connected to a router XBee that you configured as described above, try the following sketch that reads in the returned value. Figure 7-25 shows a diagram of the connections. Note that it doesn't do anything with the returned value; that part is for you to write with your own purposes in mind (you must replace <your server URL here> with the hostname and path to wherever you uploaded XIG_download_example.php):

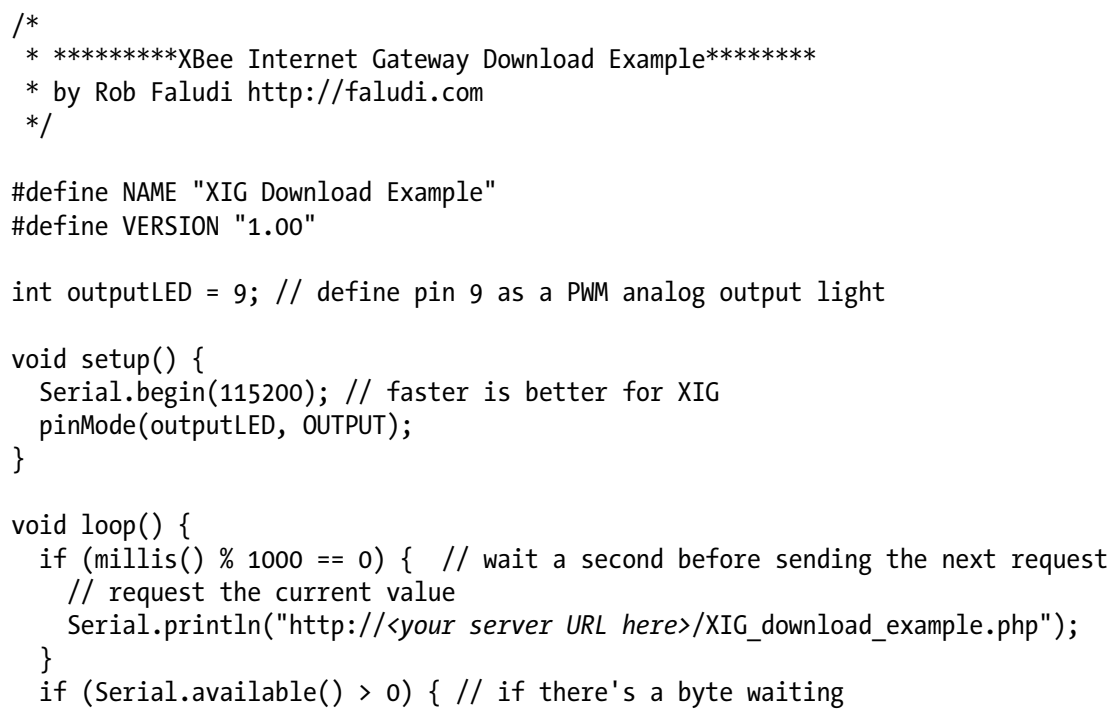


int value $=$ Serial. $\operatorname{read}() ; / /$ read a single byte

analogWrite(value, outputLED); // set an LED's brightness

// to match the value

$/ / * * *$ OTHER USEFUL THINGS COULD BE DONE WITH THE VALUE VARIABLE HERE ***

\}

You've seen how to download data using the XIG—a significant task that can be accomplished in a small amount of code. Examples for controlling output and uploading data are available on the XIG site that's linked from the book's website (see the Preface). Given your freshly obtained networking powers, you are probably wondering, "How can I use my new skills to display a real-time stream of celebrity gossip?" Glad you asked.

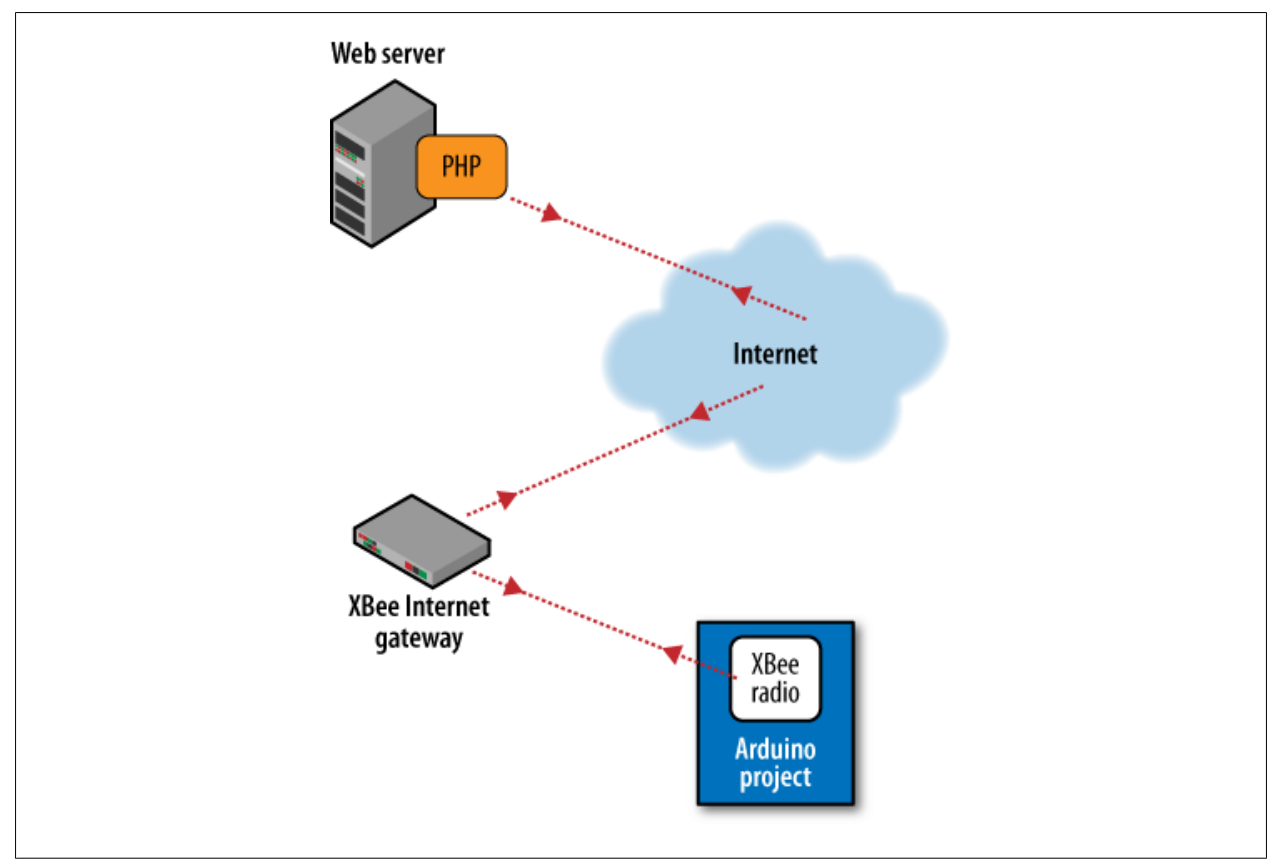

Figure 7-25. System diagram for XBee Internet Gateway connecting an Arduino project to PHP on a web server

\section{Twitter Reader}

These days, no event seems truly real until it has been reported on Twitter. From the minor details of everyday life to the tectonic shifts of the continents, an endless stream of information gushes forth from "tweeters" around the world. These missives are typically viewed on a computer screen or in a text message, but there's no reason they can't 
be unshackled and invited to join us off-screen out in the physical world. Why not display your Twitter feed on your office door, so people know why you aren't available? Perhaps you'd like to enjoy a feed of haikus while riding the elevator in the morning. Or maybe your tastes run a little less zen and you want to know Julia Roberts' matrimonial status as scrutinized by US Magazine. In all cases, the Twitter Reader example is here to help. It displays the latest message from any tweet feed wirelessly on a standard 32-character LCD display. The Reader downloads from a specially designed "twansform" online application via the XBee Internet Gateway. Twansform was written in Google App Engine by Jordan Husney. The web service used by our project can be found at http://twansform.appspot.com and the code is available at http://code.google $. \mathrm{com} / \mathrm{p} /$ twansform/. It uses simple URL requests that include the feed name. In the code below the feed is usweekly, great for keeping tabs on the stars. Of course you can easily change it to any other account you like. For example you could use the earthquake feed to keep tabs on tremors, or try out schnitzeltruck if you have a hankering for flat meat in Manhattan. The Twitterverse's offerings are endless, so let's get started reading feeds.

\section{Parts}

- One solderless breadboard (large size) (AF 239, DK 438-1045-ND, RS 276-002, SFE PRT-00112)

- Hookup wire (assorted colors are particularly helpful for this project) (AF 153, DK 923351-ND, SFE PRT-00124)

- One Arduino Uno (SFE DEV-09950, AF 50) (If you use an older model, be sure it is using the new ATMEGA328 chip!)

- USB A-to-B cable for Arduino (AF 62, DK 88732-9002, SFE CAB-00512)

- Assorted 5 mm LEDs (DK 160-1707-ND, RS 276-041, SFE COM-09590)

- One 10K $\Omega$ potentiometer (panel mount) (DK P3C3103-ND, RS 271-1715, SFE COM-09288)

- One $16 \times 2$ character LCD display (with HD44780 parallel interface) (AF 181, DK 67-1758-ND, SFE LCD-00255)

- 16-pin single-row male header (generally sold in longer breakaway strips) (DK S1012E-36-ND, SFE PRT-00116)

- One ConnectPort X2 - ZB, running the XBee Internet Gateway software (Digi X2Z11-EC-A is the new version; DK 602-1173-ND is the older version)

- One XBee radio (Series 2/ZB firmware) configured as a ZigBee Router API mode (Digi: XB24-Z7WIT-004, DK 602-1098-ND)

- One XBee breakout board with male headers and $2 \mathrm{~mm}$ female headers installed (AF 126 (add SFE PRT-00116), SFE BOB-08276, PRT-08272, and PRT-00116)

- XBee USB serial adapter (XBee Explorer, Digi Evaluation board, or similar) (AF 247, SFE WRL-08687) 
- USB cable for XBee adapter (AF 260, SFE CAB-00598)

- Wire strippers (AF 147, DK PAL70057-ND, SFE TOL-08696)

\section{Prepare Your ConnectPort with XBee Internet Gateway}

Follow the instructions for "Installing and Configuring XIG" on page 216 to install and configure the ConnectPort X2 with the XBee Internet Gateway software. If this is a new installation, use the instructions from "Testing XIG" on page 218 to test it using a terminal program from your computer.

\section{Prepare Your Router Radio}

1. Follow the instructions under "Reading Current Firmware and Configuration" on page 35 to configure your Twitter Reader radio as a ZigBee Router AT.

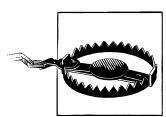

Your router radio will use the AT firmware so it can pass messages in plain text to the XIG on the ConnectPort. Be sure you select the AT version for your router!

2. Label the router radio with an "R" so that you know which one it is later on.

\section{Configure Your Router Radio}

Using the CoolTerm terminal program and an XBee Explorer USB adapter, you can set up your router radio for the Twitter Reader:

1. Select the router XBee you've labeled with an "R" and place it into the XBee Explorer.

2. Plug the XBee Explorer into your computer.

3. Run the CoolTerm program and press the Options button to configure it.

4. Select the appropriate serial port, and check the Local Echo box so you can see your commands as you type them.

5. Click on the Connect button to connect to the serial port.

6. Type +++ to go into command mode. You should receive an OK reply from the radio.

7. Type ATID followed by AAAA, the PAN ID for the XIG on the ConnectPort, and press Enter on the keyboard. You should receive OK again as a reply.

8. Every ZigBee coordinator always has 0 as its 16-bit network address, and that's the default destination address for any newly configured XBee radio. To use 16-bit addressing, the high part of your radio's destination address will be zero. Type ATDH $\mathbf{0}$ and press Enter on the keyboard. You should receive an OK response. 
9. Enter ATDL followed by the low part of your radio's destination address, in this case also a zero because that's the fixed address for the coordinator. Type ATDL $\mathbf{0}$ and press Enter. You should receive an OK response.

10. Enter ATJV1 to ensure that your router attempts to rejoin a coordinator on startup.

11. Save your new settings as the radio's default by typing ATWR and pressing Enter.

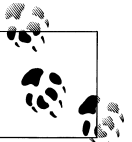

Tweets are very short, so for this project we are fine to avoid additional configuration steps and stick with the default serial commu2. nications rate of 9,600 baud (ATBD3, in case you changed it earlier). Projects that download larger datafiles will benefit from using higher baud rates.

\section{Prepare the Twitter Reader Board}

Your base station radio will use a breadboard connected to an Arduino board.

\section{Connect power from Arduino to breadboard}

1. Hook up a red wire from the $5 \mathrm{~V}$ output of the Arduino to one of the power rails on the breadboard. This is a higher voltage than we used in previous projects. The 5-volt supply is required for running the LCD screen. We will send the XBee 3.3 volts separately, directly from the Arduino as described below.

2. Hook up a black wire from either ground (GND) connection on the Arduino to a ground rail on the breadboard.

3. Hook up power and ground across the breadboard so that the rails on both sides are live.

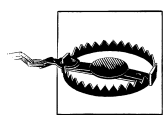

Make sure you are using $5 \mathrm{~V}$ power to supply the main breadboard.

\section{XBee connection to Arduino}

1. With the router XBee mounted on its breakout board, position the breakout board toward one end of your large breadboard so that the two rows of male header pins are inserted on opposite sides of the center trough. Leave enough free space on the breadboard for the LCD screen!

2. Use a long red hookup wire to connect pin 1 (VCC) of the XBee directly to the Arduino board's 3.3-volt output.

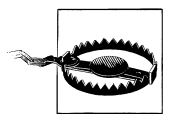

Make sure you are supplying 3.3 V power to the XBee. 
3. Use black hookup wire to connect pin 10 (GND) of the XBee to ground.

4. Use yellow (or another color) hookup wire to connect pin 2 (TX/DOUT) of the XBee to digital pin 6 on your Arduino.

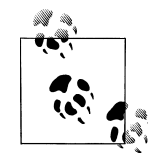

This project does not use the Arduino's hardware serial pins ( 0 and

1) because it employs the NewSoftSerial library. This handy library

1. enables any two digital pins to be used for serial communications. Avoiding the hardware serial pins allows us to reprogram the Arduino successfully without removing any wiring. Using the NewSoftSerial library requires a little more sophisticated code, which is why we didn't do it in earlier examples. You're ready now.

5. Finally, use blue (or another color) hookup wire to connect pin 3 (RX/DIN) of your XBee to digital pin 7 on your Arduino.

\section{Liquid crystal display (LCD) output}

Tweets will be displayed on a 16-character-wide, 2-row LCD with a standard HD44780 parallel interface. These displays are very common and generally have a 16-pin interface. Displays without a backlight typically omit the last two pins. The instructions below are for backlit displays. If your LCD doesn't have one, simply ignore anything to do with pins 15 and 16. Here is a typical data sheet for a 16×2 HD44780 display: http://www.xmocular.com/upload_img/2008013150690297.pdf.

1. Trim your male headers down to 16 (or 14) pins to match the number of connection holes available on your LCD. Solder the row of male headers into the LCD as shown in Figure 7-26.

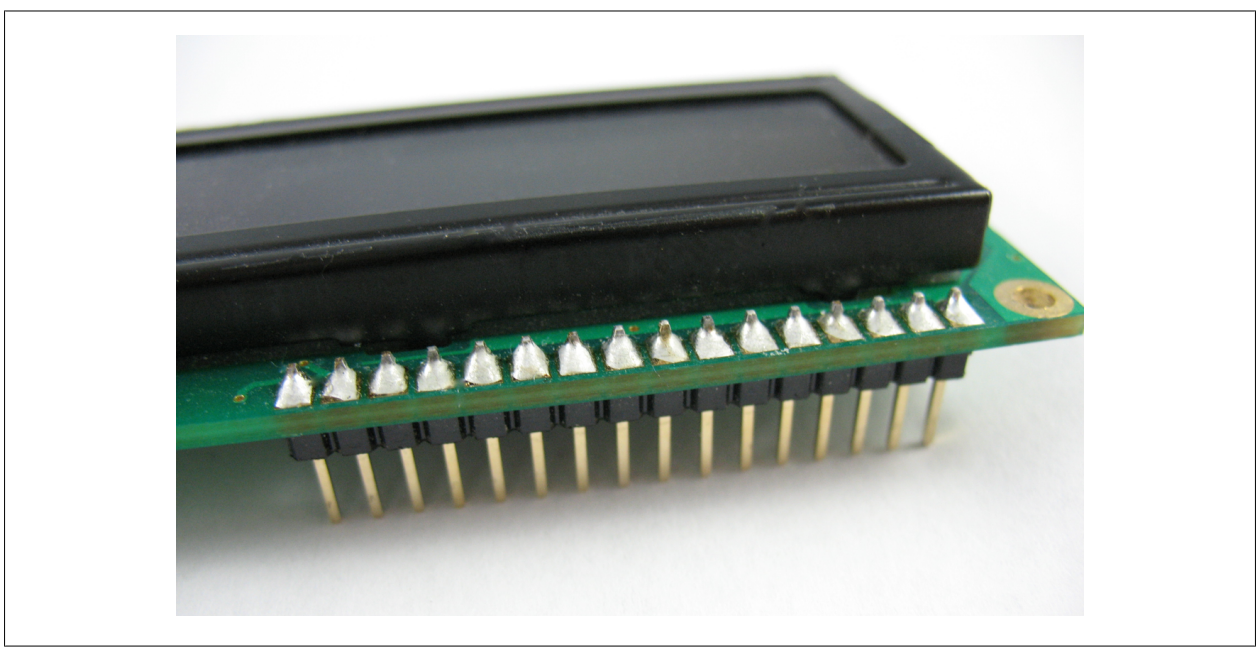

Figure 7-26. Male headers soldered to LCD 
2. Insert the LCD into your breadboard. It takes up quite a bit of room, which is why you are using a larger breadboard for this project!

3. On most LCD units, the first or last pin is labeled on at least one side of its circuit board. If not, you can always consult the data sheet. Locate physical pin 1 and use a black wire to connect the LCD's physical pin 1 to one of the ground rails.

4. Use a red wire to connect the LCD's physical pin 2 to one of the power rails.

5. Attach the potentiometer to the breadboard near the LCD. Some models of potentiometer will fit right in the breadboard, while others may need jumper wires soldered onto them to make that connection happen. There are three pins, two terminals (typically the outer pins), and a wiper pin. Connect one terminal of the potentiometer to power and the other to ground. It doesn't matter which one. Connect the wiper (typically the center pin) to the LCD's physical pin 3.

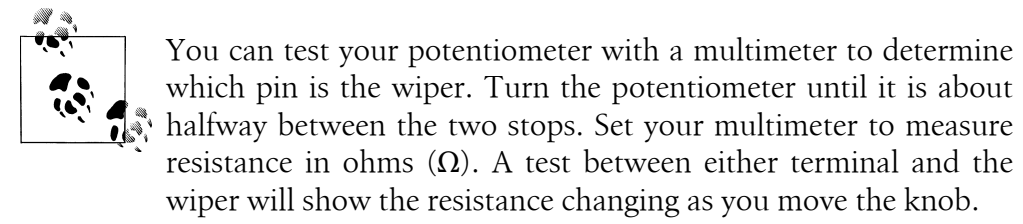

6. Hook up the remaining LCD pins as shown in Table 7-1. You can also use the diagram in Figure 7-27 and the schematic in Figure 7-28 as a guide.

Table 7-1. LCD pin connections for Twitter Reader project

$\begin{array}{lll}\text { LCD pin \# } & \text { LCD pin name } & \text { Connection } \\ 1 & \text { GND } & \text { Ground } \\ 2 & +5 V & 5 \text {-volt power } \\ 3 & \text { Contrast adjustment } & \text { Potentiometer wiper } \\ 4 & \text { Register select } & \text { Arduino digital 12 } \\ 5 & \text { Read/Write } & \text { Ground } \\ 6 & \text { Enable } & \text { Arduino digital 11 } \\ 7 & \text { Data bus } & \text { No connection } \\ 8 & \text { Data bus } & \text { No connection } \\ 9 & \text { Data bus } & \text { No connection } \\ 10 & \text { Data bus } & \text { No connection } \\ 11 & \text { Data bus } & \text { Arduino digital 5 } \\ 12 & \text { Data bus } & \text { Arduino digital 4 } \\ 13 & \text { Data bus } & \text { Arduino digital 3 } \\ 14 & \text { Data bus } & \text { Arduino digital 2 } \\ 15 & \text { Backlight power (if available) } & +5 \text {-volt power }\end{array}$




\begin{tabular}{lll} 
LCD pin \# & LCD pin name & Connection \\
16 & Backlight GND (if available) & Ground \\
\hline
\end{tabular}

7. When everything is set up, plug the Arduino into your computer using the USB cable. If your LCD has a backlight, you should see it come on. You can also try adjusting the contrast on the display by turning the potentiometer so that the rectangles behind each character position just barely disappear.

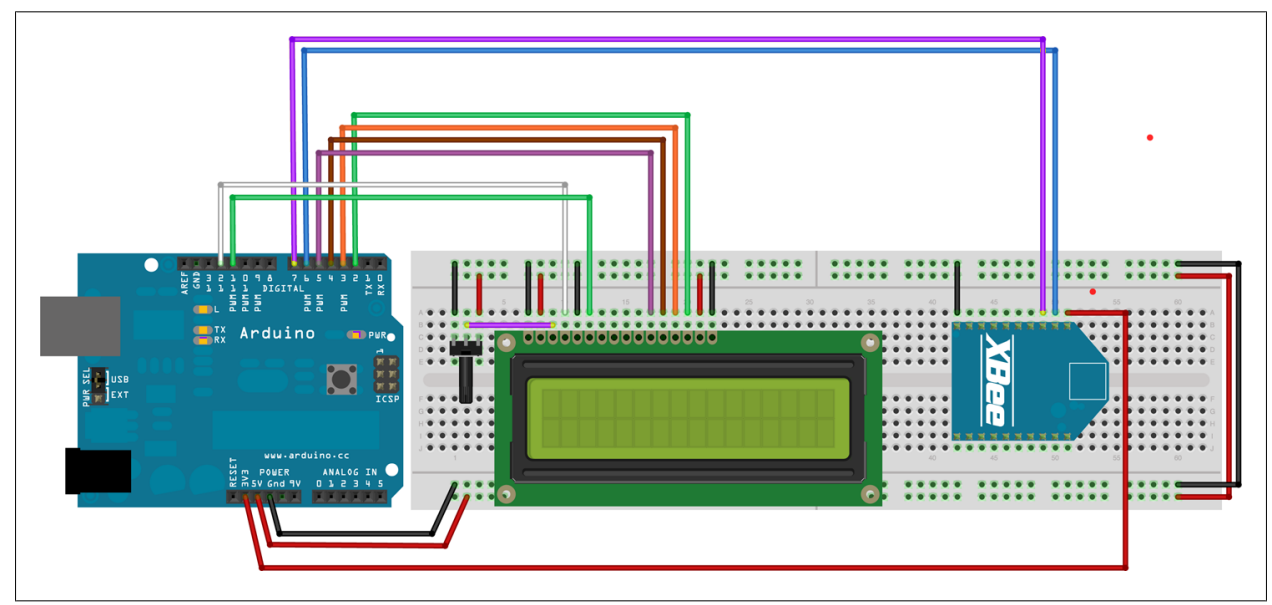

Figure 7-27. Twitter Reader breadboard layout

\section{Program the Arduino}

The Twitter Reader uses the Arduino sketch shown later in this section. You'll also need the NewSoftSerial library.

\section{Installing the NewSoftSerial library}

Download the library from http://arduiniana.org/libraries/newsoftserial and unzip it. It will be in a NewSoftSerial folder that contains NewSoftSerial.h, NewSoftSerial.cpp, keywords.txt, and an Examples subfolder.

Open your Arduino sketchbook folder (if you're not sure where this is, open a saved sketch, choose Sketch $\rightarrow$ Show Sketch Folder, then go up to its parent directory). There is probably already a folder there called libraries, but if not you can create one. Place the entire NewSoftSerial folder inside the libraries folder.

If Arduino is already running, quit it and then start it up again. You should see NewSoftSerial listed on the Sketch $\rightarrow$ Import Library menu. 


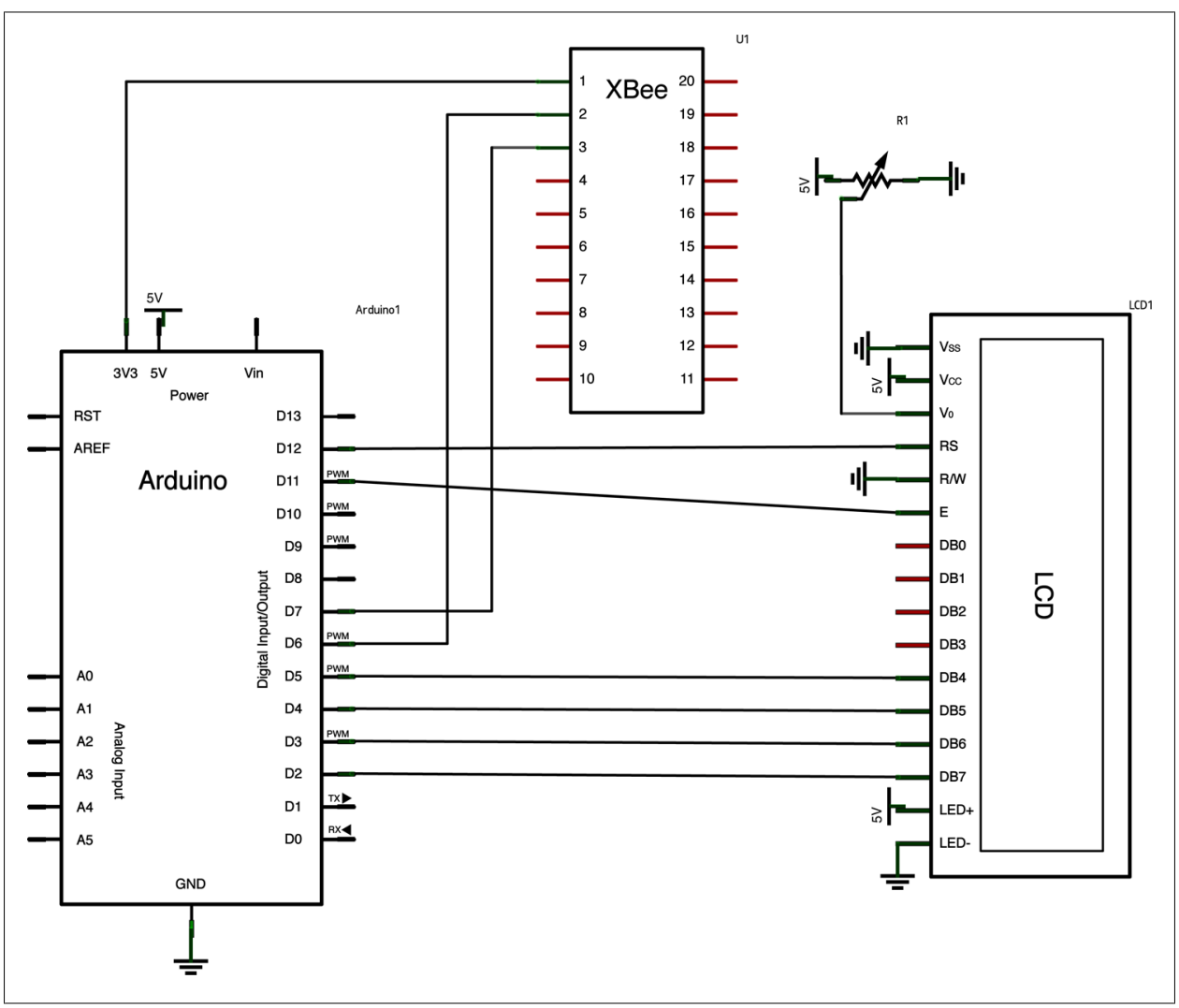

Figure 7-28. Twitter Reader schematic

If you get a message like error: NewSoftSerial.h: No such file or directory when you compile your program or load it, you probably don't have a folder in the right place. Try going through the above instructions again or check $h t t p: / / w w w$.arduino.cclen/Reference/Libraries for more information on adding libraries to Arduino.

Once you've loaded the files and directories onto your computer, open Twitter_Reader.pde (full code listed below, or you can download it from the website listed in the Preface) in Arduino, press the Upload button (labeled with a right arrow) to upload the code to your Arduino. The code will run and should briefly show the words "Twitter Reader" and a version number on the LCD screen. After that the phrase loading... will be displayed while the system attempts to connect to the Internet via the XBee Internet Gateway. Here are the two lines of code that send that URL lookup request to a special Google App Engine program that parses the Twitter feed:

mySerial.print("http://twansform.appspot.com/usweekly/text/1");

mySerial.print(" $\backslash r ")$; 
And here's the bit of code that reads the reply received back from the XIG into a text string:

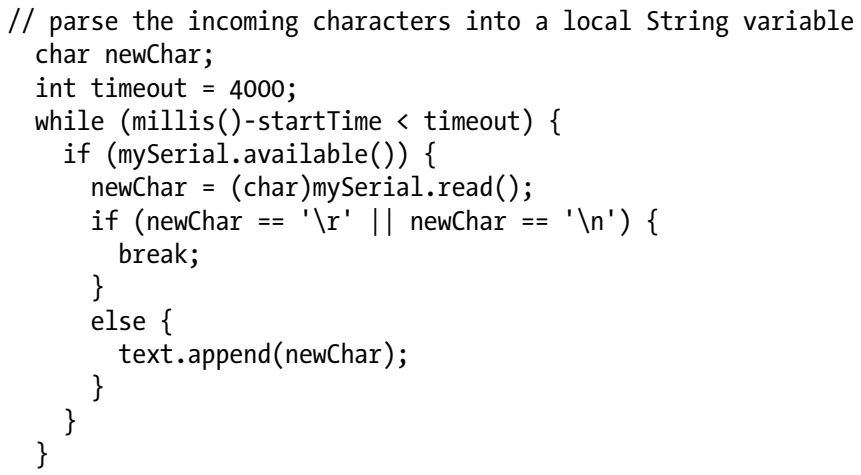

When the lookup succeeds, you will see the latest tweet for that feed displayed. The vast majority of the program is devoted to properly displaying the tweet on the LCD, including splitting up the message properly and adding line breaks between words for maximum readability. Getting the message from the Internet is easy, thanks to the XIG! (See Figures 7-29 through 7-31.)

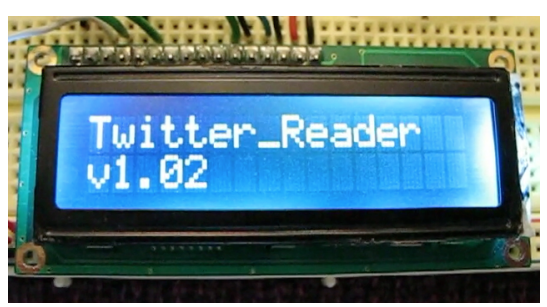

Figure 7-29. Twitter Reader startup display

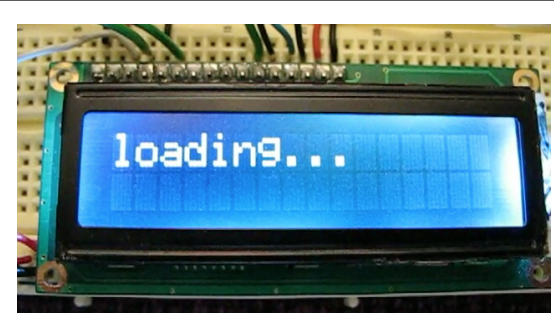

Figure 7-30. Twitter Reader shows "loading..." message while accessing the Twansform URL via XBee Internet Gateway 


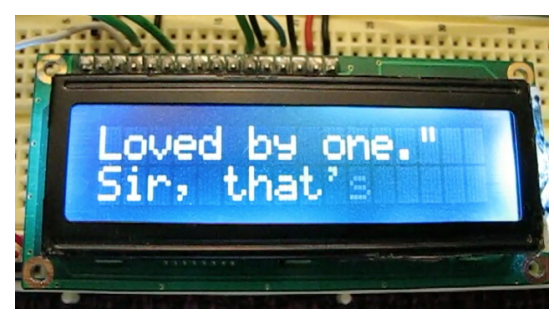

Figure 7-31. Twitter Reader displaying a tweet

\section{Twitter Reader code}

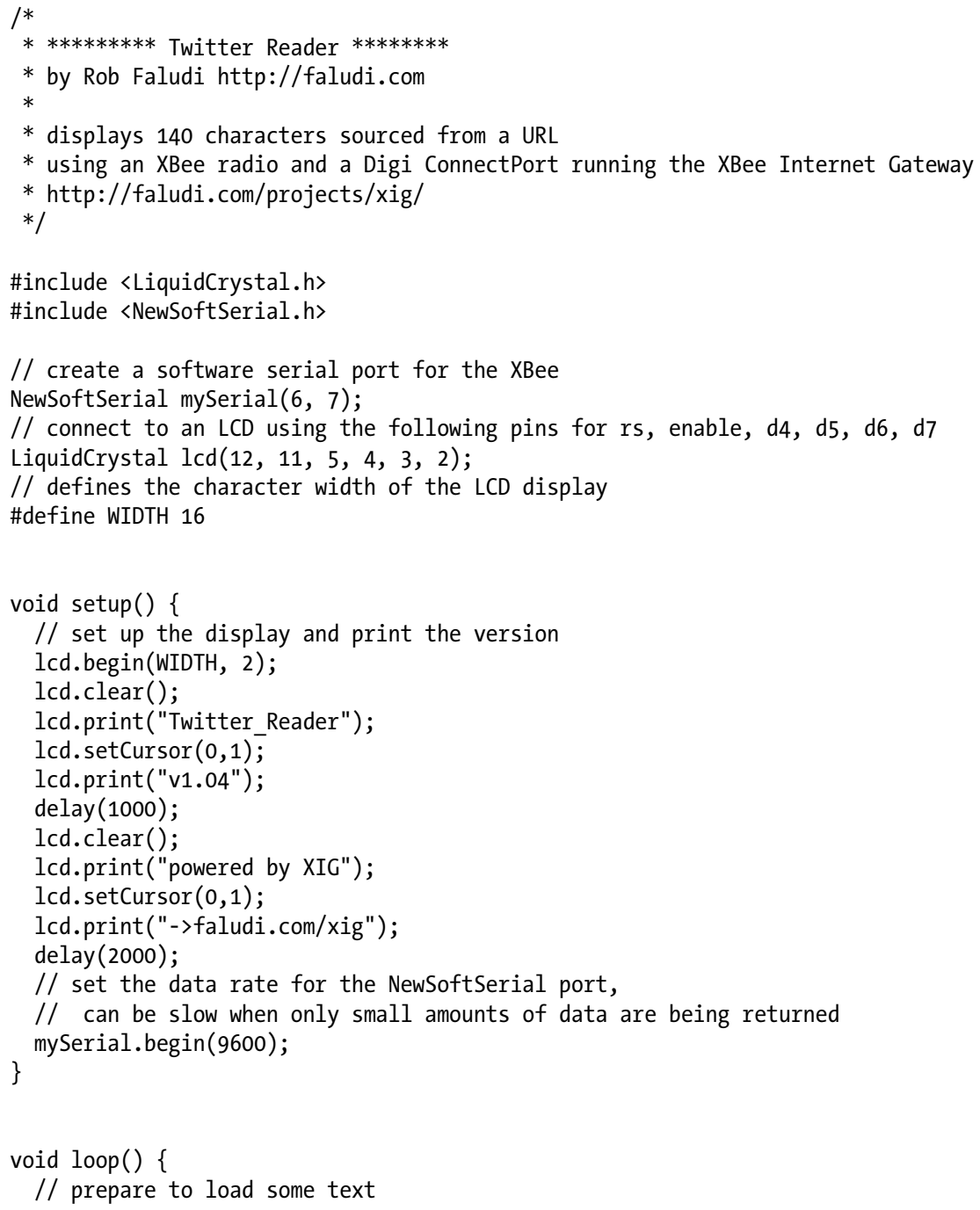




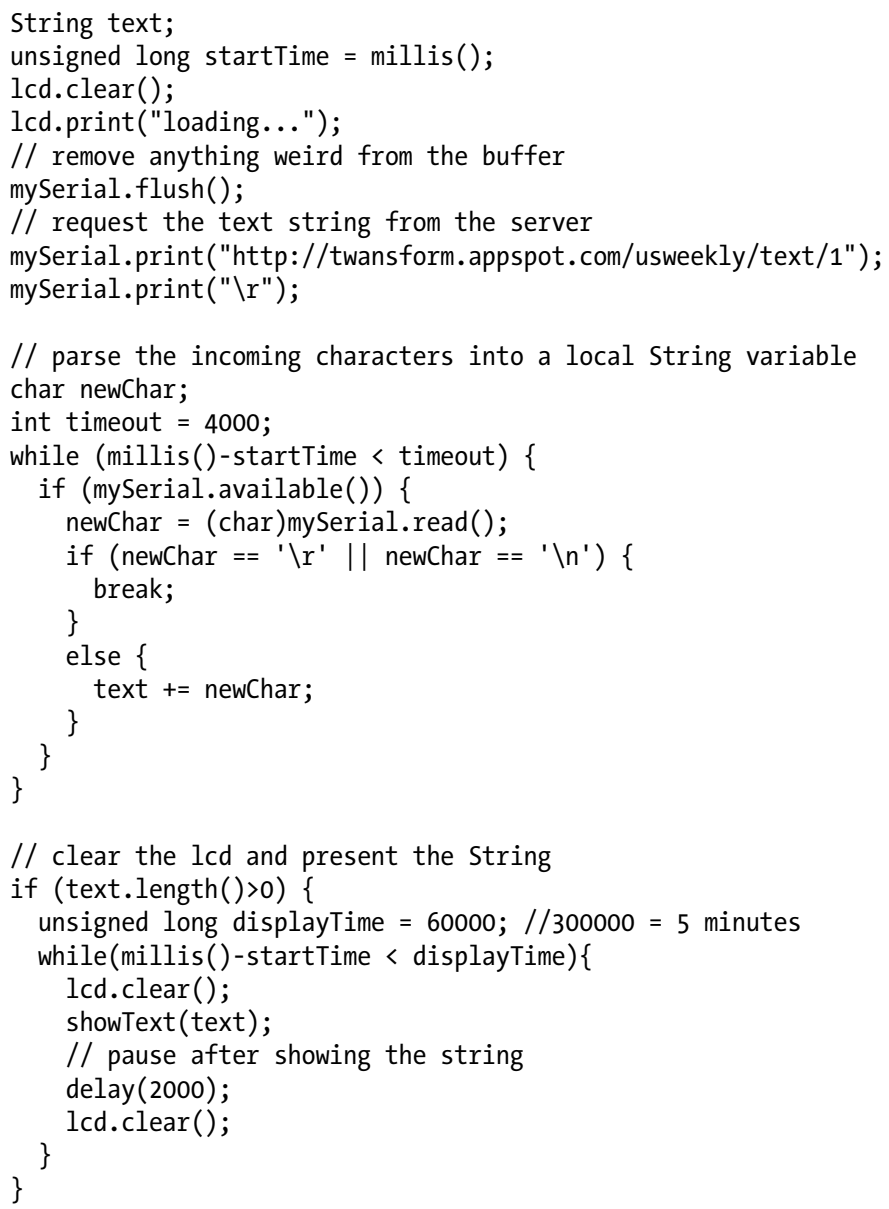

// displays the text on an lcd with correct line breaks between words void showText(String theText) \{

String text; // String variable for the text we are displaying text $+=$ theText; // puts the incoming text into our String variable String lineBuffer; // temporary storage for the last displayed line int cpos=0; // keeps track of the current cursor position

int line=0; // keeps track of the current line

// step through the text one character at a time

for (int $i=0 ; i<$ text.length ()$; i++)\{$

$/ /$ in general, don't make a linefeed

boolean linefeed = false;

if (text $[i]=='$ ') \{

// if the current character is a space, then make a line feed

linefeed = true;

// ...but check first that there isn't another space before

// the edge of the screen 


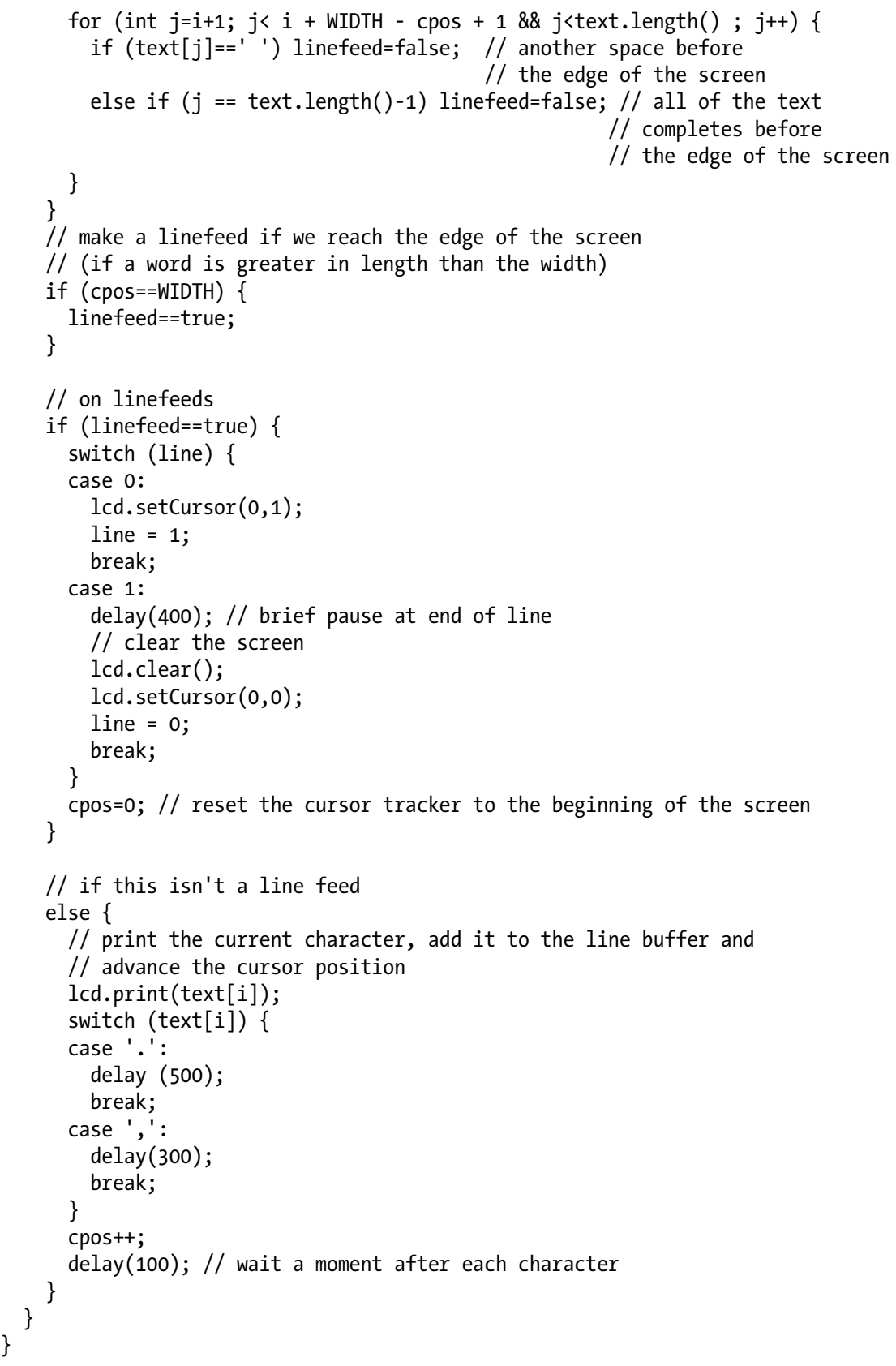




\section{Troubleshooting}

If things don't work at first, here are some steps to try:

1. Check all your electrical connections to make sure there are no loose wires and that all the components are connected properly.

2. Check the router configuration in X-CTU to confirm that the correct modem type (XB24-ZB) and function set (ZigBee Router AT) have been selected. Also check that the PAN ID, baud rate, destination high, and destination low are configured as you expect, and that ATJV has been configured as described above.

3. An LED placed from the ASSOC pin of the reader's XBee (physical pin 15) to ground should show a flashing light.

4. Check the XBee Network screen in the ConnectPort's management window to see if it shows the Twitter Reader's XBee when you Refresh the listing. The Extended ID is the same number that is printed on the back of the XBee radio.

5. Make sure you see "Twitter Reader" and version information on your LCD when the system starts up. If not, check your connections and make sure that you have adjusted the contrast properly with the potentiometer.

6. The Twitter Reader will generally run best on an Arduino using the ATMEGA328 chipset. This will be printed on the large black microchip. You may have inconsistent results with the ATMEGA168 chipset, which only has 1K of RAM and can't always handle long strings of text.

7. We are not always able to see our own mistakes. Have a friend check everything for you. Sometimes only a second pair of eyes will catch the one or more issues that are standing in the way of success.

8. When all else fails: Try taking a break and coming back to the project after a good night's rest. Remember, many of midnight's intractable puzzles are morning's simple fixes.

\section{Moving Forward}

We're coming near the end of our fast journey through the vast world of wireless networking. You now know how to reach beyond local ZigBee environments to traverse many other systems, most notably the Internet. We've given you a great way to get started on making connections using the XBee Internet Gateway, and a full example of that connection being used in an Arduino project. The next and last chapter will give you a starting point for further expeditions into the ZigBee protocol, and outline a few advanced features that will help you in your explorations going forward. This will not be the end of the road; it's merely a stepping-off point to begin your own journey. 

Our final chapter serves as a broader introduction to sensor networking. You are now prepared to explore advanced communication techniques, share your data, and develop your own projects. Good job! Some of the ideas and techniques we look at in this chapter are pretty technical. That's why they come here at the end, when you are in the best position to understand them. We'll start with an overview of ZigBee application messaging, examine alternate routing techniques, peruse security options, and shine a light on serial flow control. To make sure that your data doesn't languish on some local hard drive, this chapter also offers an example project for publishing your sensor network results publicly. Finally, we wrap up with a peek at the future of ZigBee and some suggestions for sharing your results with others.

Enjoyed what came before? There's more to adore. Let's go take a dip in the deep end!

\section{Advanced ZigBee}

While much of advanced ZigBee is beyond the general scope of this book, there are several things worth knowing when you need to enable larger systems or pursue interoperation. You are now ready to meet these higher-level concepts and consider their implications for your projects. We'll discuss ZigBee stack layers, standardized APS messaging, routing scenarios, security, and serial flow control. Finally we'll point you toward reference material on the ZigBee standard.

Each of these sections is intended as a jumping-off point to get you started. Need to know more? You'll find additional detailed technical documentation in the Product Manual for the XBee ZB radios, available at: $h t t p: / / w w w$.digi.com/support/supporttype .jsp?tp=3. 


\section{ZigBee Stack Layers}

As discussed in Chapter 2, the ZigBee protocol is divided into layers (Figure 8-1). These are portions of the protocol that do distinct jobs, and come together to create the entire mesh communications structure in an organized and flexible fashion.

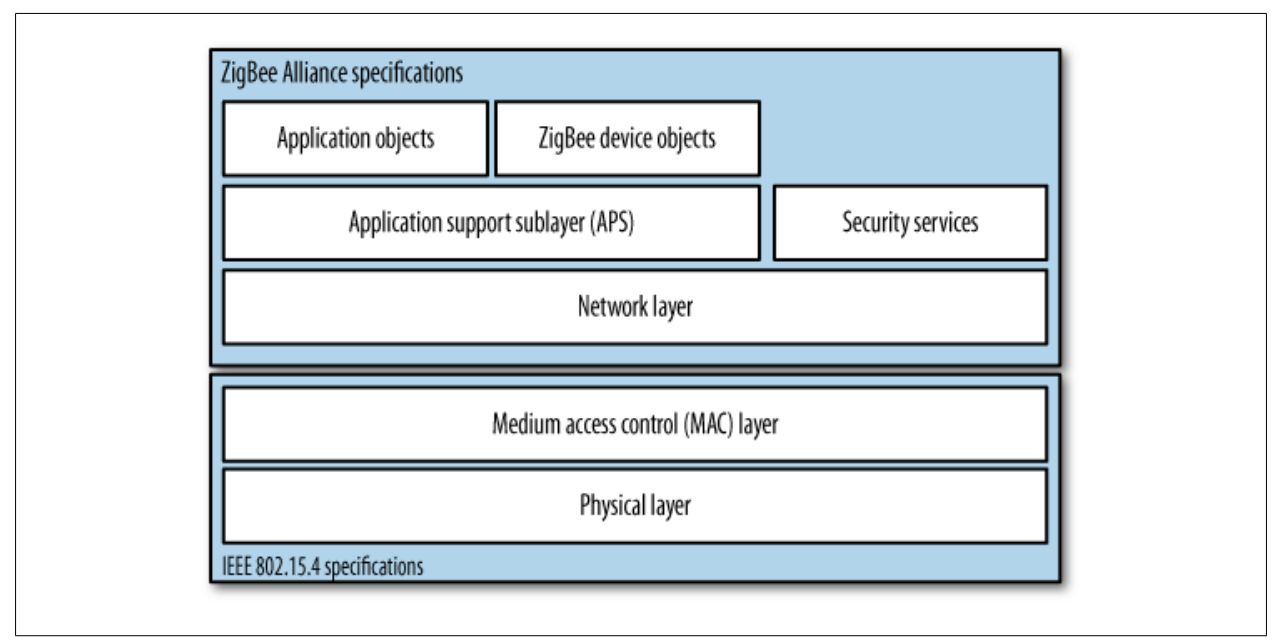

Figure 8-1. ZigBee protocol stack showing the PHY, MAC, Network, APS, and ZDO layers

We briefly covered ZigBee's physical layer (PHY) in Chapter 2, as well as the media access controller (MAC) that defines low-level addressing, among other things. Both are part of the 802.15.4 protocol that underlies ZigBee. We've also described the ZigBee Network layer that handles routing information from place to place, creating full mesh networking. There are two higher levels in the protocol that were somewhat beyond the scope of our initial forays, but may become important to you as your projects evolve and perhaps connect to other systems. They're like the frosting and decorations on the ZigBee cake:

\section{Application Support Sublayer (APS)}

The APS layer defines standardized messaging for specific tasks where ZigBee radios are commonly employed. By creating standard messages, ZigBee devices created by different manufacturers can all carry on conversations with each other and collaborate seamlessly in predefined applications such as home automation. We will describe this layer in more detail shortly.

ZigBee Device Objects (ZDO)

The ZDO layer is an application profile for dealing with the radios themselves. It provides device and service discovery along with certain network management capabilities. We won't discuss this layer in any more detail here and only note it for completeness. If you find yourself in a situation where requesting routing or endpoint information from a neighboring radio becomes necessary to your application, 
you can learn more about it in the XBee ZB Product Manual under "ZDO Transmissions."

\begin{abstract}
These higher layers will matter when you're talking to other brands of radios, or when you're building something that needs to interact with ZigBee-certified device profiles such as Smart Energy, Home Automation, Consumer Electronics, or Health Care (see "Profiles" on page 237). If your projects only involve XBees talking to each other, then keep it simple! Read these sections purely for entertainment and don't fret about implementing the concepts.
\end{abstract}

\title{
Application Support Layer
}

The APS layer implements application profiles, clusters, and endpoints. You can think of these as describing the general kind of thing you're doing (profile), the more specific action you're taking (cluster), and the location within a device where that action will be carried out (endpoint). On the XBee, these APS messages are all sent and received by special API frames (see "Next steps" on page 239). We'll go through the concepts one by one to make it easier to understand them.

\section{Profiles}

ZigBee application profiles are collections of common definitions and protocols that allow various devices to work together in a particular domain, such as home automation. Each profile defines device types and required functionality. The most interesting profiles are the public ones that are developed and maintained by the ZigBee Alliancethe official standards organization for the ZigBee protocol—so that all ZigBeecertified devices from any manufacturer can interoperate in systems for:

- Health Care

- Home Automation

- Building Automation

- Smart Energy

- Telecommunication Services

- Consumer Electronics

- ....and new profiles that are added on a regular basis

For example, in the case of Smart Energy, the profile allows different brands of electric meters, thermostats, appliances, and in-home display units to share a common language. Any Smart Energy-certified brand of thermostat can request pricing data through any brand of certified electric meter to decide whether an extra three degrees of cooling is an affordable comfort or a pricey luxury. Similarly, any ZigBee Health Care-certified patient sensor can communicate medical data to any other brand of 
Heath Care-certified patient monitor. Public profiles are available to all manufacturers who want to participate in these networks.

Manufacturers can define their own profiles internally as well. These are referred to as private profiles and are generally used within one company's products or shared in business partnerships.

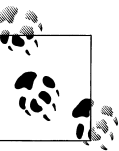

The XBee ZB communications you have been using are all part of Digi's private, manufacturer-specific Drop-in Networking profile. This is how your radios can communicate about proprietary data like analog I/O readings.

Each public or private profile has a name and a 16-bit numeric identifier to tag its messages. For example, the Smart Energy profile uses 0x0109. Any device that wants to support the Smart Energy profile is required to provide certain standard functions and to identify its messages with 0x0109 to all other devices.

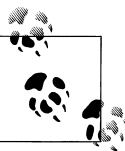

XBee-brand radios pass profile, cluster, and endpoint information inside Explicit ZigBee API frames. See "Next steps" on page 239 for

references to detailed information in the Product Manual.

Every profile defines a number of clusters, which describe how two radios in a profile interact with one another. Many clusters can exist on a single endpoint. We'll look at those next.

\section{Endpoints}

In ZigBee, endpoints exist so that a device can implement multiple profiles. For example, you can have a device that belongs to both the Home Automation and Consumer Electronics profiles. It's an endpoint's job to describe applications that are running on a particular device. You can think of endpoints as mailboxes in an apartment building. To access a particular apartment, you need to know the building address but also its mailbox number. Devices frequently have many endpoints. (Readers with a basic knowledge of Internet protocols may recognize that ZigBee endpoints function similarly to port numbers in TCP/IP.) All ZigBee APS messages are sent from some endpoint on one device to some other endpoint on another device. Endpoints contain a number of clusters that are specific to a profile. Each endpoint is identified with an 8-bit number from $0 \mathrm{x} 0$ to $0 \mathrm{xF} 0$.

\section{Clusters}

In ZigBee, device profiles interact with one another through their clusters. ZigBee clusters are collections of functionality that applications can perform within a profile. Each cluster is associated with a particular action or service. For example, in the Smart Energy 
profile one of the clusters is devoted to price, including the various attributes and actions around setting, changing, and labeling different energy pricing scenarios and states. There are two types of clusters, client clusters and server clusters. A radio implementing a particular service - such as sending pricing information-implements the server cluster. When it has a price to transmit, it interacts with the client cluster on another radio. Likewise, client clusters may send commands that manipulate attributes or perform commands on a corresponding server cluster. Each cluster has a 16-bit ID (price messages are cluster $0 \mathrm{x} 0700$ ) and can contain various attributes and command types identified by other numeric IDs. On the XBee, for example, an API frame publishing an energy price will contain command ID 0x00 and pass a payload that includes all of the following: Provider ID, Rate Label, Issuer Event ID, Current Time, Unit of Measure, Currency, Price Trailing Digit \& Price Tier, Number of Price Tiers \& Register Tier, Start Time, Duration In Minutes, and finally Price. Programmers may recognize that clusters are a little like software objects, in that both contain bundles of related states and behaviors.

\section{The ZigBee Cluster Library}

Many ZigBee application profiles use a specific protocol called the ZigBee Cluster Library (or ZCL) protocol. The ZCL defines both a method for how clusters talk to one another and a collection of common actions that can be used in multiple ZigBee application profiles. For example, someone defining a new ZigBee profile for electric automobiles (a Vehicle Area Network) may import clusters of functionality from the ZCL that are already used in Home Automation or Smart Energy profiles. The ZCL exists to promote reuse, both the reuse of ideas and the reuse of source code.

The ZCL protocol encourages people to define clusters as collections of data attributes, each with a specific data type. For example, a vehicle may define a cluster of attributes for the dashboard, including the vehicle speed, the engine speed, and the amount of charge left in the battery_each defined as a whole number. The ZCL allows devices to discover which attributes are available on a cluster, read attributes, write attributes, receive periodic reports about attributes, or even receive an update when an attribute changes.

The ZCL builds on top of ZigBee clusters. Each ZCL protocol command is simply an operation sent from one cluster on one radio to another cluster on another a radio. Most commands— such as the ZCL Attribute Read command — are sent from a client cluster to a corresponding server cluster.

\section{Next steps}

Now that you know some of the basic concepts used in ZigBee APS layer communications, you can learn more by looking in the XBee ZB Product Manual (available from $h t t p: / / w w w$.digi.com/support/supporttype.jsp?tp=3) under the API section for:

- Explicit Addressing ZigBee Command Frames 
- ZigBee Explicit Rx Indicator

- Sending ZigBee Device Objects (ZDO) Commands

- Sending ZigBee Cluster Library (ZCL) Commands

- Sending Public Profile Commands with the API

Also check the latest ZigBee Specification and Public Application Profile documentation at http://www.zigbee.org/Products/DownloadZigBeeTechnicalDocuments.aspx.

\section{Routing}

To get data from one place in the network to another, ZigBee employs several different routing methods. The first is familiar to you and is the default that's available at all times. The next two are being introduced for the first time. They must be specified using the AR command and, in some cases, special API frames:

\section{Ad hoc On-demand Distance Vector (AODV) mesh routing}

This default method that we've been using all along automatically creates routing paths between every source and destination radio as needed. These AODV paths can hop through multiple router nodes as necessary, with every intermediate hop discovering the next step on the way to the destination address. Limitations in space for internal routing tables mean that repeated route discoveries usually need to take place to keep messages moving properly.

\section{Many-to-one routing}

The purpose of a sensor network is often to route data messages in from a large number of remote nodes to one central location. Many-to-one routing is optimized for this situation. The central location broadcasts a single routing configuration message out to the network, allowing all remote devices to set up and save a reverse path back toward the central destination node. After this path is created, no more discoveries are needed for information to be properly delivered. The AR command is used to enable many-to-one broadcasting on an XBee device.

\section{Source routing}

The purpose of other networks is to send messages from a central location out to multiple remote nodes. Source routing allows the central location to discover and store individual routes to a large number of remote nodes. These routes are not stored on the central location's radio, but obtained by the device or computer controlling it using the XBee's Route Record API frame. When the time comes to send a message to one particular remote node, the central location specifies a route to the remote node with the Create Source Route API frame. It then includes the data and the destination address in an API Transmit Request. Routes include the address of each intermediate hop that messages need to pass through to reach their destination.

Each routing method has its pros and cons, outlined here in Table 8-1. 
Table 8-1. ZigBee routing methods compared

\begin{tabular}{lll}
$\begin{array}{l}\text { Routing } \\
\text { method }\end{array}$ & Pros & Cons \\
\hline AODV routing & $\begin{array}{l}\text { Default method, automatically creates routes, } \\
\text { works on any network topology. }\end{array}$ & $\begin{array}{l}\text { Poor performance on large networks (more than } 40 \\
\text { nodes) due to overhead for repeated routing requests. }\end{array}$ \\
$\begin{array}{l}\text { Many-to-one } \\
\text { routing }\end{array}$ & $\begin{array}{l}\text { Excellent performance for multiple paths in- } \\
\text { bound to a single central location. }\end{array}$ & $\begin{array}{l}\text { Not appropriate for messages outbound from a central } \\
\text { location ornetworks withremote-to-remotemessaging. } \\
\text { Requires custom configuration. }\end{array}$ \\
$\begin{array}{l}\text { Source } \\
\text { routing }\end{array}$ & $\begin{array}{l}\text { Excellent performance for outbound messages } \\
\text { from one or more central locations, especially } \\
\text { from highly capable devices such as computers. } \\
\text { Good for networks of more than 40 nodes. }\end{array}$ & $\begin{array}{l}\text { Requires considerable preconfiguration, including spe- } \\
\text { cial device programming and offboard route storage. } \\
\text { Routes must be programmatically acquired, stored, and } \\
\text { recovered for sending. }\end{array}$ \\
\hline
\end{tabular}

The good news is that you won't need to worry too much about advanced routing methods on networks that are smaller than 40 nodes. However, as your networks grow, more skills with routing will become essential.

\section{Next steps}

This overview of ZigBee routing serves as a guide to learning more. You can find additional information in the XBee ZB Product Manual. Look in the Transmission, Addressing, and Routing section for RF packet routing. You'll see a detailed discussion of and instructions for:

- Link status transmission

- AODV mesh routing

- Many-to-one routing

- Source routing

In the API section look at the frame types for:

- Route record indicator

- Many-to-one route request indicator

- Create source route

Finally, check the AR, NI, and DN commands in the XBee Command Reference Tables, as these also relate to source routing. Take your time and work through your setup slowly. The concepts may not initially seem intuitive but they are very powerful and can help you build networks that are extremely large while still being remarkably efficient. 


\section{Security}

ZigBee users are often anxious to employ encryption and security on their wireless networks. Security can be essential when communications or the network itself needs to be protected. Security can also be a liability, in terms of network resources and added development effort, so always consider your project. What is at risk? Do the benefits of adding security outweigh the costs? If you are transferring financial billing information, clearly your network needs all sorts of protection. On the other hand, if you are creating interactive kitty toys, adding security is only going to slow down your efforts, bog down your network, and bore your cat.

\section{Network and link keys}

ZigBee uses mathematical keys to encrypt data that is passed over the wireless network. There are two kinds of key-based security that can be used at the same time if desired: network keys and link keys.

Network keys protect your data frames as they pass between nodes. Each packet of data gets encrypted, sent to the next hop in the network, and decrypted before being reencrypted and passed along to the following node. Network security is hop-to-hop and fully protects your transmissions on private networks where all the radios are under the control of one entity. Encrypting and decrypting the packet at every hop in the route does add some transmission delay or latency. In addition, 18 bytes of overhead are required for the key, so data packet size is decreased from 72 to 54 . This means more packets have to be sent to convey the same amount of information.

Link keys provide an added layer of end-to-end protection. Data is encrypted by the sender and remains secure as it hops along the network. Each packet is only decrypted when it reaches its destination. Use link keys to prevent intermediary hops from examining your data-useful on a shared network where individual nodes can't be trusted. For example, if you were sending personal information across a ZigBee network set up at your school, you might want to secure that data from being seen unencrypted by some radio that was legitimately a part of the network, but that you didn't control. The coordinator's link key should also be used to encrypt the distribution of the network key. Latency increases slightly and packet size is also decreased some more, so there's an additional cost to this type of layered security.

\section{Technical details}

Network security applies to data and routing messages, but not to the lower MAC-level beacon requests used by radios to first join the network. The network key is either preselected on the coordinator or set there randomly. Packets are encrypted and authenticated using 128-bit Advanced Encryption Standard (AES), a symmetric-key encryption standard adopted by the U.S. government's National Institute of Standards. A frame counter protects against replay attacks but tops out at 4 million (32 bits). If you are going to send more than 4 million messages (unlikely, as even at 10 messages 
per second it would take 13 full years), check the radio documentation for advanced suggestions about getting beyond that limit - for example, automatically leaving and rejoining the network.

APS link security (end-to-end) is also 128-bit AES. It can't be used in broadcast mode. Both network and APS link security can be used at the same time, and often are since they provide different types of protection.

\section{Fast guide to turning on XBee network security}

1. Set ATEE (Encryption Enable) to 1 for all devices on the network.

2. Set ATNK (Network Key) to 0 only on the coordinator. 0 is the default and selects a random key, which is usually fine. You could also pick a 16-position hexadecimal. The network key will be distributed to all nodes automatically. Keys are 128 bits long and the NK register is write-only.

3. Set ATKY (link KeY) to any 16-position hexadecimal (0x0000000000000001 to OxFFFFFFFFFFFFFFFF). Use the same key for all radios on the network. Manually setting the coordinator's link key on each radio allows encrypted distribution of the network key. Keys are 128 bits long and the KY register is write-only.

\section{Using optional APS encryption}

APS end-to-end encryption can be selected on a per-packet basis by setting the enable APS options bit in the API transmit frame (see Chapter 5). Using APS encryption decreases the maximum data payload size by 9 additional bytes. This is a good example of how security is a trade-off, because it adds network latency and reduces communications efficiency. Only use it if you need it! For most, network-key-based security will be enough.

\section{ZigBee Protocol References}

If this section's taste of complexity has you hungry for more, here's where to find a cornucopia of information and documentation on the ZigBee protocol:

ZigBee Alliance Protocol Documentation http://www.zigbee.org/Products/DownloadZigBeeTechnicalDocuments.aspx

ZigBee Alliance White Papers http://www.zigbee.org/LearnMore/WhitePapers.aspx

XBee Product Manuals http://www.digi.com/support http://www.digi.com/support/supporttype.jsp?tp=3

Digi White Papers

http://www.digi.com/learningcenter/literature/whitepapers.jsp 
Other ZigBee technical books

http://www.zigbee.org/LearnMore/BooksGuides.aspx

\section{Serial Flow Control}

Throughout this book we have been using two pins on the XBee to handle serial communications. In some situations, simple TX and RX connections aren't enough to ensure your data gets delivered intact. In these cases, serial flow control can help make sure nothing is lost in transmission. Let's get some background to help understand this.

Data is held in buffers inside the XBee module during the transmit and receive process. Buffers are temporary memory locations that accumulate and hold information until it is ready to be sent to the radio antenna or serial port. They are limited in size and therefore sometimes need to be actively managed to prevent losing important data.

The serial transmit buffer inside the XBee holds data that is waiting to go out via radio over the antenna. Information is accumulated there until a full packet is ready to go or enough time has passed that the XBee decides that no more information is coming right away. Data will also wait in the transmit buffer while the XBee is receiving information, because it can't talk and listen at the same time.

The serial receive buffer holds information that is waiting to be sent from the XBee's serial port (TX pin) to the host computer or microcontroller. Computers usually can receive all the information the XBee has to send; however, in some cases microcontrollers won't always be ready to process incoming data due to limited buffer size or during times when the microcontroller program is busy doing other things.

\section{RTS and CTS}

The RTS (physical pin 16) and CTS (physical pin 12) pins on the XBee act like electronic traffic lights to control the flow of information over the RX and TX pins:

CTS

Clear to send data to the XBee. When this pin is low, it is OK for the host computer or microcontroller to proceed with sending serial information. For example, you can connect this pin to one of the Arduino's digital inputs and then read its state before trying to send serial output, something like this:

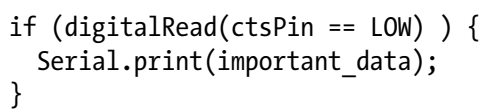

The CTS pin is de-asserted (set high) by the XBee when its serial receive buffer is almost full so that incoming data from the host doesn't overflow it and get lost. 
Request to send data from the XBee. When the RTS pin is low, it is OK for the XBee to send serial information back to the host computer or microcontroller. The RTS pin is de-asserted (set high) by the host during times when it is not able to receive data into its own buffers. For example, you could attach one of the Arduino's digital outputs to the RTS pin, and bring the pin high anytime you are not ready to process incoming data:

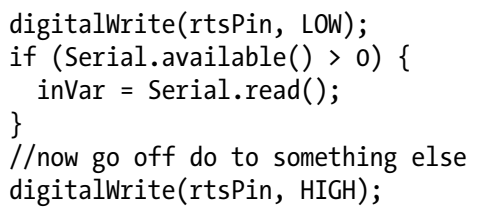

Arduino does not currently include a native implementation of hardware flow control. These basic code examples are provided for clarity. They do not cover all the complexities you might run into when using RTS and CTS together in the same application. Check the Arduino Forums if you want to learn more: http://arduino.cc/forum.

\section{Sharing Data}

You've put a lot of effort into collecting data from your sensor networks. There are plenty of reasons to share the interesting information you've acquired. By putting your data someplace accessible, you'll be able to strut your stuff, share with colleagues, and possibly pipe your information into other projects all over the world. You'll also be able to monitor your own data remotely. There are a myriad of methods for sharing your data, so we'll start you off with a popular one and let that serve as your guide to self-promotion, collaboration, and cooperation.

\section{Pachube}

The Pachube site (pronounced "patch-bay") offers public upload, download, and display of data for Internet-connected sensor networks. Device data, building information, energy readings, and environmental results can be stored, shown, displayed, and downloaded from anywhere in the world. The system is free to start with as long as you make all of your data publicly available and don't upload too much at once. Privacy and extended features are available with paid accounts.

Pachube offers a wide variety of upload and download formats, including a full web services API; downloads in XML, CSV, and JSON; online graphs of various kinds; and the RSS format to track tags for new updates. Much of the data is available in Extended Environmental Markup Language, a type of XML documented at $h t t p: / / e e m l . o r g /$ You can get started with Pachube at http://www.pachube.com/, shown in Figure 8-2. 


\section{pachưbe \\ About us | Tutorials | API Documentation | Libraries \& Examples}

Store, share \& discover realtime sensor, energy and environment data from objects, devices \& buildings around the world.

Pachube is a convenient, secure \& scalable platform that helps you connect to \& build the 'internet of things'. SICNUP
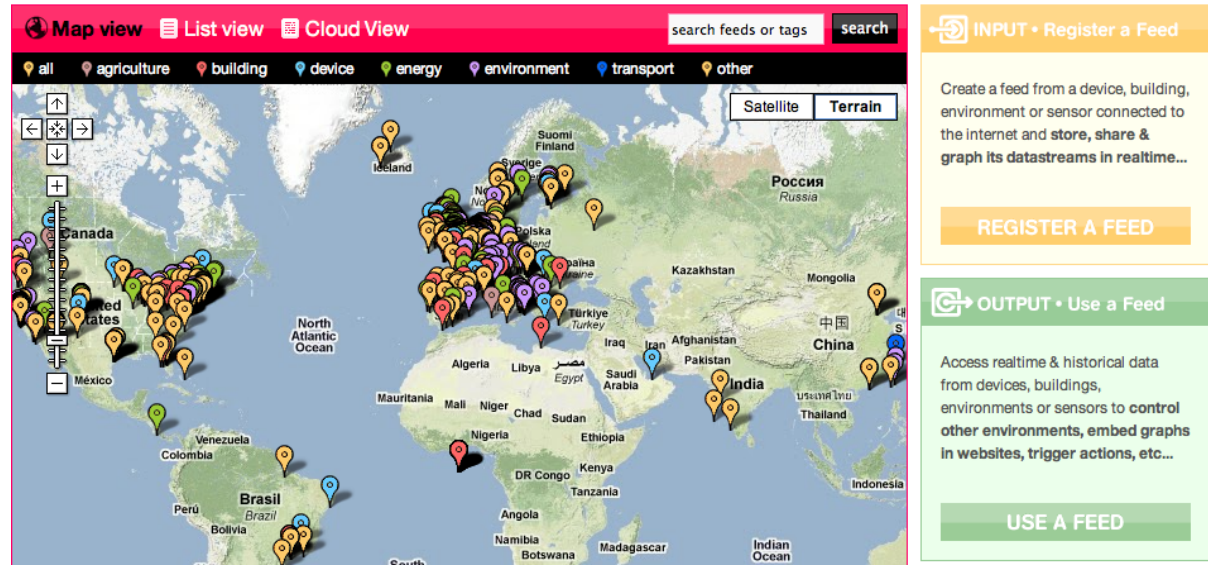

Figure 8-2. Pachube's home page, where you can sign up and register a feed

The next project modifies the simple sensor network you made in Chapter 5 to push temperature data up to Pachube for sharing. The upload code is intentionally kept very simple to show that sharing your data can be accomplished even without complex error handling, state tracking, and the like. All you need to do is create data streams and push your sensor information out to them on a regular basis.

\section{Simple Sensor Network with Pachube}

Sharing your data on the Pachube site involves setting up an account, obtaining some ID numbers, downloading an additional Processing library, and running some lightly amended Processing code that pushes your temperature network data to the site.

You'll need to sign up for a Pachube account at http://www.pachube.com/signup (see Figure 8-3). Accounts are free and require very little information, just a username, email address, and password to get going.

Once you are signed up, the first thing to do is register a new feed using the Register a Feed link on the home page. A feed is any collection of related data. For this project you'll use manual mode, meaning that your program will manually contact the Pachube site every time there is data it wants to place there. Your feed will need a title, and you can optionally enter other descriptive information as you see fit (see Figure 8-4). 


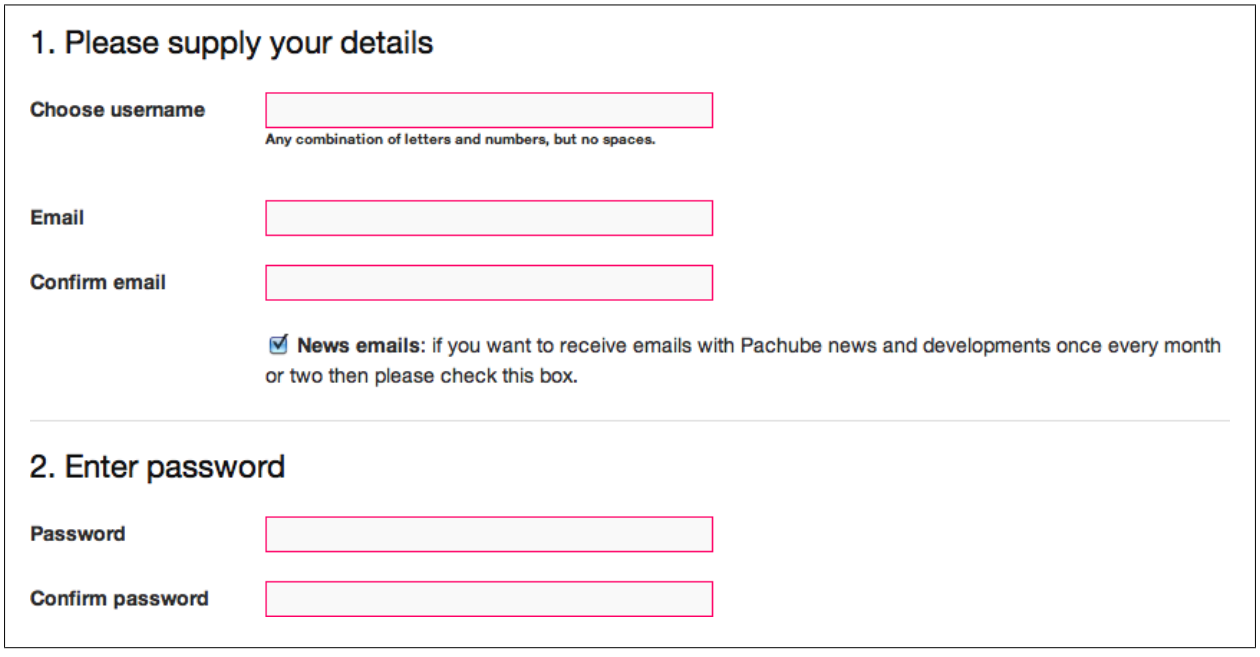

Figure 8-3. Pachube signup is free for a basic account

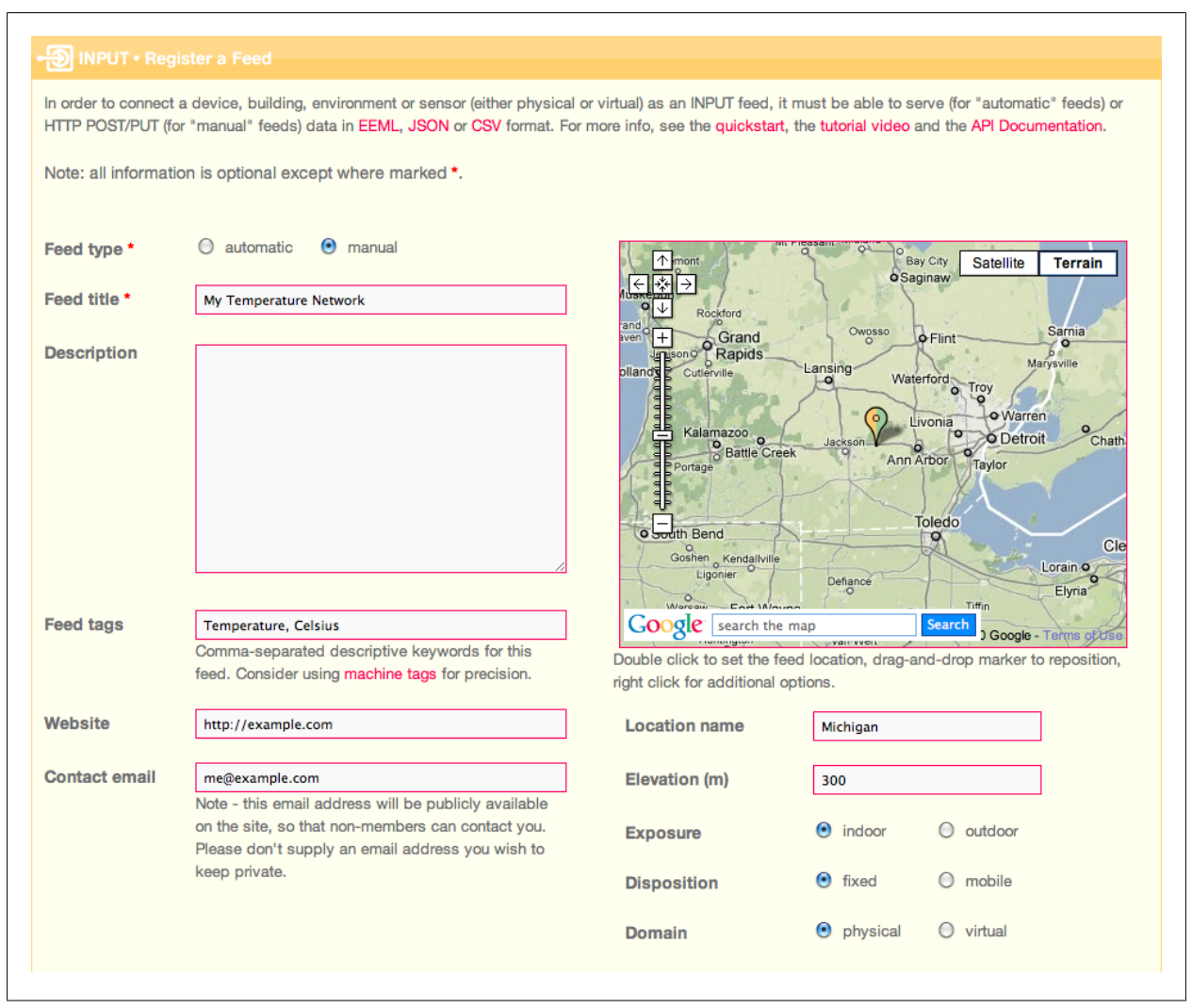

Figure 8-4. Pachube Input screen for registering a feed-any collection of related data streams 
When you are done entering data, click the Save button at the bottom of the screen. This takes you to the Output window that shows you the basic feed information and several URLs for accessing data in XML, CSV, and JSON formats (Figure 8-5).

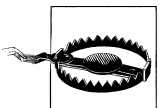

The number in each URL is your feed ID—for example http://api.pa chube.com/v2/feeds/10298.xml. Make a note of that feed ID number. You will need to enter it in your code for the project below!

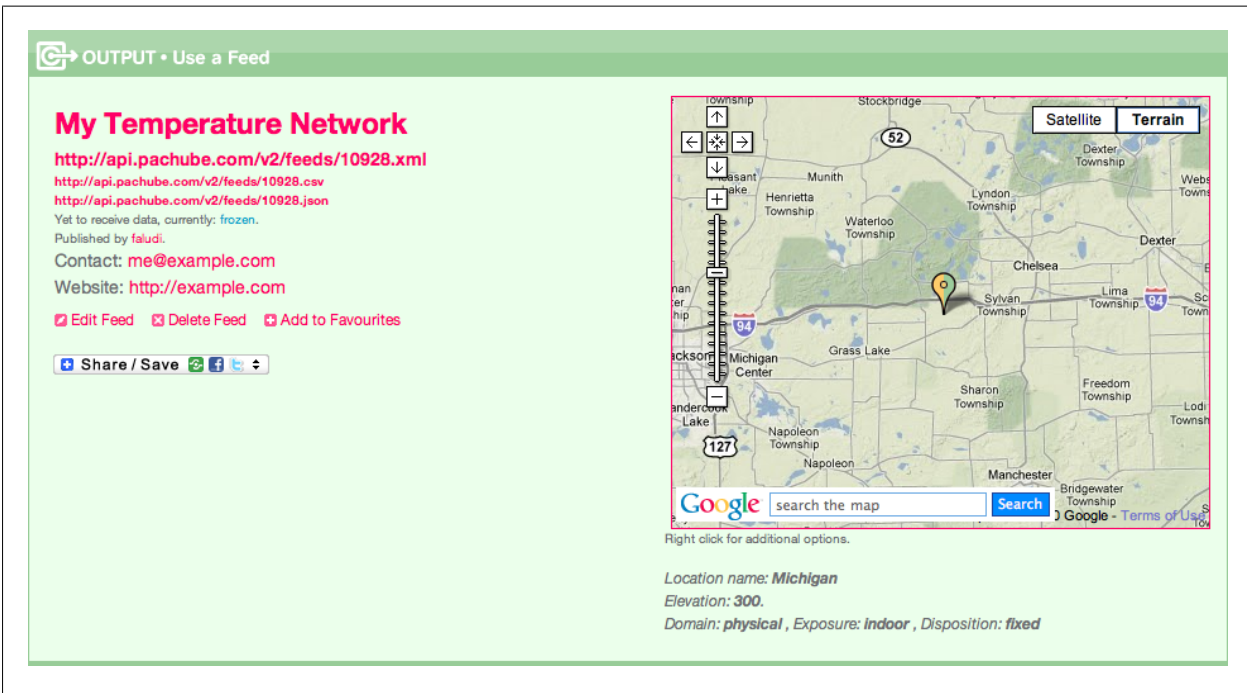

Figure 8-5. Pachube's Feed Output screen shows information about the feed, with links to receive that data in XML, CSV, or JSON formats. The number listed in the URL is the feed ID.

\section{API Key}

To access your feed remotely, Pachube requires that you pass it an API key, which is really just a long, private number to identify that it's really you uploading the data. Once you are signed in, you can get your API key by clicking on the My Profile link and selecting the Settings tab (see Figure 8-6). You'll want to copy this master API key so that you can paste it into your Processing program later.

\section{Build the Simple Sensor Network in Chapter 5}

Create the hardware and configure all the radios exactly as you did for Chapter 5's Simple Sensor Network project. Don't use the Processing code for the Chapter 5 project though! The program sketch and associated libraries in Processing are different for this Pachube version. 


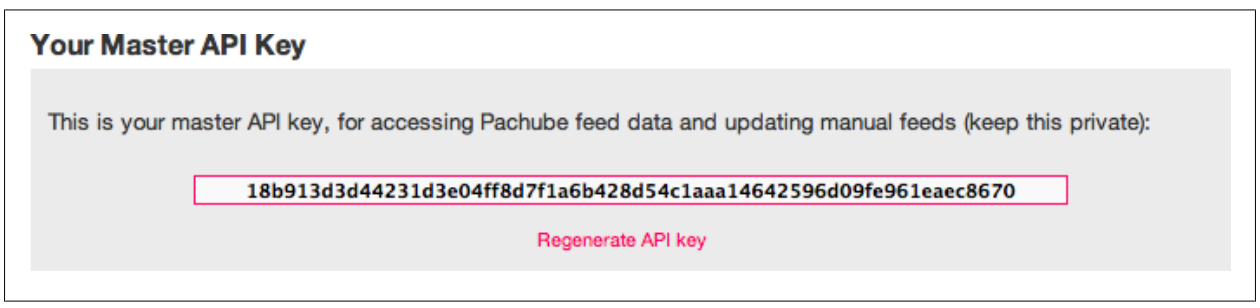

Figure 8-6. Pachube Master API Key screen at the My Profile $\rightarrow$ Settings tab; your personal API key must be entered in your program's code and passed on each time you update feeds manually

\section{Program the Base Station}

The simple sensor network Pachube base station uses the Processing program below. Download the ZIP file of all the libraries and resources from this book's website (see the Preface for more information). Inside the Processing sketch folder for the Simple Sensor Network are two subdirectories called code and data (see Figure 8-7). The code folder contains the log4j.jar and xbee-api-0.5.5.jar library files, just like in Chapter 5 . There's also a new library here called JPachube that handles connections with Pachube. The JPachube library is available at http://code.google.com/p/jpachube/, where you can check for the latest version if necessary. The data folder still holds the log4j.properties file, required by $\log 4 j$.jar, and the font file for the sans serif 10-point font used for screen display.

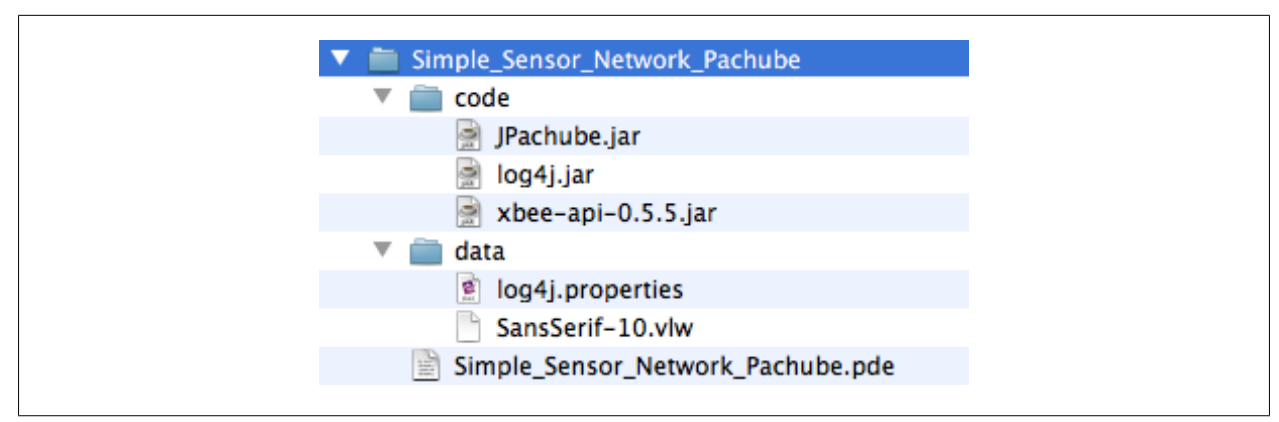

Figure 8-7. Directory structure for the Processing sketch program Simple Sensor Network Pachube, including the JPachube library used for sharing data online

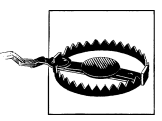

You must replace the COM port listed in this code with your actual COM port. Look for it in the code around line 27. Port names are listed in the console in Processing, as your program starts up.

You must also enter your API key and the Feed ID you created in this code. Look for a string variable called apikey and an integer variable called feedID starting near line 36 . 
Once you've loaded the files and directories onto your computer and opened Simple_Sensor_Network_Pachube.pde in Processing, press the Run button (labeled with a triangle) to launch the display code. It will open in a new window and show a thermometer for each sensor node detected. Every minute, it will attempt to upload a reading to Pachube.

If all goes well, your data will now start showing up in Pachube. Check your feed at the Pachube home page $\rightarrow$ My Feeds tab. Clicking on the feed title will show the Output screen (Figure 8-5).

The latest data will be displayed at the bottom of the Output page, with an entry for each data stream, in this case one for each temperature sensor, as shown in Figure 8-8.

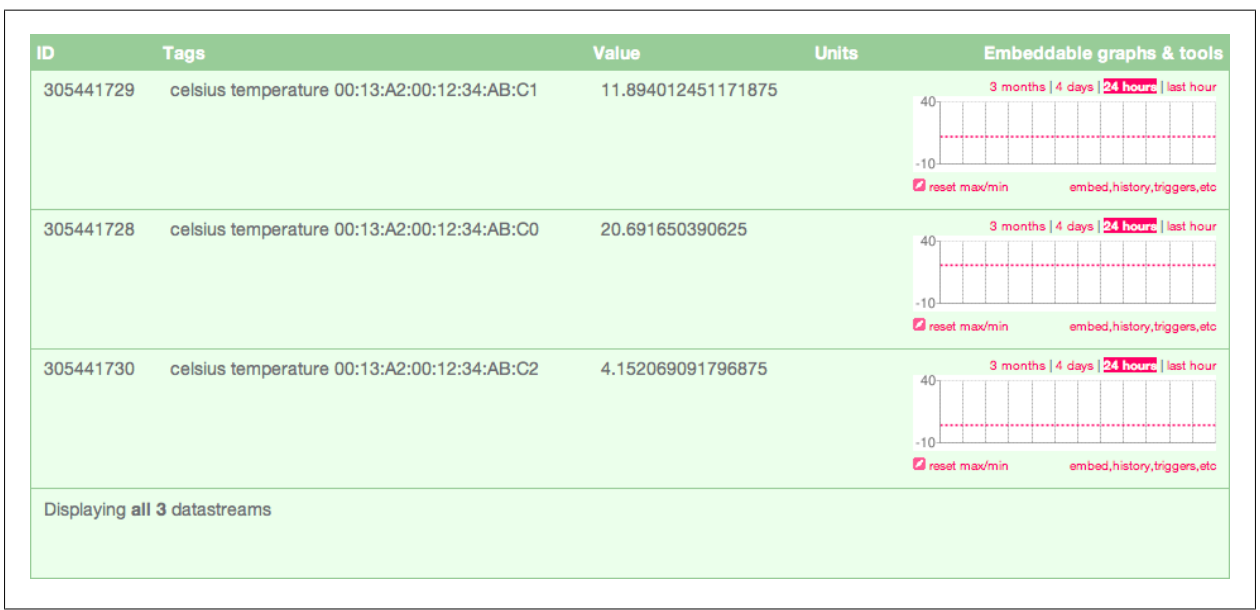

Figure 8-8. Pachube data streams are at the bottom of the Output screen for each Feed. In this case the ID created is the decimal version of the XBee's 64-bit ID, so that each ID is unique.

\section{Simple Sensor Network Pachube Code in Processing}

Here's the source code for the Processing sketch. The comments shown in bold about the serial port, API key, and feed ID highlight essential changes. Other commented instructions are only important if you didn't download the source from the website listed in the Preface:

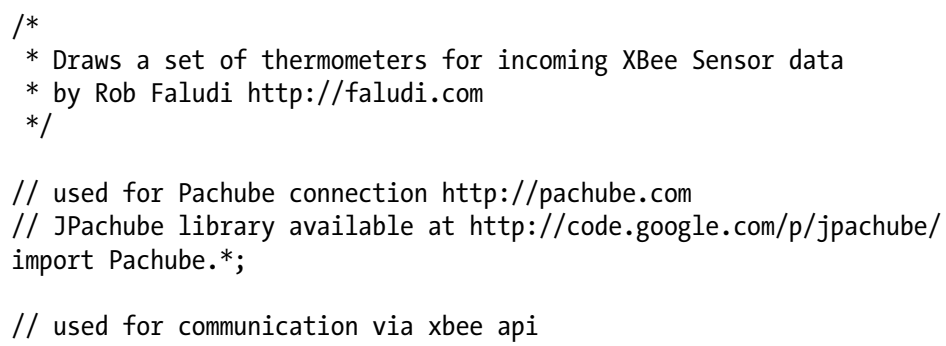


import processing.serial.*;

// xbee api libraries available at http://code.google.com/p/xbee-api/

// Download the zip file, extract it, and copy the xbee-api jar file

$/ /$ and the log $4 j . j$ ar file (located in the lib folder) inside a "code"

// folder under this Processing sketch's folder (save this sketch, then

// click the Sketch menu and choose Show Sketch Folder).

import com.rapplogic.xbee.api.ApiId;

import com.rapplogic.xbee.api.PacketListener;

import com.rapplogic.xbee.api.XBee;

import com.rapplogic.xbee.api.XBeeResponse;

import com.rapplogic.xbee.api.zigbee.ZNetRxIoSampleResponse;

String version = "1.04";

// *** REPLACE WITH THE SERIAL PORT (COM PORT) FOR YOUR LOCAL XBEE ***

String mySerialPort = "/dev/tty.usbserial-A1000iMG";

// *** REPLACE WITH YOUR OWN PACHUBE API KEY AND FEED ID $* * *$

String apiKey="your_api_key_here";

int feedID=your_feed_id_heré;

// create and initialize a new xbee object

$\mathrm{XBee}$ xbee $=$ new $\mathrm{XBee}()$;

int error $=0$;

// used to record time of last data post

float lastUpdate;

// make an array list of thermometer objects for display

ArrayList thermometers $=$ new $\operatorname{ArrayList}()$;

// create a font for display

PFont font;

void $\operatorname{setup}()\{$

size $(800,600)$; // screen size

smooth(); // anti-aliasing for graphic display

// You'll need to generate a font before you can run this sketch.

// Click the Tools menu and choose Create Font. Click Sans Serif,

// choose a size of 10 , and click OK.

font $=$ loadFont ("SansSerif-10.vlw");

textFont(font); // use the font for text

// The $\log 4 j$.properties file is required by the xbee api library, and

// needs to be in your data folder. You can find this file in the xbee // api library you downloaded earlier

PropertyConfigurator.configure(dataPath(" ")+"log4j.properties");

// Print a list in case the selected one doesn't work out

println("Available serial ports:"); 


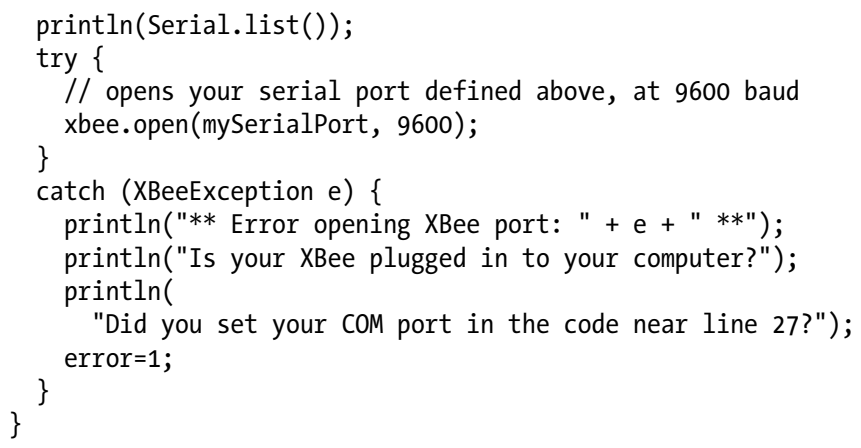




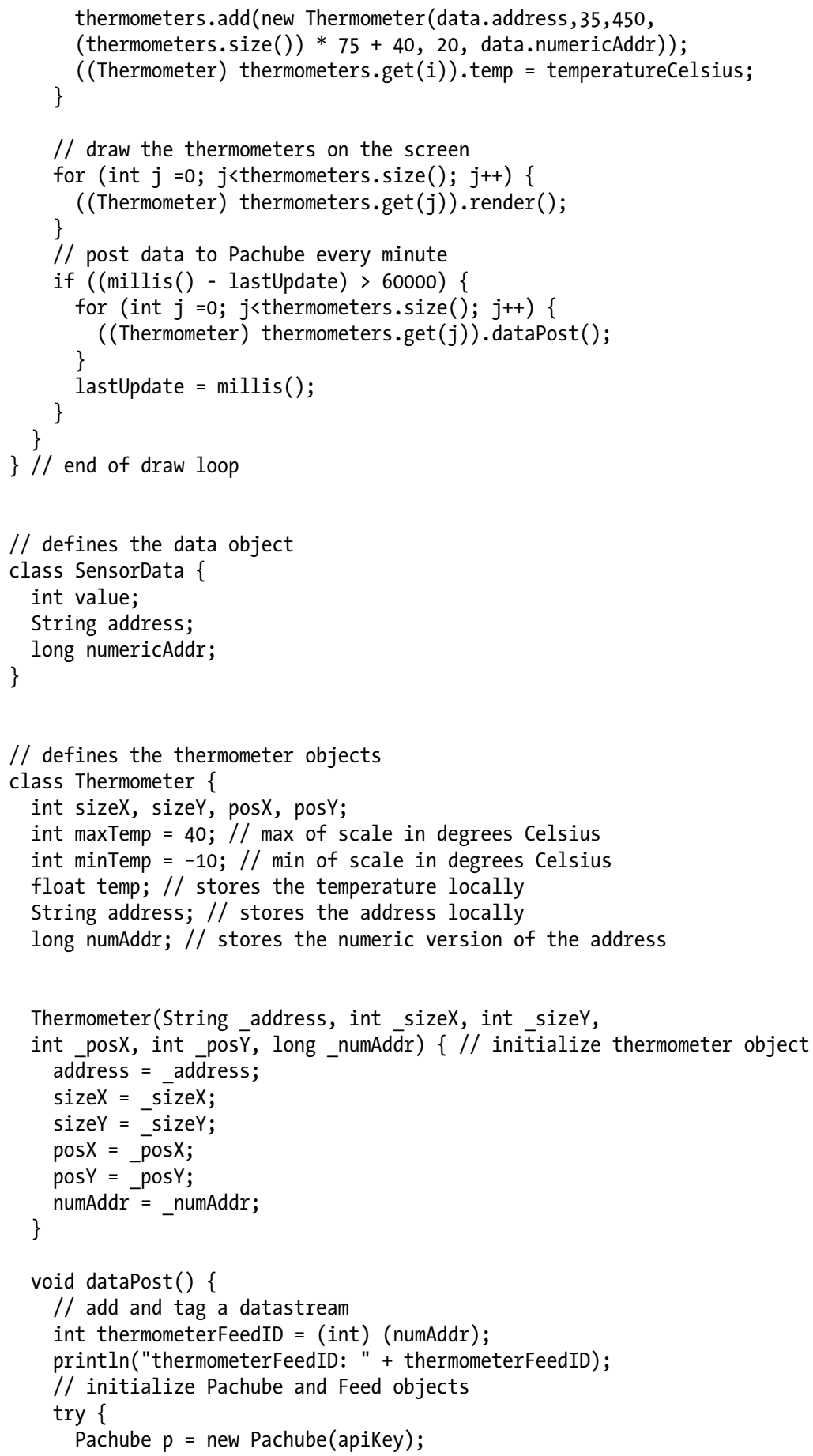




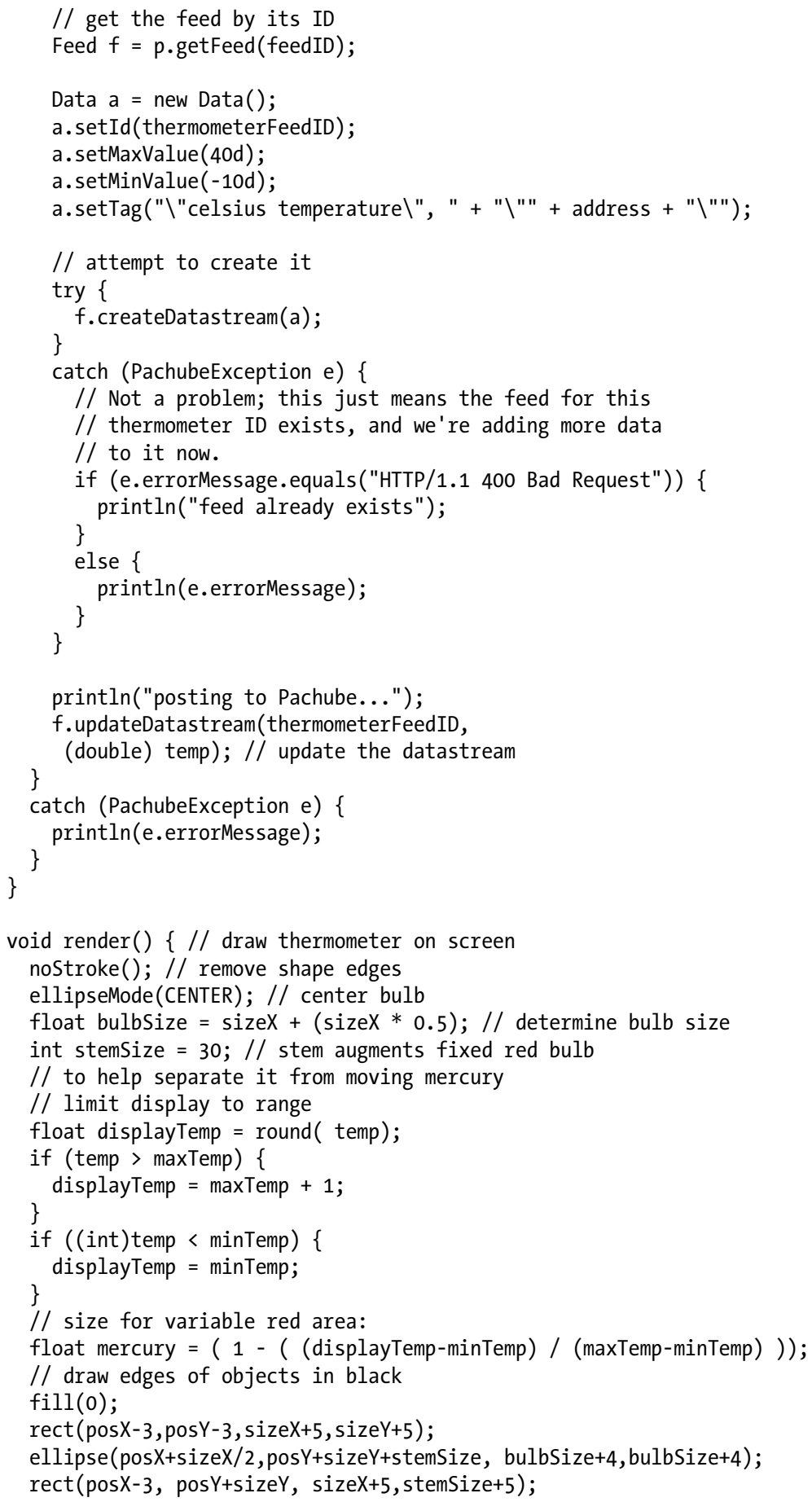




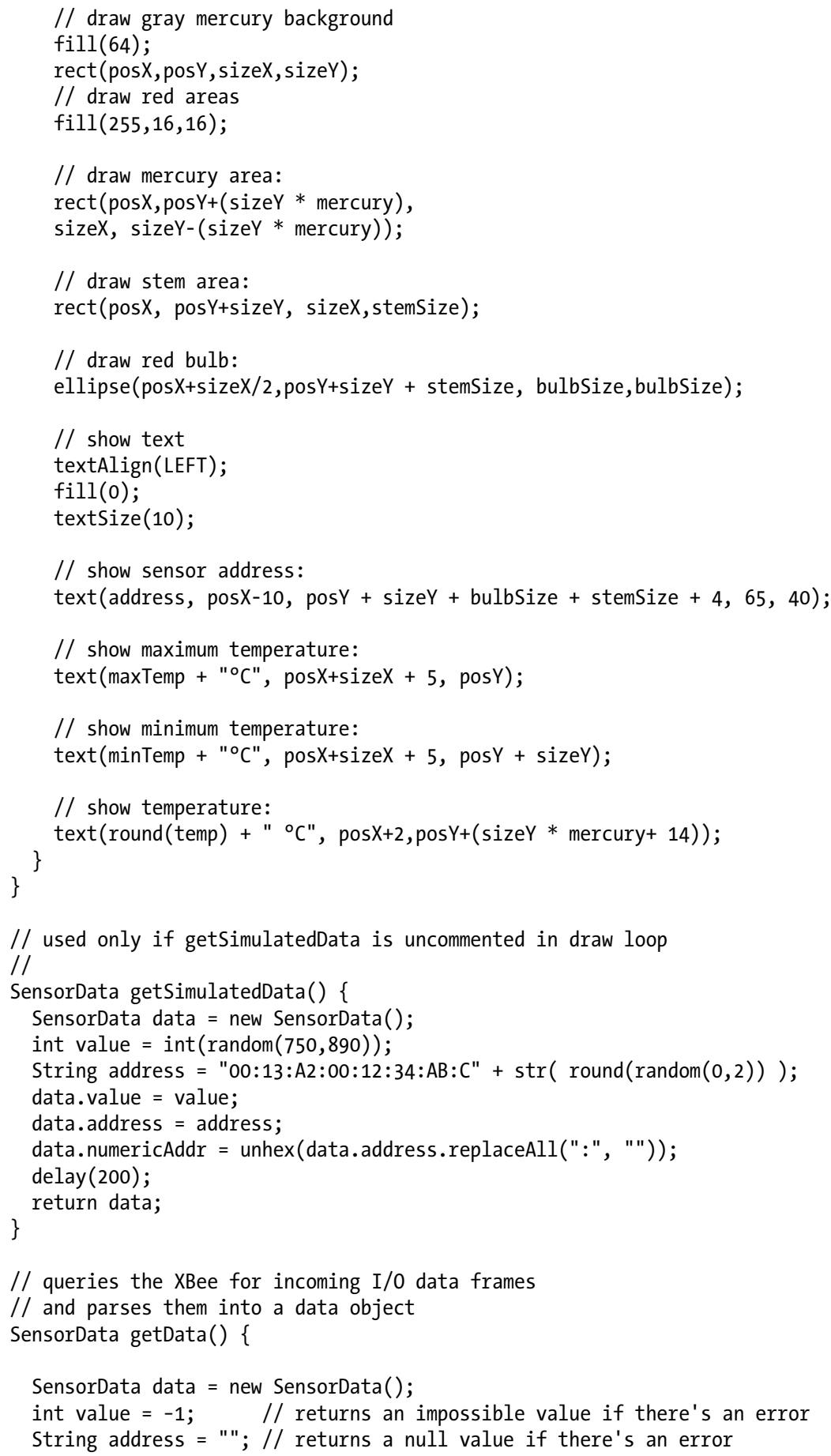




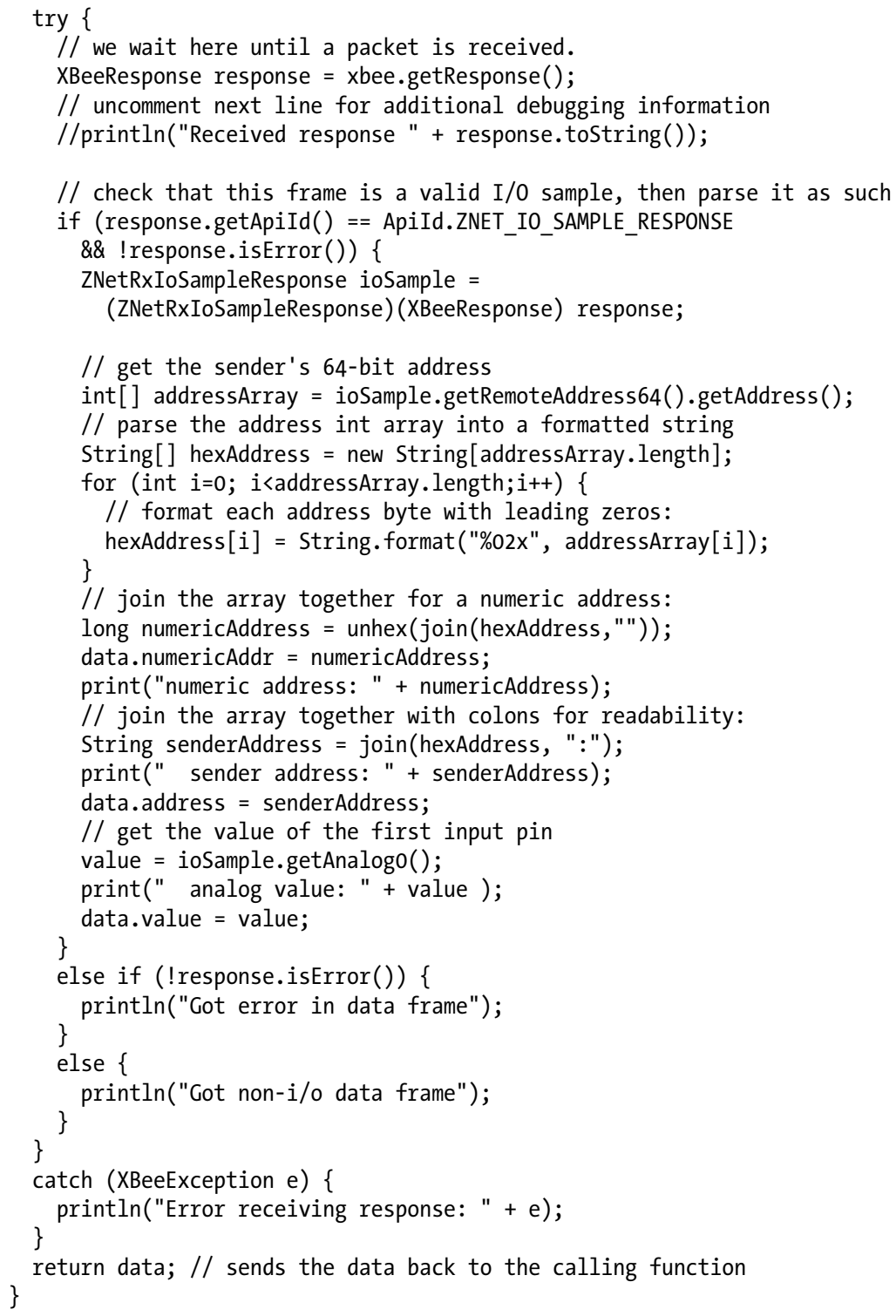

\section{Troubleshooting}

If things don't work at first, here are some steps to try:

1. Run through all the troubleshooting steps in Chapter 5 to ensure that the basic project's electronics and configuration are functioning properly.

2. Confirm that you entered your API key correctly in the Processing code. 
3. Check that the feed ID you entered in the code matches the ID number of the feed you set up in Pachube.

4. Make sure you entered the correct port information for your XBee adapter in the Processing code.

5. Third-party services often change without warning. Check the Pachube website and the JPachube code library site to see if there have been any updates that might alter the way Pachube or the library functions.

\section{The Future of ZigBee}

One thing is for certain: ZigBee won't stay the way it is for long. The protocol is finding its way into lots of new markets so new capabilities are bound to develop quickly. One of the most interesting ventures from the ZigBee Alliance is a new protocol being developed in cooperation with the HomePlug Alliance: ZigBee Smart Energy 2.0. This new standard is broadly envisioned as a networking and application integration platform for messages between customer devices and energy services providers. The stated goal of the ZigBee+HomePlug collaboration is to "Develop a common system architecture and application profile interfaces for home energy devices, supported by a comprehensive certification process that delivers secure, robust, reliable, plug and play interoperability with AMI and Smart Grid applications."

It is reasonable to expect that innovations made in the Smart Energy 2.0 specification will inform other application profiles and, therefore, the path of ZigBee going forward. While the specifications are still being drafted, here are some of the most interesting features being discussed:

- Plans to add support for additional networking protocols, including WiFi (802.11), and HomePlug powerline. These will be in addition to the existing wireless 802.15.4. support.

- Communications over both wireless and wired networks.

- Internet Protocol addressing, including the new IPv6 standard that allows for 128bit addresses. This creates an addressable space large enough for every device in the world to have a unique address.

- Standard UDP and TCP support so that seamless interconnections with the Internet will become possible.

- HTTP or other RESTful application protocols are expected to be included to extend these popular interconnection standards for the Web to home area devices.

This push to adopt and incorporate addressing, protocols, and interconnection standards from the global Internet should mean that direct communications between devices anywhere will become much easier to implement. Look for a future where common household objects like lamps and wall clocks can join Internet conversations as easily 
as a teenager can join Facebook. ZigBee stands to be a strong player in giving sensors and devices access to the world at large.

\section{Next Steps for You}

You've finally come to the end of the book, but your journey is just beginning! There's no end to the projects you can create-and plenty of excellent resources that can guide you in making your new creations.

\section{Making Stuff}

To whet your appetite, here's a list of 20 whimsically envisioned projects that could be brought into being with ZigBee radios and sensor networks. Hopefully one or more will inspire a creation of your own:

- Manage a model airplane competition.

- Make a room into a musical instrument.

- Create an electronic game of hide-and-seek.

- Monitor and display your electrical use to help reduce your bills.

- Entertain or perturb your pets.

- Keep track of open spots in a parking lot.

- Keep an eye on your grandmother's home health care.

- Link a feed of worldwide earthquakes to your massage chair.

- Network your sailboat.

- Create sock sensors to detect cold feet.

- Design reactive furniture.

- Bring a swarm of toy dinosaurs to life and simulate their migration.

- Record rainfall in an apple orchard.

- Make bracelets that sparkle when you're with your best friend.

- Create an interactive haunted house.

- Track air quality in a nearby forest.

- Send secret signals to your friends.

- Plant a garden that cares for itself.

- Sensor suit + robotic puppet $=$ new form of ballet.

- Link a wind gauge to a fan in your cubicle and bring the outside in.

$\bullet$ own idea is the one that will change the world!). (because your 


\section{Sharing Your Work}

Networks aren't just for devices. By reading this book and creating some of its systems, you have joined a community of makers-people who include artists, engineers, crafters, scientists, hobbyists, students, teachers, entrepreneurs, hackers, and inventors. You are also one of many people using Arduino, XBee, Processing, and Python. Communities require communication and, just like networks, they work best when the whole is greater than the sum of its parts. You will find that sharing your process and projects can net feedback and recommendations from all over the world. This can make the outcome of your hard work even better. To share your projects, your code, and your hard-won wisdom:

- Participate in the forums linked from this book's website.

- On Twitter: use \#BWSN to tag your tweets.

- For Instructables: use keyword BWSN.

- In Flickr: tag your photos with BWSN and add them to the BWSN group.

- For Pachube: tag your feed with BWSN.

- On YouTube and other video sites: tag your videos with BWSN.

Makers everywhere will be looking forward to seeing what you invented and hearing about how your projects made the journey from imagination to reality. Best of luck with your fabulous creations, and happy networking! 

You've savored the glamour and glitz of wireless networking; now here's some extra substance to ensure that you're fully satisfied with your high-tech meal. Since you have already launched yourself into the wireless mesh, to keep the book useful we've included links to online resources for learning more about Arduino, Processing, Python, and of course, ZigBee. There's a list of recommended books that can help you flesh out your technical library. And because every project sees its share of errors and glitches, you will find a handy troubleshooting guide to get you unstuck from common mistakes. There are tables to use as a fast daily reference of Digi radio flavors, other brands of ZigBee modules, network analyzers, packet sniffers, and XBee connectors and shields. There are also cross-referenced tables for hexadecimals, binary numbers, and ASCII codes to help keep your bytes organized, and finally, a complete guide to AT commands for the $\mathrm{ZB}$ radio modules.

Remember that URLs and offerings will change as time goes by, so check the book's website (listed in the Preface) for updated references to new resources.

\section{Arduino Resources}

Here are some Arduino resources you'll find useful:

Arduino Blog (http://arduino.cc/blog/)

This is the official blog, featuring Arduino news, announcements, cool projects, and more.

Arduino Forum (http://www.arduino.cc/cgi-bin/yabb2/YaBB.pl)

This is the official Arduino forum site, which contains subforums on many Arduino topics such as troubleshooting, programming, cool projects, and more.

Arduino Playground (http://www.arduino.cc/playground/)

The Playground is a wiki containing Arduino tutorials, circuits, and code. This is a good place to start if you're trying to connect Arduino to an unfamiliar device. 
Ladyada.net Arduino Tutorials (http://www.ladyada.net/learn/arduino/)

This is a great set of lessons from the folks behind Adafruit Industries.

Make: Online's Arduino section (http://blog.makezine.com/archive/arduino/)

This section of Make: Online's blog is devoted to all things Arduino.

Make: Projects' Arduino section (http://makeprojects.com/Topic/Arduino)

Make: Projects is an editable wiki full of projects.

\section{Processing Resources}

Here are some resources for the Processing language:

Processing Wiki (http://wiki.processing.org/w/Main_Page)

This wiki features documentation, example programs, reference material, and other resources.

The Processing Feed (http://feed.processing.org/)

Keep up-to-date on the latest news from the world of Processing.

Tutorials (http://processing.org/learning/)

These tutorials will get you up and running with Processing and also teach you advanced Processing topics.

Processing examples (http://processing.org/learning/topics/)

This section of the site contains many short Processing examples that can run in your browser.

\section{Python Resources}

Here are some resources for the Python language:

Python Documentation (http://www.python.org/doc/)

This documentation covers all the currently supported versions of Python and has

links to various learning resources.

Python News (http://www.python.org/news/)

Keep up-to-date on the latest news from the Python community.

Community (http://www.python.org/community/)

This page links to various mailing lists, wikis, user groups, and more.

The Python Tutorial (http://docs.python.org/tutorial/)

This is the official Python tutorial. 


\section{ZigBee Resources Online}

The Internet has plenty of important ZigBee resources, including specifications and information from the various standards organizations, interesting white papers, community forums, and additional tidbits compiled by other organizations.

\section{Standards Organizations}

ZigBee Alliance (http://www.zigbee.org)

The Alliance is an association of companies that defines the protocol layers for

ZigBee and promotes the standard itself as a brand.

Official ZigBee Specifications \& Public Application Profiles (http://www.zigbee.org/Prod ucts/DownloadZigBeeTechnicalDocuments.aspx)

These documents contain the official definitions for how the ZigBee protocol and

ZigBee public application profiles operate.

ZigBee Alliance White Papers (http://www.zigbee.org/LearnMore/WhitePapers.aspx)

The white papers contain longer narrative explanations of application profiles, interoperation, and vision for the ZigBee project going forward.

ZigBee Alliance guide to technical books (http://www.zigbee.org/LearnMore/Book sGuides.aspx)

This guide is a short list of technical books and resources related to ZigBee.

ZigBee Alliance guide to testing and development solutions (http://www.zigbee.org/Prod ucts/TestDevelopmentSolutions.aspx)

Here you'll find tools and devices for testing new ZigBee products for official certification.

IEEE 802.15.4 (http://standards.ieee.org/getieee802/download/802.15.4-2006.pdf)

This is the official specification for the network layers that live below ZigBee.

6LoWPAN ITEF (http://tools.ietf.org/html/rfc4944)

This is the initial specification that will define ZigBee IP addressing in the future using IPv6 for low-power wireless personal area networks.

6LoWPAN document library (http://datatracker.ietf.org/wg/6lowpan)

This library contains additional documents on 6LoWPAN.

\section{Digi International Resources}

Digi Developer Wiki (http://www.digi.com/wiki/developer)

Here you'll find evolving documentation and examples for working with Digi products.

Digi Forums (http://www.digi.com/support/forum/listforums?category=16)

This is the official discussion area for XBee-brand ZigBee radios. 
White paper: Demystifying 802.15.4 E ZigBee (http://www.digi.com/pdf/wp_zigbee.pdf)

Read a cogent explanation of how ZigBee is related to its underlying 802.15.4 layer.

White paper: Untangling the Mesh (http://www.digi.com/pdf/wp_untanglingthemesh .pdf)

This guide discusses mesh networks, including wireless network basics, and gives an overview of mesh-related technologies.

White paper:Source Routing (http://www.digi.com/wiki/developer/index.php/Large_Zig Bee_Networks_and_Source_Routing)

If you need to use the advanced source routing protocol discussed in Chapter 8, this guide can help you implement it on XBee ZB radio modules.

White paper: Antenna Considerations (http://ftp1.digi.com/support/images/XST -AN019a_XBeeAntennas.pdf)

This is an older but still relevant guide to the different antenna options available for XBee modules.

\section{Additional Online Resource Lists}

Daintree Networks ZigBee Information (http://www.daintree.net/resources/index.php) This guide includes useful white papers, a comparison matrix for older versions of the ZigBee protocol, and a glossary of terms.

Palo Wireless ZigBee resources (http://www.palowireless.com/zigbee/tutorials.asp)

This is an interesting article list that focuses on the rationale behind ZigBee and gives some comparisons to other standards, including Bluetooth.

Webcom's ZigBee Resource Guide (http://www.zigbeeresourceguide.com)

Webcom publishes this commercially sponsored guide to ZigBee resources that includes advertising from various industry players.

ZDNet ZigBee Topics (http://www.zdnet.com/topics/zigbee)

This is a collection of articles published by ZDNet and tagged as ZigBee-related.

\section{Recommended Books}

The following publications can help you learn more about some of the topics briefly covered in this book:

- Programming PHP by Rasmus Lerdorf, et al. (O’Reilly)

- The Visual Display of Quantitative Information by Edward Tufte (Graphics Press)

- The Design of Everyday Things by Donald Norman (Basic Books)

- Getting Started with Arduino by Massimo Banzi (O'Reilly)

- Learning Processing: A Beginner's Guide to Programming Images, Animation, and Interaction by Daniel Shiffman (Morgan Kaufmann) 
- Making Things Talk: Physical Computing with Sensors, Networks, and Arduino by Tom Igoe (O'Reilly)

- Getting Started with Processing by Casey Reas and Ben Fry (O'Reilly)

- Practical Electronics for Inventors by Paul Scher (McGraw-Hill)

- Make: Electronics by Charles Platt (O’Reilly)

- Physical Computing: Sensing and Controlling the Physical World with Computers by Tom Igoe and Dan O'Sullivan (Course Technology PTR)

\section{Sidewalk Telescopes}

At the end of the Preface, I mention sidewalk telescopes are a source of inspiration. If you'd like to build a sensor that detects the universe, here are some links to get you started:

- http://www.telescopesineducation.com/dobson/index.html

- http://www.sfsidewalkastronomers.org

- http:/quanta-gaia.org/dobson/

\section{Troubleshooting}

When it's late at night and you just can't get your network working, more than likely a simple solution is waiting in the wings. Here are some tips for resolving issues typically encountered when working with XBee and Arduino systems.

\section{Common XBee Mistakes}

If your project won't work, check through this list of common mistakes that both beginners and experts make:

- Not using the correct firmware (choose coordinator, router, end device, and AT or API mode).

- Using ZNet 2.5 firmware, which is obsolete and will not interoperate with ZB firmware.

- Forgetting that AT commands use hexadecimals.

- Hitting Return after +++ (or otherwise not respecting the 1-second default guard times).

- Conversely, not hitting Return after an AT command.

- Letting the XBee time out of command mode before issuing an AT command (you'll know because you get no response).

- Forgetting to write the configuration to firmware with ATWR (unless your application configures the radio interactively). 
- Not using ATRE (restore factory defaults) before reconfiguring a previously used radio. Previous settings lurk unless you manually reset them all.

- Using a voltage regulator without decoupling capacitors $(10 \mu \mathrm{F}$ on input, $1 \mu \mathrm{F}$ on output is usually good).

- Mixing up TX and RX pins. The fastest way to check this is to switch the wires and see if things start working.

- Trying to read more than 1.2 volts on the ZB analog inputs (1.2 V is the upper limit).

- Buying PRO radios when you don't need them. Pros cost more, are bigger, and use a lot more battery power.

- Deciding the XBees are flaky. (You may not be using them correctly; they are very reliable.)

- Deciding an XBee is burned out when it's set to a different baud rate. Check that the ON and ASSOC lights are functioning to confirm proper operation.

- Deciding an XBee is burned out when it is just sleeping. Check the ON light to see if it blinks occasionally.

- Forgetting to supply power or ground. (The ON light may go on and ASSOC light may blink, but both will be significantly dimmer.)

- Neglecting to check whether you are joined to the right network using ATAI to test for joining, and/or ATND to discover other network nodes.

- Not enabling rejoining for radios on smaller-sized networks (by setting ATJV to 1).

- Not contacting Digi sooner for support, especially if your radio seems dead or you keep getting an error you don't understand.

\section{XBee Arduino Mistakes}

Here are a few more mistakes commonly made during XBee projects that work with Arduino:

- Sending values continuously without any delay. (Try a 10 ms delay in case you are overwhelming the receiving end.)

- Not removing RX and TX connections before uploading code. (Arduino will give an error.)

- Not removing the RX connection when resetting, if you are continuously receiving data. (Arduino will never reset.)

\section{Reference Tables}

The information in this section provides a reference to radio modules, useful tools, numbering systems, and all of the XBee AT command set for the ZB radios. 


\section{Other ZigBee Modules}

Although Digi's XBee radios are certainly the most popular option for certain markets, they are hardly the only option for ZigBee modules. Table A-1 shows some of the many manufacturers and components you might consider for your projects, including some of the ZigBee integrated circuit (IC) chips that modules use internally.

Table A-1. ZigBee module options

\begin{tabular}{|c|c|c|}
\hline Manufacturer & URL & Components \\
\hline Atmel & http://www.atmel.com & ICs, modules, development environment \\
\hline California Eastern Laboratories & http://www.cel.com/ & ICs, modules \\
\hline Digi International & http://www.digi.com & Modules, development kits and environments \\
\hline Freescale & http://www.freescale.com & ICs, development kits and environments \\
\hline Ember & http://www.ember.com & ICs, development kits and environments \\
\hline Jennic & http://www.jennic.com & ICs, modules, development environments \\
\hline Laird Technologies & http://www.lairdtech.com & Modules, development kits \\
\hline LS Research & http://www.lsr.com/ & Modules, development kits, test environments \\
\hline Microchip & http://www.microchip.com & Modules \\
\hline Panasonic & http://www.panasonic.com & Modules \\
\hline Radiocrafts & http://www.radiocrafts.com & Modules \\
\hline RadioPulse & http://www.radiopulse.co.kr & ICs \\
\hline Radiotronix & http://www.radiotronix.com/ & Modules \\
\hline Telegesis & http://www.telegesis.com/ & Modules, development kits, USB dongles \\
\hline Telit & http://www.telit.com & Modules, development environments, USB dongles \\
\hline Texas Instruments & http://www.ti.com & ICs, development kits and environments \\
\hline
\end{tabular}

\section{ZigBee Packet Sniffers}

Table A-2 shows devices that detect and capture 802.15.4 and ZigBee radio signals for analysis. Packet sniffers are somewhat expensive but indispensable troubleshooting tools, typically used in professional network design. If you get really serious about ZigBee, you'll want to own one. 
Table A-2. Network analyzers and packet sniffers

$\begin{array}{lll}\text { Device } & \text { Manufacturer } & \text { URL } \\ \text { Perytons Analyzer } & \text { Perytons } & \text { http://www.perytons.com/products_perytonS.php } \\ \text { Sensor Network Analyzer } & \text { Daintree Networks } & \text { http://www.daintree.net/sna/sna.php } \\ \text { WiSens } & \text { BzWorks Ltd. } & \text { http:///bzworks.com/wisenssoftware.htm } \\ \text { ZENA Network Analyzer } & \text { Microchip Technology } & \text { http://www.microchip.com/stellent/idcplg?ldcService=SS_GET_PAGE\& } \\ & & \text { nodeld=1406\&dDocName=en520682 }\end{array}$

\section{Digi XBee Radio Modules}

Table A-3 shows the various XBee radio modules that are available.

Table A-3. Guide to XBee radio module versions

\begin{tabular}{|c|c|c|c|}
\hline XBee radio type & Protocol & Frequency & Notes \\
\hline XBee ZB & ZigBee PRO & $2.4 \mathrm{GHz}$ & Regular and PRO high-power versions are available. \\
\hline XBee ZB SMT & ZigBee PRO & $2.4 \mathrm{GHz}$ & $\begin{array}{l}\text { Surface mount for soldering directly to printed circuit } \\
\text { boards. Regular and PRO versions available. }\end{array}$ \\
\hline XBee ZNet 2.5 & $\begin{array}{l}\text { ZigBee (obsolete } \\
\text { version!) }\end{array}$ & $2.4 \mathrm{GHz}$ & $\begin{array}{l}\text { Obsolete, but firmware can be replaced with newer ZB } \\
\text { ZigBee PRO version in X-CTU. }\end{array}$ \\
\hline XBee DigiMesh & DigiMesh & $2.4 \mathrm{GHz}$ & Proprietary protocol. Regular and PRO versions available. \\
\hline $\begin{array}{l}\text { XBee-PRO DigiMesh } \\
900\end{array}$ & DigiMesh & $900 \mathrm{MHz}$ & Proprietary protocol. Only the PRO version is available. \\
\hline XBee-PR0 868 & Proprietary & $868 \mathrm{MHz}$ & Licensed for use in Europe only. \\
\hline XBee 802.15 .4 & IEEE 802.15 .4 & $2.4 \mathrm{GHz}$ & Regular and PRO versions are available. \\
\hline XBee-PRO XSC & Proprietary & $900 \mathrm{MHz}$ & $\sim 10 \mathrm{~km}$ outdoor range. \\
\hline
\end{tabular}

\section{XBee Connectors and Shields}

Chapters 1 and 3 showed you some excellent options for connecting the XBee to computers via USB and directly to Arduino. Table A-4 is more comprehensive list of components that you can use to link the XBee to other devices. 
Table A-4. Connectors and shields for XBee

\begin{tabular}{|c|c|c|c|}
\hline Component & Connection & Manufacturer & URL \\
\hline XBee Adapter Kit & $\begin{array}{l}\text { Computer via FTDI } \\
\text { cable, breadboard }\end{array}$ & Adafruit & $\begin{array}{l}\text { http://www.adafruit.com/index.php?main_page }= \\
\text { product_info\&products_id }=126\end{array}$ \\
\hline Arduino XBee Shield & Arduino & Arduino/Libelium & http://arduino.cc/en/Main/ArduinoXbeeShield \\
\hline $\begin{array}{l}\text { USB Development } \\
\text { Board (part of kit) }\end{array}$ & Computer & Digi International & $\begin{array}{l}\text { http://store.digi.com/index.cfm?fuseaction=product } \\
. \text { display\&Product_ID }=2352\end{array}$ \\
\hline FIO & Arduino & Funnel & http://funnel.cc \\
\hline XBee to USB Adapter & Computer & Gravitech & http://store.gravitech.us/xbtousbad.html \\
\hline XBee Dongle & Computer & New Micros & $\begin{array}{l}\text { http://www.newmicros.com/cgi-bin/store/order.cgi } \\
\text { ?form=prod_detail\&part=USB-XBEE-DONGLE-CAR } \\
\text { RIER }\end{array}$ \\
\hline USB XBee Adapter & $\begin{array}{l}\text { Computer, } \\
\text { breadboard }\end{array}$ & Parallax & $\begin{array}{l}\text { http://www.parallax.com/Store/Accessories/Commu } \\
\text { nicationRF/tabid/161/ProductID/643/List/O/Default } \\
\text {.aspx }\end{array}$ \\
\hline LilyPad XBee & $\begin{array}{l}\text { Wearables (e.g., } \\
\text { clothing) }\end{array}$ & $\begin{array}{l}\text { SparkFun } \\
\text { Electronics }\end{array}$ & $\begin{array}{l}\text { http://www.sparkfun.com/commerce/product_info } \\
\text {.php?products_id=8937 }\end{array}$ \\
\hline $\begin{array}{l}\text { Seeeduino XBee } \\
\text { Shield }\end{array}$ & Arduino & Seeed Studio & $\begin{array}{l}\text { http://www.seeedstudio.com/depot/xbee-shield } \\
\text {-v11-by-seeedstudio-p-419.htm/ }\end{array}$ \\
\hline XBee Explorer & $\begin{array}{l}\text { Computer, } \\
\text { breadboard }\end{array}$ & $\begin{array}{l}\text { SparkFun } \\
\text { Electronics }\end{array}$ & $\begin{array}{l}\text { http://www.sparkfun.com/commerce/product_info } \\
\text {.php?products_id=8687 }\end{array}$ \\
\hline XBee Explorer Serial & $\begin{array}{l}\text { Computer with } \\
\text { RS-232 serial }\end{array}$ & $\begin{array}{l}\text { SparkFun } \\
\text { Electronics }\end{array}$ & $\begin{array}{l}\text { http://www.sparkfun.com/commerce/product_info } \\
\text {.php?products_id=9111 }\end{array}$ \\
\hline $\begin{array}{l}\text { SparkFun XBee } \\
\text { Shield }\end{array}$ & Arduino & $\begin{array}{l}\text { SparkFun } \\
\text { Electronics }\end{array}$ & $\begin{array}{l}\text { http://www.sparkfun.com/commerce/product_info } \\
\text {.php?products_id =9588 }\end{array}$ \\
\hline
\end{tabular}

\section{Hex, Decimal, and Binary}

Table A- 5 shows the numbers 0 through 32 represented in three different numeric bases: base 16 (hexadecimal), base 10 (decimal), and base 2 (binary). 
Table A-5. The numbers 0 through 32 in hex, decimal, and binary

\begin{tabular}{|c|c|c|}
\hline Hexadecimal & Decimal & Binary \\
\hline $0 \times 00$ & 00 & 00000000 \\
\hline $0 \times 01$ & 01 & 00000001 \\
\hline $0 \times 02$ & 02 & 00000010 \\
\hline $0 \times 03$ & 03 & 00000011 \\
\hline $0 \times 04$ & 04 & 00000100 \\
\hline $0 \times 05$ & 05 & 00000101 \\
\hline $0 \times 06$ & 06 & 00000110 \\
\hline $0 \times 07$ & 07 & 00000111 \\
\hline $0 \times 08$ & 08 & 00001000 \\
\hline $0 \times 09$ & 09 & 00001001 \\
\hline $0 x 0 a$ & 10 & 00001010 \\
\hline $0 \times 0 b$ & 11 & 00001011 \\
\hline $0 \times 0 c$ & 12 & 00001100 \\
\hline $0 \times 0 d$ & 13 & 00001101 \\
\hline $0 \times 0 e$ & 14 & 00001110 \\
\hline $0 \times 0 f$ & 15 & 00001111 \\
\hline $0 \times 10$ & 16 & 00010000 \\
\hline 0x11 & 17 & 00010001 \\
\hline $0 \times 12$ & 18 & 00010010 \\
\hline $0 \times 13$ & 19 & 00010011 \\
\hline 0x14 & 20 & 00010100 \\
\hline $0 \times 15$ & 21 & 00010101 \\
\hline $0 \times 16$ & 22 & 00010110 \\
\hline $0 \times 17$ & 23 & 00010111 \\
\hline $0 \times 18$ & 24 & 00011000 \\
\hline 0x19 & 25 & 00011001 \\
\hline $0 \times 1 a$ & 26 & 00011010 \\
\hline $0 \times 1 b$ & 27 & 00011011 \\
\hline $0 \times 1 c$ & 28 & 00011100 \\
\hline 0x1d & 29 & 00011101 \\
\hline 0x1e & 30 & 00011110 \\
\hline $0 \times 1 f$ & 31 & 00011111 \\
\hline $0 \times 20$ & 32 & 00100000 \\
\hline
\end{tabular}




\section{ASCII Codes}

Table A- 6 shows hexadecimal and decimal ASCII codes. The first 32 codes ( 0 through 31) are control codes used to signify changes in transmission (such as end-of-file) or special characters embedded in strings, such as tabs and line feeds. Some control codes are not in common use. These are shown in italic in the "ASCII character" column.

Table A-6. ASCII chart

\begin{tabular}{|c|c|c|}
\hline Hexadecimal & Decimal & ASCII character \\
\hline $0 \times 00$ & 0 & nul (null) \\
\hline $0 \times 01$ & 1 & soh (start of heading) \\
\hline $0 \times 02$ & 2 & stx (start of text) \\
\hline $0 \times 03$ & 3 & etx (end of text) \\
\hline $0 \times 04$ & 4 & eot (end-of-transmission or end-of-file) \\
\hline $0 \times 05$ & 5 & enq (enquiry) \\
\hline $0 \times 06$ & 6 & ack (acknowledge) \\
\hline $0 \times 07$ & 7 & bel (bell/beep) \\
\hline $0 \times 08$ & 8 & bs (backspace) \\
\hline $0 \times 09$ & 9 & ht (horizontal tab) \\
\hline 0x0a & 10 & nl (line feed or newline) \\
\hline 0x0b & 11 & $v t$ (vertical tab) \\
\hline $0 \times 0 c$ & 12 & np (form feed, page break, or new page) \\
\hline 0x0d & 13 & $\operatorname{cr}$ (carriage return) \\
\hline 0x0e & 14 & so (shift out) \\
\hline $0 \times 0 f$ & 15 & si (shift in) \\
\hline $0 \times 10$ & 16 & dle (data link escape) \\
\hline $0 \times 11$ & 17 & $d c 1$ (device control one) \\
\hline $0 \times 12$ & 18 & $d c 2$ (device control two) \\
\hline $0 \times 13$ & 19 & $d c 3$ (device control three) \\
\hline $0 \times 14$ & 20 & $d c 4$ (device control four) \\
\hline $0 \times 15$ & 21 & nak (negative acknowledge) \\
\hline $0 \times 16$ & 22 & syn (synchronous idle) \\
\hline $0 \times 17$ & 23 & etb (end transmission block) \\
\hline $0 \times 18$ & 24 & can (cancel) \\
\hline $0 \times 19$ & 25 & em (end-of-medium) \\
\hline 0x1a & 26 & sub (substitute) \\
\hline $0 \times 1 b$ & 27 & esc (escape) \\
\hline
\end{tabular}




\begin{tabular}{|c|c|c|}
\hline Hexadecimal & Decimal & ASCII character \\
\hline $0 \times 1 c$ & 28 & fs (file separator) \\
\hline 0x1d & 29 & gs (group separator) \\
\hline 0x1e & 30 & rs (record separator) \\
\hline 0x1f & 31 & us (unit separator) \\
\hline $0 \times 20$ & 32 & sp (space) \\
\hline $0 \times 21$ & 33 & $!$ \\
\hline $0 \times 22$ & 34 & $"$ \\
\hline $0 \times 23$ & 35 & $\#$ \\
\hline $0 \times 24$ & 36 & $\$$ \\
\hline $0 \times 25$ & 37 & $\%$ \\
\hline $0 \times 26$ & 38 & $\&$ \\
\hline $0 \times 27$ & 39 & ' \\
\hline $0 \times 28$ & 40 & ( \\
\hline $0 \times 29$ & 41 & ) \\
\hline $0 \times 2 a$ & 42 & * \\
\hline $0 \times 2 b$ & 43 & + \\
\hline $0 \times 2 c$ & 44 & , \\
\hline $0 \times 2 d$ & 45 & - \\
\hline $0 \times 2 e$ & 46 & . \\
\hline $0 \times 2 f$ & 47 & l \\
\hline $0 \times 30$ & 48 & 0 \\
\hline $0 \times 31$ & 49 & 1 \\
\hline $0 \times 32$ & 50 & 2 \\
\hline $0 \times 33$ & 51 & 3 \\
\hline $0 \times 34$ & 52 & 4 \\
\hline $0 \times 35$ & 53 & 5 \\
\hline $0 \times 36$ & 54 & 6 \\
\hline $0 \times 37$ & 55 & 7 \\
\hline $0 \times 38$ & 56 & 8 \\
\hline $0 \times 39$ & 57 & 9 \\
\hline $0 \times 3 a$ & 58 & : \\
\hline $0 \times 3 b$ & 59 & ; \\
\hline $0 \times 3 c$ & 60 & $<$ \\
\hline $0 \times 3 d$ & 61 & $=$ \\
\hline $0 \times 3 e$ & 62 & $>$ \\
\hline
\end{tabular}




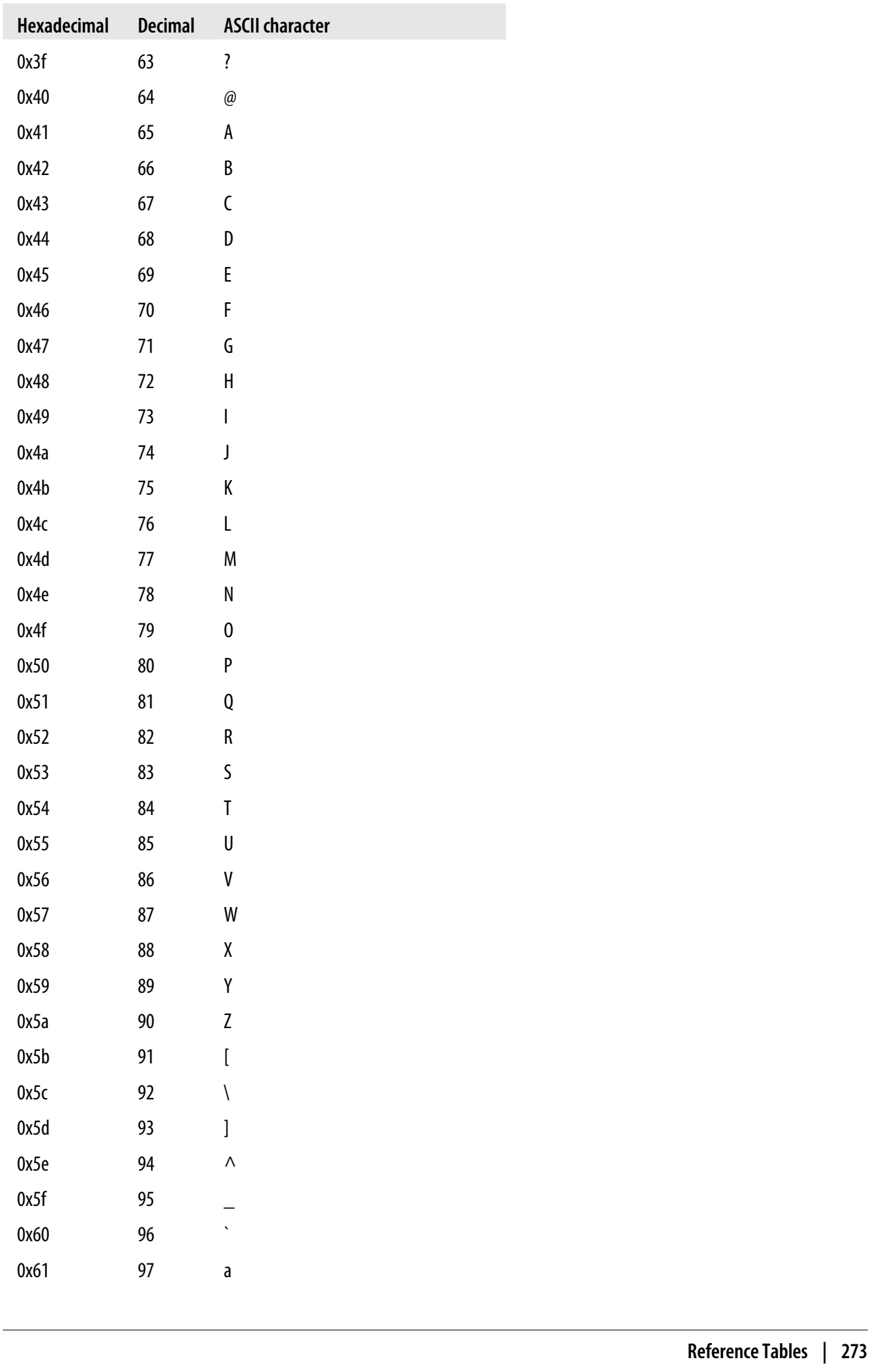




\begin{tabular}{|c|c|c|}
\hline Hexadecimal & Decimal & ASCII character \\
\hline $0 \times 62$ & 98 & $b$ \\
\hline $0 \times 63$ & 99 & $c$ \\
\hline $0 \times 64$ & 100 & $d$ \\
\hline $0 \times 65$ & 101 & e \\
\hline $0 \times 66$ & 102 & $f$ \\
\hline $0 \times 67$ & 103 & $g$ \\
\hline $0 \times 68$ & 104 & $\mathrm{~h}$ \\
\hline $0 \times 69$ & 105 & $\mathrm{i}$ \\
\hline $0 \times 6 a$ & 106 & j \\
\hline $0 \times 6 b$ & 107 & k \\
\hline $0 \times 6 c$ & 108 & I \\
\hline $0 \times 6 d$ & 109 & $\mathrm{~m}$ \\
\hline $0 \times 6 e$ & 110 & $\mathrm{n}$ \\
\hline $0 \times 6 f$ & 111 & 0 \\
\hline $0 \times 70$ & 112 & $p$ \\
\hline $0 \times 71$ & 113 & $q$ \\
\hline $0 \times 72$ & 114 & $\mathrm{r}$ \\
\hline $0 \times 73$ & 115 & s \\
\hline $0 \times 74$ & 116 & $\mathrm{t}$ \\
\hline $0 \times 75$ & 117 & u \\
\hline $0 \times 76$ & 118 & v \\
\hline 0x77 & 119 & w \\
\hline $0 \times 78$ & 120 & $x$ \\
\hline $0 \times 79$ & 121 & $y$ \\
\hline $0 \times 7 a$ & 122 & $\mathrm{z}$ \\
\hline $0 \times 7 b$ & 123 & \{ \\
\hline $0 \times 7 c$ & 124 & | \\
\hline $0 \times 7 d$ & 125 & \} \\
\hline $0 \times 7 e$ & 126 & $\sim$ \\
\hline $0 \times 7 f$ & 127 & del (delete) \\
\hline
\end{tabular}




\section{XBee Command Reference}

The tables in this section describe all the commands available to you on the XBee ZB firmware as of version $2 \times 70$. They are:

\section{Table A-7}

These addressing commands allow you to specify and retrieve destinations, endpoints, parent addresses, and more. Several are discussed in Chapters 2 and 7.

Table A-8

Networking commands allow you to work with settings such as network (PAN) IDs, router configuration, and channel configuration. Chapter 2 discusses a few of these commands.

Table A-9

You can use the security settings to configure various encryption options. Security is covered in Chapter 8.

Table A-10

These RF interfacing commands let you configure power settings and retrieve the RSSI (received signal strength indication) for the last packet received.

Table A-11

Serial interfacing commands allow you to work with serial settings, switch into API mode, and more. See Chapters 7 and 8.

Table A-12

You can use the I/O commands to work with features such as PWM, digital I/O, and analog input. I/O is the focus of Chapter 4.

Table A-13

These diagnostic commands let you consult the firmware and hardware versions, and also to check whether the module has associated with a network. Association indication is discussed in Chapter 7.

Table A-14

The AT command settings let you configure how the module handles AT commands that you send it. Chapter 2 discusses several of these.

\section{Table A-15}

Use these commands to configure the module's sleep mode, as covered in Chapter 6.

Table A-16

The execution commands let you restore defaults, write the current settings to nonvolatile memory, and more. Execution commands are noted throughout the book.

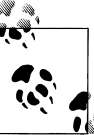

For each command, the "Node type" column indicates which node types support the command: $\mathrm{C}=$ Coordinator, $\mathrm{R}=$ Router, $\mathrm{E}=\mathrm{End}$ Device. 
Table A-7. Addressing commands

\begin{tabular}{|c|c|}
\hline $\begin{array}{l}\text { AT } \\
\text { command }\end{array}$ & Name and description \\
\hline DH & $\begin{array}{l}\text { Destination Address High. Sets/gets the upper } 32 \\
\text { bits of the 64-bit destination address. When com- } \\
\text { bined with DL, it defines the 64-bit destination ad- } \\
\text { dress for data transmission. Special definitions for } \\
\text { DHandDL include } 0 \times 000000000000 \text { FFFF (broadcast) } \\
\text { and 0x0000000000000000 (coordinator). }\end{array}$ \\
\hline $\mathrm{DL}$ & $\begin{array}{l}\text { Destination Address Low. Sets/gets the lower } 32 \\
\text { bits of the 64-bit destination address. When com- } \\
\text { bined with DH, it defines the 64-bit destination ad- } \\
\text { dress for data transmissions. Special definitions for } \\
\text { DHandDL include } 0 \times 000000000000 F F F F \text { (broadcast) } \\
\text { and 0x0000000000000000 (coordinator). }\end{array}$ \\
\hline
\end{tabular}

MY 16-bit Network Address. Reads the 16-bitnetwork address of the module. A value of $0 x F F F E$ means the module has not joined a ZigBee network.

16-bit Parent Network Address. Reads the 16-bit network address of the module's parent. A value of OxFFFE means the module does not have a parent.

NC Number of Remaining Children. Reads the number of end device children that can join the device. If $\mathrm{NC}$ returns 0 , then the device cannot allow any more end device children to join.

Serial Number High. Reads the high 32 bits of the module's unique 64-bit address.

$\mathrm{SL}$

Serial Number Low. Reads the low 32 bits of the module's unique 64-bit address.

NI Node Identifier. Stores a string identifier. The register only accepts printable ASCII data. In AT command mode, a string cannot start with a space. A carriage return ends the command. The command will automatically end when maximum bytes for the string have been entered. This string is returned as part of the ND (Node Discover) command. This identifier is also used with the DN (Destination Node) command. In AT command mode, an ASCll comma ( $0 \times 2 \mathrm{C})$ cannot be used in the NI string.

SE

\footnotetext{
SourceEndpoint. Sets/reads theZigBeeapplication layer source endpoint value. This value will be used as the source endpoint for all data transmissions. SE is onlysupported in ATfirmware. The defaultvalue (0xE8) is the Digi data endpoint.
}

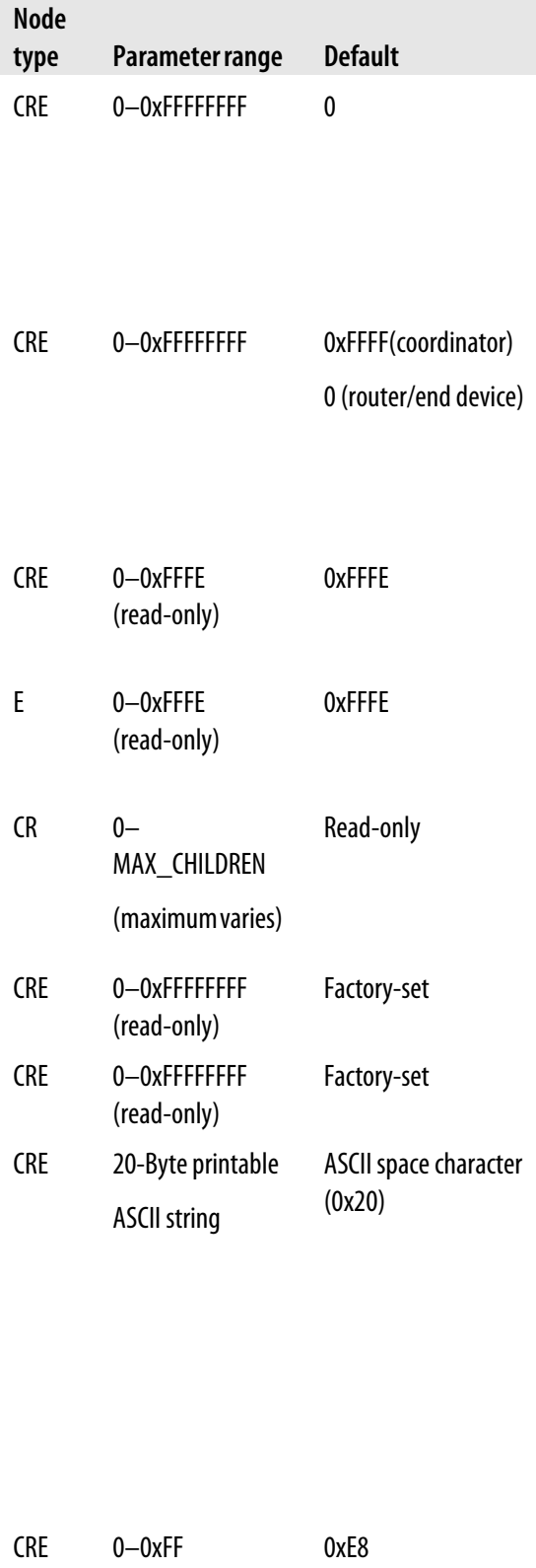




\begin{tabular}{|c|c|c|c|c|}
\hline $\begin{array}{l}\text { AT } \\
\text { command }\end{array}$ & Name and description & $\begin{array}{l}\text { Node } \\
\text { type }\end{array}$ & Parameter range & Default \\
\hline $\mathrm{DE}$ & $\begin{array}{l}\text { Destination Endpoint. Sets/reads ZigBee applica- } \\
\text { tion layer destination IDvalue. This valuewill beused } \\
\text { asthedestination endpointforalldatatransmissions. } \\
\text { DE isonlysupportedinATfirmware. The defaultvalue } \\
\text { (0xE8) is the Digi data endpoint. }\end{array}$ & CRE & $0-0 x F F$ & $0 x E 8$ \\
\hline $\mathrm{CI}$ & $\begin{array}{l}\text { Cluster Identifier. Sets/reads ZigBee application } \\
\text { layer cluster ID value. This value will be used as the } \\
\text { cluster ID for all data transmissions. CI is only sup- } \\
\text { ported in AT firmware. The default value (0x11) is the } \\
\text { transparent data cluster ID. }\end{array}$ & CRE & $0-0 x F F F F$ & $0 \times 11$ \\
\hline \multirow[t]{2}{*}{ NP } & $\begin{array}{l}\text { Maximum RF Payload Bytes. This value returns } \\
\text { the maximum number of RF payload bytes that can } \\
\text { be sent in a unicast transmission. If APS encryption } \\
\text { is used (API transmit option bit enabled), the maxi- } \\
\text { mum payload size is reduced by } 9 \text { bytes. If source } \\
\text { routing is used (AR < 0xFF), the maximum payload } \\
\text { size is reduced further. }\end{array}$ & CRE & $0-0 x F F F F$ & (read-only) \\
\hline & $\begin{array}{l}\text { Note: NP returns a hexadecimal value (e.g., if NP } \\
\text { returns } 0 \times 54 \text {, this is equivalent to } 84 \text { bytes). }\end{array}$ & & & \\
\hline \multirow[t]{15}{*}{$\mathrm{DD}$} & $\begin{array}{l}\text { Device Type Identifier. Stores a device type value. } \\
\text { This value can be used to differentiate different XBee- } \\
\text { based devices. Digi reserves the range 0-0xFFFFFF. }\end{array}$ & CRE & 0-0xFFFFFFFF & $0 \times 30000$ \\
\hline & $\begin{array}{l}\text { For example, Digi currently uses the following DD } \\
\text { values to identify various ZigBee products: }\end{array}$ & & & \\
\hline & 0x30001 - ConnectPort X8 Gateway & & & \\
\hline & 0x30002 - ConnectPort X4 Gateway & & & \\
\hline & 0x30003 - ConnectPort X2 Gateway & & & \\
\hline & 0x30005 - RS-232 Adapter & & & \\
\hline & 0x30006 - RS-485 Adapter & & & \\
\hline & 0x30007 - XBee Sensor Adapter & & & \\
\hline & 0x30008 - Wall Router & & & \\
\hline & 0x3000A - Digital I/0 Adapter & & & \\
\hline & 0x3000B - Analog I/0 Adapter & & & \\
\hline & 0x3000C - XStick & & & \\
\hline & 0x3000F - Smart Plug & & & \\
\hline & 0x30011 - XBee Large Display & & & \\
\hline & 0x30012 - XBee Small Display & & & \\
\hline
\end{tabular}


Table A-8. Networking commands

$\begin{array}{ll}\text { AT } & \\ \text { command } & \text { Name and description } \\ \mathrm{CH} & \text { Operating Channel. Reads the channel number used for } \\ & \text { transmitting and receiving between RF modules. Uses } \\ & \text { 802.15.4 channel numbers. A value of 0 means the device has } \\ \text { not joined a PAN and is not operating on any channel. }\end{array}$

not joined a PAN and is not operating on any channel.

$\begin{array}{lll}\begin{array}{lll}\text { Node } \\ \text { type }\end{array} & \text { Parameter range } & \text { Default } \\ \text { CRE } & \text { XBee } & \text { (Read- } \\ & \text { 0,0x0B-0x1A } & \text { only) } \\ & \text { (Channels 11-26) } & \end{array}$

XBee-PRO (S2)

$0,0 \times 0 \mathrm{~B}-0 \times 18$

(Channels 11-24)

XBee-PRO (S2B)

$0,0 \times 0 B-0 \times 19$

(Channels 11-25)

CRE

$0-$

OxFFFFFFFFFFFFFFFF

set to 0 , the coordinator will select a random extended PAN

ID, and the router / end device will join any extended PAN ID. Changes to ID should be written to nonvolatile memory using the WR command to preserve the ID setting if a power cycle occurs.

Operating Extended PAN ID. Reads the 64-bit extended PAN ID. The OP value reflects the operating extended PAN ID that the module is running on. If ID $>0, O P$ will equal ID.

$\mathrm{NH}$ Maximum Unicast Hops. Sets/reads the maximum hops limit. This limit sets the maximum broadcast hops value (BH) and determines the unicast timeout. The timeout is computed as $(50 * \mathrm{NH})+100 \mathrm{~ms}$. The default unicast timeout of 1.6 seconds ( $\mathrm{NH}=0 \times 1 \mathrm{E})$ is enough time for data and the acknowledgment to traverse about 8 hops.

BH Broadcast Hops. Sets/reads the maximum number of hops for each broadcast data transmission. Setting this to 0 will use the maximum number of hops.

OI

Operating 16-bit PAN ID. Reads the 16-bit PAN ID. The OI value reflects the actual 16-bit PAN ID the module is running on.

NT Node Discovery Timeout. Sets/reads the node discovery timeout. When the network discovery (ND) command is issued, the NT value is included in the transmission to provide all remote devices with a response timeout. Remote devices wait a random time, less than NT, before sending their response.

NO forthenetworkdiscoverycommand Theoptionsbitfieldvalue can change the behavior of the ND (network discovery)

$\begin{array}{lll}\text { CRE } & \begin{array}{l}\text { 0x01- } \\ \text { 0xFFFFFFFFFFFFFFF }\end{array} & \begin{array}{l}\text { (Read- } \\ \text { only) }\end{array} \\ \text { CRE } & 0-0 x F F & 0 x 1 E \\ \text { CRE } & 0-0 x 1 E & \\ & & 0 \\ \text { CRE } & 0-0 x F F F F & \begin{array}{l}\text { (Read- } \\ \text { only) }\end{array}\end{array}$
CRE $\quad 0 \times 20-0 \times \mathrm{FF} \quad 0 \times 3 \mathrm{C}$
$(\times 100 \mathrm{msec})$

CRE $\quad 0-0 \times 03$ (bit field) $\quad 0$ 
AT

command

Name and description

command and/or change what optional values are returned in any received ND responses or API nodeidentification frames. Options include:

$0 \times 01=$ Appends DD value (to ND responses or API node identification frames)

$002=$ Local device sends ND response frame when ND is issued

SC

Scan Channels. Sets/reads the list of channels to scan.

Coordinator - Bit field list of channels to choose from prior to starting network.

Router/End Device - Bit field list of channels that will be scanned to find a coordinator/router to join.

Changes to SC should be written using the WR command to preserve the SC setting if a power cycle occurs.

Bit (Channel):

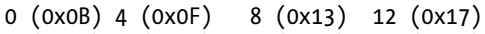

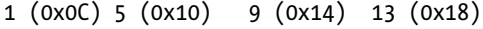

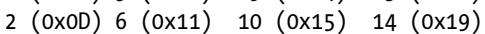

$$
\begin{aligned}
& 3 \text { (OXOE) } 7 \text { (0x12) } 11(0 \times 16) \quad 15(0 \times 1 \mathrm{~A})
\end{aligned}
$$

\section{Node}

type

Parameter range

Default

CRE

XBee

1FFE

1-0xFFFF (bit field)

XBee-PRO (S2)

1-0x3FFF (bit field)

(bits 14,15 not

allowed)

XBee-PRO (S2B)

1-0x7FFF

(bit 15 is not allowed)

CRE $\quad 0-7$ (exponent) Changes to SD should be written using the WR command.

Coordinator - Duration of the Active and Energy Scans (on each channel) that are used to determine an acceptable channel and Pan ID for the coordinator to start up on.

Router/End Device - Duration of Active Scan (on each channel) used to locate an available coordinator/router to join during association.

Scan Time is measured as: (\# Channels to Scan) ${ }^{*}(2 \wedge$ SD) * $15.36 \mathrm{~ms}$ - the number of channels to scan is determined by the SC parameter. The XBee can scan up to 16 channels (SC $=0 \mathrm{xFFFF}$ ).

Sample Scan Duration times (13 channel scan):

$$
\begin{aligned}
& \text { If } \mathrm{SD}=0, \text { time }=0.200 \mathrm{sec} \\
& \text { If } \mathrm{SD}=2, \text { time }=0.799 \mathrm{sec} \\
& \text { If } \mathrm{SD}=4, \text { time }=3.190 \mathrm{sec} \\
& \text { If } \mathrm{SD}=6, \text { time }=12.780 \mathrm{sec}
\end{aligned}
$$

SD influences the time the MAC listens for beacons or runs an energy scan on a given channel. The SD time is not a good estimate of the router/end device joining-time requirements. ZigBee joining adds additional overhead including beacon processing on each channel, sending a join request, etc., that extend the actual joining time. 
AT

command Name and description

ZS

NJ

JV

NW

JN

AR join the same network. association.
ZigBee Stack Profile. Sets/reads the ZigBee stack profile value. This must be set the same on all devices that should

Node Join Time. Sets/reads the time that a coordinator/ router allows nodes to join. This value can be changed at runtime without requiring a coordinator or router to restart. The time starts once the coordinator or router has started. The timer is reset on power-cycle or when $\mathrm{NJ}$ changes.

For an end device to enable rejoining, $N J$ should be set less than 0xFF on the device that will join. If $\mathrm{NJ}<0 \mathrm{xFF}$, the device assumes the network is not allowing joining and first tries to join a network using rejoining. If multiple rejoining attempts fail, or if $\mathrm{NJ}=0 \mathrm{xFF}$, the device will attempt to join using

Channel Verification. Sets/reads the channel verification parameter. If $\mathrm{JV}=1$, a router will verify the coordinator is on its operating channel when joining or coming up from a power cycle. If a coordinator is not detected, the router will leave its current channel and attempt to join a new PAN. If $\mathrm{JV}=0$, the router will continue operating on its current channel even if a coordinator is not detected.

Network Watchdog Timeout. Sets/reads the network watchdog timeout value. If $\mathrm{NW}$ is set $>0$, the router will monitor communication from the coordinator (or data collector) and leave the network if it cannot communicate with the coordinator for three NW periods. The timer is reset each time data is received from or sent to a coordinator, or if a many-toone broadcast is received.

Join Notification. Sets/reads the join notification setting. If enabled, the module will transmit a broadcast node identification packeton power-upand whenjoining. Thisaction blinks the Association LED rapidly on all devices that receive the transmission, and sends an API frame out the UART of API devices. This feature should be disabled for large networks to prevent excessive broadcasts.

Aggregate Routing Notification. Sets/reads the time between consecutive aggregate route broadcast messages. If used, AR should be set on only one device to enable many-toone routing to the device. Setting AR to 0 sends only one broadcast.

$\begin{array}{lll}\begin{array}{l}\text { Node } \\ \text { type } \\ \text { CRE }\end{array} & \text { Parameter range } & \text { Default } \\ & 0-2 & 0 \\ \text { CR } & 0-0 x F F & \text { 0xFF } \\ & (\times 1 \text { sec) } & \begin{array}{l}\text { (always } \\ \text { allows } \\ \text { joining) }\end{array}\end{array}$

$\mathrm{R}$

0 - Channel verifica- $\quad 0$ tion disabled

1 - Channel verification enabled

0-0x64FF

0

( $\times 1$ minute)

(disabled)

(up to over 17 days)

RE

$0-1$

0

CR $\quad 0-0 x F F$

OxFF 
Table A-9. Security commands

\begin{tabular}{|c|c|c|c|c|}
\hline $\begin{array}{l}\text { AT } \\
\text { command }\end{array}$ & Name and description & $\begin{array}{l}\text { Node } \\
\text { type }\end{array}$ & $\begin{array}{l}\text { Parameter } \\
\text { range }\end{array}$ & Default \\
\hline \multirow[t]{2}{*}{$\mathrm{EE}$} & Encryption Enable. Sets/reads the encryption enable setting. & CRE & $\begin{array}{l}0 \text { - Encryption } \\
\text { disabled }\end{array}$ & 0 \\
\hline & & & $\begin{array}{l}1 \text { - Encryption } \\
\text { enabled }\end{array}$ & \\
\hline \multirow[t]{3}{*}{ EO } & $\begin{array}{l}\text { Encryption Options. Configures options for encryption. Unused } \\
\text { option bits should be set to } 0 . \text { Options include: }\end{array}$ & CRE & $0-0 x F F$ & -- \\
\hline & $0 \times 01$ - Send the security key unsecured over the air during joins & & & \\
\hline & $0 \times 02$ - Use trust center (coordinator only) & & & \\
\hline NK & $\begin{array}{l}\text { Network Encryption Key. Sets the } 128 \text {-bit AES network encryp- } \\
\text { tion key. This command is write-only; NK cannot be read. If set to } \\
0 \text { (default), the module will select a random network key. }\end{array}$ & C & 128-bit value & 0 \\
\hline KY & $\begin{array}{l}\text { Link Key. Sets the } 128 \text {-bit AES link key. This command is write- } \\
\text { only; } K Y \text { cannot be read. Setting KY to } 0 \text { will cause the coordinator } \\
\text { to transmit the network key in the clear to joining devices, and will } \\
\text { cause joining devices to acquire the network key in the clear when } \\
\text { joining. }\end{array}$ & CRE & 128-bit value & 0 \\
\hline
\end{tabular}

Table A-10. RF interfacing commands

\begin{tabular}{lllll}
$\begin{array}{l}\text { AT } \\
\text { command }\end{array}$ & Name and description & $\begin{array}{l}\text { Node } \\
\text { type }\end{array}$ & Parameter range & Default \\
\hline PL & $\begin{array}{l}\text { Power Level. Selects/reads the power level at } \\
\text { which the RF module transmits conducted power. }\end{array}$ & CRE & XBee & 4 \\
& $\begin{array}{l}\text { For XBee-PRO (S2B) Power Level } 4 \text { is calibrated and } \\
\text { the other power levels are approximate. }\end{array}$ & (boost mode disabled) & \\
& & $0=-8 \mathrm{dBm}$ \\
& $1=-4 \mathrm{dBm}$ \\
& $2=-2 \mathrm{dBm}$ \\
& $3=0 \mathrm{dBm}$ \\
& $4=+2 \mathrm{dBm}$
\end{tabular}

XBee-PRO (S2)

$4=17 \mathrm{dBm}$

XBee-PRO (S2)

(International Variant)

$4=10 \mathrm{dBm}$

XBee-PRO (S2B)

(Boost mode enabled)

$4=18 \mathrm{dBm}$ 


\begin{tabular}{|c|c|c|c|c|}
\hline $\begin{array}{l}\text { AT } \\
\text { command }\end{array}$ & Name and description & $\begin{array}{l}\text { Node } \\
\text { type }\end{array}$ & Parameter range & Default \\
\hline & & & $3=16 \mathrm{dBm}$ & \\
\hline & & & $2=14 \mathrm{dBm}$ & \\
\hline & & & $1=12 \mathrm{dBm}$ & \\
\hline & & & $0=10 \mathrm{dBm}$ & \\
\hline & & & XBee-PRO (S2B) & \\
\hline & & & (International Variant) & \\
\hline & & & (Boost mode enabled) & \\
\hline & & & $4=10 \mathrm{dBm}$ & \\
\hline & & & $3=8 \mathrm{dBm}$ & \\
\hline & & & $2=6 \mathrm{dBm}$ & \\
\hline & & & $1=4 \mathrm{dBm}$ & \\
\hline & & & $0=2 \mathrm{dBm}$ & \\
\hline PM & Power Mode. Sets/reads the power mode of the & CRE & $0-1$ & 1 \\
\hline & $\begin{array}{l}\text { device. Enabling boost mode will improve the re- } \\
\text { ceive sensitivity by } 1 \mathrm{~dB} \text { and increase the transmit } \\
\text { power by } 2 \mathrm{~dB} \text {. }\end{array}$ & & $\begin{array}{l}0=\text { Boost mode disabled, } 1= \\
\text { Boost mode enabled }\end{array}$ & \\
\hline & $\begin{array}{l}\text { Note: Enabling boost mode on the XBee-PRO (S2) } \\
\text { will not affect the output power. Boost mode im- } \\
\text { poses a slight increase in current draw. }\end{array}$ & & & \\
\hline DB & Received Signal Strength. This command reports & CRE & $0-0 x F F$ & \\
\hline & data packet. The DB command only indicates the & & Observed range for & \\
\hline & signal strength of the last hop. It does not provide & & XBee-PRO: & \\
\hline & $\begin{array}{l}\text { an accurate quality measurement for a multihop } \\
\text { link. DB can be set to } 0 \text { to clear it. The DB command }\end{array}$ & & $0 \times 1 A-0 \times 58$ & \\
\hline & value is measured in $-\mathrm{dBm}$. For example, if DB re- & & For XBee: & \\
\hline & $\begin{array}{l}\text { turns } 0 \times 50 \text {, then the } R S S l \text { of the last packet received } \\
\text { was }-80 \mathrm{dBm} \text {. As of } 2 \times 6 \times \text { firmware, theDB command } \\
\text { value is also updated when an APS acknowledgment } \\
\text { is received. }\end{array}$ & & $0 \times 1 A-0 \times 5 C$ & \\
\hline PP & $\begin{array}{l}\text { Peak Power. Reads the dBm output when maxi- } \\
\text { mum power is selected (PL4). }\end{array}$ & CRE & $0 \times 0-0 \times 12$ & $\begin{array}{l}\text { (Read- } \\
\text { only) }\end{array}$ \\
\hline
\end{tabular}


Table A-11. Serial interfacing commands

$\begin{array}{ll}\begin{array}{l}\text { AT } \\ \text { command }\end{array} & \text { Name and description } \\ \text { AP } & \text { API Enable. Enables API mode. } \\ & \text { The AP command is only supported when } \\ & \text { using API firmware:21xx (API coordinator), } \\ & \text { 23xx(API router), and29xx(APlenddevice). } \\ & \\ \text { A0 } & \begin{array}{l}\text { API Options. Configures options for API. } \\ \text { Current options select the type of receive } \\ \text { API frame to send out the UART for received } \\ \text { RF data packets. }\end{array}\end{array}$

BD

NB

SB

RO
Interface Data Rate. Sets/reads the serial interface data rate for communication between the module serial port and host.

Any value above $0 \times 07$ will be interpreted as an actual baud rate. When a value above $0 \times 07$ is sent, the closest interface data rate represented by the number is stored in the $\mathrm{BD}$ register.

Serial Parity. Sets/reads the serial parity setting on the module.

Stop Bits. Sets/reads the number of stop bits for the UART. (Two stop bits are not supported if mark parity is enabled.)

Packetization Timeout.Sets/readsnumber of character times of intercharacter silence required before packetization. Set $(\mathrm{RO}=0)$ totransmitcharactersas theyarrive

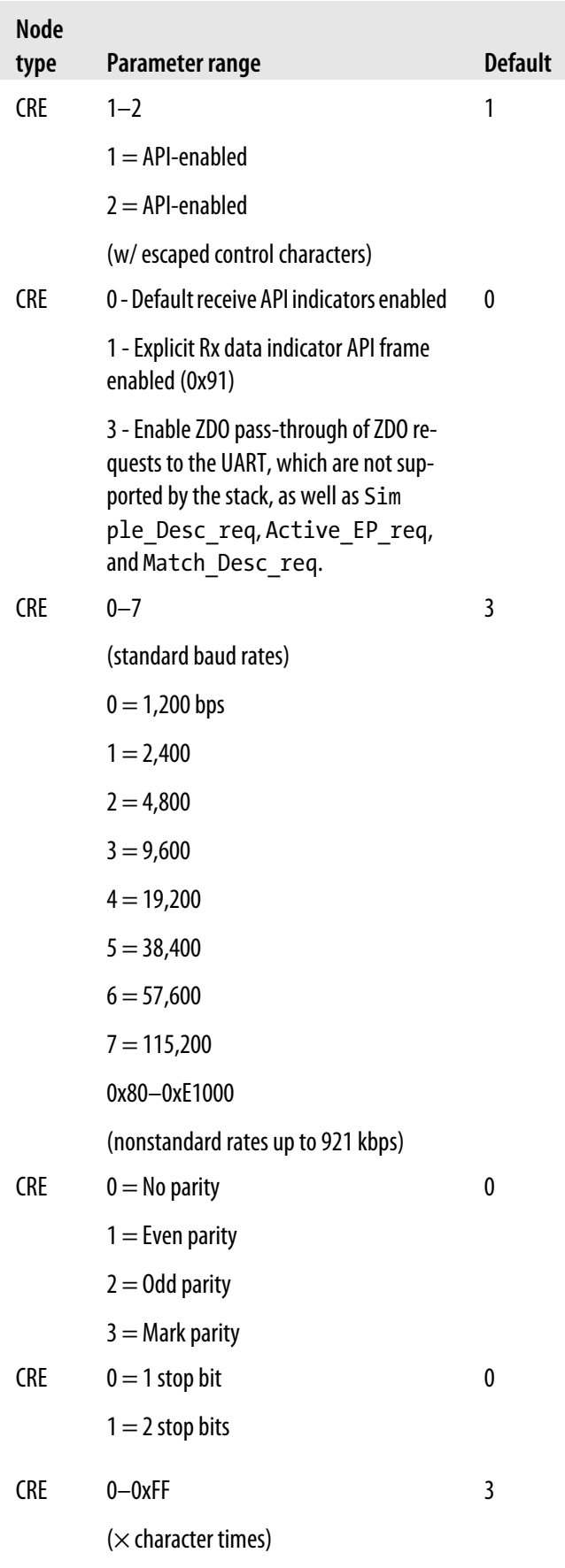




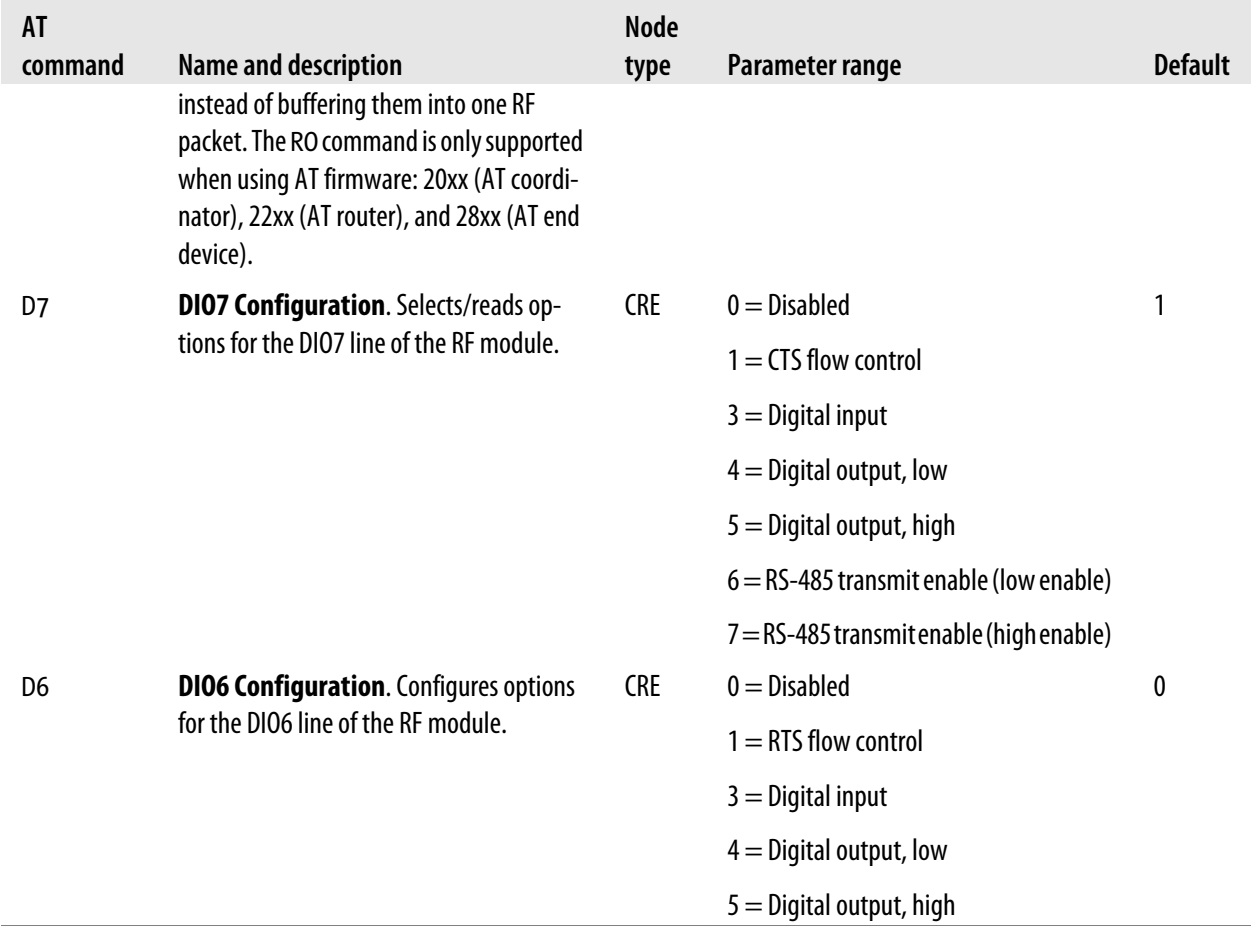

Table A-12. I/O commands

\begin{tabular}{|c|c|c|c|c|}
\hline $\begin{array}{l}\text { AT } \\
\text { command }\end{array}$ & Name and description & $\begin{array}{l}\text { Node } \\
\text { type }\end{array}$ & Parameter range & Default \\
\hline IR & $\begin{array}{l}\text { I0 Sample Rate. Sets/reads the I0 sample rate to } \\
\text { enable periodic sampling. For periodic sampling to } \\
\text { be enabled, IR must be set to a nonzero value, and } \\
\text { at least one module pin must have analog or digital } \\
\text { I0 functionality enabled (see DO-D8, PO-P2 } \\
\text { commands). The sample rate is measured in } \\
\text { milliseconds. }\end{array}$ & CRE & 0,0x32:0xFFFF (ms) & 0 \\
\hline \multirow[t]{3}{*}{ IC } & $\begin{array}{l}\text { I0 Digital Change Detection. Sets/reads the dig- } \\
\text { ital IO pins to monitor for changes in the I0 state. } \\
\text { IC works with the individual pin configuration } \\
\text { commands (DO-D8, PO-P2). If a pin is enabled as } \\
\text { a digital input/output, the IC command can beused } \\
\text { to forcean immediate IO sample transmission when } \\
\text { the DI0 state changes. IC is a bit mask that can be } \\
\text { used to enable or disable edge detection on indi- } \\
\text { vidual channels. Unused bits should be set to } 0 \text {. }\end{array}$ & CRE & $0-0 x F F F F$ & 0 \\
\hline & Bit (10 pin): & & & \\
\hline & 0 (DI00) 4 (DI04) 8 (DI08) & & & \\
\hline
\end{tabular}




\begin{tabular}{|c|c|c|c|c|}
\hline $\begin{array}{l}\text { AT } \\
\text { command }\end{array}$ & Name and description & $\begin{array}{l}\text { Node } \\
\text { type }\end{array}$ & Parameter range & Default \\
\hline & 1 (DI01) 5 (DI05) 9 (DI09) & & & \\
\hline & 2 (DI02) 6 (DI06) 10 (DI010) & & & \\
\hline & 3 (DI03) 7 (DI07) 11 (DI011) & & & \\
\hline Po & PWMO Configuration. Selects/reads function for & CRE & 0 - Disabled & 1 \\
\hline & PWM0. & & 1 - RSSI PWM & \\
\hline & & & 3- Digital input, monitored & \\
\hline & & & 4 - Digital output, default low & \\
\hline & & & 5 - Digital output, default high & \\
\hline P1 & DI011 Configuration. Configures options for the & CRE & 0 - Unmonitored digital input & 0 \\
\hline & & & 3- Digital input, monitored & \\
\hline & & & 4 - Digital output, default low & \\
\hline & & & 5 - Digital output, default high & \\
\hline P2 & DI012 Configuration. Configures options for the & CRE & 0 - Unmonitored digital input & 0 \\
\hline & DI012 line of the RF module. & & 3 - Digital input, monitored & \\
\hline & & & 4 - Digital output, default low & \\
\hline & & & 5 - Digital output, default high & \\
\hline P3 & DI013 Configuration. Sets/reads function for & CRE & $0,3-5$ & -- \\
\hline & DI013. This command is not yet supported. & & 0 - Disabled & \\
\hline & & & 3 - Digital input & \\
\hline & & & 4 - Digital output, low & \\
\hline & & & 5 - Digital output, high & \\
\hline Do & $\begin{array}{l}\text { ADO/DIO0 Configuration. Selects/reads function } \\
\text { for ADO/DI00. }\end{array}$ & CRE & $\begin{array}{l}1 \text { - Commissioning button } \\
\text { enabled }\end{array}$ & 1 \\
\hline & & & 2 - Analog input, single-ended & \\
\hline & & & 3 - Digital input & \\
\hline & & & 4 - Digital output, low & \\
\hline & & & 5 - Digital output, high & \\
\hline D1 & AD1/DI01 Configuration. Selects/reads function & CRE & $0,2-5$ & 0 \\
\hline & for AD1/DI01. & & 0 - Disabled & \\
\hline & & & 2-Analog input, single-ended & \\
\hline & & & 3 - Digital input & \\
\hline & & & 4 - Digital output, low & \\
\hline & & & 5 - Digital output, high & \\
\hline
\end{tabular}




\begin{tabular}{|c|c|c|c|c|}
\hline $\begin{array}{l}\text { AT } \\
\text { command }\end{array}$ & Name and description & $\begin{array}{l}\text { Node } \\
\text { type }\end{array}$ & Parameter range & Default \\
\hline $\mathrm{D} 2$ & $\begin{array}{l}\text { AD2/DI02 Configuration. Selects/reads function } \\
\text { for AD2/DI02. }\end{array}$ & CRE & $\begin{array}{l}0,2-5 \\
0 \text { - Disabled } \\
2 \text { - Analog input, single-ended } \\
3 \text { - Digital input } \\
4 \text { - Digital output, low } \\
5 \text { - Digital output, high }\end{array}$ & 0 \\
\hline D3 & $\begin{array}{l}\text { AD3/DI03 Configuration. Selects/reads function } \\
\text { for AD3/DI03. }\end{array}$ & CRE & $\begin{array}{l}0,2-5 \\
0 \text { - Disabled } \\
2 \text { - Analog input, single-ended } \\
3 \text { - Digital input } \\
4 \text { - Digital output, low } \\
5 \text { - Digital output, high }\end{array}$ & 0 \\
\hline D4 & $\begin{array}{l}\text { DI04 Configuration. Selects/reads function for } \\
\text { DI04. }\end{array}$ & CRE & $\begin{array}{l}0,3-5 \\
0 \text { - Disabled } \\
3 \text { - Digital input } \\
4 \text { - Digital output, low } \\
5 \text { - Digital output, high }\end{array}$ & 0 \\
\hline D5 & $\begin{array}{l}\text { DI05 Configuration. Configures options for the } \\
\text { DI05 line of the RF module. }\end{array}$ & CRE & $\begin{array}{l}0 \text { - Disabled } \\
1 \text { - Associated } \\
\text { indication LED } \\
3 \text { - Digital input } \\
4 \text { - Digital output, default low } \\
5 \text { - Digital output, default high }\end{array}$ & 1 \\
\hline D8 & $\begin{array}{l}\text { DI08Configuration. Sets/reads function for DI08. } \\
\text { This command is not yet supported. }\end{array}$ & CRE & $\begin{array}{l}0,3-5 \\
0 \text { - Disabled } \\
3 \text { - Digital input } \\
4 \text { - Digital output, low } \\
5 \text { - Digital output, high }\end{array}$ & \\
\hline LT & $\begin{array}{l}\text { Assoc LED Blink Time. Sets/reads the Association } \\
\text { LED blink time. If the Association LED functionality } \\
\text { is enabled ( } D 5 \text { command), this value determines } \\
\text { the on and off blink times for the LED when the } \\
\text { module has joined a network. If } L T=0 \text {, the default } \\
\text { blink rate will be used ( } 500 \mathrm{~ms} \text { for coordinator, } 250\end{array}$ & CRE & $0,0 \times 0 \mathrm{~A}-0 \times \mathrm{xFF}(100-2,550 \mathrm{~ms})$ & 0 \\
\hline
\end{tabular}


AT

command

Name and description

ms for router/end device). For all other LT values, $\mathrm{LT}$ is measured in $10 \mathrm{~ms}$.

PR

Pull-up Resistor. Sets/reads the bit field that configures the internal pull-up resistor status for the I/Olines. "1"specifiesthe pull-up resistorisenabled. "0" specifies no pull-up. (30k pull-up resistors.)

Bits:

0 - DI04 (Pin 11)

1 - AD3 / DI03 (Pin 17)

2 - AD2 / DI02 (Pin 18)

3 - AD1 / DI01 (Pin 19)

4 - ADO / DI00 (Pin 20)

5 - RTS / DI06 (Pin 16)

6 - DTR / Sleep Request / DI08 (Pin 9)

7 - DIN / Config (Pin 3)

8 - Associate / DI05 (Pin 15)

9- On/Sleep / DI09 (Pin 13)

10 - DI012 (Pin 4)

11 - PWMO / RSSI / DI010 (Pin 6)

12 - PWM1 / D1011 (Pin 7)

13 - CTS / DI07 (Pin 12)

RP

RSSI PWM Timer. Number of times the RSSI signal will be output on the PWM after the last RF data reception or APS acknowledgment. When $\mathrm{RP}=$ OxFF, output will always be on.

$\% \mathrm{~V}$

Supply Voltage. Reads the voltage on the Vccpin. Scale by $1200 / 1024$ to convert to $\mathrm{mV}$ units. For example, a \% reading of $0 \times 900$ (2,304 decimal) represents $2,700 \mathrm{mV}$ or $2.70 \mathrm{~V}$.

V+
Node

type

Parameter range

Default

CRE

0-0x3FFF

0

0x1FFF
CRE $\quad 0-0 x F F(\times 100 \mathrm{~ms}) \quad 0 \times 28$

(40d)

CRE - -0x-0xFFFF (read-only)

CRE $\quad 0-0 x F F F F$

0 threshold is set with the $\mathrm{V}+$ command. If the measured supply voltage falls below or equal to this threshold, the supply voltage will be included in the I0 sampleset. $V+$ issetto 0 by default(donotinclude the supply voltage). Scale $\mathrm{mV}$ units by $1,024 / 1,200$ to convert to internal units. For example, for a 2,700 $\mathrm{mV}$ threshold enter $0 \times 900$. 


\begin{tabular}{|c|c|c|c|c|}
\hline \multirow[t]{5}{*}{$\begin{array}{l}\text { AT } \\
\text { command }\end{array}$} & Name and description & $\begin{array}{l}\text { Node } \\
\text { type }\end{array}$ & Parameter range & Default \\
\hline & $\begin{array}{l}\text { Given the operating Vcc ranges for different plat- } \\
\text { forms, and scaling by } 1,024 / 1,200 \text {, the useful pa- } \\
\text { rameter ranges are: }\end{array}$ & & & \\
\hline & XBee $2,100-3,600 \mathrm{mV}, 0,0 \times 0700-0 \times 0<00$ & & & \\
\hline & PRO 3,000-3,400 mV, 0,0x0a00-0x0b55 & & & \\
\hline & $\mathrm{S} 2 \mathrm{~B} 2,700-3,600 \mathrm{mV}, 0,0 \times 0900-0 \times 0 \mathrm{C} 00$ & & & \\
\hline \multirow[t]{2}{*}{ TP } & $\begin{array}{l}\text { Reads the module temperature in degrees Celsius. } \\
\text { Accuracy }+/-7 \text { degrees. }\end{array}$ & CRE & $0 \times 0-0 \times F F F F$ & -- \\
\hline & $\begin{array}{l}1^{\circ} \mathrm{C}=0 \times 0001 \text { and }-1^{\circ} \mathrm{C}=0 \times \text { xFFF. Command is only } \\
\text { available in PRO } 22 \mathrm{~B} \text {. }\end{array}$ & & & \\
\hline
\end{tabular}

Table A-13. Diagnostics commands

\begin{tabular}{|c|c|c|c|c|}
\hline $\begin{array}{l}\text { AT } \\
\text { command }\end{array}$ & Name and description & $\begin{array}{l}\text { Node } \\
\text { type }\end{array}$ & $\begin{array}{l}\text { Parameter } \\
\text { range }\end{array}$ & Default \\
\hline VR & $\begin{array}{l}\text { Firmware Version. Reads firmware version of the module. } \\
\text { The firmware version returns } 4 \text { hexadecimal values ( } 2 \text { bytes) "ABCD". } \\
\text { Digits " } A B C \text { " are the main release number and " } D \text { " is the revision } \\
\text { number from the main release. " } B \text { " is a variant designator. } \\
\text { XBee and XBee-PRO ZB modules return: } \\
\text { OX2xxx versions. } \\
\text { XBee and XBee-PRO ZNet modules return: } \\
\text { Ox1xxx versions. ZNet firmware is not compatible with ZB firmware. }\end{array}$ & CRE & $\begin{array}{l}\text { 0-0xFFFF } \\
\text { (read-only) }\end{array}$ & $\begin{array}{l}\text { Factory- } \\
\text { set }\end{array}$ \\
\hline HV & $\begin{array}{l}\text { Hardware Version. Reads the hardware version of the module. This } \\
\text { command can be used to distinguish among different hardware } \\
\text { platforms. The upper byte returns a value that is unique to each } \\
\text { module type. The lower byte indicates the hardware revision. } \\
\text { XBee ZB and XBee ZNet modules return the following (hexadecimal) } \\
\text { values: } \\
\text { 0x19xx - XBee module } \\
\text { 0x1Axx - XBee-PRO module }\end{array}$ & CRE & $\begin{array}{l}\text { 0-0xFFFF } \\
\text { (read-only) }\end{array}$ & $\begin{array}{l}\text { Factory- } \\
\text { set }\end{array}$ \\
\hline AI & $\begin{array}{l}\text { Association Indication. Reads information regarding last node } \\
\text { join request: } \\
\text { 0x00 - Successfully formed or joined a network. (Coordinators form } \\
\text { a network, routers and end devices join a network.) } \\
\text { 0x21 - Scan found no PANs. } \\
\text { 0x22-Scanfound no validPANs based on currentSC and ID settings. }\end{array}$ & CRE & $\begin{array}{l}0-0 x F F \\
\text { (read-only) }\end{array}$ & -- \\
\hline
\end{tabular}




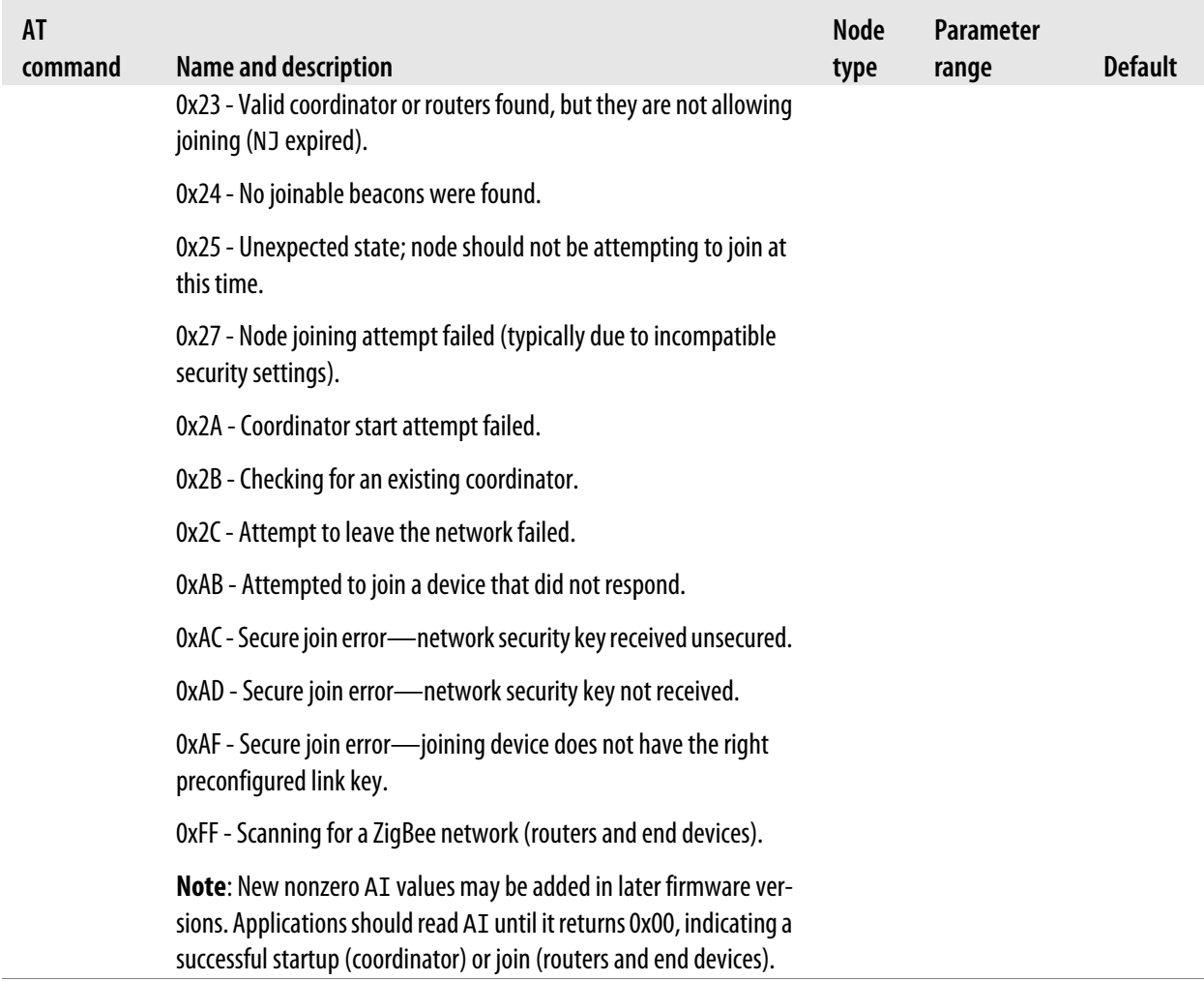

Table A-14. AT Command Options commands

\begin{tabular}{|c|c|c|c|c|}
\hline $\begin{array}{l}\text { AT } \\
\text { command }\end{array}$ & Name and description & $\begin{array}{l}\text { Node } \\
\text { type }\end{array}$ & $\begin{array}{l}\text { Parameter } \\
\text { range }\end{array}$ & Default \\
\hline CT & $\begin{array}{l}\text { Command Mode Timeout. Sets/reads the period of inactivity (no } \\
\text { valid commands received) after which the RF module automatically } \\
\text { exits AT command mode and returns to idle mode. }\end{array}$ & CRE & $\begin{array}{l}2-0 \times 028 \mathrm{~F} \\
(\times 100 \mathrm{~ms})\end{array}$ & $\begin{array}{l}0 \times 64 \\
(100 d)\end{array}$ \\
\hline $\mathrm{CN}$ & $\begin{array}{l}\text { Exit Command Mode. Explicitly exits the module from AT command } \\
\text { mode. }\end{array}$ & CRE & -- & -- \\
\hline GT & $\begin{array}{l}\text { Guard Times. Sets required period of silence before and after the } \\
\text { Command Sequence Characters of the AT Command Mode Sequence } \\
(G T+C C+G T) \text {. The period of silence is used to prevent inadvertent } \\
\text { entrance into AT command mode. }\end{array}$ & CRE & $\begin{array}{l}\text { 1-0x0CE4 } \\
\text { (×1 ms) } \\
\text { (max of } 3.3 \\
\text { decimal sec) }\end{array}$ & $\begin{array}{l}0 \times 3 E 8 \\
(1,000 d)\end{array}$ \\
\hline CC & $\begin{array}{l}\text { Command Sequence Character. Sets/reads the ASCII character } \\
\text { value to be used between Guard Times of the AT Command Mode } \\
\text { Sequence (GT }+C C+G T) \text {. The AT Command Mode Sequence enters } \\
\text { the RF module into AT command mode. }\end{array}$ & CRE & $0-0 x \mathrm{FF}$ & $\begin{array}{l}0 \times 2 B \\
\left({ }^{\prime}+' A S C I I\right)\end{array}$ \\
\hline & $\begin{array}{l}\text { The CC command is only supported when using AT firmware: } \\
20 x x \text { (AT coordinator), 22xx (AT router), and 28xx (AT end device). }\end{array}$ & & & \\
\hline
\end{tabular}


Table A-15. Sleep commands

$\begin{array}{ll}\begin{array}{l}\text { AT } \\ \text { command }\end{array} & \text { Name and description } \\ \text { SM } & \text { Sleep Mode. Sets the sleep mode on the RF module. An XBee } \\ & \text { loaded with router firmware can be configured as either a router } \\ & (\text { SM set to } 0) \text { or an end device }(S M>0) \text {. Changing a device from } \\ & \text { a router to an end device (or vice versa) forces the device to leave } \\ \text { the network and attempt to join as the new device type when } \\ \text { changes are applied. }\end{array}$

SN

Number of Sleep Periods. Sets the number of sleep periods to not assert the 0 n/Sleep pin on wake-up if no RF data is waiting for the end device. This command allows a host application to sleep for an extended time if no RF data is present.

SP Sleep Period. This value determines how long the end device will sleep at a time, up to 28 seconds. (The sleep time can effectively be extended past 28 seconds using the SN command.) On the parent, this value determines how long the parent will buffer a message for the sleeping end device. It should be set at least equal to the longest SP time of any child end device.

ST

Time Before Sleep. Sets the time-before-sleep timer on an end device. The timer is reset each time serial or RF data is received. Once the timer expires, an end device may enter low-power operation. Applicable for cyclic sleep end devices only.

SO

Sleep Options. Configures options for sleep. Unused option bits should be set to 0 . Sleep options include:

0x02 - Always wake for ST time

0x04 - Sleep entire SN * SP time

Sleep options should not be used for most applications. See Chapter 6 for more information.

WH

Wake Host. Sets/reads the wake host timer value. If the wake host timer is set to a nonzero value, this timer specifies a time (in E $\quad 0-0 x F F F F$ millisecond units) that the device should allow after waking from

$\begin{array}{lll}\begin{array}{l}\text { Node } \\ \text { type }\end{array} & \begin{array}{l}\text { Parameter } \\ \text { range }\end{array} & \text { Default } \\ \text { RE } & \begin{array}{l}0 \text {-Sleep disa- } \\ \text { bled (router) }\end{array} & 0 \text { - Router } \\ & \begin{array}{l}\text { - End } \\ \text { 1- Pin sleep } \\ \text { enabled }\end{array} & \text { device } \\ \end{array}$

4 - Cyclic sleep enabled

5 - Cyclic sleep, pin wake

CRE

1-0xFFFF

CRE $\quad 0 \times 20-0 \times A F 0 \quad 0 \times 20$

$(\times 10 \mathrm{~ms})$

(Quarter-second

resolution)

1-0xFFFE

0x1388 (5

( $\times 1 \mathrm{~ms}) \quad$ seconds)

sleep before sending data out the UART or transmitting an 10 sample. If serial characters are received, the WH timer is stopped immediately.

SI Sleep Immediately. See Table A-16.

PO Polling Rate. Sets/reads the end device poll rate. Setting this to 0 (default) enables polling at $100 \mathrm{~ms}$ (default rate). Adaptive 0-0x3E8 $\quad 0 \times 00(100$ polling may allow the end device to poll more rapidly for a short msec) time when receiving RF data. 
Table A-16. Execution commands

$\begin{array}{ll}\text { AT } \\ \text { command } & \text { Name and description } \\ \text { AC } & \text { Apply Changes. Applies changes to all command registers, causing } \\ & \text { queued command register values to be applied. For example, changing } \\ \text { the serial interface rate with the BD command will not change the UART } \\ \text { interface rate until changes are applied with the AC command. The CN } \\ \text { command and Ox08 API command frame also apply changes. }\end{array}$

WR Write. Writes parameter values to nonvolatile memory so that parameter modifications persist through subsequent resets.

Note: Once WR is issued, no additional characters should be sent to the module until after the "OKIr" response is received. The WR command should be used sparingly. The EM250 supports a limited number of write cycles.

RE Restore Defaults. Restores module parameters to factory defaults.

FR Software Reset. Resets module. Responds immediately with an OK status, and then performs a software reset about 2 seconds later.

NR Network Reset. Resets network layer parameters on one or more modules within a PAN. Responds immediately with an "OK," then causes a network restart. All network configuration and routing information is consequently lost.

If $\mathrm{NR}=0$ : Resets network layer parameters on the node issuing the command.

If $\mathrm{NR}=1$ : Sends broadcast transmission to reset network layer parameters on all nodes in the PAN.

SI Sleep Immediately. Causes a cyclic sleep module to sleep immediately rather than wait for the ST timer to expire.

CB Commissioning Pushbutton. This command can be used to simulate

Node Parameter
type range Default

CRE -- - -

CRE -- $\quad--$

CRE -- $\quad--$

CRE -- $\quad--$

CRE $\quad 0-1 \quad--$

CRE commissioning button presses in software. The parameter value should be set to the number of button presses to be simulated. For example, sending the ATCB1 command will execute the action associated with one commissioning button press.

ND Node Discover. Discovers and reports all RF modules found. The following information is reported for each module discovered:

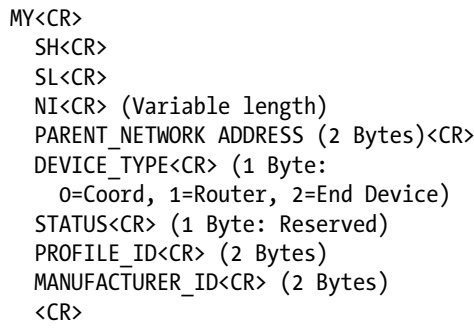

Optional 20- -byte NI or MY value 
AT

command

Name and description

After $\left(\mathrm{NT}^{*}{ }^{*} 100\right)$ milliseconds, the command ends by returning a $\langle\mathrm{CR}\rangle$. ND also accepts a Node Identifier (NI) as a parameter (optional). In this case, only a module that matches the supplied identifier will respond.

If ND is sent through the API, each response is returned as a separate AT_CMD_Response packet. The data consists of the above-listed bytes without the carriage return delimiters. The NI string will end in a " $0 \times 00$ " null character. The radius of the ND command is set by the $\mathrm{BH}$ command.

DN

Destination Node. Resolves an NI (Node Identifier) string to a physical address (case-sensitive). Thefollowing events occur after the destination node is discovered:

AT Firmware

1. DL and DH are set to the extended (64-bit) address of the module with the matching NI (Node Identifier) string.

2. OK (or ERROR) $\backslash r$ is returned.

3. Command mode is exited to allow immediate communication.

API Firmware

The 16-bit network and 64-bit extended addresses are returned in an API Command Response frame.

If there is no response from a module within (NT * 100) milliseconds or a parameter is not specified (left blank), the command is terminated and an "ERROR" message is returned.

In the case of an ERROR, command mode is not exited. The radius of the DN command is set by the BH command.

IS Force Sample. Forces a read ofall enabled digital and analoginput lines.

$1 \mathrm{~S}$

XBee Sensor Sample. Forces a sample to be taken on an XBee sensor
Node Parameter

type range

Default

CRE

Up to 20

byte

printable

ASCll string device. This command can only be issued to an XBee sensor device using an API remote command. 


\section{Index}

\section{Symbols}

16-bit addressing, 29, 126

$3 \mathrm{G}$ and $4 \mathrm{G}$ protocol, 191

64-bit addressing, 28, 126

128-bit Advanced Encryption Standard (AES), 242

A

accelerometer, 86

acoustic sensor, 86, 87

actuation, xiv

(see also direct actuation; remote actuation)

ad hoc network creation, 26

Ad hoc On-demand Distance Vector (AODV) mesh routing, 240

AD0...AD3 Analog Input pins, 15, 89

Adafruit electronics kits, 63

Adafruit XBee Adapter Kit, 8

adapter

Arduino board for, 12-15

breakout board, 10-11

buying, 6-15

Digi evaluation board, 7

drivers for, 8,33

ports for, 40

USB adapter, 7-9

addresses, network, 28-29

addressing commands, 276-277

AES (Advanced Encryption Standard), 242

Analog Input pins (AD0...AD3), 15, 89

animism, xii

antennas, 4-5

AODV (Ad hoc On-demand Distance Vector) mesh routing, 240
API (application programming interface), 111$112,116-119$

API frame, 117-119

AT Command frame, 120-122

AT Response frame, 122-124

checksum, 118

data bytes, 118

I/O Data Sample Rx Indicator frame, 131135

length bytes, 118

parsing, API code for, 138-140

parsing, libraries for, 141

Remote AT Command Request frame, 135137

Remote Command Response frame, 137138

start delimiter, 117

types of, 119-142

ZigBee Receive Packet frame, 129-131

ZigBee Transmit Request frame, 124-127

ZigBee Transmit Status frame, 127-129

API protocol, 116-119

application layer, 30

application profiles, 237-238

application programming interface (see API)

APS (Application Support Sublayer) layer, 236, 237-240

APS encryption, 243

APS link security, 243

AR command, 240

Arduino \& C/C++ library, 141

Arduino board, 57-65

adapter hack for, $12-15$

buying, 59

cable for, 59, 60

We'd like to hear your suggestions for improving our indexes. Send email to index@oreilly.com. 
connecting to, 65

electronics supplies for, 61-63

IDE software for, 60

programming language for, 60-61

resources for, 59, 61, 64-65, 261

troubleshooting, 266

Arduino FIO, 269

Arduino Uno, 59

Arduino XBee Shield, 269

ASCII codes, 271

ASSOC pin, 15

AT command, 48

AT Command frames, 120-122

AT commands, 47-49, 90-93, 275-292

addressing commands, 276-277

command option commands, 289

diagnostic commands, 288-289

execution commands, 291-292

I/O commands, 284-288

networking commands, 278-280

RF interfacing commands, 281-282

security commands, 281

serial interfacing commands, 283-284

sleep commands, 290

AT Response frame, 122-124

AT\%V (Supply Voltage), 93, 287

AT1S (XBee Sensor Sample), 292

ATAC (Apply Changes), 291

ATAI (Association Indication), 288

ATAO (API Options), 283

ATAP (API Enable), 283

ATAR (Aggregate Routing Notification), 280

ATBD (Interface Data Rate), 283

ATBH (Broadcast Hops), 278

ATCB (Commissioning Pushbutton), 291

ATCC (Command Sequence Character), 289

ATCH (Operating Channel), 278

ATCI (Cluster Identifier), 277

ATCN (Exit Command Mode), 48, 289

ATCT (Command Mode Timeout), 289

ATD0...ATD8 (Digital I/O Configuration), 91, 284, 285

ATDB (Received Signal Strength), 282

ATDD (Device Type Identifier), 277

ATDE (Destination Endpoint), 277

ATDH (Destination Address High), 48, 276

ATDL (Destination Address Low), 48, 276

ATDN (Destination Node), 203, 292

ATEE (Encryption Enable), 281
ATEO (Encryption Options), 281

ATFR (Software Reset), 291

ATGT (Guard Times), 289

ATHV (Hardware Version), 288

ATIC (I/O Digital Change Detection), 93, 284

ATID (Extended PAN ID), 48, 49, 278

ATIR (I/O Sample Rate), 91, 284

ATIS (Force Sample), 292

ATJN (Join Notification), 280

ATJV (Channel Verification), 280

ATKY (Link Key), 281

ATLT (Assoc LED Blink Time), 286

Atmel components, 267

ATMP (16-bit Parent Network Address), 276

ATMY (16-bit Network Address), 48, 276

ATNB (Serial Parity), 283

ATNC (Number of Remaining Children), 276

ATND (Node Discover), 202, 291

ATNH (Maximum Unicast Hops), 278

ATNI (Node Identifier), 202, 276

ATNJ (Node Join Time), 280

ATNK (Network Encryption Key), 281

ATNO (Network Discovery Options), 278

ATNP (Maximum RF Payload Bytes), 277

ATNR (Network Reset), 291

ATNT (Node Discovery Timeout), 278

ATNW (Network Watchdog Timeout), 280

ATOI (Operating 16-bit PAN ID), 278

ATOP (Operating Extended PAN ID), 278

ATP0...ATP3 (Digital I/O Configuration), 91, 285

ATPL (Power Level), 281

ATPM (Power Mode), 282

ATPO (Polling Rate), 290

ATPP (Peak Power), 282

ATPR (Pull-up Resistor), 93, 287

ATRE (Restore Defaults), 291

ATRO (Packetization Timeout), 283

ATRP (RSSI PWM Timer), 287

ATSB (Stop Bits), 283

ATSC (Scan Channels), 279

ATSD (Scan Duration), 279

ATSE (Source Endpoint), 276

ATSH (Serial Number High), 48, 276

ATSI (Sleep Immediately), 290, 291

ATSL (Serial Number Low), 48, 276

ATSM (Sleep Mode), 163-164, 166, 290

ATSN (Number of Sleep Periods), 165, 166, 290 
ATSO (Sleep Options), 166, 167, 290

ATSP (Sleep Period), 164, 166, 290

ATST (Time Before Sleep), 165, 166, 290

ATTP (Module Temperature), 288

ATV+ (Voltage Supply Monitoring), 287

ATVR (Firmware Version), 288

ATWH (Wake Host), 166, 167, 290

ATWR (Write), 48, 91, 291

ATZS (ZigBee Stack Profile), 280

\section{B}

battery life, determining, 163

Bluetooth protocol, 189, 190

board-level serial (see TTL serial protocol)

books, recommended, 264

breadboard, 9

breakout board, 10-11

bytes, 114,135

California Eastern Laboratories, 267

CAN (Controller-Area Networking) protocol, 190

CAN-bus protocol, 190

capacitance sensor, 86

cellular data connections, 194

channels, network, 29

chat session, 50-56

addresses for, 50

coordinator for, 51

with one computer, 54

parts for, 50

router for, 53

with two computers, 53

checksum, 116, 118

cluster tree network, 28

clusters, 238-239

code examples, permission to use, $\mathrm{xv}$

color sensor, 86

command mode, 43, 44, 46-47

COMMISioning pin, 15

communication settings, terminal program, 40

computer, as Internet gateway, 195

ConnectPort gateways, 195-202

configuring, 198-202

configuring on iDigi server, 209-210

connecting to iDigi server, 206-209 firmware updates using iDigi server, 210 212

remote management of, 203-214

setting up, 197-198

contact information for this book, xvi

contents ID, in stream, 115-116

context phenomena, 87

Controller-Area Networking (CAN) protocol, 190

conventions used in this book, $\mathrm{xv}$

CoolTerm program, 18, 43-46

coordinator device, 26

CTS pin, 15, 244

D

data

presentation on Internet, 193

reasons for collecting, 85

sharing, 245-257

storage on Internet, 193

data bytes, API frame, 118

dedicated gateways, 195

diagnostic commands, 288-289

Digi evaluation board, 7

Digi International, 1, 6, 263, 267

DigiKey, 6, 63

DIN pin, 15

DIO0...DIO12 Digital I/O pins, 15, 89

direct actuation, 171-187

base station for, 177-180

code for, 180-187

coordinator for, 173

parts for, 172

routers for, 174-177

direct I/O, 88-89

direct phenomena, 86

distal phenomena, 87

distance, sensors for, 86

doorbell, 67-84

breadboard for, connecting, 68

breakout board for, connecting, 68-69

button input for, $72-73,77,81$

buzzer output for, 73, 78, 83

coordinator for, 68

feedback for, 80-83

nap doorbell, 83

parts for, 67

programs for, $77-80,81-83$

router for, 68 
troubleshooting for, 78

XBee radios, configuring, 75-77

XBee radios, connecting, 69-70

DOUT pin, 15

drivers for adapter, 8,33

DTR pin, 15

\section{E}

EEML (Extended Environmental Markup Language), 245

electrical power, testing for, 79

electromagnetic spectrum, 24

electronic sensors (see sensors)

electronics supplies, buying, 61-63

embedded gateways, 190-192

Ember components, 2, 267

end device, 27, 161-163

endpoints, 238

errors (see troubleshooting)

Ethernet connections, 194

Ethernet protocol, 190

events, triggering (see direct actuation; remote actuation)

exploratory data analysis, 85

Explorer adapter, 8

Extended Environmental Markup Language (EEML), 245

\section{$\mathbf{F}$}

feedback doorbell, 80-83

FIO, Arduino, 269

firmware, 15

configuring, 35-39

update software for, 17, 32-39

updating from iDigi server, 210-212

flex sensor, 86

fonts used in this book, $x v$

force sensor, 86

frame (see API frame)

Freescale components, 1, 267

FTDI drivers, 8, 33

\section{G}

galvanic skin response (GSR) sensor, 86 gas sensor, 86

gateways, 189-195, 189

(see also XIG (XBee Internet Gateway))

ConnectPort gateways, 195-202 embedded gateways, 190-192

Internet gateways, 192-195

GND pin, 15

GPRS connections, 194

GPRS protocol, 191

Gravitech XBee to USB Adapter, 9

GSR (galvanic skin response) sensor, 86

gyroscope sensor, 86

H

Hall effect sensor, 86

Hayes command set, 47

hexadecimal notation, 30-32

conversion table for, 269

pin states represented as, 134

HomePlug Alliance protocol, 191, 257

human protocols, 113

HyperTerminal program, 18

I

I/O (input/output), 87-93

AT commands for, 90-92, 284-288

XBee radio capabilities for, 87,89

I/O Data Sample Rx Indicator frame, 131-135

iDigi server, 203-214

adding ConnectPort to, 206-209

administration of, 204-206

configuring, 209-210

firmware updates using, 210-212

viewing remote XBee networks, 212-214

IEEE 802.15.4 standards, 26, 263

indirect phenomena, 87

input/output (see I/O)

Internet gateways, 192-195, 214

(see also XIG)

inverse square law, 24

J

Jameco, 63

Jennic components, 267

JPachube library, 249

K

key-based security, 242

L

Laird Technologies, 267 
large numbers, representing as bytes, 135

layers, ZigBee protocol, 25-26, 30, 236-240

LEDs, testing for power, association, or signal using, 79

length bytes, 115, 118

libraries for API, 141

JPachube, 249

NewSoftSerial, 225, 227

xbee api, 250

light

doorbell feedback using, 81

romantic lighting sensor, 93-109

sensors for, 86

LilyPad XBee, 269

link keys, 242

Linux

adapter port, determining, 40

terminal program for, 20, 40

X-CTU program with, 33, 36

Linux:downloading Processing IDE, 150

local interactions, 112

logic-level serial (see TTL serial protocol)

LS Research, 267

M

MAC (media access controller) layer, 236

MAC address, 207

Macintosh

adapter port, determining, 40

configuring XBee radio, 43-46

downloading Processing IDE, 150

serial port for, 60

terminal program for, 18, 19, 20

magnetic fields, sensor for, 86

MAKE: magazine, 6

Maker SHED, 6, 62

many-to-one routing, 240

Max/MSP library, 141

media access controller (MAC) layer, 236

Mega, Arduino, 59

mesh networking, 2, 26, 28, 236

messages stored for sleeping devices, 161-162

Microchip components, 267

microcontroller, 57

(see also Arduino)

external, 58

not using, 88

microphone sensor, 86,87

Mini, Arduino, 59 mobile data connections, 194

motion sensor, 86

Mouser, 63

multimeter, 13, 79, 104, 147, 159, 176, 226

N

nap doorbell, 83

network keys, 242

Network layer, 26, 236

networking commands, 278-280

networks, 27

(see also wireless sensor networks; ZigBee network)

connectivity between (see gateways)

protocols for, 189

New Micros XBee Dongle, 9

NewSoftSerial library, 225, 227

\section{0}

ON pin, 15

P

Pachube site, 245-257

account for, signing up, 246

API key for, 248

program for, 249-256

registering a feed, 246

troubleshooting sensor network using, 256

packet sniffers, 267

pair network, 27

PAN addresses, 29, 216

Panasonic, 267

parent device, messages stored by, 161-162

(see also coordinator device; router device)

photocell, 86, 87

PHP code, running on XIG, 220-221

PHY (physical) layer, 25, 236

picocom program, 20, 40

pin configurations, 15, 89

ports, for adapter, 40

position, sensors for, 86

potentiometer, 86

power (VCC) pin, 15

pressure sensor, 86

PRO version, XBee radio, 2

Processing \& Java library, 141

Processing IDE, 150-152, 262

profiles, application, 237-238 
protocols, 113-119

proximal phenomena, 86

pulse sensor, 86

PureData library, 141

PWM0 pin, 15

Python language

library for, 141

resources for, 262

used for XIG, 215

\section{$\mathbf{R}$}

radio, 23-24, 23

(see also XBee radio)

Radio Frequency ID (RFID), 191

radio signal, testing for, 79

radio waves, 24

Radiocrafts components, 267

RadioPulse components, 267

Radiotronix components, 267

ranging sensor, 86

real-world events, triggering (see direct actuation; remote actuation)

remote actuation, 193

Remote AT Command Request frame, 135137

Remote Command Response frame, 137-138

remote control (see direct actuation)

remote interactions, 112

remote management of ConnectPort gateways, 203-214

RESET pin, 15

RF interfacing commands, 281-282

RF without protocols, 191

RFID (Radio Frequency ID), 191

romantic lighting sensor, 93-109

base station for, 99-104, 106

coordinator for, 94

feedback for, 104-109

parts for, 94

program for, 106-109

router for, 96, 105

sensor board for, 97-99, 104

troubleshooting, 103

rotary encoder, 86

rotation, sensors for, 86

router device, 26

routing, 26, 240-241

RSSI pin, 15

RTS pin, 15, 244
RX pin, 15

\section{$S$}

screen program, 20

security, 242-243

AT commands for, 281

protocols for, 29

Seeeduino XBee Shield, 269

self-healing mesh, 26

sensors, types of, 85-87

serial flow control, 244-245

serial interfacing commands, 283-284

serial terminal program, 15-20, 40

Series 1 hardware, XBee radio, 1, 3

Series 2 hardware, XBee radio, 2, 3

sharing data, 245-257

shields, 12, 268

sidewalk telescopes, 265

simple actuator network, 178-187

simple sensor network, 142-160, 246-257

skin response, sensor for, 86

sleep mode, 161-167

advantages of, 162

AT commands for, 290

configuring, 163-167

constraints of, 162

end devices in, 161-163

resetting a device to wake it, 170

storage of messages for sleeping devices, 161-162

for temperature sensor network, 167-171

waking device, 166, 167, 170

SLEEP/ON pin, 15

SLEEP_RQ pin, 15

Smart Energy 2.0 specification, 257

smoke sensor, 86

solderless breadboard, 9

sound, sensor for, 86

source routing, 240

SparkFun Electronics, 6, 63

SparkFun XBee Explorer, 8

SparkFun XBee Shield, 269

stack layers, ZigBee protocol, 236-237

star network, 27

start bytes, 114

start delimiter, API frame, 117

stretch sensor, 86

subtext phenomena, 87

switch sensor, 86, 87 
Telegesis components, 267

telescopes, sidewalk, 265

Telit components, 267

temperature sensor network, 142-160

base station for, 148-150

coordinator for, 143-144

parts for, $142-143$

program for, $152-160$

router for, 144

sensor boards for, 144-148

sharing data with Pachube, 246-257

sleep mode for, 167-171

troubleshooting, 158-160

temperature, sensor for, 86

Tera Term program, 19, 41-43

terminal program (see serial terminal program)

Texas Instruments, 267

thermistor, 86

tilt sensor, 86

transparent mode, 46-47

troubleshooting

Arduino board, common mistakes using, 266

chat session problems, 55

command mode, failure to enter, 46

ConnectPort gateway problems, 197, 201, 202

diagnostic commands, 288

doorbell problems, 78

electrical power, testing for, 79

modem configuration file not found, 36

Pachube data sharing in sensor network, 256

radio signal, testing for, 79

resetting a device, 170

romantic lighting sensor, 103

temperature sensor network, $158-160$

Twitter reader problems, 233

"Unable to communicate with modem" error, 35

wake-up problems, 170

XBee radio, common mistakes using, 265

XIG connection problems, 219

TTL serial protocol, 190

Twitter reader, 221-233

Arduino board for, preparing, 224-227

Arduino board for, programming, 227-233

LCD output for, 225-227 parts for, 222

router for, 223-224

troubleshooting, 233

TX pin, 15

\section{U}

Uno, Arduino, 59

USB (Universal Serial Bus) protocol, 191

USB adapters, 7-9

USB Development Board, 269

USB XBee Adapter, 269

\section{V}

VCC (power) pin, 15

vendors, XBee radio, 6

VREF pin, 15

\section{W}

waking a sleeping device, 166, 167, 170

website for this book, xvi

WiFi connections, 194

WiFi protocol, 192

Windows

adapter port, determining, 40

configuring XBee radio, 41-43

downloading Processing IDE, 150

serial port for, 60

setting up ConnectPort, 197

terminal program for, 18, 19

wireless sensor networks

benefits of, xi

chat session project, 50-56

direct actuation project, 172-187

doorbell project, 67-84

romantic lighting sensor project, 93-109

sharing projects with others, 259

suggested projects for, 258

temperature sensor project, 142-160, 167$171,246-257$

Twitter reader project, 221-233

$\mathrm{X}$

X-10 protocol, 192

X-CTU program, 17, 33-39

XBee Adapter Kit, 269

XBee API frame (see API frame)

xbee api library, 250 
XBee API protocol (see API protocol)

XBee Dongle, 269

XBee Explorer, 269

XBee Explorer Serial, 269

XBee Internet Gateway (see XIG)

$\mathrm{XBee}$ radio

acting as gateway, 190

addresses for, 202

antennas, 4-5

buying, 1-6

command mode, 43, 44, 46-47

configuring, 40-56

connectors for, 268

direct I/O with, 88-89

documentation for, 235

input/output capabilities of, 87, 89

limitations of, 88

modules for, 268

naming, 202

pin configurations, 15, 89

PRO version, 2

Series 1 hardware, 1, 3

Series 2 hardware, 2, 3

shields for, 12, 268

transparent mode, 46-47

troubleshooting, 265

vendors, 6

XBee remote network, viewing, 212-214

XBee to USB Adapter, 269

XIG (XBee Internet Gateway), 214-233 code for, 216

installing and configuring, 216-218

PHP code running on, 220-221

security for, 218

testing, 218-219

\section{Z}

Z-Wave Alliance protocol, 192

ZCL (ZigBee Cluster Library), 239

ZDO (ZigBee Device Objects) layer, 236

ZigBee Alliance, xii, 237, 243, 263

ZigBee Cluster Library (ZCL), 239

ZigBee Device Objects (ZDO) layer, 236

ZigBee network, 25-32

ad hoc creation of, 26

addresses for, 28-29

channels for, 29

devices in, 26

inverse square law and, 24 modules for, 267

packet sniffers for, 267

security for, 242-243

self-healing mesh of, 26

topology of, 27-28

ZigBee protocol, xi, 25

future of, 257

layers of, 25-26, 30, 236-240

resources for, 243, 263-264

ZigBee Receive Packet frame, 129-131

ZigBee Transmit Request frame, 124-127

ZigBee Transmit Status frame, 127-129

ZTerm program, 19 


\section{About the Author}

Robert Faludi is an NYU professor, SVA professor, and an expert consultant on commercial projects, including large-scale home energy monitoring. His work has appeared in The New York Times, on CNET, on Good Morning America, and elsewhere. He is a co-creator of the LilyPad XBee wearable radios, and of Botanicalls, a system that allows thirsty plants to place phone calls for human help.

\section{Colophon}

The animals on the cover of Building Wireless Sensor Networks are dachshunds. The short-legged, elongated dogs were first bred for hunting in the 17th century in Germany; in fact, the name literally means "badger dog." Dachshunds are officially classified as members of the hound family in the United States, though there are some that argue that many varieties, especially wire-haired types, look and behave more like members of the terrier group. The World Canine Organization, which boasts 86 member countries, takes a middle road and specifies a separate group for dachshunds apart from both terriers and other scent hounds.

Further disagreement has arisen involving the official varieties of the dachshund breed. The World Canine Organization defines three sizes: standard, miniature, and rabbit. The American Kennel Club, on the other hand, recognizes only the standard and miniature sizes, arguing that so-called "rabbit" dachshunds are just comparatively smaller miniature varieties. Three coat types are universally recognized, however: smoothhaired, long-haired, and wire-haired.

Though dachshunds are popular pets in the United States, the dogs are perhaps most strongly prized in and associated with Germany. While dachshunds there are generally called Dackel, worthy specimens that are able to pass blood-tracking tests earn the moniker Teckel and are held in higher regard. Furthermore, the animal is so popular that a dachshund, named Waldi, was chosen as the official mascot of the 1972 Summer Olympics in Munich.

The cover image is from Lydekker's Royal History. The cover font is Adobe ITC Garamond. The text font is Linotype Birka; the heading font is Adobe Myriad Condensed; and the code font is LucasFont's TheSansMonoCondensed. 